\title{
Alexandri de figuris sententiarum et verborum
}

\author{
Inauguraldissertation \\ zur Erlangung des Grades eines Doktors der Philosophie \\ der Georg - August- Universität
}

vorgelegt von

Ahn Jaewon

aus Seoul, Südkorea

Januar, 2004 
Inhaltsverzeichnis

Einleitung

I-XLII

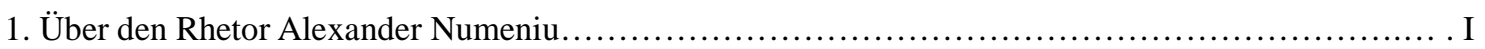

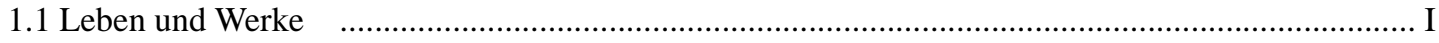

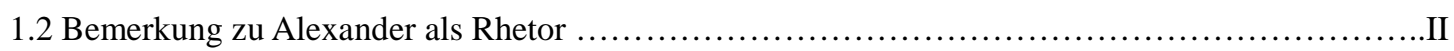

2. Über das Werk De figuris sententiarum et verborum......................................... VII

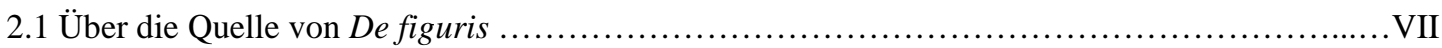

2.2 Über die Rezeption von De figuris ...............................................

2.3 Über die Struktur von De figuris .....................................................

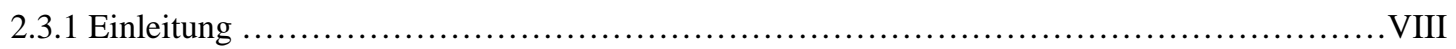

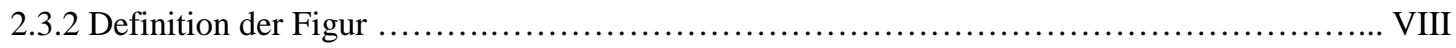

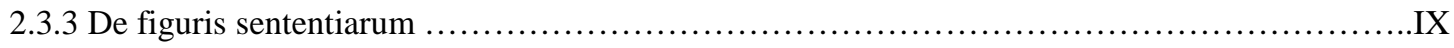

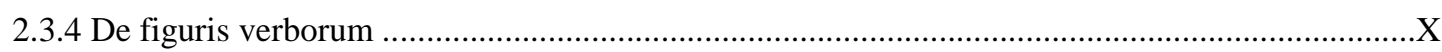

2.4 Über den Stil von De Figuris......................................................

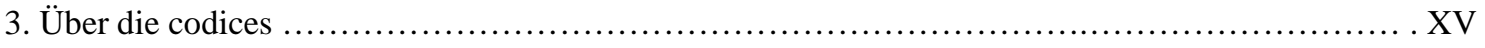

3.1 Paris. 1741 und Marc. 512 .........................................................

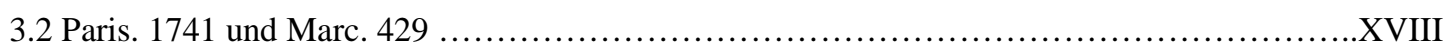

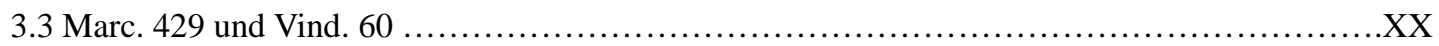

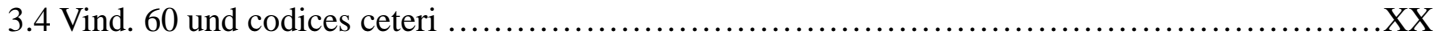

3.5 Paris. 1656 und die editio princeps Aldina .............................................

3.6 Paris 2087 und Notae Marginales in Paris. 1741 .......................................................................XXIII

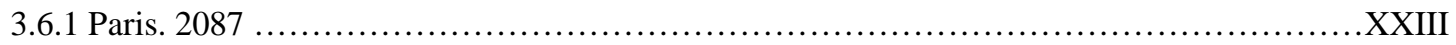

3.6.2 Die Familie des Paris. 2087 ....................................................XIX

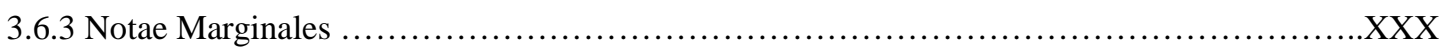

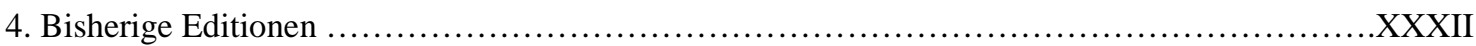

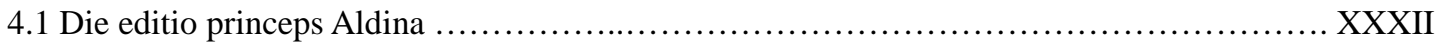

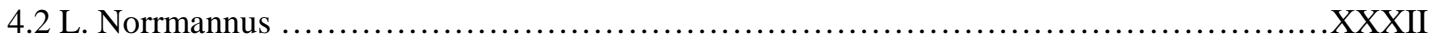

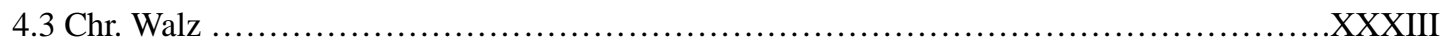

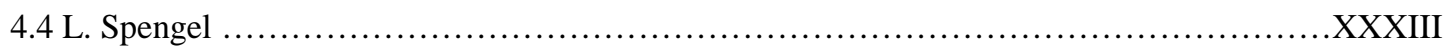

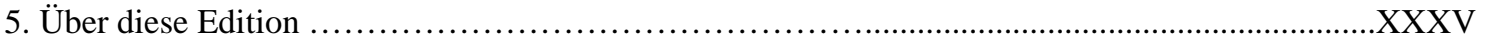

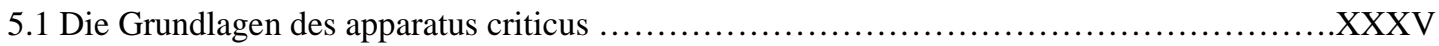

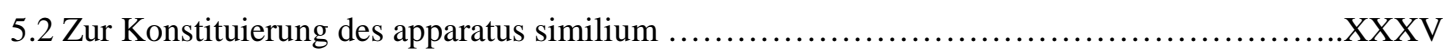

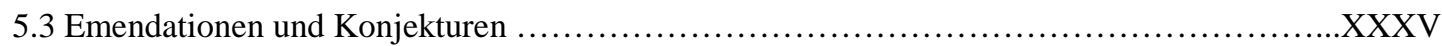

5.4 Kleine Bemerkungen zu Grammatik und Orthographien .............................XXVI

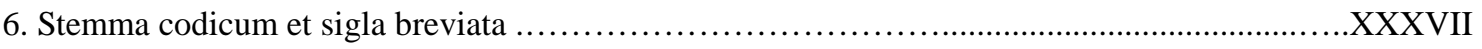

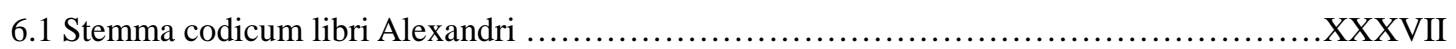




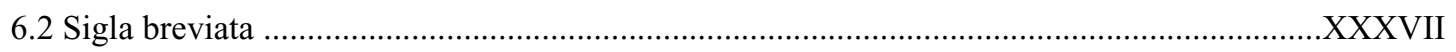

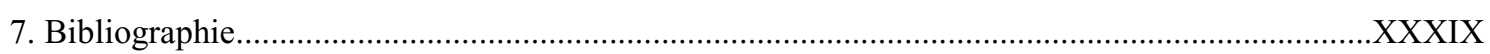

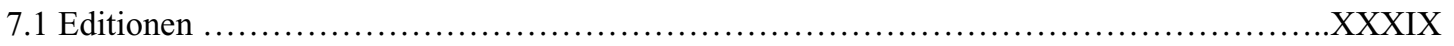

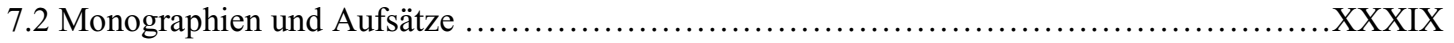

Alexandri de figuris sententiarum et verborum............................................................................ 1-78

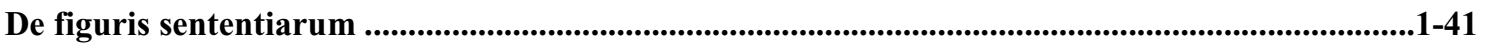

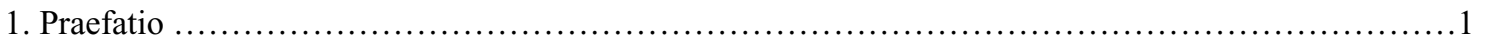

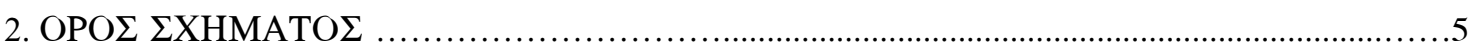

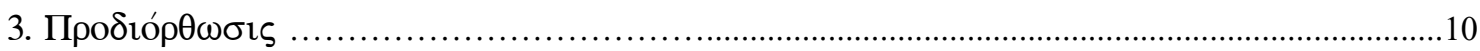

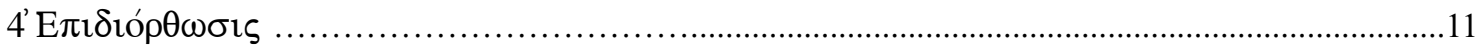

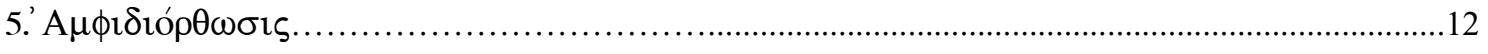

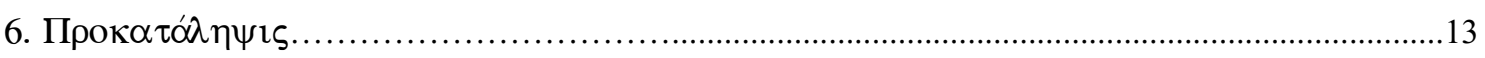

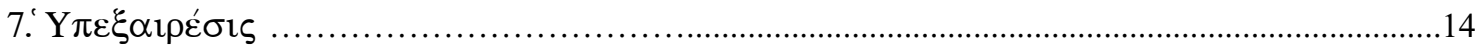

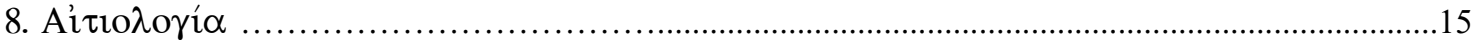

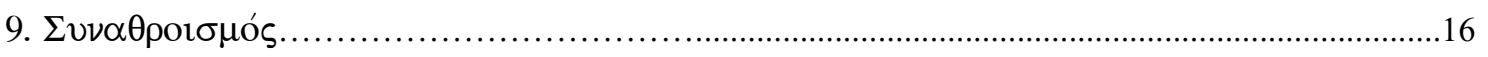

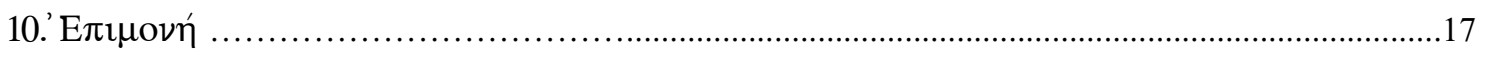

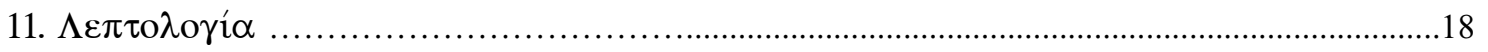

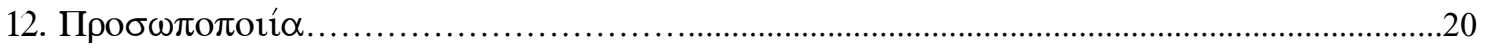

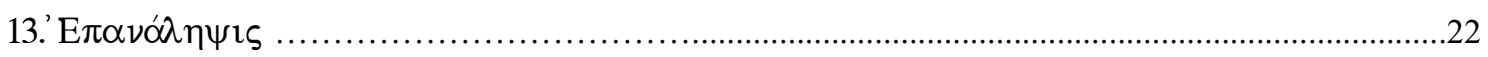

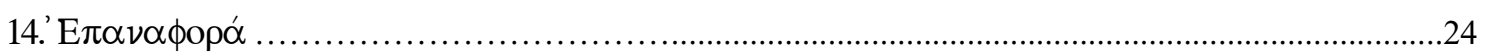

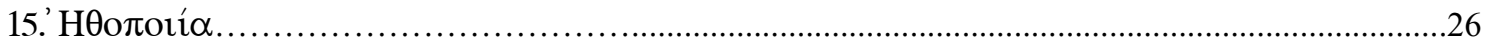

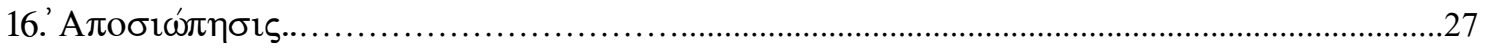

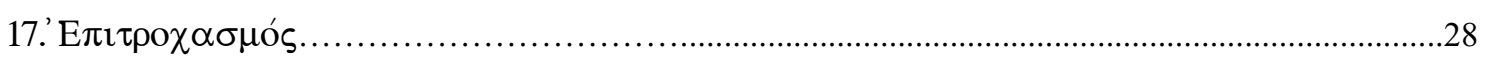

18. Eıр

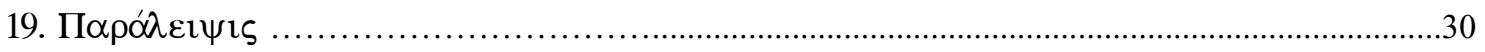

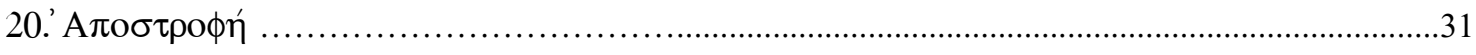

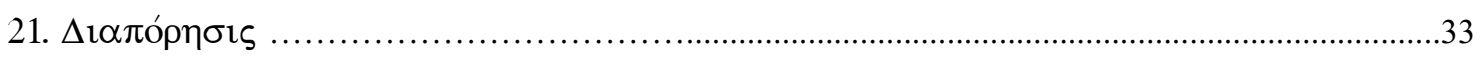

22.' Eр

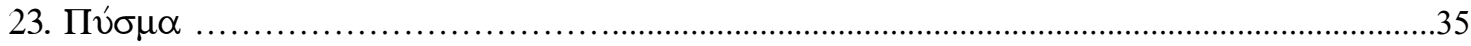

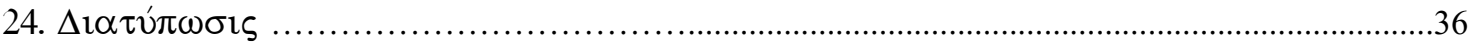

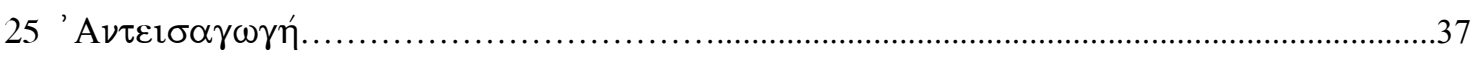

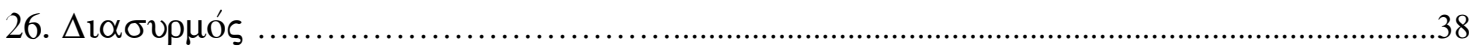

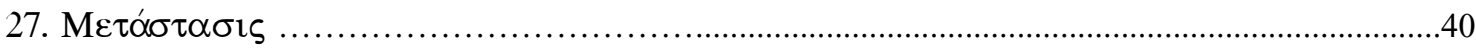

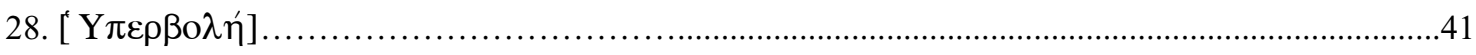




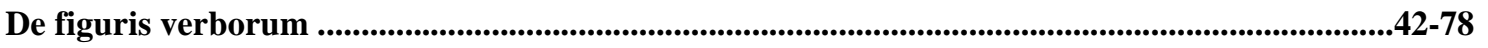

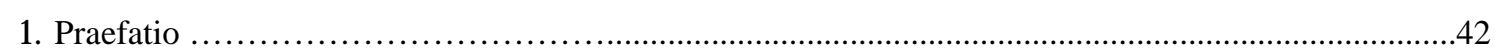

2. A nadipl ws ij hlP a I il ogi

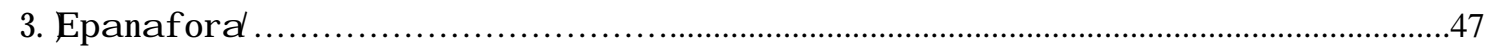

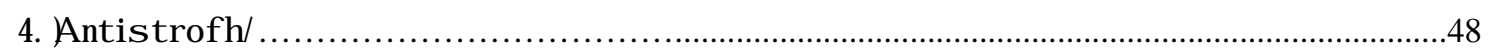

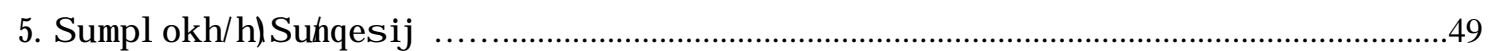

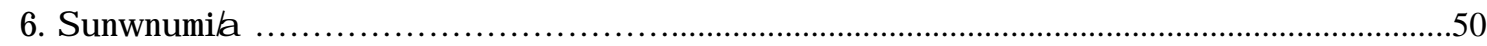

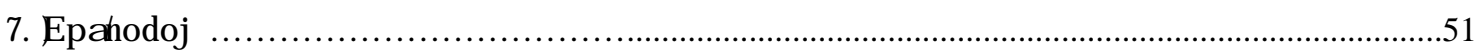

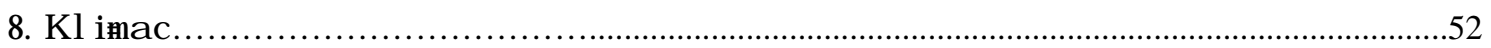

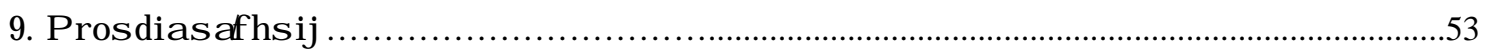

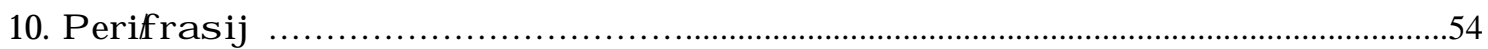

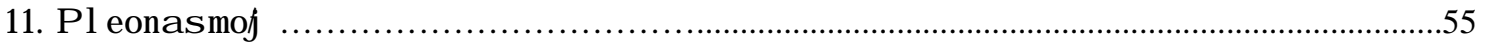

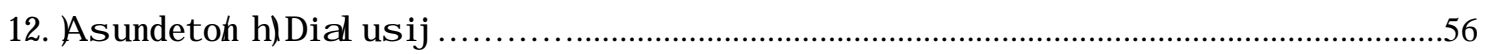

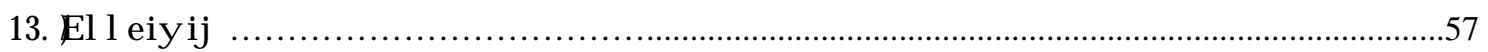

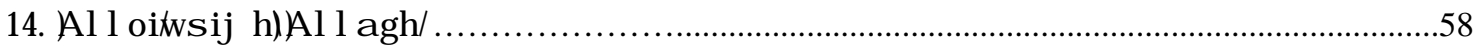

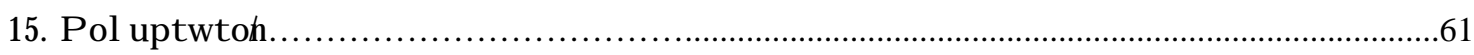

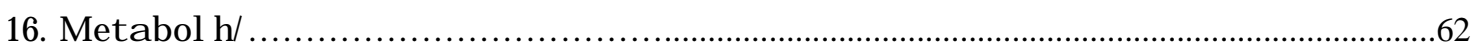

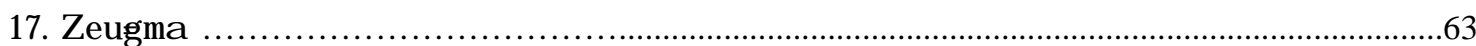

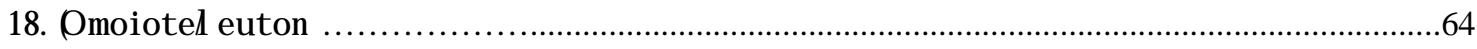

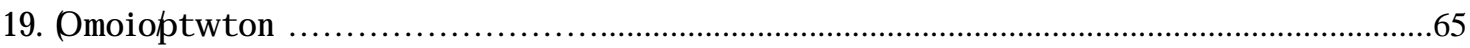

20. P a r onoma s i

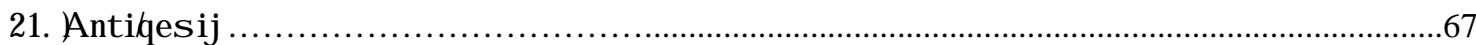

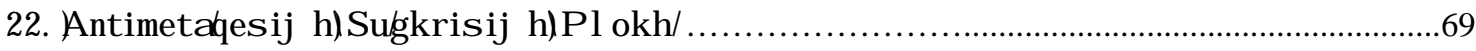

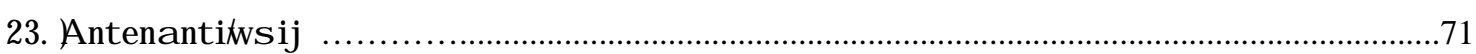

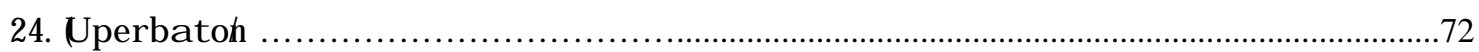

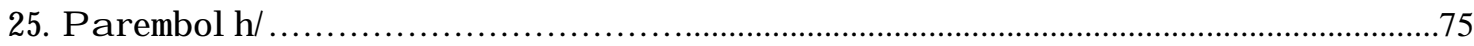

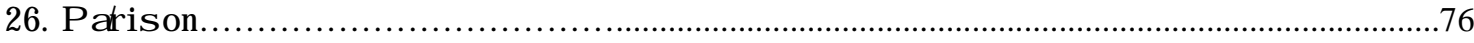

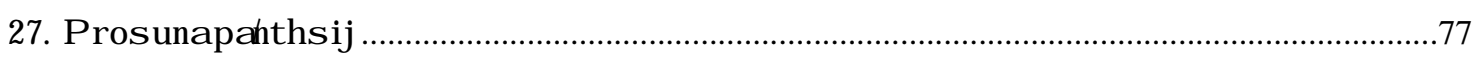

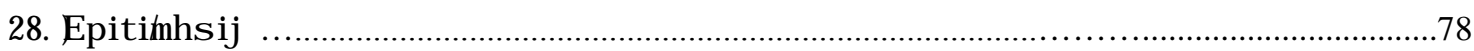

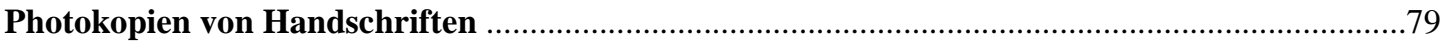

Indices 


\section{1. Über den Rhetor Alexander Numeniu}

\subsection{Leben und Werke}

Alexander war Sohn des Numenios, der eine Trostrede für Kaiser Hadrian (reg. 117 - 138 n. Chr.) über den Tod des Antinoos gehalten hat ${ }^{1}$. Alexander war also als Rhetor in posthadrianischer Zeit tätig. Leider aber wissen wir sonst gar nichts über sein Leben. Auch die $S u d a^{2}$ stellt ihn nur mit einer

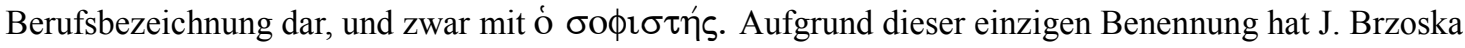
behauptet, daß Alexander „ein hervorragender Rhetor‘3 ${ }^{\text {‘3 }}$ war. Es ist uns aber nicht bekannt, in welchem Ausmaß Alexander als Redner wirksam geworden ist. Die Behauptung von Brzoska ist deshalb nur eine Vermutung. Klar ist jedoch, daß Alexander ein bedeutender Verfasser rhetorischer Schriften war. Denn man kann den Namen Alexander häufig bei späteren Technographen finden, wie bei Iulius Rufinianus: Hactenus Aquila Romanus ex Alexandro Numenio: exinde ab eo praeteritas (sc. figuras), aliis quidem proditas subtexuimus (sc. Rufinianus). ${ }^{4}$ Auch in verschiedenen Rhetoriktraktaten anonymer Autoren erscheint der Name Alexanders, so in:

\section{ANONYMOY ПPO $\Lambda$ EOMENA T $\Omega$ N EYPE $\Sigma E \Omega N^{5}$, ANONYMOY $\Sigma$ XO $\Lambda$ IA EI $\Sigma$ EPMOГENOY $\Sigma$ EYPE $\Sigma$ E $\Omega$ N TOMOY $\Sigma^{6}$,

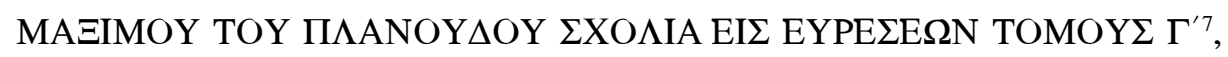 ПРО $\Lambda$ ЕОМЕNA TH $\Sigma$ EPMOГЕNOY $\Sigma$ PHTOPIKH $\Sigma^{8}$,}

\section{ANONYMI SEGUERIANI TEXNH RHTORIKH ${ }^{9}$.}

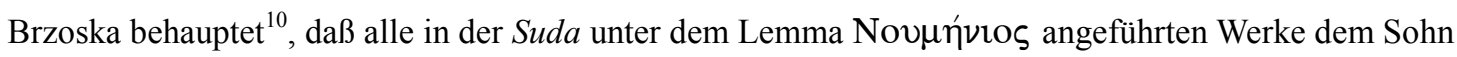

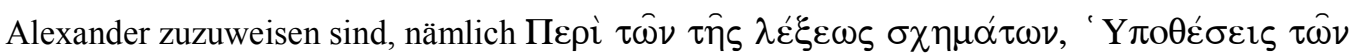

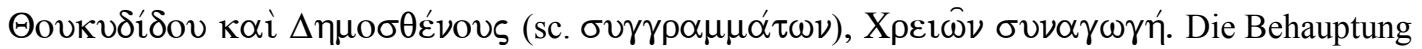
Brzoskas ist akzeptabel. Dafür gibt es zwei Gründe:

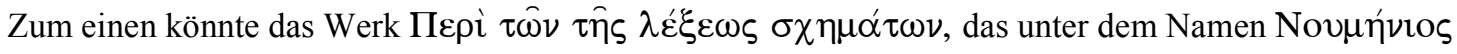
aufgezählt worden ist, der zweite Teil von De figuris sententiarum et verborum sein. Ein Beleg dafür

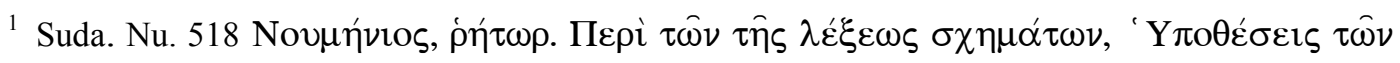

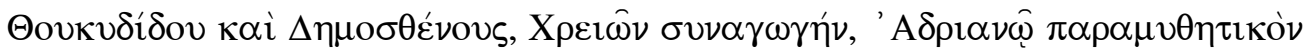
Eis 'Avtívoov.

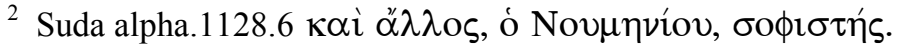

${ }^{3}$ Vgl. J. Brzoska, s.v. ,Alexandros', RE I 2, 1894, S. 1456-1459.

${ }^{4}$ Iulii Rufiani DE FIGURIS SENTENTIARUM ET ELOCUTIONIS, RLM, ed. K. Halm, Leipzig 1863, S.38.

${ }^{5}$ RG VII pars I, ed. Chr. Walz, Stuttgart 1833, S. 53, 11.

${ }^{6}$ RG VII pars II, ed. Chr. Walz, Stuttgart 1833, S.763, 14.

7 RG V, ed. Chr. Walz, Stuttgart 1833, S. 403, 22.

${ }^{8}$ RG IV, ed. Chr.Walz, Stuttgart 1833, S. 35, 8.

9 RG I 1, ed. Spengel/Hammer, Leipzig 1894, S. 352-398.

${ }^{10}$ Siehe dazu J. Brzoska, s.v. ,Alexandros', RE I 2, 1894, S. 1456-1459. 
scheint der Codex Parisinus BN gr. 2762 zu sein, der nur den ersten Teil von De figuris sententiarum et verborum überliefert: De figuris sententiarum et verborum könnte also zunächst vollständig abgeschrieben worden sein, der erste Teil dann aber verlorengegangen sein. Es ist zu vermuten, daß der Verfasser der Suda nur den zweiten, ,kopflosen', d.h. anonymen Teil von De figuris sententiarum et verborum gelesen und ihn dann dem Vater N o u mh hi oj zugewiesen hat. Denn der Verfasser der Suda hat unter dem Namen Alexander keine Werke erwähnt, obwohl Alexander mindestens drei rhetorische Werke verfasst hat, die die späteren griechischen und lateinischen Schulrhetoriker wie Phoebammon oder Aquila beeinflußten. $^{11}$

Zum anderen könnte Alexander auch die Upoqek eij tw Q oukudidou ka ilDhmos qehouj (sc. s uggra mma t w $n$ ) geschrieben haben. Denn Alexander ist derjenige, der im Gegensatz zu Cicero eine große Vorliebe für den Stil von Historikern wie Herodot, Xenophon und insbesondere Thukydides entwickelte. Nach Cicero ist der Stil von Thukydides für einen Redner nicht geeignet, da die von Thukydides verfaßten Reden in der Praxis nur schwer zu verstehen seien. ${ }^{12}$ Alexander hat aber oft Thukydides - Sätze als Beispiele verwendet. So gibt es etwa zu Beginn von De figuris sententiarum et verborum ein Beispiel, das der Historia des Thukydides entnommen ist. ${ }^{13}$

Sicher ist, daß Alexander ein grundlegendes Buch über die Rhetorik verfaßt hat, das als Vorlage des Textes des Anonymus Seguerianus gilt ${ }^{14}$. Er hat sich auch mit einer anderen rhetorischen Schrift $^{15}$ beschäftigt, die fragmentarisch unter dem Namen eines Menander überliefert ist. ${ }^{16}$ Schließlich hat er den Figurentraktat De figuris sententiarum et verborum (= De figuris) geschrieben, der auf der Lehre des Caecilius von Kaleakte fußt.

\subsection{Bemerkung zu Alexander als Rhetor}

Alexander ist Theodoreer: Es ist charakteristisch für die Rhetoriklehre Alexanders, daß sie von der Lehre der Apollodoreer ${ }^{17}$ abweicht $^{18}$ und identisch oder ähnlich mit der Lehre der Theodoreer ${ }^{19}$ ist. Belege

11 Vgl. dazu C. Walde, Alexandros 25, DNP I, 1996, S. 479.

12 Cic. Orat. 30: Thucydides autem res gestas et bella narrat et proelia, graviter sane et probe, sed nihil ab eo transferri potest ad forensem usum et publicum: ipsae illae contiones ita multas habent obscuras abditasque sententias, vix ut intellegantur; quod est in oratione civili vitium vel maximum.

13 Vgl. Alexander, De figuris sententiarum et verborum, RG III, ed. Spengel/Hammer, Leipzig 1894, S.10, 17-19.

14 Vgl. dazu G. Kennedy, The Art of Rhetoric in the Roman World, Princeton 1972, S. 618.

15 Alexandri P E RI RH T O RIK W N A F O RM W N, RG III, ed. ed. Spengel/Hammer, Leipzig 1894, S. 16.

${ }^{16}$ Hoc Alexandri fragmentum primae Menandri parti per i legk w mi w $n$ fol. 610 Ald. propter simile argumentum ei ut videtur insertum adhaeret, disiunxit Walzius titulo addito peri $r$ h t or i $\mathrm{k}$ W af or mw \#. nos (Spengel) Alexandri libro de exornationibus praemisimus. L. Spengel, wie Anm. 15, Praefatio (S. III).

17 Die Apollodoreer sind diejenigen, die ,nullam oratori relinquebant libertatem, qua ille usus regulas artis ad tempora causas occasiones necessitates accommodare posset. Omnia in eorum aridissimis libris erant provisa, praescripta, certis finita praeceptis.“(K. Morawski, De Dionysii et Caecili studiis rhetoricis, RhM 34 /1879, S. 371). Siehe dazu G. Ballaira, La dottrina delle figure retoriche in 
dafür zeigen sich in der folgenden Gegenüberstellung ${ }^{20}$ :

\begin{tabular}{|c|c|c|c|}
\hline & Alexander & Theodoreer $^{21}$ & Apollodoreer \\
\hline $\begin{array}{l}\text { Auffassung der } \\
\text { Rhetorik }\end{array}$ & 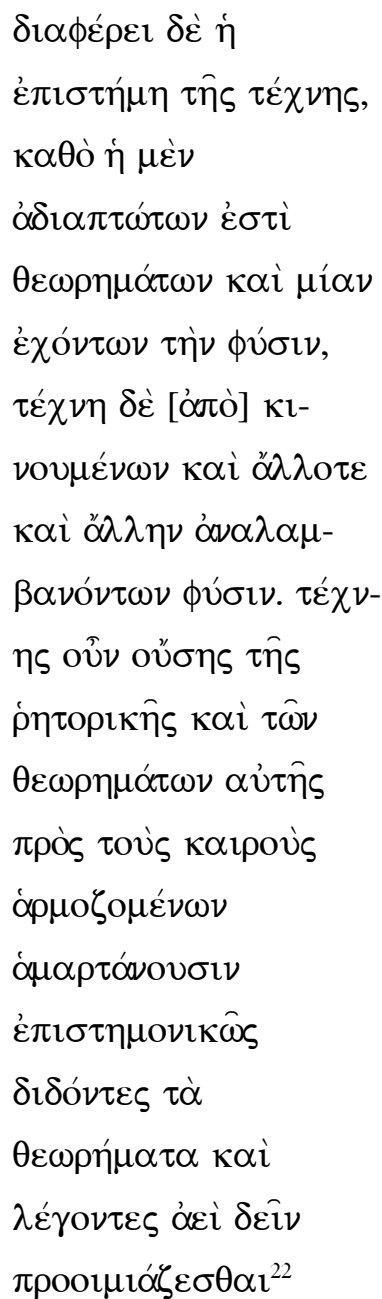 & 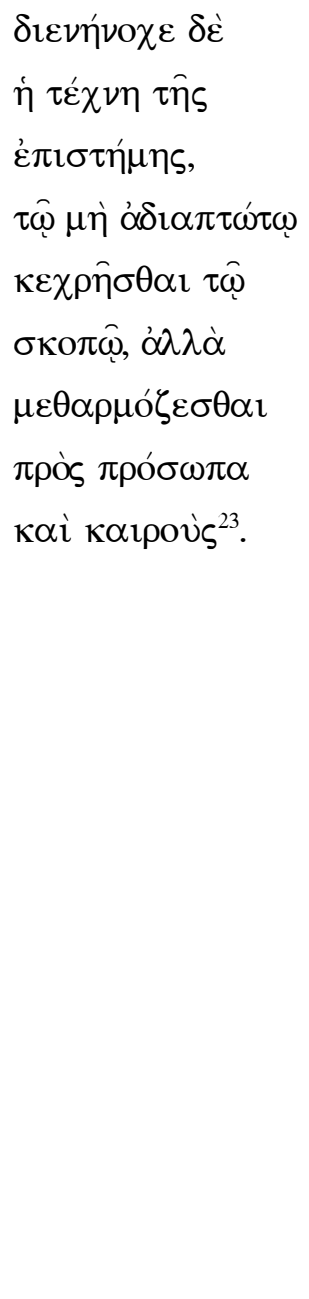 & 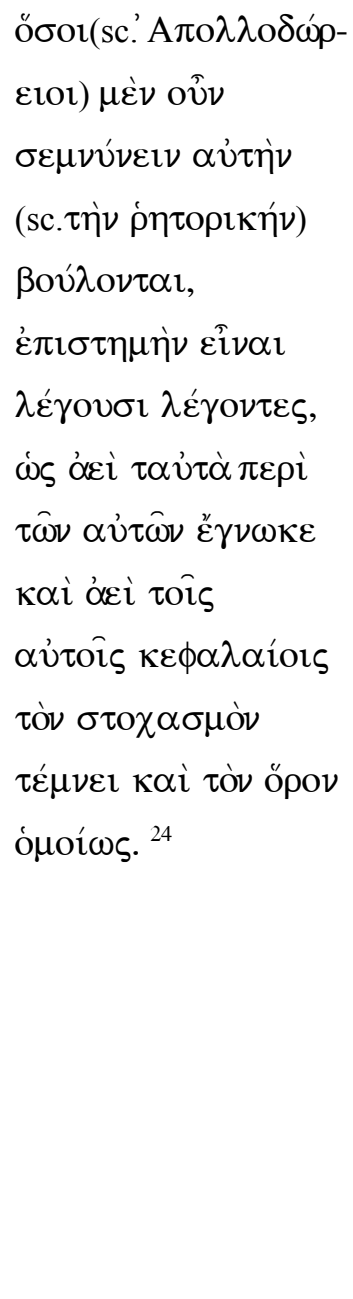 \\
\hline
\end{tabular}

Apollodoros di Pergamon, in: Quaderni Urbinati di cultura classica 5 (1968), S. 37-91.

${ }^{18}$ Vgl. dazu G. Kennedy wie Anm. 14, S. 618 f.

19 Die Theodoreer sind diejenigen, bei denen „Vieles dem freien Ermessen anheimgegeben ist“ (M.

Schanz, Die Apollodoreer und die Theodoreer, Hermes 25 (1897), S. 49). Siehe dazu G.M.A. Grube,

Theodoros of Gadara, in: AJP 80 (1959), S. 337-365.

${ }^{20}$ In diesem Schema werden diejenigen Beispiele zum Vergleich herangezogen, die deutlich zeigen, daß Alexander Theodoreer ist. Vgl. dazu M. Schanz, Die Apollodoreer und die Theodoreer, Hermes 25 (1897), S. 36-54.

${ }^{21}$ Siehe dazu M. Schanz, Die Apollodoreer und die Theodoreer, Hermes 25 (1897), S. 49: „Der Unterschied bei den Schulen bezieht sich nicht auf untergeordnete Fragen der Technik, sondern auf den Mittelpunkt aller rhetorischen Thätigkeit, nämlich auf die Rede.“

${ }^{22}$ AN $\Omega$ NYMOY TEXNH PHTOPIKH, RG I 1, ed. Spengel/Hammer, Leipzig 1894, S. 358, 23 - 359, 4.

${ }^{23}$ Sopatros, RG V, ed. Chr. Walz, Stuttgart 1833, S. 4, 7f. Vgl. dazu M. Schanz wie Anm. 21, S. 52.

${ }^{24}$ Wie Anm. 23, S. 4, 28f. 


\begin{tabular}{|c|c|c|c|}
\hline $\begin{array}{l}\text { Frage der } \\
\text { Narratio }^{25}\end{array}$ & 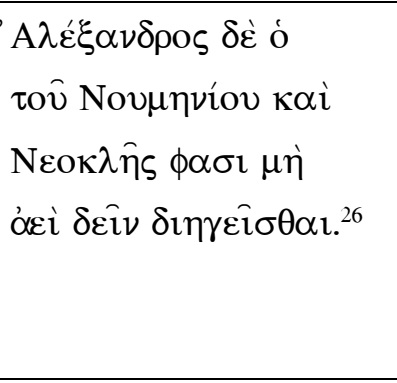 & 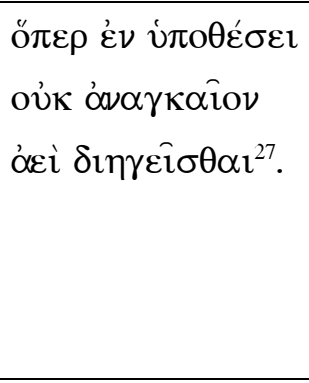 & 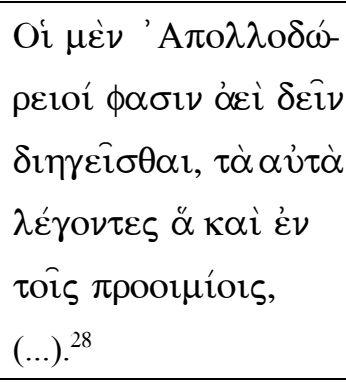 \\
\hline Problem der Figuren ${ }^{29}$ & 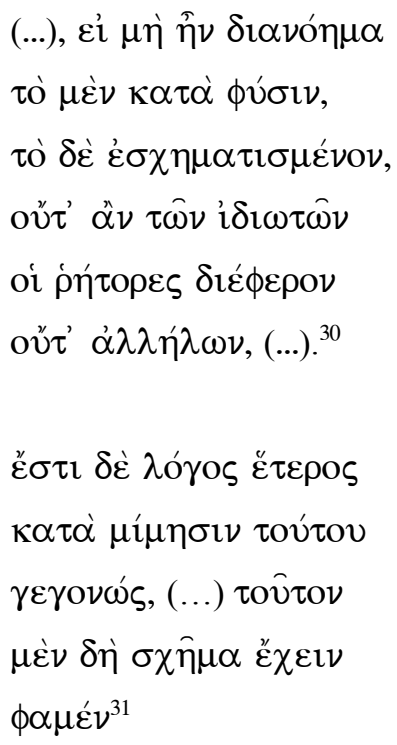 & & 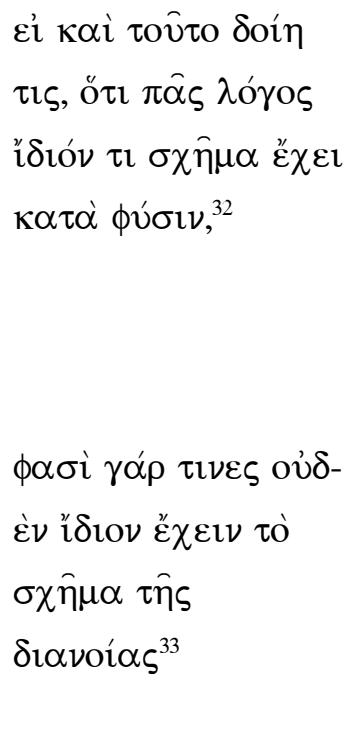 \\
\hline
\end{tabular}

Alexander ist ein selbständiger Theoretiker. Sicher ist, wie der Vergleich gezeigt hat, daß Alexander in der Tradition der Theodoreer steht. Interessant ist jedoch, daß Alexander auch an Theodoros Kritik übt. Dies wird deutlich an der folgenden Gegenüberstellung:

${ }^{25}$ „Auch Quintilian kennt diese Streitfrage, er stellt sich auf Seite der Theodoreer; denn er sagt (Inst. orat. 4.2.4): plerique semper narrandum putaverunt quod falsum esse pluribus coarguitur. Sunt enim ante omnia quaedam tam breves causae, ut propositionem potius habeant quam narrationem." (M. Schanz wie Anm. 21, S. 42).

${ }^{26}$ ANSNYMOY TEXNH PHTOPIKH, RG I 1, ed. Spengel/Hammer, Leipzig 1894, S. 372, 18.

27 Theon Rhet. Aelius, Progymnasmata, RG II, ed. Spengel/Hammer, Leipzig 1894, S. 76, 13.

${ }^{28}$ AN $\Omega N Y M O Y$ TEXNH PHTOPIKH, RG I 1, ed. Spengel/Hammer, Leipzig 1894, S. 372, 1.

${ }^{29}$ Quintilian erwähnt auch diese Streitfrage (Inst. orat. 9.1.12): Quo si contenti sumus, non inmerito Apollodorus, si tradenti Caecilio credimus, incomprehensibilia partis huius existimavit. Interessant ist aber, daß Alexander die Erörterung über Figuren eben mit dieser Streitfrage beginnt und sogar Quintilians Wort „,incomprehensibilia“ mit dem Ausdruck Alexanders „„র $\pi \varepsilon \rho i ́ \lambda \eta \pi \tau \alpha^{\prime \prime}$ (siehe S.1, 8) identisch ist. Vgl. dazu M. Schanz wie Anm. 21, S. 50-51.

${ }^{30}$ Alexander, De figuris sententiarum et verborum, RG III, ed. Spengel/Hammer, Leipzig 1894, S. 11, $30 \mathrm{ff}$.

${ }^{31}$ Wie Anm. 30, S. 12, 22.

${ }^{32}$ Wie Anm. 30, S, 11, 20

33 Wie Anm. 30, S. 12, 15. 


\begin{tabular}{|c|c|}
\hline Theodoros & Alexander \\
\hline 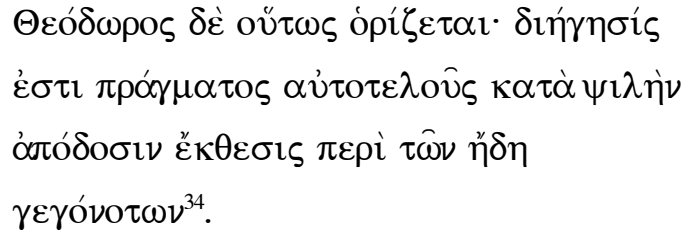 & 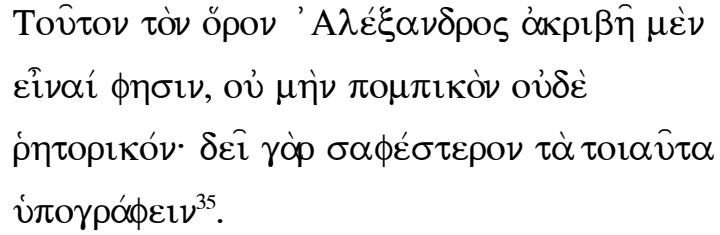 \\
\hline
\end{tabular}

Alexander distanziert sich also von der Lehre der Theodoreer. Dies weist darauf hin, daß Alexander nicht ein fanatischer Theodoreer, sondern ein kritischer und selbständiger Theoretiker ist.

In diesem Zusammenhang zu erwähnen ist die Argumentationslehre Alexanders. Diese Lehre orientiert sich an Aristoteles. Dies zeigt sich an folgendem Vergleich deutlich:

\begin{tabular}{|c|c|}
\hline Alexander & Aristoteles \\
\hline 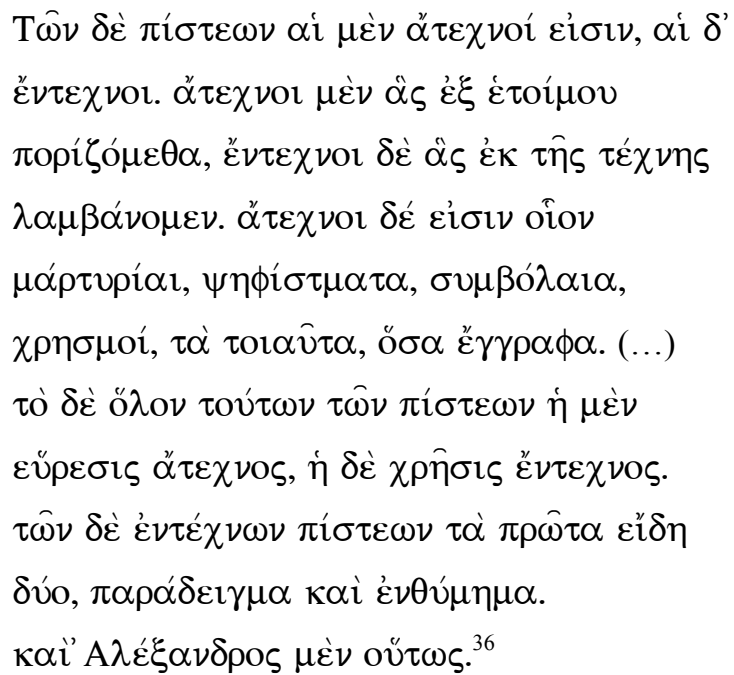 & 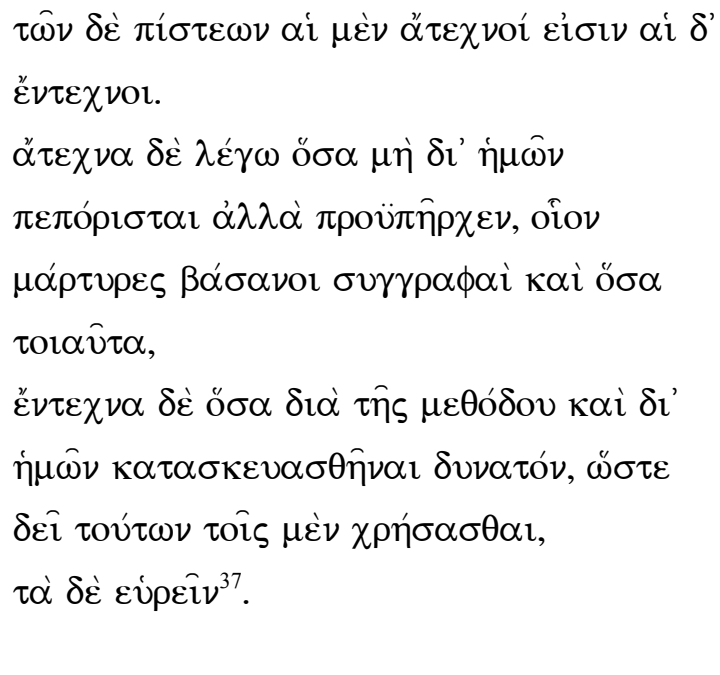 \\
\hline
\end{tabular}

Wie der Vergleich zeigt, ist die Aussage Alexanders fast identisch mit der Formulierung des Aristoteles. Aber Alexander folgt nicht bloß Aristoteles, sondern er versucht deutlich zu machen, was unklar ist. Ein

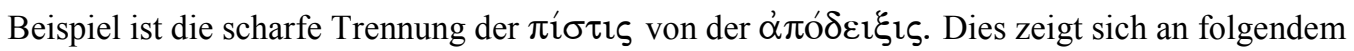
Vergleich deutlich:

\footnotetext{
${ }^{34}$ AN $\Omega$ NYMOY TEXNH PHTOPIKH, RG I 1, ed. Spengel/Hammer, Leipzig 1894, S. 363, 1.

${ }^{35}$ Wie Anm. 34, S. 363, 3.

${ }^{36}$ Wie Anm. 34, S. 378, 4.

37 Arist. Rhet. A. 2. 1355 b 35.
} 


\begin{tabular}{|c|c|}
\hline Alexander $^{38}$ & Aristoteles $^{39}$ \\
\hline 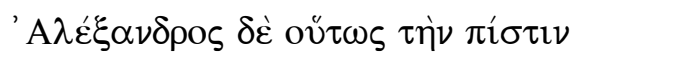 & 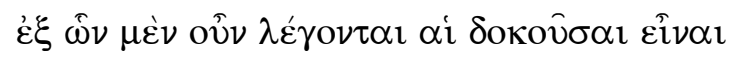 \\
\hline 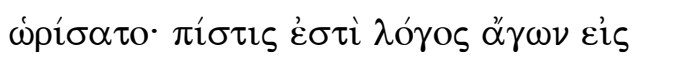 & 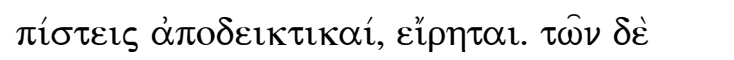 \\
\hline 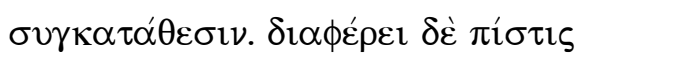 & 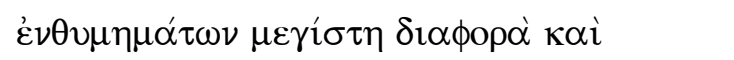 \\
\hline 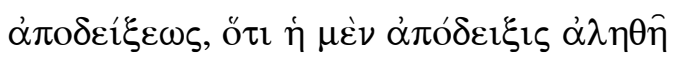 & 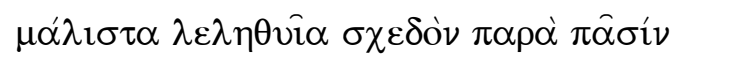 \\
\hline 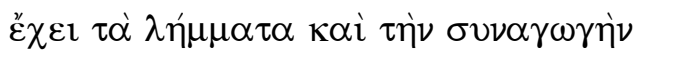 & 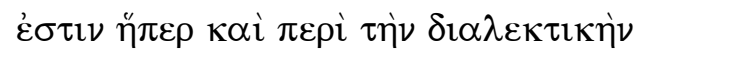 \\
\hline 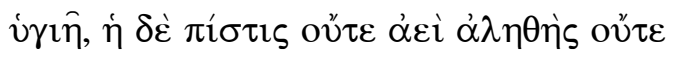 & $\mu \dot{\varepsilon} \theta \mathrm{o} \delta \mathrm{o} \nu \tau \hat{\omega} \nu \sigma v \lambda \lambda \mathrm{o} \gamma 1 \sigma \mu \omega \hat{\omega} \nu \cdot(\ldots) \lambda \dot{\varepsilon} \gamma \omega \gamma \alpha \grave{\rho} \rho$ \\
\hline 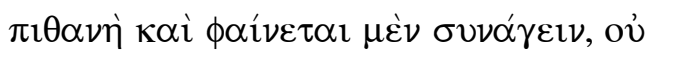 & 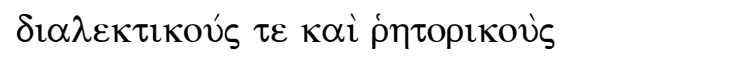 \\
\hline 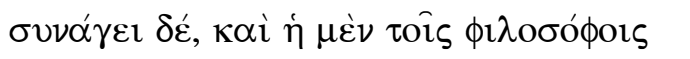 & 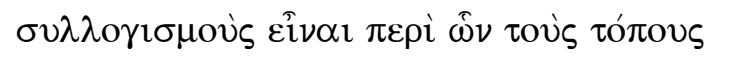 \\
\hline 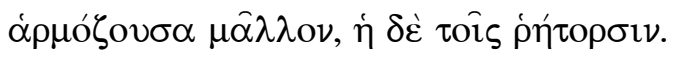 & $\lambda \varepsilon \dot{\gamma o \mu \varepsilon v . ~}$ \\
\hline
\end{tabular}

Es ist klar, daß Alexander von der Lehre des Aristoteles offen abweicht. Dieser Abweichung liegt aber die

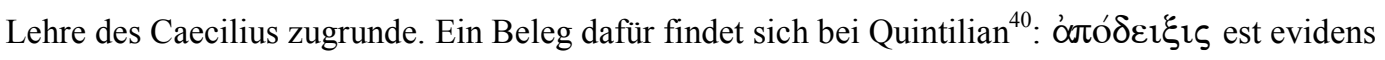

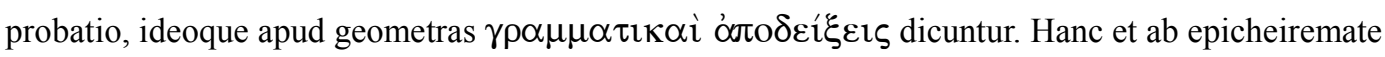
Caecilius putat differe solo genere conclusionis et esse apodixin imperfectum epicheirema eadem causa qua diximus enthymema a syllogismo distare. $\mathrm{Zu}$ diesem Zitat ist noch zu sagen, daß die Differenzierung Alexanders zwar ähnlich der des Caecilius ist, aber sich inhaltlich ein wenig unterscheidet von der des

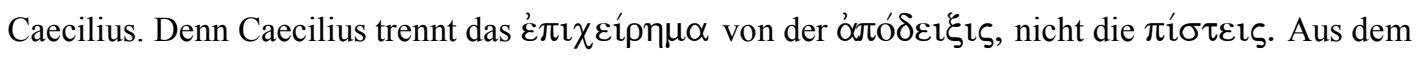
Zitat Quintilians ist festzuhalten, daß die Trennung des Caecilius nicht so scharf wie die Alexanders ist. Dies bestätigt, daß Alexander ein selbständiger Theoretiker ist.

${ }^{38}$ AN $\Omega$ NYMOY TEXNH PHTOPIKH, RG I 1, ed. Spengel/Hammer, Leipzig 1894, S. 377, 22 - 378 , 3.

39 Arist. Rhet. A.2. 1358 a 1.

${ }^{40}$ Quint. Inst. orat. 5.10.7. 


\section{2. Über das Werk De figuris}

\section{1 Über die Quelle von De figuris}

Quintilian nennt in seiner Figurendiskussion im 9. Buch der Institutio oratoria gleich mehrere Verfasser ${ }^{41}$ von Figurenlehrbüchern, die er zwar benutzt hat, die aber nicht auf uns gekommen sind. Zwei von ihnen sind wichtig für die Figurenlehre im Bereich der Schulrhetorik ${ }^{42}$. Da ist zunächst einmal der jüngere Gorgias, der Lehrer von Ciceros Sohn Marcus in Athen war und der eine Figurenlehre in vier Büchern verfaßt haben soll. Diese vier Bücher wurden von Rutilius Lupus zusammengefaßt ins Lateinische übersetzt. Die zwei Bücher umfassenden Ausführungen des Lupus über die Wortfiguren sind in der Form eines Schullehrbuches überliefert worden ${ }^{43}$. Der andere Autor ist Caecilius von Kaleakte ${ }^{44}$. Caecilius war in spätaugusteischer Zeit tätig und hat ein ähnliches, nicht erhaltenes Werk ${ }^{45}$ in zwei Büchern geschrieben, die sich jeweils zur Hälfte auf Sinn- und Wortfiguren bezogen haben sollen. Auf der Grundlage dieser Figurenlehre sind De figuris von Alexander Numeniu und De figuris Demosthenicis von Tiberius $^{46}$ verfaßt. Diese beide Figurentraktate sind also miteinander verwandt.

Das Verhältnis von Alexander und von Tiberius zu Caecilius legte Th. Schwab eingehend in seiner Dissertation $^{47}$ dar. Tiberius benutzte den Caecilius in unselbständiger Weise, so Th. Schwab ${ }^{48}$, Alexander in selbständiger Weise. Nach ihm ist die Selbständigkeit Alexanders im folgenden zu sehen:

„Alexander hingegen (sc. gegen Tiberius) schaltet mit dem Material, das ihm Kaikilios bietet, in freier Weise. Bei manchen Figuren schließt er sich in Einzelheiten, wie in Beispielen oder Erklärungen, an diese Vorlage an, während er das ganze Kapitel in selbständiger Weise umarbeitet, eine andere Systematisierung versucht, auch Stellen aus anderen Vorlagen einarbeitet. Den Wortlaut der Definition pflegt er von Kaikilios unabhängig zu bilden, was für die Behandlung späterer Kapitel z.B. der $\delta \imath \alpha \tau u ́ \pi \omega \sigma \iota \varsigma$ wichtig ist. In einem Falle ( $\pi \alpha \rho \varepsilon \mu \beta о \lambda \eta ́)$ wenigstens wahrte er, soweit sich feststellen ließ, die von Kaikilios herrührende Anordnung und Verknüpfung der Figuren v́ $\pi \varepsilon \rho \beta \alpha \tau o ́ v$ und $\pi \alpha \rho \varepsilon \mu \beta о \lambda \eta ́ ;$ ob auch bei der Anordnung der übrigen Figuren Kaikilios das Vorbild war, läßt sich infolge unserer mangelhaften Kunde von kaikilischen Werken nicht mehr erkennen. “49

41 Institutio oratoria 9. 3. 89: ,et proprie libros huic operi dedicaverunt, sicut Caecilius, Dionysius, Rutilius, Cornificius, Visellius aliique non pauci (sed non minor erit eorum qui vivunt gloria).“

42 Siehe dazu U. Schindel, Die Rezeption der hellenistischen Theorie der rhetorischen Figuren bei den Römern, Göttingen 2001, S. 7 f.

43 Rhetores Latini Minores, ed. K. Halm, Leipzig 1863, S. 3-21.

44 Siehe dazu J. Kennedy, The Art of Rheoric in the Roman World, Princeton 1972, S. 364-369.

45 Die Fragmente des Caecilius sind von E. Ofenloch gesammelt: Caecilii Calactini fragmenta, ed. E. Ofenloch, Leipzig 1907 (ND 1967).

46 Tiberii De figuris Demosthenicis cum deperditorum operum fragmentis, ed. G. Ballaira, Rom 1968.

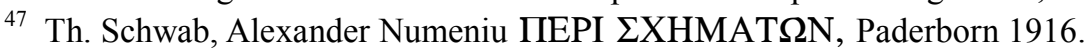

48 Wie Anm. 47, S. 30.

49 Wie Anm. 47, S. 30. 


\section{2 Über die Rezeption von De figuris}

Aquila Romanus soll sich an Alexander Numeniu orientiert haben, wie schon in der Spätantike Iulius Rufinianus meinte ${ }^{50}$. Auch hat man ermittelt, daß das Carmen de figuris (RLM Nr. IV) teilweise auf Alexanders Figurenlehre fußt ${ }^{51}$. Ebenso dient Alexanders De figuris teilweise zur Vorlage für spätere Autoren wie Herodianus ${ }^{52}$, Phoebammon ${ }^{53}$, Martianus Capella ${ }^{54}$, Anonymus Ecksteinii ${ }^{55}$ und Eugraphius $^{56}$.

\section{3 Über die Struktur von De figuris}

Das Werk De figuris besteht aus zwei Groß-Einheiten, und zwar aus den Teilen de figuris sententiarum und de figuris verborum. Bei genauerer Betrachtung ist das Werk De figuris aber in vier Teilen gegliedert: 1. Einleitung (S.1 - 4), 2. Definition der Figur (S. 5 - 9), 3. de figuris sententiarum (S. 10 - 41), 4. de figuris verborum ${ }^{57}$ (S. $\left.42-78\right)$.

\subsubsection{Einleitung (S. 1 - 4)}

In der Einleitung behandelt Alexander folgende Probleme:

a. Schwierigkeit der Abhandlung der Figuren nach Zahl und Wesen, Abgrenzung der Figurenlehre hinsichtlich figura und tropos (S. 1, 1-2, 5).

b. Abgrenzung der Figurenlehre von der Grammatik: Unterschiede zwischen grammatischen Fehlern (Barbarismus und Soloecismus) und rhetorischen Vorzügen (Tropus und Figur) (S. 2, 5 - 17).

c. Unterschiede zwischen Tropus und Figur (S. 3, 1 - 9).

d. Unterschiede zwischen Sinnfiguren und Wortfiguren (S. 3, 9 - 4, 9).

\subsubsection{Definition der Figur (S. 5 - 9)}

Im zweiten Teil erörtert Alexander drei Themen:

a. Die allgemeine Definition der Figur, die sowohl den Sinnfiguren als auch den Wortfiguren gemeinsam ist (S. 5, 2 - 15).

${ }^{50}$ Iulii Rufiniani De figuris sententiarum et elocutionis, in: Rhetores Latini Minores, ed. K. Halm, Leipzig 1863, S. 38: „Hactenus Aquila Romanus ex Alexandro Numenio : exinde ab eo praeteritas, aliis quidem proditas, subtexuimus."

${ }^{51}$ U. Schindel, Entstehungsbedingungen eines spätantiken Schulbuchs: Zum 'Carmen de figuris'(RLM 63-70), in: S. Döpp (Hrsg.), Antike Rhetorik und ihre Rezeption, Stuttgart 1998, S. 85-98.

${ }_{53}$ Herodiani De figuris, RG III, ed. L. Spengel, Leipzig 1853-1856, S. 83-109.

${ }^{53}$ Phoebammonis De figuris, RG III, ed. L. Spengel, Leipzig 1853-1856, S.41-56.

${ }^{54}$ Martianus Capella, ed. A. Dick, BT ${ }^{2}$ 1978, 5, 523-537.

55 Anonymus Ecksteinii, Schemata dianoias quae ad rhetores pertinent, ed. U. Schindel, NGG Phil.-hist. Kl. 1987, 7, S. 107-173 (=S. 1-67).

${ }_{56}^{56}$ Eugraphius, Commentum Terenti, ed. P. Wessner, BT 1908 (ND 1966) (= Donatus, Bd. 3).

${ }^{57}$ Im Unterschied zu dem Teil de figuris sententiarum beginnt der Teil de figuris verborum mit der Einleitung, die die Definitionen von $\pi \varepsilon p i ́ \delta \delta o \varsigma, ~ \kappa \omega \hat{\lambda} \mathrm{ov}$ und $\kappa o ́ \mu \mu \alpha$ und deren Beispiele zum Thema behandelt. 
b. Die Streitfrage, ob die Sinnfiguren Figuren sind (S. 5, 15 - 6, 6), und Argumente gegen diejenigen, die

Sinnfiguren nicht für Figuren halten (S. 6, 7 - 9, 16):

$\alpha$. Funktion der Figuren bei tüchtigen Rednern (S. 6, 7 - 6, 14).

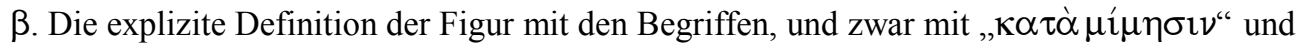

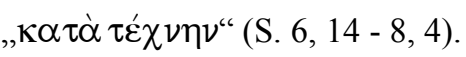

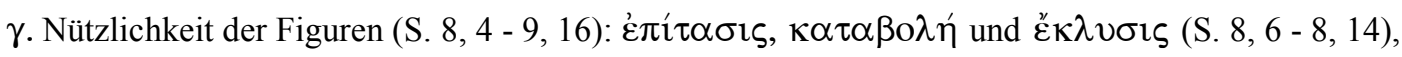

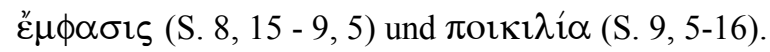

\subsubsection{De figuris sententiarum (S. 10 - 41)}

Die Zahl der Sinnfiguren ist 28. In bezug auf die Anordnung der Figuren ist es zwar schwierig festzustellen, nach welcher Regel Alexander vorgegangen ist. Gleichwohl gibt es zwei mögliche Anordnungsprinzipien, nach denen Alexander die Figuren behandelt hat: Zum einen könnte Alexander die Sinnfiguren grosso modo unter vier Produktionsmerkmalen klassifiziert haben, und zwar

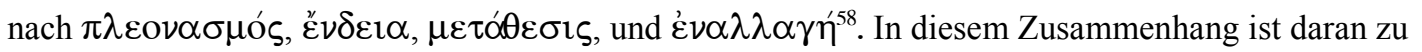
erinnern, daß Quintilian diese vier Kennzeichen bereits zur Klassizifierung der Wortfiguren verwendet hat. Diesbezüglich sagt Quintilian (Inst.orat. 9.3.27): Haec schemata (sc. schemata verborum), aut his similia quae erunt per mutationem adiectionem detractionem ordinem, (...). Zwar läßt sich nicht ausdrücklich belegen, daß Alexander die Figuren nach diesen Anordnungsprinzipien geordnet hätte, aber auch Alexander könnte diese vier Produktionsmerkmale gekannt haben. Dafür gibt es zwei Indizien. Das eine ist die Klassizifierung der Sinnfiguren durch Phoebammon ${ }^{59}$, die offenbar aus Alexanders De figuris entnommen sind. Phoebammon hat die Alexander entnommenen Sinnfiguren ohne große Änderung ${ }^{60}$ so unter den vier Katergorien untergeordnet, wie Alexander sie angeordnet hatte. Das andere sind zwei Aussagen Alexanders, die deutlich darauf hinweisen, daß er die vier Kategorien gut kannte :

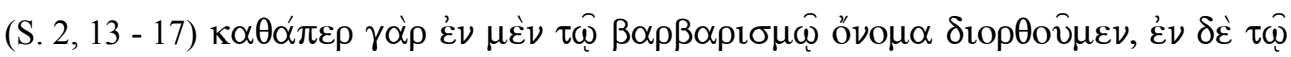

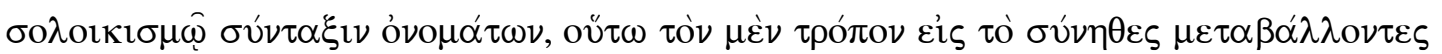

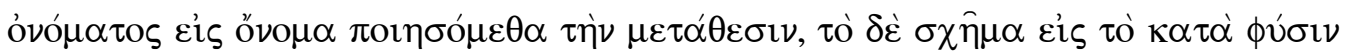

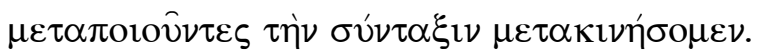

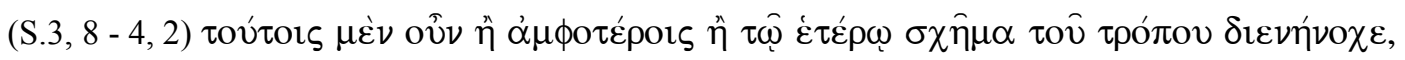

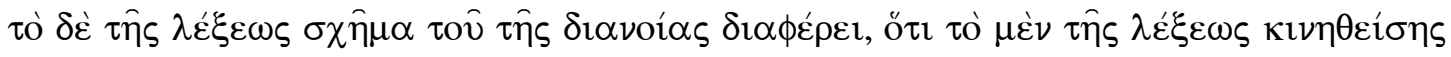

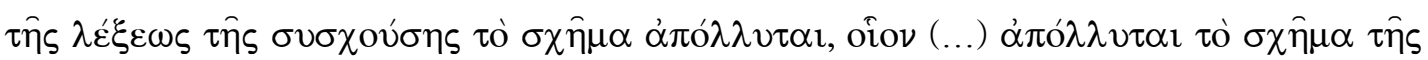

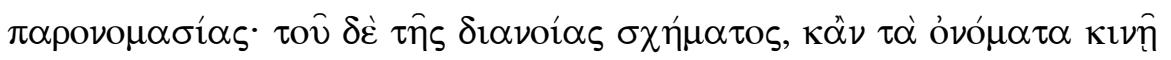

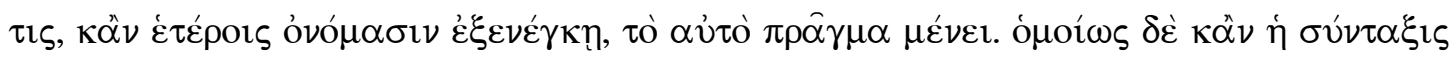

\footnotetext{
58 Vgl. dazu W. Ax, Quadripertita ratio: Bemerkungen zur Geschichte eines aktuellen Kategoriensystems (adiectio-detractio-transmutatio-immutatio), in: Histographia Linguistica 13 (1986), Amsterdam, jetzt in: Lexis und Logos, Studien zur antiken Grammatik und Rhetorik, 2000, S. 190-208.

59 Vgl. dazu Phoebammonis De Figuris II, RG III, ed. L. Spengel, Leipzig 1853-1856, S.50-56.

${ }^{60}$ Phoebammon hat die Gruppe der detractio vor die Kategorie der additio gestellt.
} 


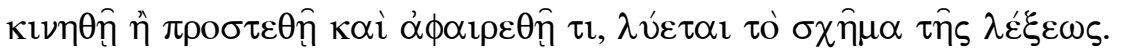

Die Vorstellung, daß Figuren aufgrund von Änderungsoperationen entstehen, ist hier klar erkennbar.

Doch ob sie als Ordnungskategorien dienen, folgt daraus nicht zwingend.

Zum anderen handelt sich es darum, daß Alexander auf kleine Distanz diejenigen Figuren nebeneinander an einem Platze zusammengestellt hat, die von Definition und Funktion her miteinander verwandt sind.

Nach den vier Produktionsmerkmalen ist zunächst der Teil von de figuris sententiarum grosso modo wie folgt zu gliedern:

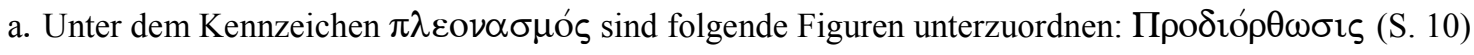
- $\Lambda \varepsilon \pi \tau 0 \lambda \mathrm{o} \boldsymbol{\gamma}^{\prime} \alpha^{61}$ (S. 19).

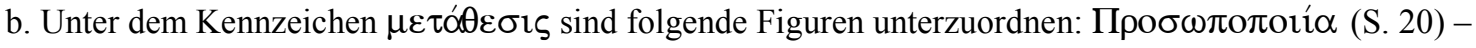

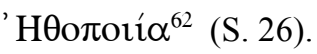

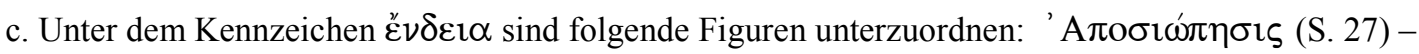

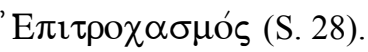

d. Unter dem Kennzeichen $\dot{\varepsilon} \nu \alpha \lambda \lambda \alpha \gamma \eta \dot{n}$ sind folgende Figuren unterzuordnen: Eip $\omega \nu \varepsilon i ́ \alpha$ (S. 29) -

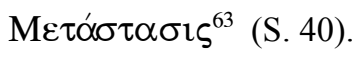

Beispiele nach der Verwandtschaftsregel sind dann folgende:

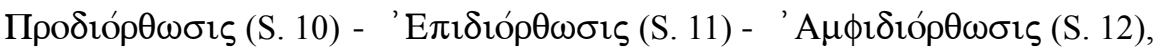

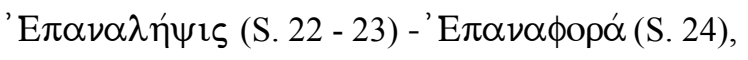

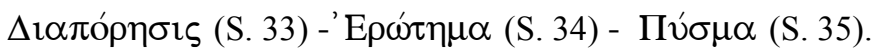

2.3.4 de figuris verborum (S. 42 - 78)

Der letzte Teil besteht aus zwei Unterteilen: a. Einleitung, b. die figurae verborum.

a. Einleitung

In der Einleitung wird die Wortfügungslehre behandelt:

$\alpha$. Einführung in die Abhandlung der Wortfiguren und der Grund dafür, weswegen Alexander in der

Einleitung die Wortfügungslehre betrachtet hat (S. 42, 2 - 9).

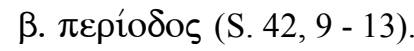

${ }^{61}$ Phoebammon nahm aber drei Figuren weg, und zwar 'A $\mu \phi \imath \delta$ เóp$\theta \omega \sigma ı \varsigma$ (S. 12), 'Y

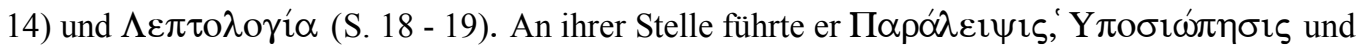
$\Delta \mathrm{l} \alpha \tau v i \omega \sigma \iota \varsigma$ an. Siehe Phoebammonis De figuris II, RG III, ed. L. Spengel, Leipzig 1853-1856, S. 50-51.

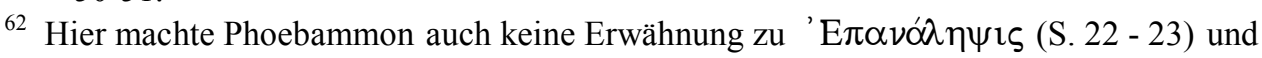

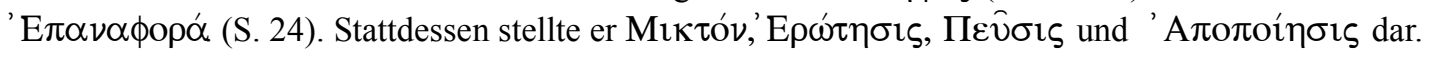
Siehe Phoebammonis De figruris II, RG III, ed. L. Spengel, Leipzig 1853-1856, S. 52-53.

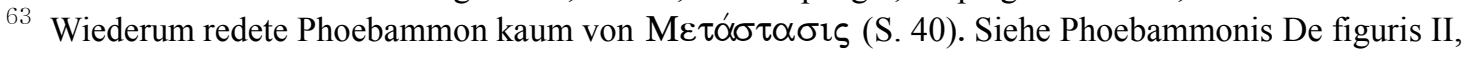
RG III, ed. L. Spengel, Leipzig 1853-1856, S. 53-56. 


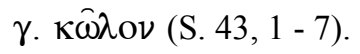

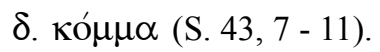

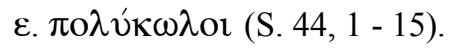

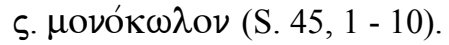

b. Die figurae verborum (S. 46 - 78)

Die Zahl der Wortfiguren ist $30^{64}$. Die Anordnung der Wortfiguren wird ebenso wie bei den Sinnfiguren nach zwei Regeln vorgenommen. Nach den vier Produktionsmerkmalen sind auch die Wortfiguren wie folgt zu klassifizieren:

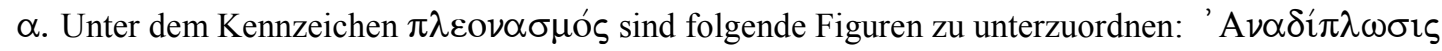

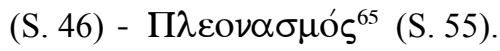

$\beta$. Unter dem Kennzeichen $\varepsilon ้ \nu \delta \varepsilon 1 \alpha^{66}$ sind folgende Figuren unterzuordnen: 'A $\sigma u ́ v \delta \varepsilon \tau O \nu$ (S. 56) ${ }^{\prime} \mathrm{E} \lambda \lambda \varepsilon i \psi 1 \varsigma^{67}$ (S. 57).

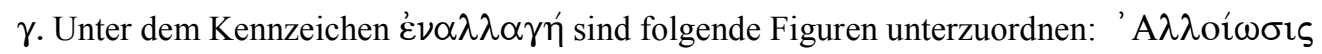

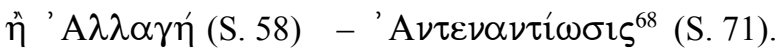

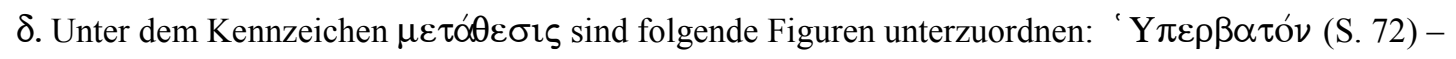
'E $\pi \imath \tau i ́ \mu \eta \sigma \iota \varsigma^{69}$ (S. 78).

Beispiele nach der Verwandtschaftsregel sind folgende:

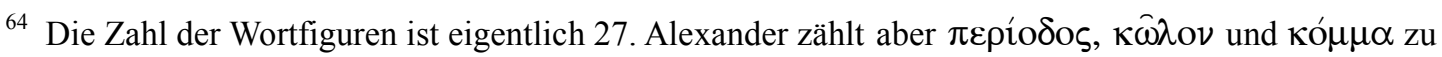

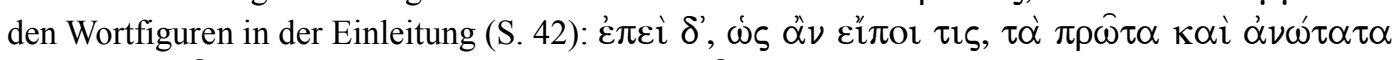

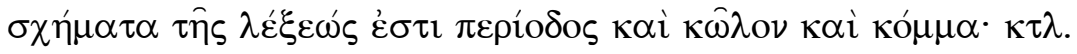

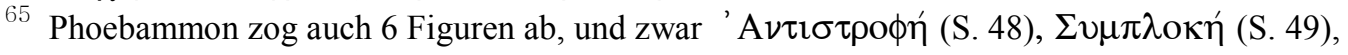

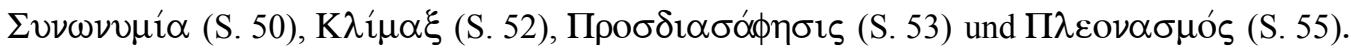

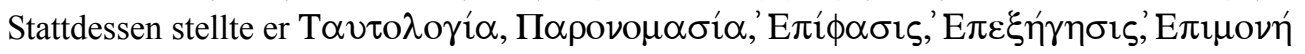
und'E $\pi i ́ \tau \alpha \sigma i \varsigma$ dar (Siehe Phoebammonis De figuris II, RG III, ed. L. Spengel, Leipzig 1853-1856, S. 46-48). Die Anordnung der Wortfiguren Alexanders, die Phoebammon beiseite gelassen hat, erklärt sich aber durch die Verwandtschaftsregel.

${ }^{66}$ Wie bei Sinnfiguren hat Phoebammon die Gruppe der detractio vor der Kategorie der additio gestellt.

${ }^{67}$ Hierzu fügt Phoebammon' A $\pi$ ò Kolvov̂ hinzu. Siehe Phoebammonis De figuris II, RG III, ed. L. Spengel, Leipzig 1853-1856, S. 45-46.

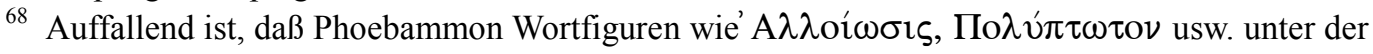
Kategorie der $\dot{\varepsilon} \nu \alpha \lambda \lambda \alpha \gamma \eta \dot{\eta}$ aber mit einer ganz anderen Benenungsweise klassifiziert : $\tau \hat{\eta} \varsigma$

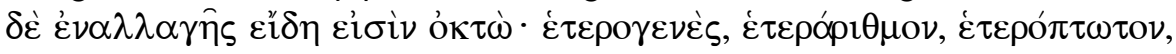

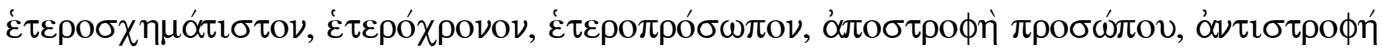
(Phoebammonis De figuris II, RG III, ed. L. Spengel, Leipzig 1853-1856, S. 45).

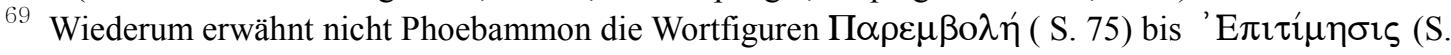

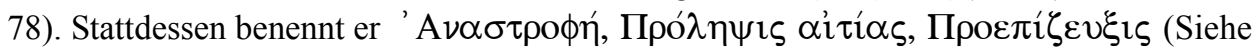
Phoebammonis De figuris II, RG III, ed. L. Spengel, Leipzig 1853-1856, S.45). Diese Angabe stimmt nicht mit den Wortfiguren überein, die im Cap. IV. ПEPI T $\Omega$ N TH $\Sigma$ META@E $\Sigma E \Omega \Sigma$ dargestellt worden sind. Hier sind behandelt 'Y

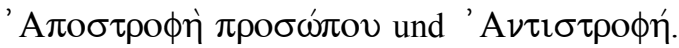




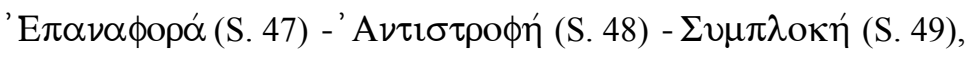

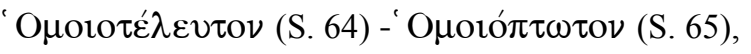

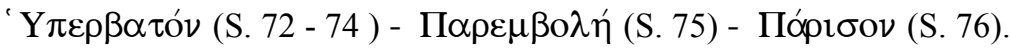

2.4 Über den Stil von De figuris

Bezüglich der stilistischen Form verdienen zwei Dinge besondere Erwähnung: Zum einen die

Schullehrbuchscharakter, zum anderen einige besondere redaktionelle Charakteristika Alexanders.

a. Die Schullehrbuchscharakter

Typisch für Alexanders scholastische Darstellungsweise ist, daß sie Einleitung und eine katalogartige

Reihe von Perikopen der Einzelfiguren umfaßt. Jede Perikope besteht aus Titel, aus Definition, aus

Beispielen und aus weiteren Bemerkungen ${ }^{70}$. Das Werk De figuris zeigt also den typischen Aufbau eines

Schullehrbuchs ${ }^{71}$. Es genügt, als Beleg dafür ein Beispiel zu geben aus de figuris verborum:

$\alpha$. Titel: $\theta^{\prime}$. ПЕPI ПРО $\Sigma \Delta \mathrm{IA} \Sigma \mathrm{A} \Phi \mathrm{H} \Sigma \mathrm{E} \Omega \Sigma$ (S. 53)

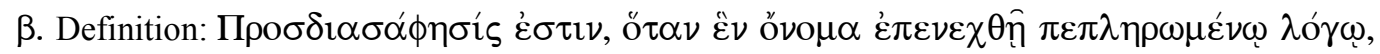
$\pi \rho \circ \sigma \delta 1 \alpha \sigma \alpha \phi \circ \hat{\nu} \pi \lambda \varepsilon \dot{\delta} \sigma \nu \tau$,

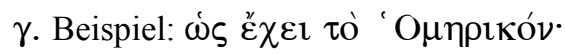

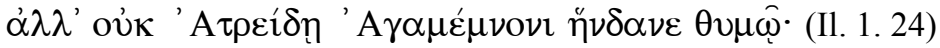

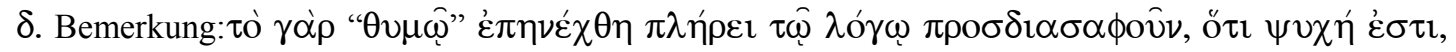

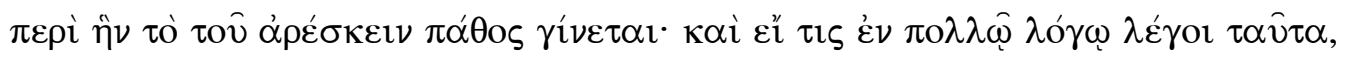

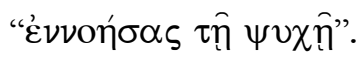

b. Besondere redaktionelle Charakteristika Alexanders

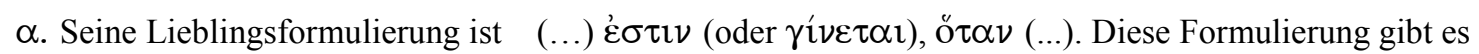
aber nicht allein bei Alexander, sondern auch bei anderen Rhetoren wie Tiberius oder Phoebammon, allerdings nicht so einheitlich.

ß. Er ist ein flexibler Theoretiker. Es gibt dafür drei Indizien:

Das erste Indiz ist, daß Alexander einigermaßen von Systemzwang frei ist. Zwar kennt er das System der Figurenlehre, wie in den oben genannten vier Anordungskategorien sichtbar ist, aber er versucht nicht, seine Lehre zwanghaft zu systematisieren. Das zeigt ein Vergleich mit Herodian, der sehr stark systematisiert:

${ }^{70}$ Vgl. dazu M. Fuhrmann, Das systematische Lehrbuch. Ein Beitrag zur Geschichte der Wissenschaften in der Antike, Göttingen 1960, S.11 - 69.

${ }^{71}$ Dies ist auch bei den anderen rhetorischen Traktaten zu belegen. Vgl. dazu RG I - III, ed. L. Spengel, Leipzig 1853 - 1856. 


\begin{tabular}{|c|c|}
\hline Alexander (S. 8, 6 -17) & Herodian \\
\hline 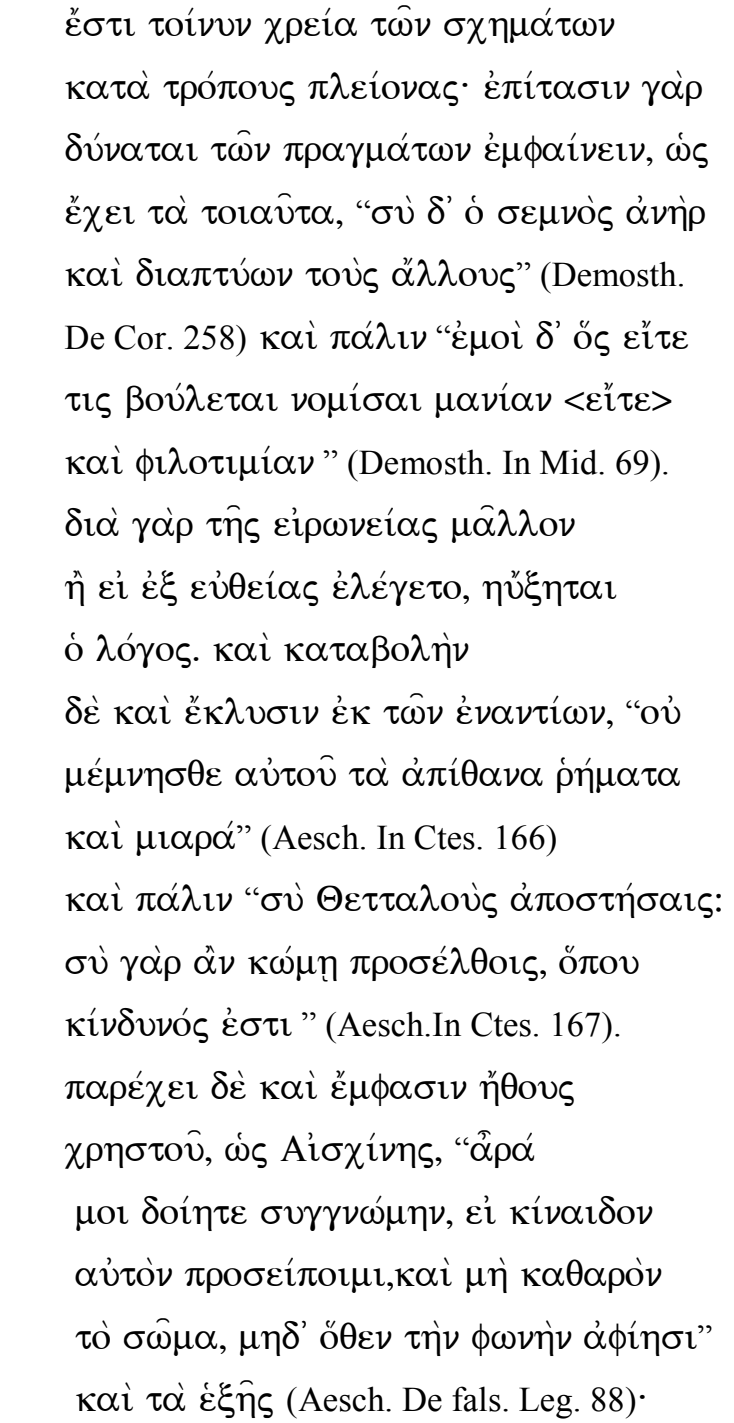 & 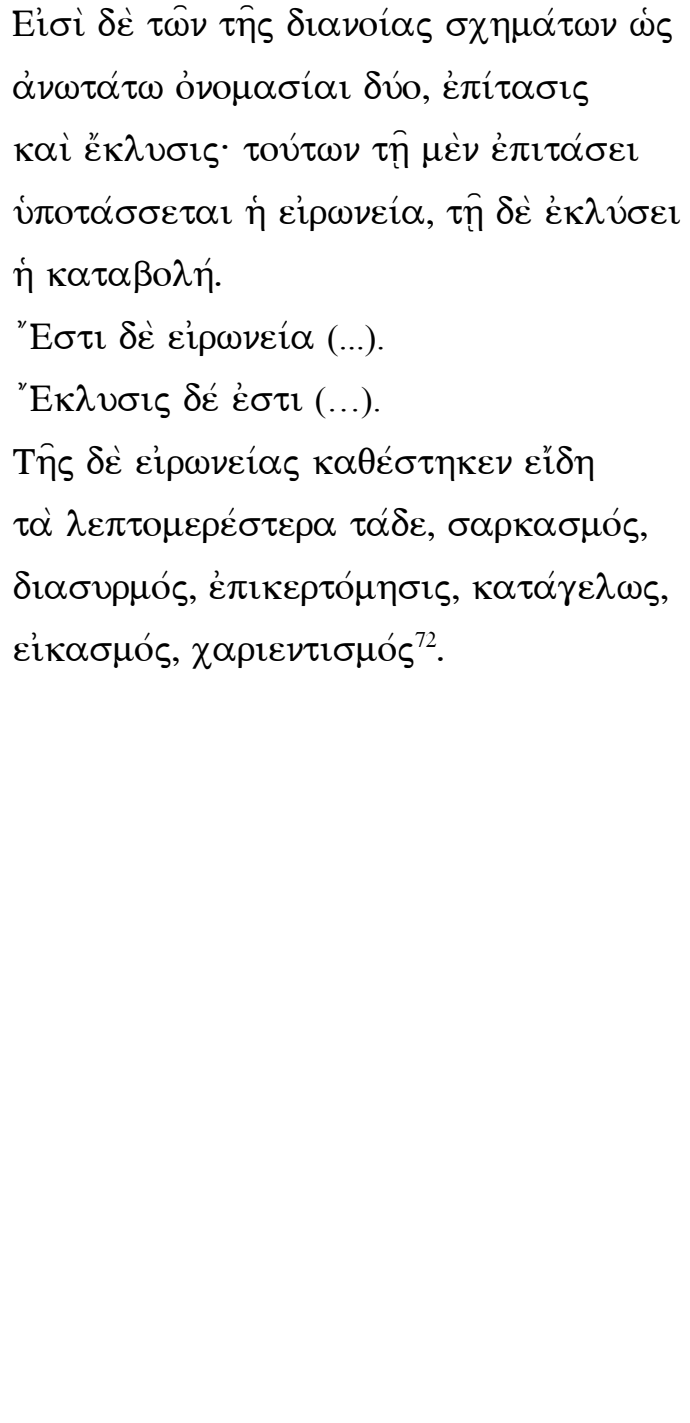 \\
\hline
\end{tabular}

Das zweite Indiz ist seine Vorgehensweise bei der Beispielsanwendung. Es ist zu beobachten, daß manche zitierten Beispielssätze zwar identisch mit den originalen Textstellen sind, aber es gibt auch manche Stelle, die Alexander absichtlich stark verkürzt und verändert hat. In diesem Zusammenhang muß man mit Emendationen vorsichtig sein, denn es könnte auch an der Textüberlieferung liegen. Nach meinen paläographischen Studien und Kollationen der Handschriften bin ich aber davon überzeugt, daß es sich zwar auch oft um Überlieferungsprobleme handelt, wenn es z.B. um kleine Änderungen geht. Aber Alexander hat auch Textstellen verändert, wenn die betroffenen Urtextstellen nicht geeignete oder unnötige Inhalte umfassten oder zu lang waren. Außerdem ist festzustellen, daß viele veränderte Textstellen auch grammatisch richtig sind. Dies zeigt, daß Alexander sie mit Absicht verkürzt und

${ }^{72}$ Herodiani De figuris II, RG III, ed. L. Spengel, Leipzig 1853 - 1856, S. 91 - 92. 
verändert hat. Zum Vergleich zwischen den verkürzten und veränderten Beispielen und den Urtext habe ich je nach Gegebenheiten die originalen Textstellen im apparatus similium angeführt. Der Klarheit wegen ist ein Exempelvergleich vorzunehmen:

\begin{tabular}{|c|c|}
\hline Alexander (S. 48) & Aischines (In Ctes. 198) \\
\hline 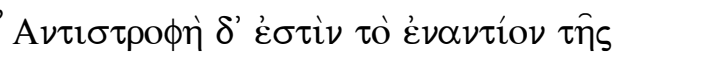 & 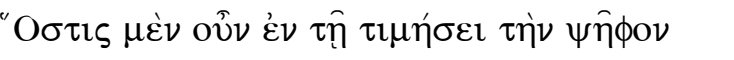 \\
\hline 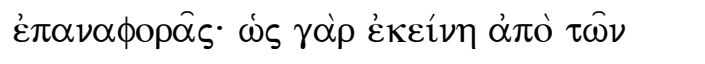 & 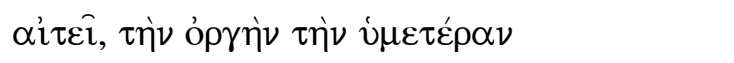 \\
\hline 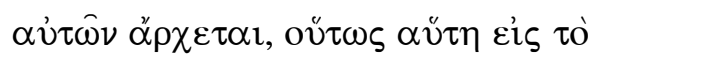 & 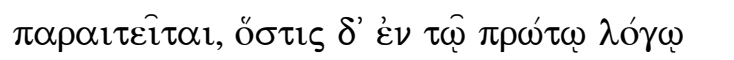 \\
\hline 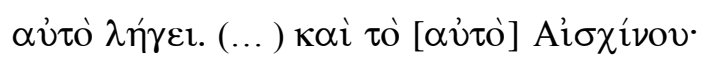 & 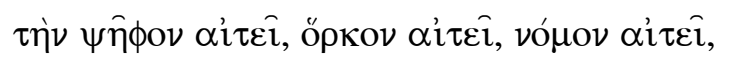 \\
\hline 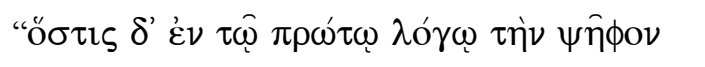 & 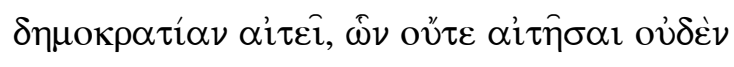 \\
\hline 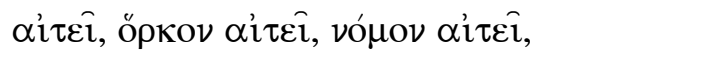 & 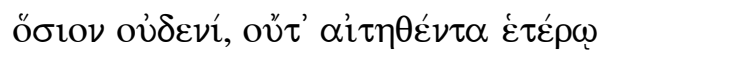 \\
\hline$\delta \eta \mu о \kappa \rho \alpha \tau i ́ \alpha \nu \alpha i \tau \varepsilon \hat{\imath} "$ (Aisch. In Ctes. 198). & 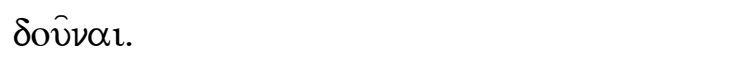 \\
\hline
\end{tabular}

Das letzte Indiz ist, daß Alexander selbst Beispiele zur Anwendung für die betroffenen Figuren dann generiert, wenn es entweder keine Musterstellen für den Fall gibt, oder daß ihm ein angemessenes Beispiel im Moment nicht einfiel. Hierbei aber nennt er sich als Urheber nicht ausdrücklich, sondern

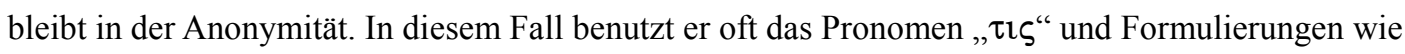

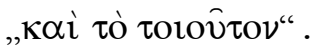




\section{3. Über die codices}

Die Schrift De figuris ist in folgenden Handschriften erhalten, die unter dem Namen Alexanders auf uns gekommen sind :

Cambridge. Queens College Lib. gr. 20 (=Horne 33), 3. Alexandrou peri ton tes dianoias schematon kai peri ton tes lexeos schematon (f. 60-76a), saec. XV/XVI. (=Cantabr. 20)

Florenz. Med. Laurent. gr. Plut. 57.7, Alexandri de figuris sensus ac dictionis (f. 334 sequ.), saec. XV.

(=Laurent. 57)

Paris. BN gr. 1656, Alexandri rhetoris de figuris sententiae et elocutionis (f. 73v - 83v), saec. XV. (=P $\mathrm{P}^{3}$ )

Paris. BN gr. 1741, Alexandri opusculum de figuris sententiarum atque elocutionis (f. 106v - 120v), saec.

X. $\left(=\mathrm{P}^{1}\right)$, cum notis marginalibus (= N.M.) (f. 106v - 120v), saec. XIII ?

Paris. BN gr. 2087, Alexandri opusculum de figuris sententiarum et elocutionis (f. 247v - 258v),

saec. XIV. $\left(=\mathrm{P}^{2}\right)$

Paris. BN gr. 2762, Alexandri opusculum de figuris sententiarum et elocutionis (f. 4v - 11v), saec. XV. $(=$ Par. 2762)

Paris. BN gr. S. 552, Alexandri sophistae opusculum de figuris sententiae et dictionis (f. 574v - 588v), saec. XVII . $\left(=\mathrm{Ald}^{\mathrm{S}}\right)$

Rom. Angel. gr. 54 (D. 5.8), 3. Alexander, de figuris dictionis et sententiarum (ff. 105), saec. XV/XVI. (=Angel. 54)

Rom. Vat. Pal. gr. 66, 3. Alexander, de figuris dictionis et sententiarum (f. 118r - 131v), saec. XVI. (=Pal.)

Rom. Vat. gr. 1881, 3. Alexandri rhetoris de schematibus (f. 94-99), saec. XIII. (= Vat. 1881)

Venedig. Marc. gr. 429, IV. Alexander Numenii filius De figuris (f. 87v - 95v), saec. XV (ca. 1468). $\left(=\mathrm{M}^{1}\right)$

Venedig. Marc. gr. 512, II. Alexander $<$ Numenius $>$, De figuris rhetoricis (f. 9-25), saec. XIII. $\left(=\mathrm{M}^{2}\right)$

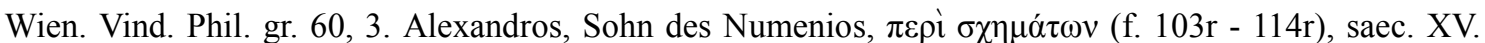
$(=\mathrm{V})$

Die oben aufgezählten Handschriften sind in Hinblick auf die Echtheitsproblematik und die Autorschaft ins Zweierlei zu unterscheiden. Die einen sind diejenigen, die zwar unter dem Namen Alexander überliefert, aber nicht von Alexander als Autor verfaßt worden sind. ${ }^{73}$ Es sind wie folgt: Paris. 2087, Paris. 2762, Notae marg. in Paris. 1741, Med. Laurent. Plut. 57 und Vat. 1881. Die anderen sind diejenigen, die tatsächlich Alexander gehören. Es sind folgende ${ }^{74}$ : Paris. 1741, Marc. 429, Vind. 60 , Paris.

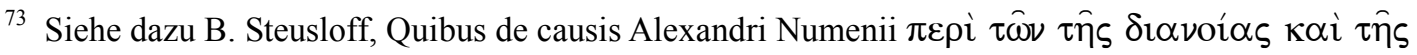

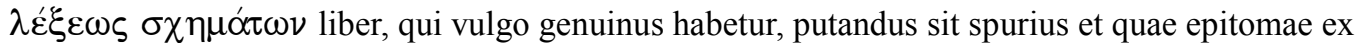
deperdito Alexandri libro excerptae supersint, demonstratur, Diss. Phil. Breslau 1861, S. 49. Darauf werde ich auch im Paragraph 3. 6 ausführlich eingehen.

${ }^{74}$ Vgl. dazu E. Drerup, Eine alte Blattversetzung bei Alexander Numeniu, Philologus 71 (1912), S. 392 , 
1656, Cantabr. 20, Angel. 54, Vat. Pal. 66, Paris. S. 552 und Marc. gr. 512. Zuerst ist von den eigentlichen Alexander - Handschriften zu handeln:

3.1 Paris. $1741\left(=\mathrm{P}^{1}\right)$ und Marc. $512\left(=\mathrm{M}^{2}\right)$

a. Alexander hat zwei Überlieferungszweige. Der eine ist die Familie von Paris. 1741, aus dem Marc. 429, Vind. 60 , Paris. 1656, Cantabr. 20, Angel. 54, Vat. Pal. 66 und

Paris. S. 552 stammen. Der andere ist durch Marc. 512 repräsentiert. Diese beiden Familien haben einen verlorenen, schon korrupten und stark verkürzten Archetypus $\Omega^{75}$. Der entscheidende Beleg dafür, daß Marc. 512 eine von der anderen Familie unabhängige Überlieferung bewahrt, ist die Lücke im Abschnitt ‘ $\zeta^{\prime}$. ПЕPI ZEYГМАTO $\Sigma$, die nur im Marc. 512 gefüllt ist ${ }^{76}$ :

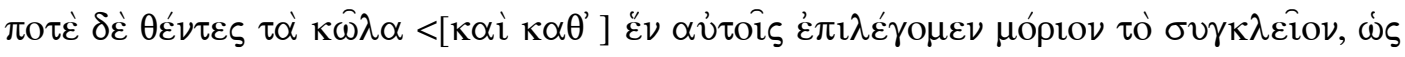

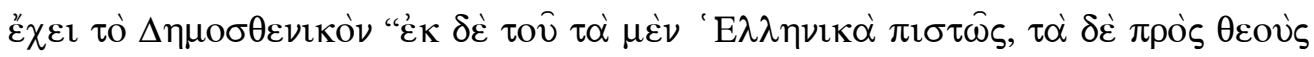

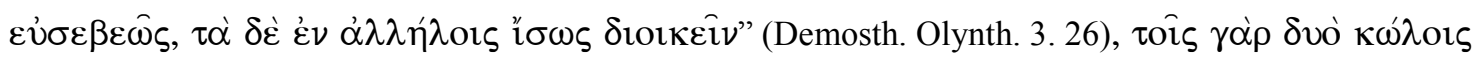

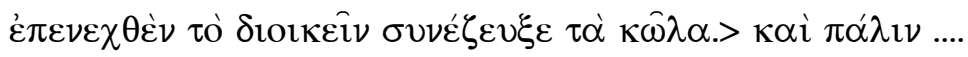

Die Lücke könnte grundsätzlich auch mit Hilfe von Paris. 2087 gefüllt worden sein - zumal das Demosthenische Beispiel Olynth. 3.26 von daher stammen könnte. Doch spricht das überflüssige $\kappa \alpha i ̀ ~ \kappa \alpha \theta^{\prime}$ gegen eine selbständige Konjektur oder Ergänzung des Schreibers von $\mathrm{M}^{2}$ : wenn er schon ergänzte, hätte er es vermieden, Sinnloses einzuschieben. Da er das sinnlose $\kappa \alpha i ̀ ~ \kappa \alpha \theta^{\prime}$ schreibt, hat er es in seiner Vorlage gelesen. Und das Demosthenische Exempel hat er nicht aus Paris. 2087, sondern eben aus seiner - besseren - Vorlage, deren Existenz durch Paris. 2087 bestätigt wird.

Weitere Belege dafür finden sich je nach Gegebenheiten in apparatus criticus. Zur Verdeutlichung führe ich noch eine Kollation zwischen Paris. $1741\left(=\mathrm{P}^{1}\right)$ und Marc. $512\left(=\mathrm{M}^{2}\right)$ an, die zweifach gegliedert ist:

. Gemeinsame Lesarten, die zeigen, daß Paris. 1741 und Marc. 512 auf der gleichen Urhandschrift fußen:

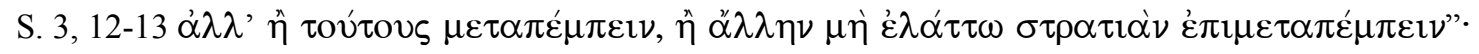

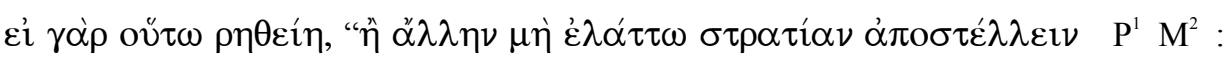

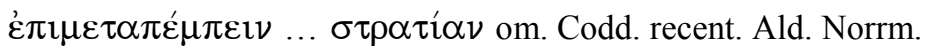

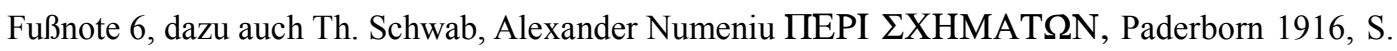
1-3 und W. Tröbst, Quaestiones Hyperideae et Dinarcheae I, in: Schulprogramm Hameln (1881), S. $1-27$.

75 Auch Tröbst vertritt die Position, daß es zwei Überlieferungsklassen gab, die auf einer gemeinsamen Urhandschrift $\Omega$ fußen. Nach ihm ist aber die eine Klasse durch Paris. 1741, die andere durch Vind. 60 repräsentiert. Diese Position ist falsch, denn Tröbst hat bei seinen Kollationen den Marc. 429 nicht geprüft, der direkt aus dem Paris. 1741 stammt und von dem Vind. 60 abgeschrieben wurde, und er kennt auch den Marc. 512 nicht, der die andere Klasse vertritt. Vgl. dazu W. Tröbst, Quaestiones Hyperideae et Dinarcheae I, in: Schulprogramm Hameln (1881), S. 9-20.

76 Siehe S. 63, 5-9. Die Entstehung der Lücke durch Sprungfehler ist evident. 
S. 4, 5 દ’ $\delta \dot{\varepsilon} \tau \imath \varsigma \mathrm{P}^{1} \mathrm{M}^{2}$ : $\tau \iota \varsigma$ om. Codd.recent. Edd.

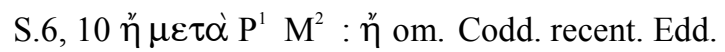

S.14, 10 O' $\chi \alpha \lambda \imath \hat{\eta} \iota \mathrm{P}^{1} \mathrm{M}^{2}$ : O'i $\chi \alpha \lambda \imath \hat{\eta} \iota$ om. Codd. recent. Edd.

S. 36, $3 \pi \alpha \rho \varepsilon 1 \sigma \alpha \gamma \omega \gamma \eta \dot{\nu} \mathrm{P}^{1} \mathrm{M}^{2}: \pi \alpha \rho \alpha \sigma \nu \nu \alpha \gamma \omega \gamma \eta \dot{\nu} \nu$ Codd. Recent. Edd.

S.43, $9 \theta \hat{\omega} \mathrm{P}^{1} \mathrm{M}^{2}: \theta \varepsilon \hat{\omega}$ Codd. recent. Edd.

$\mathrm{S} .49,2 \tau \varepsilon \mathrm{P}^{1} \mathrm{M}^{2}$ : om. Codd. recent. Edd.

S.55, $6 \pi \rho \circ \sigma \kappa \varepsilon i ́ \mu \varepsilon v o v \mathrm{P}^{1} \mathrm{M}^{2}: \pi \rho \circ \kappa-$ Codd. recent. Ald.

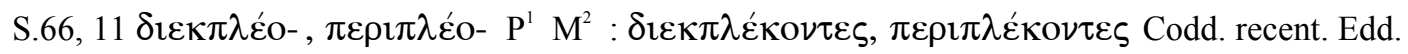

ß. Trennfehler (Minuskel- Verlesungen, falsche Auflösung von Abbreviaturen oder Kompendien,

Lücken), die zeigen, daß Marc. 512 nicht von Paris. 1741 abhängt.

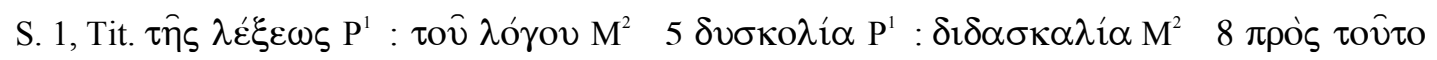

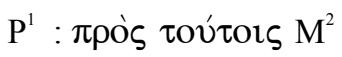

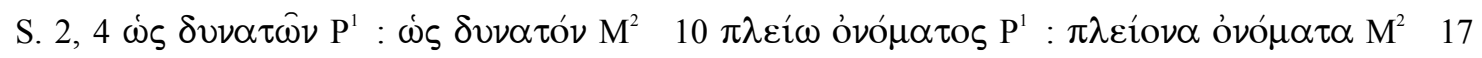

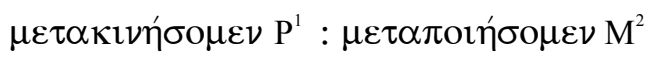

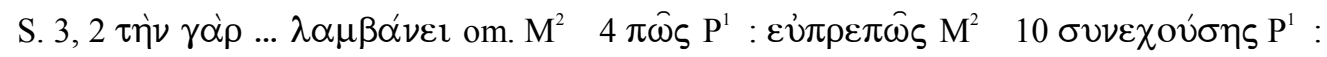

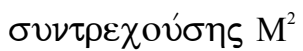

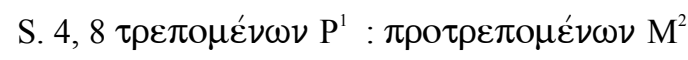

etc.

b. Der Marc. 512 enthält gleichwohl viele Verbesserungen, zu denen ein gebildeter Schreiber auch ohne Vorlage fähig ist: Der Schreiber des Marc. 512 war offenbar ein Mann mit scharfem Auge und philologischer Bildung ${ }^{77}$. Deshalb gelingen ihm viele richtige Konjekturen und Emendationen. Aber diese Eingriffe sind oft auch Trivialisierungen und Korrekturen, die den Autor nur vermeintlich verbessern.

Beispiele dafür sind folgende:

$\alpha$. Die selbständige Behandlung der 'E $\pi \alpha \nu \alpha \delta i \pi \lambda \omega \sigma \iota \varsigma$ : Die 'E $\pi \alpha \nu \alpha \delta i ́ \pi \lambda \omega \sigma \iota \varsigma$ ist ein wichtiger Beweis für die rekonstruierende Arbeitsweise des Schreibers des Marc. 512: Aus der am Schluß

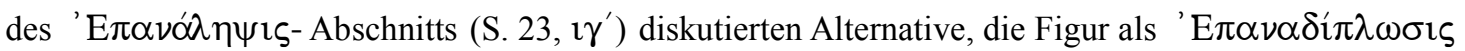
zu bezeichnen, hat er eine selbständige Perikope mit eigener Überschrift gemacht - sicher nicht im Sinne

${ }^{77}$ Der Schreiber von Alexanders Traktat im Marc. 512 war der Besitzer der Handschrift. Nach Mioni wurden einige rhetorische Traktate und einige Traktate von Tryphon und anderen Autoren von dem Besitzer selbst geschrieben (Siehe E. Mioni, Codices Graeci Manuscripti II, Roma 1985, S. 369). Dazu gehört Alexanders Figuren- Traktat. Dies ist an der Schriftform des Traktats erkennbar, die identisch ist mit dem Пív $\boldsymbol{\xi}$ und den anderen Traktaten des Marc. 512 ist, die von dem Besitzer selbst geschrieben worden waren. 
Alexanders, der auch in anderen Fällen konkurrierende Terminologie miteinbezieht ${ }^{78}$.

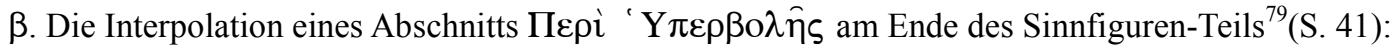
Fraglich ist, ob die 'Y $Y \varepsilon \rho \beta о \lambda \eta ́$ von Alexander als eine Sinnfigur betrachtet wurde. In bezug darauf ist G. Ballaira der Auffassung ${ }^{80}$, daß sie zu Alexanders originalem Bestand gehört. Als Beleg dafür erwähnt Ballaira Quintilians Erörterung zu 'Y $\pi \varepsilon \rho ß o \lambda \eta$. Das Problem liegt aber schon darin, daß Quintilian die 'Y $\pi \varepsilon \rho ß o \lambda \eta ́$ nicht als eine Sinnfigur, sondern als Tropus betrachtet ${ }^{81}$. Es kommt hinzu, daß auch die Autoren, die mit Caecilius in Verbindung stehen, wie Tiberius oder der Autor des Paris. 2087, und die Verfasser, die sich an Alexander orientierten, wie Aquila und Rufinianus, und teilweise von Alexander beeinflußt worden sind, die 'Y $\pi \varepsilon \rho \beta 0 \lambda \eta$ nicht als Sinnfigur betrachten. Das Problem liegt schließlich darin, daß es außer dem Marc. 512 keine Alexander - Handschrift gibt, die die 'Y $\pi \varepsilon \rho ß o \lambda \eta ́$ als Sinnfigur überliefert. Es könnte sein, daß sie zwar von Alexander thematisiert worden ist, aber nicht im Figurentraktat, sondern im Themenbereich der Argumentation oder im Bereich der Tropoi wie bei Quintilian. Einen interessanten Beleg dafür liefert Rufinianus, der diese Figur zu dem Bereich gezählt hat, wo auch Figuren der Argumenation erörtert worden $\operatorname{sind}^{82}$. Hieraus ist zu schließen, daß die Y $\tau \varepsilon \varepsilon \rho ß o \lambda \eta ́$ von Alexander im Bereich der Argumentation thematisiert worden sein könnte. Auf dieser Grundlage dürfte sie im vorliegenden Text eine Interpolation sein.

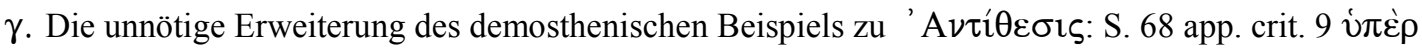

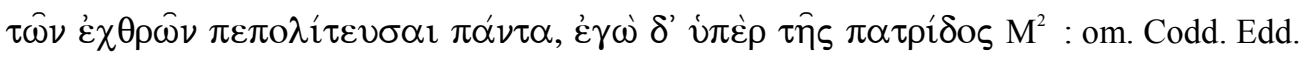

\subsection{Paris. 1741 und Marc. 429}

Der Paris. 1741 ist die älteste Handschrift. Diese Handschrift ist sehr wichtig nicht allein für Alexander, sondern auch für andere technographische Autoren wie Aristoteles, Dionysios von Halikarnassos, Demetrios, Apsines und Aristides. Auch die Poetik des Aristoteles ist in diesem Codex enthalten. Aus diesem Grund ist er berühmt und gut erschlossen. Der Paris. 1741 wurde in der Mitte des 10. Jahrhunderts

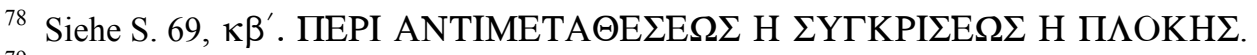

${ }^{79}$ Der Abschnitt erweist sich durch seinen einleitenden Satz als nachgeschobene Ergänzung; es fehlen die Definition und Beispiele, also die konventionellen Bestandteile der übrigen Figuren-Artikel. Andererseits sind sowohl die sachliche Erörterung der Figur als auch das verwendete Vokabular

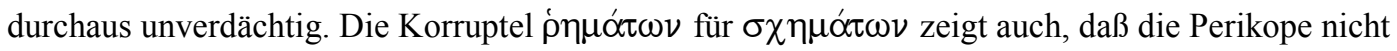
vom Schreiber des Marc. 512 erfunden ist. Man wird sie deshalb am besten als delendum im Text behalten als nicht unerhebliches Überlieferungszeugnis.

${ }^{80}$ G. Ballaira, Una Figura Inedita del $\pi \varepsilon p i ̀ ~ \sigma \chi \eta \mu o ́ x t \omega \nu$ di Alessandro di Numenio e le sue Affinità con Quintiliano (Inst. 8, 6, 67-76), RhM 119 (1976), S. 323-328.

${ }^{81}$ Siehe Quint. Inst. orat. 8.6.67: Hyperbolen audacioris ornatus summo loco posui.

${ }^{82}$ Iulii Rufiniani De figuris sententiarum et elocutionis, in: Rhetores Latini Minores, ed. K. Halm, Leipzig 1863, S. 47. 
in Konstantinopel geschrieben. ${ }^{83}$ Wann und wie er nach Italien gelangte, ist nicht bekannt. ${ }^{84}$ Sicher ist nur, daß der Codex sich noch kurz vor 1427 in Konstantinopel befand. Dort hat er als Vorlage für die Codices Laurent. 60.18 und Laurent. 60.21 gedient, die Filelfo im Jahre 1427 nach Italien mitbrachte. ${ }^{85}$ Der Paris. $1741^{86}$ wird zum ersten Mal im 15. Jahrhundert in einem undatierten Brief ${ }^{87}$ Bessarions erwähnt. Bessarion „,bittet in diesem Briefe einen nicht genannten Adressaten, ihm sukzessiv Teile einer

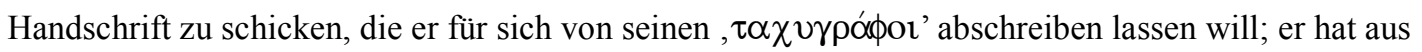
dieser Handschrift bereits Dionys von Halikarnaß, De compositione verborum geschickt bekommen und bittet jetzt um die Sendung der Ars rhetorica von Apsines. Rhetorik und Poetik von Aristoteles, die ebenfalls in dem erwähnten Manuskript enthalten seien, benötige er nicht, da er diese Handschriften schon besitze (vgl. Lobel, S. 6-7). Die Abfassungszeit dieses Briefes fällt, wie Lobel plausibel nachgewiesen hat, in die Jahre 1457 bis 1468. Als Adressaten vermutet Mohler Theodoros Gazes, während Mioni (in: Cento codici Bessarionei. Catalogo di mostra a cura di T. GASPARRINI LEPORACE ed. E. Mioni, Venedig 1968, S.7) Michael Apostolios angibt. Beide gehören zum Kreis des Bessarion und kämen als Empfänger in Frage, in erster Linie wohl Michael Apostolios, mit dem Bessarion zahlreiche Handschriftengeschäfte abgewickelt hat. Es ist allerdings auch durchaus denkbar, daß der Brief an einen Dritten gerichtet ist; daher läßt sich nicht mit Sicherheit sagen, wer in der fraglichen Zeit der Besitzer unseres Manuskriptes war.“ 88

In dieser Zeit wurde der Marc. 429 von den „, $\alpha \chi v \gamma \rho \alpha ́ \phi o{ }^{\prime \prime}$ geschrieben. In Bezug darauf sagt E. Lobel folgendes: „(...) Marc. 429 (which contains the only Apsines given by Bessarion) was part of the collection handed over in May 1468،“89 Hieraus läßt sich vermuten, daß der Marc. 429 am Anfang des

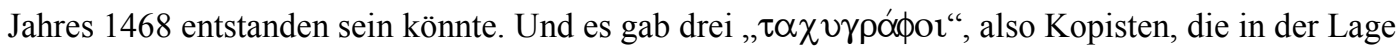
waren, schnell zu schreiben. Nach Mioni ${ }^{90}$ waren es Cosmas Hieromonachus (f. 4-99v), Georgius Trivizias (f. 100-166) und ein ,adhuc ignotus“ (f. 166-202v). In diesem Marc. 429 ist der Text Alexanders (f. 87-95v) enthalten. Cosmas Hieromonachus war also der Schreiber des Textes von Alexander. In der

${ }^{83}$ W. Schmid hat festgestellt, daß die Sammlung des cod. Par. 1741 im byzantinischen Schulunterricht während des 10. Jh. entstanden war. Vgl. W. Schmid, Die sogenannte Aristidesrhetorik, in: RhM 72 (1917/1918), S. 113-149 und dazu E. Lobel, The Greek manuscrits of Aristotle's Poetics, Oxford 1933, S. 6-7; ferner D. Harlfinger und D. Reinsch, Die Aristotelica des Parisinus Gr. 1741, in:

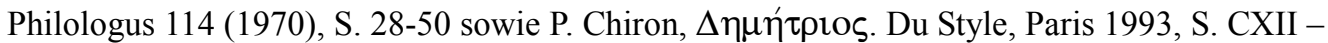
CXIII.

84 Über das weitere Schicksal des Paris. 1741 vgl. D. Harlfinger und D. Reinsch, Die Aristotelica des Parisinus Gr. 1741, in: Philologus 114 (1970), S. 34-37.

85 Siehe dazu P. Chiron, Démétrios. Du Style, Paris 1993, S. CXIII.

${ }^{86}$ Wie Anm. 84, S. 35.

87 L. Mohler, Kardinal Bessarion, Bd. III, Paderborn 1942, S. 483.

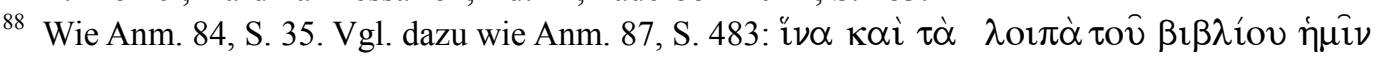

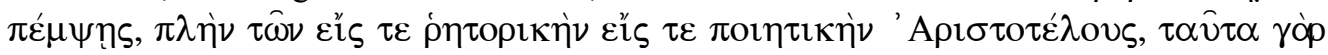

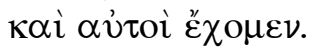

${ }^{89}$ E. Lobel, The Greek manuscrits of Aristotle's Poetics, Oxford 1933, S. 6, Fußnote 4.

90 E. Mioni, Codices Graeci Manuscripti II, Roma 1985, S. 196. 
Tat ist Alexanders De figuris sehr schnell abgeschrieben worden. ${ }^{91}$

3.3 Marc. $429\left(=\mathrm{M}^{1}\right)$ und Vind. $60(=\mathrm{V})$

Der Vind. 60 wurde von dem gerade erwähnten Georgius Trivizias (vor 1423-1485) geschrieben ${ }^{92}$, der sich auch an der Herstellung des Marc. 429 beteiligt hat. Die Abfassungszeit fällt in die Zeit von 1468 bis 1485, denn die Vorlage war der Marc. 429. ${ }^{93}$ Als Belege dafür, daß der Vind. 60 aus dem Marc. 429 stammt, sind folgende Bindefehler anzuführen, die auch die Abhängigkeit des Marc. 429 von Par. 1741 $\left(=\mathrm{P}^{1}\right)$ deutlich zeigen:

S. 1, $5 \delta v \sigma \kappa o \lambda i ́ \alpha \mathrm{P}^{1} \mathrm{M}^{1}: \delta v \sigma i \phi \lambda i ́ \alpha \mathrm{V}$ (s. u. Anm. 9494)

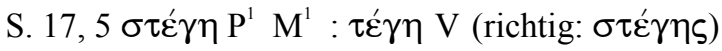

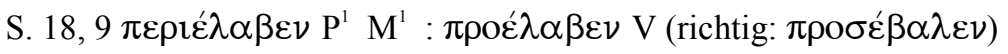

S. 21, $7 \stackrel{\omega}{\omega} \mu \dot{\varepsilon v} \mathrm{P}^{1} \mathrm{M}^{1}$ : $\omega^{\Uparrow}$ om. V (richtig: of $\mu \varepsilon \dot{\varepsilon}$ )

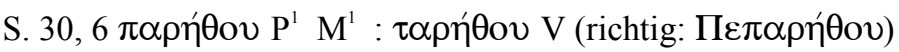

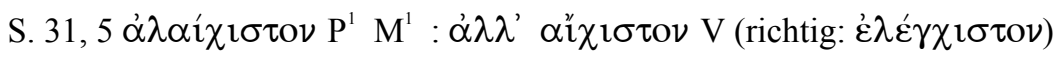

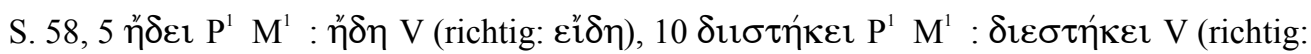
$\delta 1 \varepsilon \imath \sigma \tau \eta \dot{\kappa \varepsilon \imath)}$

3.4 Vind. 60 und codices ceteri

Die jüngeren Handschriften sind Paris. $1656\left(=\mathrm{P}^{3}\right.$ ), Vat. Pal. 66.(=Pal.), Cantabr. 20 und Angel. $54^{95}$; M.

R. James ist der Überzeugung, daß Cantabr. 20 den Paris. 1741 als Vorlage benutzt hat ${ }^{96}$. Außer dem Cantabr. $20^{97}$ stammen alle jüngeren Handschriften ${ }^{98}$ aus Vind. 60. Der Klarheit wegen stelle ich eine

${ }^{91}$ Hierfür führe ich als Beleg eine Photokopie an. Siehe Photokopie III.

${ }_{92}$ Siehe dazu M. Sicherl, Griechische Erstausgaben des Aldus Manutius, Paderborn 1997, S. 324.

${ }^{93}$ M. Sicherl ist der Auffassung, daß der Vind. 60 als Vorlage des Marc. 429 gedient hat. Die Auffassung Sicherls beruht aber auf W. Schmids Analyse (Siehe M. Sicherl, Griechische Erstausgaben des Aldus Manutius, Paderborn 1997, S. 324), die sich auf ,die sogenannte Aristidesrhetorik“ konzentriert und ein einseitiges Bild gibt (Siehe dazu W. Schmid, Die sogenannte Aristidesrhetorik, in: RhM 72 (1917/1918), S. 113-149).

${ }^{94}$ Es ist schwer, eine Entscheidung zu treffen, ob man den Buchstaben $\kappa$ im Wort $\delta v \sigma \kappa o \lambda i ́ \alpha$ des Marc. 429 als $\kappa$ oder $\phi$ lesen muß. Denn Cosmas, der Schreiber des Marc. 429, hat den Text schnell abgeschrieben. Dieses $\delta v \sigma \kappa o \lambda i ́ \alpha$ des Marc. 429 hat der Schreiber des Vind. 60 offenbar als $\delta v \sigma i \phi \lambda i ́ \alpha$ gelesen.

${ }^{95}$ Hier sind nur die Handschriften betrachtet, die auf Vind. 60 fußen. In dieser Liste der Handschriften fehlen also Paris. BN. Gr. 2762, Rom. Vat. 1881, 3 und Paris. BN. Gr. S. 552. Paris. BN. Gr. S. 552 stammt nämlich aus der editio princeps Aldina, Paris. BN. Gr. 2762, Rom. Vat. 1881, 3 und Med. Laurent. Plut. 57. 7 gehören zu der Familie von Paris. 2087. Siehe dazu unten Abschnitt 3. 6.

${ }^{96}$ R. M. James, A descriptive catalogue of the western manuscripts in the Library of Queens College, Cambridge, Cambridge 1904, S. 24.

${ }^{97}$ Diese Handschrift zu bekommen, habe ich vergeblich versucht. Es könnte sein, daß die Handschrift sich nicht in der Queens College Library, sondern in einer anderen Bibliothek in Cambridge befindet. Was den stemmatischen Zusammenhang betrifft, habe ich Zweifel, ob Cantabr. 20 wirklich aus Par. 
Fehlerliste vor, die sich am Vind. 60 orientiert:

\author{
S. 1, $5 \delta v \sigma \imath \phi \lambda i ́ \alpha$ V P Pal

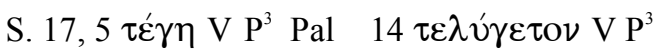

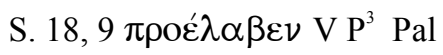

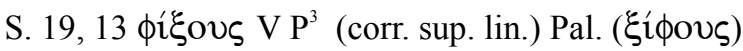 \\ S. 21, $7 \hat{\omega}$ om. V P $\mathrm{P}^{3}$ Pal.

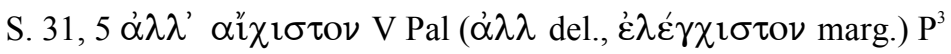 \\ S. 40, 8 ö $\sigma \tau \varsigma$ ö $\pi$ ov V P Pal. \\ S. 46, $6 \mu \dot{\varepsilon} v$ om. V P Pal. \\ S. 54, $3 \pi \varepsilon \rho 1 \pi \varepsilon \phi \alpha \gamma \mu \varepsilon ́ v \alpha$ V P Pal.

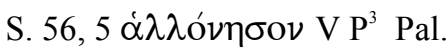

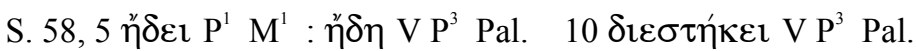

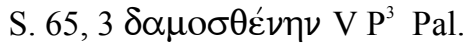

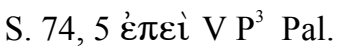

3.5 Paris. 1656 und die editio princeps Aldina

a. Fraglich ist, auf welcher Handschrift die erste gedruckte Alexander-Edition von Aldus fußt. Dazu gibt es bereits zwei Meinungen, nämlich daß der Aldina Vind. 60 zugrunde liegt oder aber daß sie Par. 1656 benutzt hat. Diese Genese liegt nahe, denn der Paris. 1656 stammt von der Hand des Zacharias Kallierges, der um die Wende vom 15. zum 16. Jahrhundert in Padua und Venedig für Aldus als Kopist und Drucker tätig war. ${ }^{99}$ W. Schmid ist dagegen der Meinung, daß die Aldina von 1508 aus Vind. 60 stammt. ${ }^{100}$ Chr. Walz hingegen betont, daß die Aldina sehr eng mit dem cod. Paris. 1656 zusammenhänge : „Par.3.(1656), cuius primum caput speciminis causa contuli, consentit cum Aldina. “101

Nach meinen eigenen Kollationen ist aber sicher, daß die Aldina aus dem Paris. 1656 entstanden ist. Dies wird am folgenden Vergleich zwischen dem Vind. 60 und dem Paris. 1656 deutlich, wobei noch die Lesarten der Aldina (Ald.) sowie die (handschriftlichen) Notate in der handschriftlichen Kopie der Aldina

1741 stammt. Denn die Reihenfolge der Autoren und der Werke auf der Titelseite stimmt mit der des Vind. 60 überein. Es könnte aber sein, daß Vind. 60 als Vorlage diente.

98 Ähnlich erfolglos wie beim Cantabr. 20 war ich mit meinem Versuch, einen Mikrofilm von Angel. 54 zu erhalten. In Bezug auf seine Stellung im Stemma gibt es aber kaum offene Fragen, weil die Stellen, die als lectio varia im apparatus criticus von Chr. Walz angeführt worden sind, zeigen, daß Angel. 54 von Vind. 60 abhängt. Außerdem ist die Reihenfolge der Autoren und der Werke auf der Titelseite des Angel. 54 identisch mit der des Vind. 60. Der Angel. 54 stammt daher aus dem Vind. 60.

99 M. Sicherl, Griechische Erstausgaben des Aldus Manutius, Paderborn 1997, S. 324-325.

100 Siehe dazu W. Schmid, Die sogenannte Aristidesrhetorik, in: RhM 72 (1917/1918), S. 113-149. Auch M. Sicherl ist der Meinung, daß die Vorlage der Aldina eine Kopie des Vind. 60 gewesen sein dürfte. Siehe dazu M. Sicherl, wie Anm. 99, S. 322 - 325.

101 Siehe dazu Chr. Walz, RG VIII, S. 415. 
$\left(\right.$ Ald $\left.^{\mathrm{s}}\right)$ einbezogen sind:

S. $1,5 \delta v \sigma \iota \phi \lambda i ́ \alpha \mathrm{V}: \delta v \sigma \imath \phi \imath \lambda i ́ \alpha \mathrm{P}^{3}$ Ald. Ald

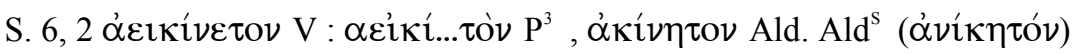

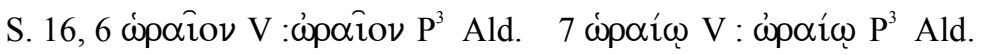

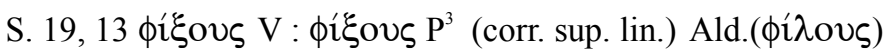

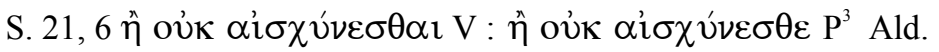

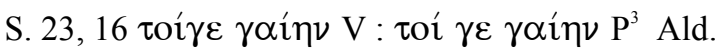

S. 37, $6 \kappa \alpha \tau \alpha \lambda \imath \pi \mathrm{o} \mu \varepsilon \dot{v} \omega \nu$ V: $\kappa \alpha \tau \alpha \lambda \varepsilon \imath \pi \mathrm{o} \mu \varepsilon^{\prime} \omega \omega \nu \mathrm{P}^{3}$ Ald.

S. $61,10 \mu \eta \dot{~ o m . ~} \mathrm{~V}: \ddot{\alpha} \mu \eta \dot{\mathrm{P}^{3}}$ Ald.

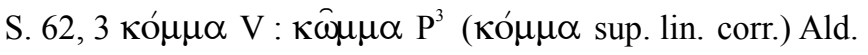

S. 65, $6 \tau \varepsilon \dot{\varepsilon} \tau \alpha \nu \tau \alpha \iota \mathrm{V}: \tau \dot{\tau} \tau \alpha \kappa \tau \alpha \mathrm{l} \mathrm{P}^{3}$ Ald.

b. Die Entstehungszeit des Paris. 1656 könnte dadurch eingegrenzt sein, daß Markos Musuros von Konstantinopel nach Italien gekommen war und sich von 1503 bis 1509 an der Universität in Padua als Lehrer der griechischen Sprache, Rhetorik und Philosophie betätigte. In dieser Zeit könnte der Paris. 1656 auf Veranlassung des Musuros aus dem Vind. 60 in Padua abgeschrieben worden sein. Denn der Vind. 60 befand sich damals in Padua ${ }^{102}$. Der Entstehungsanlaß könnte darin liegen, daß Musuros den Paris. 1656 als Vorlage für die Druckausgabe in der Druckerei des Aldus benutzen wollte. Dafür gibt es drei Indizien. Das erste ist, daß der Schreiber des Paris. 1656 im Vergleich zum Vind. 60 versucht, systematisch das Komma zu verwenden. Dies stimmt mit der Aldina überein. Ein Beispiel dafür ist das Folgende: S. 78, 9

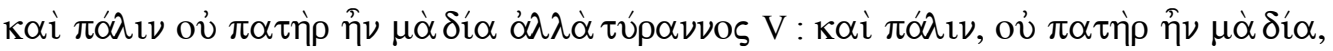
$\dot{\alpha} \lambda \lambda \dot{\alpha} \tau \hat{\rho} \rho \alpha \nu \nu o \varsigma \mathrm{P}^{3}$ Ald.. Das zweite Indiz ist, daß der Schreiber die Zeichen verwendet, mit denen man bei der Druckarbeit die Fehler kenntlich machte, die sich entweder an Vind. 60 orientierten oder die individuelle Fehler des Abschreibers waren. Es gibt drei solcher Zeichen : 1. untergesetzte Punkte ( ... ); 2. eine untergestrichene Linie (__ $)$; 3. ein Schrägstrich ( $\backslash$ ). Die Punkte sollen darauf hinweisen, daß man die so gekennzeichneten Buchstaben oder Wörter korrigieren mußte. Ein Beispiel dafür ist das

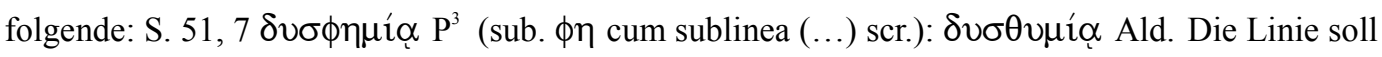
anzeigen, daß ein neues Wort zur Verbesserung von dem Abschreiber selbst ergänzt worden ist. Ein

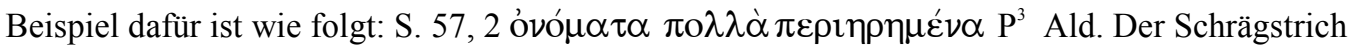
schließlich soll bedeuten, daß das von diesem gestrichene Wort wegzunehmen und zu korrigieren ist. Dies

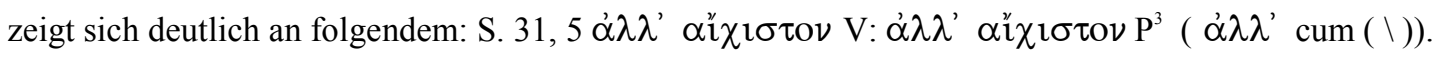
Das dritte Indiz aber bezieht sich auf die Verbesserung der Perikopen-Titel. Eigentlich war die

102 H. Hunger sagt über das Schicksal des Vind. 60: Der Besitzer war Johannes Baptista de Leone aus Padua: Johannes Sambucus kaufte den Codex im Jahre 1554 für sieben Dukaten wahrscheinlich in Padua. Bis 1554 war der Vind. 60 wahrscheinlich in Padua geblieben. Vgl. dazu H. Hunger, Katalog der Griechischen Handschriften der Österreichischen Nationalbibliothek, Wien 1961, S. 178-179. 
Betitelungsweise des Paris. 1656 sehr ungewöhnlich im Vergleich zu den älteren Handschriften, die aus dem Paris. 1741 stammen, und im Vergleich zu den jüngeren Codices wie Pal. 66, die den Vind. 60 als Vorlage benutzt haben. Ein Beispiel dafür ist folgendes: S. 10, 1 Tit. $\alpha^{\prime} \pi \rho \circ \delta$ ı́ $\rho \theta \omega \sigma ı \varsigma \mathrm{P}^{1}$ :

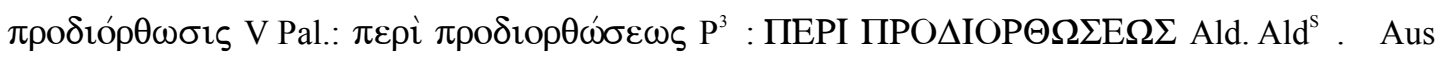
diesem Vergleich ist zu ersehen, daß Paris. 1656 eine neue Regulierung des Textbildes vorgenommen hat. Dieser Neuformatierung liegt die Absicht des Schreibers zugrunde, den Paris. 1656 als Vorlage für die Druckfassung der editio princeps zu benutzen.

3.6 Paris. $2087\left(\mathrm{P}^{2}\right)$ und Notae Marginales (N.M.) in Paris. $1741\left(\mathrm{P}^{1}\right)$

\subsubsection{Paris. 2087}

Auffallend ist, daß der Paris. 2087 christliche Beispiele hat, die aus Gregorios von Nazianz entnommen zu sein scheinen. Der Paris. 2087 hat aber diesen Namen in den meisten Fällen nicht ausdrücklich erwähnt. Genannt wird immer nur ó $\theta \varepsilon o \lambda$ ó $\gamma$ os. Aber einmal taucht der Name Gregorios ${ }^{103}$ auf:

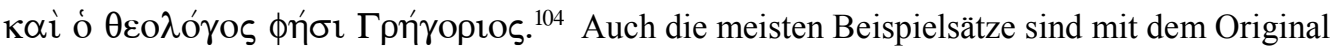
identifizierbar. Auf dieser Grundlage ist sicher, daß der Originaltext des Paris. 2087 „, scriptus est a christiano quodam vel ludi magistro ad pueros erudiendos vel ab ecclesiastico oratorum in usum sacrorum, ita ut in illustranda quaque figura definitionem et alteram exemplorum partem, nimirum ea, quae e paganis scriptoribus videmus (sc. Steusloff) petita esse, Alexandro Numenio vel ad verbum surriperet vel contracta et, ut ad ediscendum aptiora essent, conformata ederet. ${ }^{، 105}$ Die Abfassung des Originaltextes des Paris. 2087 fällt auf die Wende vom 3. zum 4. Jahrhundert n. Chr. ${ }^{106}$ Fraglich ist, auf welcher Vorlage der Urtext des Paris. 2087 fußt. Denn es gibt zwei Möglichkeiten: Zum einen könnte der Urtext des Paris. 2087 aus Alexander entstanden sein. Zum anderen ist es auch möglich, daß der Traktat sich direkt an Caecilius orientiert hat. In diesem Zusammenhang ist Steusloff der Auffassung, daß Paris. 2087 auf Alexander fußt. Dies zeigt sich deutlich an folgendem Stemma Codicum, das Steusloff als Fazit der Analyse am Ende seiner Dissertation aufgestellt hat ${ }^{107}$ :

103 Gregorios, Lexikon der antiken christlichen Literatur, S. 262-266: Gregorios wurde als Sohn des Bischofs Gregor von Nazianz in Kappadokien um 326 geboren und ist um 390 in Arianz bei Nazianz gestorben. Er wurde 380/81 Patriarch von Konstantinopel, doch da er sich mit seinen kirchenpolitischen Vorstellungen auf dem 2. Ökumenischen Konzil nicht durchsetzen konnte, trat er zurück, zog sich nach Nazianz zurück und führte später ein asketisches Leben. Von seinen Werken seien bes. die 44 Reden, die für Jahrhunderte als stilistische Vorbilder gegolten haben, die 245 Briefe und seine Gedichte erwähnt.

104 RG VIII, ed. Chr. Walz, S. 434, Fußnote 6.

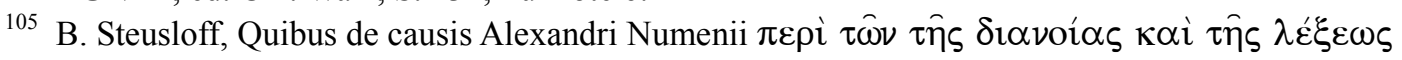
$\sigma \chi \eta \mu \alpha ́ \alpha \omega \nu$ liber, qui vulgo genuinus habetur, putandus sit spurius et quae epitomae ex deperdito Alexandri libro excerptae supersint, demonstratur, Diss. Phil. Breslau 1861, S. 11.

106 Wie Anm. 105, S. 49.

107 Wie Anm. 105, S. 49. 
(Liber Alexandri Numenii)

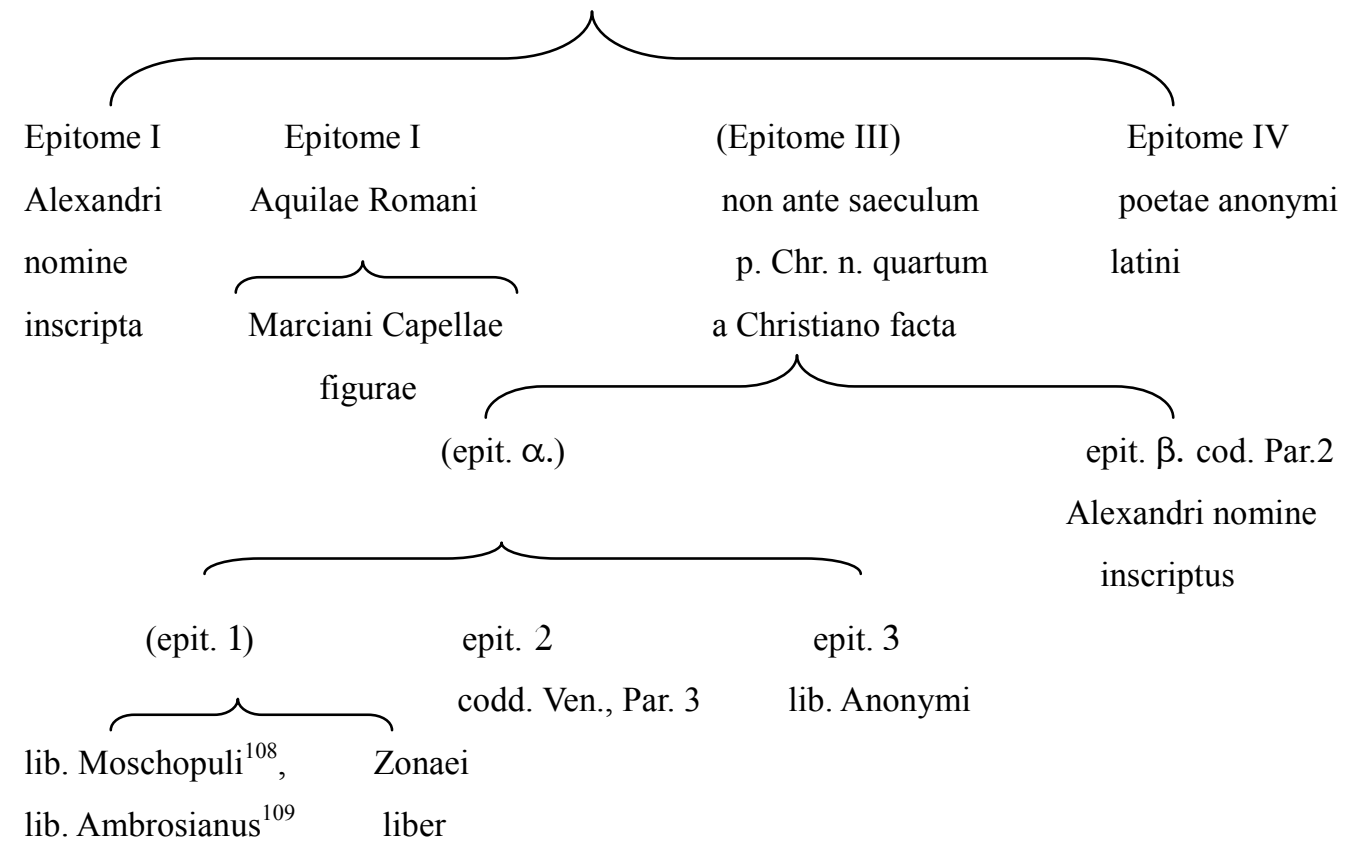

Adn. : Librorum deperditorum titulos uncinis inclusi (sc. Steusloff).

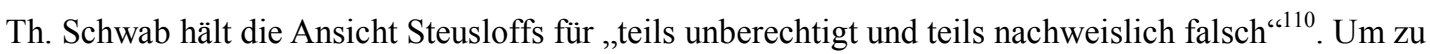
korrigieren, was Steusloff falsch dargestellt hat, vergleicht Schwab Einzelfiguren zwischen Alexander und dem Paris. 2087. ${ }^{111}$ Durch diesen Vergleich wird nachgewiesen, daß Paris. 2087 in drei Gesichtspunkten von Alexander abweicht. Die Abweichungen finden sich in den Definitionen von Einzelfiguren, in

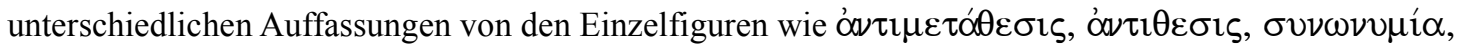

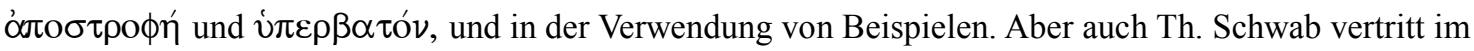
Grunde genommen die gleiche Position, wenn seiner Ansicht nach der Paris. 2087 und Aquila Romanus ,ausschließlich den echten Alexander ausgeschrieben hat.“112

In diesem Zusammenhang bin ich aber der Überzeugung, daß die Vorlage des Urtextes des Paris. 2087 nicht Alexander, sondern Caecilius sein könnte. Es gibt dafür zwei Anhaltspunkte, die ich während meiner Beschäftigung mit der Edition gefunden habe, und zwar die Abfolge der Einzelfiguren und einige Figuren, die zeigen, daß der Paris. 2087 nicht auf dem Umweg über Alexander von Caecilius abhängt.

\footnotetext{
108 Siehe dazu Zonaei De figuris, RG VIII, ed. Chr. Walz, S. 672: (...), quam (sc. commentationem) inter Moschopuli opuscula grammatica p. 60-67 edidit Titzius a. 1822 e codice nuper in Bohemia reperto, in quo sine auctoris nomine continetur (apud nos (sc. Walzium) M), cum qua ad verbum consentit cod. Ambros. A. 115 (apud nos (sc. Walzium) Ambr. vel A.).

109 Siehe Anm. 108.

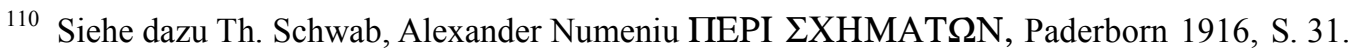

111 Wie Anm. 110, S. 31-101.

112 Wie Anm. 110, S. 3.
} 


\section{$\alpha$. Die Abfolge der Einzelfiguren}

Der Verfasser der Quelle des Paris. 2087 folgt nicht der Abfolge der Einzelfiguren Alexanders. Grosso modo ist die Abfolge der Figuren Alexanders, wie im § 2.3 gezeigt, nach den vier Produktionskategorien angeordnet. Es ist aber kaum möglich, nach diesem Ordnungsprinzip die Einzelfiguren des Paris. $2087 \mathrm{zu}$ klassifizieren. Die Abfolge des Paris. 2087 gehört also nicht in den Horizont Alexanders, sondern zu einer früheren Phase. Ein entscheidender Beleg dafür ist die Behandlungsweise der Gorgianischen Figuren,

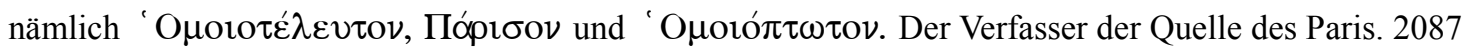
behandelt die drei Figuren im Zusammenhang, während Alexander diese Tradition nicht berücksichtigt, sondern nach neuem Prinzip die drei Figuren getrennt kategorisiert. Außerdem gibt es einige Figuren, die

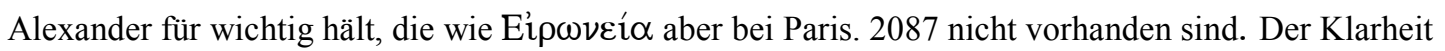
wegen fertige ich eine Synopse an, welche die Unterschiede zwischen beiden deutlich zeigt:

Alexander

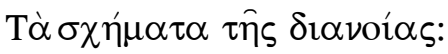

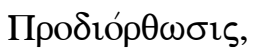

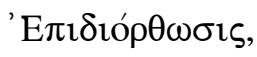

'A $\mu \phi \imath \delta$ ióp$\theta \omega \sigma t \varsigma$,

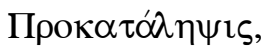

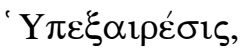

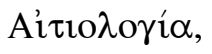

$\Sigma v v \alpha \theta \rho o \imath \sigma \mu o ́ \varsigma$,

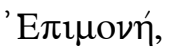

$\Lambda \varepsilon \pi \tau \mathrm{\alpha} \lambda \mathrm{o \gamma i \alpha}^{\alpha}$,

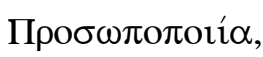

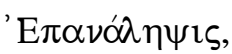

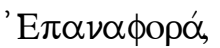

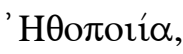

'А

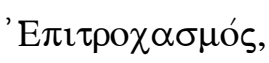

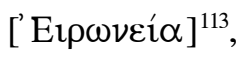

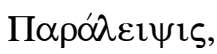

'A $\mathrm{\pi} \sigma \sigma \tau \rho \phi \emptyset$,

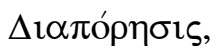

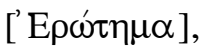

Paris. 2087

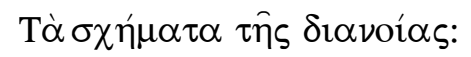

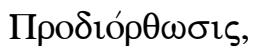

'E $\pi \mathrm{\imath} \delta \imath o ́ \rho \theta \omega \sigma \imath \varsigma$,

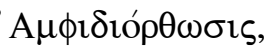

Прокатód

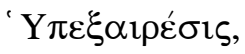

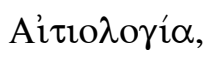

$\Sigma v v \alpha \theta \rho o \imath \sigma \mu o ́ \varsigma$,

'Елиногі,

$\Lambda \varepsilon \pi \tau \circ \lambda \circ \gamma^{i} \alpha$,

'НӨолоиí $\alpha$,

Проб

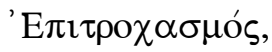

А

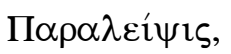

'A $\pi$ о $\tau \rho \circ \phi \eta '$,

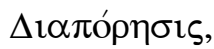

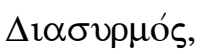

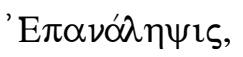

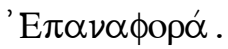

${ }^{113}$ Die Figuren, die von diesem Zeichen [ ] eingeklammert sind, sind im Paris. 2087 nicht vorhanden. 


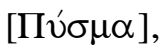

[ $\Delta \mathrm{l} \alpha \tau \dot{\pi} \pi \omega \sigma \imath \varsigma]$,

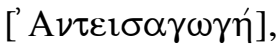

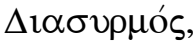

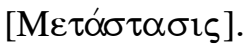

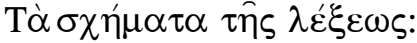

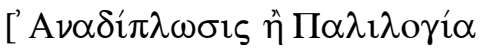

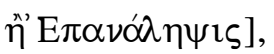

['E $\pi \alpha v \alpha \phi о \rho \alpha ́]$,

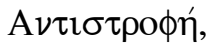

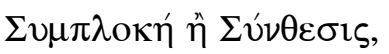

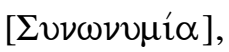

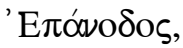

$\mathrm{K} \lambda \hat{\mathrm{\mu}} \mu \alpha \xi$,

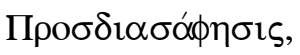

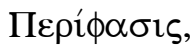

$\Pi \lambda \varepsilon \circ v \alpha \sigma \mu o ́ s$,

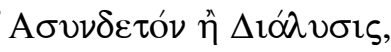

"E $\lambda \lambda \varepsilon i \psi i \varsigma$,

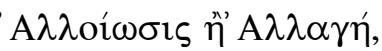

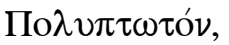

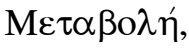

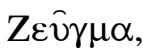

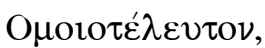

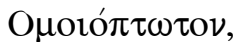

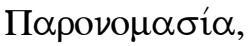

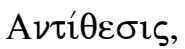

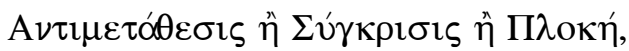

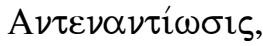

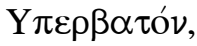

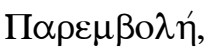

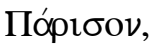

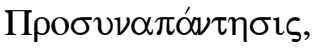

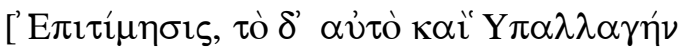

$\tau \imath v \varepsilon \varsigma \kappa \alpha \lambda \mathrm{ov} \sigma \mathrm{v}$.

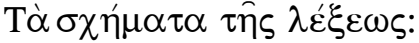

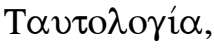

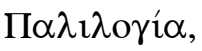

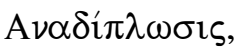

A $\nu \tau 1 \sigma \tau \rho \circ \phi \eta '$,

$\Sigma v \mu \pi \lambda$ оки́,

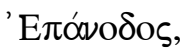

$\mathrm{K} \lambda \hat{\mathrm{u}} \mu \alpha \xi$,

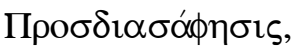

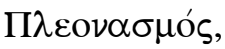

"E $\lambda \lambda \varepsilon \imath \psi \imath \iota \varsigma$,

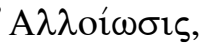

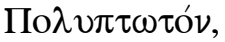

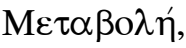

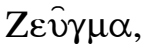

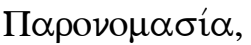

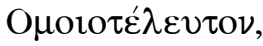

Пópiбov,

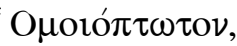

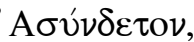

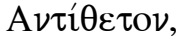

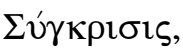

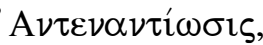

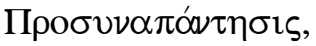

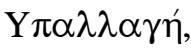

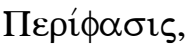

Y $\pi \varepsilon \rho \beta \alpha \tau o ́ v$,

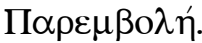


ß. Einige Figuren, die bestätigen, daß Paris. 2087 auf Caecilius fußt.

Hierfür ist der erste Beleg die П $\alpha \lambda \imath \lambda \lambda \sigma \gamma i \alpha$. Diese wurde von Alexander als' A $v \alpha \delta i ́ \pi \lambda \omega \sigma \iota \varsigma$

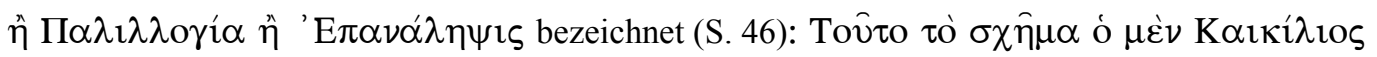

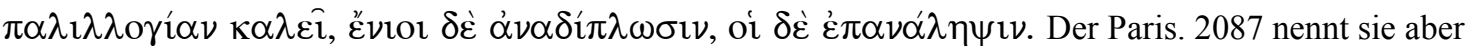
ohne Alternative $\Pi \alpha \lambda \imath \lambda \lambda \sigma \gamma i \alpha$. In diesem Zusammenhang ist Tiberius ein wichtiger Zeuge, der sagt, da $\beta$

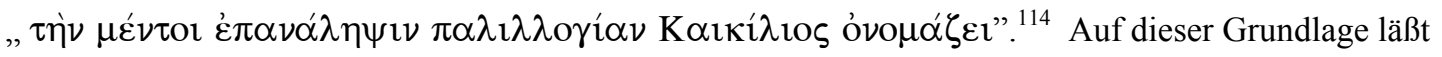
sich feststellen, daß Paris. 2087 sich an Caecilius orientiert.

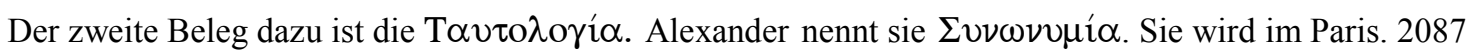
als T $\alpha v \tau o \lambda o \gamma i \alpha$ bezeichnet. Dennoch benutzen sie gemeinsam das Beispiel eines Demosthenes-Zitats. Dies zeigt sich an folgendem Vergleich:

Paris. 2087 (S. 50)

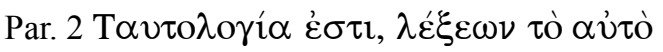

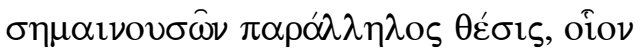

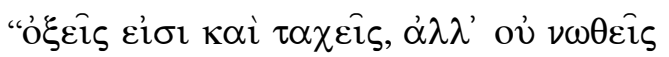

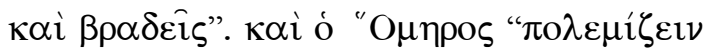

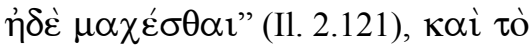

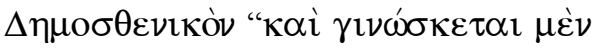

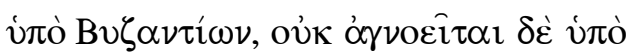

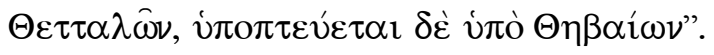

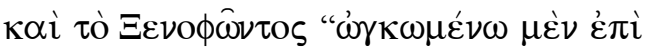

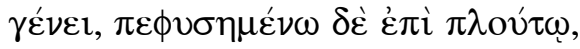

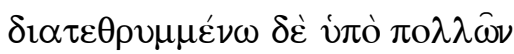

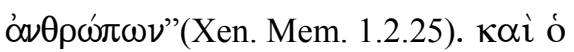

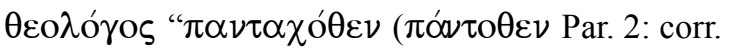

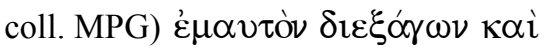

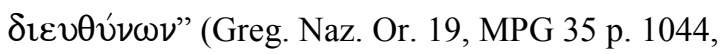

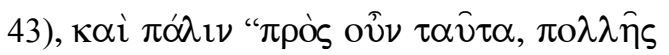

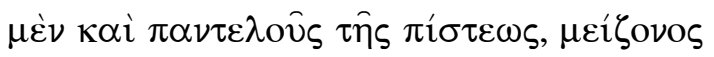
$\delta \dot{\varepsilon} \tau \hat{\eta} \varsigma \pi \alpha \rho \grave{\alpha} \theta \varepsilon o \hat{v}(\theta \nu \mu \omega \hat{~ P a r .2 ~: ~ c o r r . ~ c o l l . ~}$

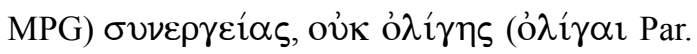
2: corr. coll. Vat. 1881) $\delta \dot{\varepsilon} \tau \hat{\eta} \varsigma$ $\eta \mu \varepsilon \tau \varepsilon \dot{\rho} \rho \varsigma$

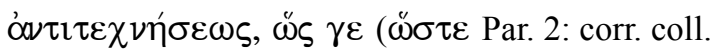

Alexander (S. 50)

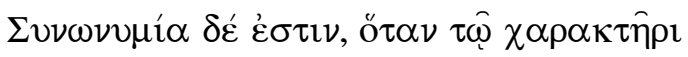

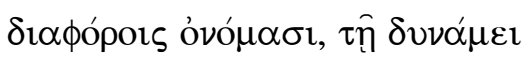

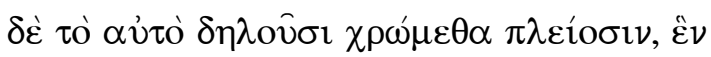

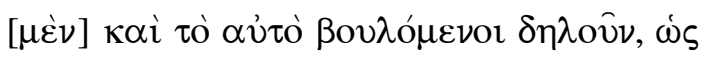

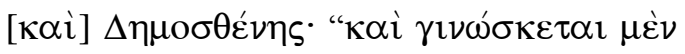

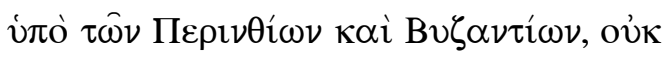

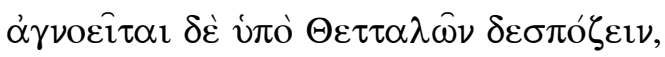

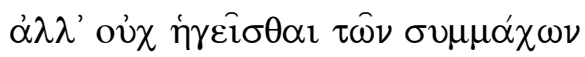

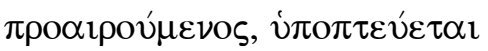
$\delta \varepsilon \dot{v} \pi \grave{o} \Theta \eta \beta \alpha i ́ \omega v . ”$ (Demosth. In Epist. Philipp. 3-4)

114 Tiberii De figuris Demosthenicis cum deperditorum operum fragmentis, ed. G. Ballaira, Rom 1968, S. 27. 


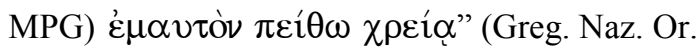

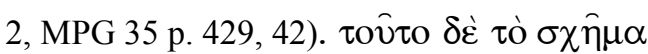

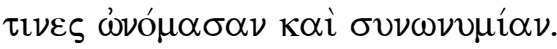

Auffallend ist das Beispiel eines Xenophon-Zitats im Paris. 2087. Denn Alexander verwendet dieses

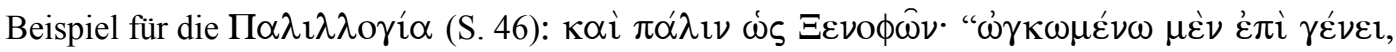

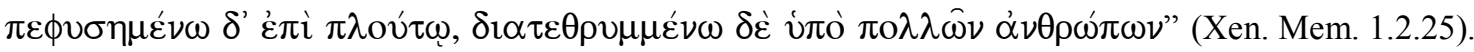

Auf dieser Grundlage ist festzustellen, daß Alexander in bewußter Opposition versucht, die FigurenTheorie des Caecilius zu korrigieren oder soweit zu verbessern, wie er sie für unangemessen hält.

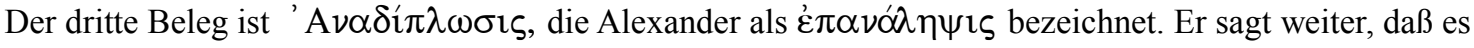

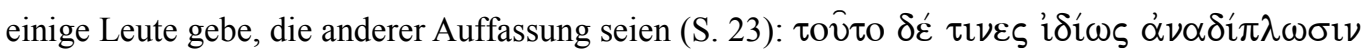

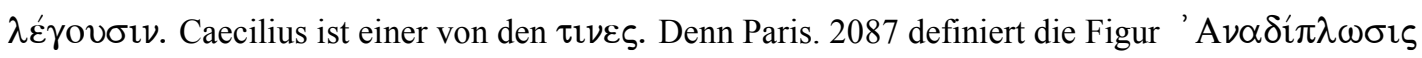

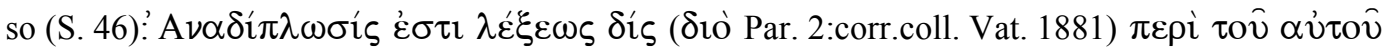

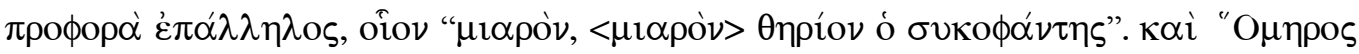

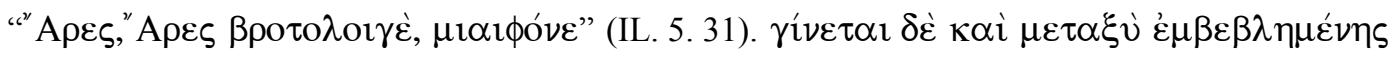

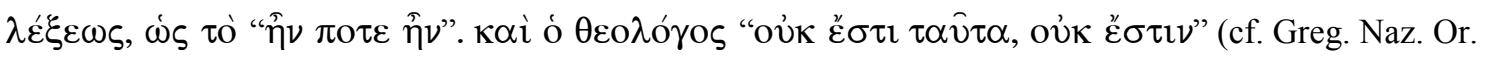

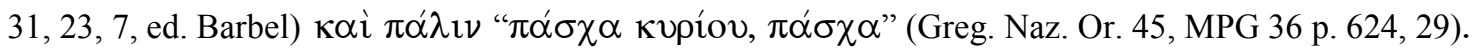
Die’ E $\pi \alpha \nu \alpha \lambda \eta ́$ $\psi \iota \varsigma$ bestätigt schließlich deutlich, daß Paris. 2087 nicht auf Alexander, sondern auf Caecilius fußt. Denn der Paris. 2087 zählt diese Figur zu den Sinnfiguren, während Alexander sie sowohl zu den Sinnfiguren als auch zu den Wortfiguren gehören läßt. Außerdem unterscheiden sich Alexander

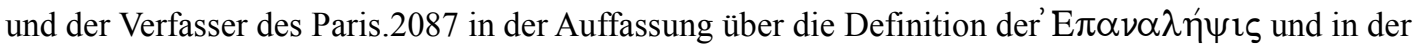

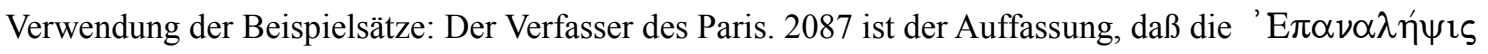
eine $\alpha v \alpha \dot{\alpha} \mu \nu \eta \sigma ı \varsigma$ ist, während Alexander sie umfangreicher und ausführlicher erörtert. Aber er erwähnt nicht die $\alpha \dot{\alpha} \alpha \dot{\mu} \nu \eta \eta \imath \varsigma$. Alexander korrigiert also das, was seiner Meinung nach unangemessen ist. Dies wird auch an folgendem Vergleich deutlich:

Paris. 2087 (S. 22)

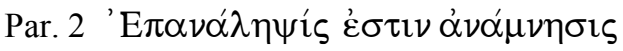

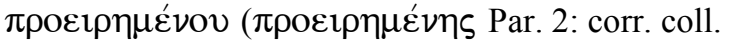
Vat. 1881) $\mu \varepsilon \tau \alpha \xi v \lambda o \gamma i \alpha \varsigma$ $\varepsilon \mu \beta \varepsilon \beta \lambda \eta \mu \varepsilon \dot{v \eta}$

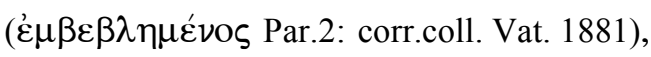

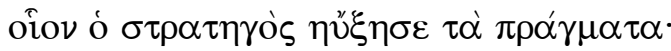

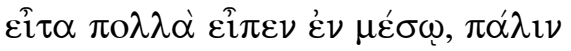

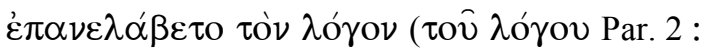

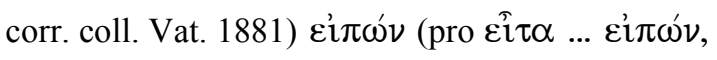

Alexander (S. 22 - 23)

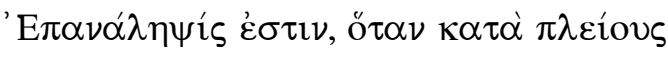

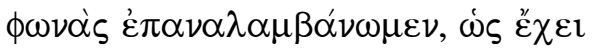

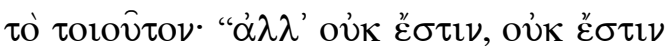

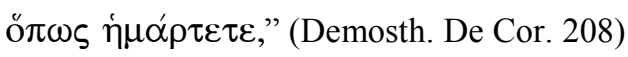

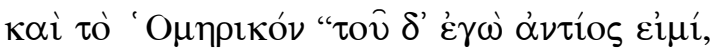

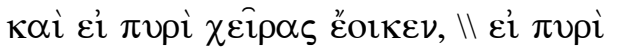

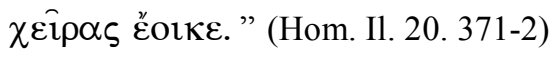

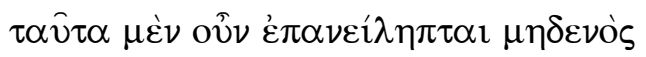




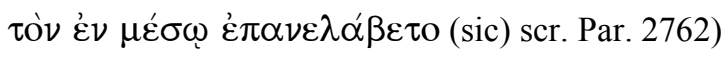

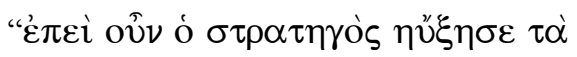

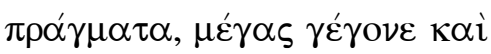

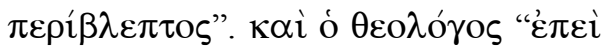

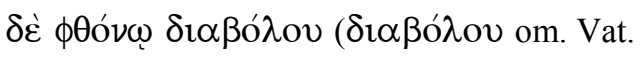

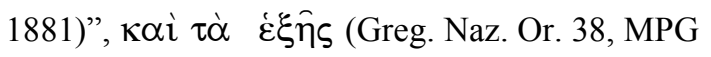
36 p. 324, 39). عi $\tau \alpha<\mu \varepsilon \tau \dot{\infty}>$ (addidi)

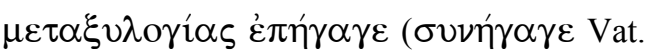

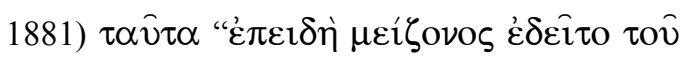

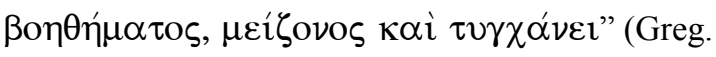
Naz. Or. 38, MPG 36 p. 325, 16).

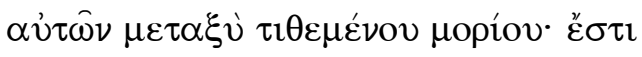

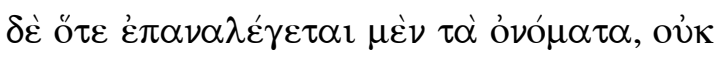

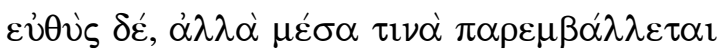

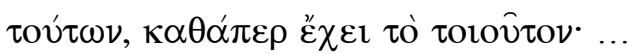

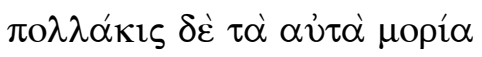

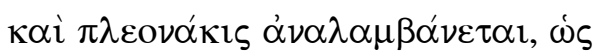

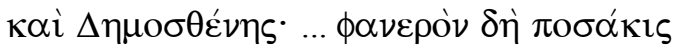

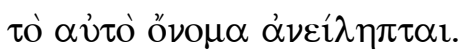

Die bisherige Darstellung läßt sich so zusammenfassen, daß der Paris. 2087 unverändert in der Tradition des Caecilius steht, während Alexander die Anordnung des Caecilius geändert hat:

Paris. 2087 (Caecilius)

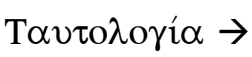

$\Pi \alpha \lambda \imath \lambda \lambda \mathrm{o} \gamma \dot{i} \alpha \rightarrow$

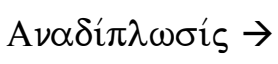

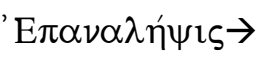

Alexander

$\varsigma^{\prime}$. ПEPI $\Sigma$ YN $\Omega N Y M I A \Sigma$ (S. 50)

$\beta^{\prime}$. ПЕPI ANA $\triangle \mathrm{I \Pi} \Lambda \Omega \Sigma \mathrm{E} \Omega \Sigma \mathrm{H}$

ПА $\Lambda \Lambda \Lambda$ ОГА $\Sigma$ Н ЕПАNА $Н \Psi Е \Omega \Sigma$ (S. 46)

$\imath \gamma^{\prime}$. ПЕРI ЕПАNА $\Lambda \Psi E \Omega \Sigma$ (S. $\left.22-23\right)$

$\imath \gamma^{\prime}$. ПЕРI ЕПАNA $\Lambda$ HЕ $\Omega \Sigma$ (S. $\left.22-23\right)$

3.6.2 Die Familie des Paris. 2087

Während meiner Beschäftigung für diese Edition habe ich drei Handschriften für die Familie des Paris. 2087 ermittelt. Sie sind Vat. 1881, Paris. 2762 und Laurent. 57. Der Vat. 1881 ist im 13. Jh. entstanden. Diese Handschrift ist also älter als der Paris. 2087. Der Paris. 2762 und der Laurent. $57^{115}$ sind eng verwandt mit dem Vat. 1881. Es könnten also der Paris. 2762 und der Laurent. 57 auf Vat. 1881 beruht haben. Ein entscheidender Beleg dafür ist eine Stelle, die Paris. 2087 nicht enthält, während die anderen

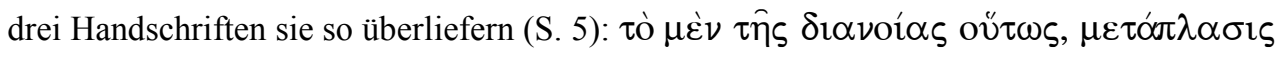

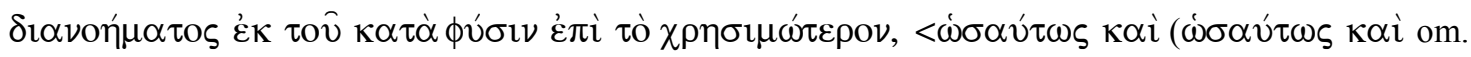

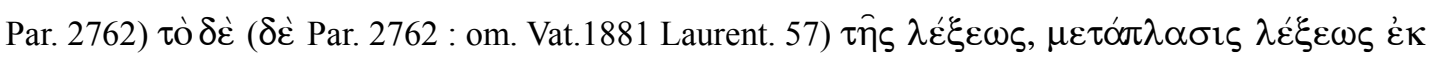

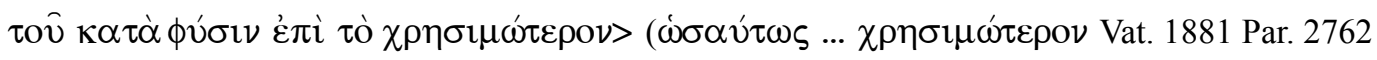

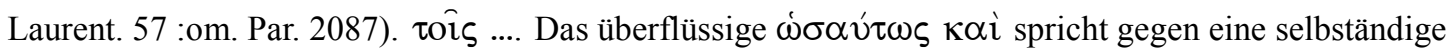
Konjektur oder Ergänzung des Schreibers von Vat. 1881: wenn er schon ergänzte, hätte er es vermieden,

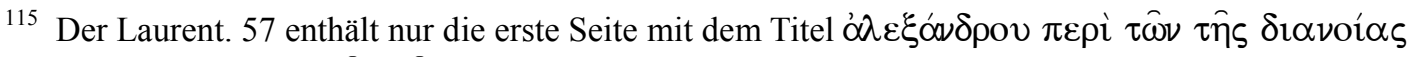

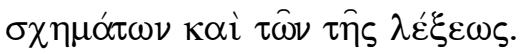




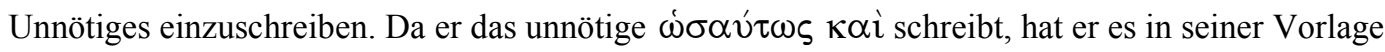
gelesen. Dieses $\omega \sigma \alpha v ́ \tau \omega \varsigma$ K $\alpha i$ enthält der Laurent. 57. Der Schreiber von Paris. 2762 hat aber das

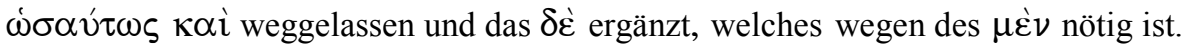

\subsubsection{Notae Marginales in Paris. 1741}

Charakteristisch für diese Randbemerkungen ist, daß sie eine stark verkürzte Epitome sind. Sie bestehen in der Regel nur aus zwei Bestandteilen, und zwar aus Definition und Beispiel. Die Definition ist teilweise mit der des Paris. 2087 und teilweise mit der des Zonaios ${ }^{116}$ identisch. Die Beispiele sind christlich und identisch mit denen des Paris. 2087. Die Abfolge der Epitomai aber unterscheidet sich von der des Paris. 2087. Denn sie folgt dem Paris. 1741, auf dessen Rand sie steht. Auf dieser Grundlage läßt sich feststellen, daß der Schreiber der Notae Marginales den Paris. 1741 mit einer bewußt christlichen Überarbeitung ergänzen möchte.

Sicher ist, daß die Vorlage der Notae Marginales im Paris. 1741 zu der Familie der Vorlage des Paris. 2087 gehört. Die Vorlage für die Notae Marginales war aber nicht der Paris. 2087 selbst. Denn es gibt Handschriften ${ }^{117}$, wie Steusloff gezeigt hat (S. 22), die wie Veneto classe. 18. 8 als Vorlage gedient haben könnten. Dieser Kodex ist im Hinblick auf den inhaltlichen Zusammenhang und die Abfolge der Epitomai beinahe identisch mit den Notae Marginales ${ }^{118}$. Es gibt dafür zwei Möglichkeiten, auf welcher Vorlage die Notae Marginales fußen, daß nämlich entweder der Schreiber der Notae Marginales den cod. Veneto classe. 18.8 benutzt haben könnte oder aber daß ein gemeinsames Manuskript als Vorlage diente, aus dem die beiden Handschriften stammen.

Auf dieser Grundlage versuche ich eine Verbesserung des stemma codicum von Steusloff zur Klärung der Frage, in welchen Zusammenhang die Notae Marginales gehören. Dies zeigt sich an folgendem Stemma:

116 Zonaei De figuris, RG VIII, ed, Chr. Walz, Stuttgart 1832-36, S.671-690.

117 Dazu gehören Paris. 2929, Paris. 2008 und Ambros. A. 115. Siehe Zonaei De figuris, RG VIII, ed. Chr. Walz, Stuttgart 1832-1836, S. 671-672.

118 Belege dafür finden sich im kritischen Apparat des Zonaios De figuris. Siehe wie Anm. 117, S.671690 . 


\section{(libri Caecilii Caleactanii)}

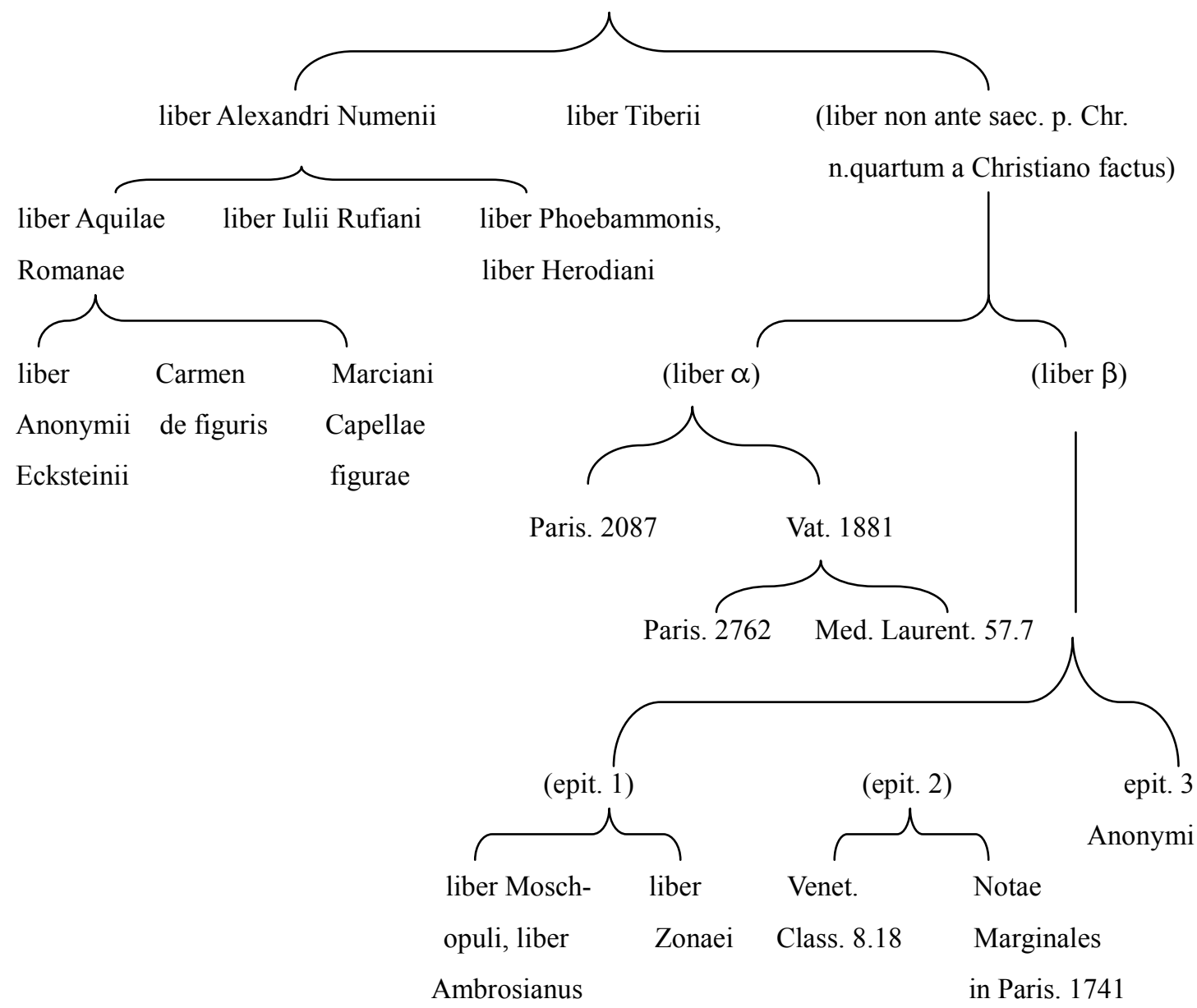




\section{Bisherige Editionen}

\subsection{Die editio princeps Aldina}

Die erste Edition ${ }^{119}$ von De figuris sententiarum et verborum des Alexander Numeniu wurde im Jahre 1508 von Aldus Manutius erstellt. ${ }^{120}$ Im Vergleich zu den Handschriften ist es charakteristisch für diese erste Druckausgabe $^{121}$, daß sie Majuskeln für die Titelangaben benutzt, die Wortumbrüche im Text mit Linien gekennzeichnet sind und bei Seitenumbrüchen das Wort vorweggenommen wird, das am Anfang der nächsten Seite steht ${ }^{122}$. Außerdem wird der Beginn der Erörterung einer jeden Einzelfigur durch Initialen, die in ein freies Feld gesetzt sind, hervorgehoben. Der Aldina liegt Paris. 1656 zugrunde, welcher von Markos Musurus zur Druckausgabe überarbeitet worden ist. Diese Edition wurde von Natale de' Conti 1557 ins Lateinische übersetzt ${ }^{123}$. Dazu gibt es eine handschriftliche Kopie der Aldina (Paris.

BN gr. S. 552).

\subsection{Norrmannus}

Der Traktat De figuris sententiarum et verborum des Alexander Numeniu wurde 1690 von Laurentius Norrmannus ${ }^{124}$ neu bearbeitet und mit einer eigenen lateinischen Übersetzung herausgegeben. ${ }^{125}$ Norrmannus hat viele Bemerkungen zu Textstellen am Rande notiert, jede Perikope numeriert und einen längeren Kommentar Notae ad Alexandrum ${ }^{126}$ hinzugefügt. Mit den Randbemerkungen stellte er zum ersten Mal sozusagen einen kritischen Apparat her. Dort sind Verbesserungsvorschläge angeführt, die auf Kollationen von Handschriften und auf Vergleichung mit dem originalen Wortlaut der Beispielsätze beruhen. Die Notae bestehen aus drei Teilen. Der erste ist eine Darstellung, die einer Praefatio entspricht. Norrmannus bespricht hier den Autor und die Überlieferungsgeschichte von De figuris sententiarum et verborum $^{127}$. Der zweite ist ein sprachlicher und sachlicher Kommentar, in dem auch erklärt wird, worauf seine Konjekturen und verbessernden Vorschläge beruhen. ${ }^{128}$ Der letzte ist mit $\pi \dot{i} v \alpha \xi$ überschrieben, was dem Index im modernen Sinne entspricht. Der $\pi i v \alpha \xi$ enthält rhetorische Termini mit Seitenangaben wie zum Beispiel:

119 Siehe M. Sicherl, Griechische Erstausgaben des Aldus Manutius, Paderborn 1997, S. 310: „Die editio princeps zahlreicher rhetorischer Traktate und Kommentare, eine Folioausgabe in zwei Bänden, von denen der erste den Kolophon des Aldus Manutius vom November 1508, der zweite vom Mai 1509 trägt, ist von dem Kreter Demetrios Dukas besorgt.“

120 Rhetores Graeci, ed. M. Aldus, Venedig 1508.

121 Vgl. dazu M. Sicherl, Griechische Erstausgaben des Aldus Manutius, Paderborn 1997, S. 310-340.

122 Hierfür führe ich als Beleg eine Photokopie an. Siehe Photokophie VII.

123 Alexandri Sophistae De schematibus, übers. von Natale de' Conti, Paris 1557.

124 L. Norrmannus (1651-1703) war als Philologe in Uppsala tätig. Er konzentrierte sich auf RhetorikTraktate und dabei besonders auf Aristides.

125 Alexandri De figuris sententiarum et verborum, ed. L. Norrmannus, Uppsala 1690.

126 Wie Anm. 125, S. 165-201.

127 Wie Anm. 125, S. 165-169.

128 Wie Anm. 125, S. 169-182. 
ПINA $\Xi^{129}$

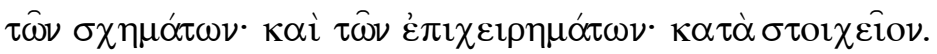

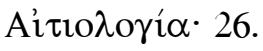

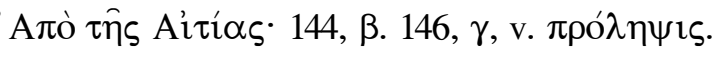

A $\lambda \lambda \eta \gamma о \rho i ́ \alpha \cdot 14$.

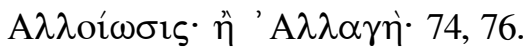

etc.

\subsection{Chr. Walz}

Die Textausgabe des Norrmannus hat Chr. Walz in der Bibliothèque Nationale in Paris um 1830 entdeckt und mit vier Handschriften verglichen. ${ }^{130}$ Eine von ihnen ist identisch mit der jetzt in der Österreichischen Nationalbibliothek in Wien befindlichen (Phil.gr. 60) ${ }^{131}(=\mathrm{V})$, während die anderen drei Handschriften in Paris geblieben sind (Nr. $\left.1741^{132}\left(=\mathrm{P}^{1}\right), 1656^{133}\left(=\mathrm{P}^{3}\right), 2087^{134}\left(=\mathrm{P}^{2}\right)\right)$. Tatsächlich aber hat Walz diese Handschriften in seiner Edition nicht berücksichtigt, wie schon L. Spengel meinte: nec Walzium, diligentissimum virum, libri scripti adiuverunt. ${ }^{135}$ Die Textverbesserungen, die Walz gibt, sind in der Regel von Norrmannus übernommen. Der kritische Apparat von Walz hat drei Funktionen. Die Notate konstituieren zuerst einen richtigen apparatus criticus: Dort wird dokumentiert, wie eine Textstelle überliefert oder korrigiert worden ist. Zweitens hat er die Funktion eines apparatus similium: Es wird nämlich der Wortlaut des Paris. 2087 zum Vergleich geboten. Dieser Text, der wie oben gezeigt, eine selbständige Caecilius - Version darstellt, ist bisher nicht systematisch dokumentiert. Dies geschieht in der vorliegenden Edition in der Form eines Similienapparats. Schließlich unternimmt es Walz, im kritischen Apparat zu erklären, worauf seine Vorschläge beruhen ${ }^{136}$; er hat also die Funktion eines Kommentars. Walz hat dabei die Handschriften verkürzt bezeichnet, um Platz zu sparen: Der Paris. 1741 wird als Par.1, der Paris.2087 als Par. 2 und der Paris. 1656 als Par. 3 bezeichnet. ${ }^{137}$

\subsection{Spengel}

Schließlich hat L. Spengel den Text von De figuris sententiarum et verborum im dritten Band der

129 Alexandri De figuris sententiarum et verborum, ed. L. Norrmannus, Uppsala 1690, S. 195-201.

130 Rhetores Graeci, Bd. 1-9, ed. Ch. Walz, Stuttgart 1832-1836.

131 H. Hunger, Katalog der griechischen Handschriften der Österreichischen Nationalbibliothek 1, Wien 1961.

132 H. Omont, Inventaire sommaire des Manuscrits Grecs de la Bibliothèque Nationale 1-4, Paris 18861898.

133 Wie Anm. 132.

134 Wie Anm. 132.

135 Rhetores Graeci, Bd. 1-3, ed. L. Spengel, Leipzig 1853-1856, Bd. 3, S. III.

136 Vgl. dazu Rhetores Graeci, Bd. VIII, ed. Chr. Walz, Stuttgart 1832-1836, S. 422, Fußnote 7.

137 Bei meiner Edition habe ich die Abkürzungen von Walz übernommen. 
Sammlung Rhetores Graeci im Jahre 1856 ediert. ${ }^{138}$ Die Edition Spengels, die noch heute als maßgebliche Textausgabe benutzt wird, ist in vielerlei Hinsicht unzulänglich. So ist es allein schon ungünstig, daß der textkritische Apparat vom Text getrennt in der Praefatio angeführt wird ${ }^{139}$, und zwar ohne die Quellenbelege der Beispielzitate, die wiederum an anderer Stelle, nämlich am Schluß des dritten Bandes ${ }^{140}$ erscheinen. Daher ist es nicht einfach, etwa Textvarianten miteinander zu vergleichen. Noch schwerer wiegt jedoch, daß die Quellenangaben der Beispielzitate manchmal ungenau, ja bisweilen ganz irreführend sind. Außerdem bleiben Textlücken vielfach unbehandelt. Zwar stellt Spengel einige Verbesserungsvorschläge im textkritischen Apparat vor, sie sind aber nicht ausreichend zur Lösung des jeweiligen Textproblems. In der Tat wurde die dritte Band der Rhetores Graeci von Spengel selbst nicht genau emendiert und in der Neuedition von Hammer nicht berücksichtigt und damit nicht ausreichend textkritisch betrachtet.

${ }_{138}^{138}$ Rhetores Graeci, Bd. 1-3, ed. L. Spengel, Leipzig 1853-1856.

139 Wie Anm. 138, Bd. 3, S. III-V.

${ }^{140}$ Wie Anm. 138, Bd. 3, S. 534-535; die Zitierweise der Autoren-Exempel ist durchgehend veraltet. 


\section{5. Über diese Edition}

\subsection{Die Grundlagen des apparatus criticus}

Zur Bearbeitung des textkritischen Apparats habe ich zunächst alle Handschriften, die mir erreichbar waren, gesammelt und miteinander verglichen. Dabei habe ich einige Handschriften ermittelt, die die Editoren von Aldus bis Spengel bei ihren Texteditionen nicht berücksichtigt haben. So habe ich eine Handschrift in der Bibliothèque Nationale in Paris (S. $552^{141}$ ) gefunden, dazu zwei weitere Handschriften in der Bibliotheca divi Marci Venetiarum in Venedig (Gr. $429^{142}$, Gr. $512^{143}$ ), darunter eine mit einer selbständigen Überlieferung, und noch eine Handschrift in der Bibliotheca Vaticana in Rom (Palat. Gr. $66^{144}$ ) und sie kollationiert.

\subsection{Zur Konstituierung des apparatus similium}

Zur Klärung der Frage, wie Alexander mit den Beispielsätzen umgeht, führe ich die Originaltextstellen im apparatus criticus an, sofern die von Alexander gegebene Form vom Original abweicht. Zur Konfrontierung mit dem Text Alexanders habe ich dann die Version des Paris. 2087 und die Notae Marginales in Paris. 1741 verwendet. Walz hat den Paris. 2087 im kritischen Apparat oft ungenau und unvollständig wiedergegeben. Zur Verbesserung habe ich zwei Handschriften (Paris. 2762 und Vat. 1881) und die Notae Marginales benutzt.

\subsection{Emendationen und Konjekturen}

a. Diese Edition verfährt nach der textkritischen Methode Lachmanns ${ }^{145}$. Der Paris. 1741 als älteste Handschrift und Vorlage aller jüngeren (mit einer Ausnahme) wird also in erster Linie berücksichtigt. Doch hat sich im Lauf der recensio der Marc. 512 als selbständige Überlieferung erwiesen. Wie im $\S 3.1$ dargestellt, habe ich zunächst nach einem Vergleich zwischen der Familie des Paris. 1741 und dem Marc. 512 die fehlerhaften Textstellen emendiert. Demnach werden oft diejenigen fehlerhaften Stellen korrigiert, die in der Familie des Paris. 1741 etwa aus der Mißdeutung der Kürzungszeichen entstanden sind. Und

141 H. Omont, Inventaire sommaire des Manuscrits Grecs de la Bibliothèque Nationale 1-4, Paris 18861898.

142 E. Mioni, Bibliothecae Divi Marci Venetiarum codices Graeci manuscripti 1. Thesaurus antiquus 1-2, Rom 1981-1985.

143 Wie Anm. 142

144 M. Sicherl, Griechische Erstausgaben des Aldus Manutius, Paderborn 1997, S. 324.

145 R. Pfeiffer, Die Klassische Philologie von Petrarca bis Mommsen, München 1976, S. 232 f.: „Lachmanns Name ist vor allem berühmt durch seine textkritische Methode, in der die ,recensio’ der Handschriften zu dem sogenannten ,Archetypus' führen sollte; ergab die Prüfung der Handschriften nicht eine überzeugende ursprüngliche Lesart, so wurde ,emendatio' notwendig. Lachmann entwickelte diese Methode in seiner Arbeit an den lateinischen Dichtern, und er brachte sie in der Erforschung der Handschriften des Neuen Testaments zu solcher Vollkommenheit, daß es ihm schließlich gelang, das Ansehen des ,textus receptus' zu erschüttern.“ Siehe dazu auch S. Timpanaro, Die Entstehung der Lachmannschen Methode, 2.Aufl. Hamburg 1971. 
wenn es scheint, daß eine Version der Familie Paris. 1741, des Marc. 512 oder eine Konjektur von Editoren als diskutable Variante zu gelten hat, dann habe ich sie als Vorschlag in den apparatus criticus aufgenommen. Auch die „Verbesserungen“ des Marc. 512, die nicht den originalen Wortlaut Alexanders herstellen, sind im apparatus criticus dokumentiert. Außerdem habe ich dort in den Text eingegriffen, wo Versionen der Notae Marginales bzw. Paris. 2087 es nahelegen, daß Alexanders Text sprachlich korrupt ist.

b. Plausible Konjekturen habe ich in der Regel von früheren Editoren übernommen. Denn diejenigen Stellen, bei denen es einer Konjektur bedurfte, sind weitgehend schon von den früheren Editoren emendiert worden. Vor allem hat L. Norrmannus viele zutreffende Konjekturen vorgeschlagen. Deshalb war es in der Regel nicht notwendig, mit Hilfe von eigenen Konjekturen Textstellen zu verbesseren. Lediglich an solchen Stellen habe ich konjiziert, wo es deutlich an grammatischen Funktionsprädikaten

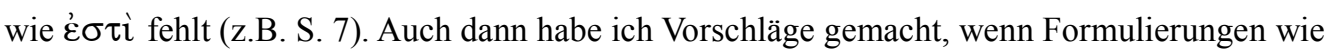

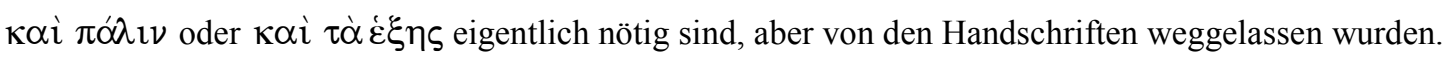
Offensichtliche Sprungfehler habe ich korrigiert.

\subsection{Kleine Bemerkungen zu Grammatik und Orthographien}

Im kritischen Apparat ist die Überlieferung dokumentiert, und es sind die Urheber der jeweiligen Verbesserungen notiert, bisweilen mit Alternativvorschlägen, die nicht in den Text aufgenommen sind. Nicht dokumentiert sind die üblichen Suspensionen und Kontraktionen der Handschriften, also

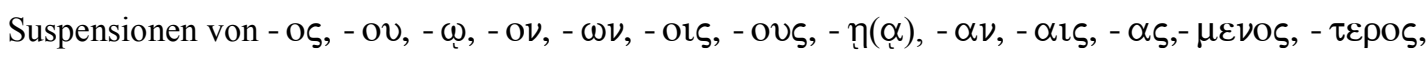

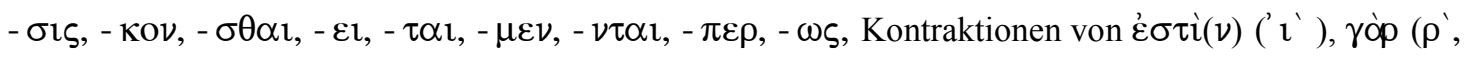

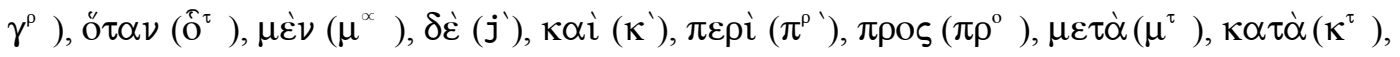

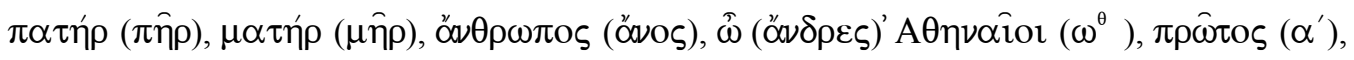
$\delta \varepsilon v ́ \tau \varepsilon \rho \circ \varsigma\left(\beta^{\prime}\right), \tau \varepsilon \dot{\varepsilon} \sigma \sigma \alpha \rho \alpha\left(\delta^{\prime}\right), \tau 0 \hat{v}\left(\tau^{v}\right), \tau \hat{\omega} \nu\left(\hat{\tau}^{v}\right), \tau \eta \dot{\nu} \nu\left(\tau^{\eta}{ }^{v}\right)$.

Auch bei der Worttrennung besteht eine gewisse Inkonsequenz in den Handschriften. Wenn die falsch durchgeführte Worttrennung einen gewissen Sinn hat und sie deshalb offensichtlich gewollt war, dann ist ein solcher Fall im kritischen Apparat notiert. Ebenso nicht dokumentiert sind die

Buchstabenverwechslungen im kritischen Apparat, da sie verbreitet sind: $\alpha-\varepsilon, \alpha-\varepsilon 1, \alpha-v, \varepsilon-\eta, \varepsilon-v$,

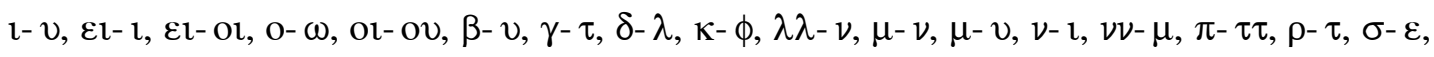
$\sigma \chi-\chi, \psi-\phi, \zeta-\xi$. Weiter sind auch bewegliche Endkonsonanten nicht notiert, da sie offensichtlich sind: $-\nu,-\varsigma,-\kappa(-\xi),-\kappa(\chi)$. Schließlich sind auch Verwechslungen zwischen Aspiration und Akzenten nicht notiert, da sie sehr verbreitet sind: ' -', '- ', '-', '-'. 


\section{Stemma codicum et sigla breviata}

6. 1 Stemma codicum libri Alexandri

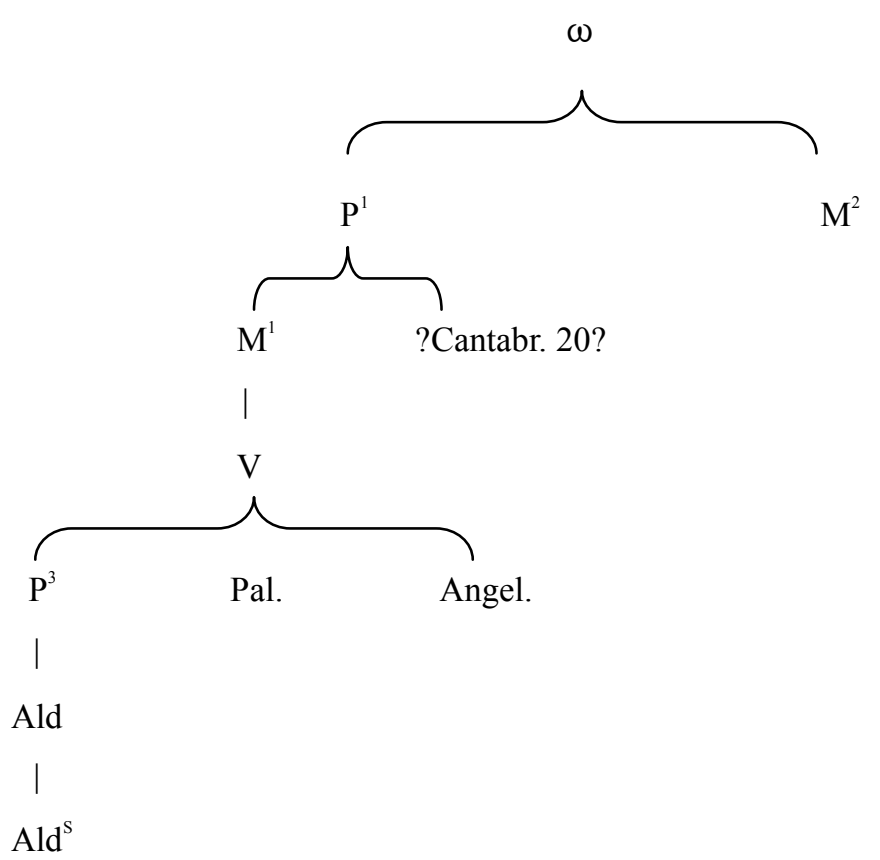

6.2 Sigla breviata

a. Codices (=Codd.) Alexandri

$\mathrm{P}^{1}$ : Paris. BN gr. 1741, Alexandri opusculum de figuris sententiarum atque elocutionis (f. 106v - 120v), saec. X.

$\mathrm{M}^{2}$ : Venedig. Marc. gr. 512, II. Alexander $<$ Numenius $>$ De figuris rhetoricis (f. 9 - 25), saec. XIII.

Codices recentiores ( $=$ Codd. recent.)

$M^{1}$ : Venedig. Marc. gr. 429, IV. Alexander Numenii filius De figuris (f. 87v - 95v), saec. XV (ca. 1468).

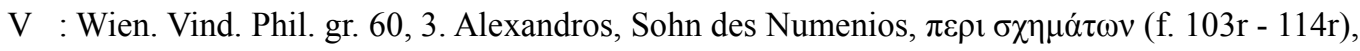
saec. XV.

$\mathrm{P}^{3}$ : Paris. BN gr. 1656, Alexandri rhetoris de figuris sententiae et elocutionis (f. 73v - 83v), saec. XV.

Pal. : Rom. Palat. - Vat. 66, 3. Alexander, de figuris dictionis et sententiarum (f. 118v-130r), saec. XVI.

Angel. : Rom. Angel. 54 (D. 5. 8), 3. Alexander, de figuris dictionis et sententiarum (ff. 105), saec XV/XVI.

Cantabr.: Cambridge. Queens' College Library, 20 RHETORICA QUAEDAM GRAECE. (=Horne 33 ), 3.

Alexandrou peri ton tes dianoias schematon kai peri ton tes lexeos schematon (f.60-76a), saec. XV/XVI.

Ald $^{\mathrm{s}}$.: Paris. BN gr. S. 552, Alexandri sophistae opusculum de figuris sententiae et dictionis (f. 574v - 
588v), saec. XVII.

Editiones (=Edd.)

Ald. : Rhetores Graeci, Alexandri De figuris sententiae atque elocutionis, ed. Aldus Manutius, Venedig 1508, S. 574-588.

Norrm. : Alexandri De figuris sententiae atque elocutionis, ed. L. Norrmannus, Uppsala 1690, S. 1-95.

Walz : Rhetores Graeci (=RG), Bd. 1-9, ed. Ch. Walz, Stuttgart 1832 - 1836, Bd.8, S.421-486.

Speng.: Rhetores Graeci (=RG), Bd. 1-3, ed. L. Spengel, Leipzig 1853 - 1856, Bd.3, S. 9-39.

Codices Anonymi cuiusdam Christiani

$\mathrm{P}^{2}$ : Paris. BN gr. 2087, Alexandri opusculum de figuris sententiarum et elocutionis (f. 247v - 258v), saec. XIV.

Par. 2762 : Paris. BN gr. 2762, Alexandri opusculum de figuris sententiarum et elocutionis (f. 4v - 11v), saec. XV.

Laurent. 57 : Florenz Med. Laurent. gr. Plut. 57.7, Alexandri de figuris sensus ac dictionis (f. 334 sequ.), saec. XV.

Vat. 1881 : Rom. Vat. gr. 1881. 3 Alexandri rhetoris de schematibus (f. 94-99), saec. XIII.

N.M. in $\mathrm{P}^{1}$ : Notae Marginales in $\mathrm{P}^{1}$, saec. XIII ? 


\section{Bibliographie}

\subsection{Editionen:}

Anonymi Carmen de figuris vel schematibus, ed. U. Schindel, in: ders., Die Rezeption der hellenistischen Theorie der rhetorischen Figuren bei den Römern, Göttingen 2001.

Anonymi cuiusdam de vitiis et virtutibus orationis liber, ed. U. Schindel, in: ders., Die lateinischen

Figurenlehren des 5. bis 7. Jahrhunderts und Donats Vergilkommentar, Göttingen 1975 („Isidorus iunior“, cod. Basil. F III 15d).

Anonymi cuiusdam de vitiis et virtutibus orationis liber, ed. U. Schindel, in: ders., Die lateinischen

Figurenlehren des 5. bis 7. Jahrhunderts und Donats Vergilkommentar, Göttingen 1975 („Sergius“, cod.

S. Paul. in valle Lavant. 2/1).

Anonymi Ecksteinii, Schemata dianoeas quae ad rhetores pertinent, ed. U. Schindel, NGG Phil.-hist. K1. 1987, 7, S. 107-173 (=S. 1-67).

Diodorus Siculus, Bibliotheca historica, lib. 1-20, ed. F. Vogel/T. Fischer, Leipzig 1888 - 1906 (ND 1964).

Eugraphius, Commentum Terenti, ed. P. Wessner, Bd. 1-2, Leipzig 1902/1908 (ND 1966).

Grammatici Latini, Bd. 1-7, ed. H. Keil, Leipzig 1857-1880.

Grégoire de Nazianze, Discours funèbres en l' honneur de son frère Césaire et de Basile de Césarée, ed. F. Boulenger, Paris 1908.

Gregorius Nazianzenus, Patrologiae cursus completus ... Series Graeca (=MPG), Bd. 35-38, ed. P. J. Migne, Paris 1858.

Gregor von Nazianz, Die fünf Theologischen Reden, ed. J. Barbel, Düsseldorf 1963.

Martianus Capella, ed. A. Dick, Stuttgart, BT² 1978.

Poetae Comici Graeci (=PCG), Bd. 1-8, ed. R. Kassel/C. Austin, Berlin 1983- 2003.

Quintilianus, Institutio oratoria, Bd. 1-2, ed. M. Winterbottom, Oxford 1970.

Rhetores Graeci, ed. Aldus Manutius, Venedig 1508.

Rhetores Graeci (=RG), Bd. 1-9, ed. Chr. Walz, Stuttgart 1832 - 1836.

Rhetores Graeci (=RG), Bd. 1-3, ed. L. Spengel, Leipzig 1853 - 1856.

Rhetores Graeci (=RG), Bd. 1, 1, ed. Spengel/Hammer, Leipzig 1894.

Rhetores Latini Minores (=RLM), ed. K. Halm, Leipzig 1863.

Tiberii De figuris Demosthenicis cum deperditorum operum fragmentis, ed. G. Ballaira, Rom 1968.

7.2 Monographien und Aufsätze

Textkritik

W. Canter, De ratione emendandi Graecos auctores syntagma, 1566.

P. Maas, Textkritik, 1927. 
G. Pasquali, Storia della tradizione e critica del testo (2. Aufl.), Florenz 1952.

S. Timpanaro, Die Entstehung der Lachmannschen Methode, 2.Aufl. Hamburg 1971.

R. Pfeiffer, Die Klassische Philologie von Petrarca bis Mommsen, München 1976.

E. Pöhlmann, Einführung in die Überlieferungsgeschichte und in die Textkritik der antiken Literatur,

Darmstadt 1994.

F. Robertello, De arte sive ratione corrigendi antiquorum libros disputatio, 1557.

M. L. West, Text Criticism and Editorial Technique, Stuttgart 1973.

Aldus Manutius und seine Editionen:

H. G. Fletcher, New Aldine Studies, San Francisco 1988.

D. J. Geanakoplos, Greek Scholars in Venice, Cambridge 1962.

P. F. Grendler, Books and Schools in the Italian Renaissance, Aldershot (Hampshire) 1995.

H. Hunger, Die hochsprachliche profane Literatur der Byzantiner, Bd. 1 - 2, München 1978.

J. E. Kenney, The Classical Text: Aspects of Editing in the Age of the Printed Book, Los Angeles 1974.

E. Legrand, Bibliographie Hellénique 1 (1476-1563), Paris 1885.

M. Lowry, The World of Aldus Manutius: Business and Scholarship in Renaissance Venice, Oxford 1979.

J. Monfasani, Byzantine Scholars in Renaissance Italy: Cardinal Bessarion and other Emigrés, Aldershot (Hampshire) 1995.

H. Rabe, Aus Rhetoren - Handschriften, in: Rheinisches Museum 62 (1907), S. 559 - 590.

H. Rabe, Aus Rhetoren - Handschriften, in: Rheinisches Museum 63 (1908), S. 127 - 151.

H. Rabe, Rhetoren - Corpora, in: Rheinisches Museum 67 (1912), S. 337 - 357.

J. B. Ross, Venetian Schools and Teachers, Fourteenth to Early Sixteenth Century: A survey and a study of Giovanni Battista Egnazio, in: Renaissance Quarterly 39 (1976), S. 521-560.

W. Schmid, Die sogenannte Aristidesrhetorik, in: Rheinisches Museum 72 (1917/1918), S. 113 - 149.

M. Sicherl, Griechische Erstausgaben des Aldus Manutius, Paderborn 1997.

Allgemeine Rhetorik und Figurenlehre:

W. Ax, Laut, Stimme und Sprache. Studien zu drei Grundbegriffen der antiken Sprachtheorie, Göttingen 1986.

W. Ax, Quadripertita Ratio: Bemerkungen zur Geschichte eines aktuellen Kategoriensystems, in: Historiographia Linguistica 13 (1986), S. 191-214.

G. Ballaira, La dottrina delle figure retoriche in Apollodoros di Pergamon, in: Quaderni Urbinati di cultura classica 5 (1968), S. 37-91.

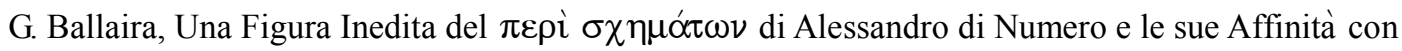
Quintiliano (Inst. 8, 6, 67-76), in: Rheinisches Museum 119 (1976), S. 323-328.

K. Barwick, Remmius Palaemon und die römische ars grammatica, Leipzig 1922 (ND. Hildesheim 1967). 
K. Barwick, Probleme der stoischen Sprachlehre und Rhetorik, Berlin 1957 (= Abhandlung der Sächs. Akademie der Wissenschaften zu Leipzig, Phil.- hist. K1. 49, 3).

A. D. Booth, The Appearance of the schola grammatici, in: Hermes 106 (1978), S. 117-125.

J. Brzoska, s.v. ,Alexandros', RE I 2, 1894, Sp. 1456-1459

P. Chiron, Démétrios. Du Style, Paris 1993.

E. Drerup, Eine alte Blattversetzung bei Alexander Numeniu, in: Philologus 71 (1912), S. 390- 413.

G. M. A. Grube, Theodoros of Gadara, in: AJP 80 (1959), S. 337-365.

M. Fuhrmann, Das systematische Lehrbuch. Ein Beitrag zur Geschichte der Wissenschaften in der Antike, Göttingen 1960.

D. Harlfinger und D. Reinsch, Die Aristotelica des Parisinus Gr. 1741, in: Philologus 114 (1970), S. 28-50.

H. Hommel, Rhetorik, in: Lexikon der Alten Welt, Zürich/Stuttgart 1965, Sp. 2611-2626.

H. Hunger, Katalog der Griechischen Handschriften der Österreichischen Nationalbibliothek, Wien 1961.

R. Jamison/J. Dyck, Rhetorik - Topik - Argumentation. Bibliographie zur Redelehre und Rhetorikforschung im deutschsprachigen Raum 1945-1979/80, Stuttgart 1983.

R. M. James, A descriptive catalogue of the western manuscripts in the Library of Queens College, Cambridge, Cambridge 1904.

R. A. Kaster, C. Suetonius Tranquillus de Grammaticis et Rhetoribus, Oxford 1995.

R. A. Kaster, Guardians of Language, The Grammarian and Society in Late Antiquity 1988.

G. Kennedy, The Art of Rhetoric in the Roman World, 300 B.C. - A.D. 300, Princeton 1972.

G. Kennedy, Greek Rhetoric under Christian Emperors, Princeton 1984.

H. Lausberg, Handbuch der literarischen Rhetorik, Bd.1 - 2, München 1990.

A. D. Leeman, Orationis ratio. The Stylistic Theories and Practice of the Roman Orators, Historians and Philosophers, Bd. 1-3, Amsterdam 1963.

E. Lobel, The Greek manuscripts of Aristotle's Poetics, Oxford 1933.

H. I. Marrou, Histoire de l'éducation dans l'antiquité, Paris 7. Aufl. 1976.

J. Martin, Antike Rhetorik. Technik und Methode, München 1974 (= Handbuch der Altertumswissenschaft 2. Abt., 3. Teil).

E. Mioni, Codices Graeci Manuscripti II, Rom 1985.

L. Mohler, Kardinal Bessarion, Bd. III, Paderborn 1942.

K. Morawski, De Dionysii et Caecili studiis rhetoricis, in: Rheinisches Museum 34 (1879).

E. Ofenloch, Caecilii Caleactini Fragmenta, Leipzig 1907.

R. Pfeiffer, History of Classical Scholarship, Oxford 1968.

H .F. Plett, Einführung in die rhetorische Textanalyse, Hamburg 1991.

M. Schanz, Die Apollodoreer und die Theodoreer, in: Hermes 25 (1897), S. 36-54.

D. M. Schenkeveld, Figures and Tropes. A Border-case between Grammar and Rhetoric, in: G. Ueding (Hrsg.), Rhetorik zwischen den Wissenschaften, Tübingen 1991, S. 149-157.

U. Schindel, Entstehungsbedingungen eines spätantiken Schulbuchs: Zum ,Carmen de figuris' (RLM 63- 
70), in: S. Döpp (Hrsg.), Antike Rhetorik und ihre Rezeption (Symposion zu Ehren von Carl Joachim Classen), Stuttgart 1998, S.85-98.

U. Schindel, Die Historiker Philistos von Syrakus und die rhetorische Figurenlehre, in: M. Janka (Hrsg.), ЕГКYК $\Lambda$ ION KНПION (Rundgärten) Zu Poesie, Historie und Fachliteratur der Antike, Leipzig 2004, p. 163-169.

U. Schindel, Die lateinischen Figurenlehren des 5. bis 7. Jahrhunderts und Donats Vergilkommentar (mit zwei Editionen), Göttingen 1975 (= Abhandlungen der Akademie der Wissenschaften in Göttingen, Phil.- hist. K1. 3, 91).

U. Schindel, Julius Rufianus - Zum Nutzen von Exzerptüberlieferung, in: Voces 4 (1993), S. 55-66.

U. Schindel, Die Quelle von Isidors ,rhetorischer' Figurenlehre, in: Rheinisches Museum NF 137 (1994), S. 374-382.

U. Schindel, Ein unedierter lateinischer Figurentraktat, in: Philologus 138 (1994), S. 335-347.

U. Schindel, Ein unidentifiziertes ,Rhetorik-Exzerpt': der lateinische Theon, in: NGG Phil.-hist. K1., 1999, 2, S. 1-27.

P. L. Schmidt, Die Anfänge der institutionellen Rhetorik in Rom, in : E. Lefevre (Hrsg.), Monumentum Chiloniense (Festschrift für Erich Burck), Amsterdam 1975, S.183-216.

H. Schrader, Schema und Tropos in den Homer-Scholien, in: Hermes 39 (1904), S. 563-603.

Th. Schwab, Alexander Numeniu ПEPI $\Sigma$ XHMAT $\Omega N$ in seinem Verhältnis zu Kaikilios, Tiberios und seinen späteren Benutzern, Paderborn 1916 (Rhetor. Studien Nr. 5).

E. Siebenborn, Die Lehre von der Sprachrichtigkeit und ihre Kriterien. Zur antiken normativen Grammatik, Amsterdam 1976.

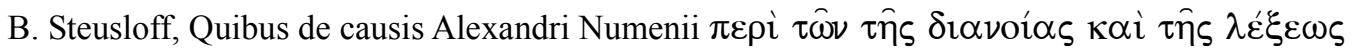

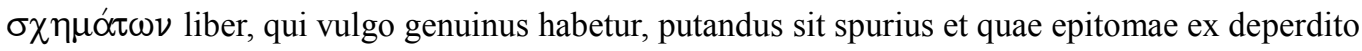
Alexandri libro excerptae supersint, demonstratur, Diss. Phil. Breslau 1861.

W. Tröbst, Quaestiones Hyperideae et Dinarcheae Pars I, in: Schulprogramm Hameln 1881, S.10-16.

C. Walde, Alexandros 25, DNP I, 1996, S. 479. 


\section{ПIEPI T $\Omega$ N TH $\Sigma \Delta$ IANOIA $\Sigma$ KAI TH $\Sigma \Lambda$ E $\Xi E \Omega \Sigma$}

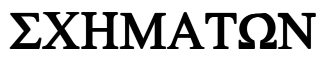

I.

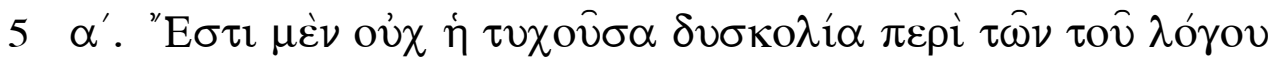

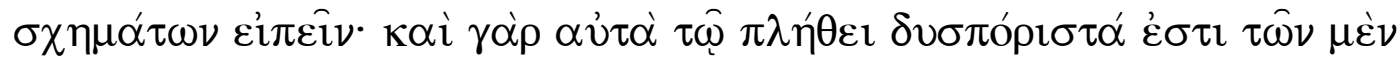

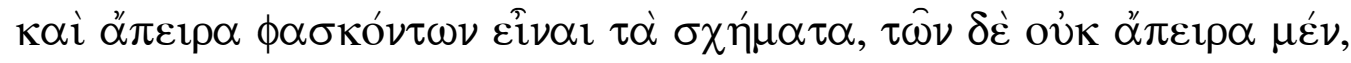

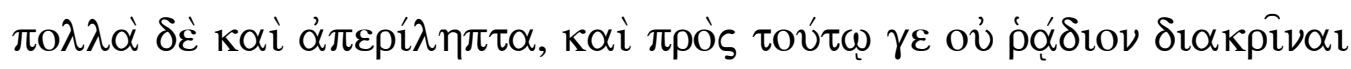

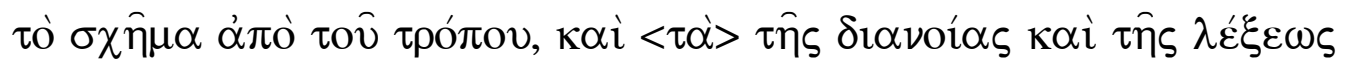
$10 \dot{\alpha}^{\prime} \dot{\alpha} \lambda \lambda \dot{\eta} \lambda \omega \nu$.

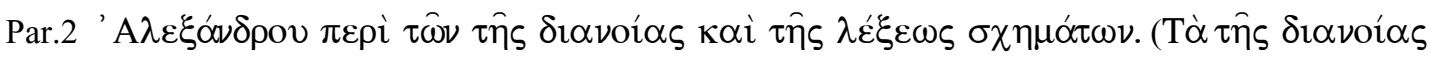

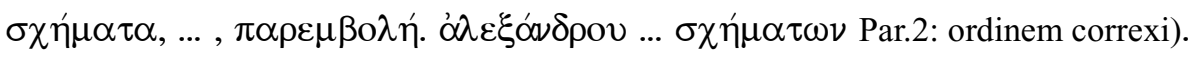

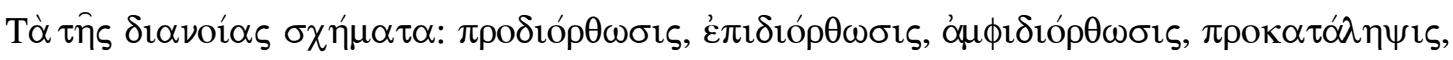

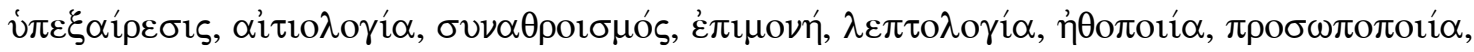

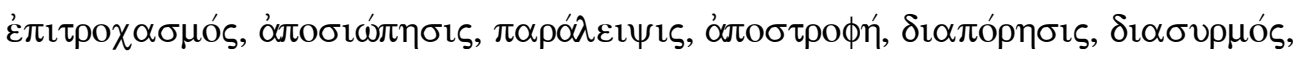

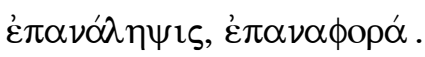

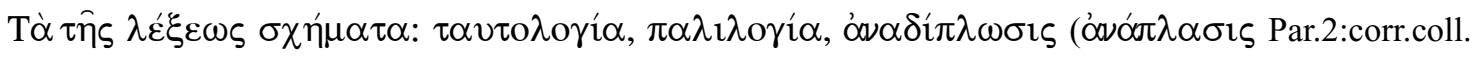

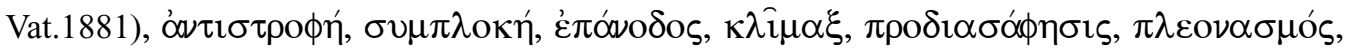

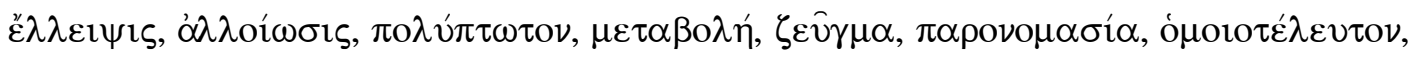

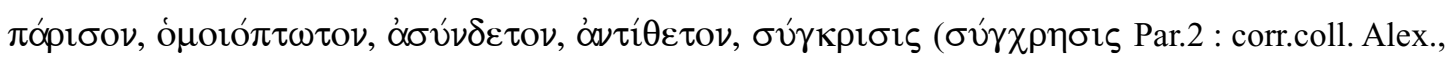

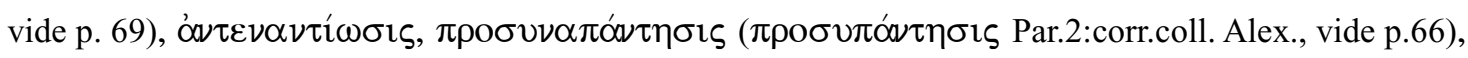

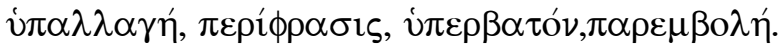

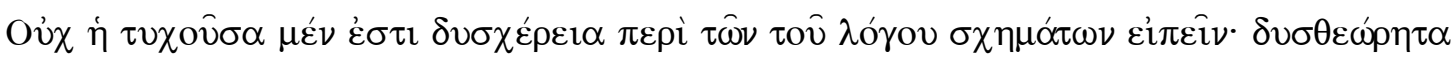

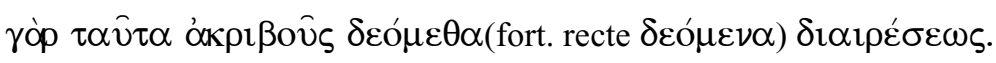

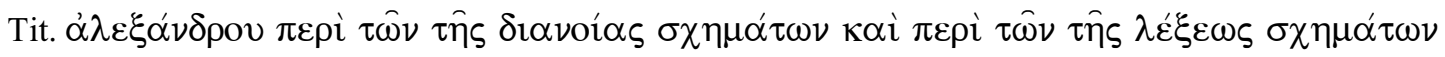

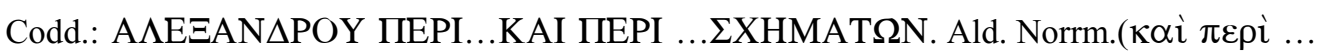

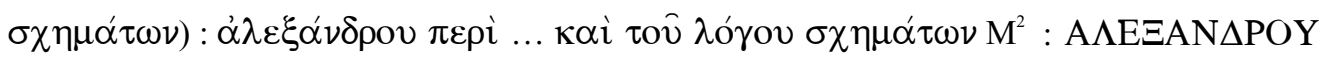
ПEPI T $\Omega$ N TH $\Sigma \Delta$ IANOIA $\Sigma$ KAI TH $\Sigma \Lambda$ E $\Xi E \Omega \Sigma \Sigma$ XHMAT $\Omega N$ Walz et Speng. cf. ${ }^{2}$ $5 \alpha^{\prime}$ om. Codd. : $\alpha^{\prime}$ ins. Norrm., $\delta v \sigma \kappa o \lambda i ́ \alpha \mathrm{P}^{1} \mathrm{M}^{1}$ Ang.: $\delta v \sigma \iota \phi \lambda i ́ \alpha \mathrm{V}$ Pal.: $\delta v \sigma \iota \phi \imath \lambda i ́ \alpha \mathrm{P}^{3}$ Ald.

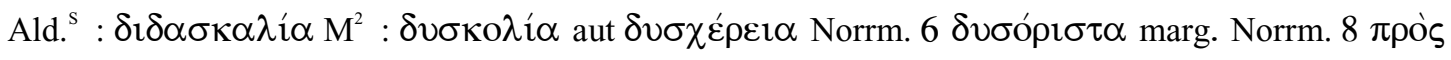

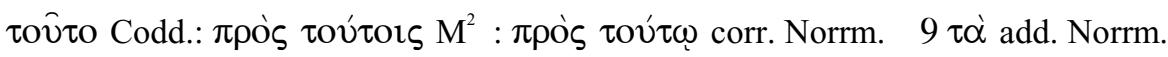




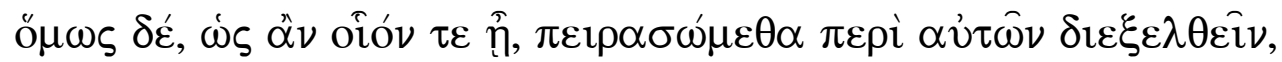

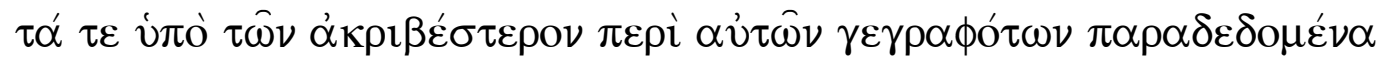

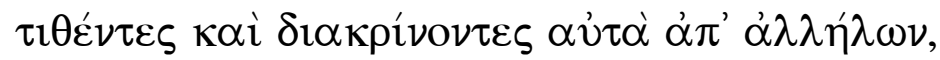

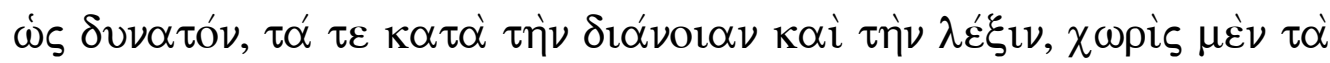

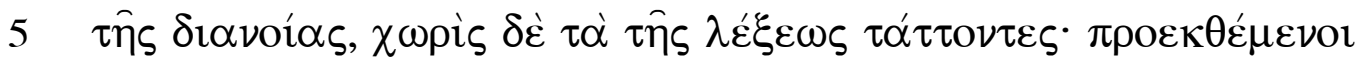

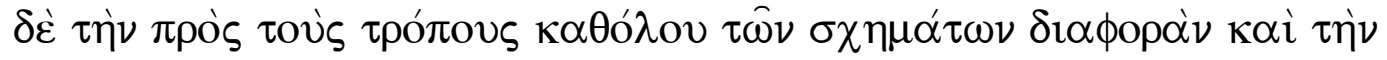

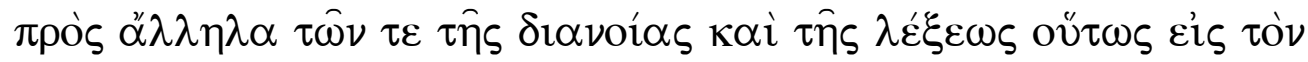

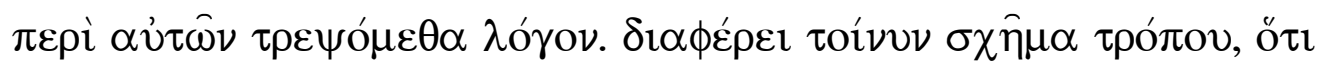

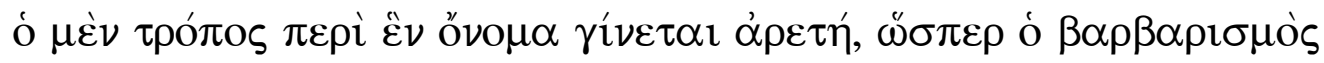

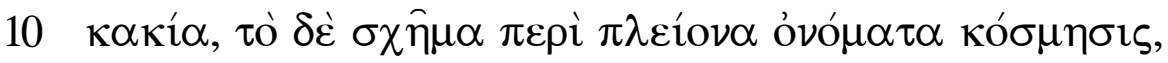

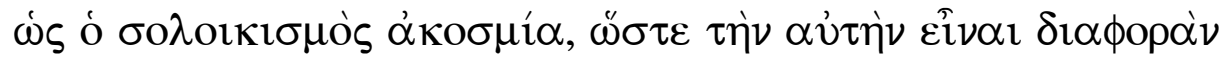

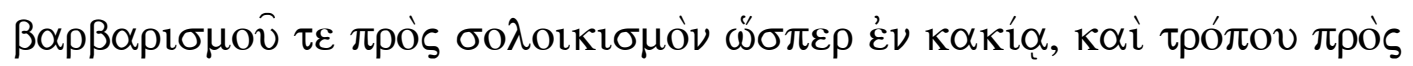

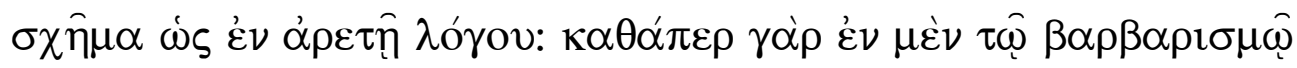

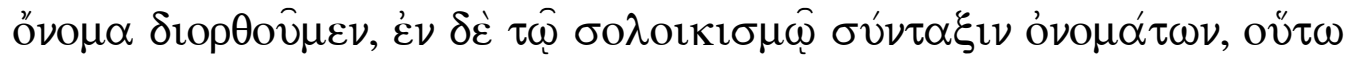

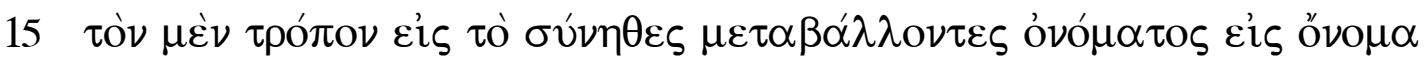

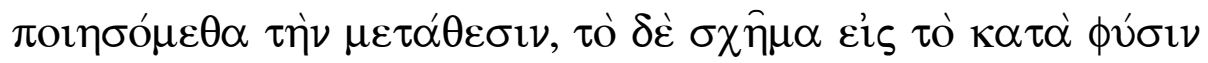

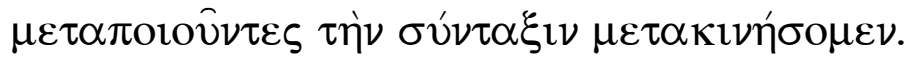

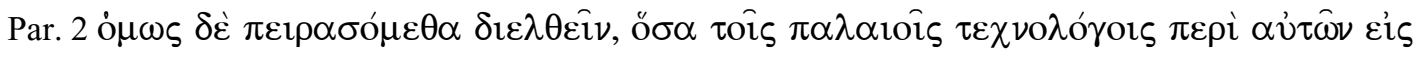

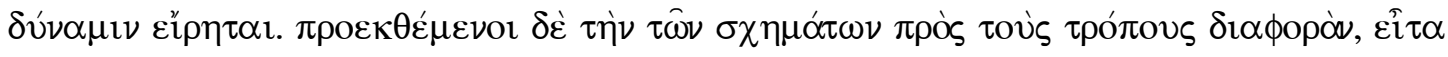

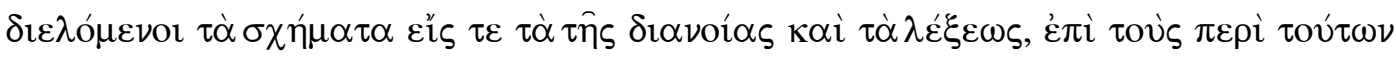

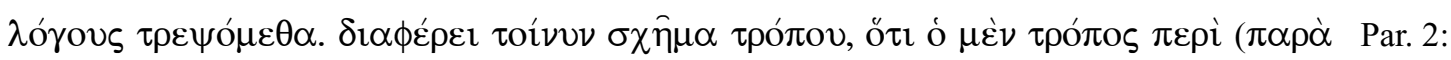

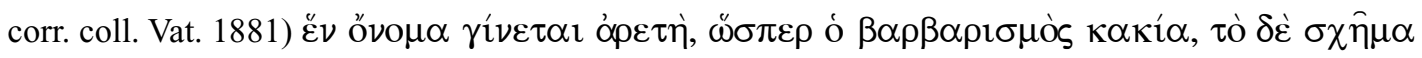

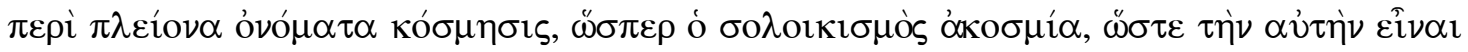

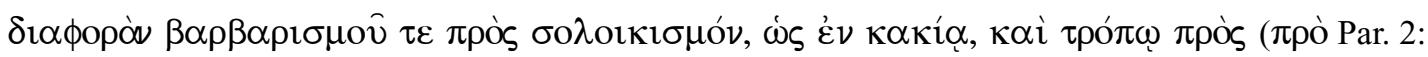

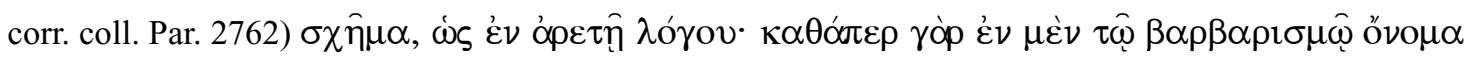

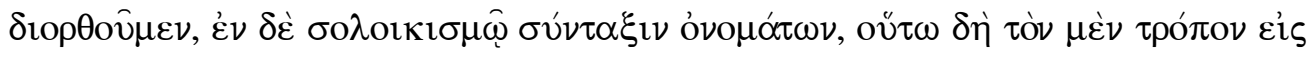

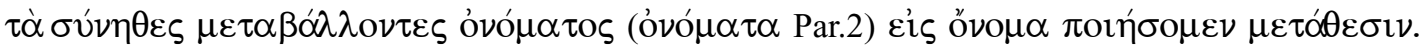

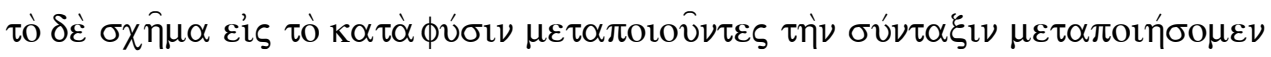

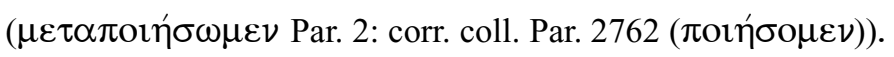

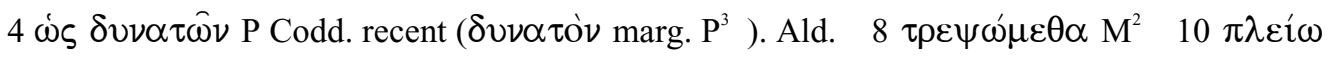

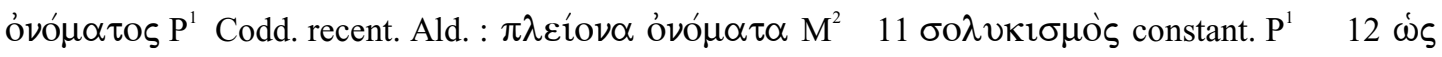

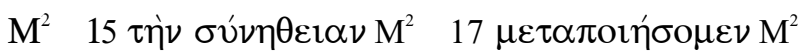




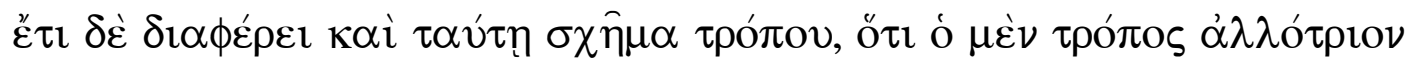

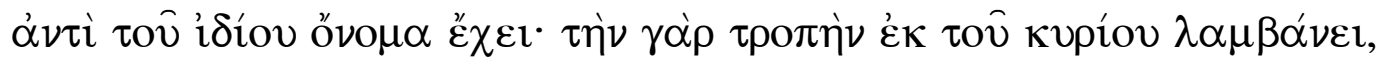

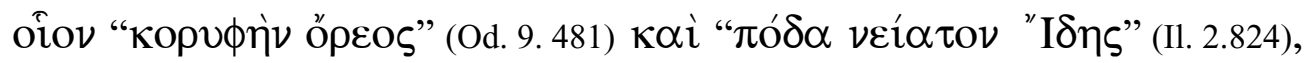

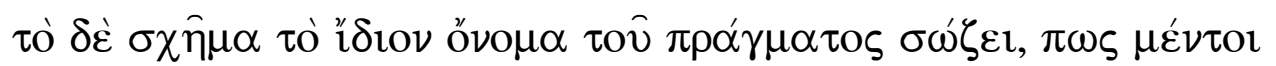

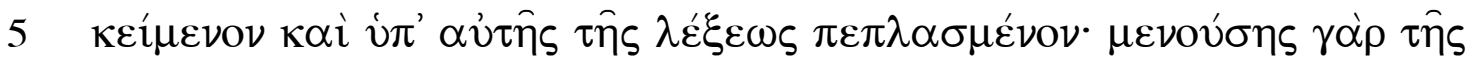

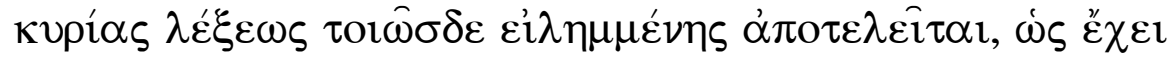

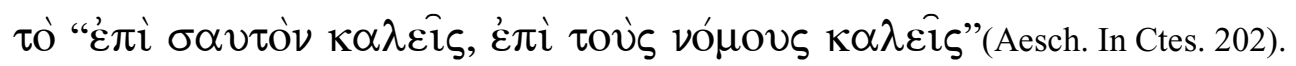

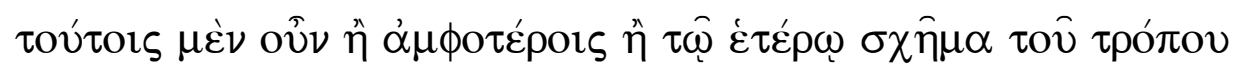

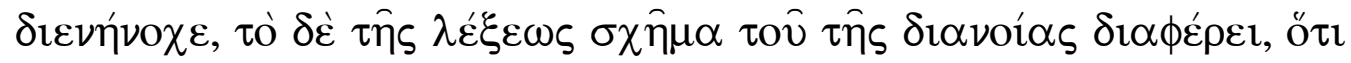

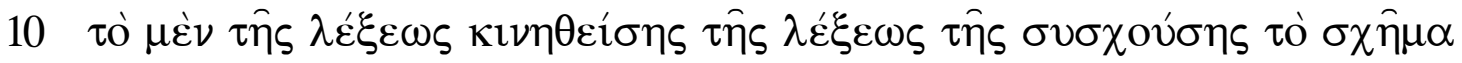

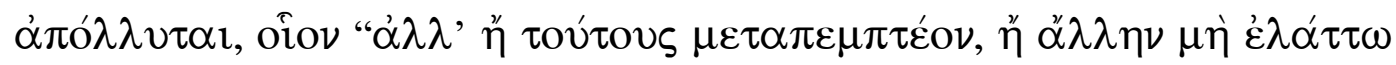

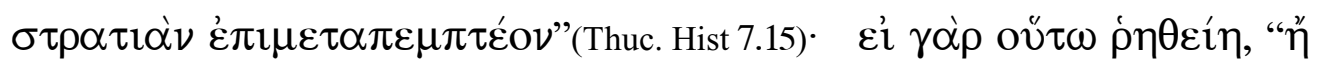

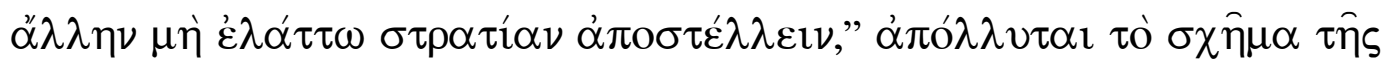

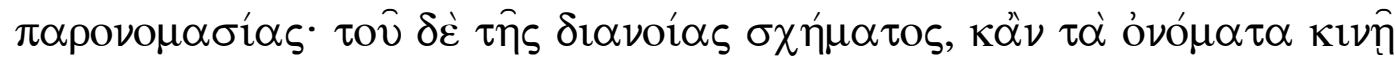

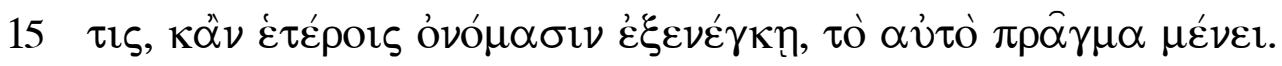

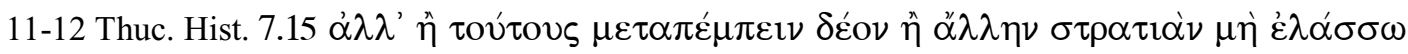
$\dot{\varepsilon} \pi \imath \pi \dot{\varepsilon} \mu \pi \varepsilon \imath \nu$.

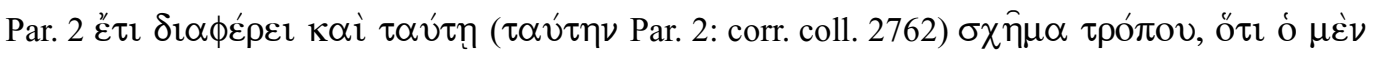

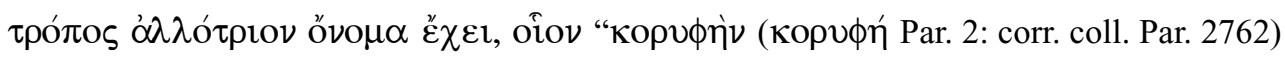

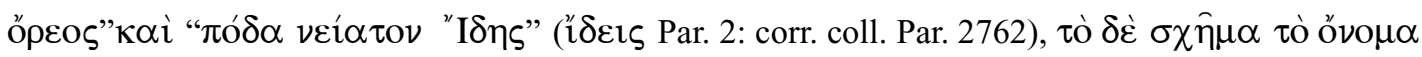

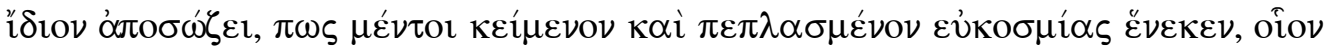

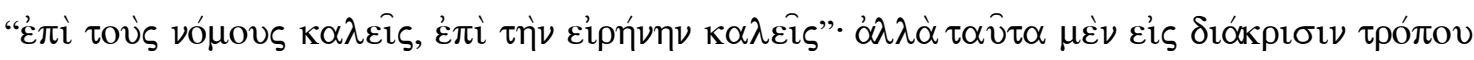

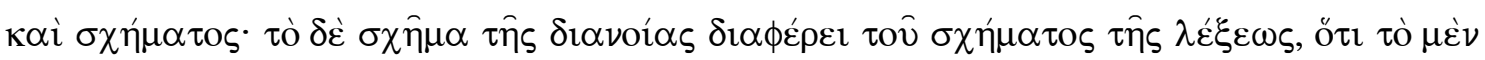

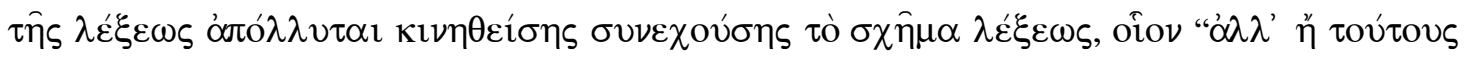

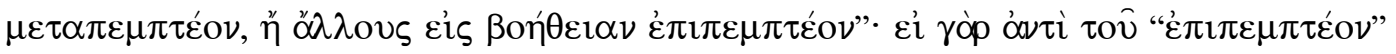

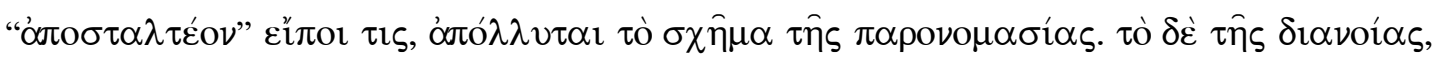

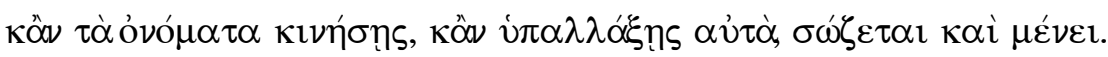

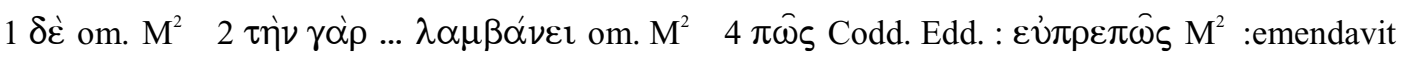

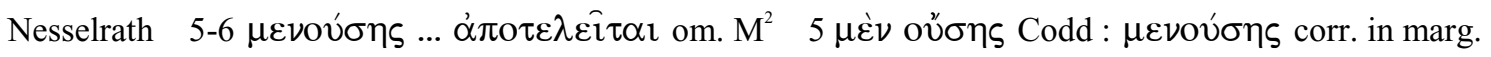

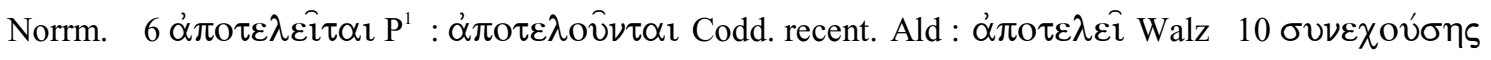

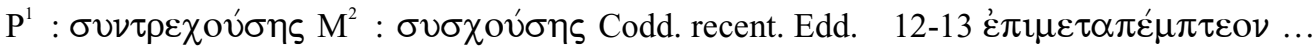

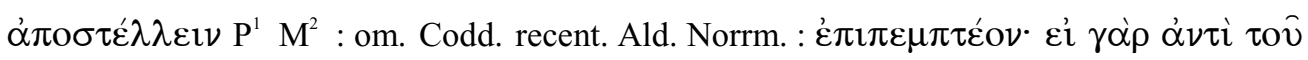

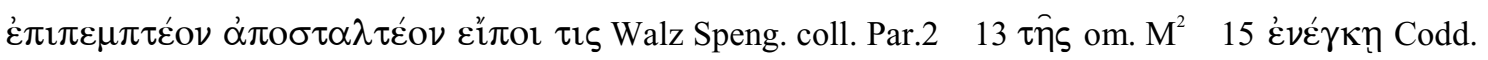
Ald : $\grave{\varepsilon} \xi \dot{\varepsilon} v \varepsilon \gamma \kappa \eta ̣$ corr.Norrm. Edd. 


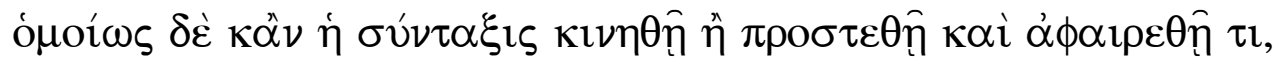

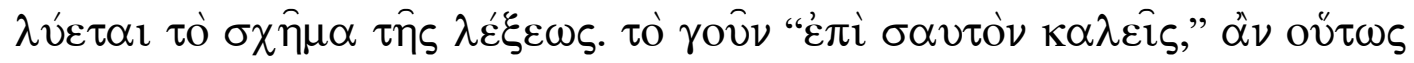

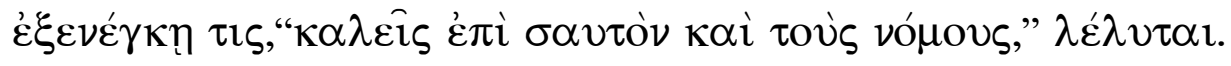

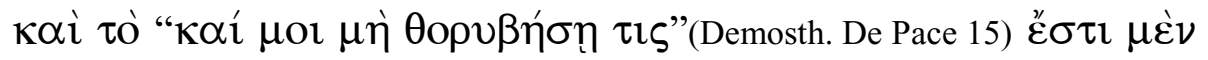

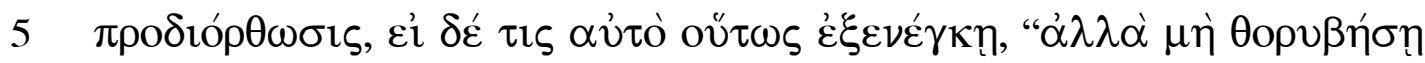

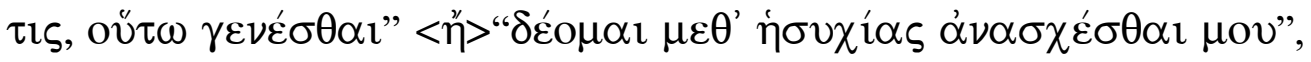

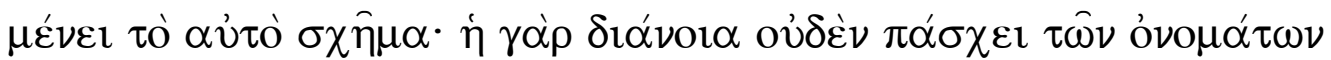

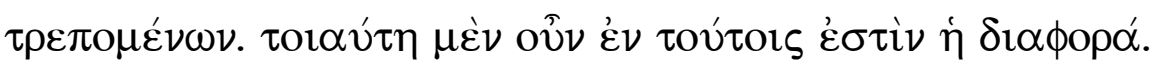

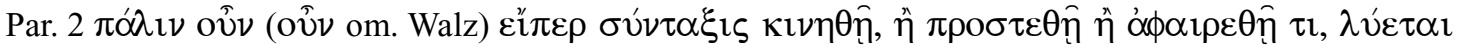

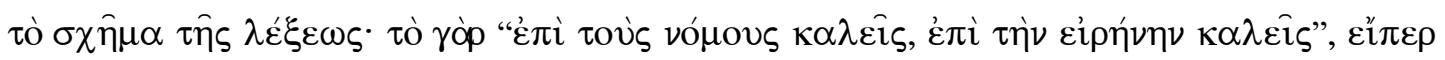

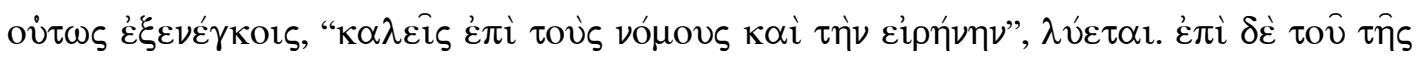

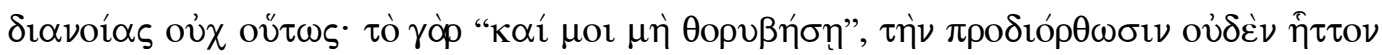

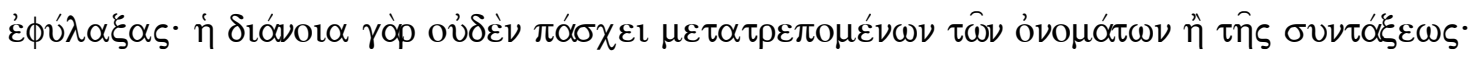

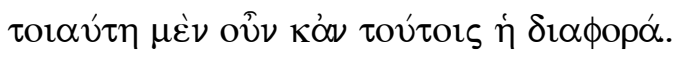

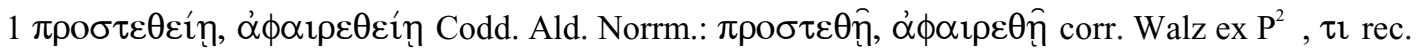

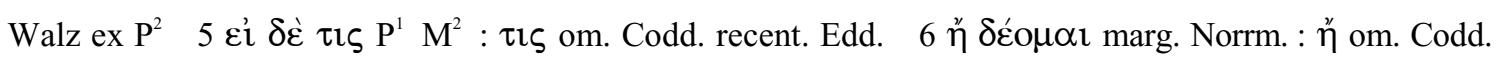
Edd. , $\mu \varepsilon \theta^{\prime}$ marg. Norrm. Edd.:om. Codd. Ald., $\tau \bar{\eta} \varsigma \grave{\eta} \sigma v \chi i \alpha \varsigma$ Codd. Edd. : $\tau \bar{\eta} \varsigma$ del. Walz,

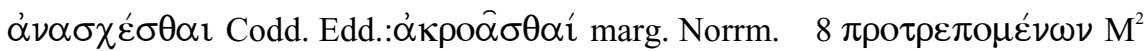




\section{$\beta^{\prime}$. OPO $\Sigma$ ХXHMATO $\Sigma$.}

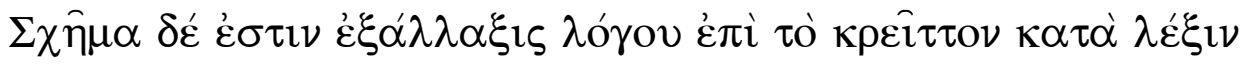

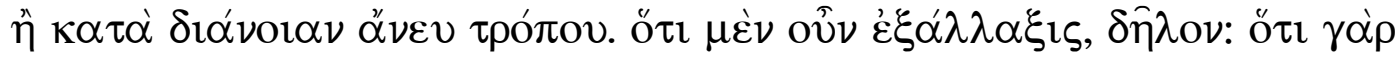

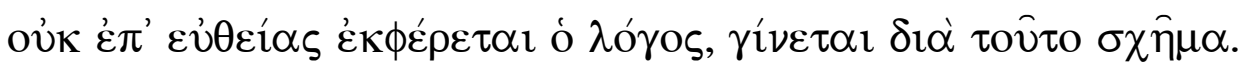

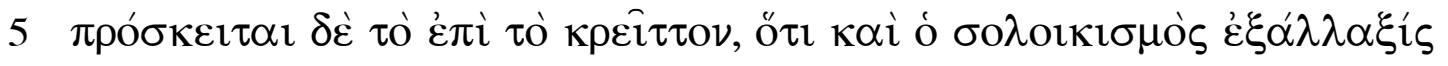

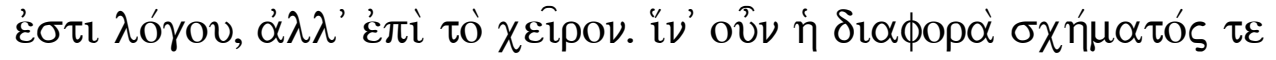

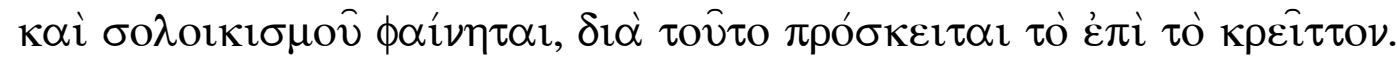

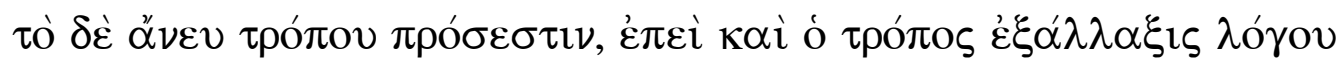

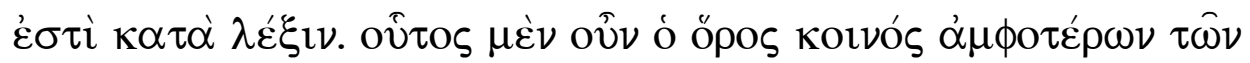

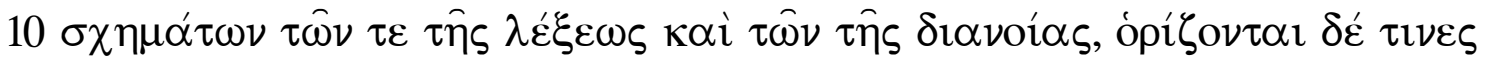

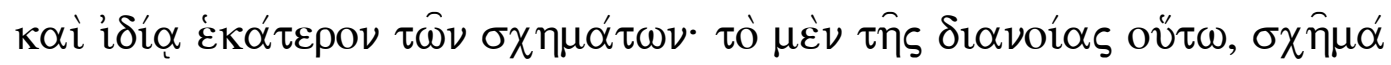

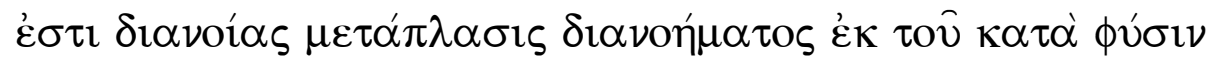

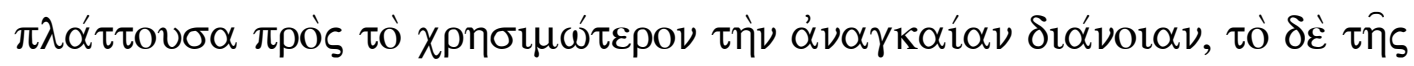

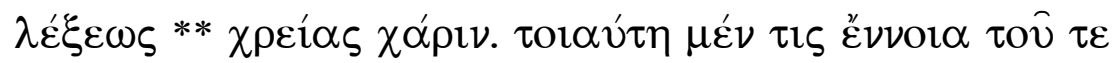

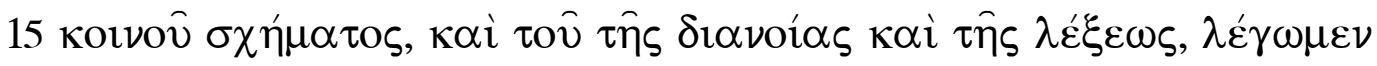

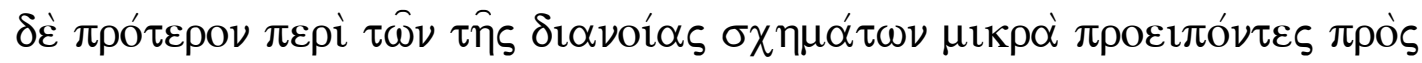

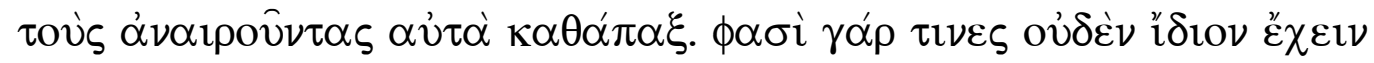

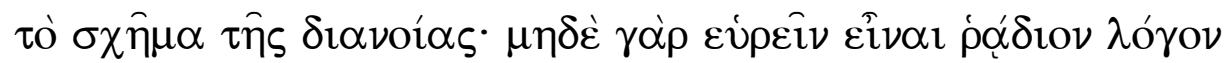

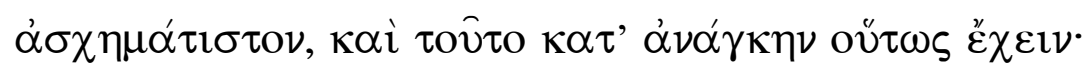

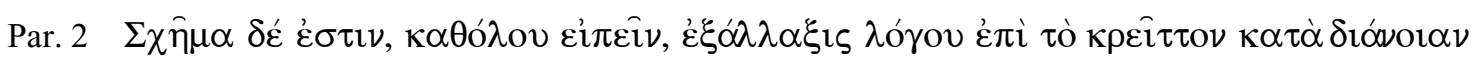

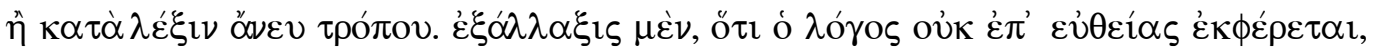

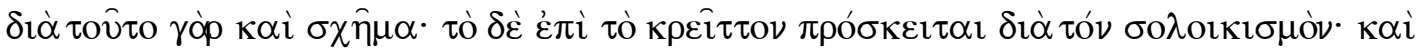

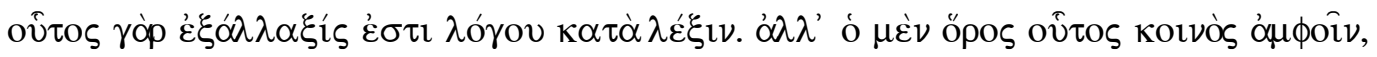

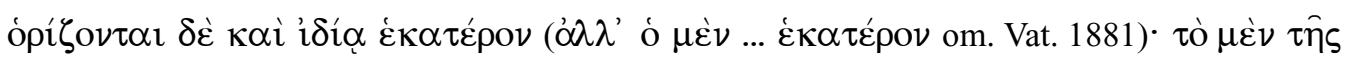

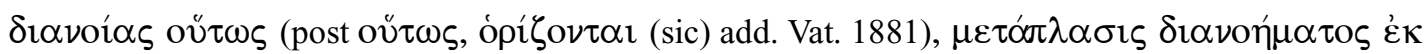

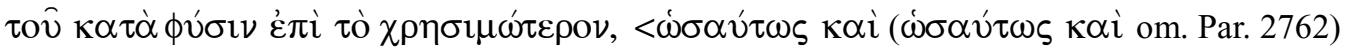

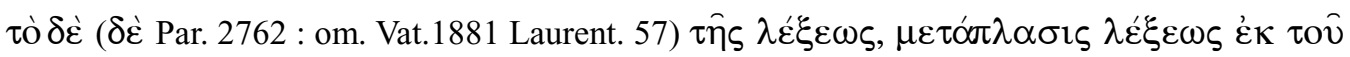

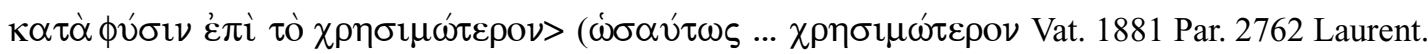

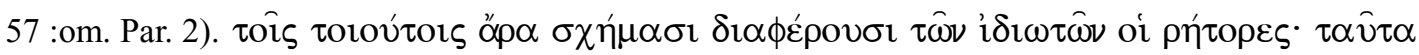

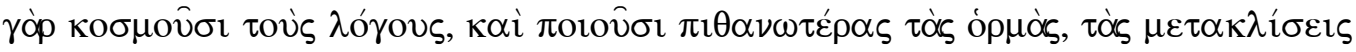

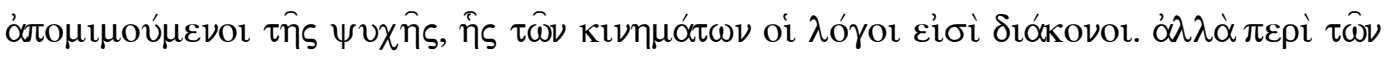

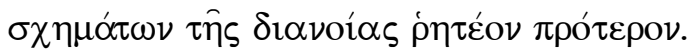

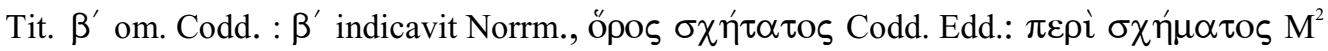

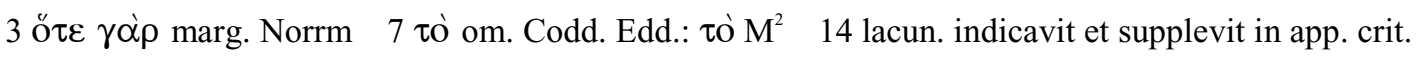

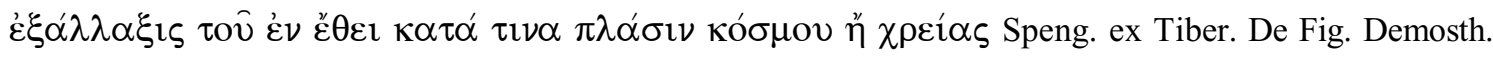
(p. 8 Ballaira) 


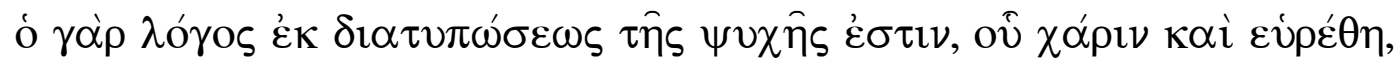

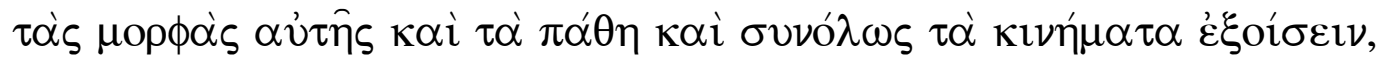

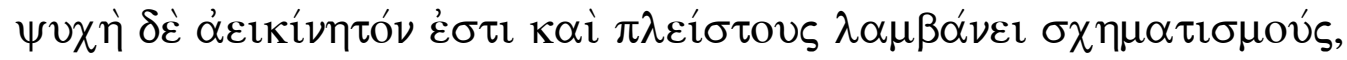
$\lambda$ ó

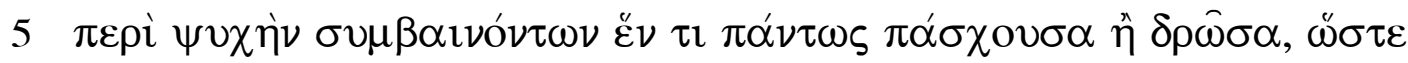

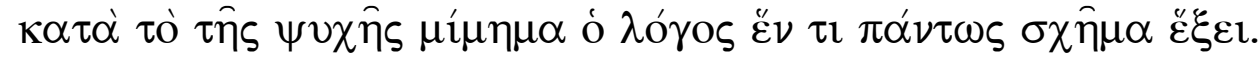

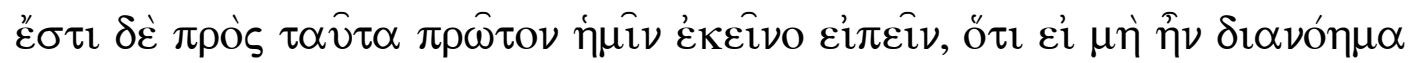

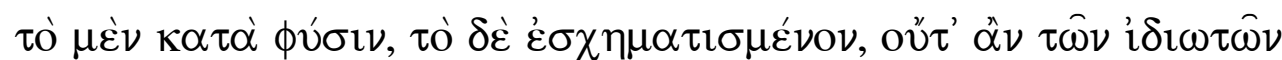
oi ṕnं

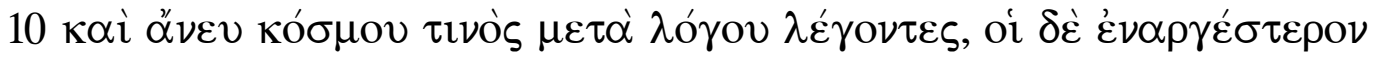

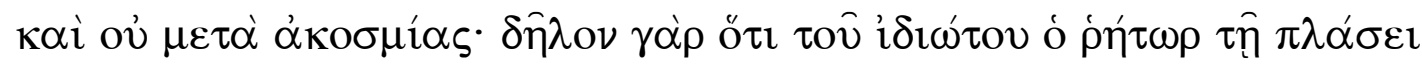

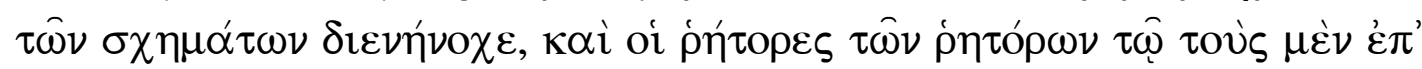

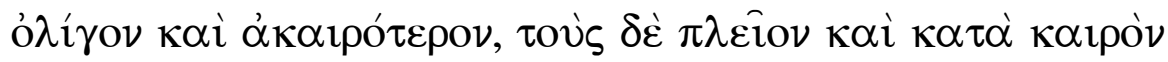

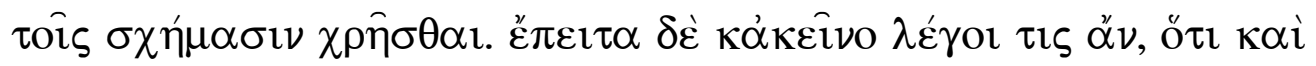

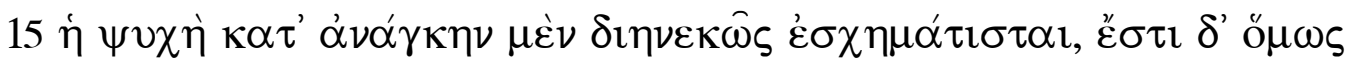

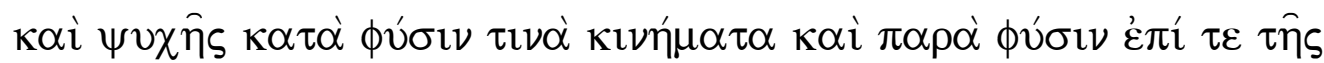

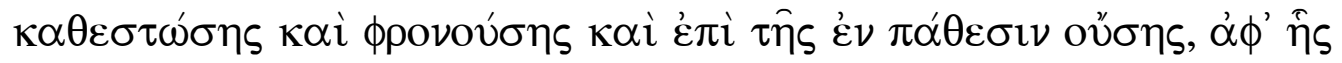

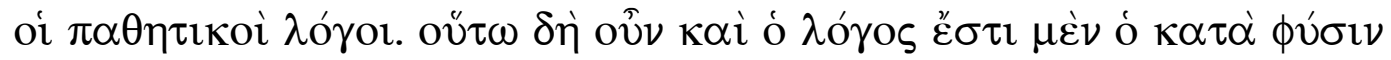

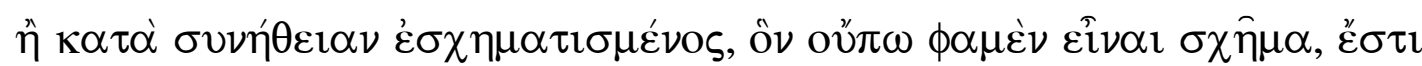

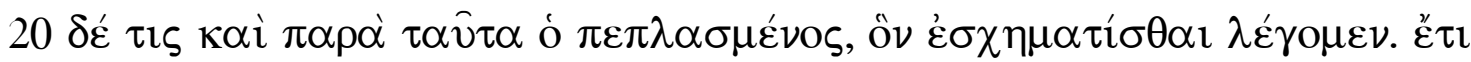

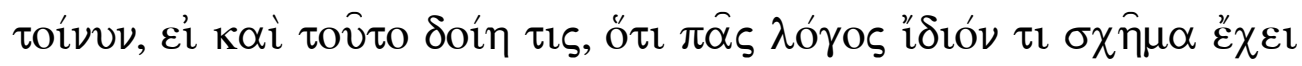

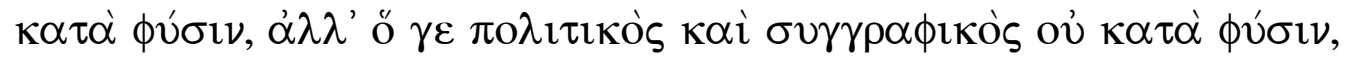

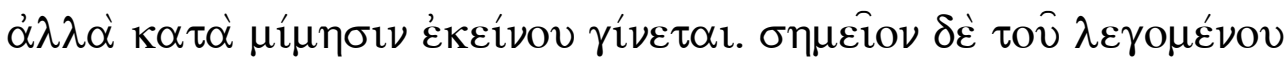

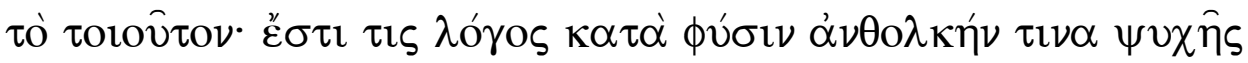

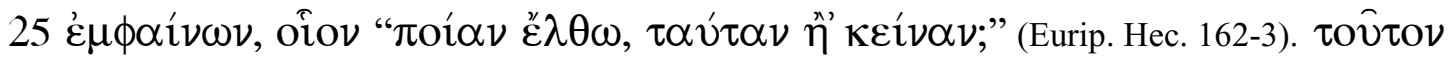

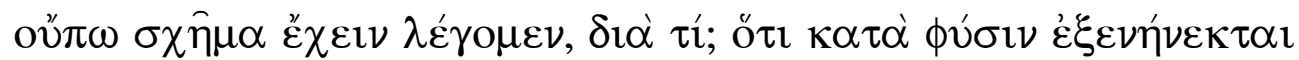

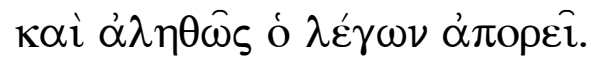

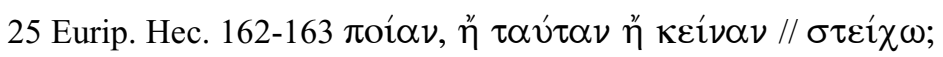

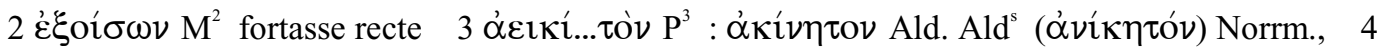

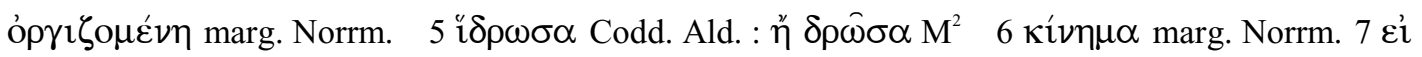

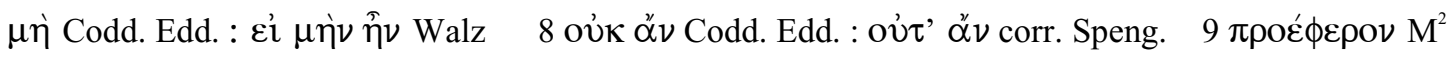

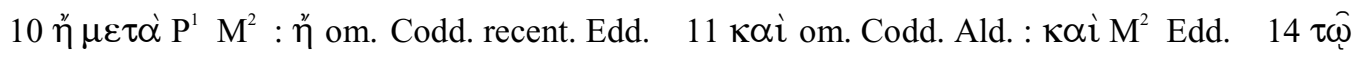

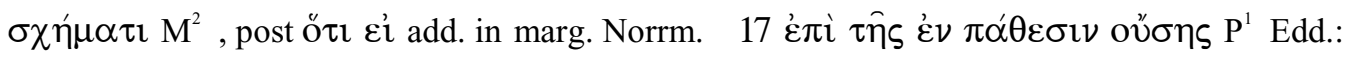

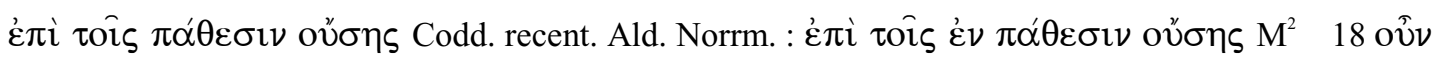

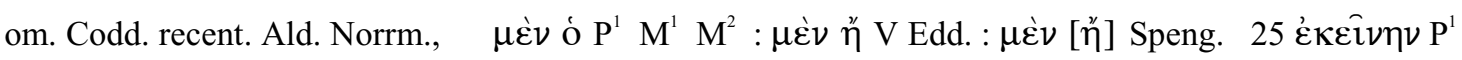

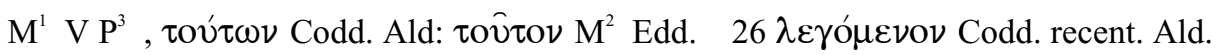




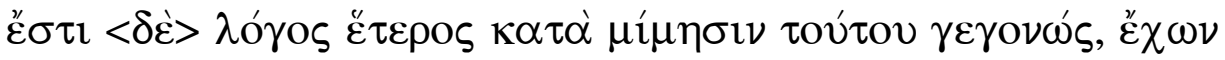

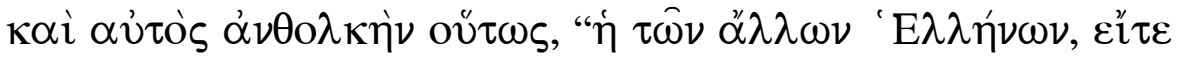

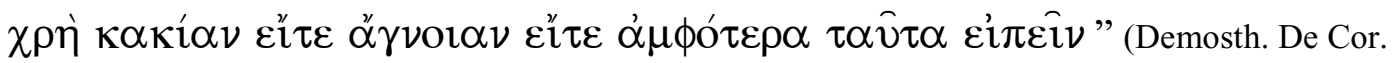

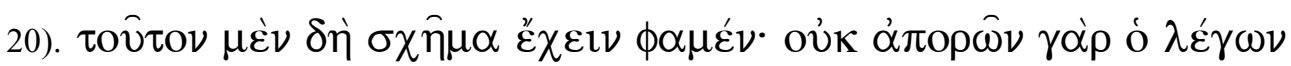

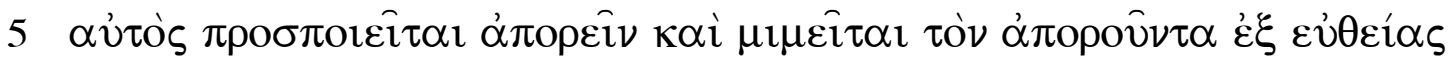

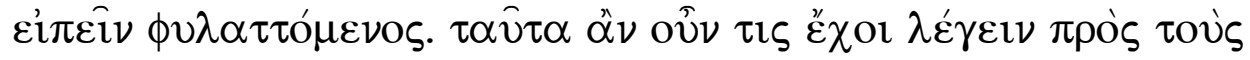

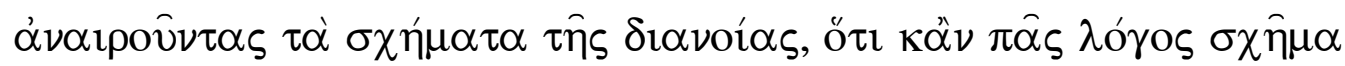

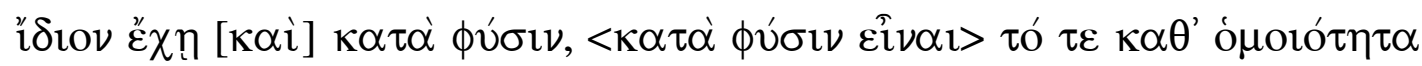

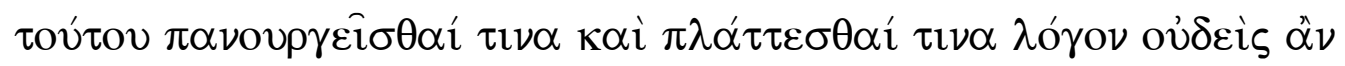

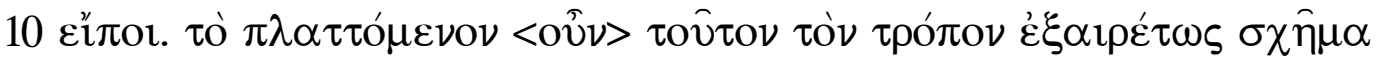

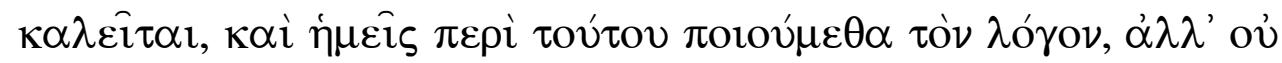

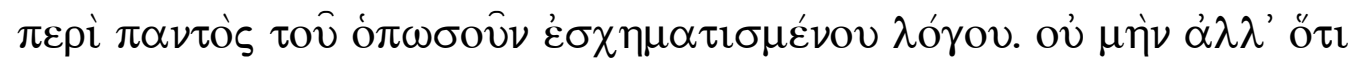

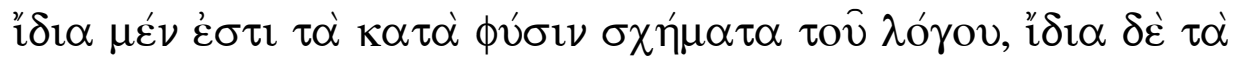

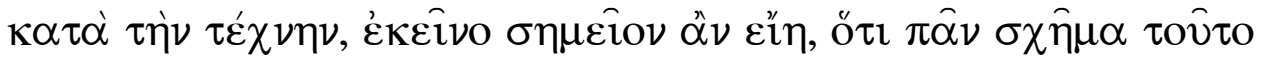

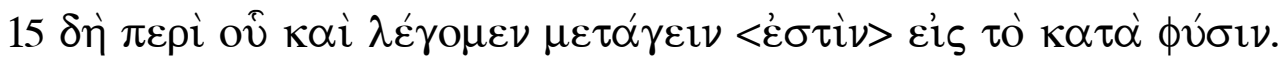

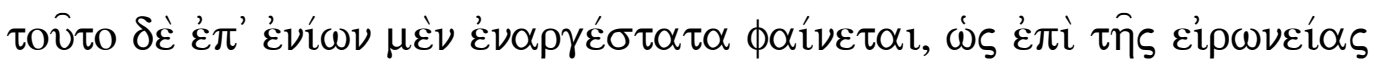

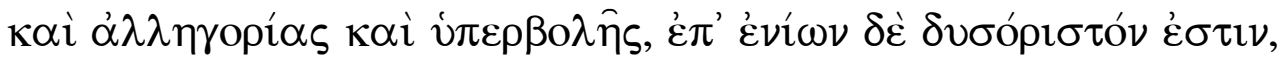

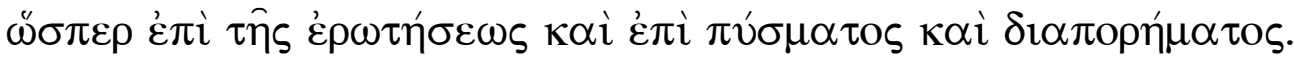

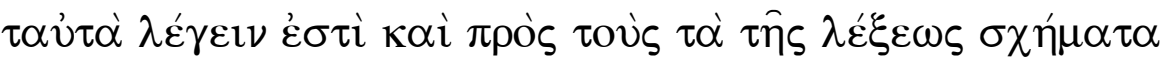

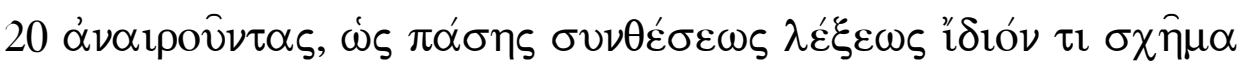

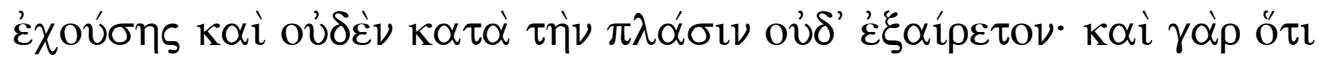

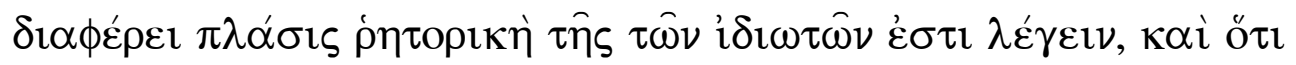

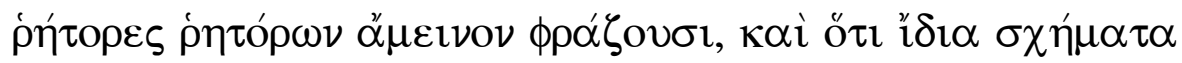

$1 \delta \dot{\varepsilon}$ ins. Ald $2 \dot{\varepsilon} \kappa \tau \hat{\omega} \nu \mathrm{P}^{1}: \kappa \alpha i ̀ \hat{\omega} \nu \mathrm{M}^{2}: \dot{\eta} \tau \hat{\omega} v$ corr. Norrm. $5 \alpha \hat{v} \tau o ̀$ Codd. Ald. Norrm. $(-v)$ :

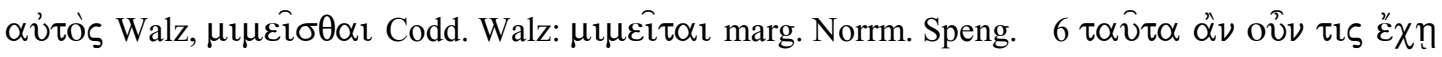

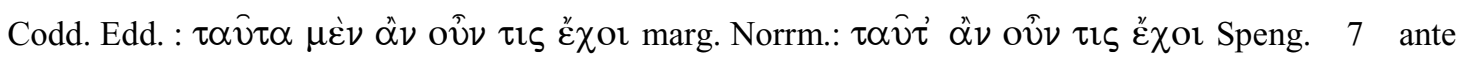

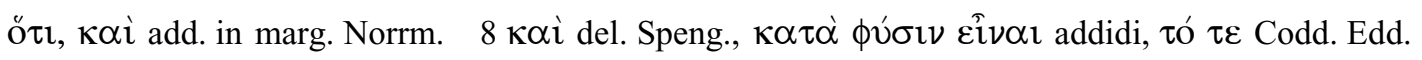

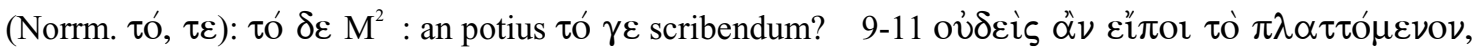

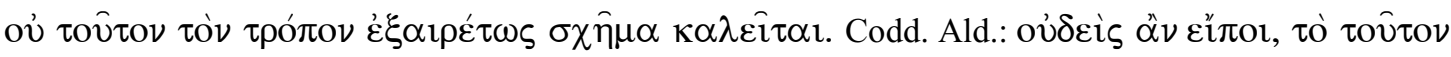

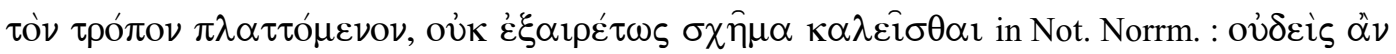

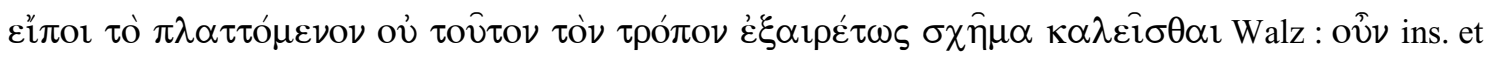
aliter interpunxit Speng. $12 \alpha^{\prime} \lambda \lambda \mathrm{o} \tau \mathrm{\tau l}$ Codd. Ald : $\alpha \lambda \lambda^{\prime}$ ö $\tau \iota \mathrm{M}^{2}$ Edd. $15 \dot{\varepsilon} \sigma \tau \grave{\nu} \nu$ add. in marg. Norrm.

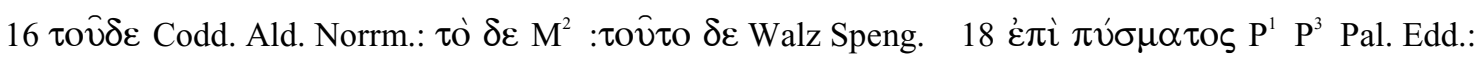

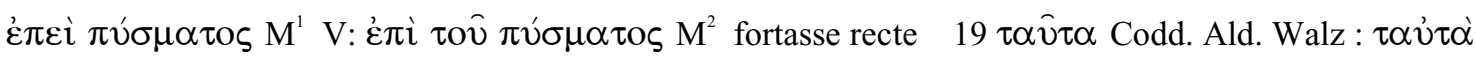
marg. Norrm. Speng. 21 post $\pi \lambda \alpha \dot{\sigma} \iota v$ fortasse excidit attributum quoddam exempli gratia $\alpha$ $\tau o \pi o \nu$ 


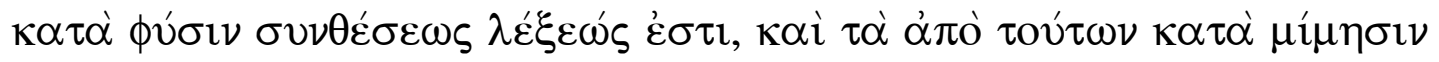

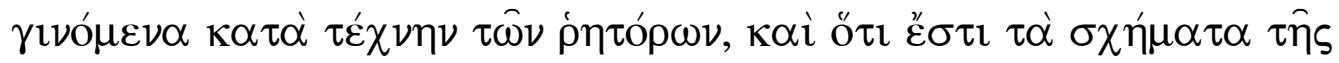

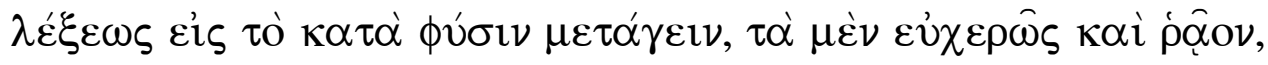

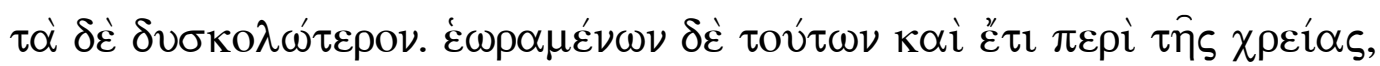

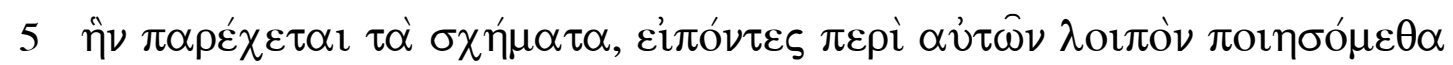

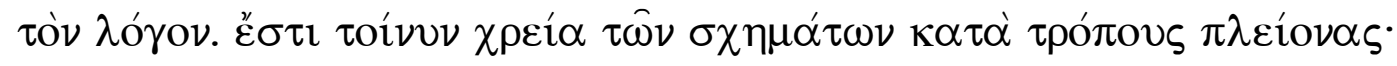

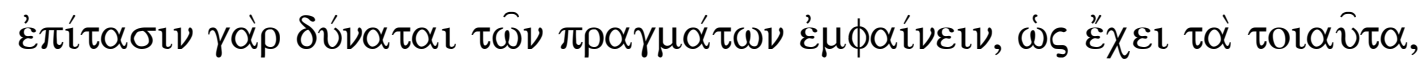

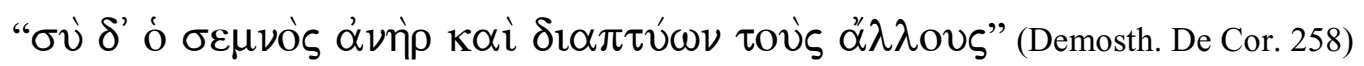

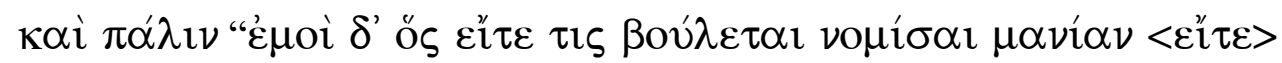

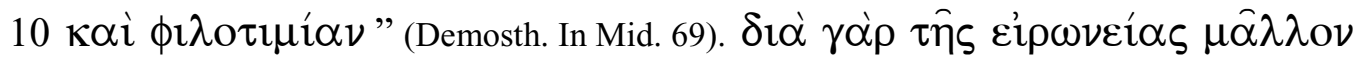

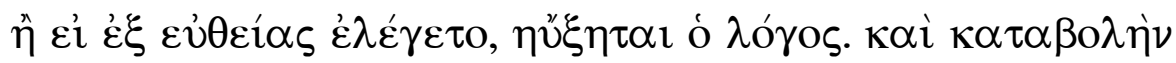

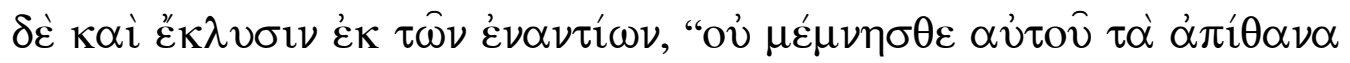

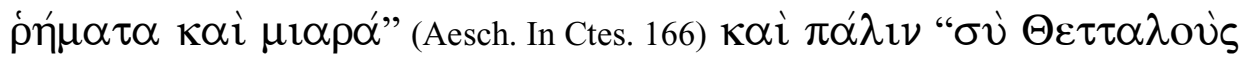

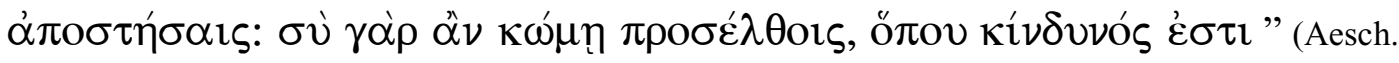

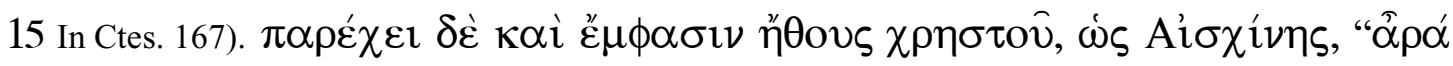

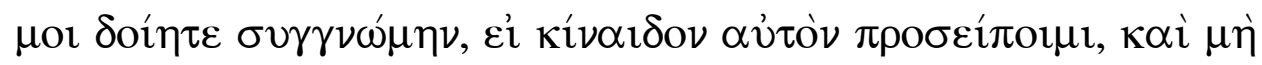

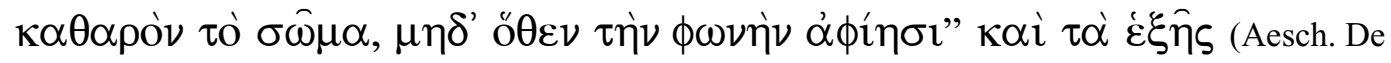
fals. Leg. 88)

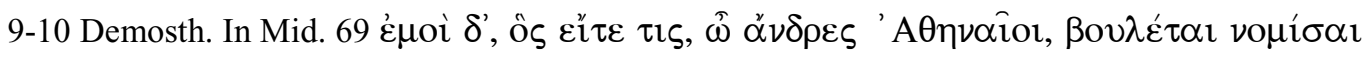

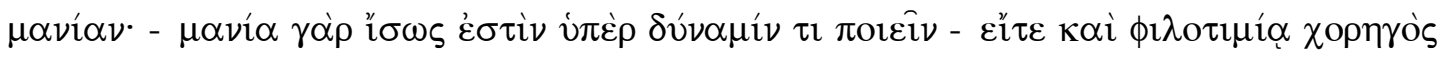

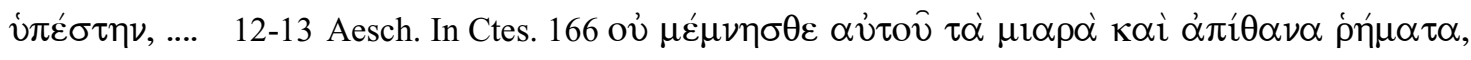

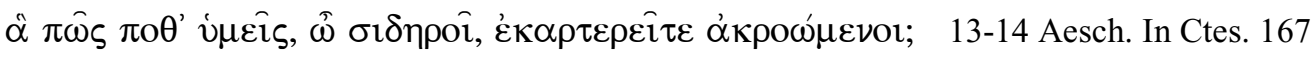

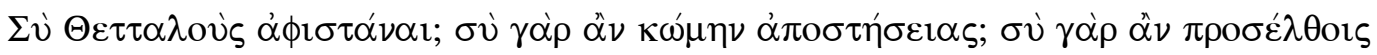

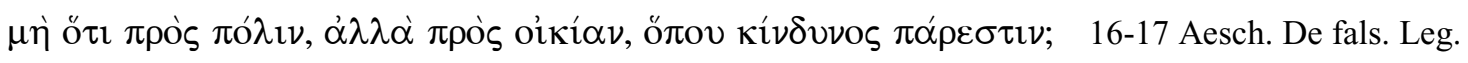

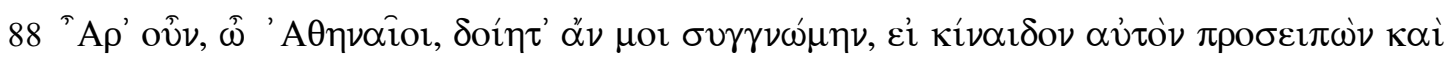

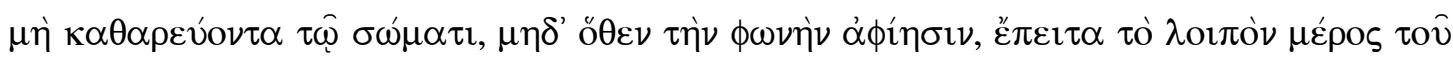

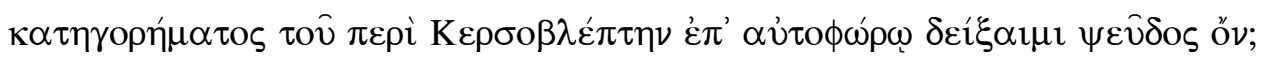

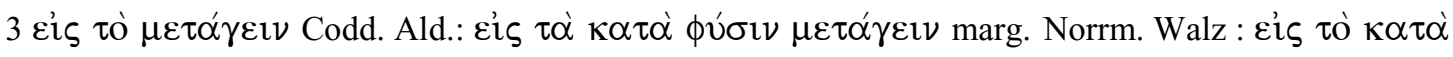

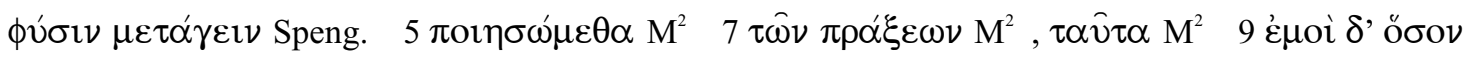

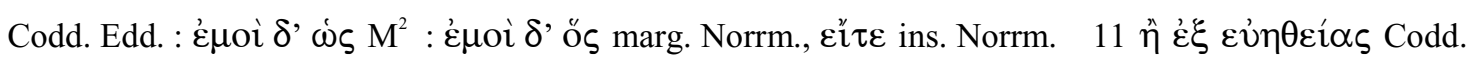

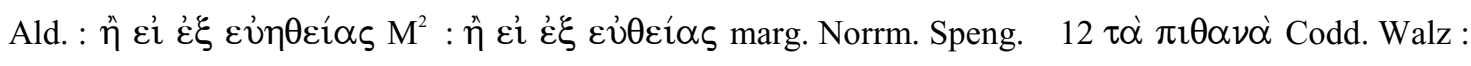

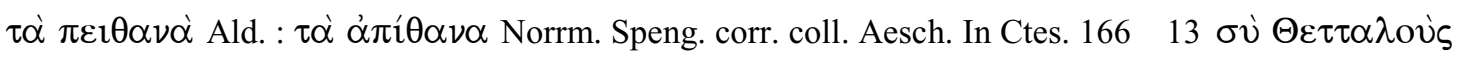

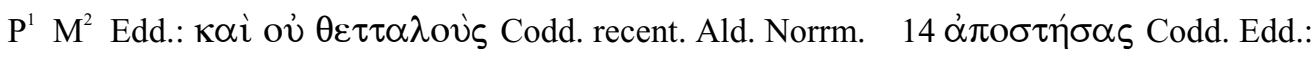

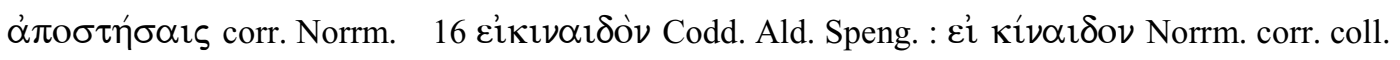

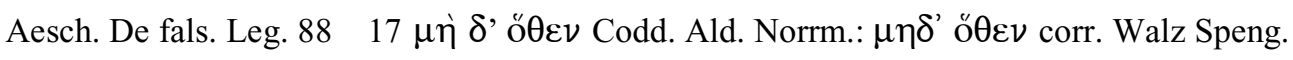




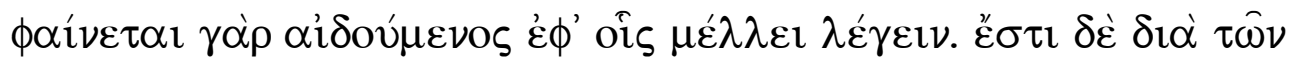

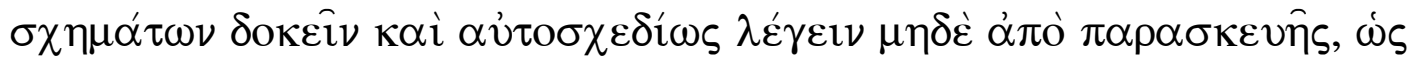

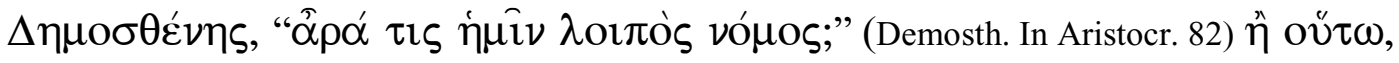

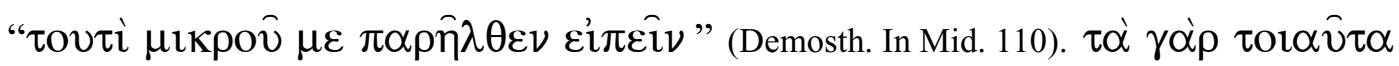

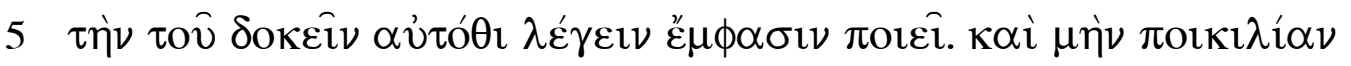

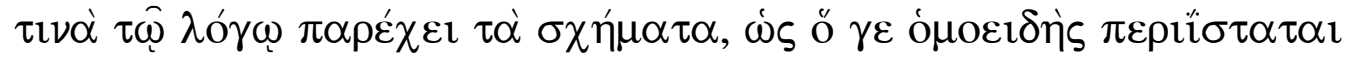

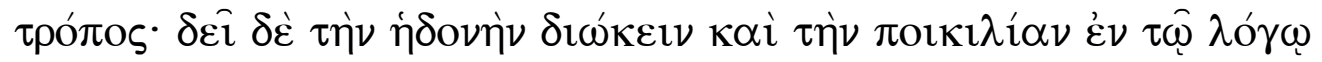

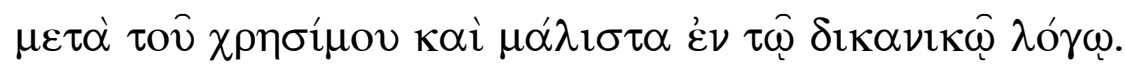

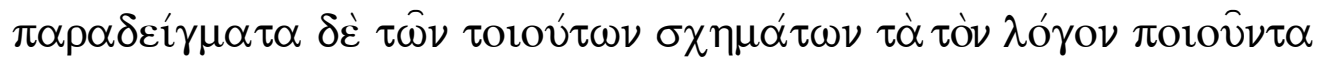

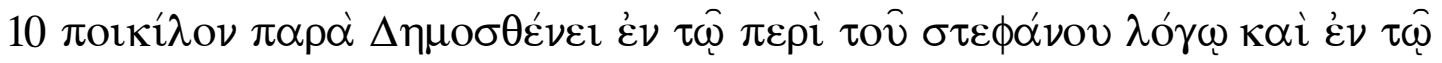

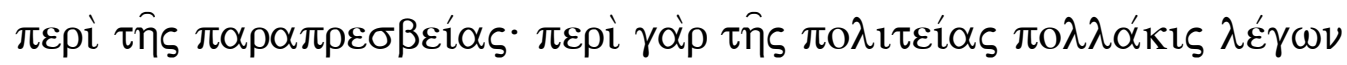

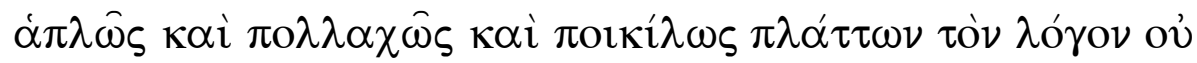

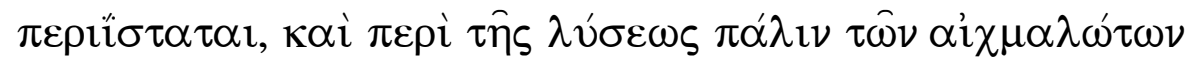

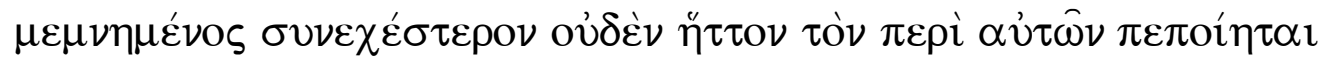

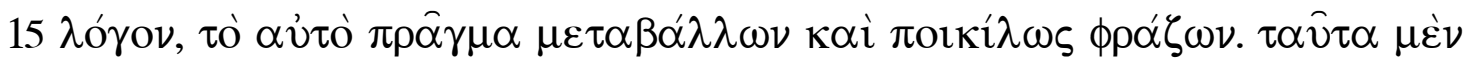

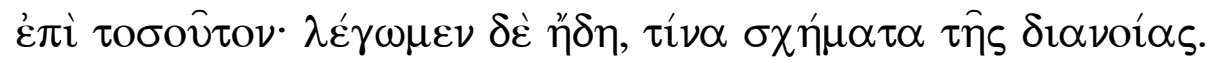

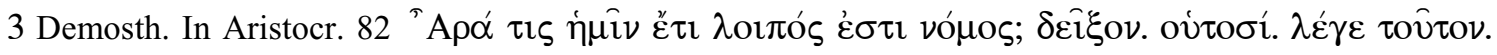

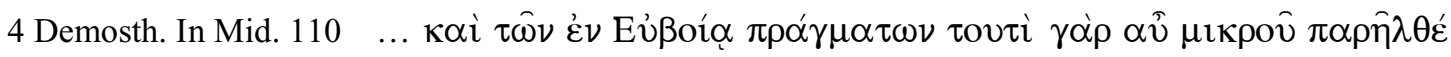

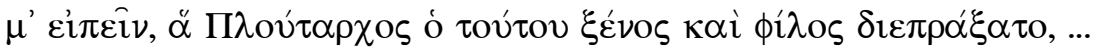
13-15 cf. Demosth. De fals. Leg. 169.

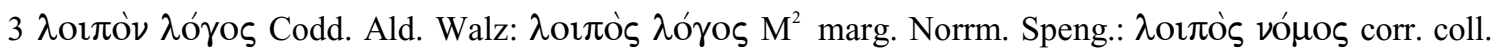

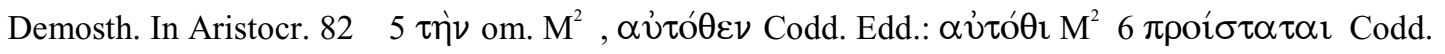

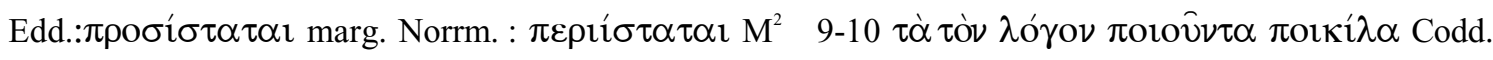

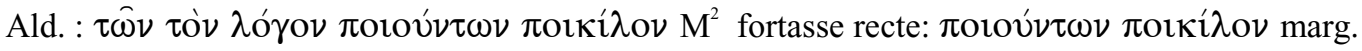

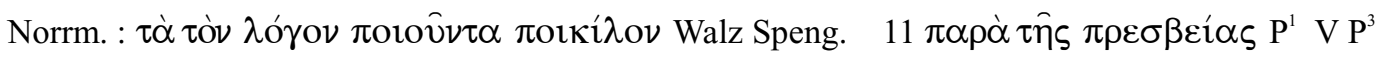
( $\lambda$ ó $\gamma \omega$ add.) : $\pi \varepsilon \rho \grave{\alpha} \tau \hat{\eta} \varsigma \pi \rho \varepsilon \sigma \beta \varepsilon i ́ \alpha \varsigma \mathrm{M}^{1}: \pi \varepsilon \rho \grave{i} \tau \hat{\eta} \varsigma \pi \rho \varepsilon \sigma \beta \varepsilon i ́ \alpha \varsigma$ Pal. Edd. : $\pi \alpha \rho \dot{\alpha} \pi \rho \varepsilon \sigma \beta \varepsilon i ́ \alpha \varsigma \mathrm{M}^{2}$ :

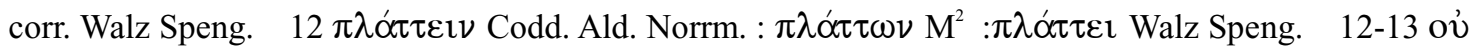
$\pi \rho o i ́ \sigma \tau \alpha \tau \alpha \iota$ Codd. Ald. : ov̉ $\pi \varepsilon p ı i ́ \sigma \tau \alpha \tau \alpha \iota$ marg. Norrm. $\mathrm{M}^{2}$ : oṽ $\pi \rho o i ́ \sigma \tau \alpha \tau \alpha \iota$ Walz Speng. 14

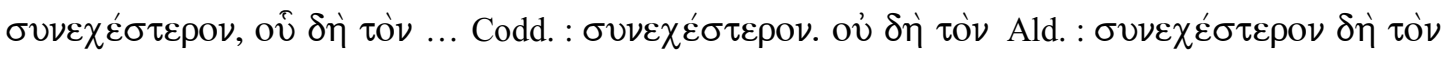

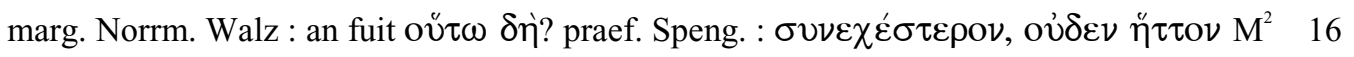

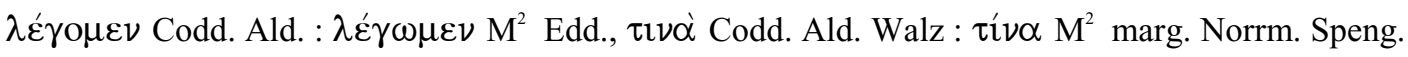




\section{$\gamma^{\prime}$. ПЕРІ ПРО $\triangle \mathrm{IOP} \Theta \Omega \Sigma \mathrm{E} \Omega \Sigma$.}

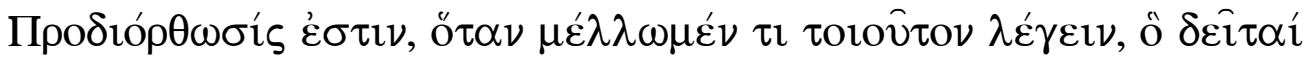

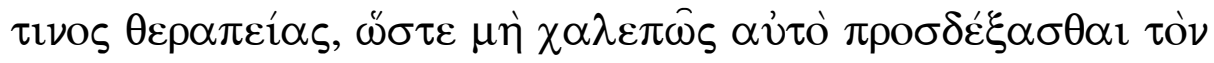

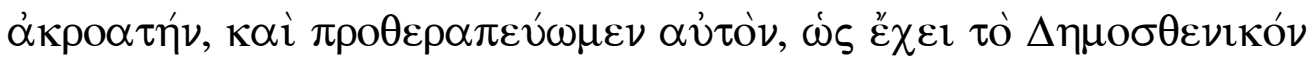

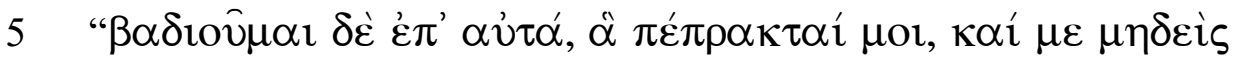

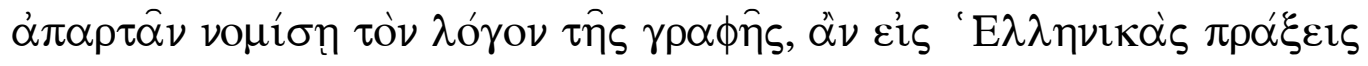

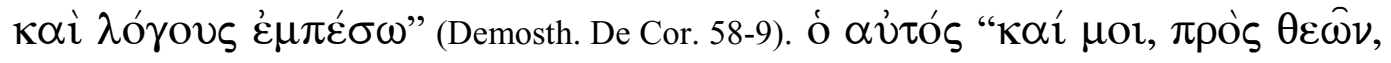

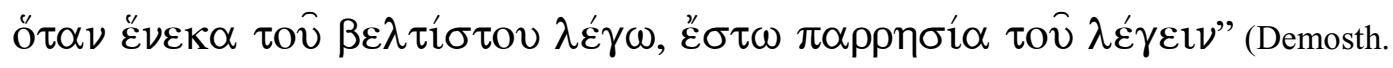

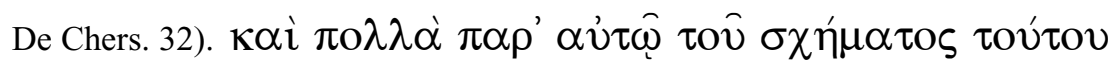

$10 \pi \alpha \rho \alpha \delta \varepsilon i ́ \gamma \mu \alpha \tau \alpha$.

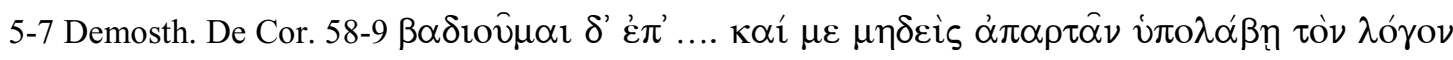

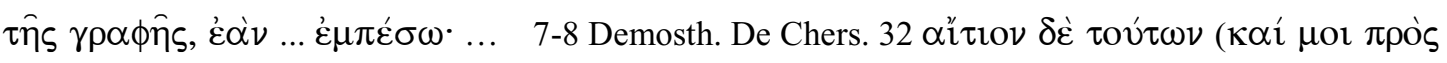

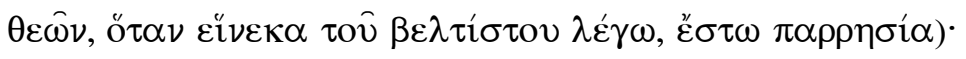

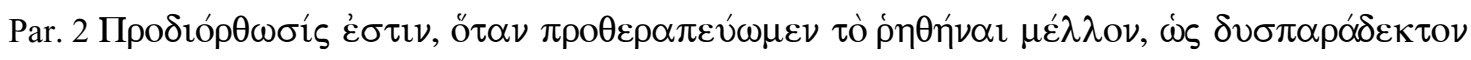

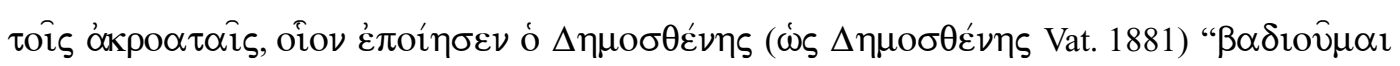

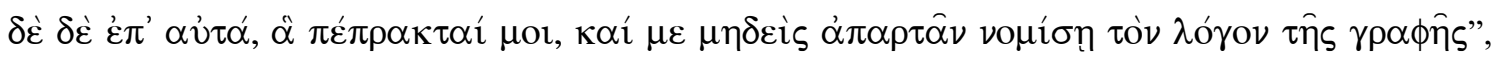

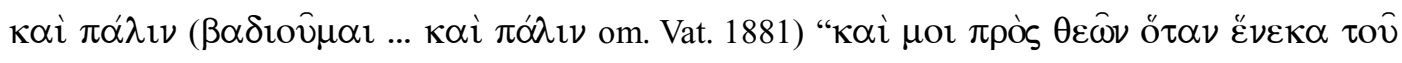

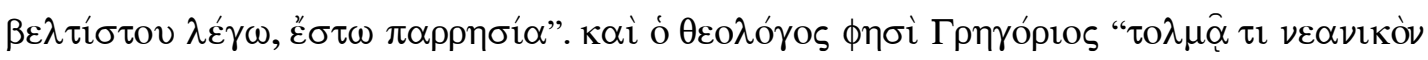

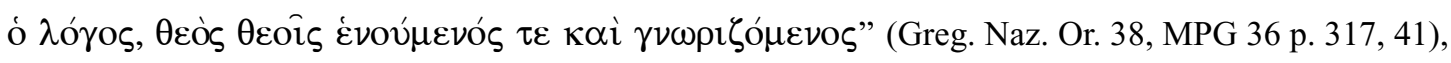

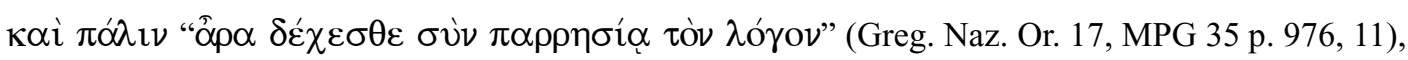

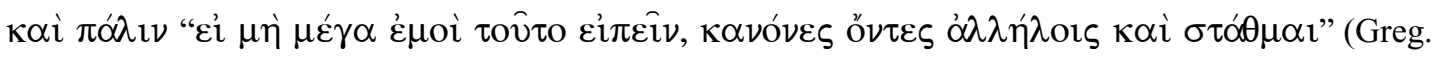

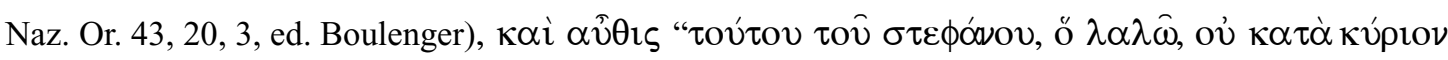

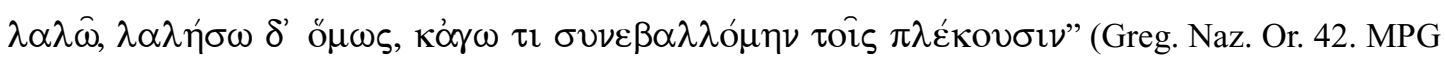
36 p. 472, 28).

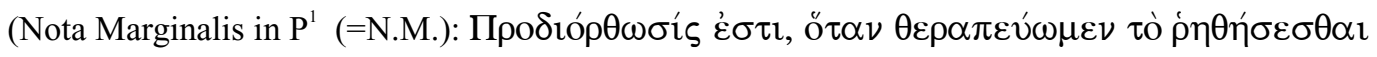

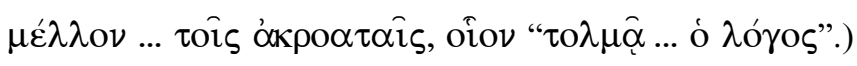

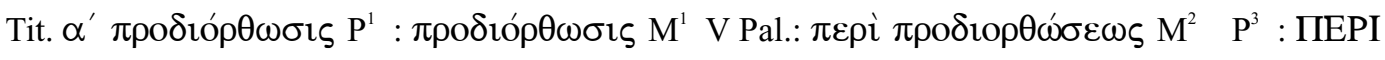
ПРО $\Delta \mathrm{IOP} \Theta \Omega \Sigma$ Е $\Omega \Sigma$ Ald. Ald ${ }^{s}:$ ПЕРI ПРО $\Delta \mathrm{IOP} \Theta \Omega \Sigma$ E $\Omega \Sigma \Gamma^{\prime}$ Norrm. : $\gamma^{\prime}$. ПЕРI $\Pi \mathrm{PO} \Delta \mathrm{IOP} \Theta \Omega \Sigma \mathrm{E} \Omega \Sigma$ Edd.

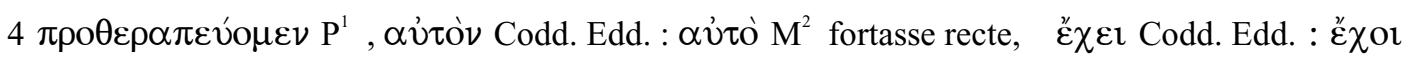

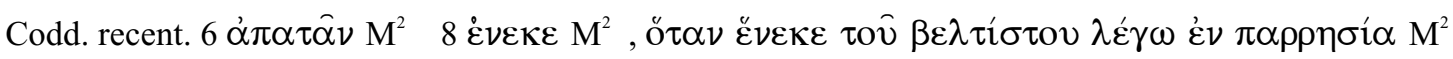




\section{$\delta^{\prime}$. ПЕРI ЕПI $\triangle \mathrm{IOP} \Theta \Omega \Sigma \mathrm{E} \Omega \Sigma$.}

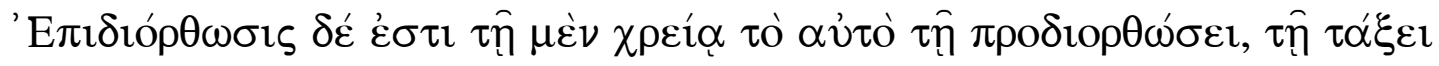

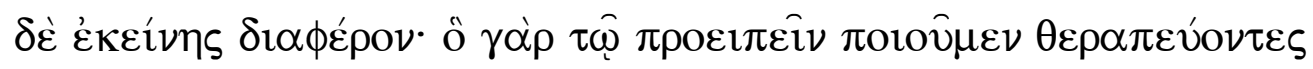

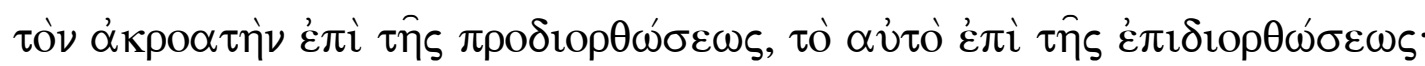

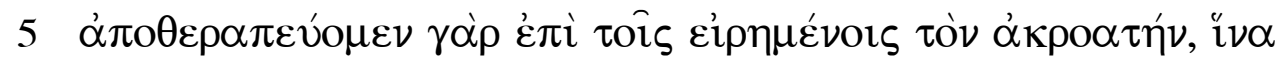

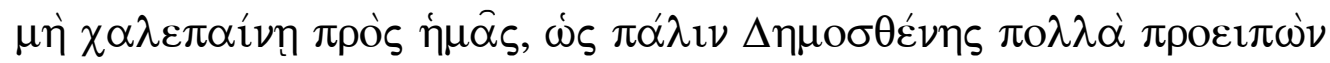

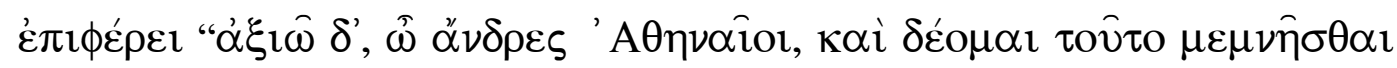

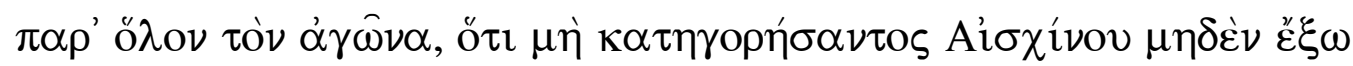

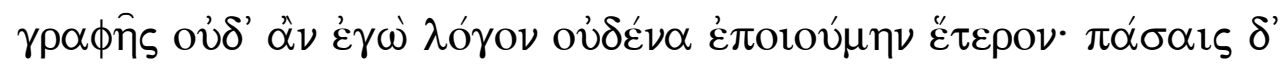

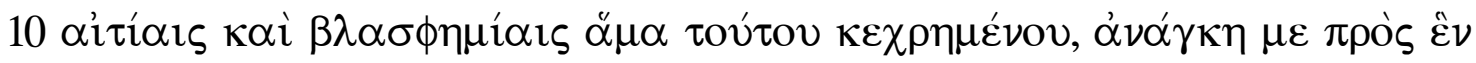

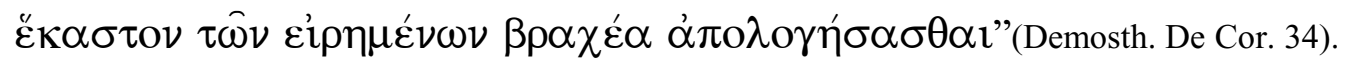

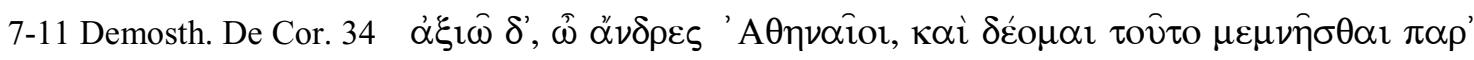

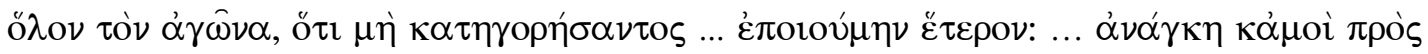

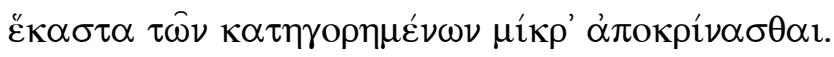

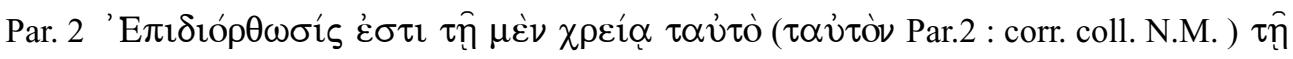

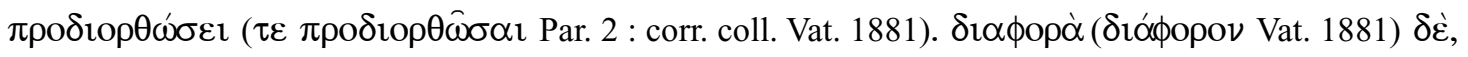

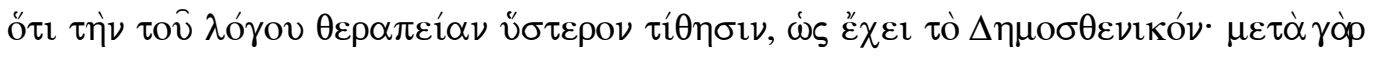

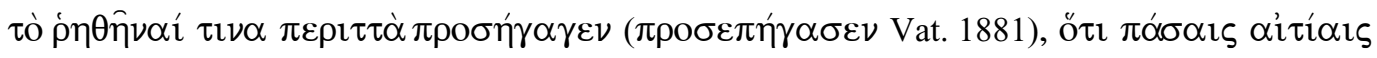

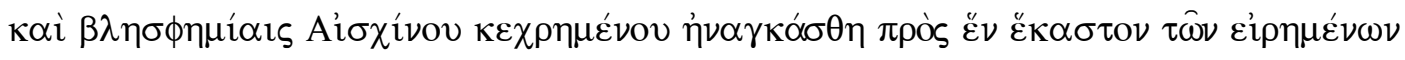

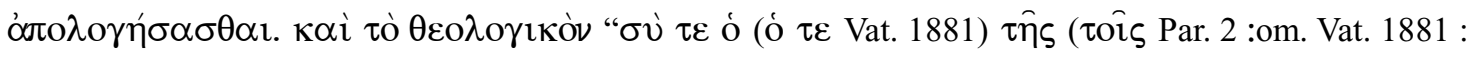

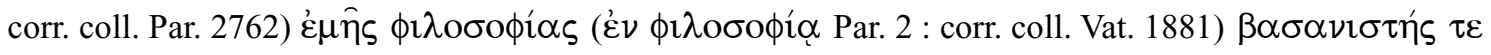

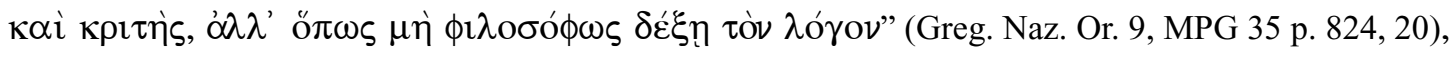

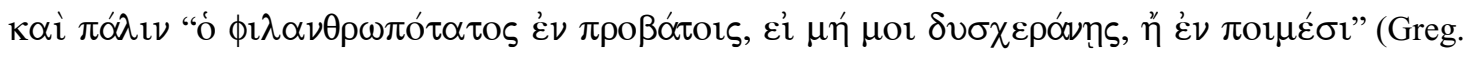

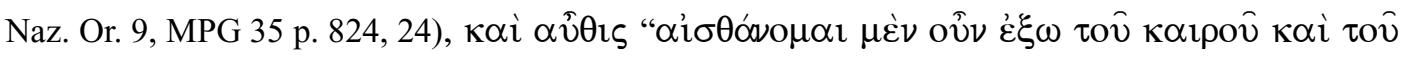

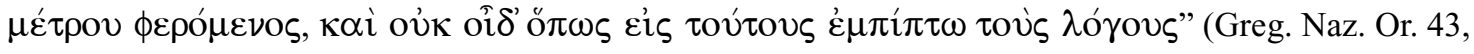

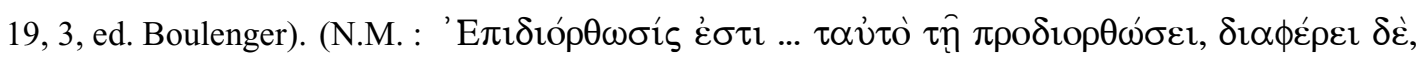

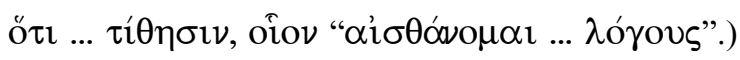

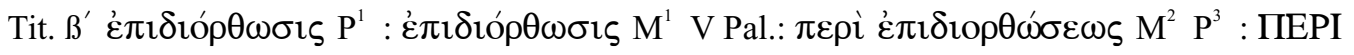
ЕПI $\Delta \mathrm{IOP} \Theta \Omega \Sigma \mathrm{E} \Omega \Sigma$ Ald. Ald ${ }^{\mathrm{s}}:$ ПЕPI ЕПI $\Delta-\Delta^{\prime}$ Norrm. : $\delta^{\prime}$. ПЕPI ЕПI $\Delta$ - Edd.

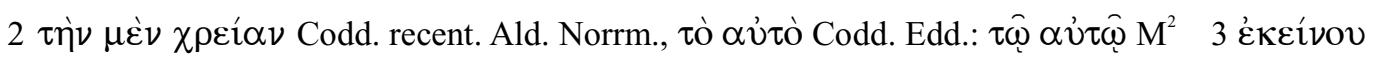

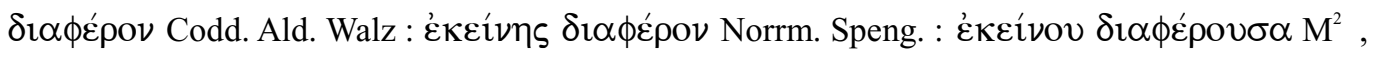

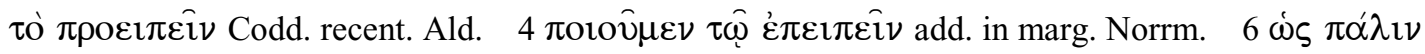

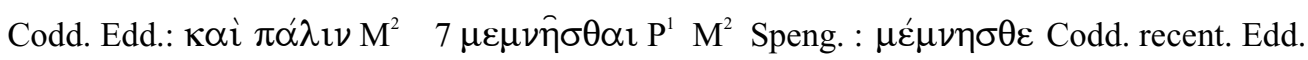

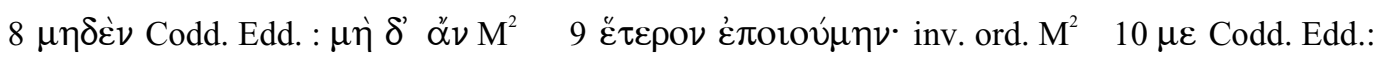

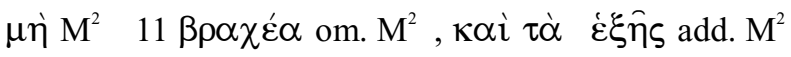




\section{$\varepsilon^{\prime}$. ПЕРI АMФI $\triangle \mathrm{IOP} \Theta \Omega \Sigma \mathrm{E} \Omega \Sigma$.}

Toṽ

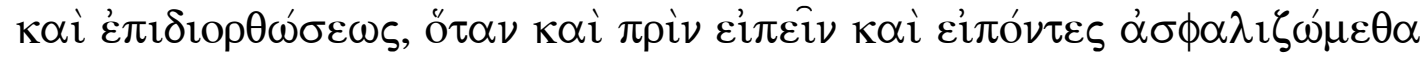

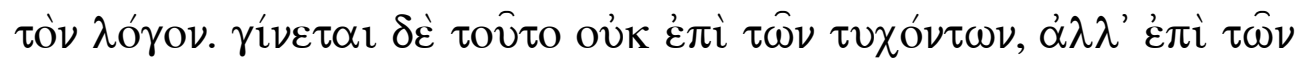

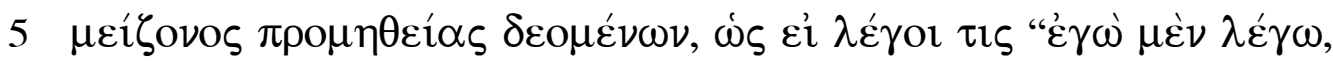

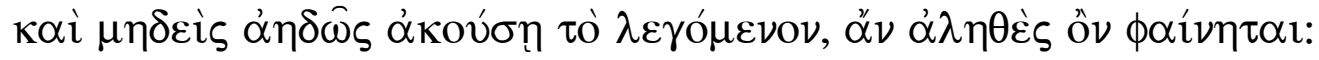

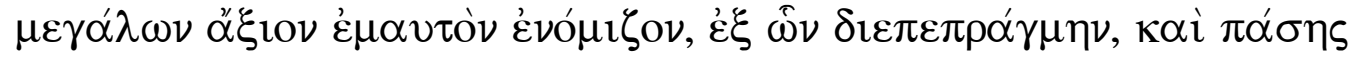

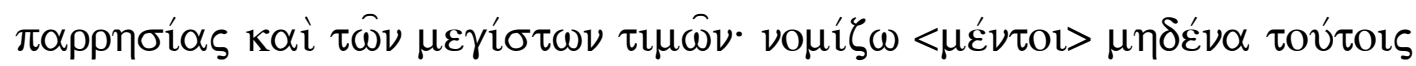

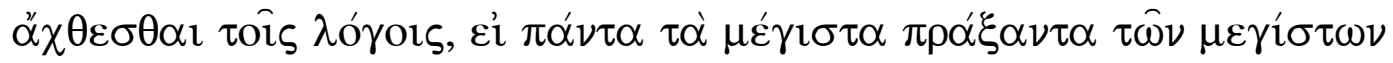

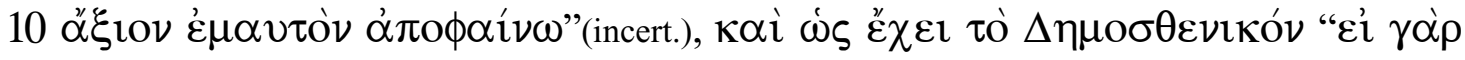

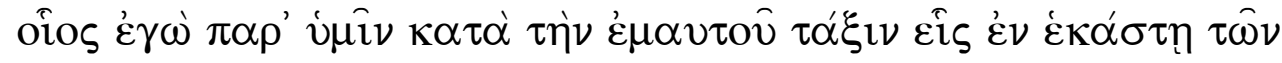

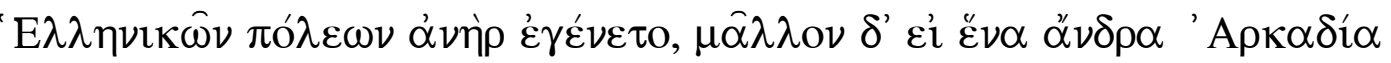

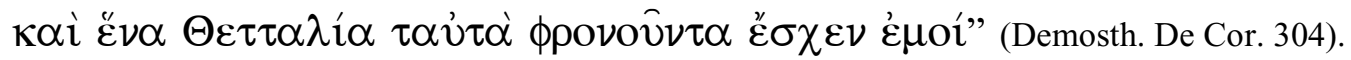

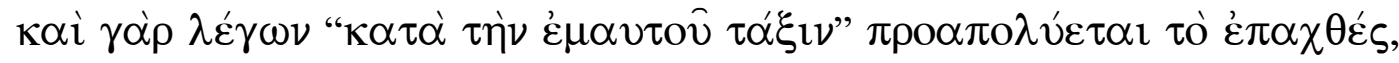

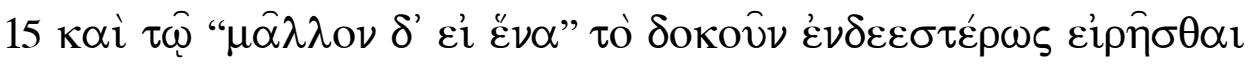
$\pi \rho \circ \sigma \varepsilon \pi \eta \nu \omega ́ \rho \theta \omega \tau \alpha$ l.

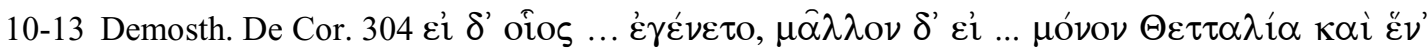

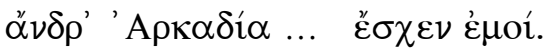

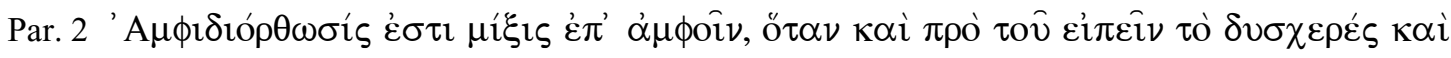

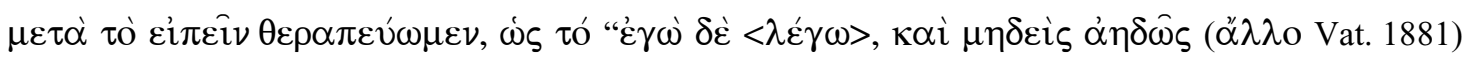

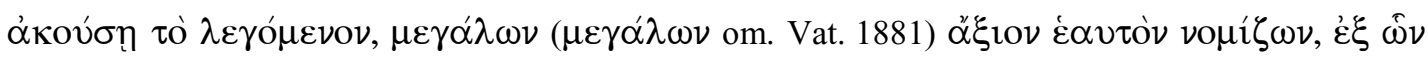

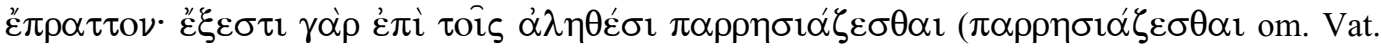

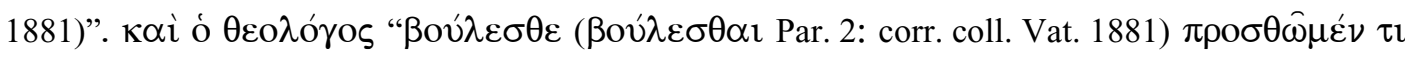

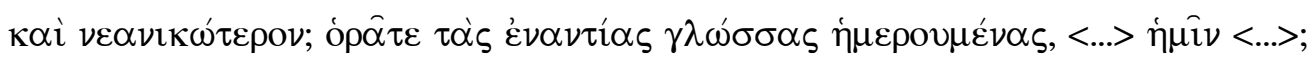

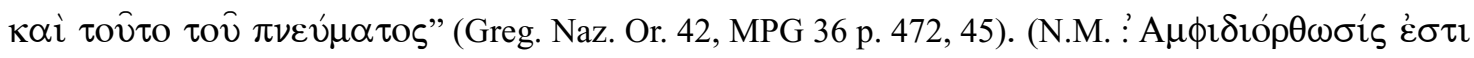

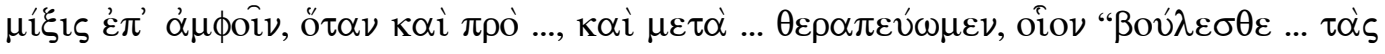

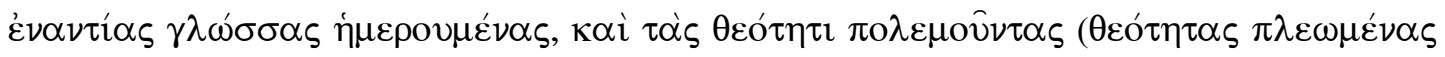

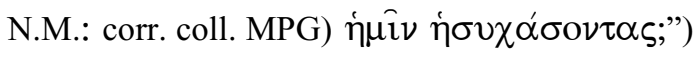

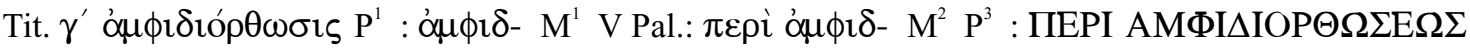

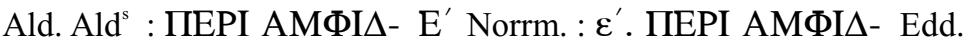

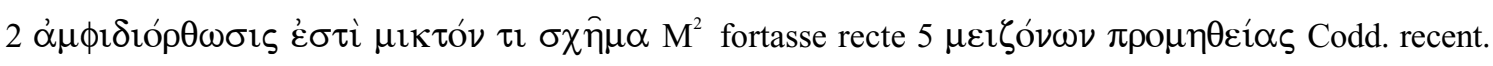

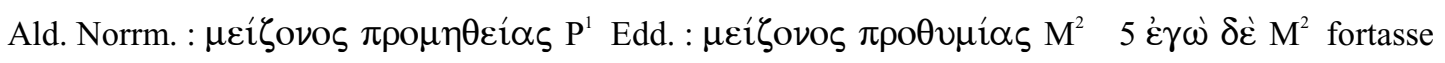

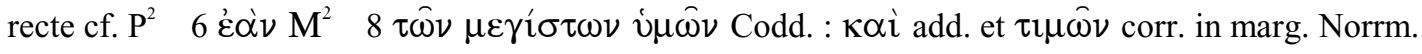

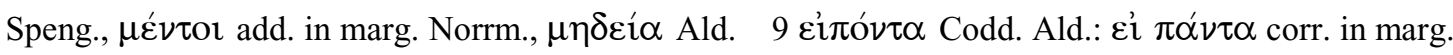
Norrm. Speng., $\pi \rho \alpha \dot{\xi} \alpha \nu \tau \alpha$ Codd. Edd. : $\pi \rho \alpha \dot{\xi} \alpha \varsigma$ Walz Speng. 11 oí $\varsigma$ V Pal. $\mathrm{P}^{3}$ Ald 13

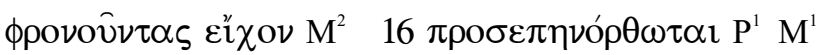




\section{$\varsigma^{\prime}$. ПЕРІ ПРОКАТААНЧЕ $\Omega \Sigma$.}

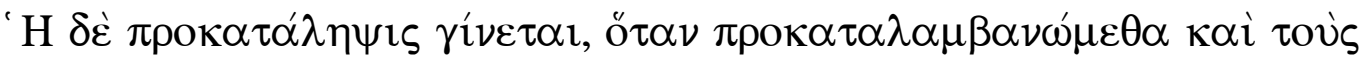

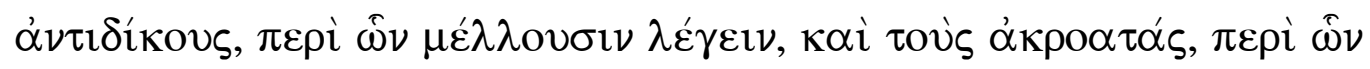

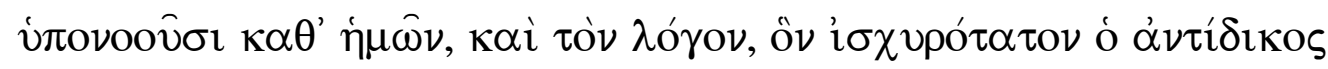

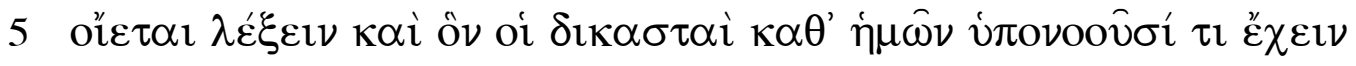

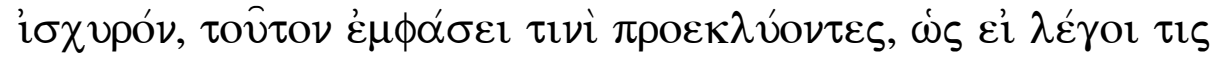

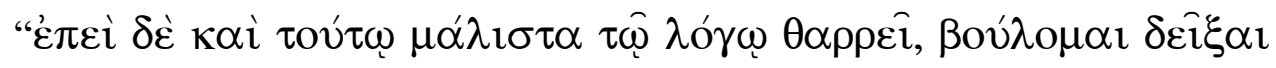

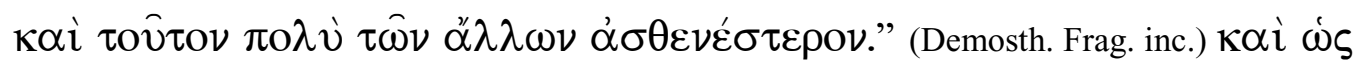

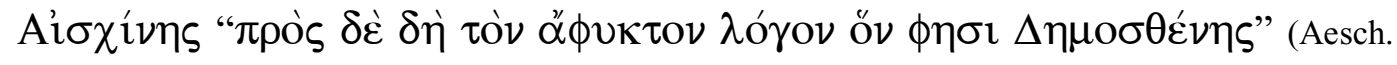
In Ctes. 17).

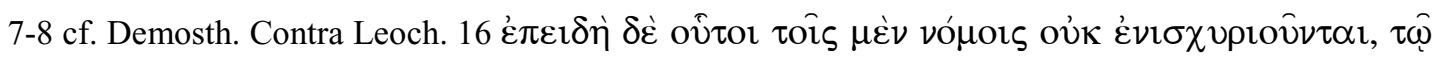

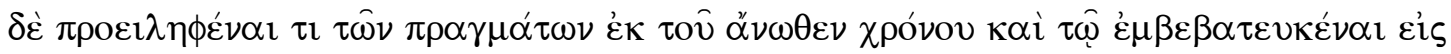

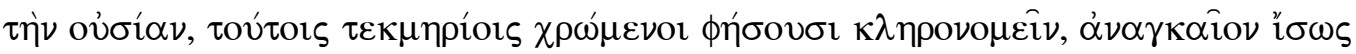

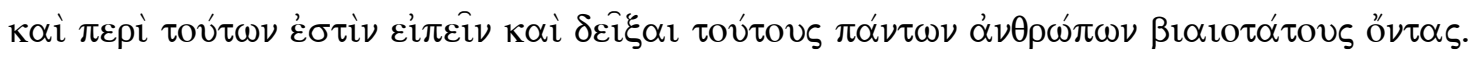

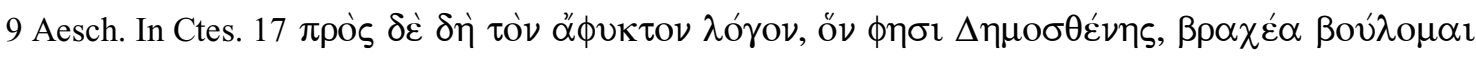
$\pi \rho \circ \varepsilon \pi \varepsilon i \bar{v}$.

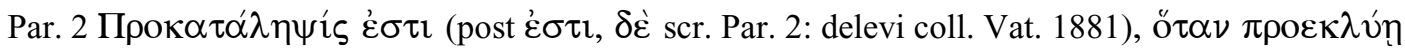

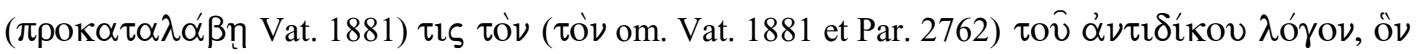

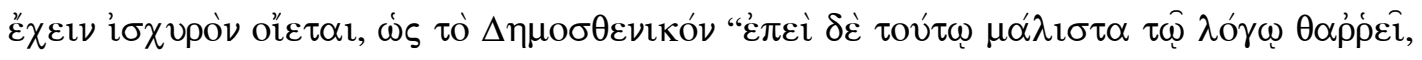

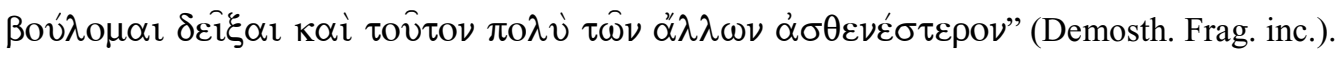

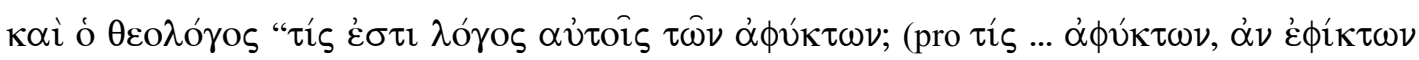

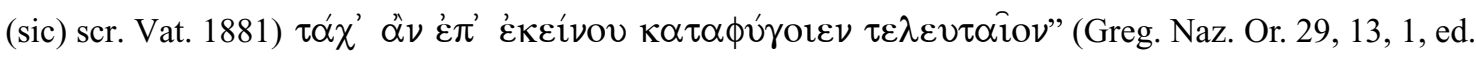

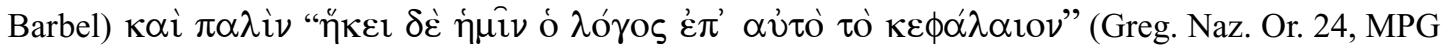
35 p. 1177, 42).

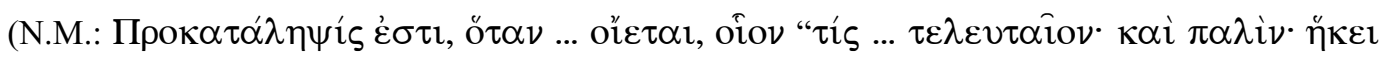

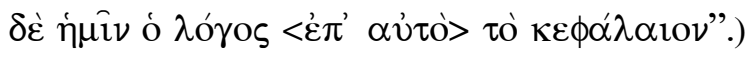

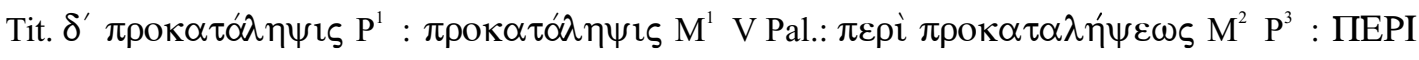

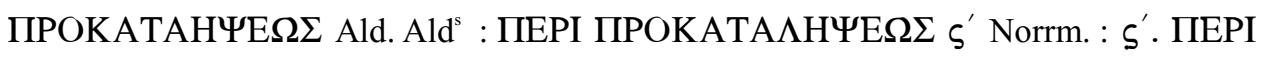
ПРОКАТА $Н \Psi Е \Omega \Sigma$ Edd.

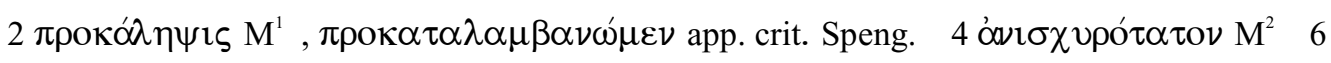

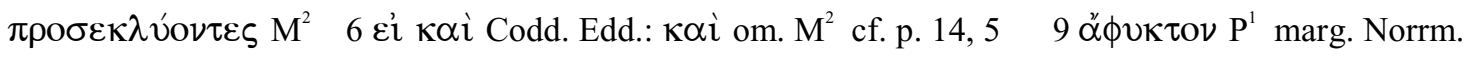
Edd. : ع̌ $\mu \phi v \tau o \nu$ Codd. recent. Ald. : $\alpha \phi v ́ \lambda \alpha \kappa \tau o \nu \mathrm{M}^{2}$ 

$\eta^{\prime}$. ПЕРI AITIO $\Lambda$ ОГIА $\Sigma$.

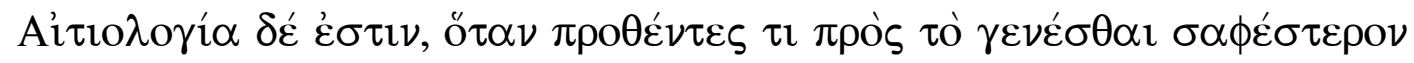

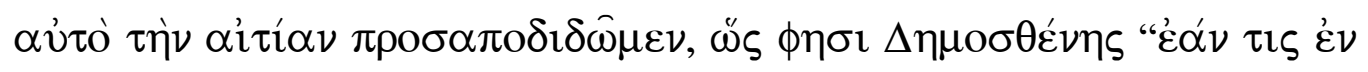

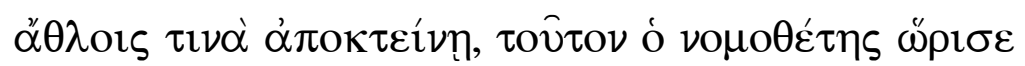

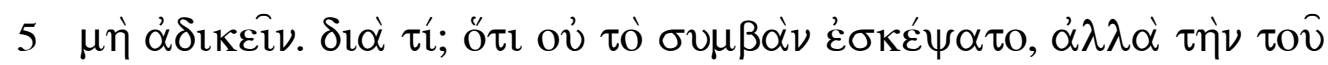

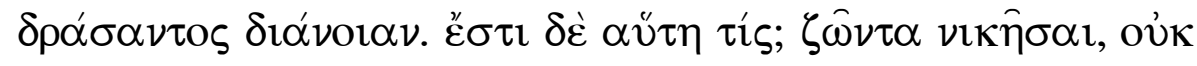

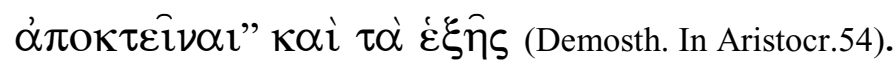

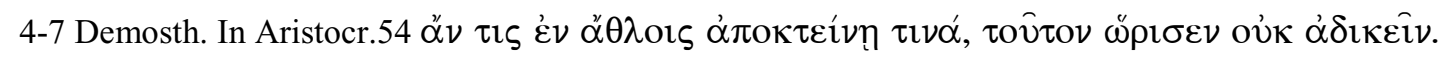

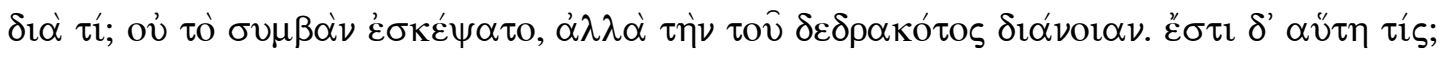

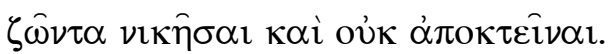

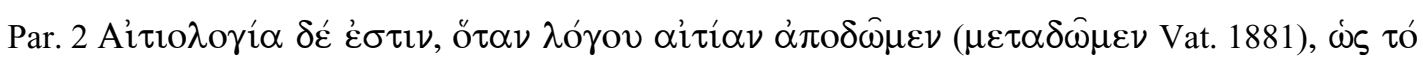

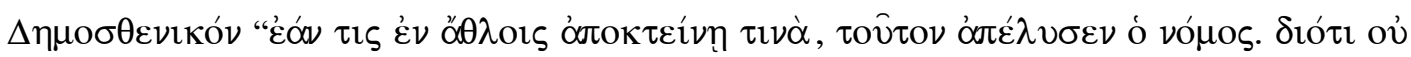

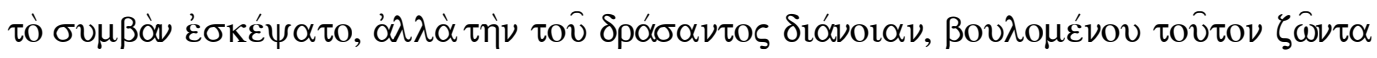

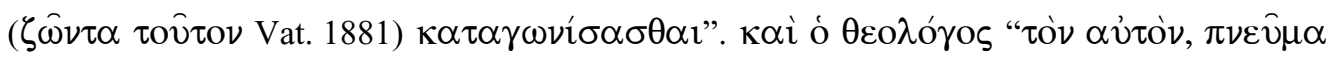

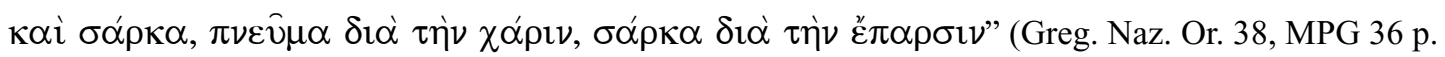

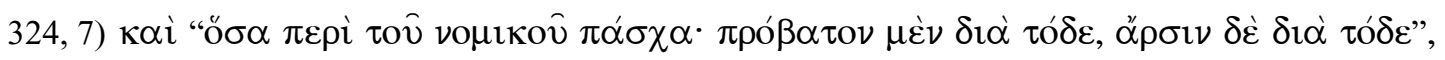

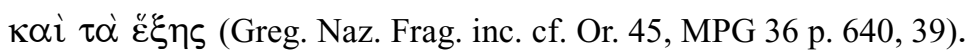

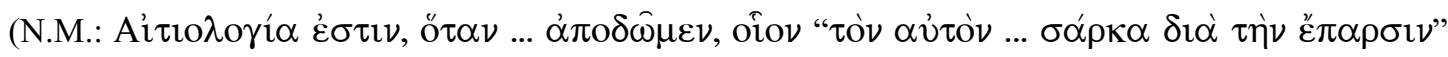

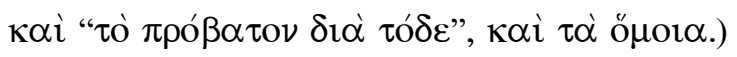

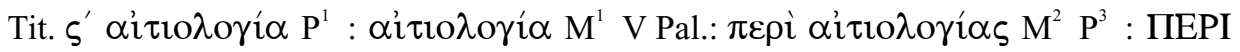
AITIO $\Lambda$ ОГIA $\Sigma$ Ald. Ald ${ }^{s}:$ ПЕРI AITIO $\Lambda$ ОГІА $\Sigma \eta^{\prime}$ Norrm. $: \eta^{\prime}$. ПЕPI АITIO $\Lambda$ ОГIA $\Sigma$ Edd.

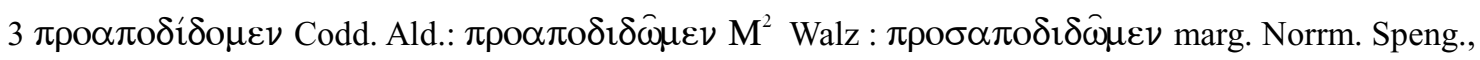

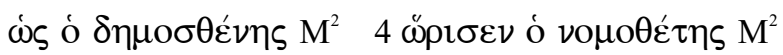




\section{$\theta^{\prime}$. ПEPI $\Sigma$ YNA $\Theta P O I \Sigma M O Y$.}

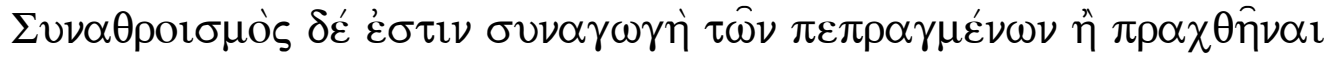

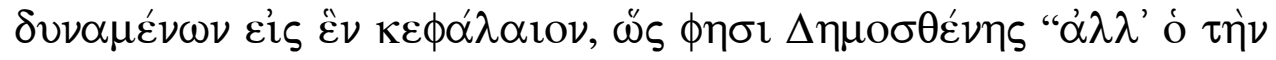

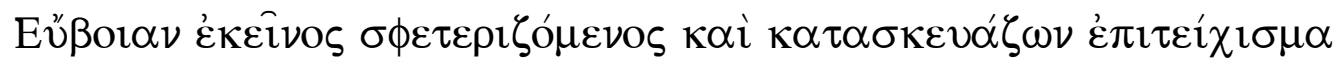

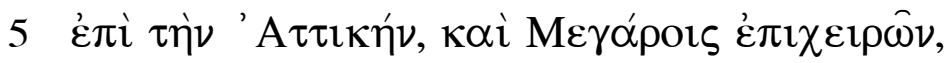

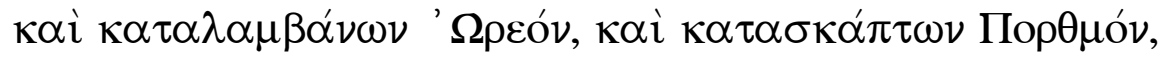

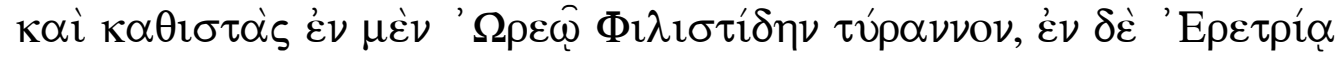

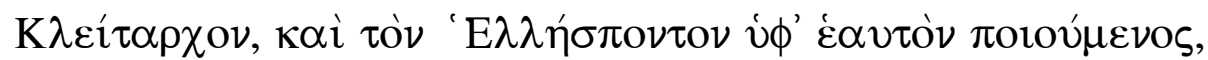

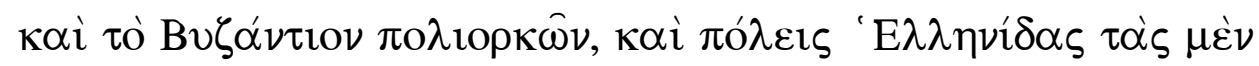

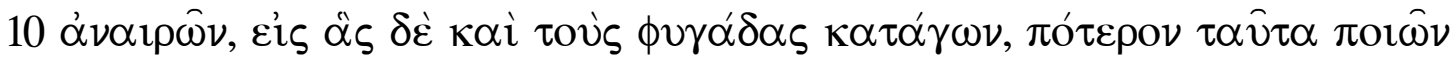

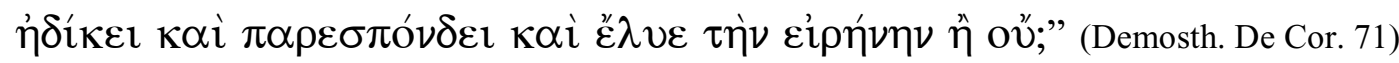

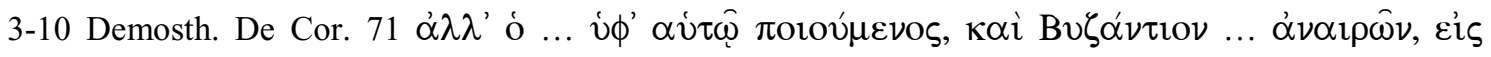

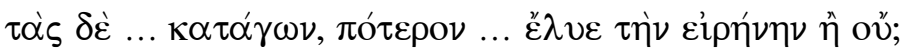

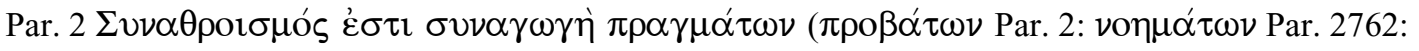

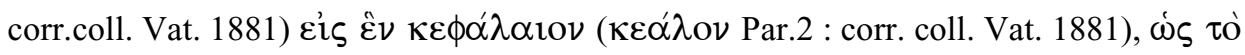

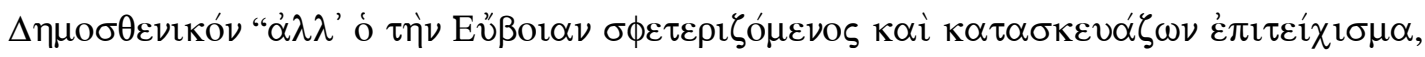

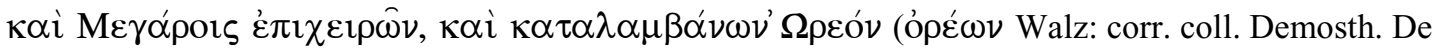

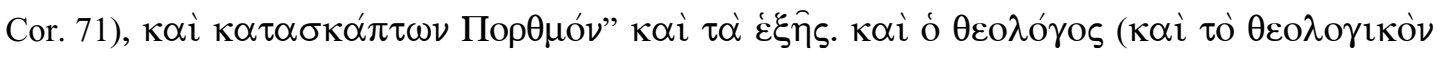

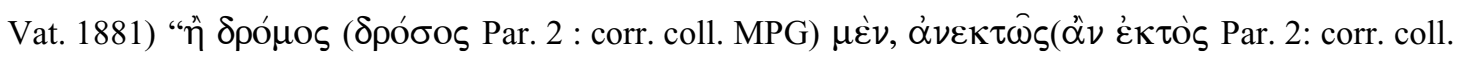

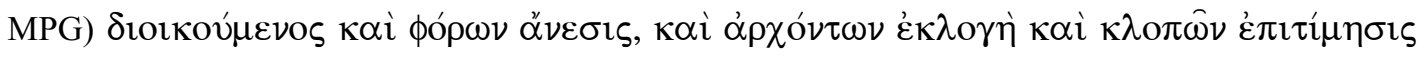

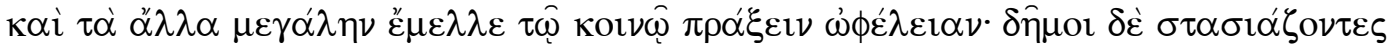

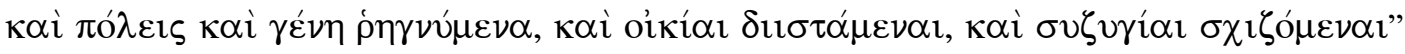

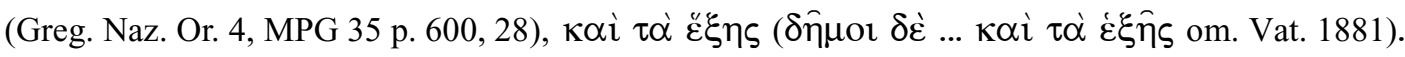

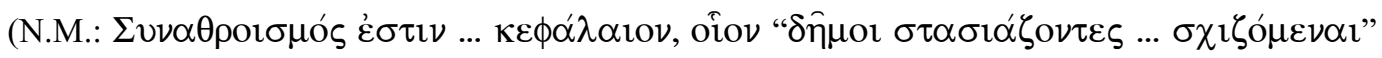

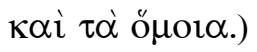

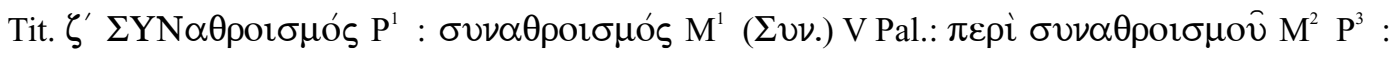

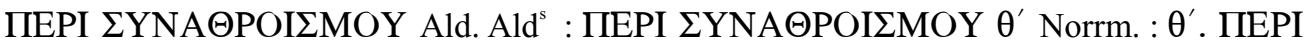
$\Sigma$ YNA@POI $\Sigma$ MOY Edd.

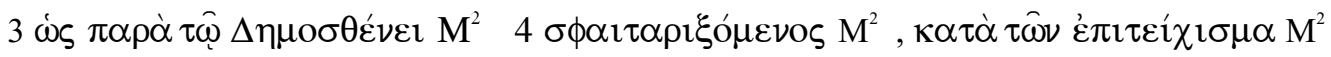

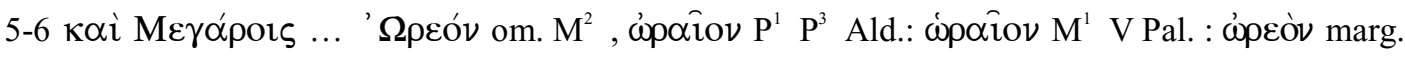

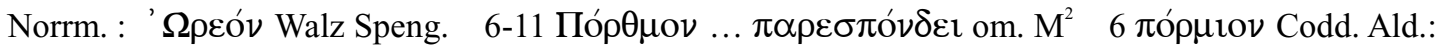

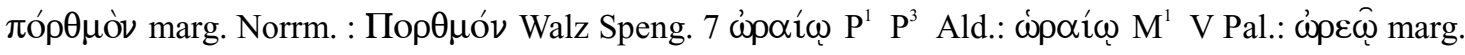

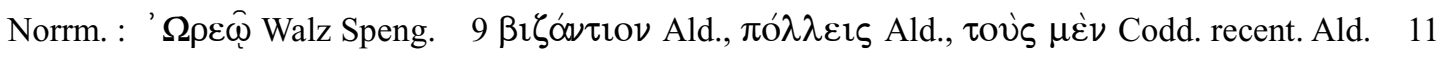

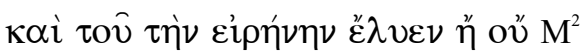




\section{$\imath^{\prime}$. ПEPI EПIMONH $\Sigma$.}

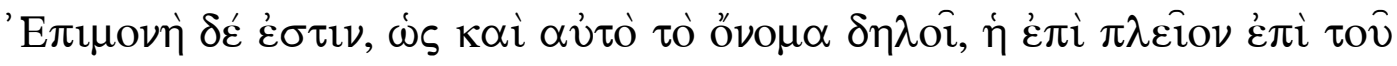

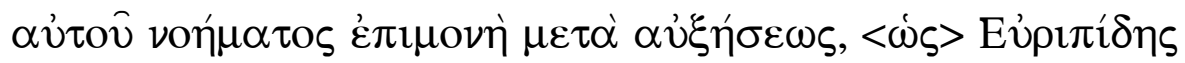

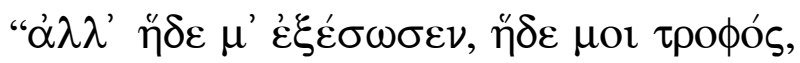

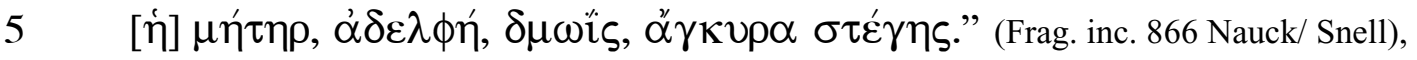

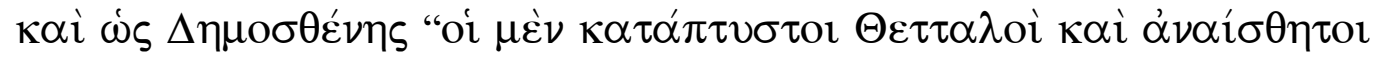

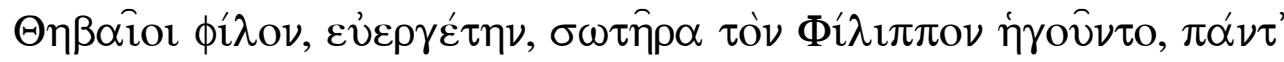

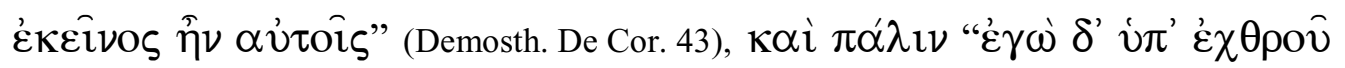

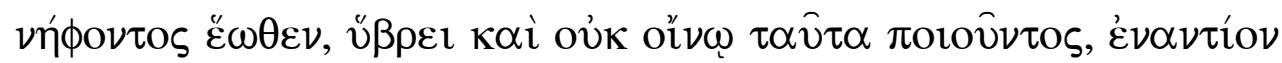

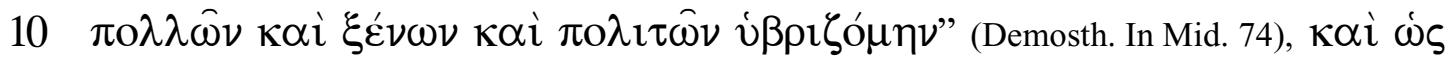

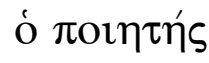

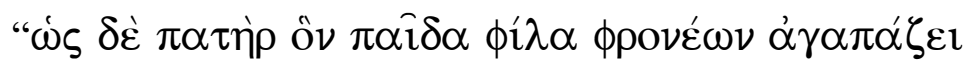

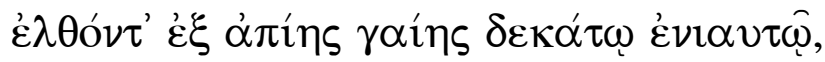

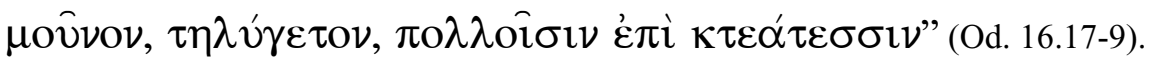

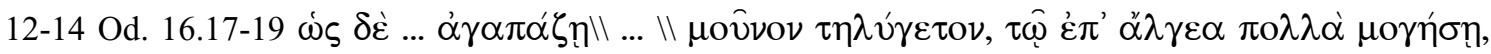

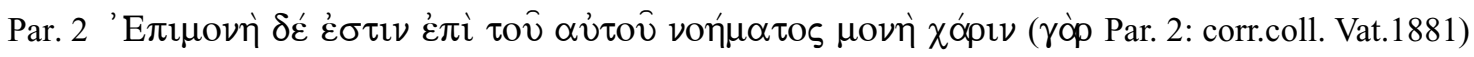

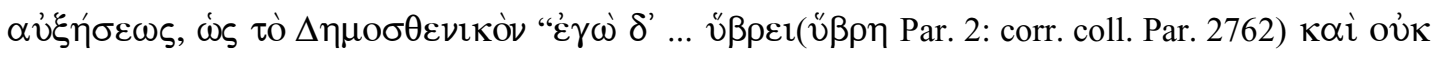

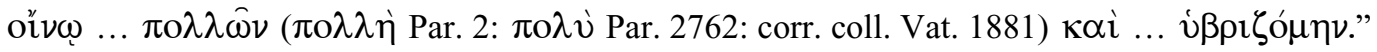

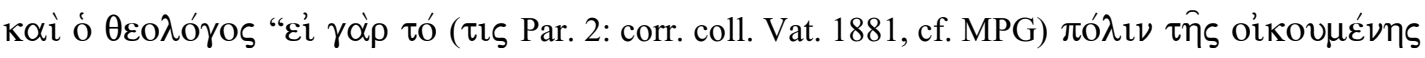

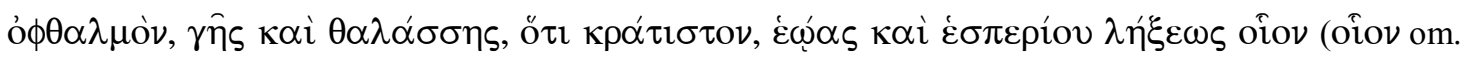

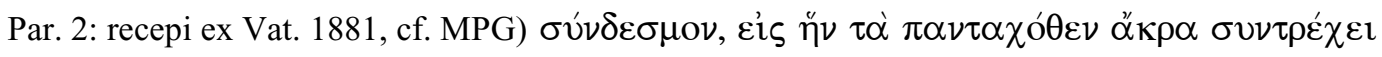

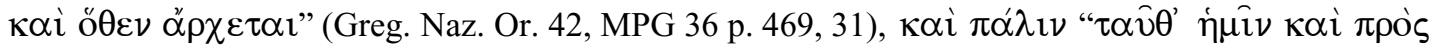

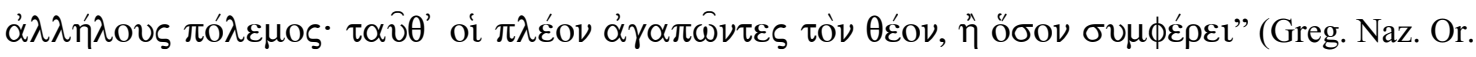

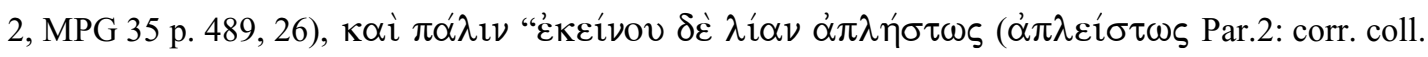

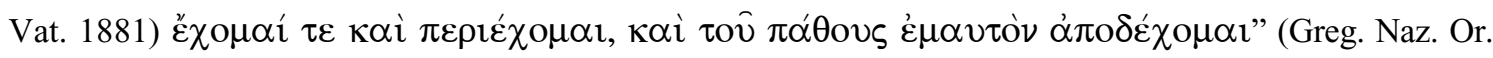

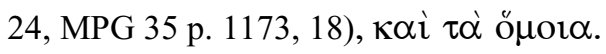

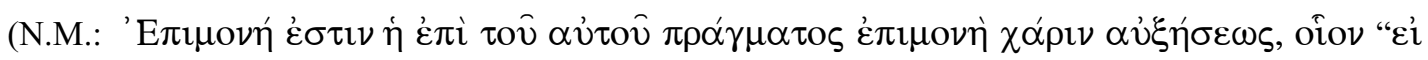

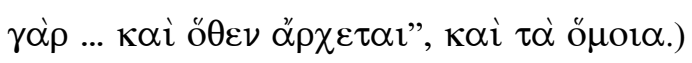

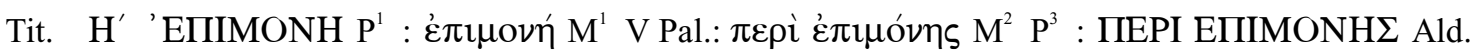
Ald $^{s}:$ ПEPI EПIMONH $\Sigma \imath^{\prime}$ Norrm. : $\imath^{\prime}$. ПЕPI EПIMONH $\Sigma$ Edd.

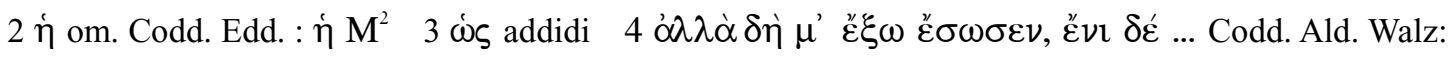

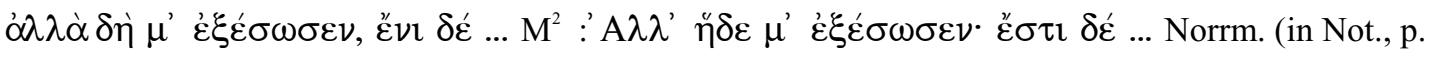

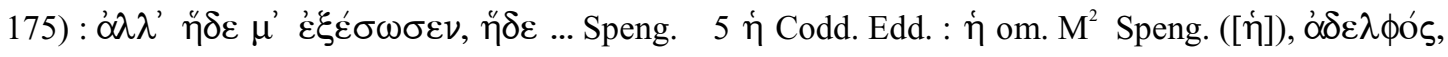

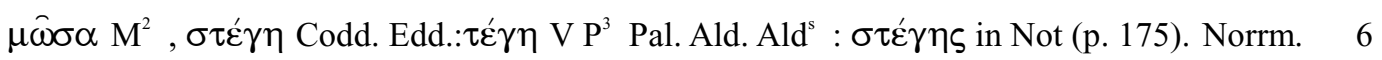

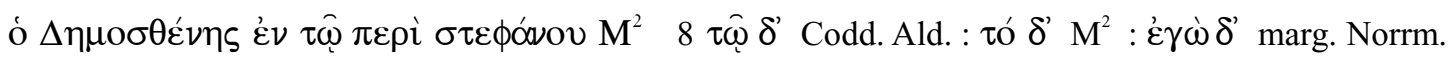

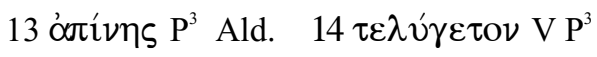


$1 \alpha^{\prime}$. ПЕРІ $\Lambda$ ЕПТО $\Lambda$ ОГАА $\Sigma$.

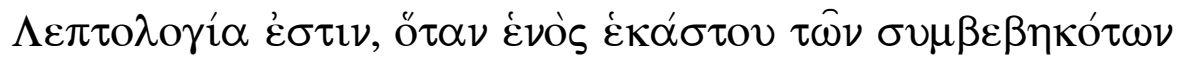

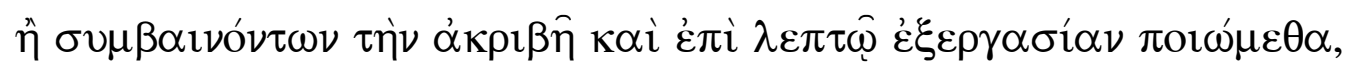
is $\pi \alpha \rho \alpha \dot{~ M \varepsilon v \alpha ́ v \delta \rho \omega ~ \tau \alpha v \tau i ́ ~}$

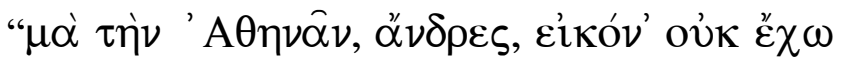

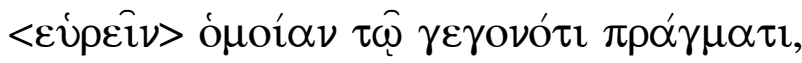

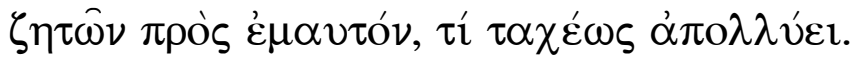

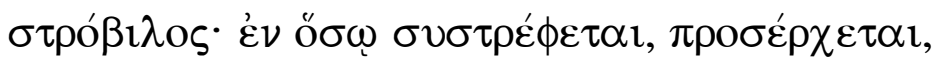

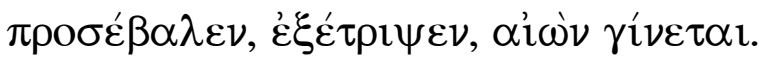

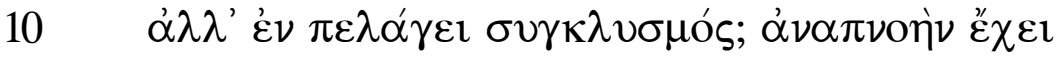

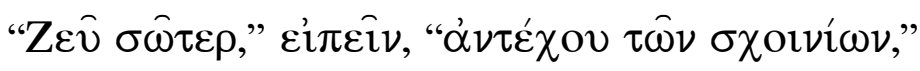

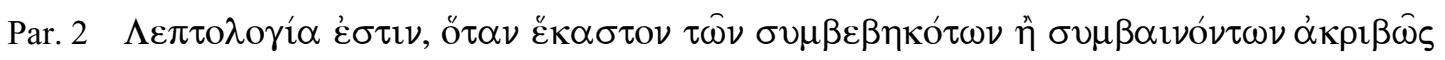

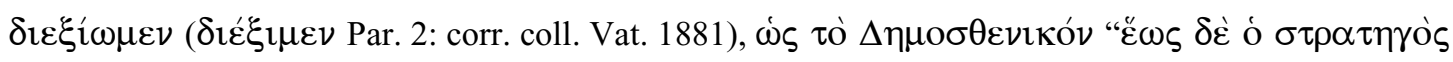

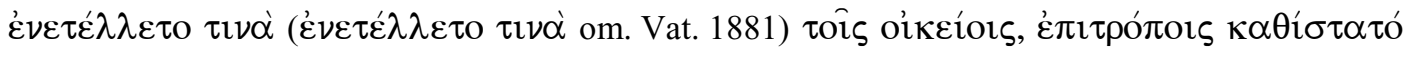

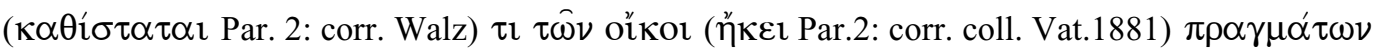

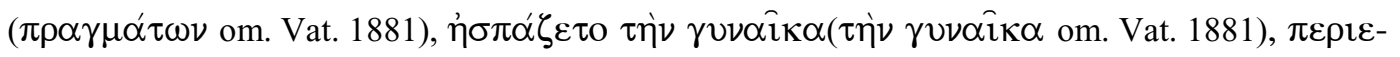

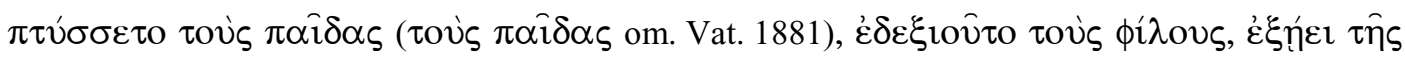

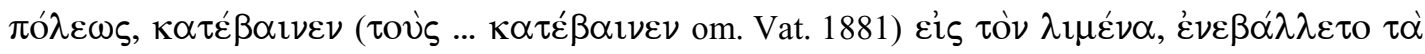

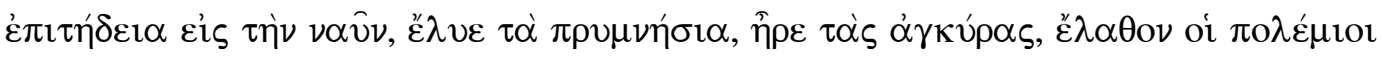

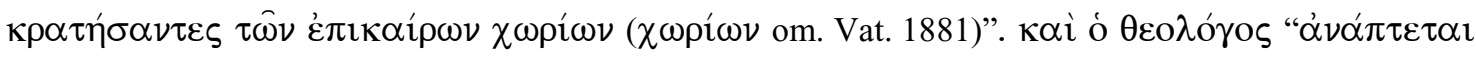

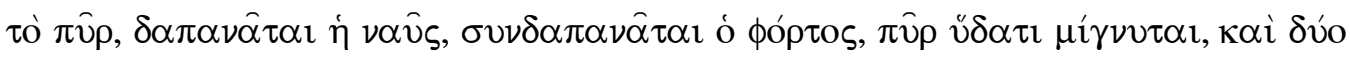

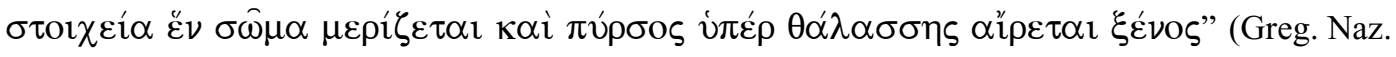

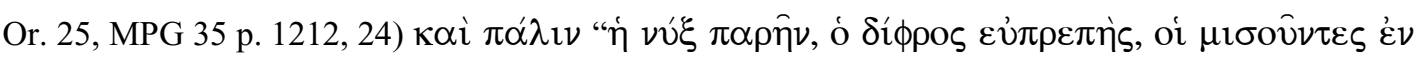

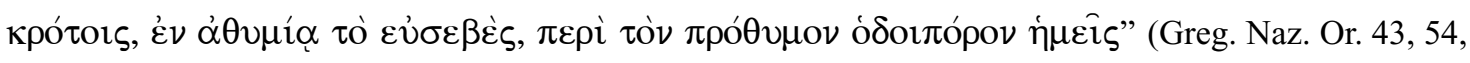

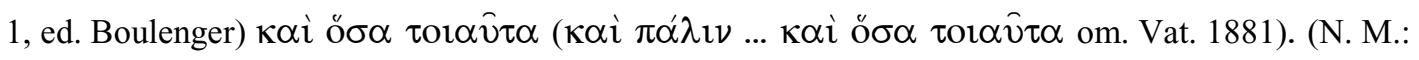

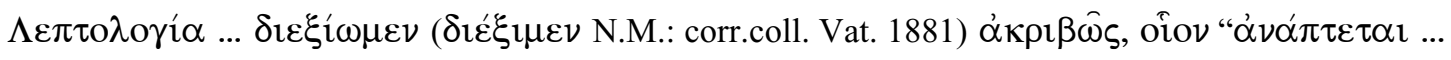

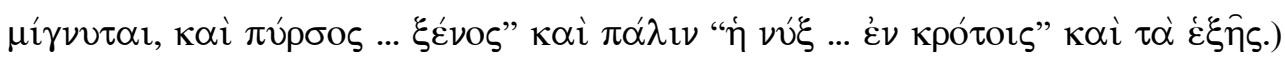

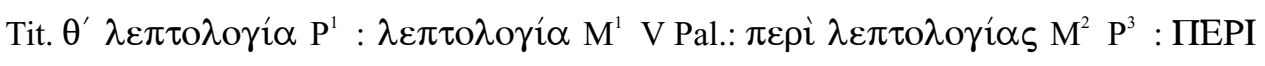
$\Lambda$ ЕПТО $\Lambda$ ОГІА $\Sigma$ Ald. Ald ${ }^{s}:$ ПЕРI $\Lambda$ ЕПТО $\Lambda$ - $1 \alpha^{\prime}$ Norrm. : $1 \alpha^{\prime}$. ПЕРI $\Lambda$ ЕПТО $\Lambda O-$ Edd.

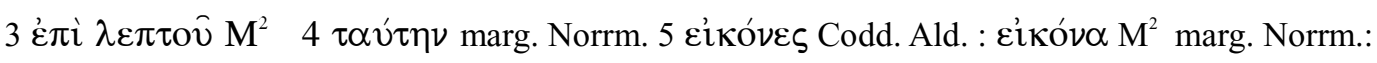

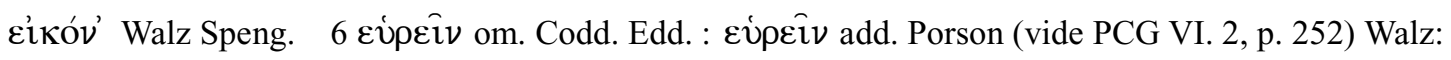

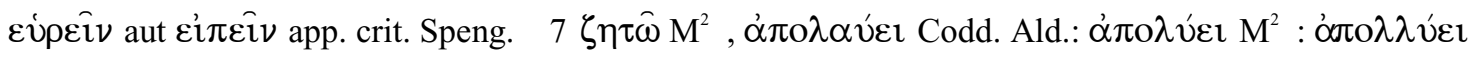

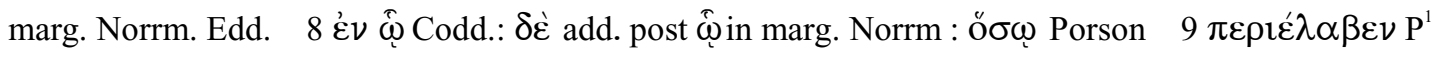

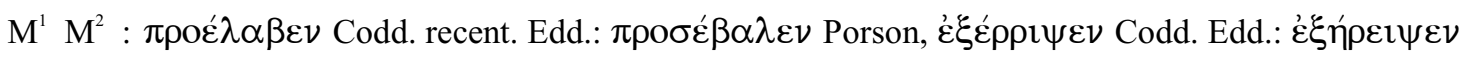

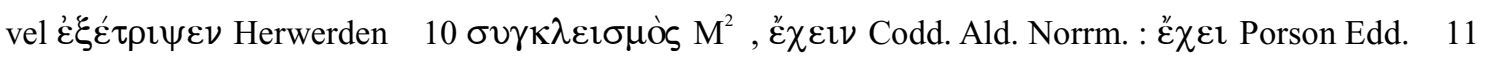

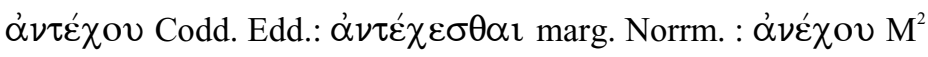




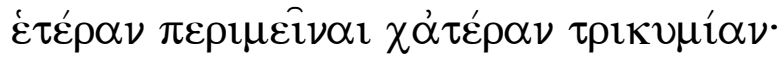

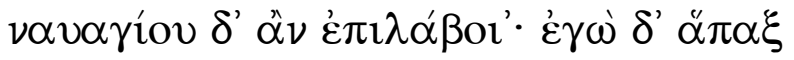

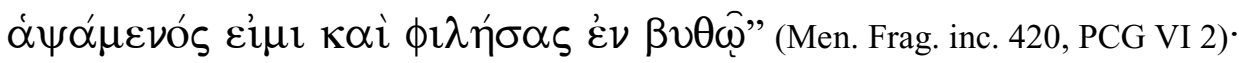

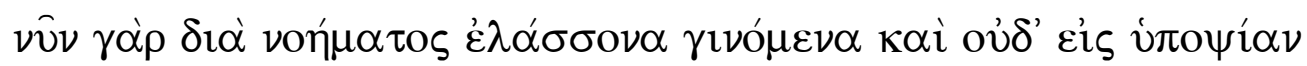

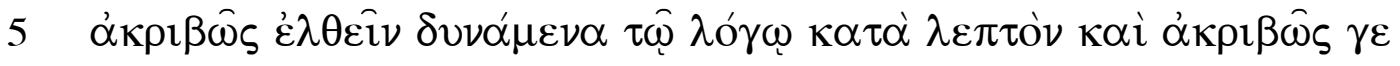

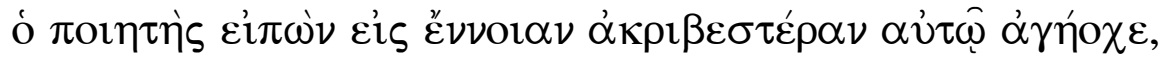

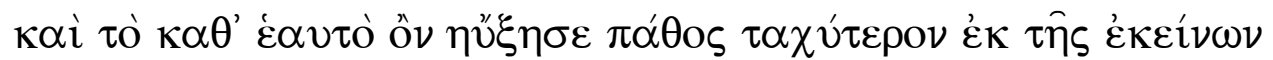

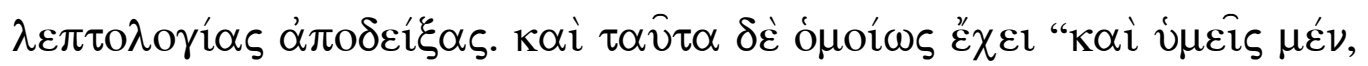

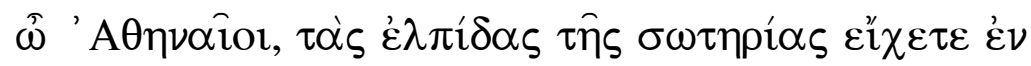

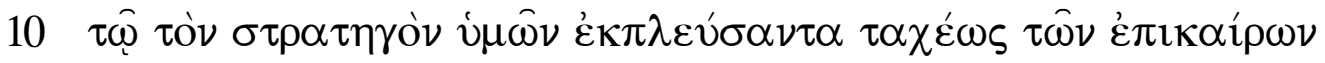

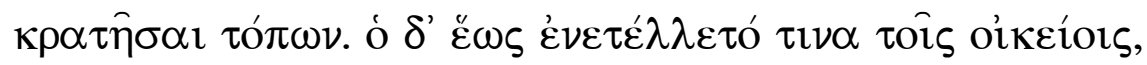

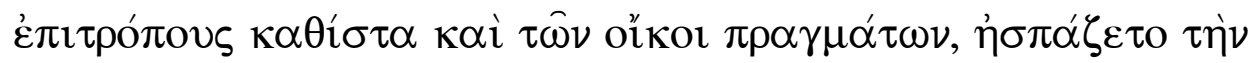

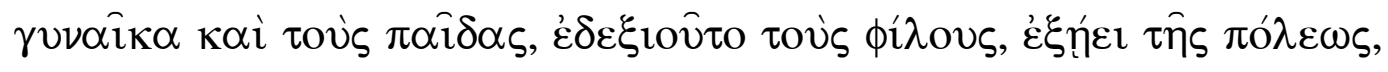

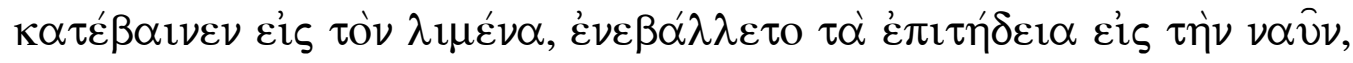

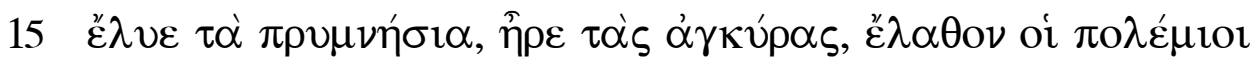

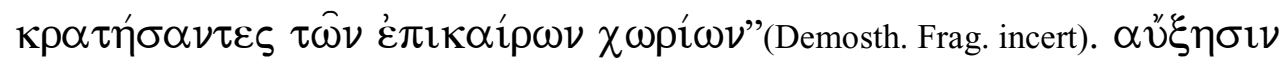

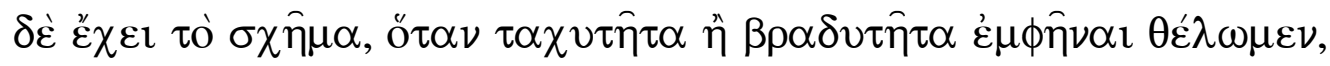

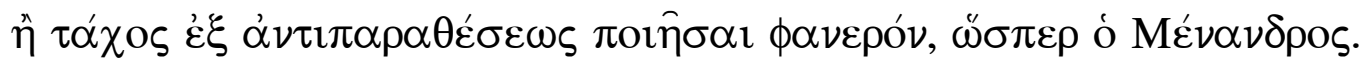

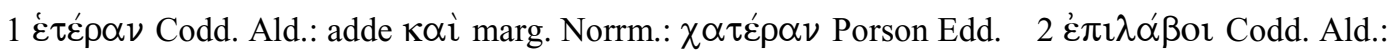

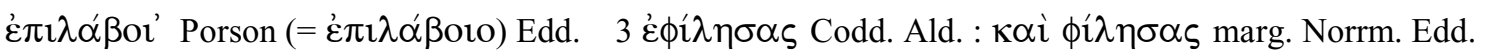

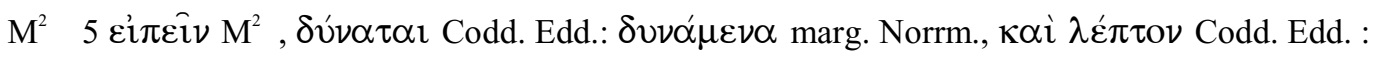
$\kappa \alpha \tau \alpha \grave{\lambda} \lambda \dot{\varepsilon} \pi \tau o \nu$ marg. Norrm: $\lambda \varepsilon \dot{\varepsilon} \pi \tau \omega \varsigma$ app. crit. Speng., $\tau \varepsilon$ Codd. Edd.: $\tau \varepsilon$ om. $\mathrm{M}^{2}: \gamma \varepsilon$ marg. Norrm.

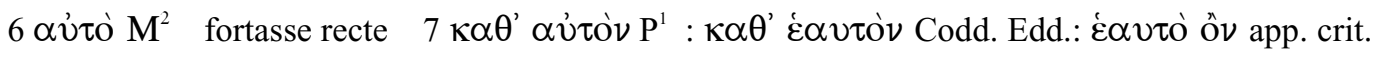

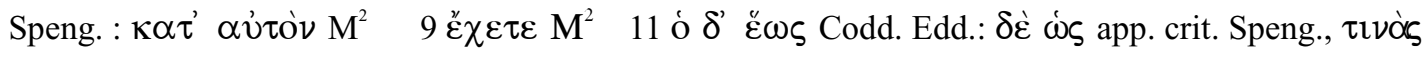

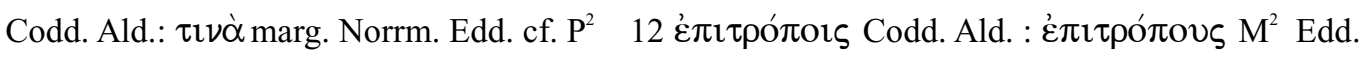

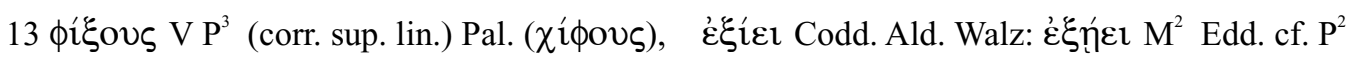

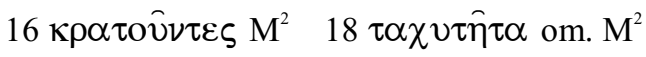




\section{$\imath \beta^{\prime}$. ПЕРІ ПРО $\Omega$ ПОПОIIА $\Sigma$.}

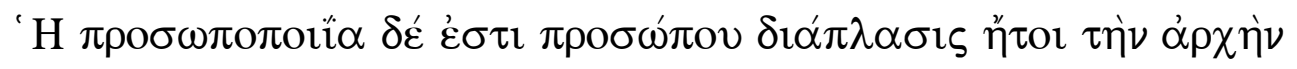

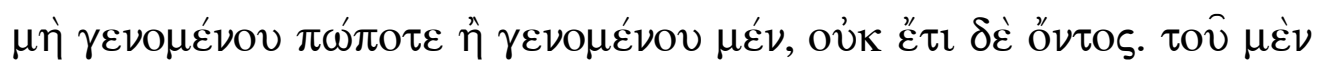

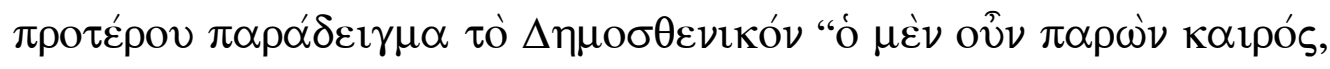

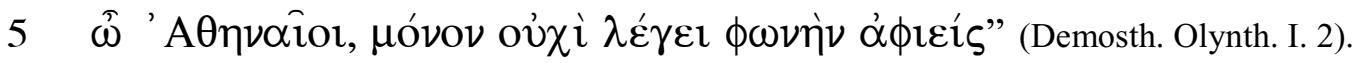

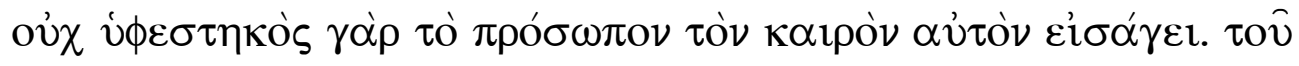

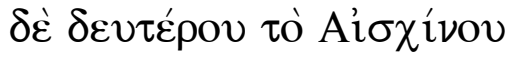

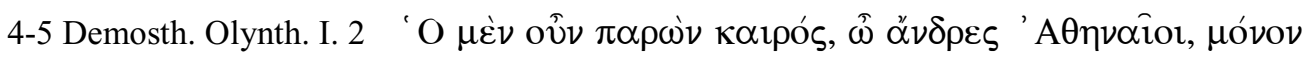

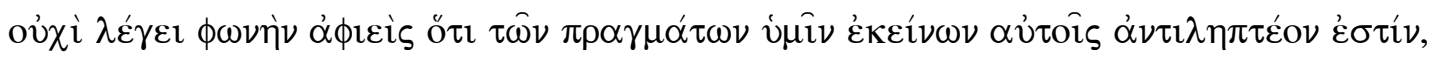

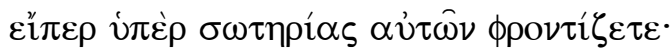

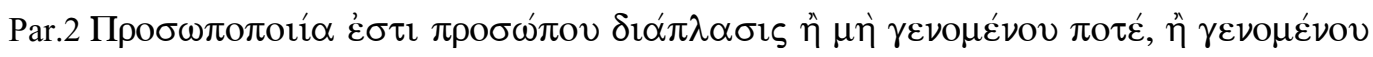

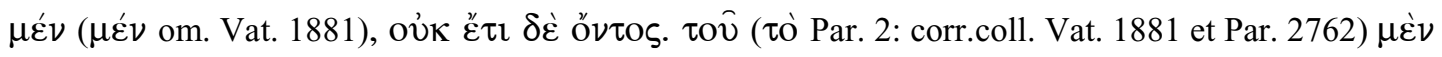

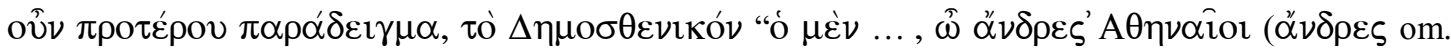

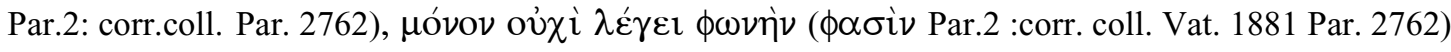

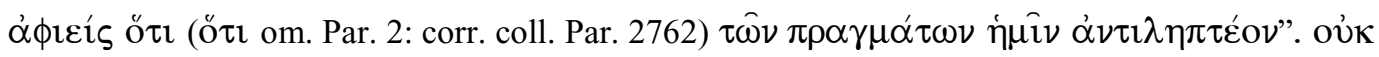

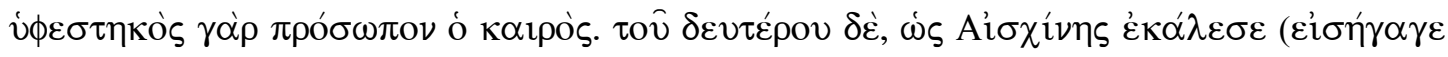

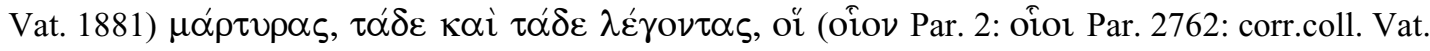

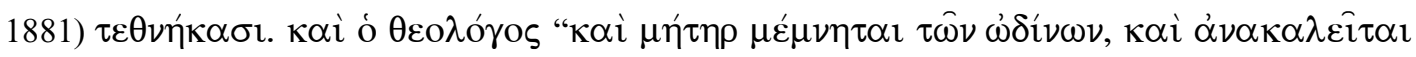

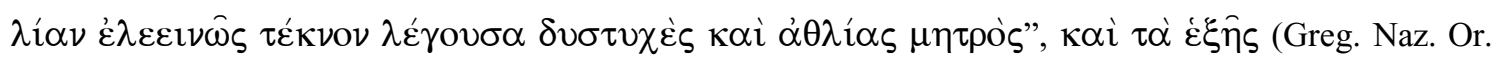

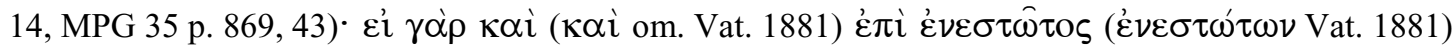

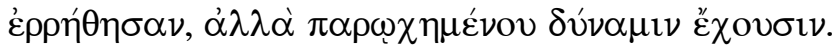

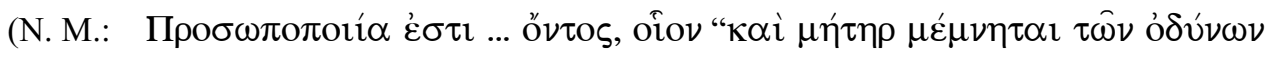

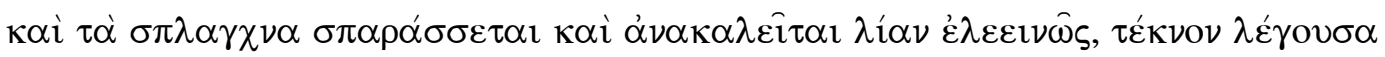

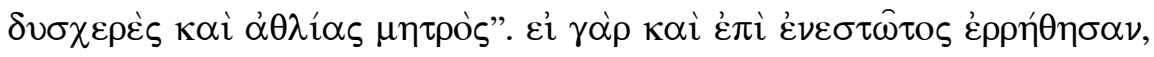

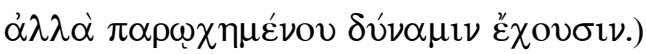

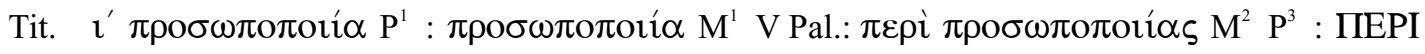
ПРО $\Sigma \Omega \Pi О П О I I A \Sigma$ Ald. Ald ${ }^{\mathrm{s}}$ : ПЕРI ПРО $\Sigma \Omega \Pi О П О I I A \Sigma \imath \beta^{\prime}$ Norrm. : $\imath \beta^{\prime}$. ПЕРI $\Pi Р О \Sigma \Omega \Pi О П О I \mathrm{~A} \Sigma$ Edd.

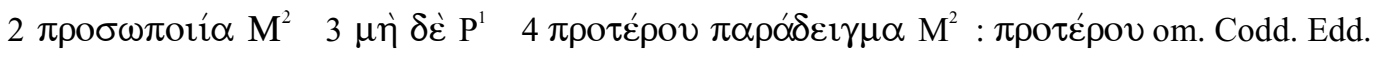

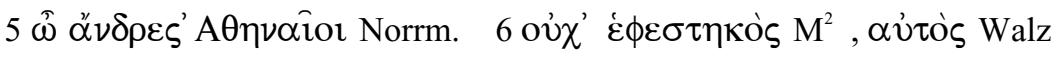




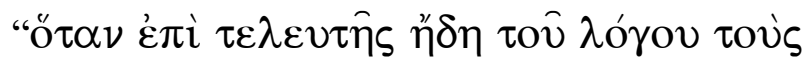

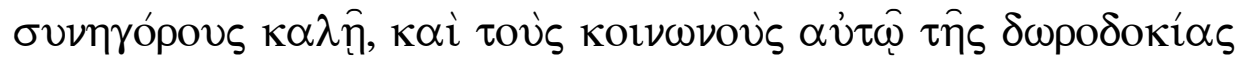

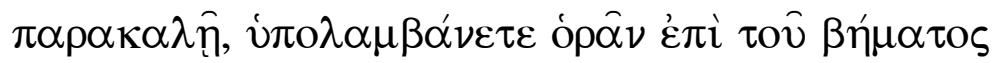

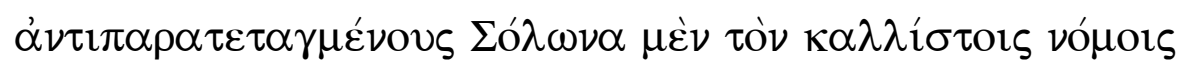

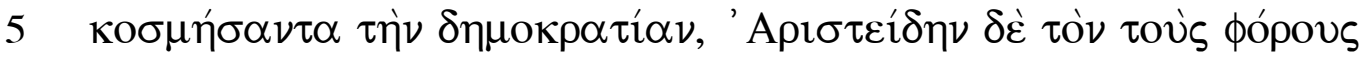

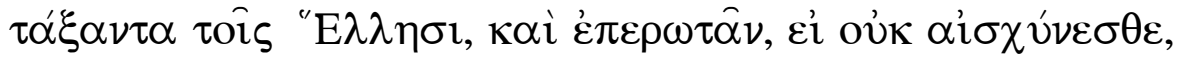

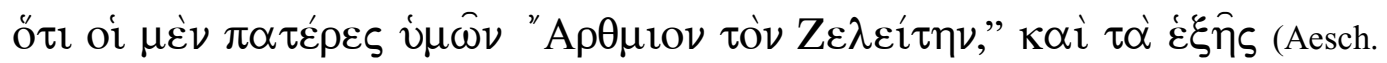
In Ctes. 257-8).

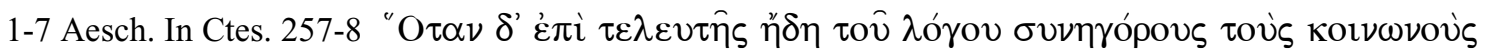

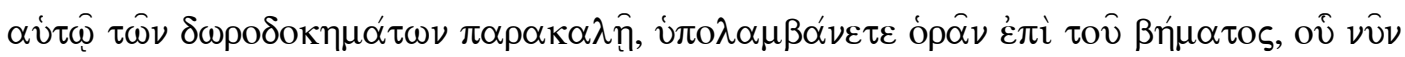

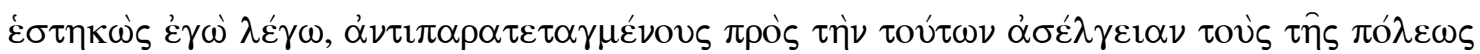

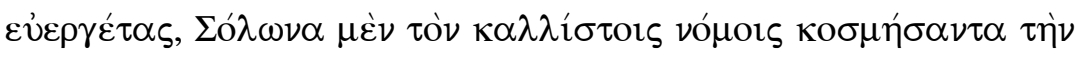

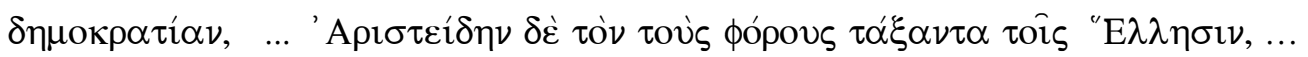

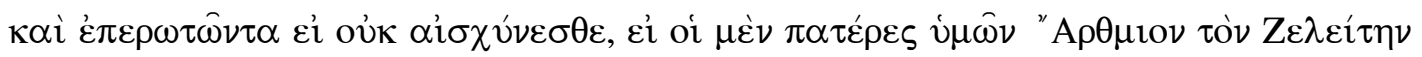

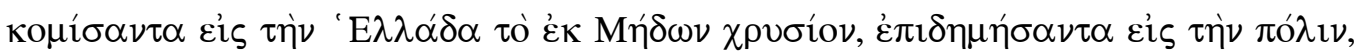

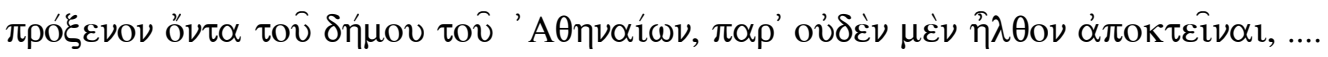

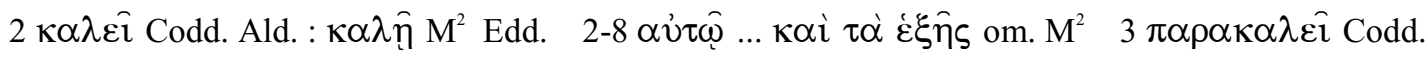

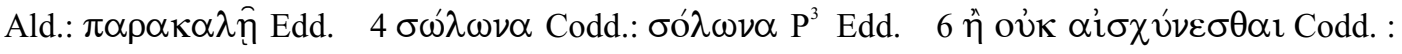

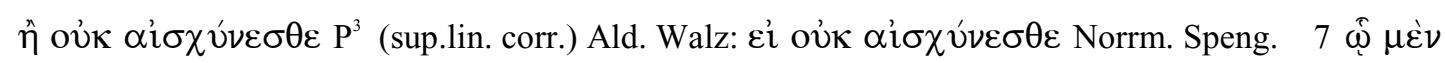

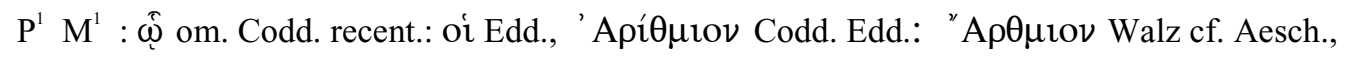

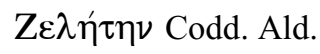


$\imath \gamma^{\prime}$. ПЕPI EПАNA $\Lambda H \Psi E \Omega \Sigma$.

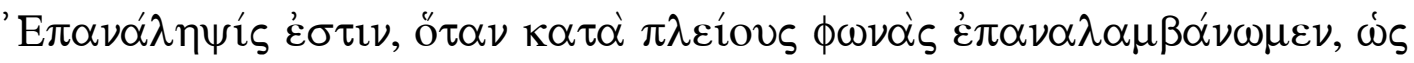

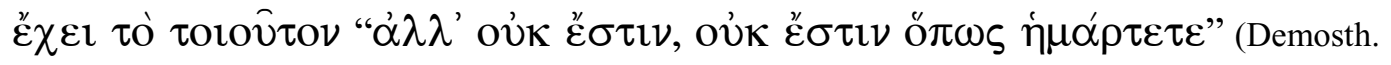

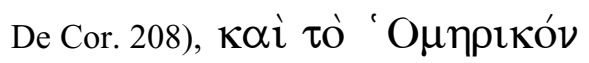

“"

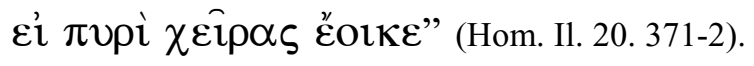

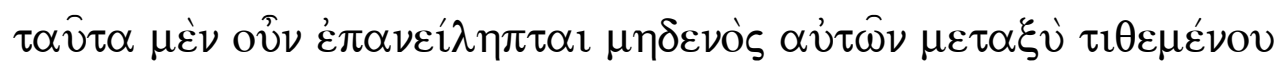

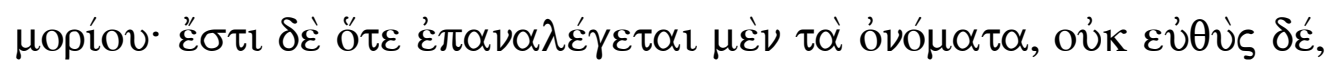

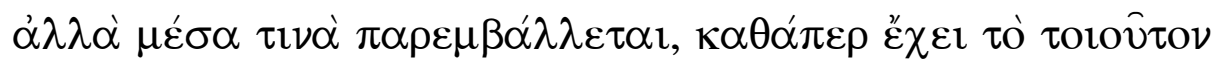

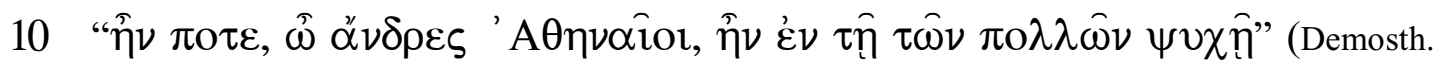

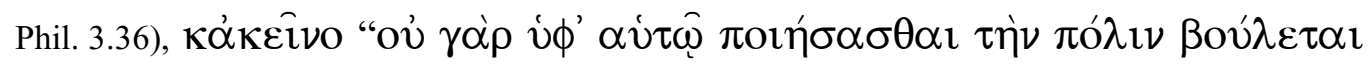

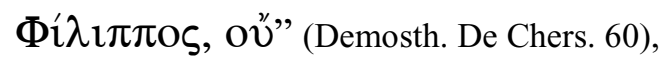

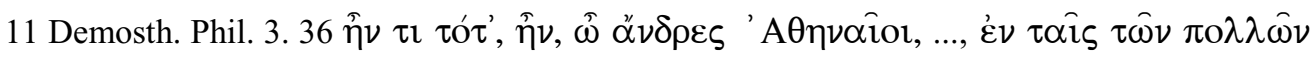

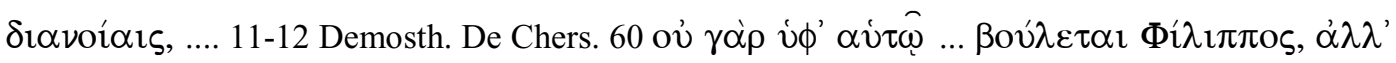

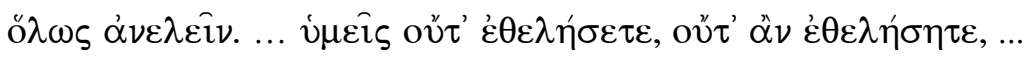

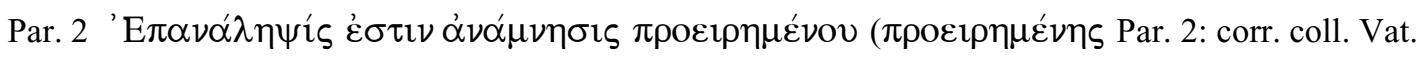

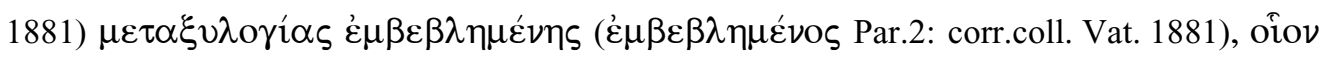

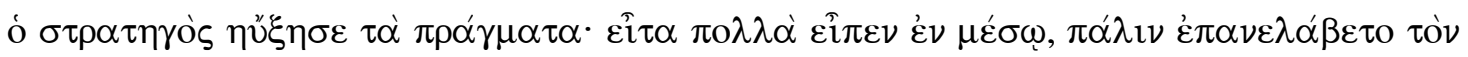

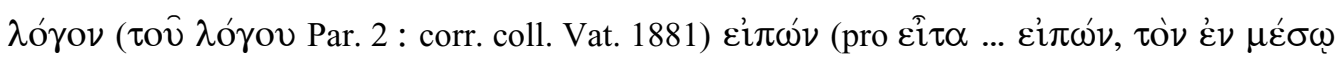

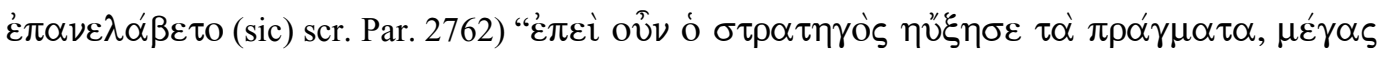

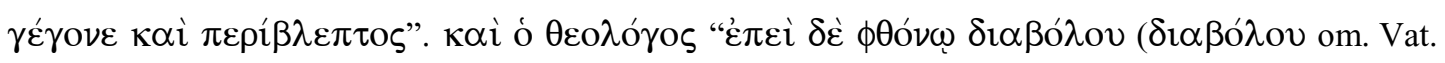

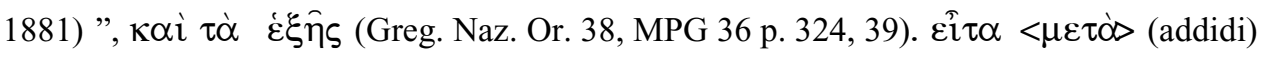

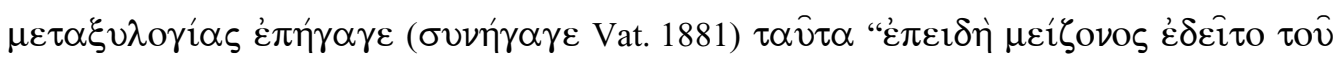

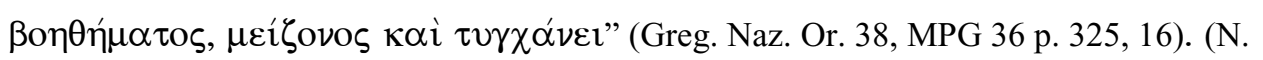

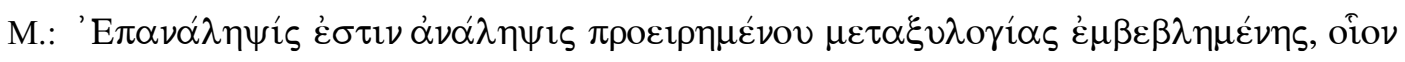

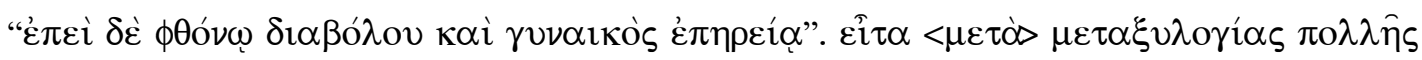

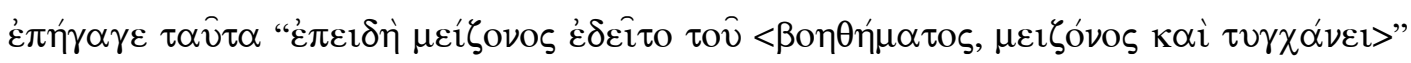
(recepi ex Ven. cf. RG VIII p. 680 app. crit. Walz).)

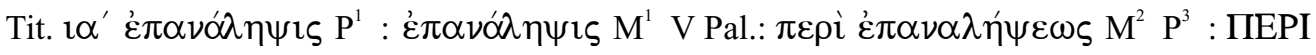
EПАNА $\Lambda$ HЕ $\Omega \Sigma$ Ald. Ald ${ }^{s}:$ ПЕPI EПAN- $\imath \gamma^{\prime}$ Norrm. : $\imath \gamma^{\prime}$. ПЕPI EПAN- Edd.

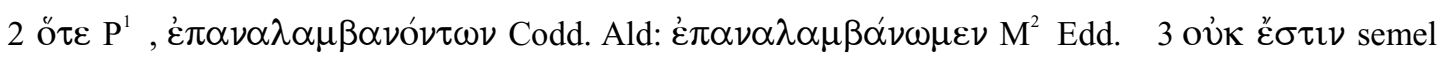

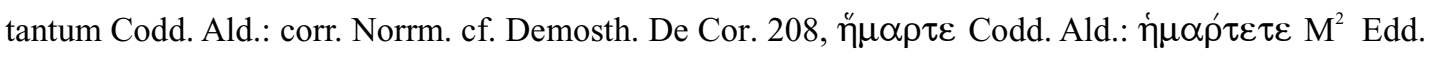

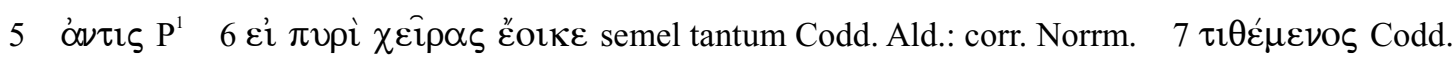

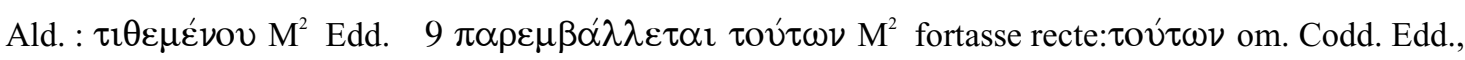

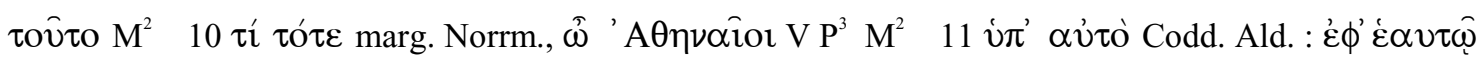

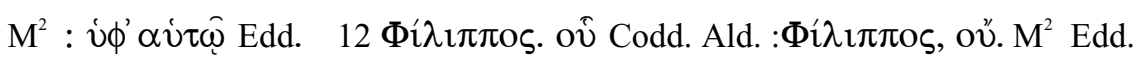




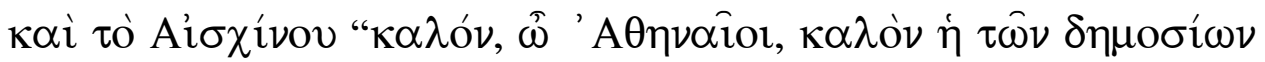

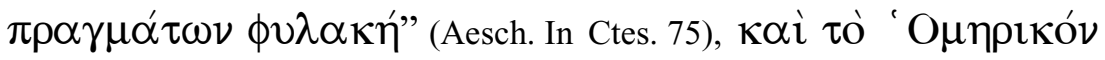

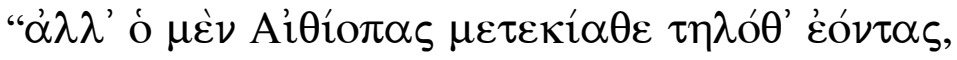

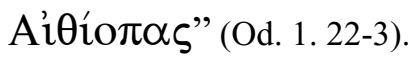

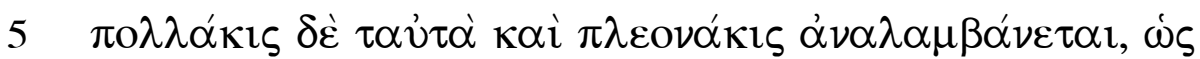

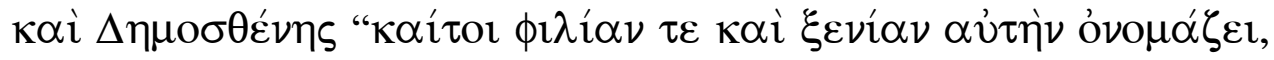

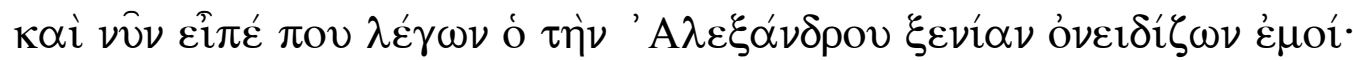

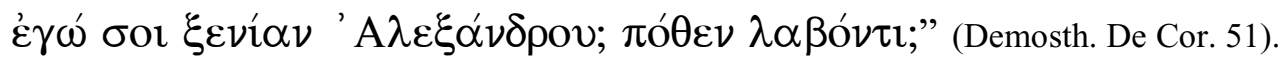

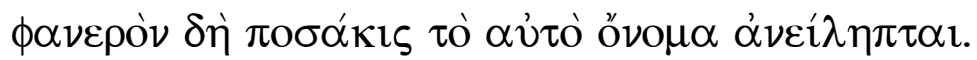

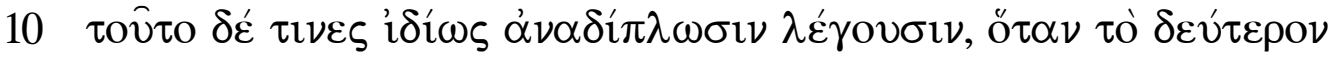

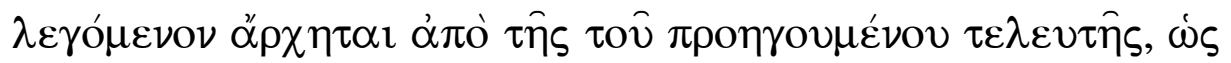

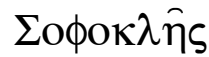

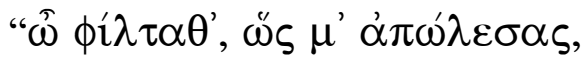

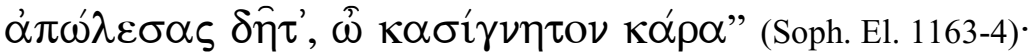

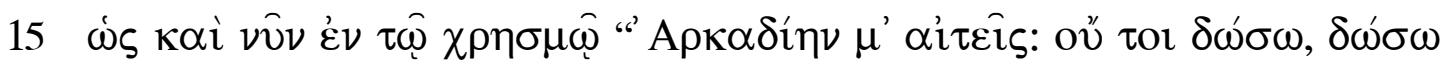

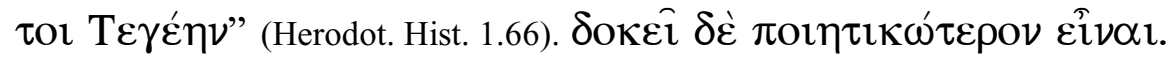

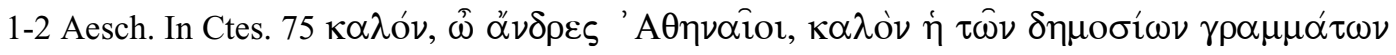

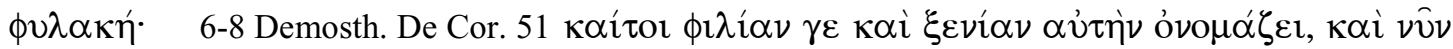

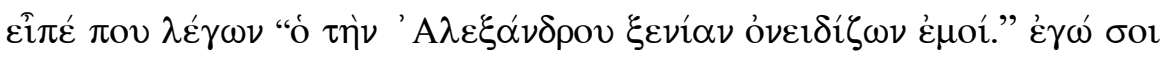

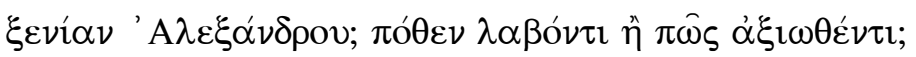

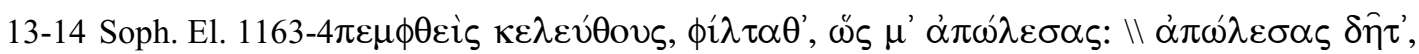

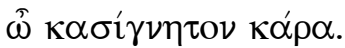

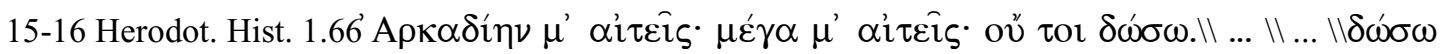

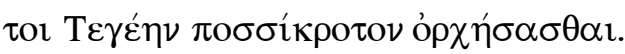

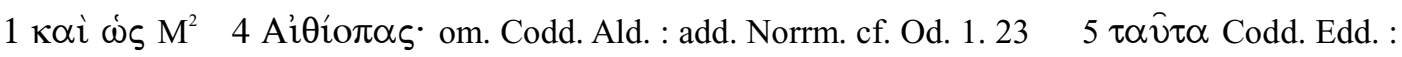

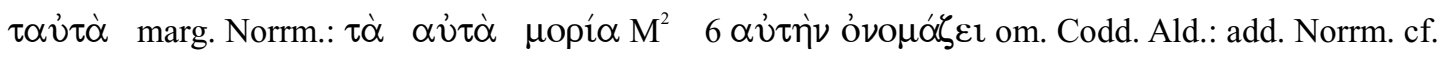

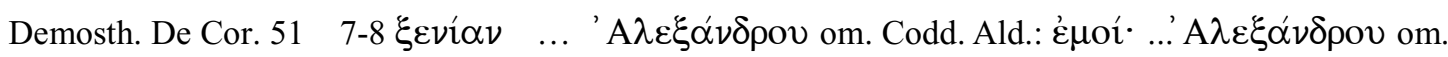

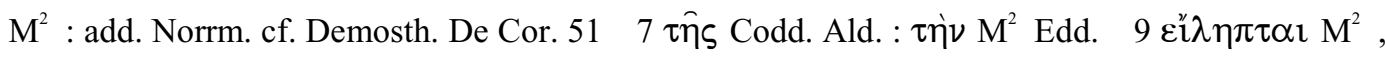

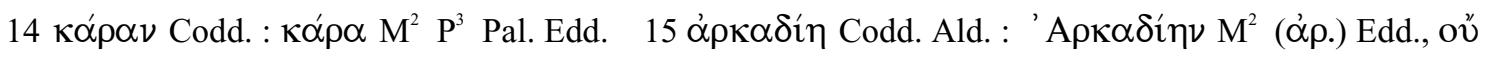

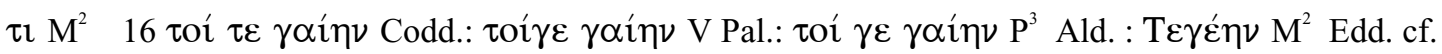
Herod. 


\section{$1 \delta^{\prime}$. ПЕРІ ЕПАNАФОРА $\Sigma$.}

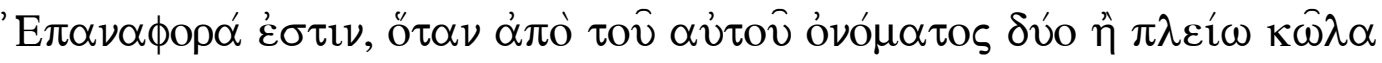

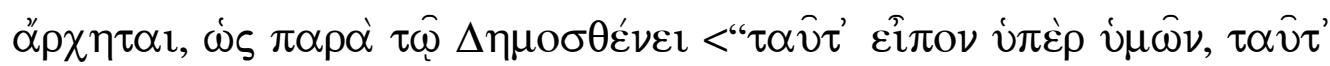

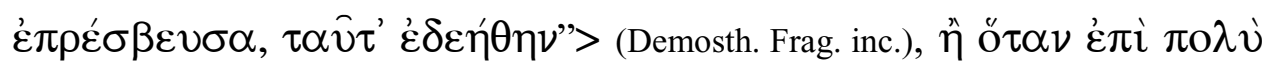

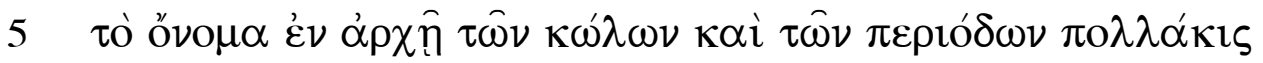

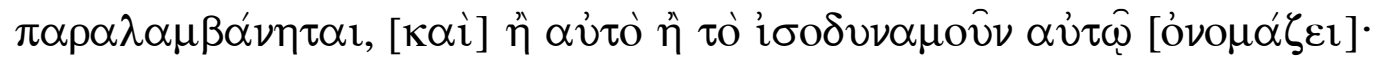

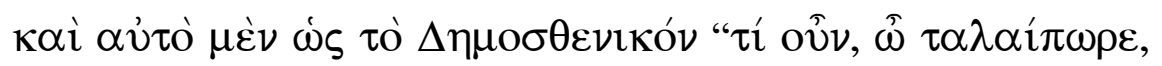

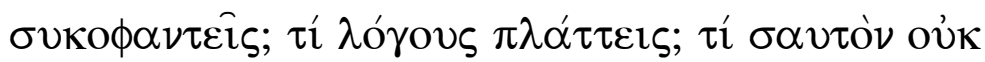
$\dot{\varepsilon} \lambda \lambda \varepsilon \beta 0 p i \zeta \varepsilon 1 \zeta ; "(D e m o s t h$. De Cor.121)

3-4 cf. Demosth. De Cor. 178

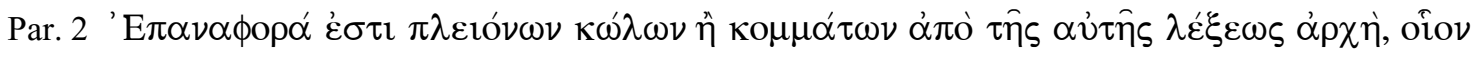

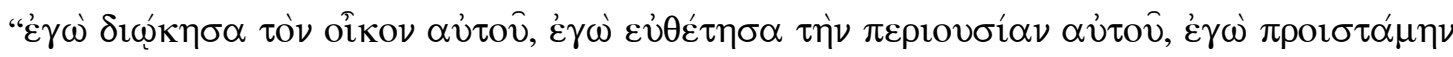

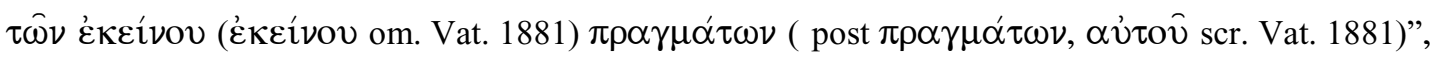

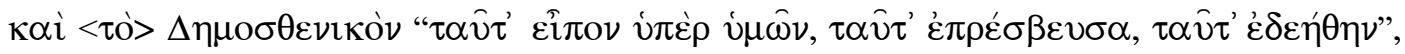

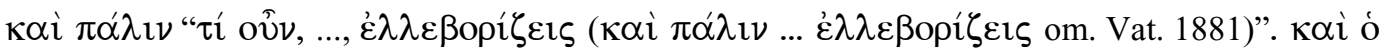

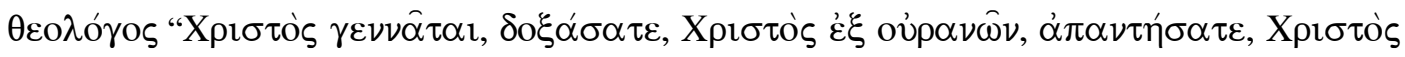

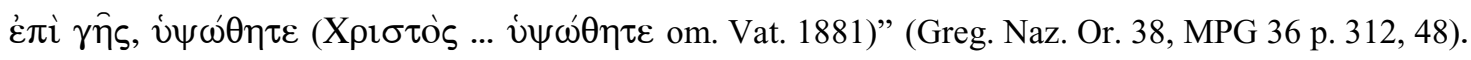

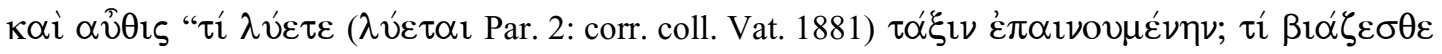

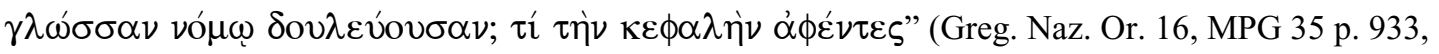

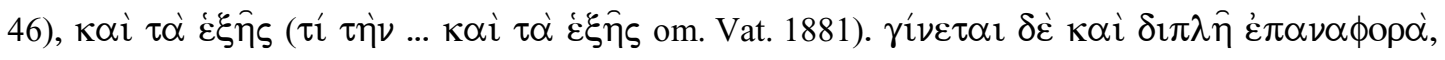

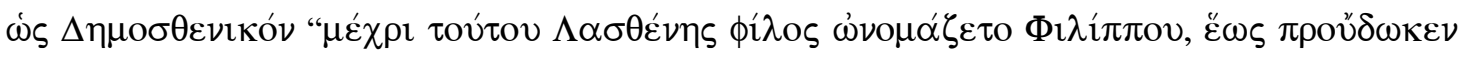

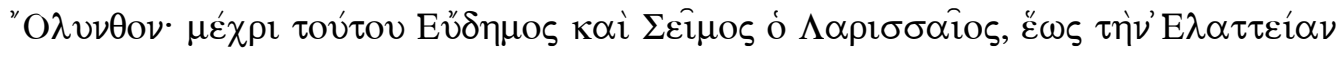

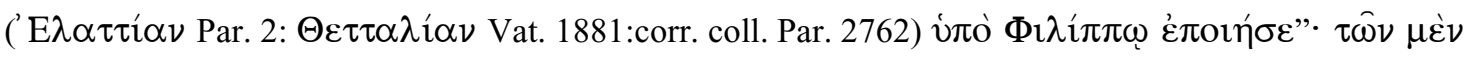

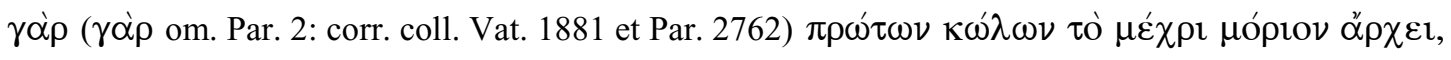

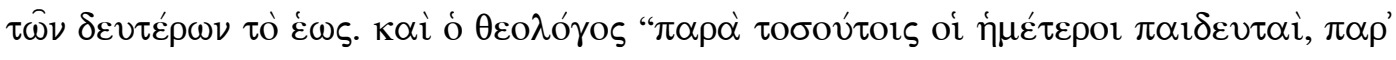

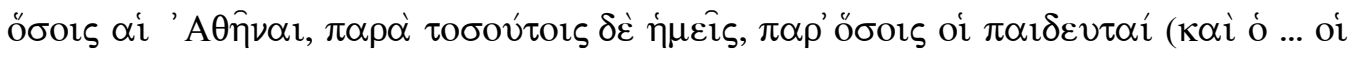

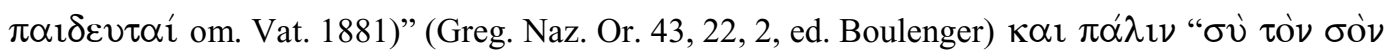

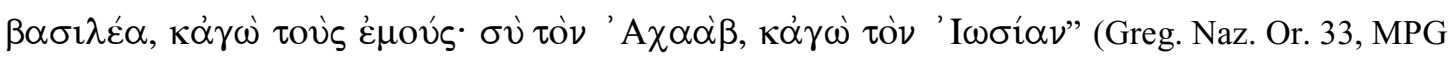

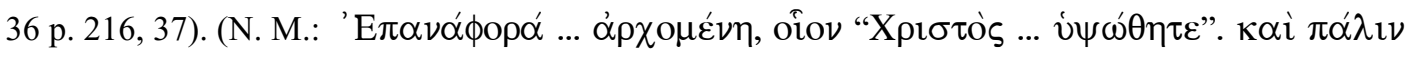

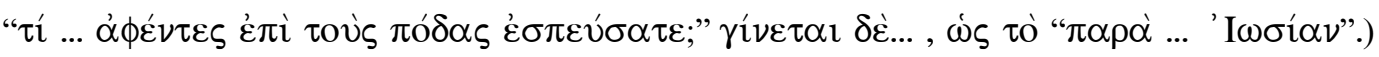

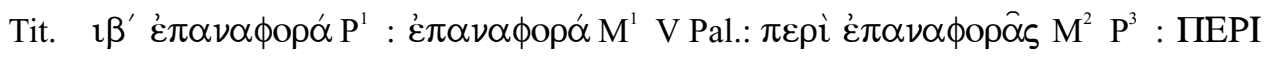
ЕПАNАФОРА $\Sigma$ Ald. Ald ${ }^{s}:$ ПЕРI ЕПАNА $\Phi-1 \delta^{\prime}$ Norrm. : $1 \delta^{\prime}$. ПЕРI ЕПАNА $\Phi-$ Edd.

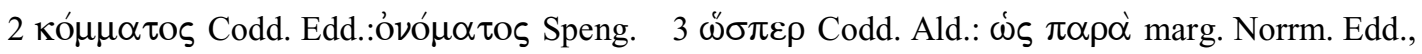

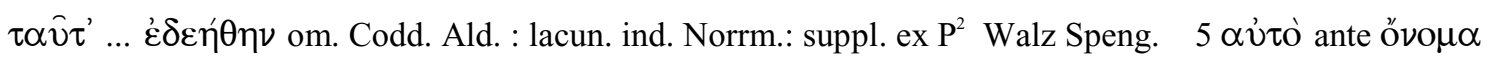

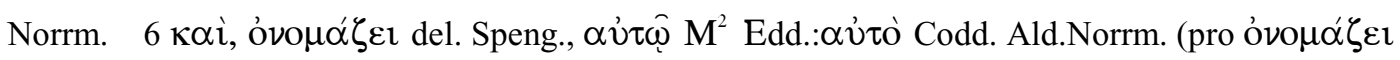

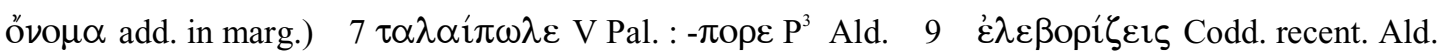




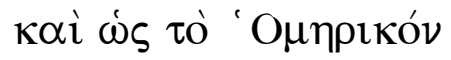

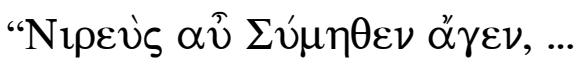

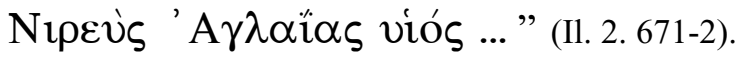

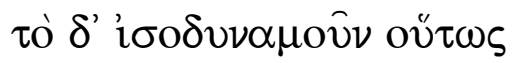

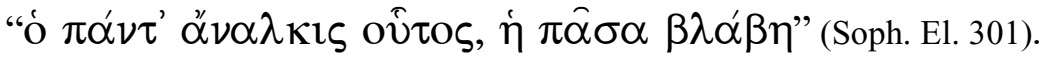

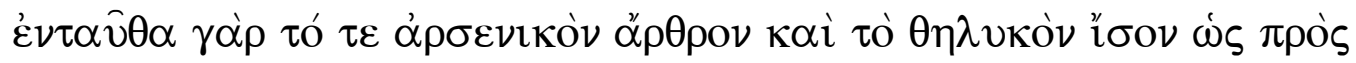

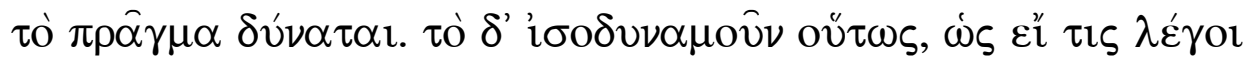

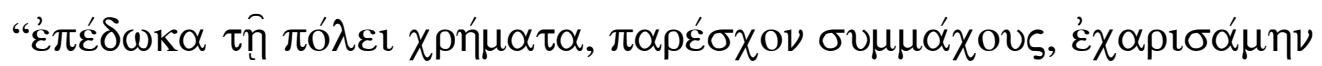

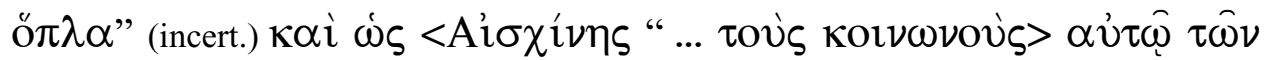

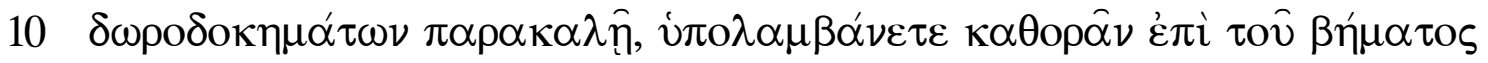

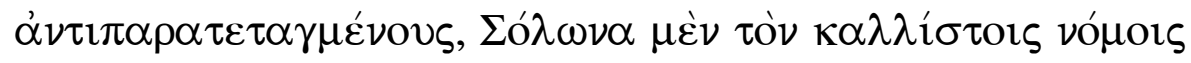

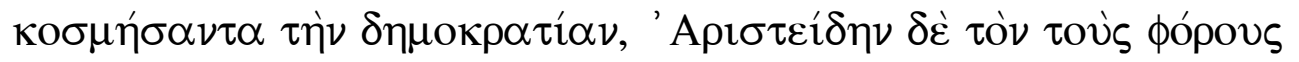

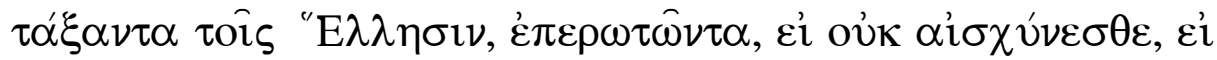

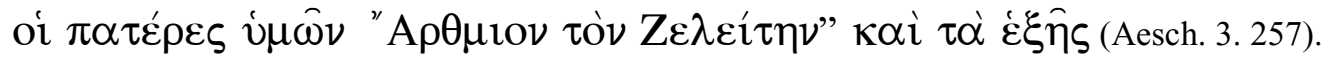

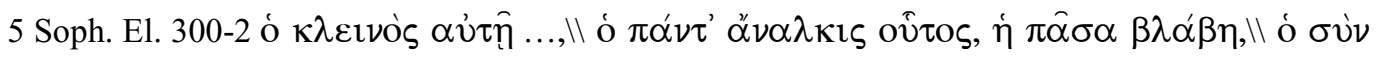

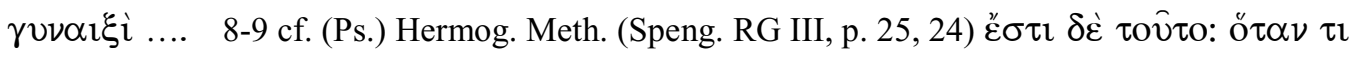

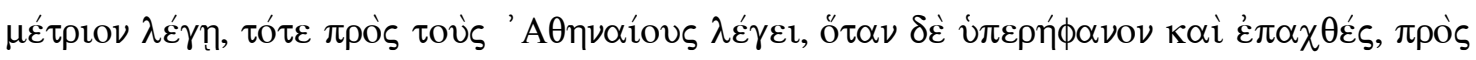

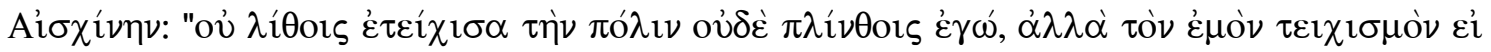

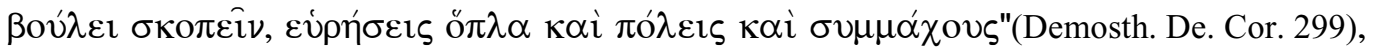

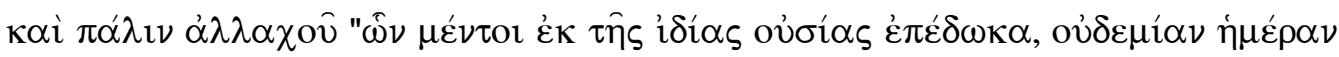

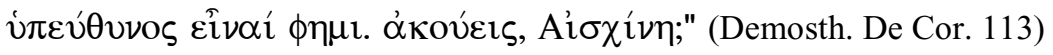

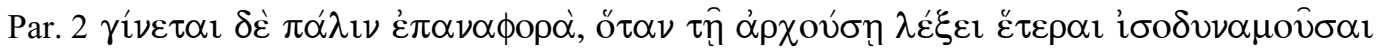

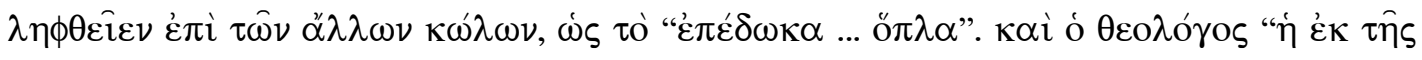

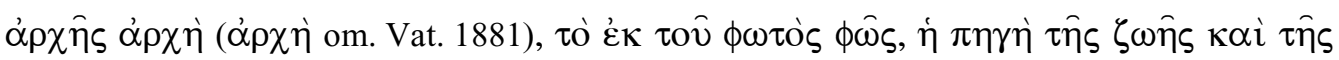

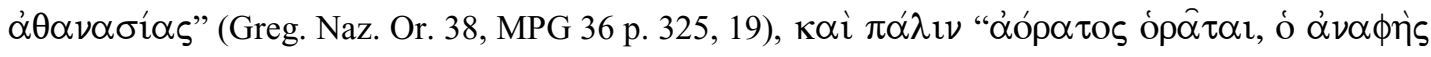

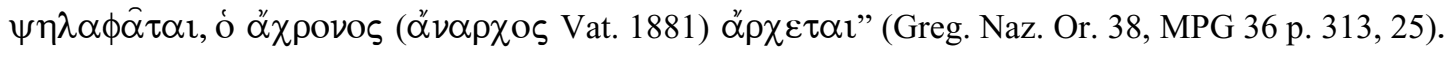

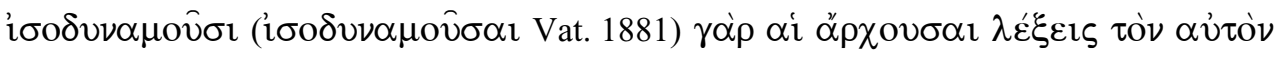

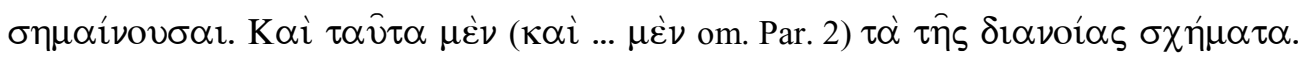

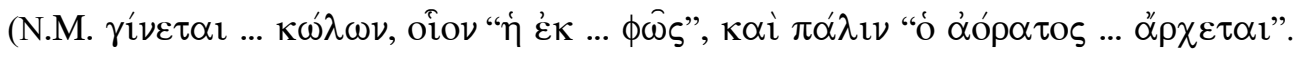

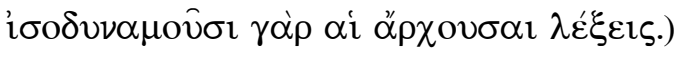

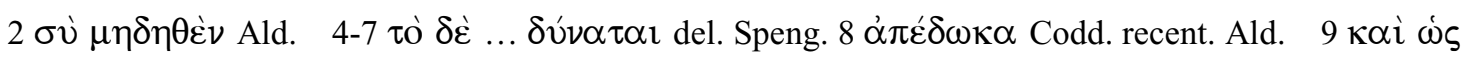

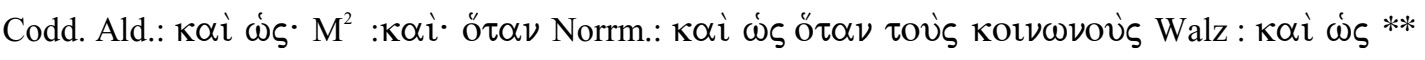

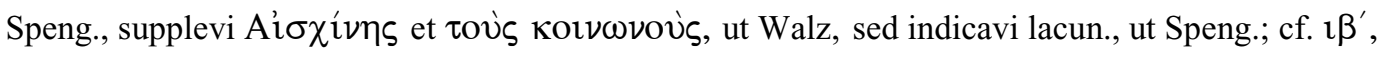

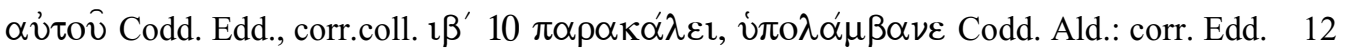

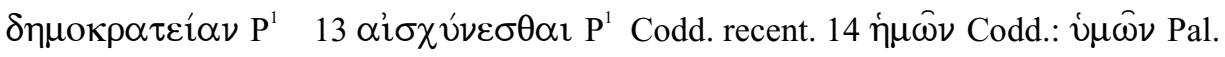

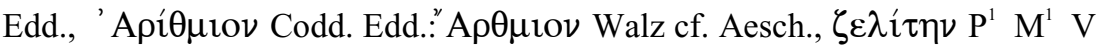




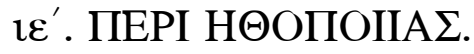

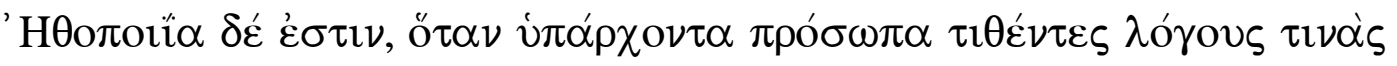

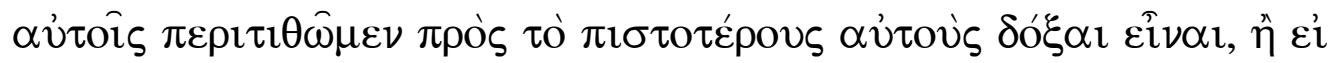

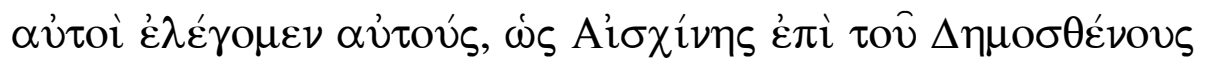

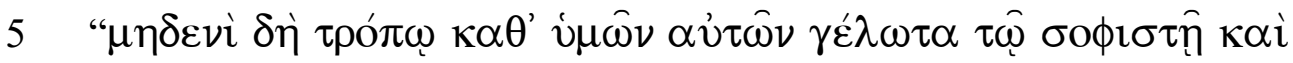

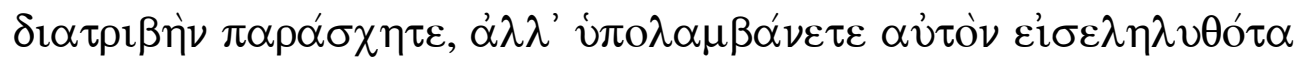

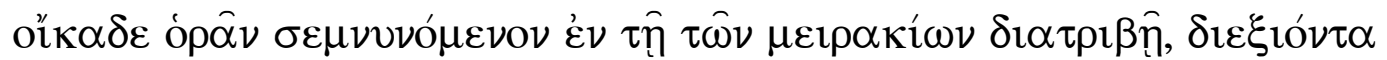

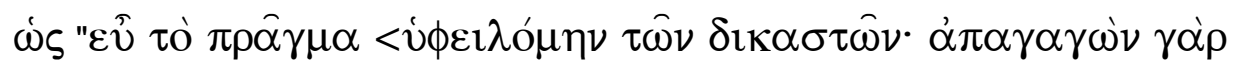

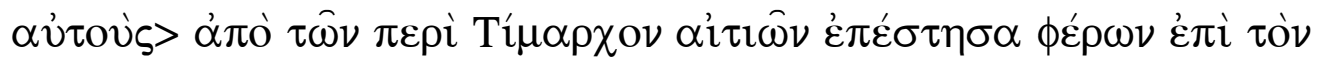

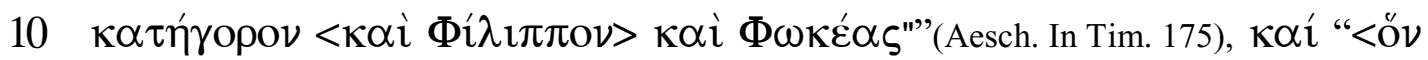

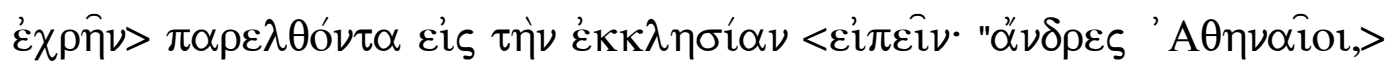

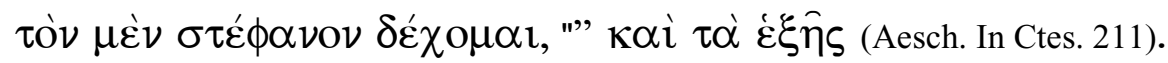

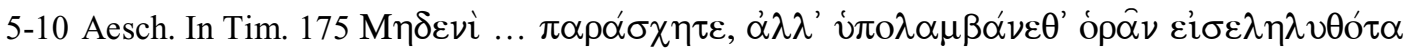

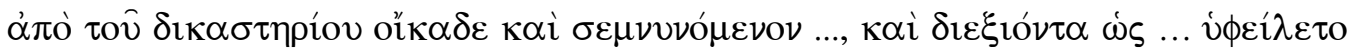

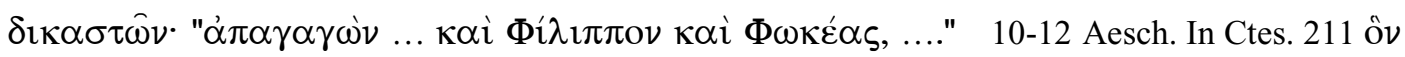

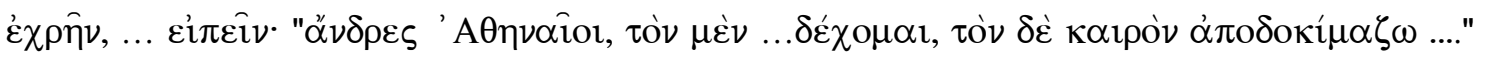

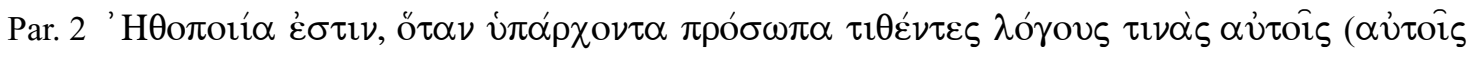

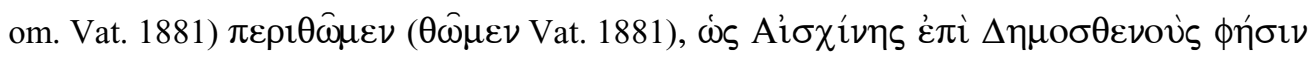
“vं

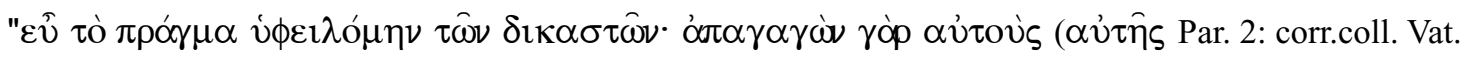

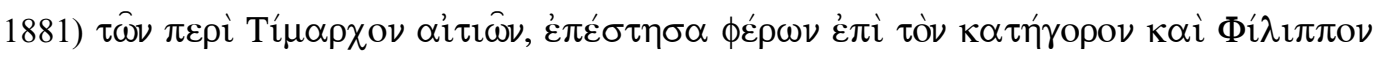

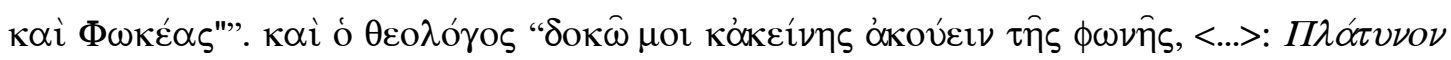

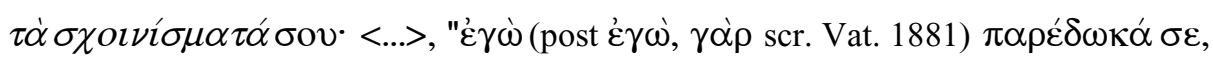

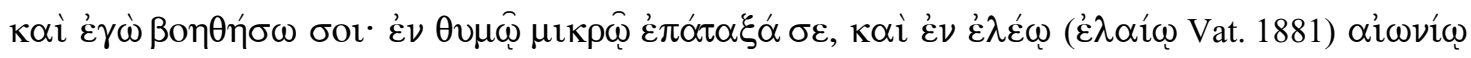

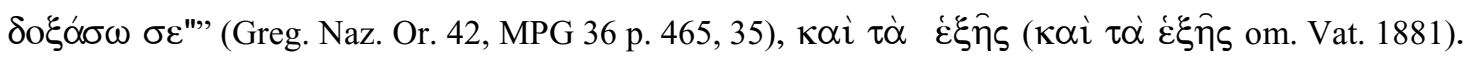

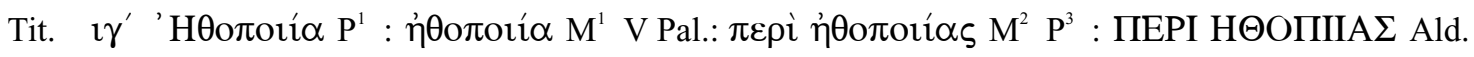

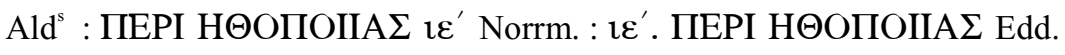

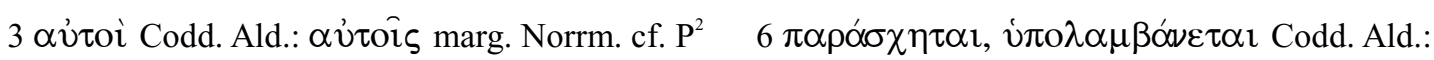

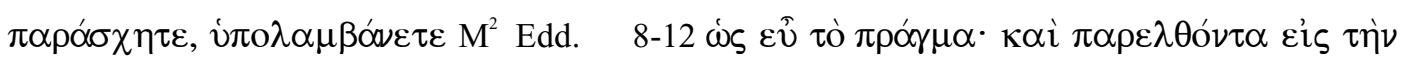

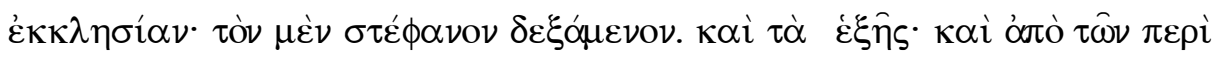

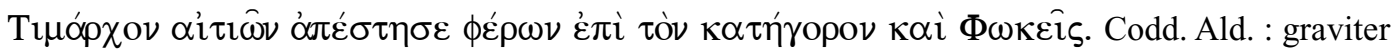

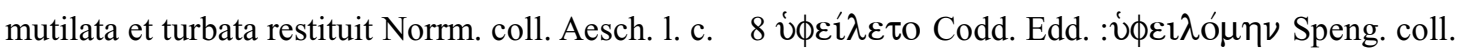

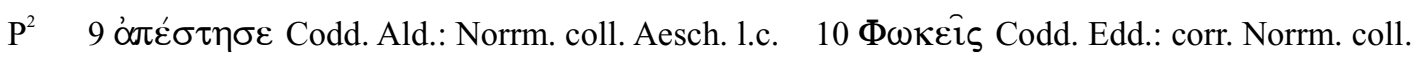

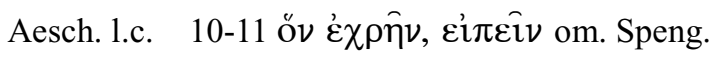


$\iota \varsigma^{\prime}$. ПЕРI AПО $\Sigma \mathrm{I} \Omega \Pi H \Sigma \mathrm{E} \Omega \Sigma$.

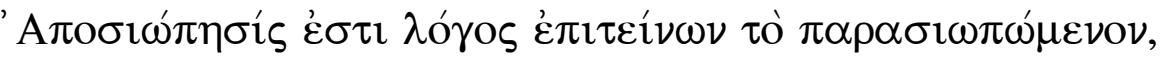

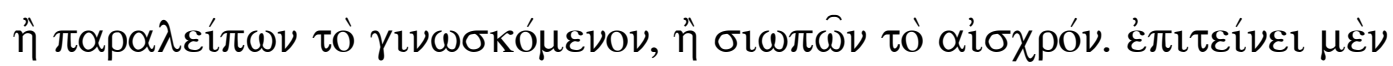

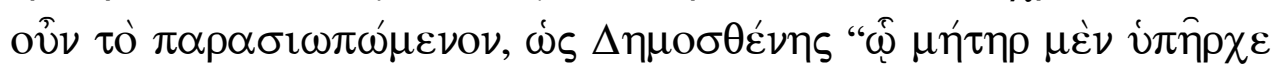

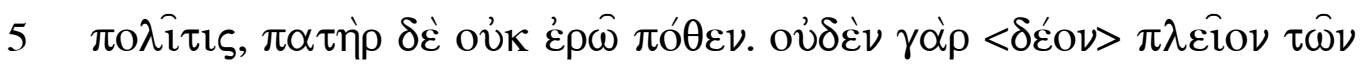

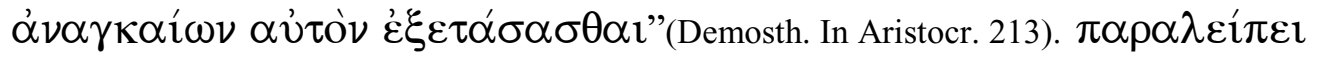

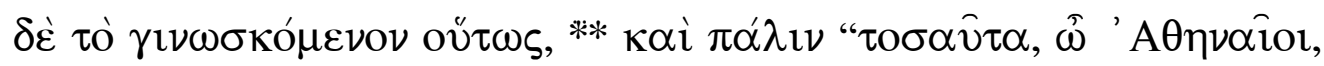

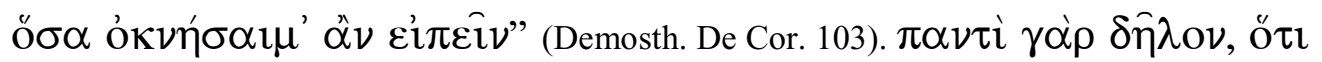

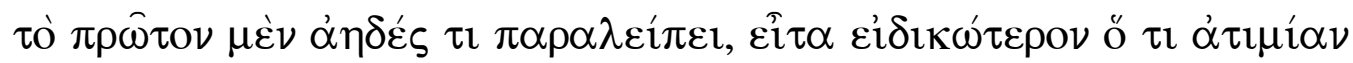

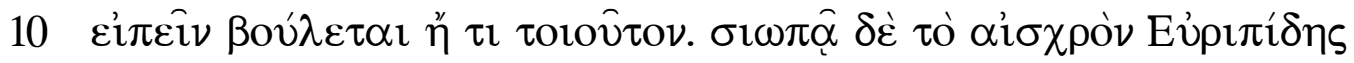

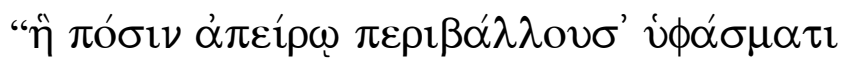

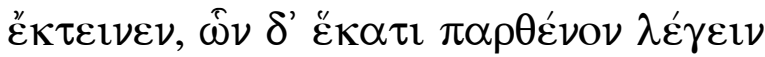

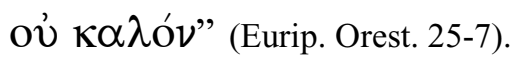

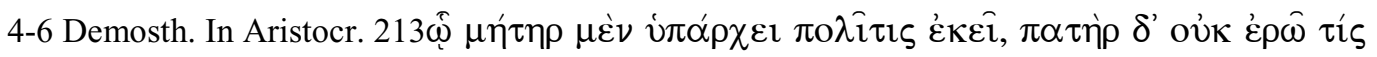

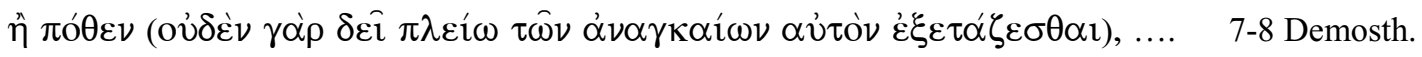

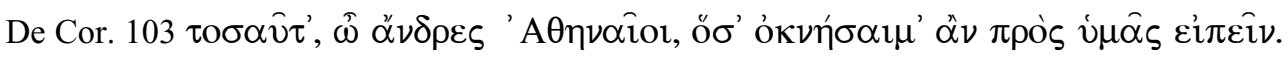

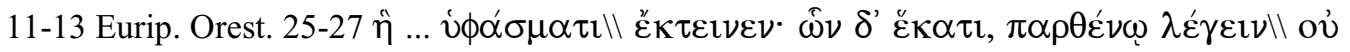

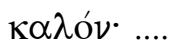

Par. 2 'A

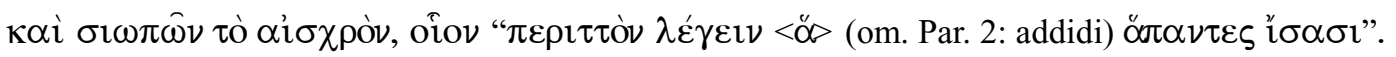

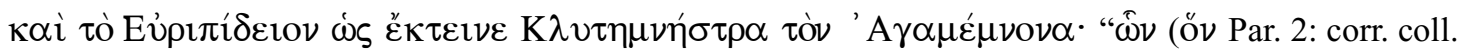

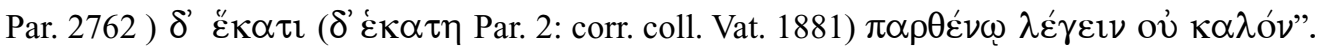

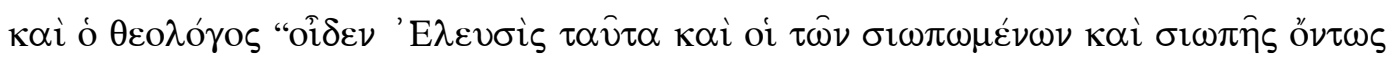

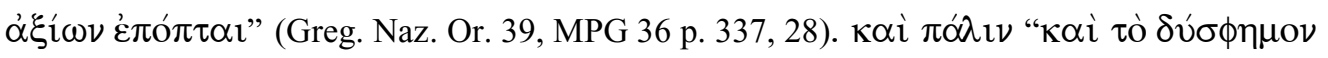

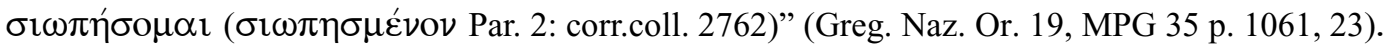

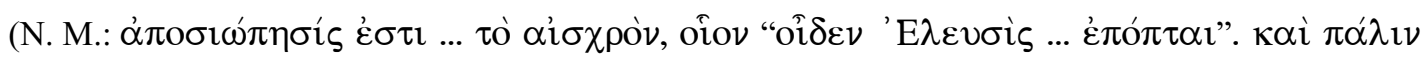

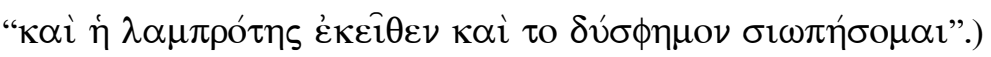

Tit. $\quad 1 \delta^{\prime}$ ó $\mathrm{A} \Pi \mathrm{I} \Omega \Omega \Pi H \Sigma \mathrm{E} \Omega \Sigma$ Ald. Ald ${ }^{s}:$ ПЕPI AПО $\Sigma \mathrm{I} \Omega \Pi H \Sigma \mathrm{E} \Omega \Sigma \iota^{\prime} \varsigma^{\prime}$ Norrm. : $\iota \varsigma^{\prime}$. ПЕPI $\mathrm{A} \Pi \mathrm{OI} \Omega \Pi \mathrm{H} \Sigma \mathrm{E} \Omega \Sigma$ Edd.

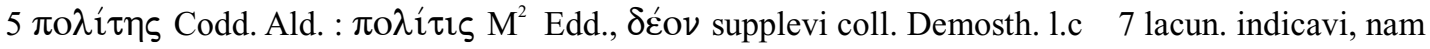
vacat exemplum, $\alpha^{\prime \prime} \nu \delta \rho \varepsilon \varsigma$ add. in marg. Norrm. coll. Demosth. 1.c. 8 ǒ $\sigma \alpha$ om. Codd. Ald.: ǒ $\sigma \alpha \mathrm{M}^{2}$

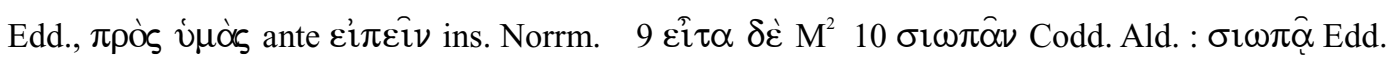




\section{$1 \zeta^{\prime}$. ПЕРІ ЕПIТРОХА $\Sigma$ MOY.}

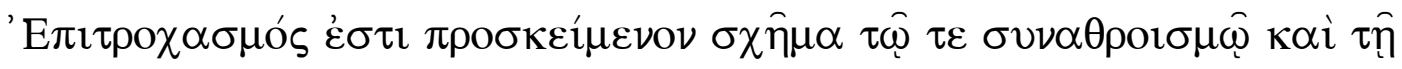

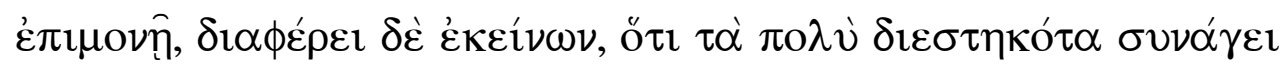

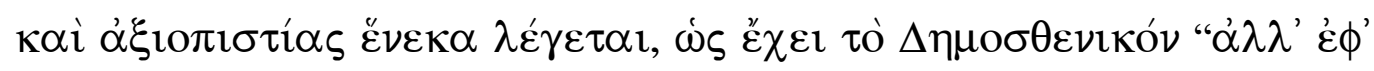

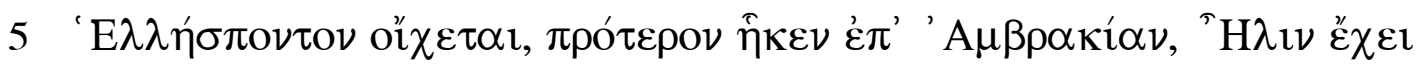

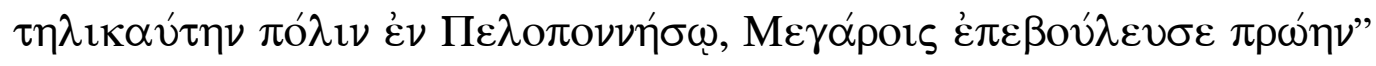
(Demosth. Phil. 3, 27 ).

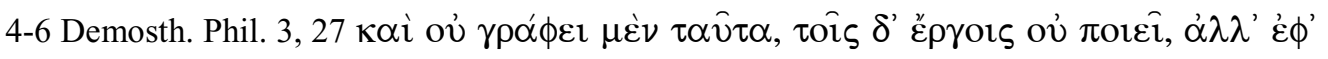

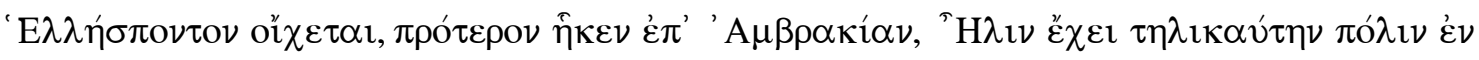

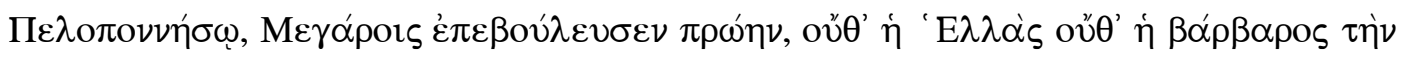

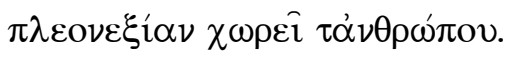

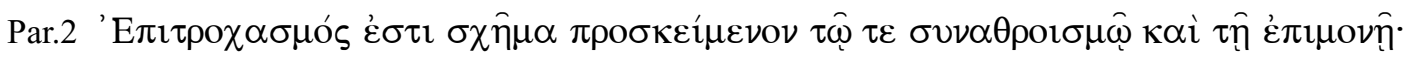

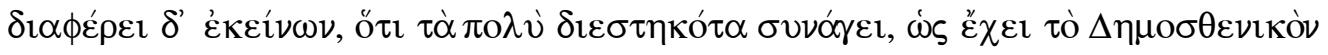

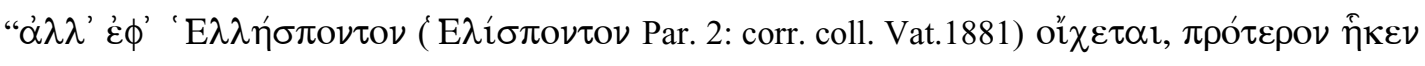

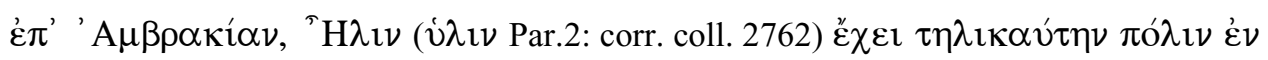

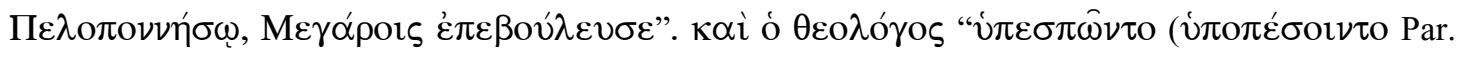

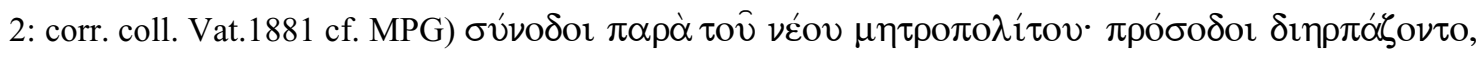

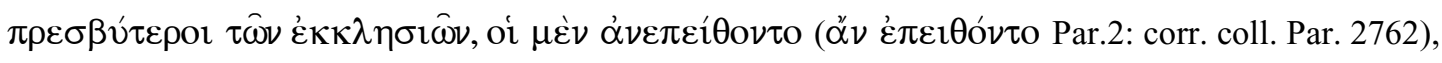

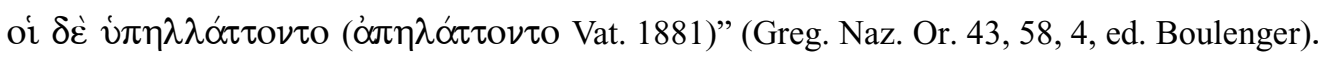

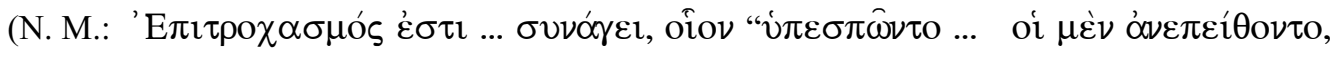

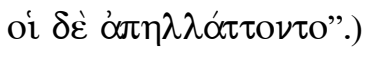

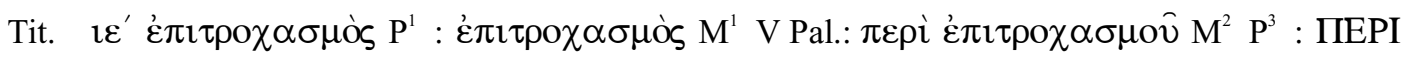
EПITPOXA $\Sigma$ MOY Ald. Ald ${ }^{s}$ : ПЕPI EПITPOXA $\Sigma$ MOY $\iota \zeta^{\prime}$ Norrm. : $\iota \zeta^{\prime}$. ПЕPI EПITPOX $\Sigma$ MOY Edd.

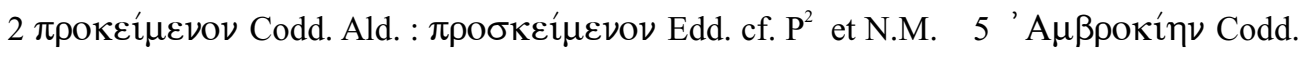

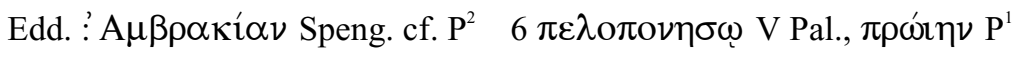


$\imath \eta^{\prime}$. MEPI EIP $\Omega N E I A \Sigma$.

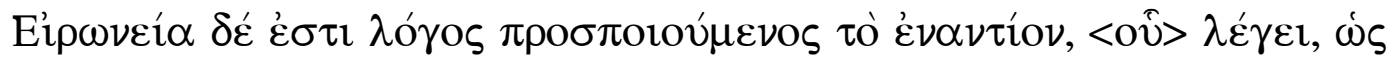

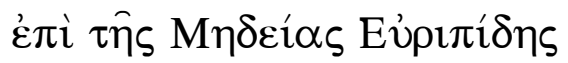

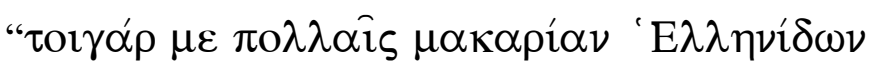

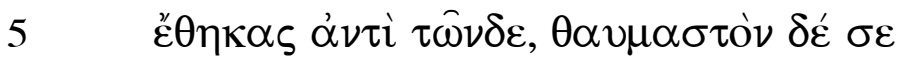

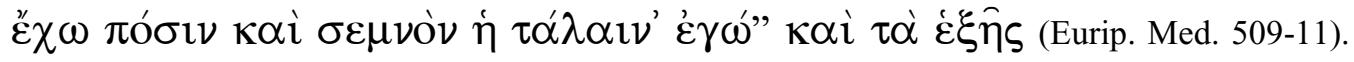

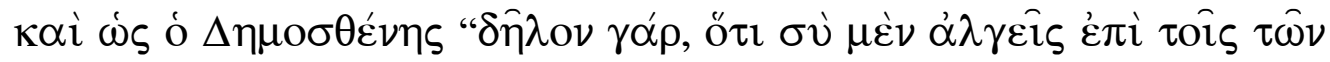

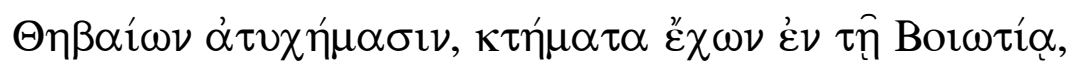

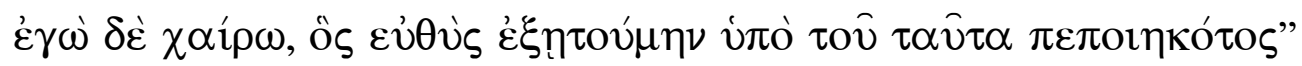

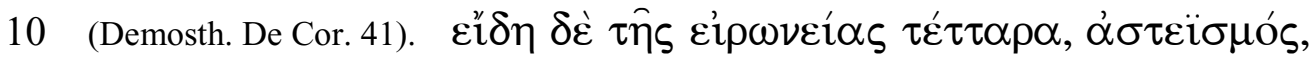

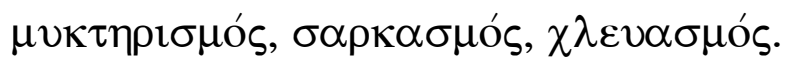

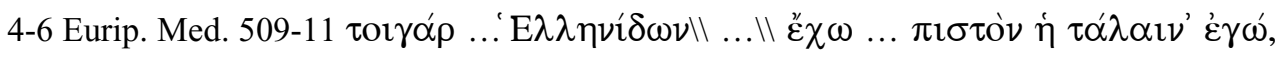

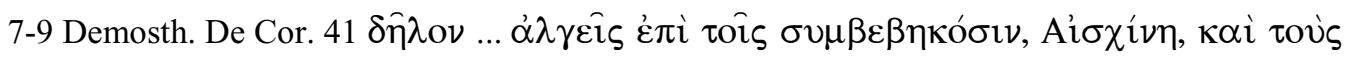

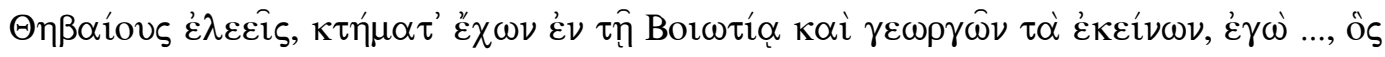

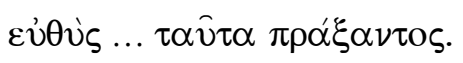

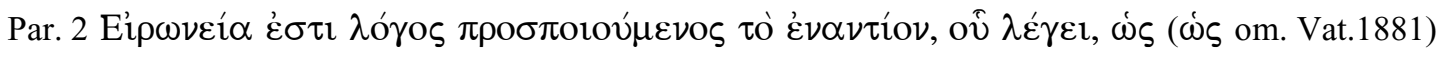

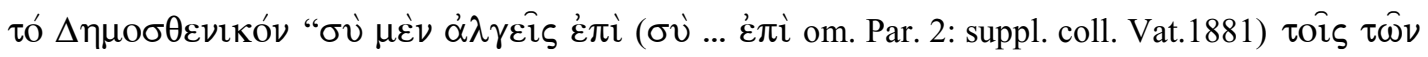

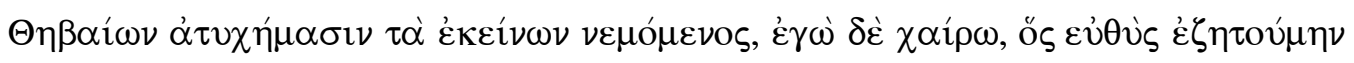

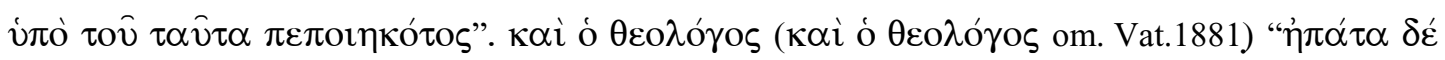

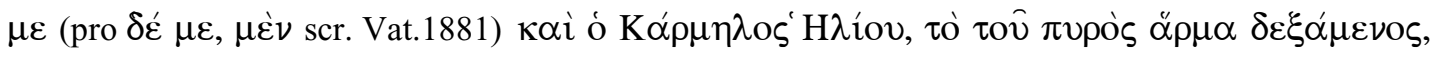

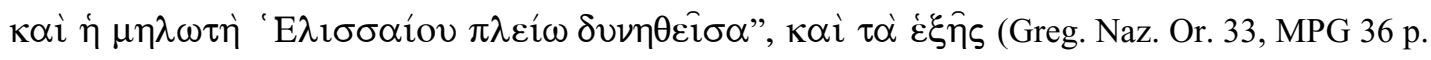
$228,7)$.

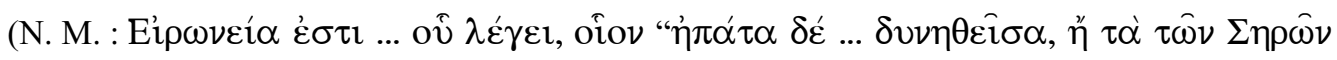
$\nu \eta \dot{\mu} \alpha \tau{ }^{\prime \prime}$.)

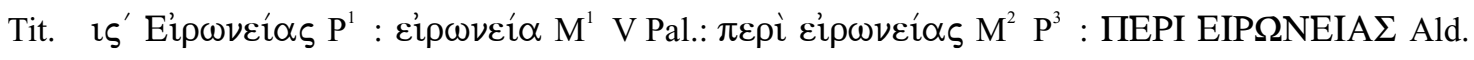

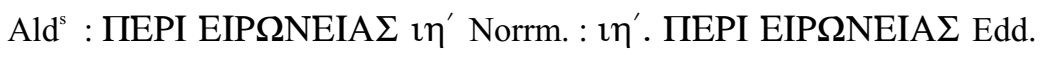

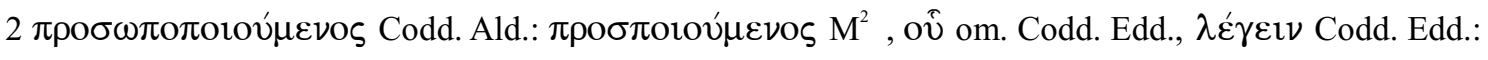

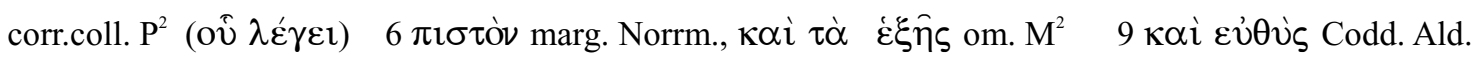

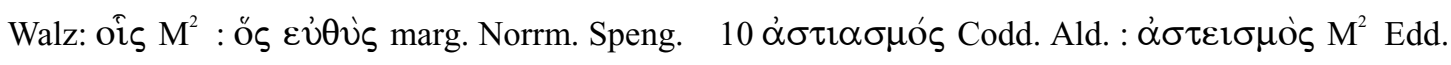

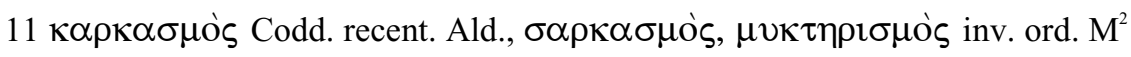




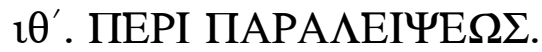

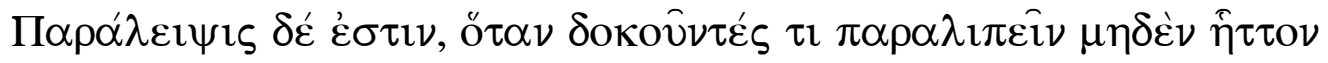

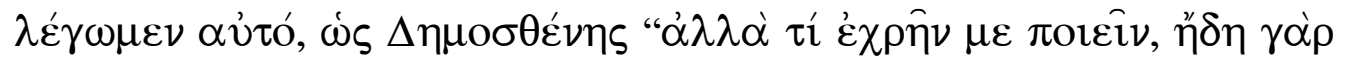

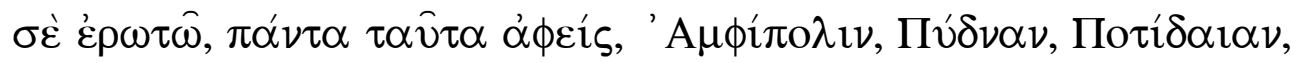

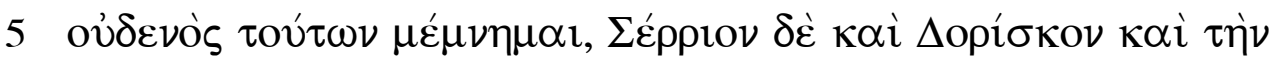

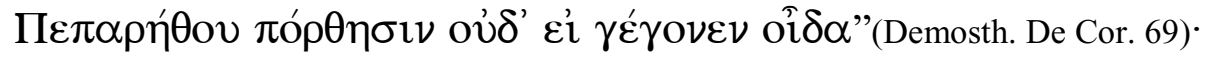

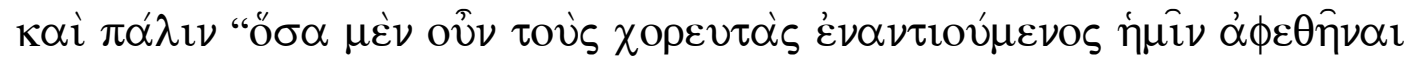

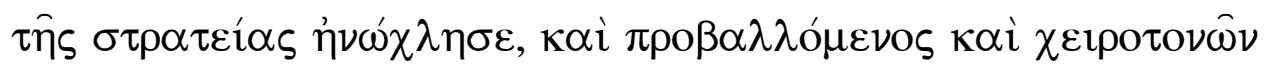

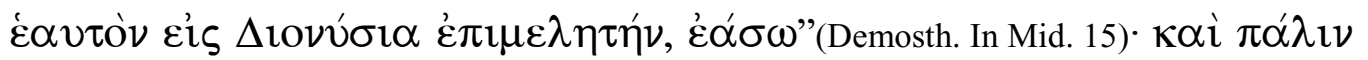

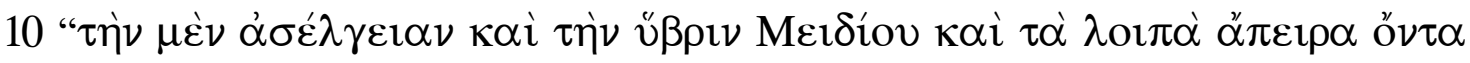

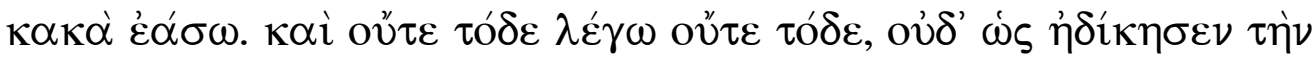

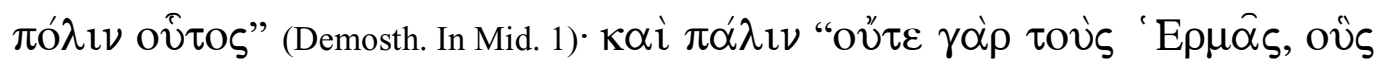

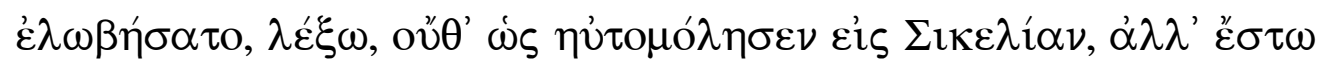

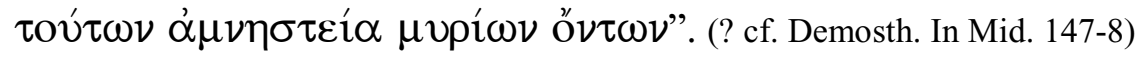

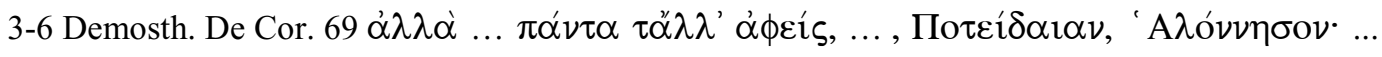

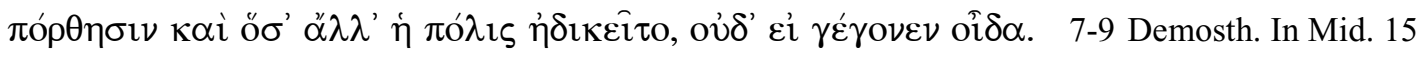

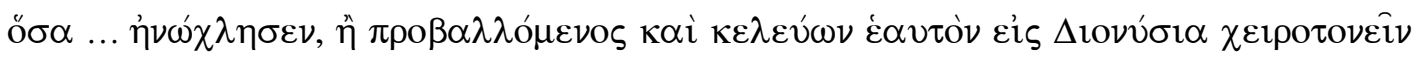

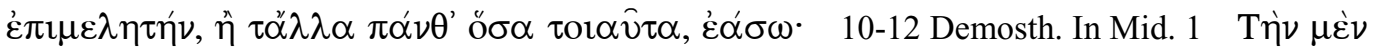

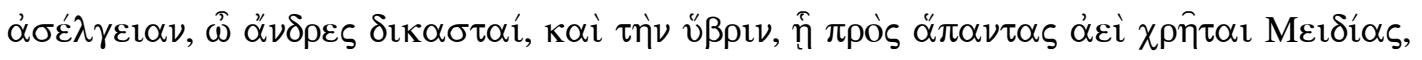

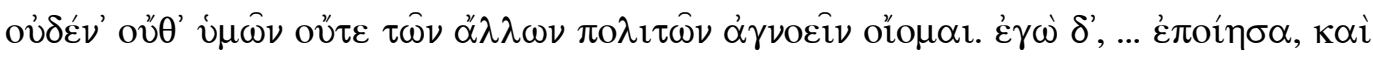

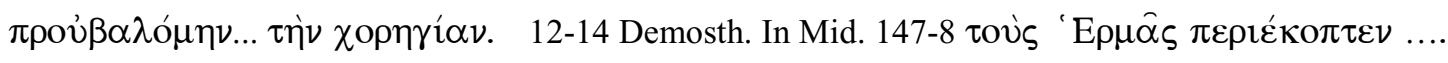

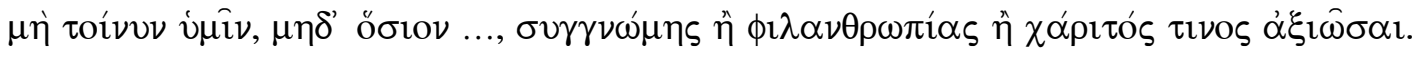

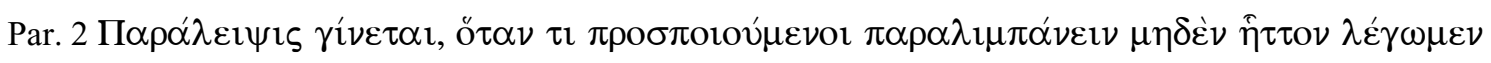

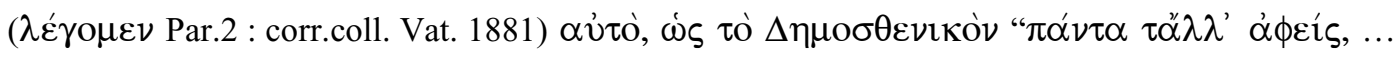

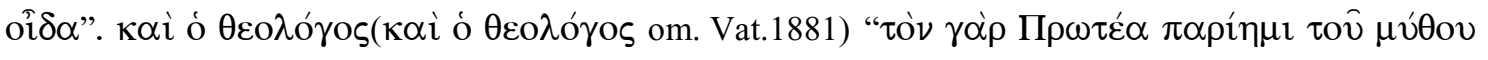

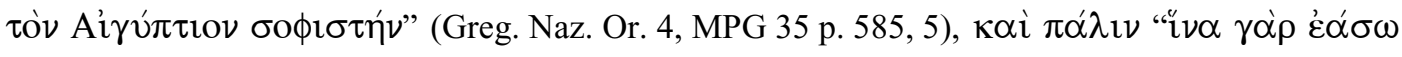

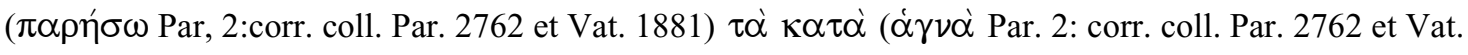

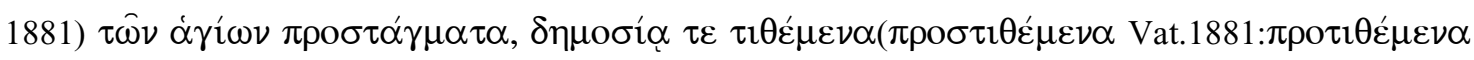

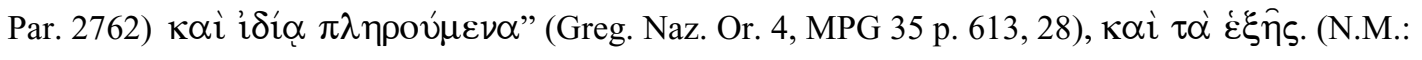

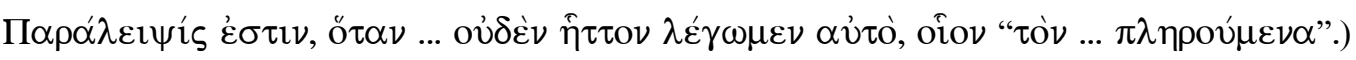

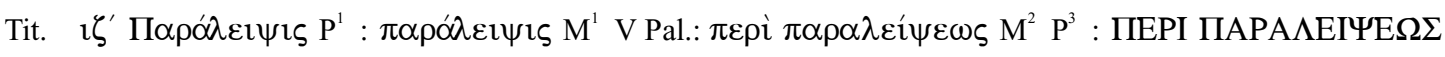
Ald. Ald ${ }^{s}$ : ПЕPI ПАРА

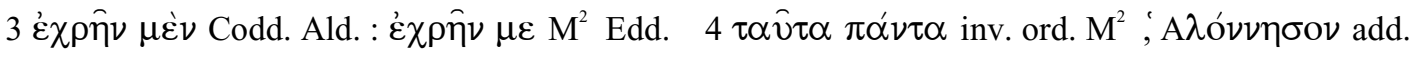

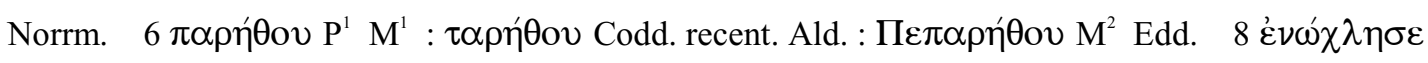

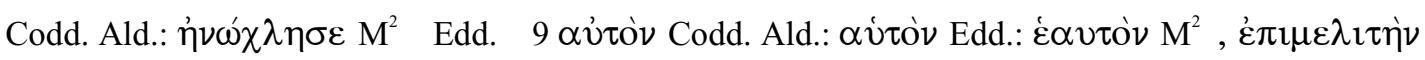

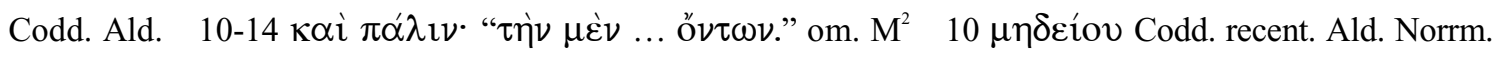


$\kappa^{\prime}$. ПЕРІ АПО $\Sigma$ ТРОФН $\Sigma$.

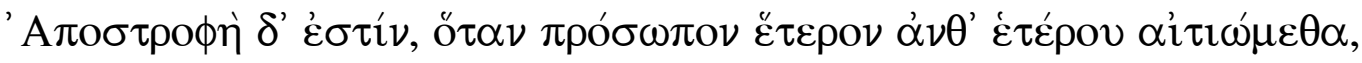

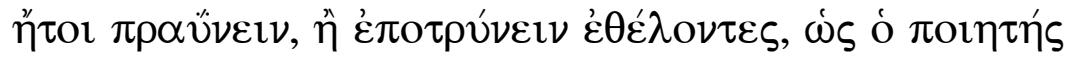

“A А

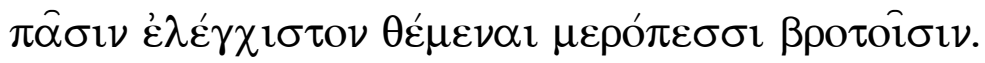

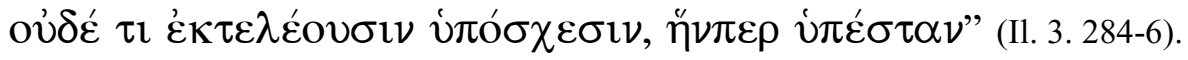

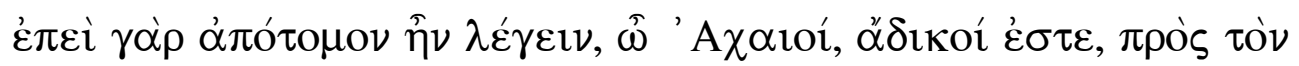

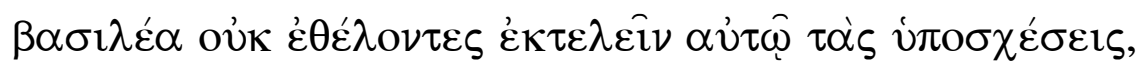

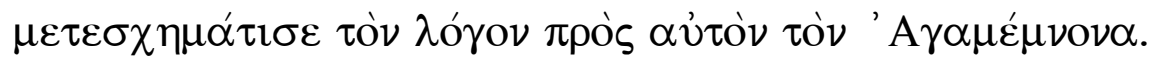

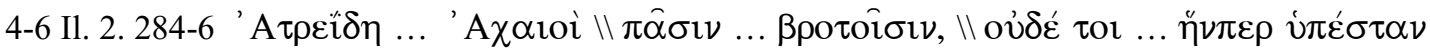

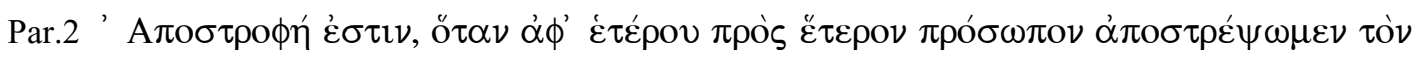

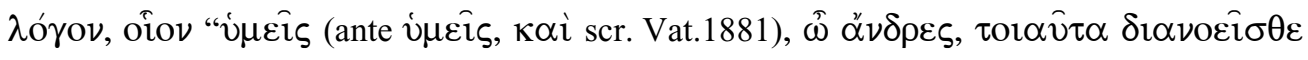

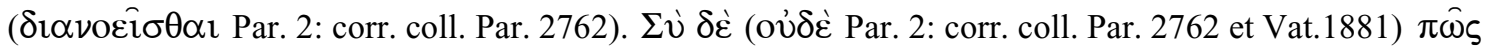

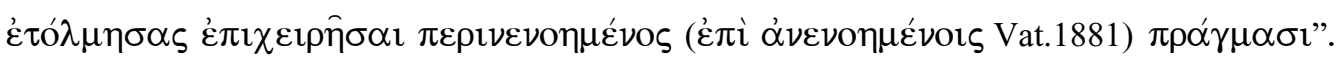

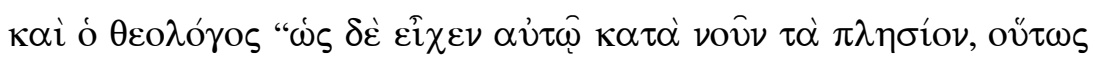

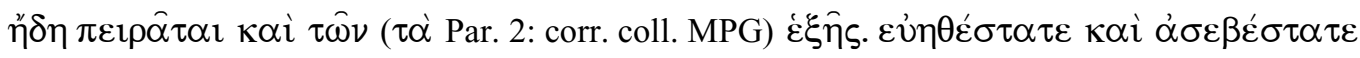

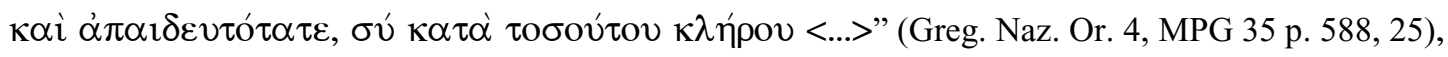

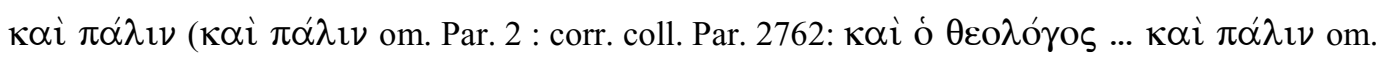

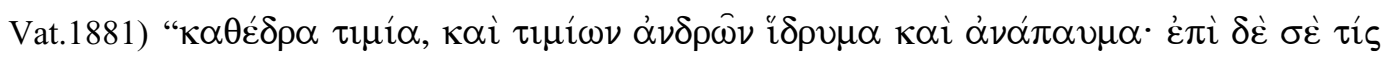

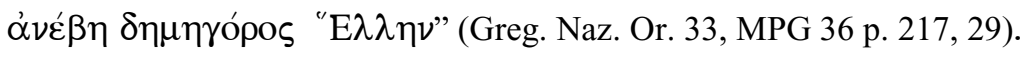

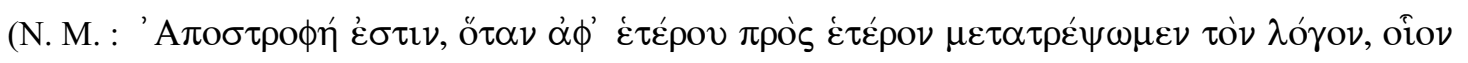

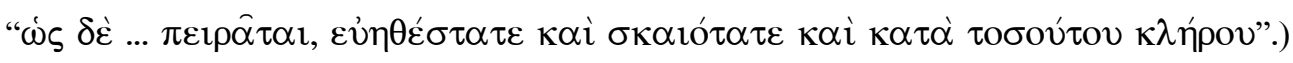

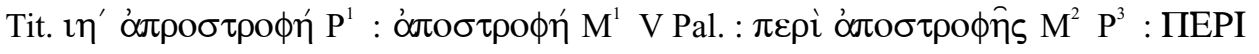
АПО $\Sigma$ TPОФН $\Sigma$ Ald. Ald ${ }^{s}:$ ПЕРI АПО $\Sigma$ TPOФН $\Sigma \kappa^{\prime}$ Norrm. : $\kappa^{\prime}$. ПЕРI АПО $\Sigma$ TPOФН $\Sigma$ Edd.

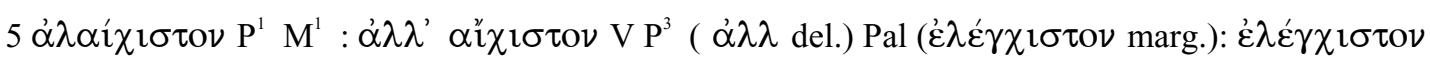
$\mathrm{M}^{2}$ Edd. 


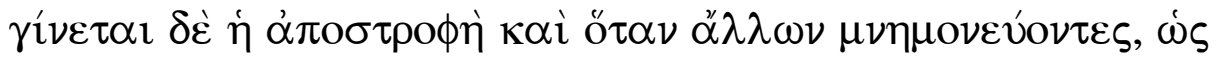

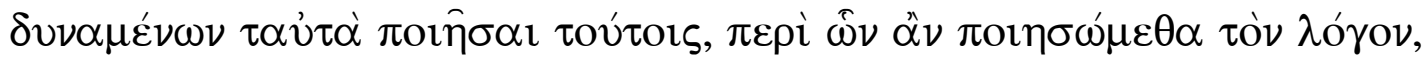

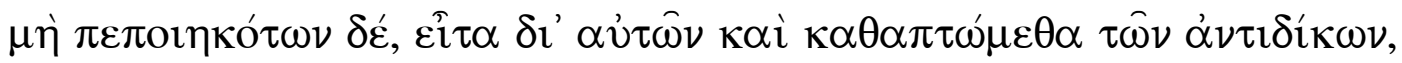
ஸ́s $\Delta \eta \mu$

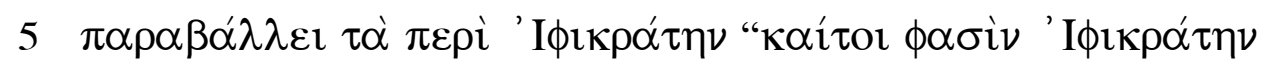

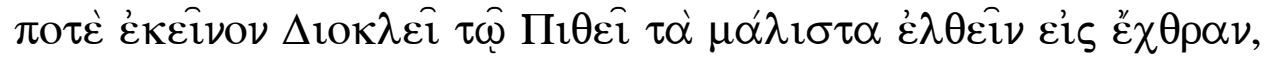

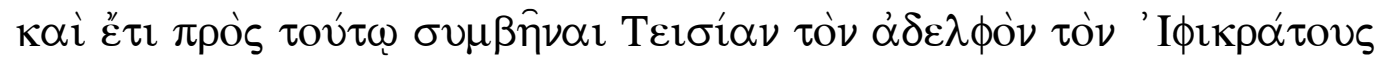

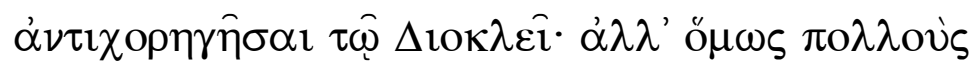

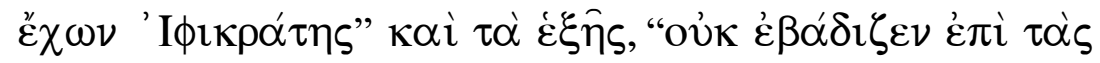

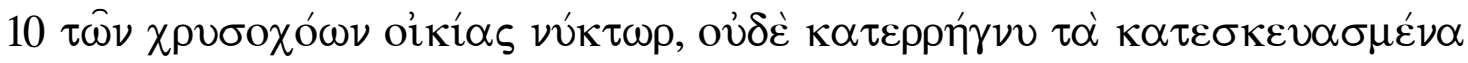

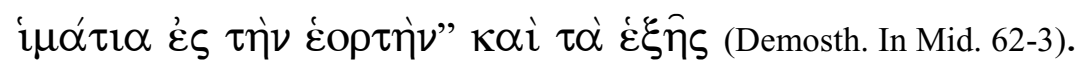

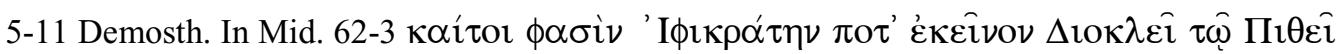

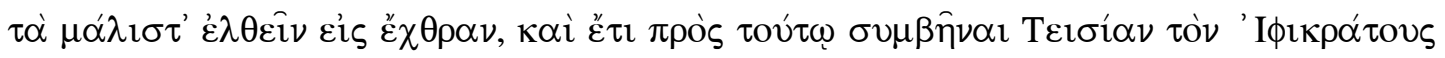

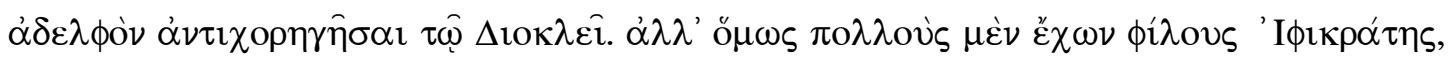

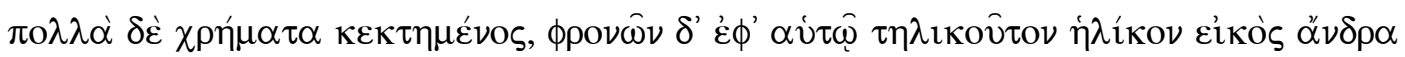

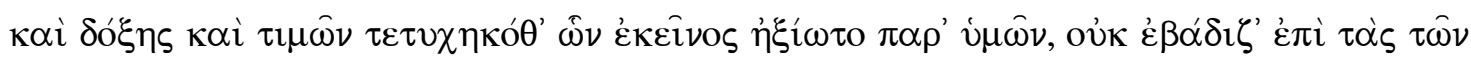

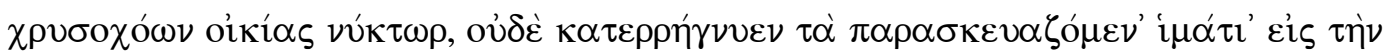

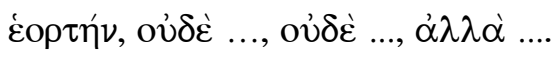

$2 \tau \alpha \hat{v} \tau \alpha$ Codd. Ald.: $\tau \alpha \hat{v} \tau \alpha \mathrm{M}^{2}$ Edd. $3 \dot{\varepsilon} \alpha v \tau \hat{\omega} \nu$ Codd. Ald.: $\alpha \hat{v} \tau \hat{\omega} \nu \mathrm{M}^{2}$ Edd. $4 \mu \eta \delta$ zíov $\mathrm{P}^{3}$ Ald.

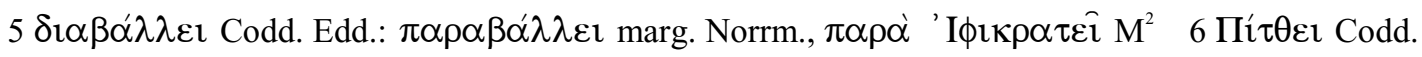

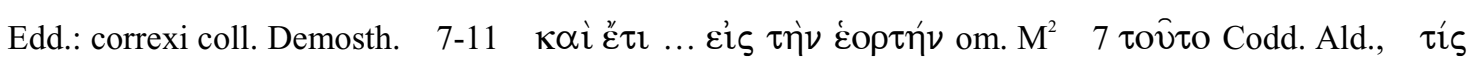

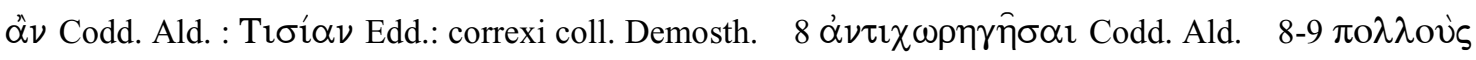

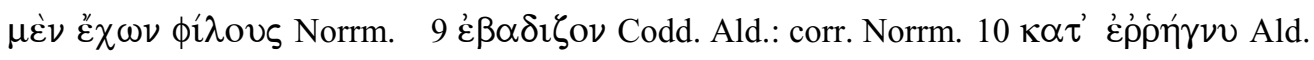


$\kappa \alpha^{\prime}$. ПЕРI $\Delta \mathrm{IA \Pi ОРН} \Sigma \mathrm{E} \Omega \Sigma$.

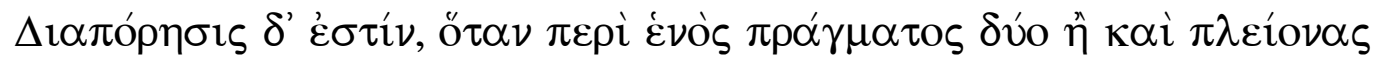

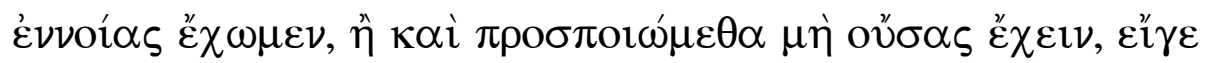

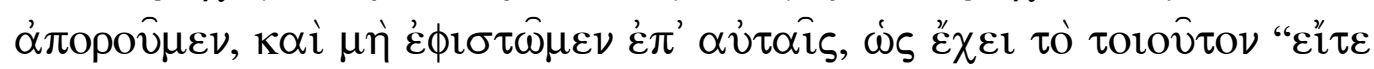

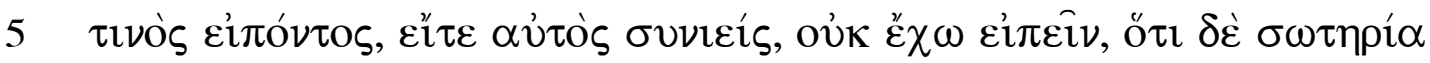

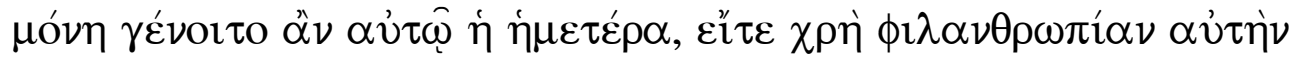

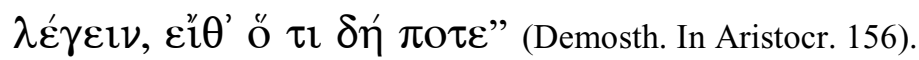

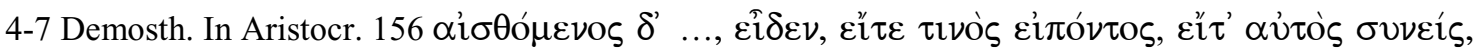

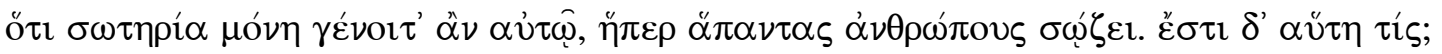

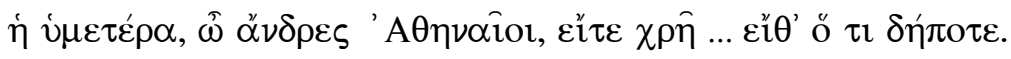

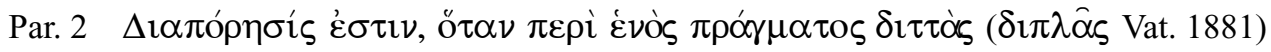

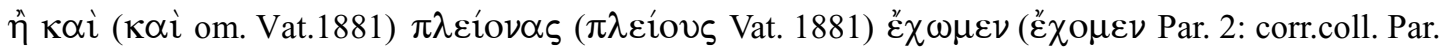

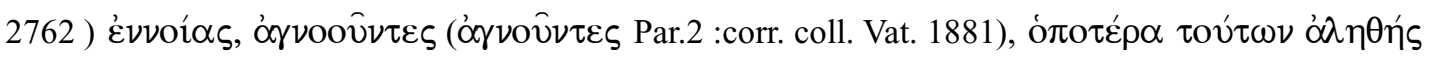

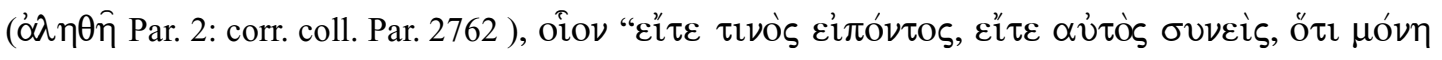

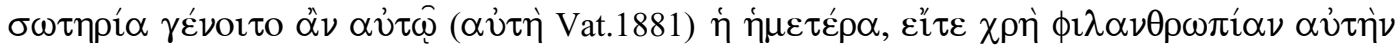

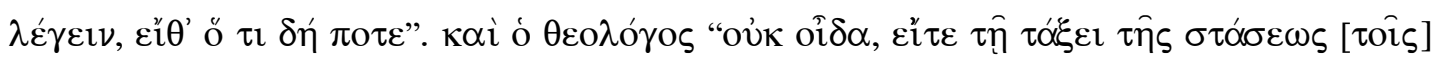

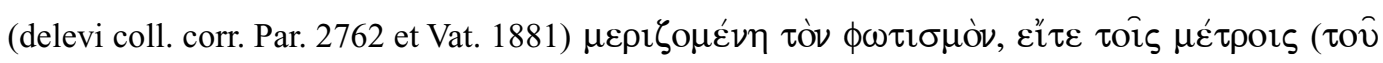

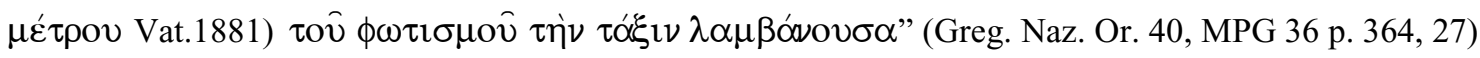

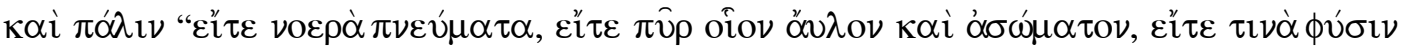

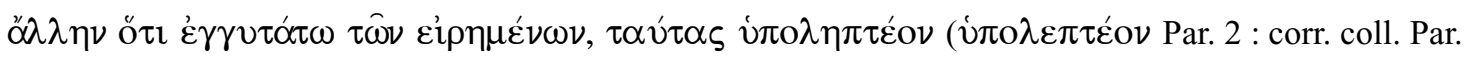

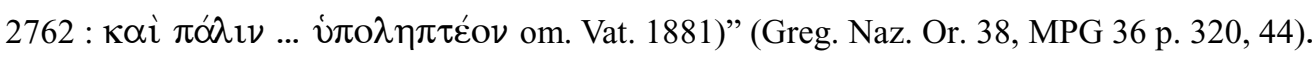

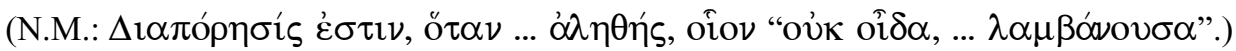

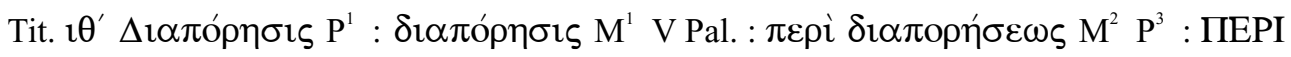
$\Delta$ IAПОРН $\Sigma$ E $\Omega \Sigma$ Ald. Ald ${ }^{s}:$ ПЕPI $\Delta$ IAПOP- $\kappa \alpha^{\prime}$ Norrm. : $\kappa \alpha^{\prime}$. ПЕPI $\Delta$ IAПOP- Edd.

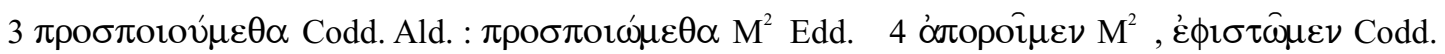

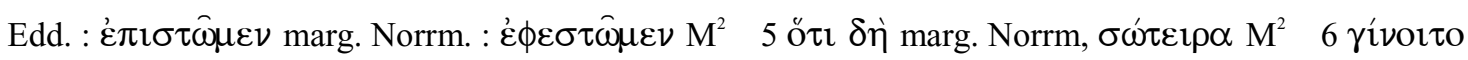

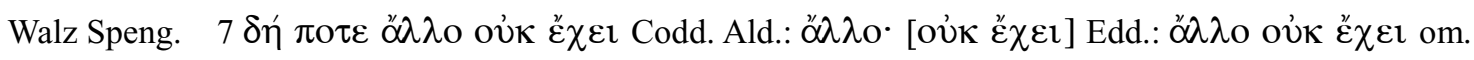

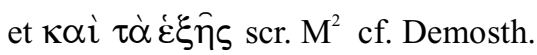


$\kappa \beta^{\prime}$. ПЕPI EP $\Omega T H M A T O \Sigma$.

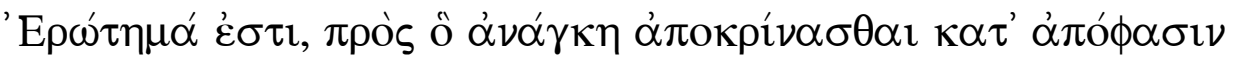

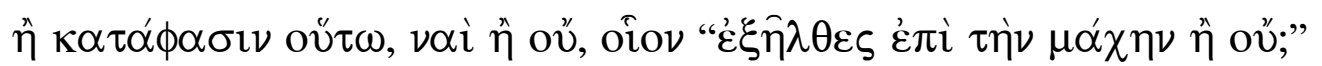

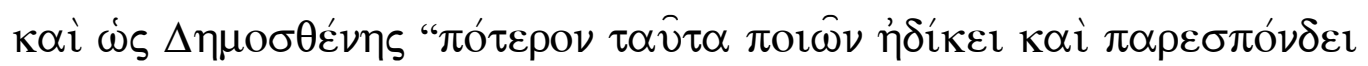

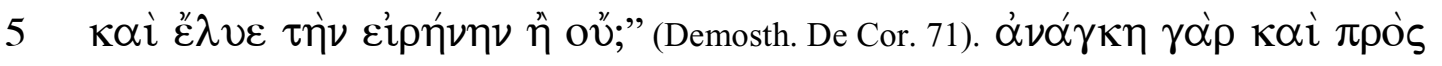

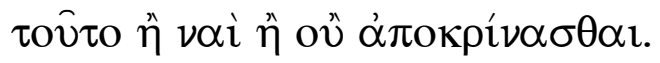

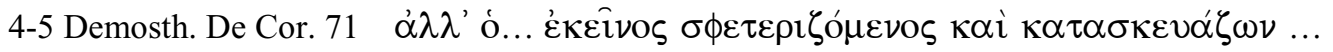

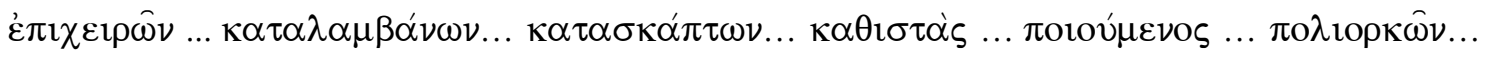

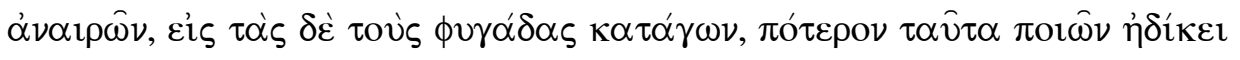

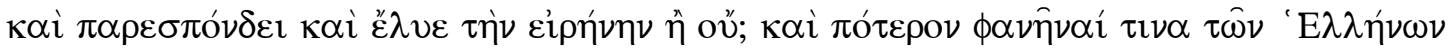

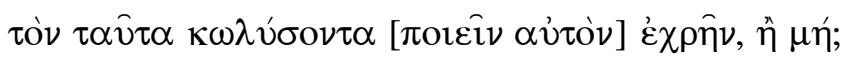

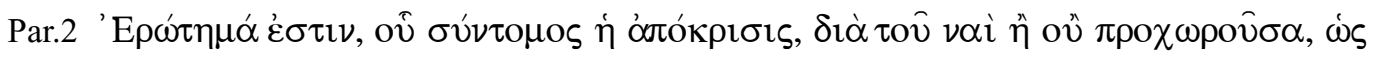

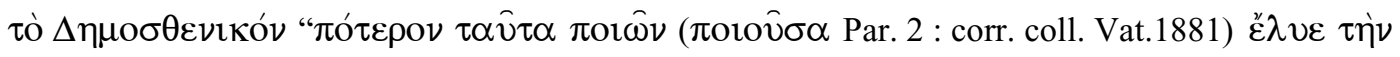

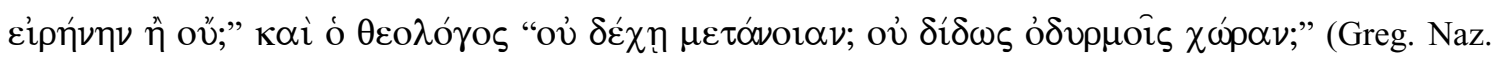
Or. 39, MPG 36 p. 356, 28).

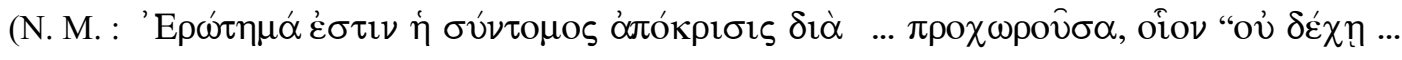

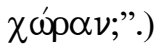

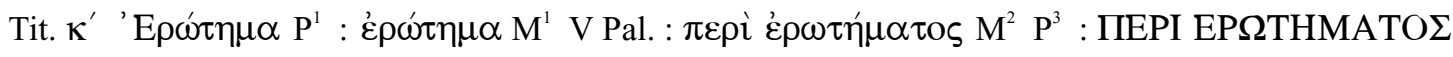
Ald. Ald ${ }^{\mathrm{s}}$ : ПЕPI EP $\Omega$ THMATO $\Sigma \kappa \beta^{\prime}$ Norrm. : $\kappa \beta^{\prime}$. ПEPI EP $\Omega T H M A T O \Sigma$ Edd.

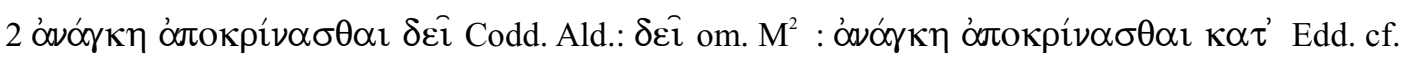

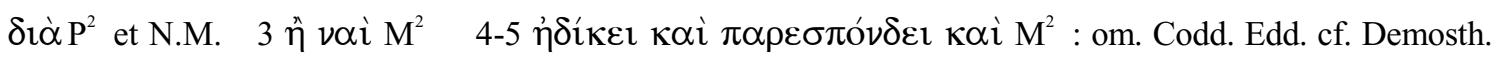

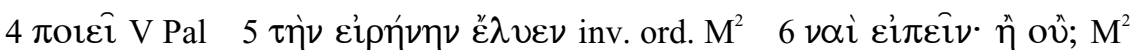


$\kappa \gamma^{\prime}$. ПЕРI ПY $\Sigma \mathrm{MATO} \Sigma$.

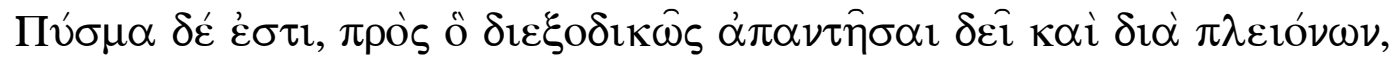

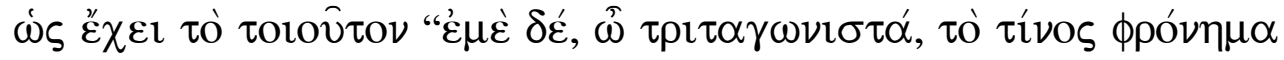

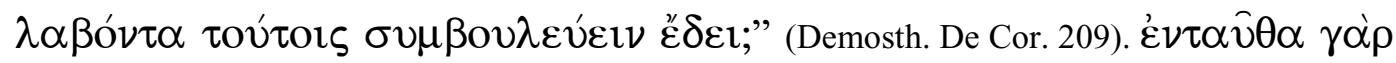

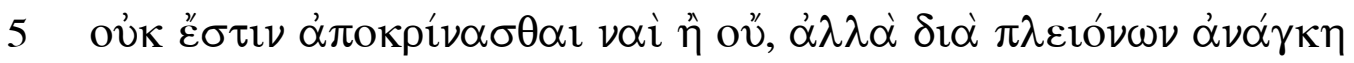
$\dot{\alpha} \pi \alpha \nu \tau \hat{\alpha} \nu \tau \hat{\omega} \tau 010 \hat{\tau} \tau \mathrm{v} \nu \dot{\varepsilon} \rho \mathrm{v} \nu \tau 1$.

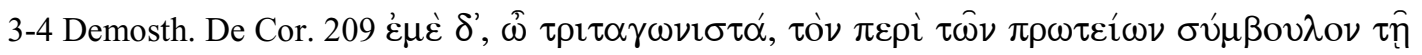

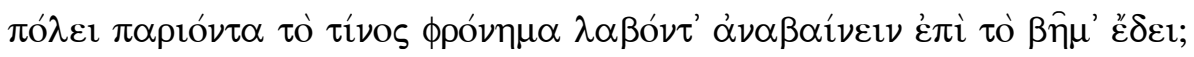

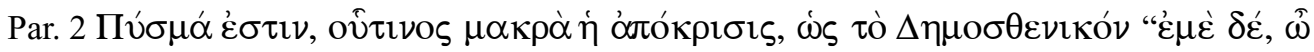

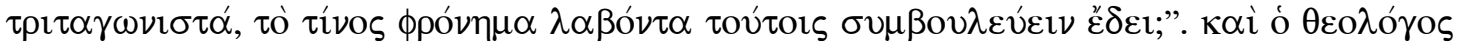

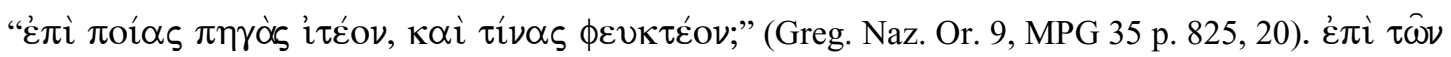

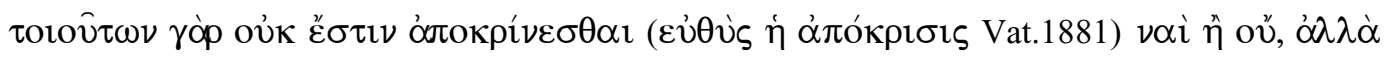

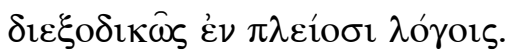

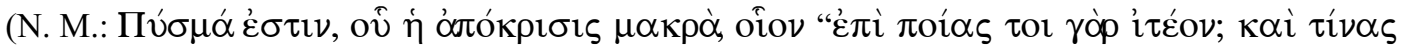

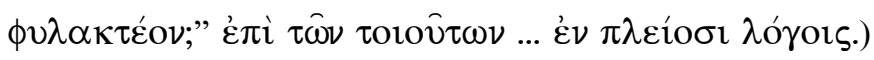

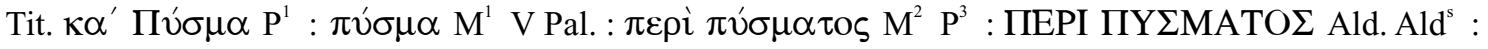
ПЕРI ПY $\Sigma$ MATO $\Sigma \kappa \gamma^{\prime}$ Norrm. : $\kappa \gamma^{\prime}$. ПЕPI ПY $\Sigma M A T O \Sigma$ Edd.

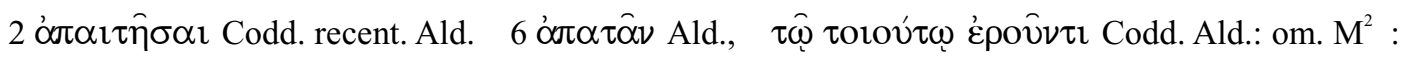

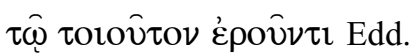


$\kappa \delta^{\prime}$. ПЕРI $\triangle \mathrm{IATY} \Omega \Sigma \mathrm{E} \Omega \Sigma$.

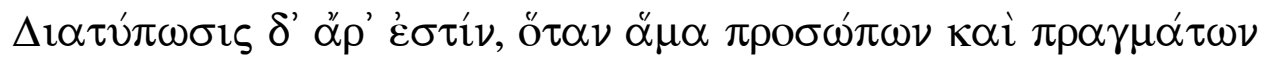

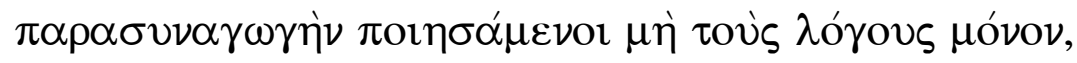

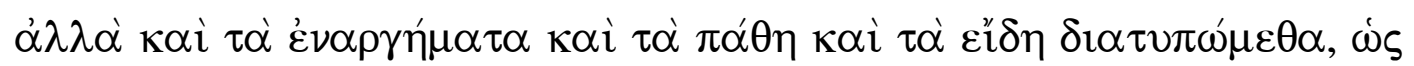

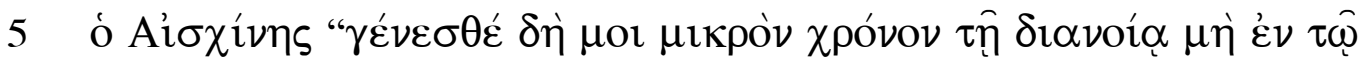

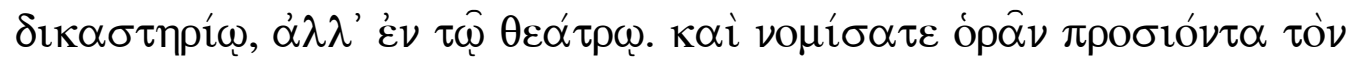

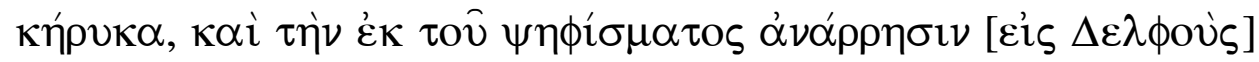

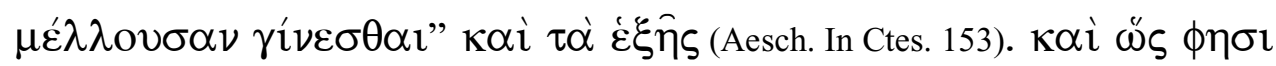

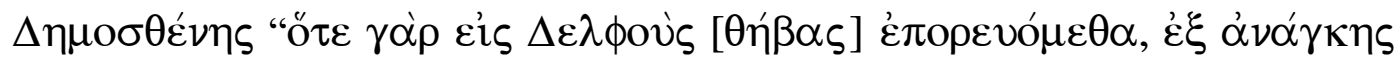

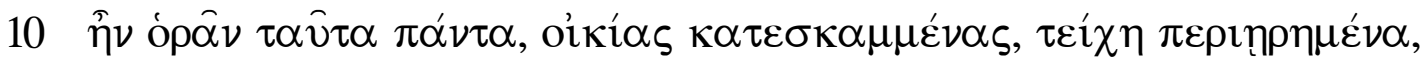

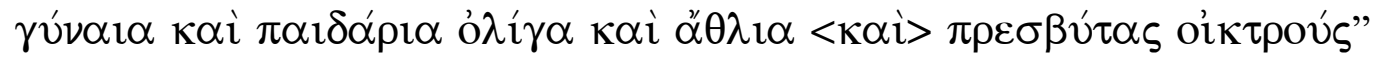
(Demosth. De fals. Legat. 65).

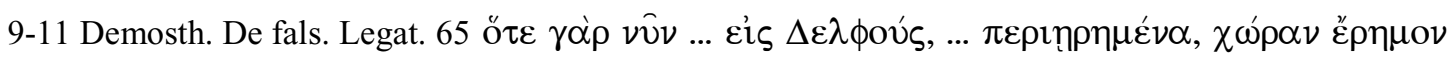

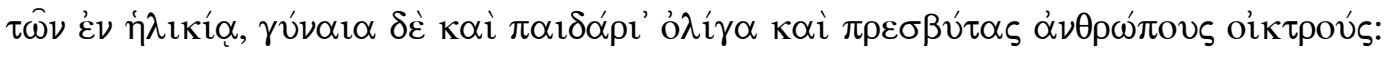

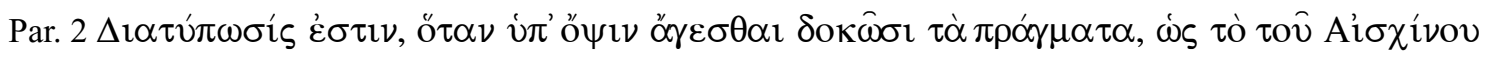

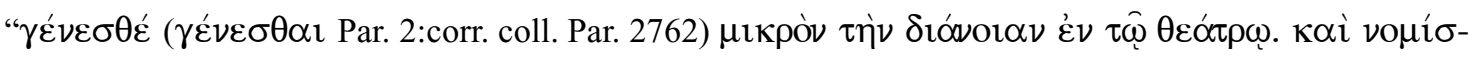

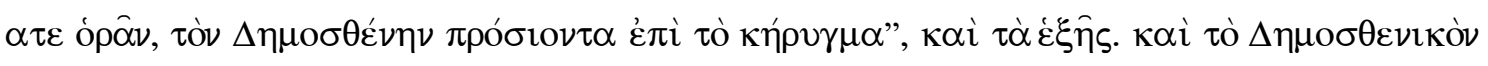

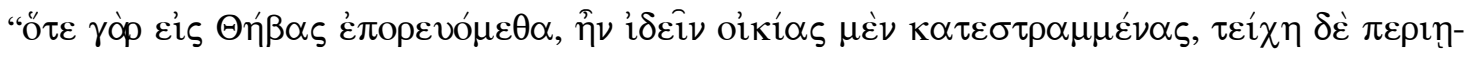

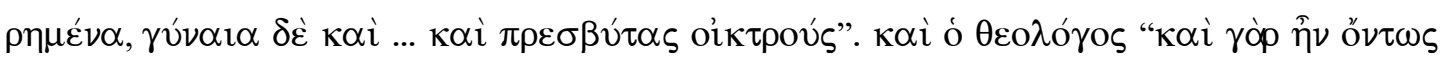

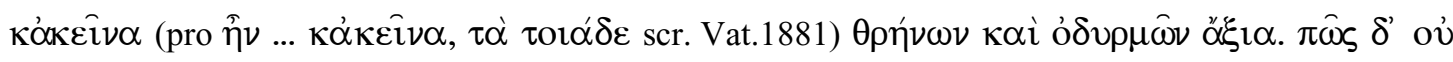

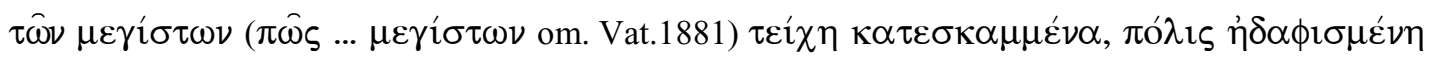

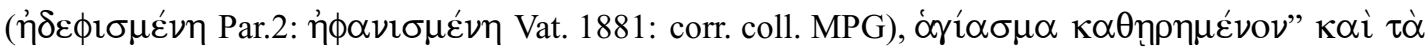

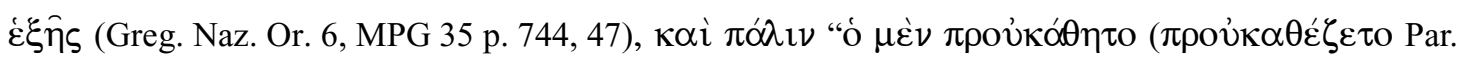

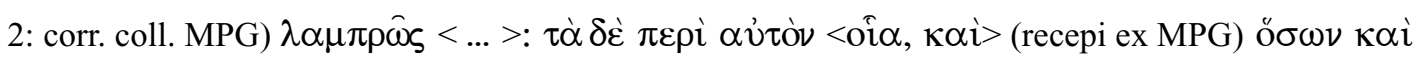

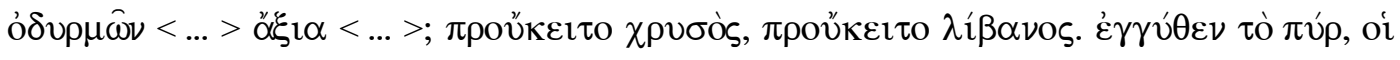

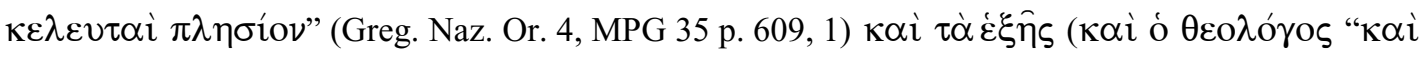

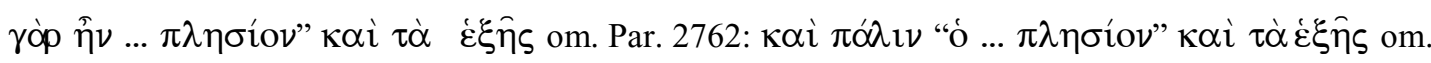

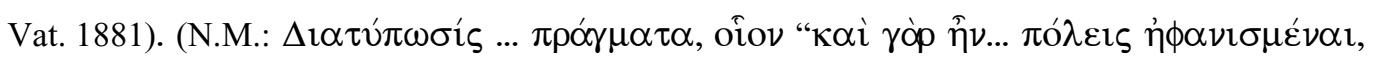

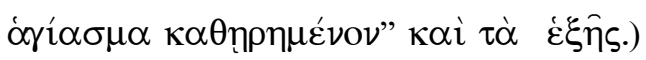

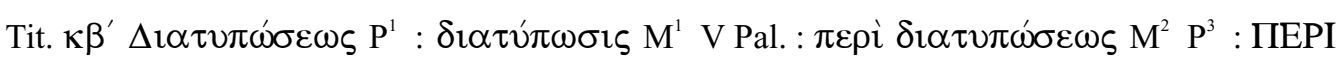
$\Delta$ IATYП $\Omega \Sigma$ E $\Omega \Sigma$ Ald. Ald ${ }^{s}:$ ПEPI $\Delta$ IATYП- $\kappa \delta^{\prime}$ Norrm. : $\kappa \delta^{\prime}$. ПEPI $\Delta$ IATYП- Edd.

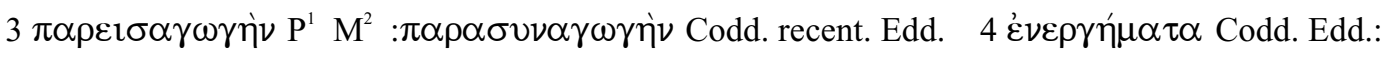

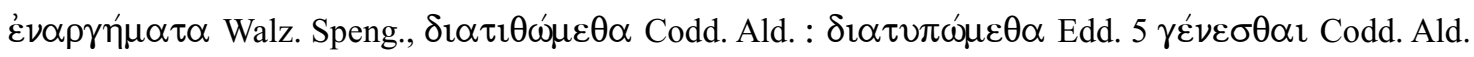

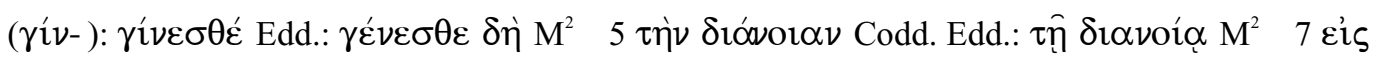

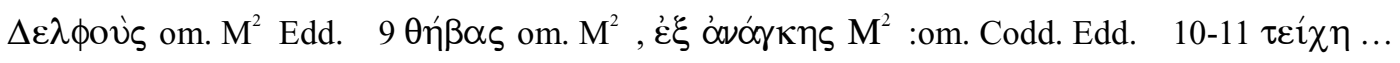

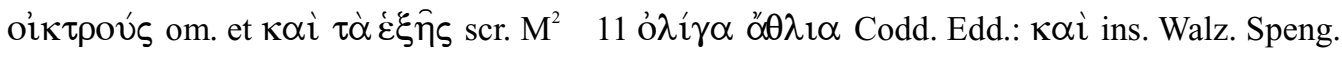


$\kappa \varepsilon^{\prime}$. ПEPI ANTEI $\Sigma \mathrm{A} \Gamma \Omega \Gamma H \Sigma$.

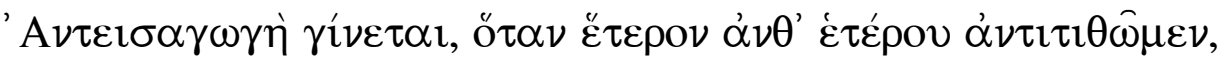

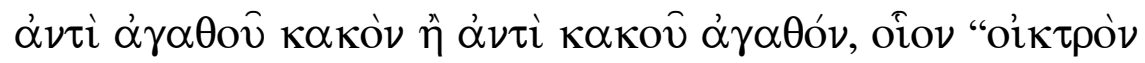

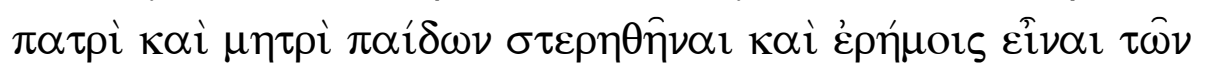

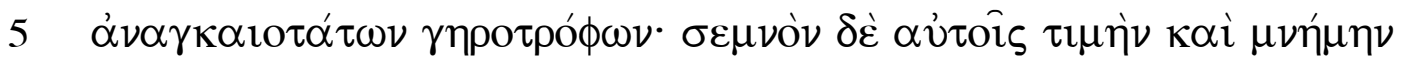

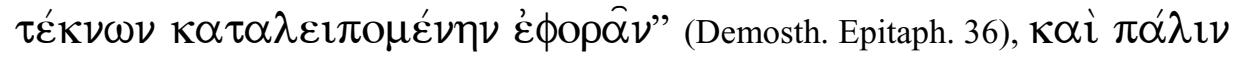

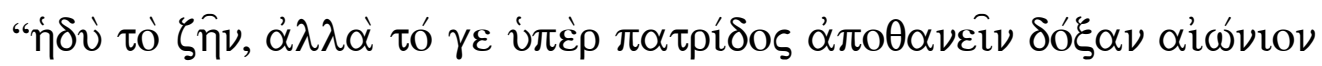
$\pi \alpha \rho \alpha \sigma \kappa \varepsilon v \alpha \dot{\zeta} \varepsilon \imath^{\prime \prime}$.

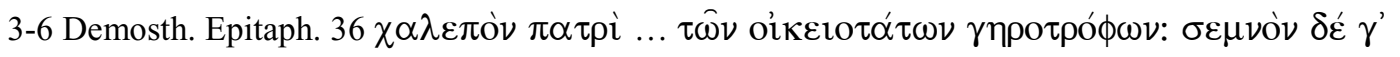

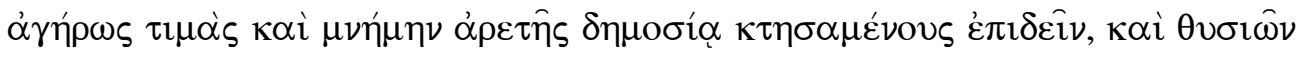

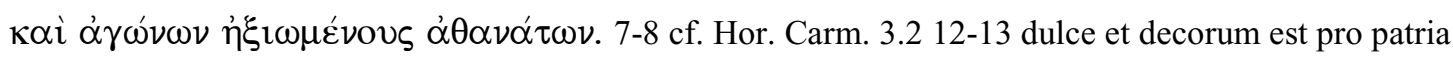

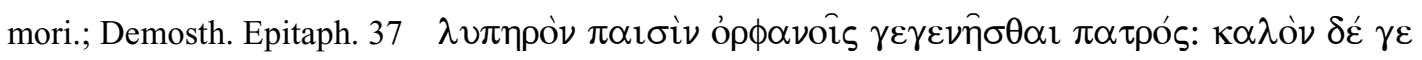

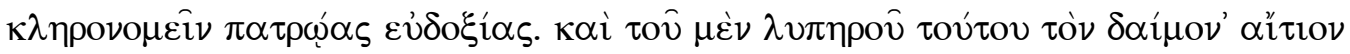

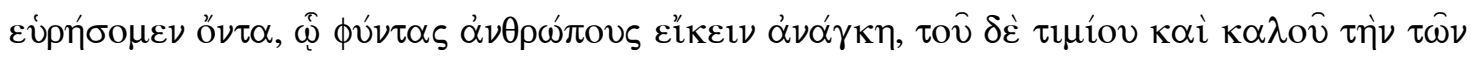

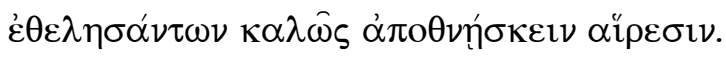

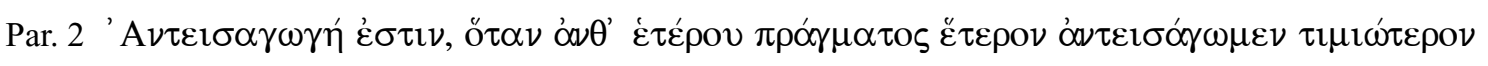

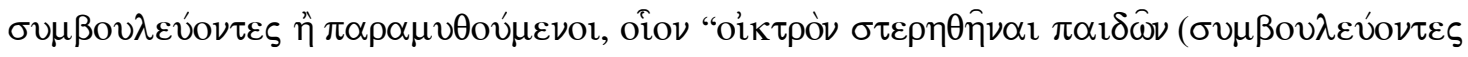

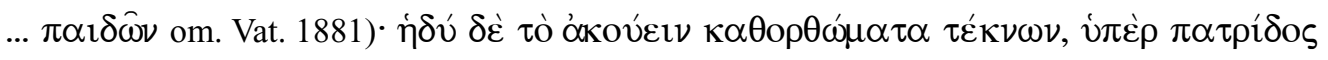

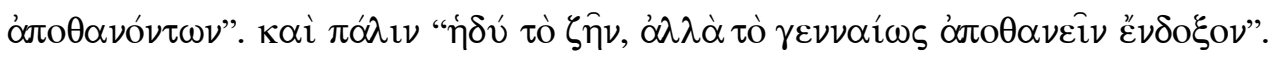

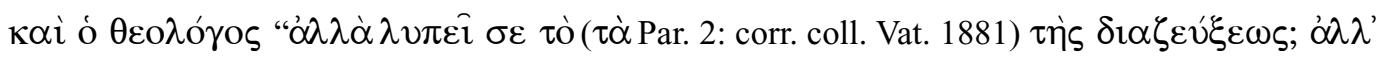

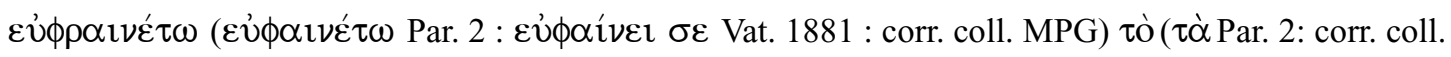

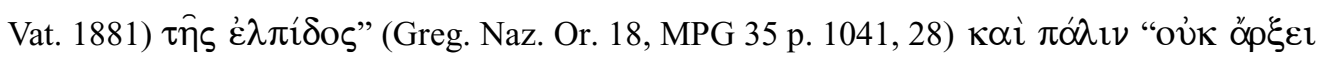

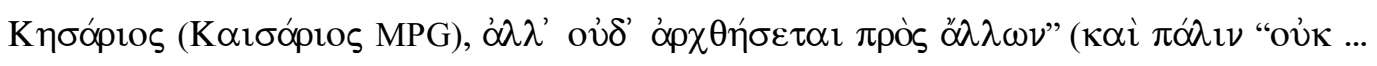

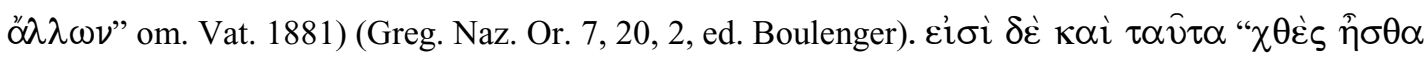

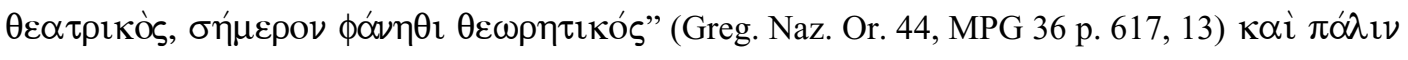

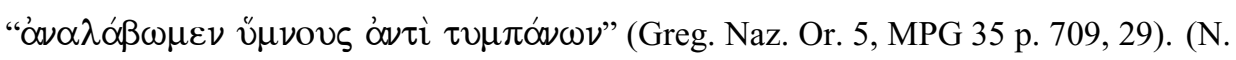

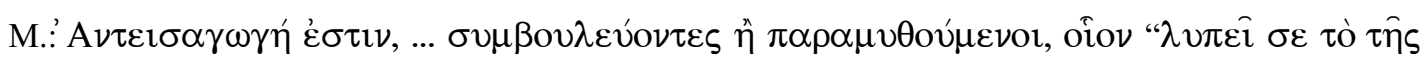

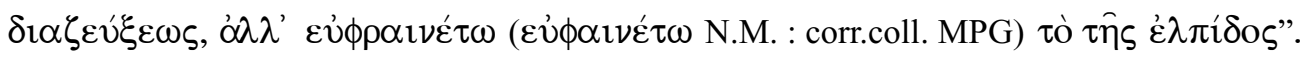

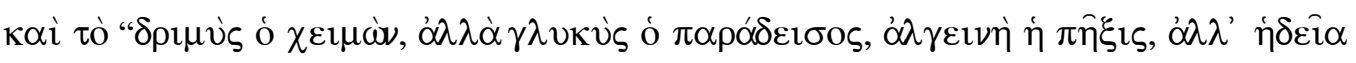

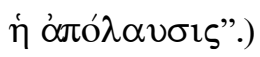

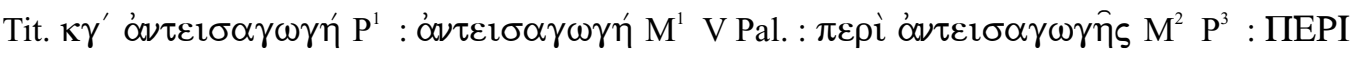
ANTEI $\Sigma$ AГ $\Omega \Gamma H \Sigma$ Ald. Ald ${ }^{\mathrm{s}}:$ ПEPI ANTEI $\Sigma$ A $\Gamma$ - $\kappa \varepsilon^{\prime}$ Norrm. : $\kappa \varepsilon^{\prime}$. ПEPI ANTEI $\Sigma A \Gamma-$ Edd.

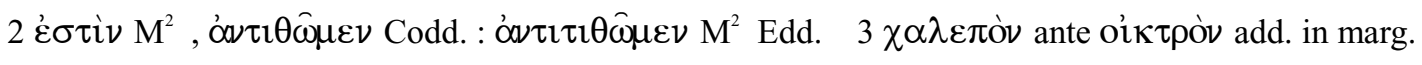

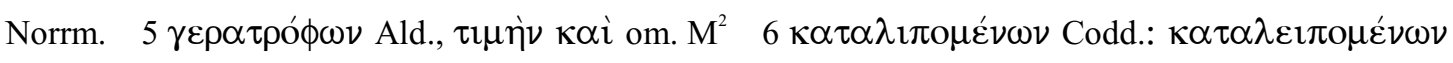
$\mathrm{P}^{3}$ Ald.: $\kappa \alpha \tau \alpha \lambda \varepsilon \imath \pi \mathrm{O} \mu \dot{\varepsilon} \nu \eta \nu \mathrm{M}^{2}$ Edd. 
$\kappa \varsigma^{\prime}$. ПЕPI $\triangle \mathrm{IA} \Sigma$ YPMOY.

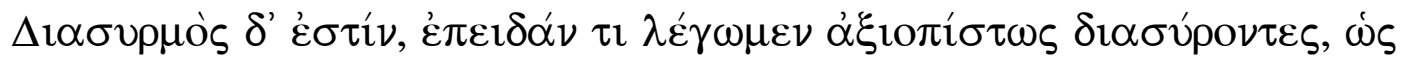

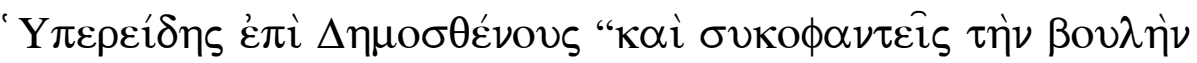

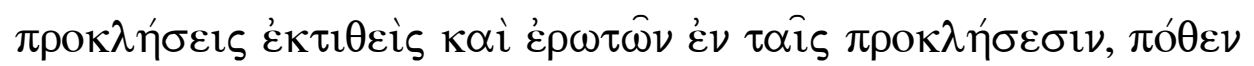

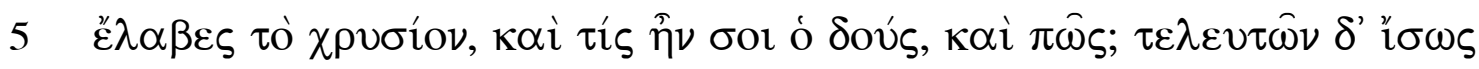

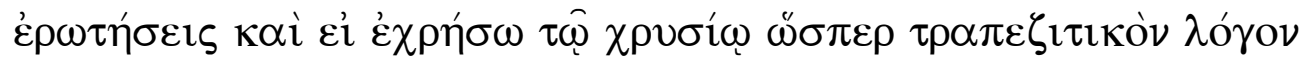

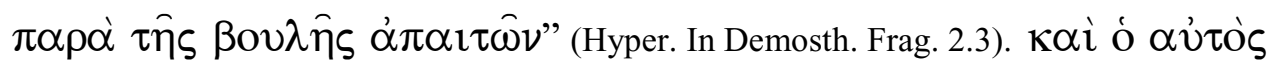

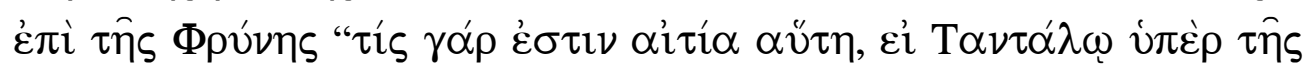

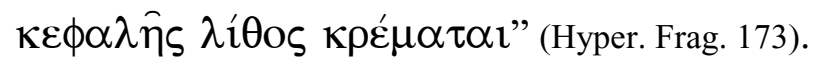

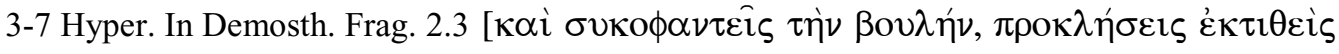

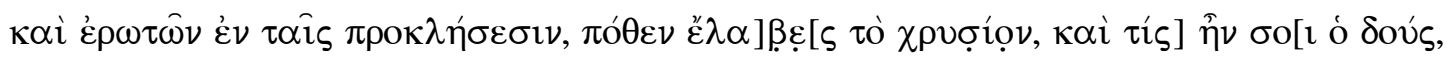

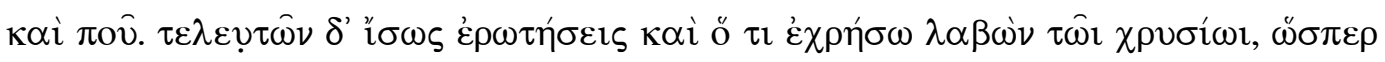

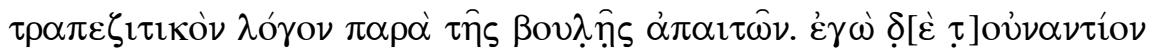

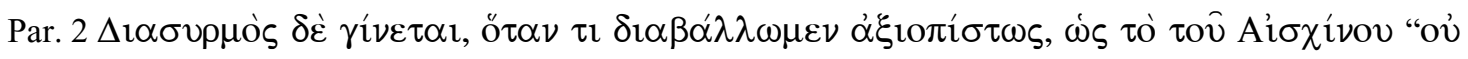

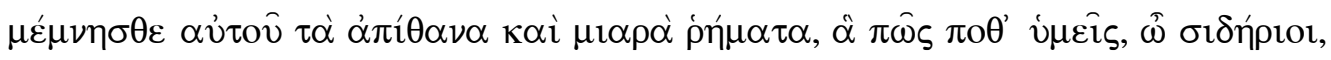

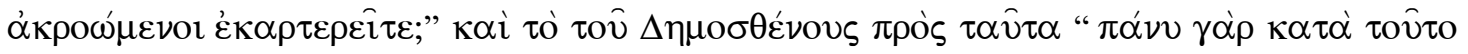

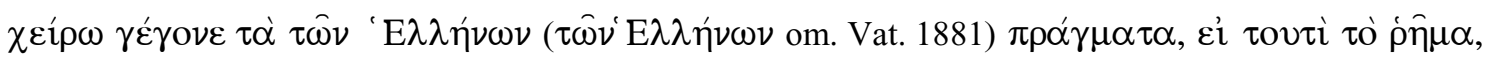

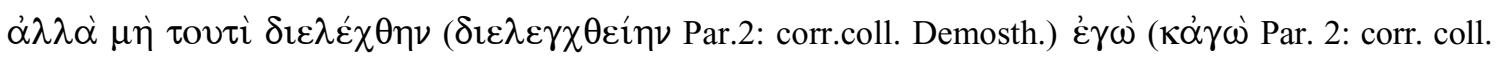

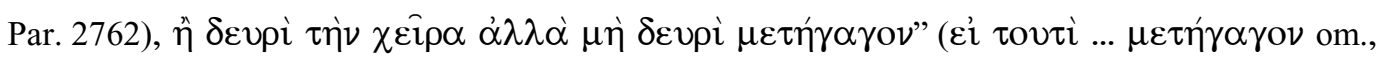

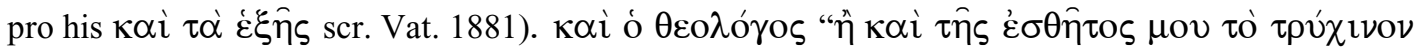

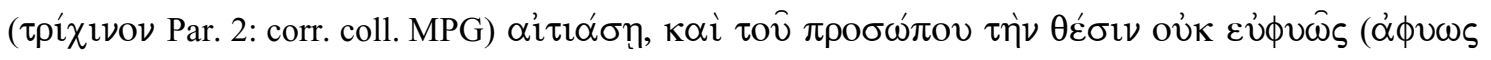

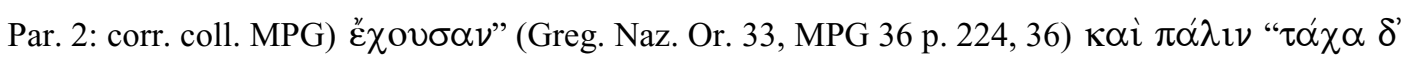

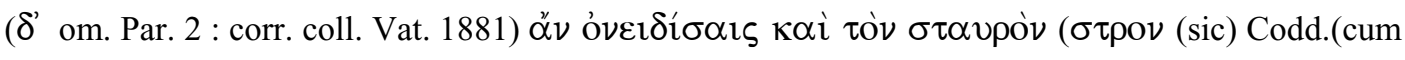

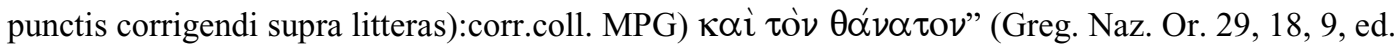
Barbel).

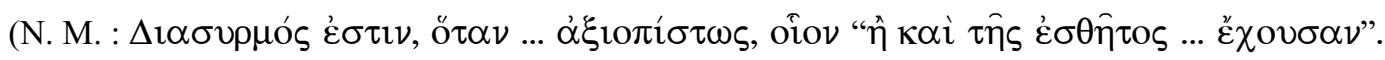

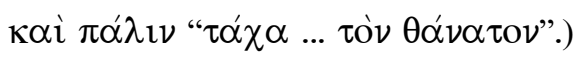

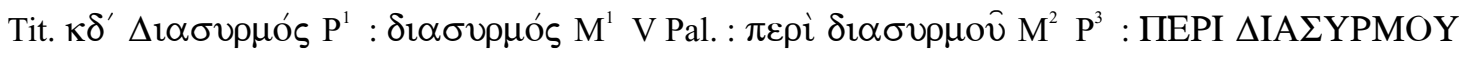
Ald. Ald ${ }^{\varsigma}:$ ПEPI $\Delta$ IA $\Sigma$ YPMOY $\kappa \varsigma^{\prime}$ Norrm. : $\kappa \varsigma^{\prime}$. ПEPI $\Delta$ IA $\Sigma$ YPMOY Edd.

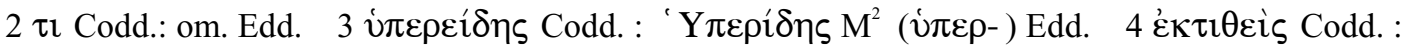

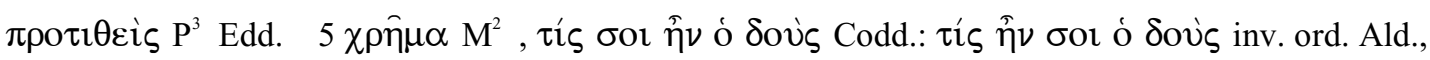

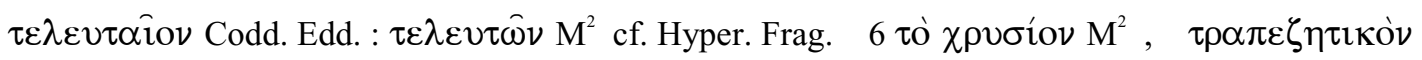

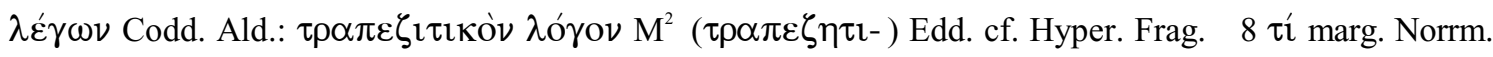




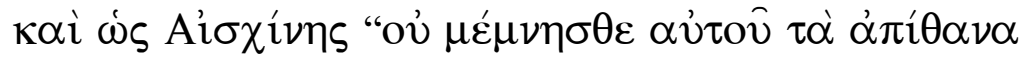

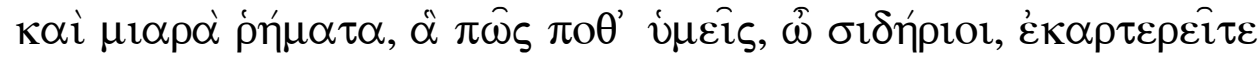

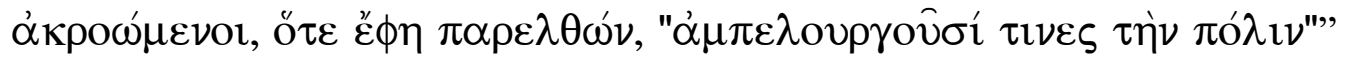

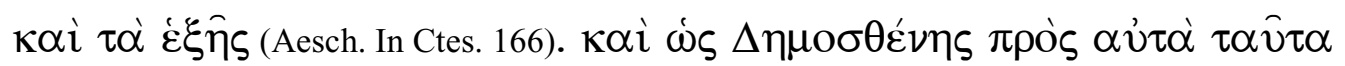

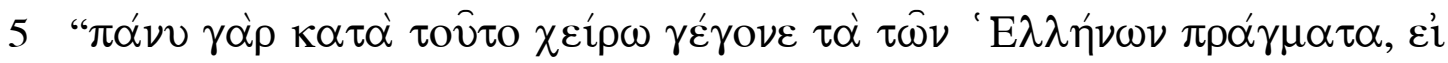

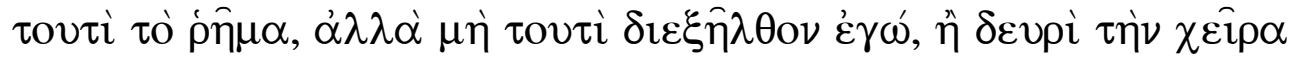

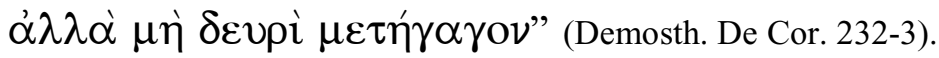

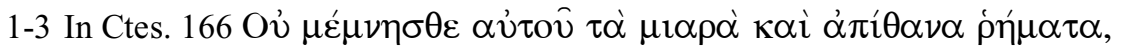

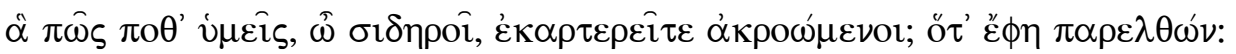

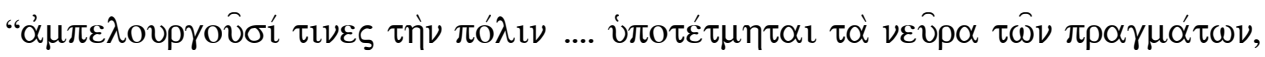

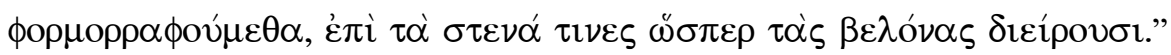

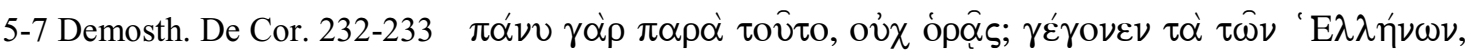

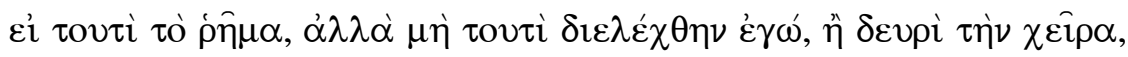

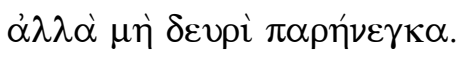

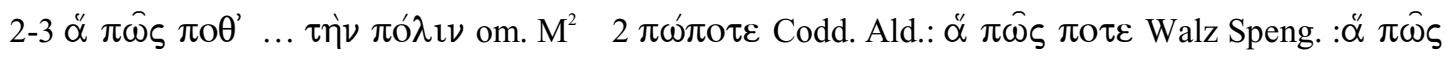

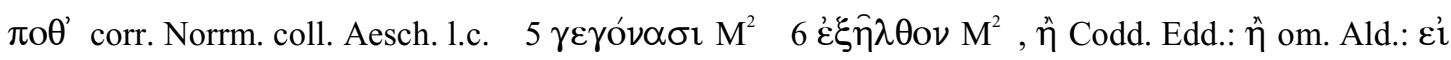
Norrm. 
$\kappa \zeta^{\prime}$. ПEPI META $\Sigma T A \Sigma E \Omega \Sigma$.

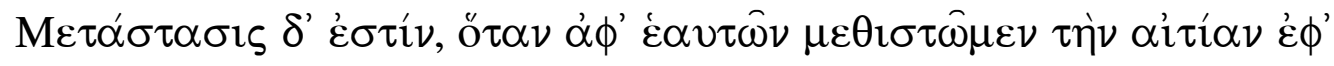

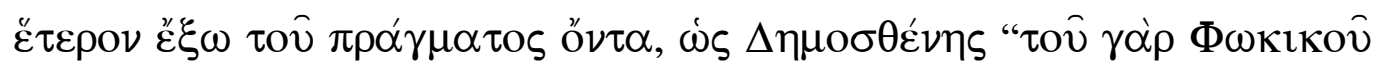

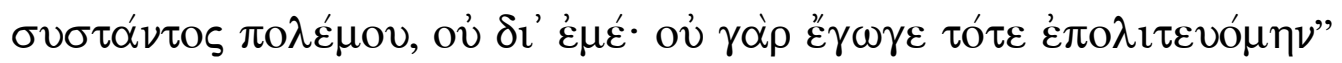

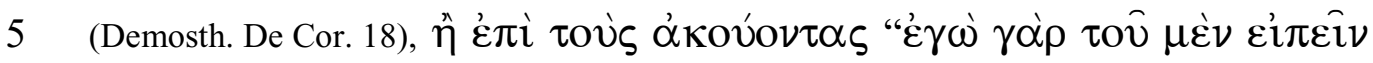

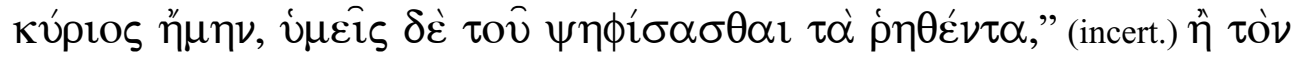

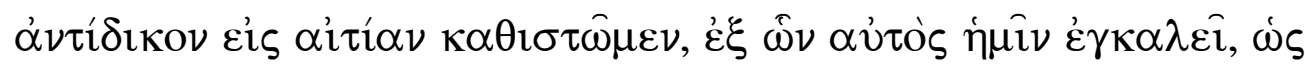

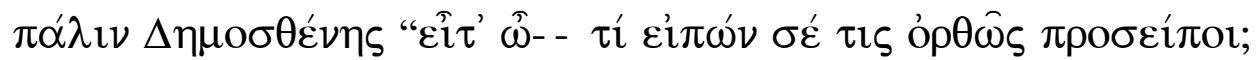

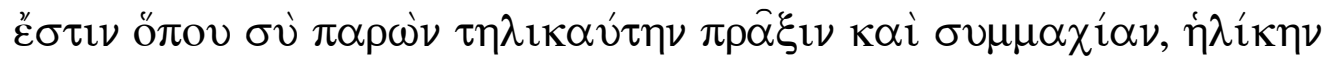

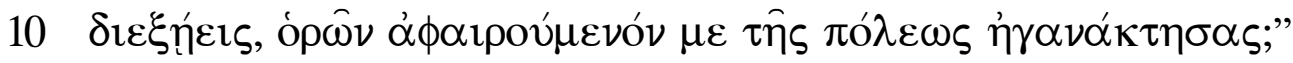

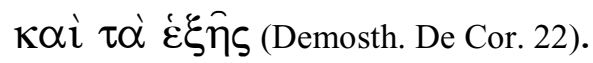

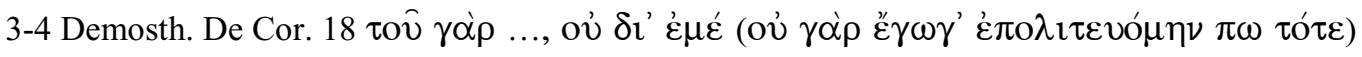

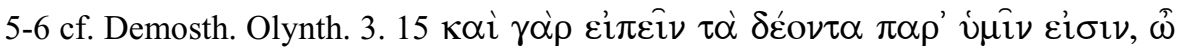

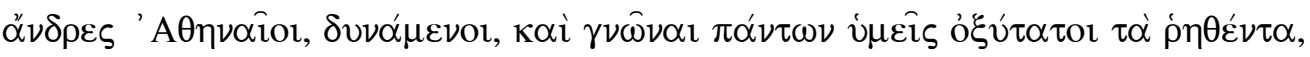

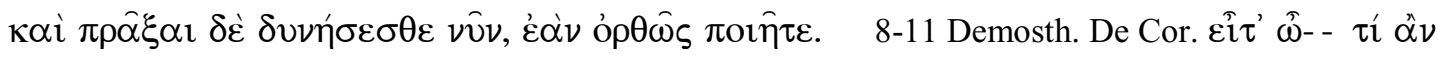

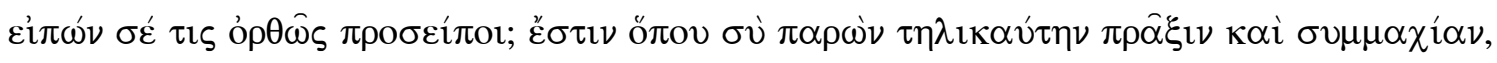

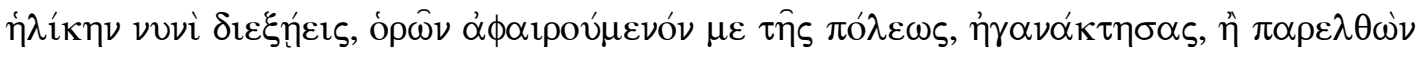

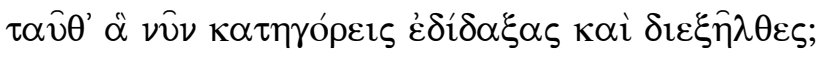

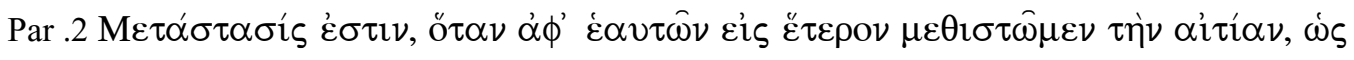

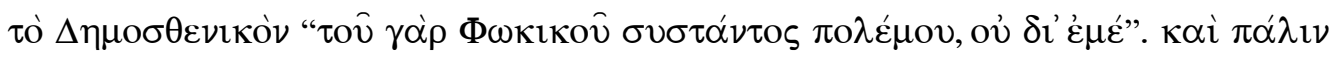

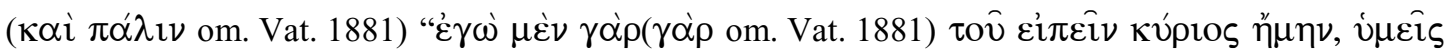

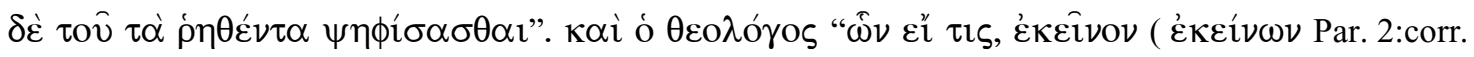

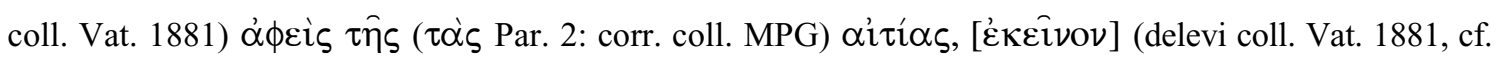

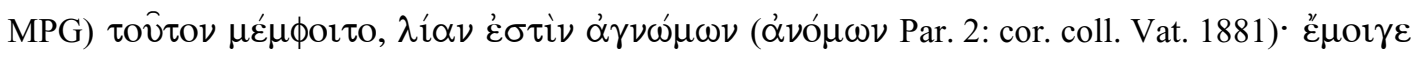

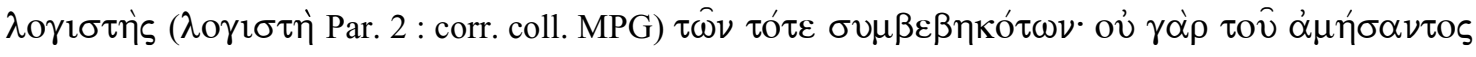

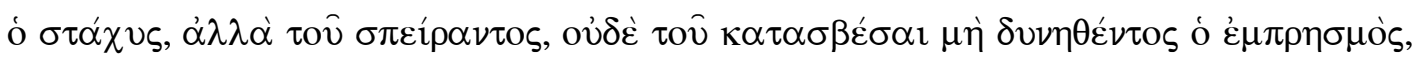

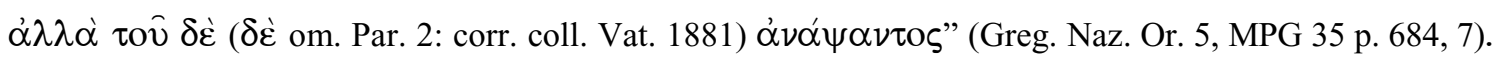

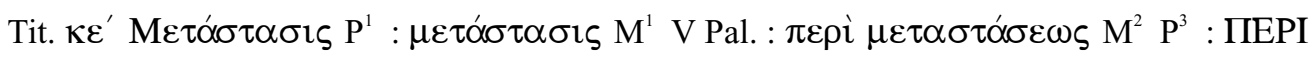
META $\Sigma T A \Sigma E \Omega \Sigma$ Ald. Ald ${ }^{s}:$ ПEPI META $\Sigma T-\kappa \zeta^{\prime}$ Norrm. : $\kappa \zeta^{\prime}$. ПEPI META $\Sigma T$ - Edd.

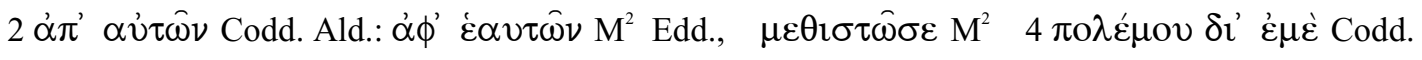

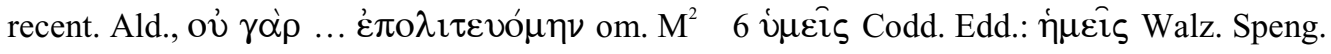

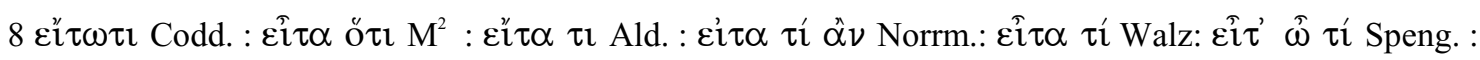

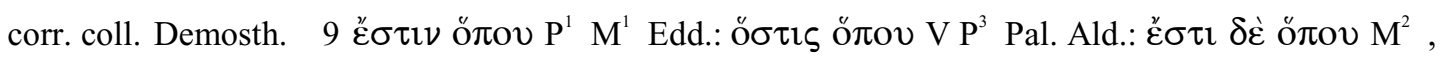

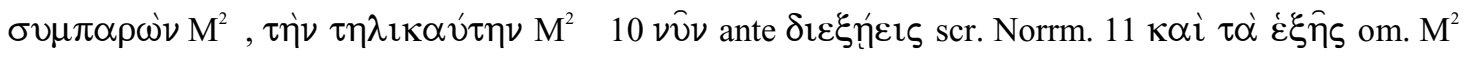


$\left[\kappa \eta^{\prime}\right.$. ПЕРI YПЕРВО $\Lambda \mathrm{H} \Sigma$.

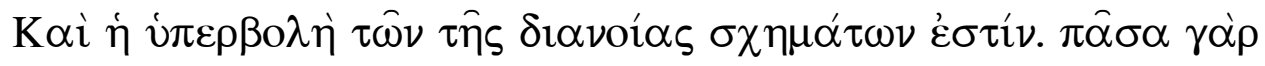

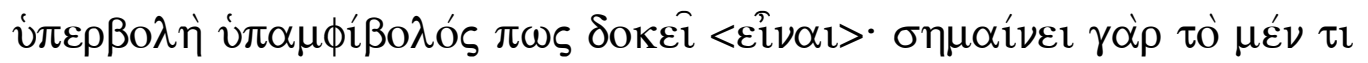

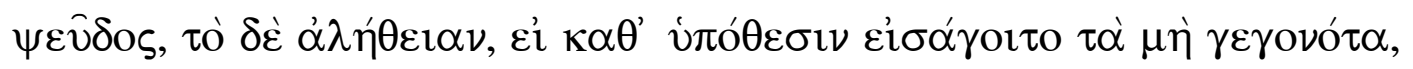

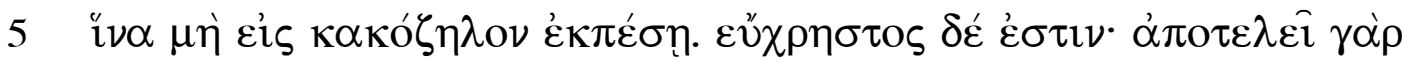

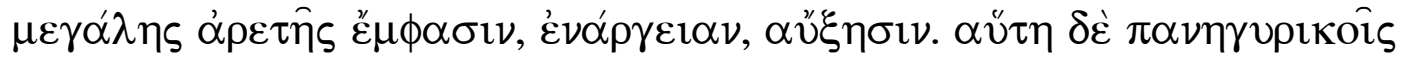

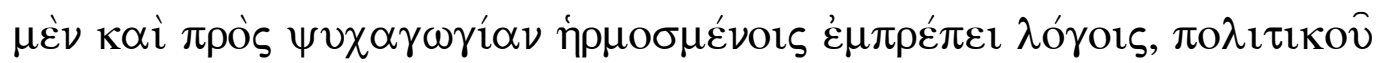

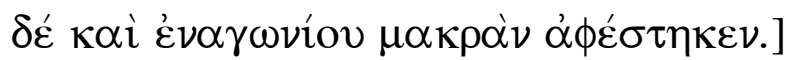

1-8 cf. Iul. Ruf. De Fig. I 38 (RLM p. 47 Halm) 'Y $₹ \varepsilon \rho \beta 0 \lambda \eta ́$ aliis tropus videtur: ceterum fit, cum excedit veritatem sententia.

Tryph. De Trop. $\alpha$ ' $\gamma^{\prime}$ (RG VIII pp. 746-7 Walz) 'Y

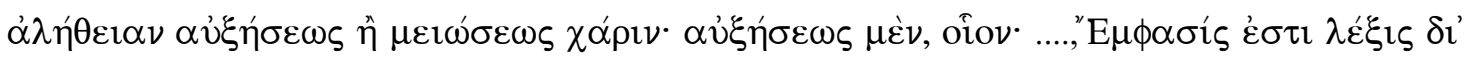

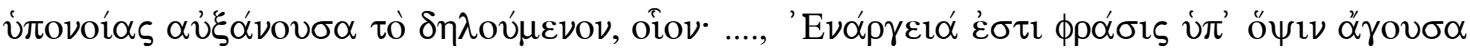

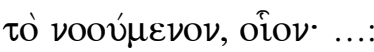

Quint, Inst. Orat. 8.4. 29 Scio posse videri quibusdam speciem amplificationis hyperbolen quoque: nam et haec in utramque (sc. amplificationis et tropi) partem valet; sed quia excedit hoc nomen, in tropos differenda est: 8. 4.15 Quas dixi per ratiocinationem fieri amplificationes viderimus : 8. 6. 67-76

Hyperbolen audacioris ornatus summo loco posui. Est haec decens veri superiectio: virtus eius ex diverso par, augendi atque minuendi. Fit pluribus modis: ... Quamvis enim est omnis hyperbole ultra fidem, non tamen esse debet ultra modum, nec alia via magis in cacozelian itur.

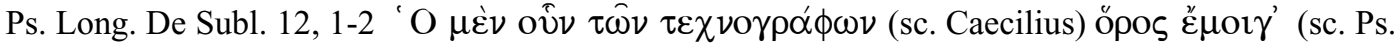

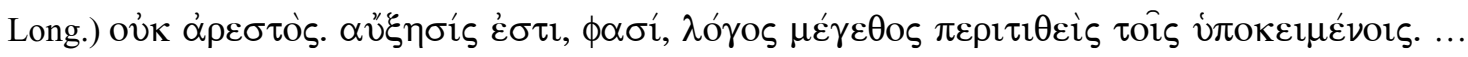

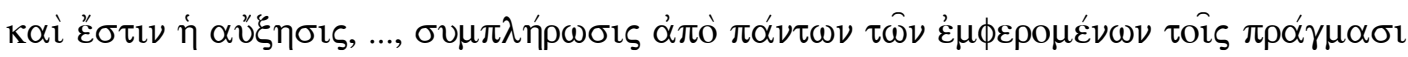

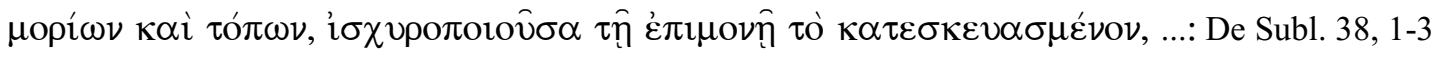

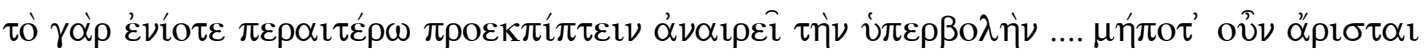

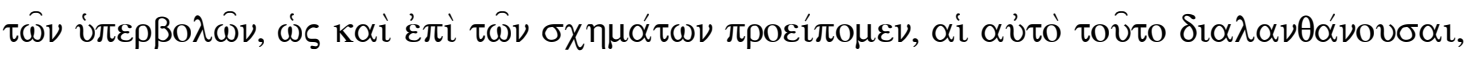

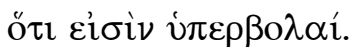

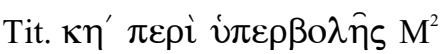

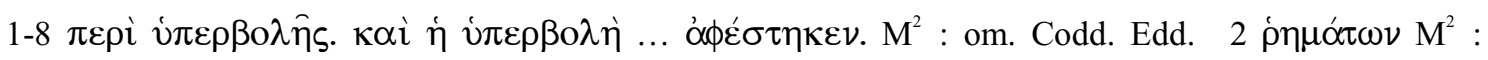

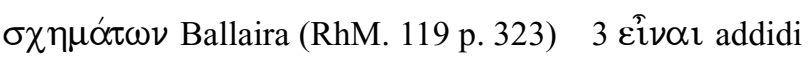


TOY AYTOY ПEPI T $\Omega N$ TH $\Sigma \Lambda$ E $\Xi E \Omega \Sigma \Sigma X H M A T \Omega N$. Speng. p. 27

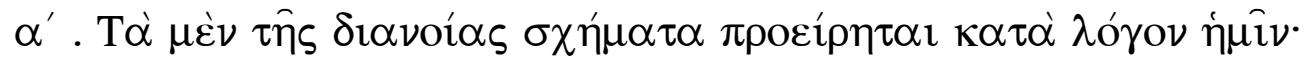

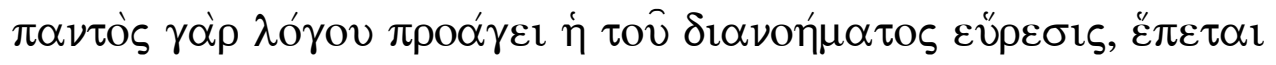

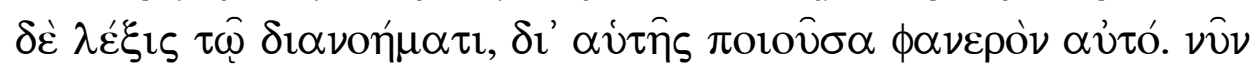

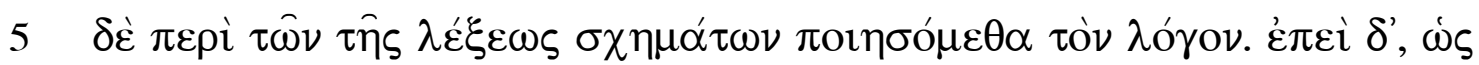

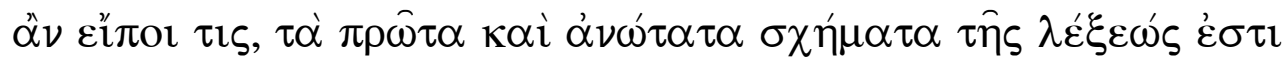

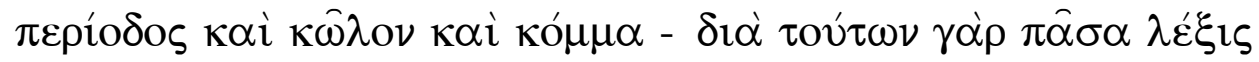

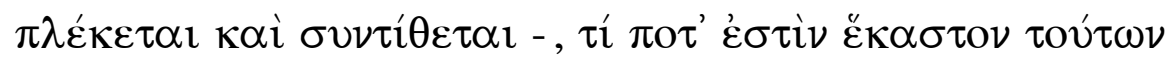

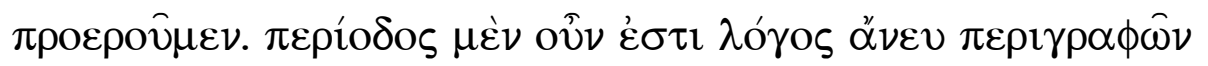

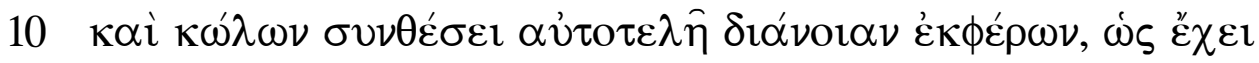

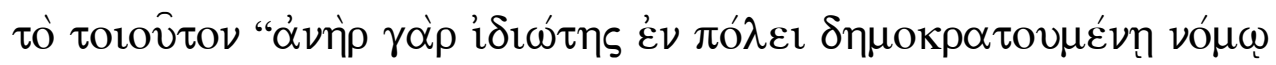

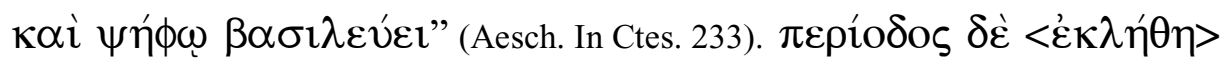

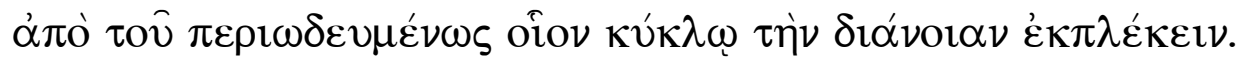

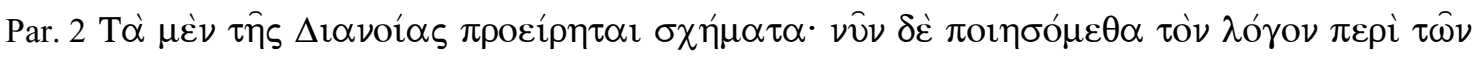

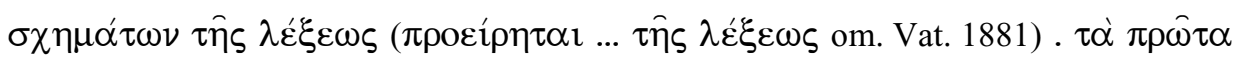

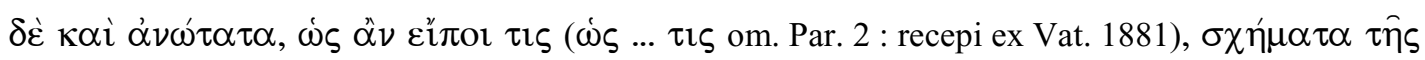

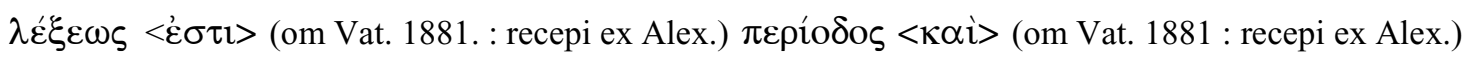

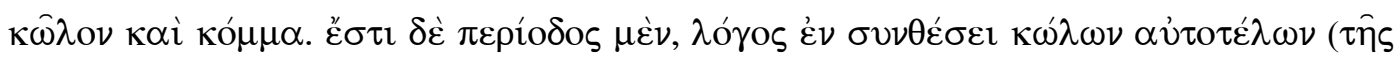

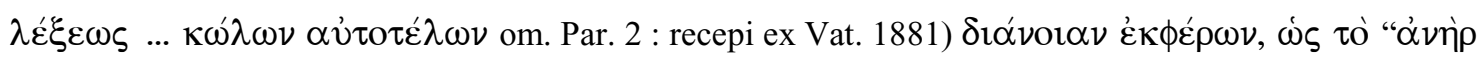

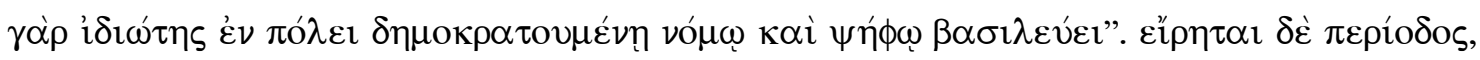

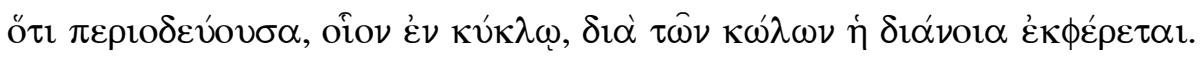

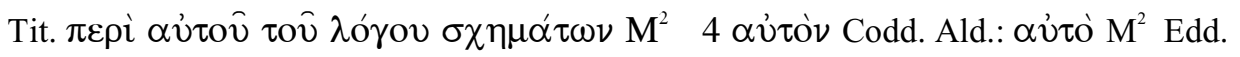

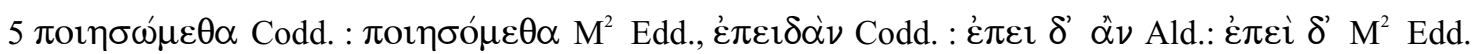

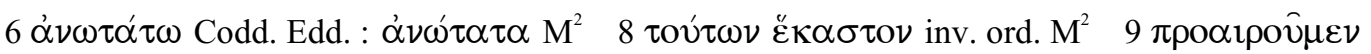

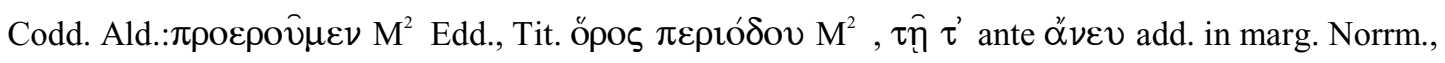

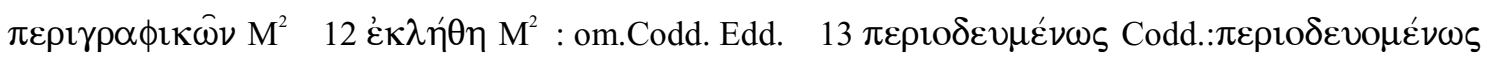
Edd.: $\pi \varepsilon \rho 1 \omega \delta \varepsilon v \mu \varepsilon ́ v \omega \varsigma$ V Pal. $\mathrm{M}^{2}$ 


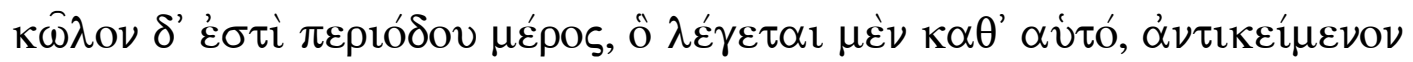

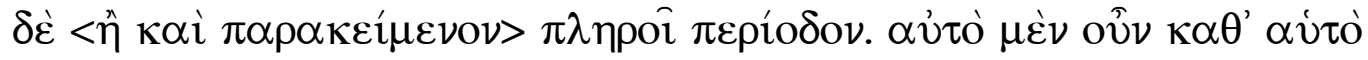

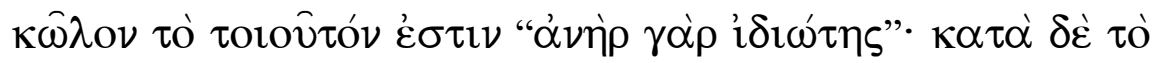

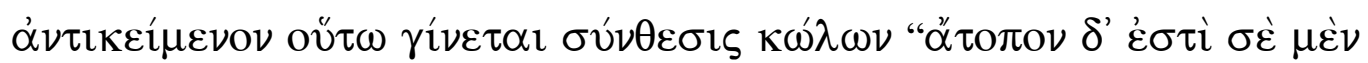

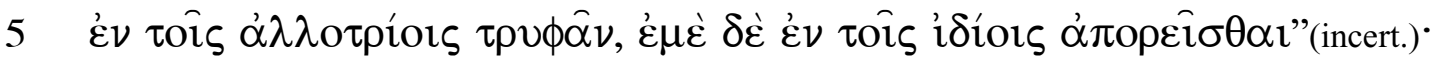

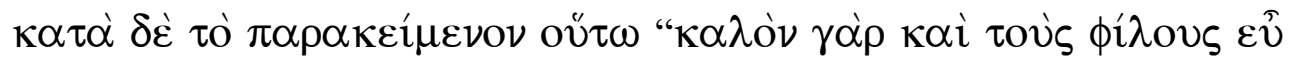

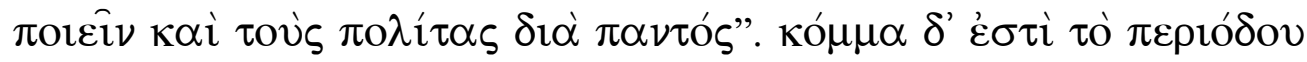

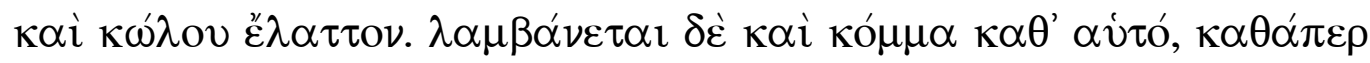

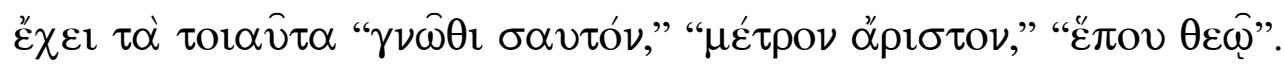

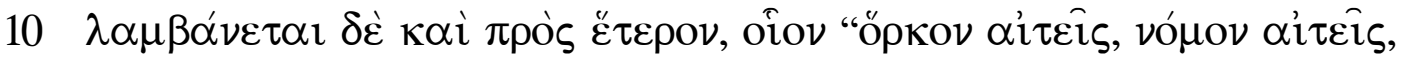
$\delta \eta \mu о \kappa \rho \alpha i ́ \alpha \nu \alpha i \tau \varepsilon i \varsigma$ ”" (Aesch. In Ctes. 198).

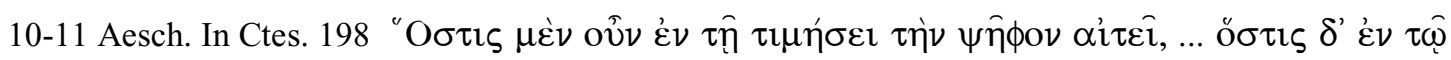

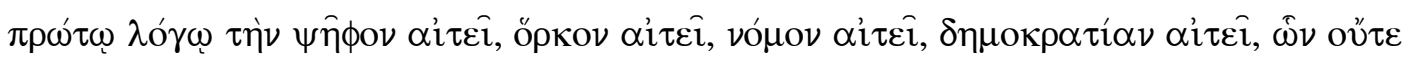

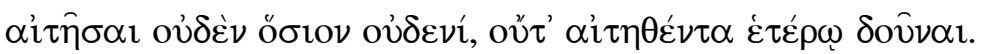

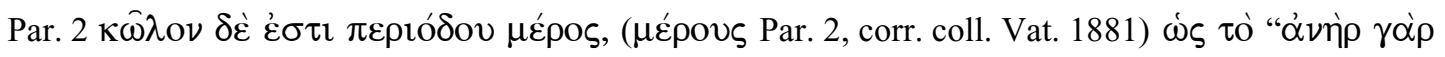

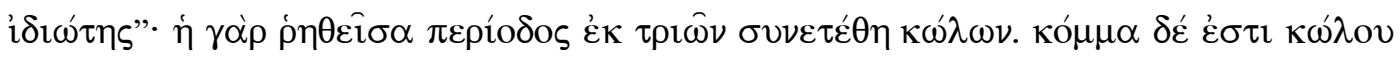

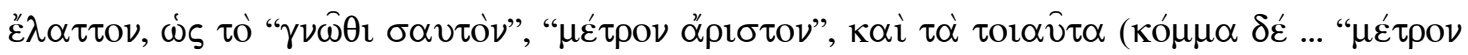

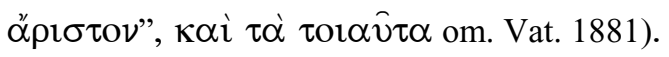

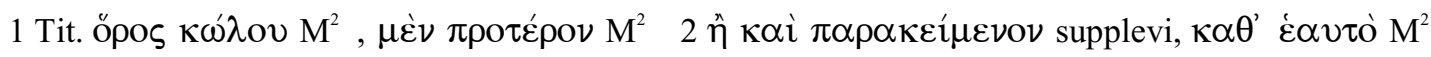

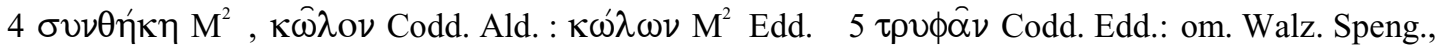

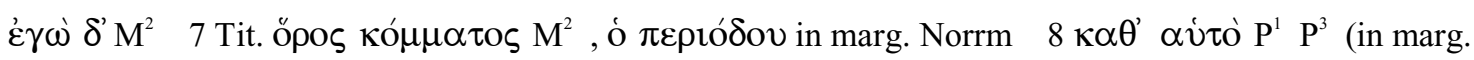

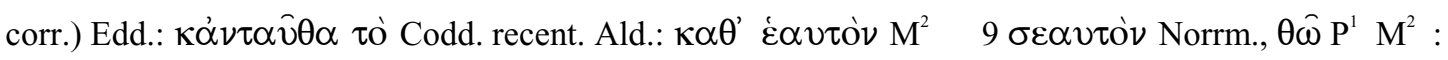
$\theta \varepsilon \omega \hat{~ C o d d . ~ r e c e n t . ~ E d d . ~} 11 \delta \eta \mu о \kappa \rho \alpha \varepsilon i ́ \alpha \nu \mathrm{P}^{1} \mathrm{M}^{1}: \delta \eta \mu о \kappa \rho \alpha \tau i ́ \alpha \nu \mathrm{M}^{2}$ Codd.recent. Edd. 


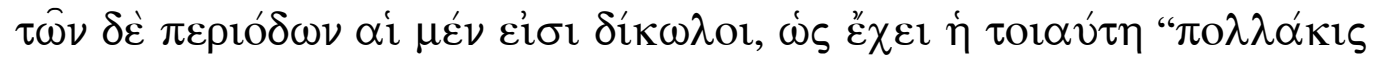

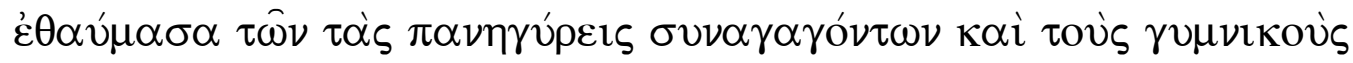

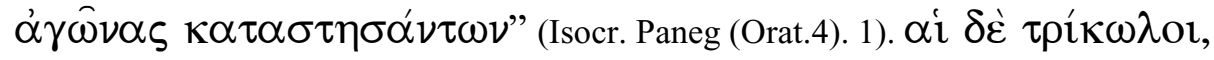

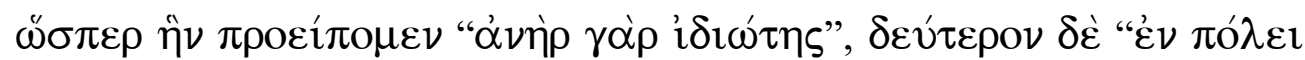

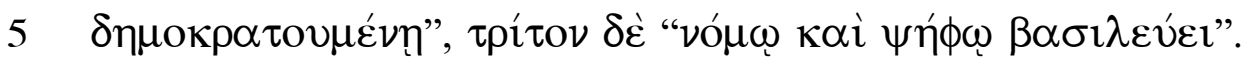

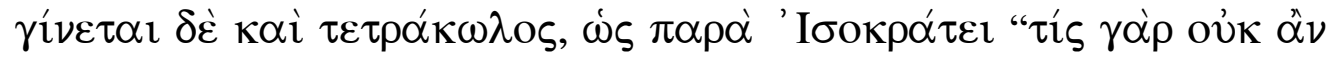

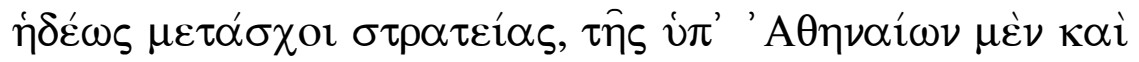

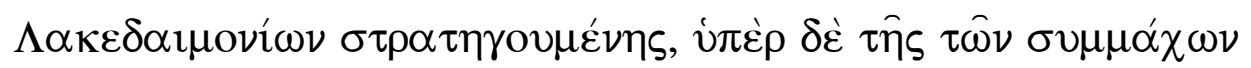

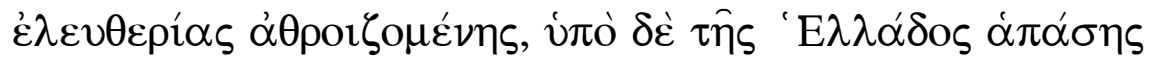

$10 \dot{\varepsilon} \kappa \pi \varepsilon \mu \pi \circ \mu \varepsilon \dot{\varepsilon} \eta \eta \varsigma$

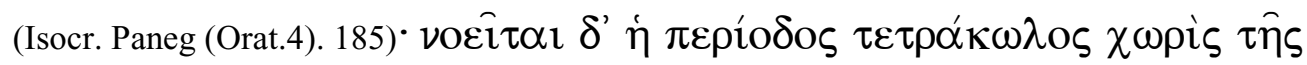

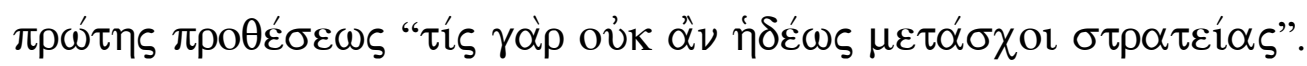

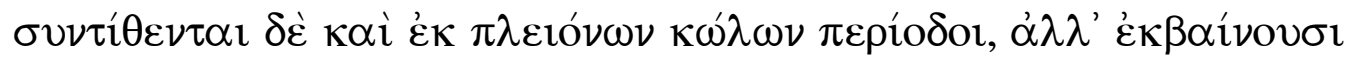

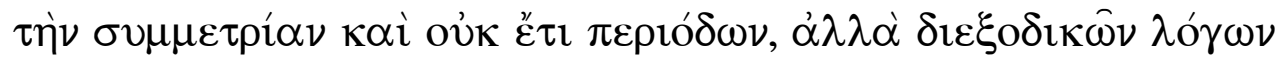

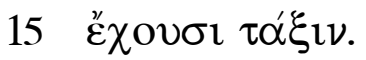

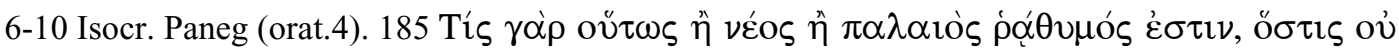

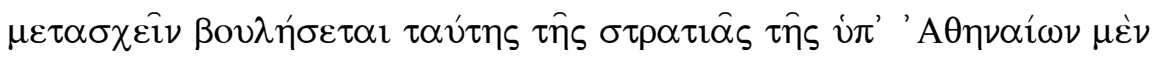

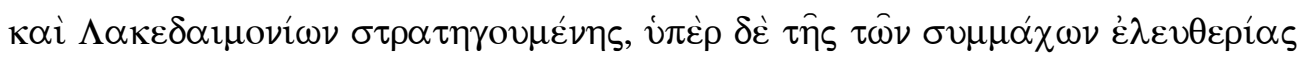

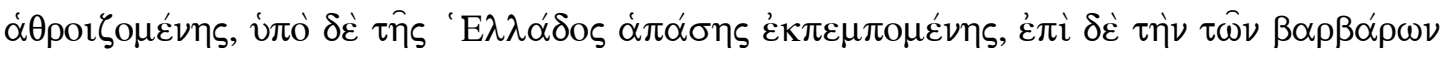

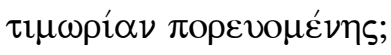

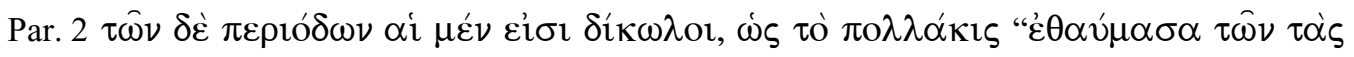

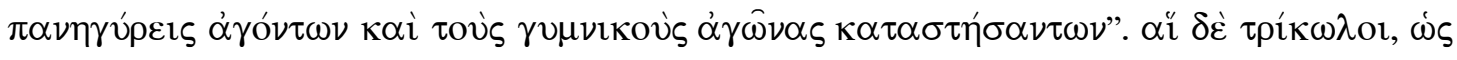

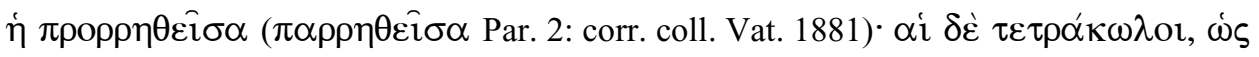

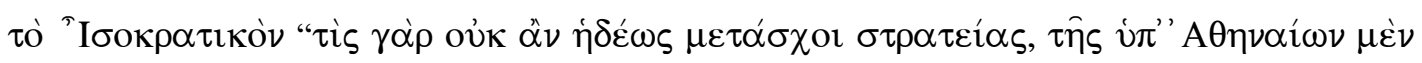

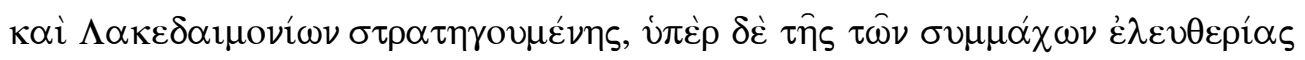

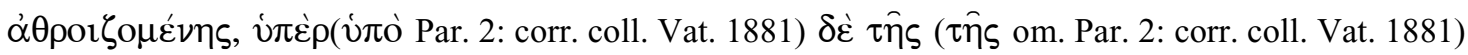

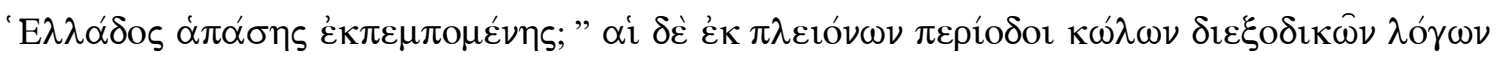

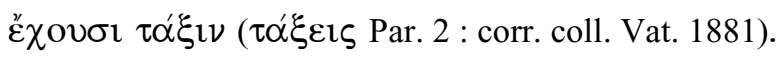

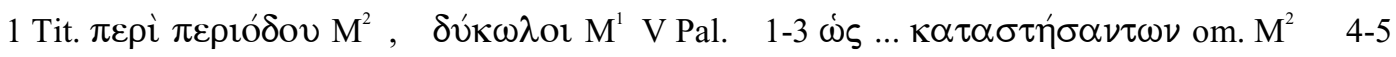

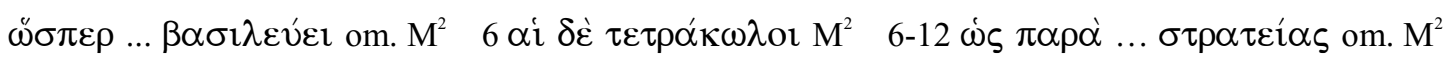

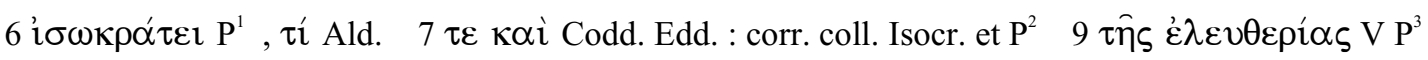

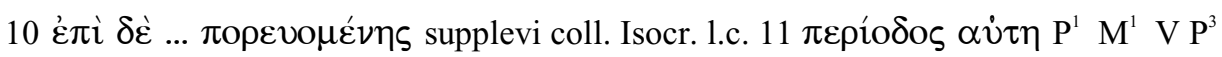




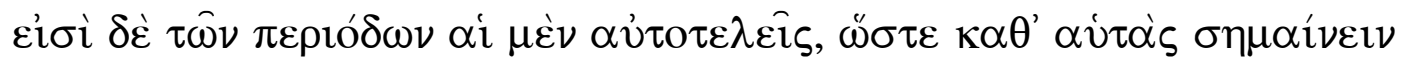

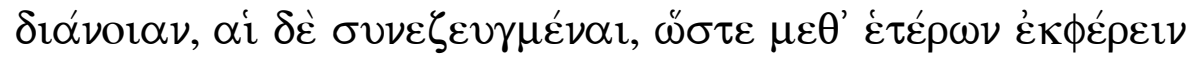

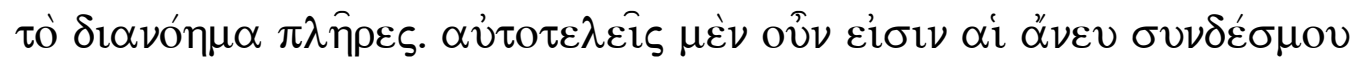

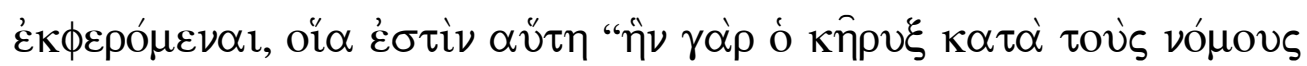

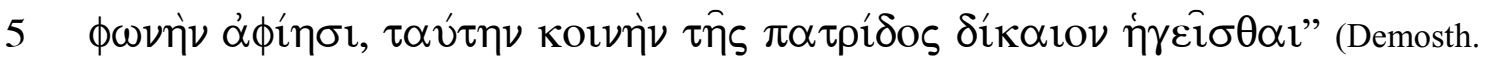

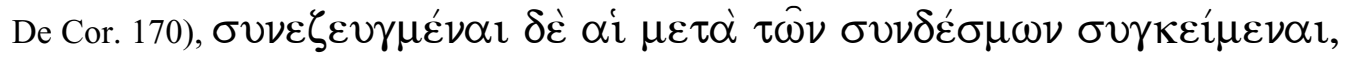

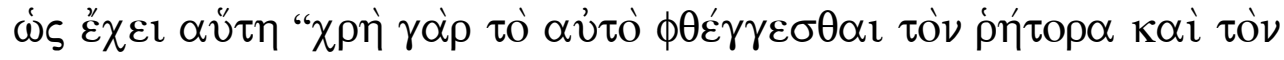

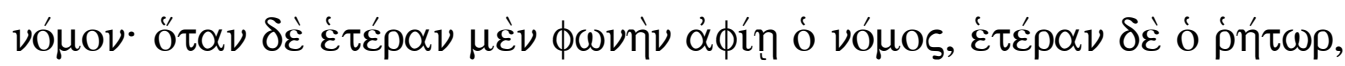

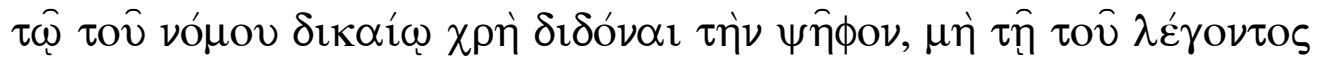

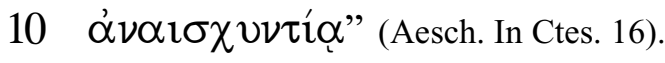

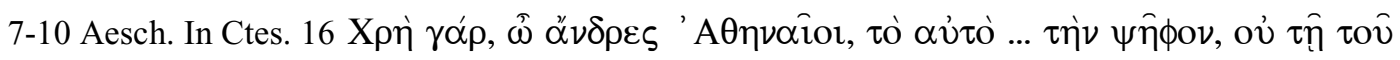

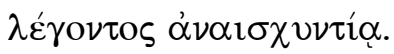

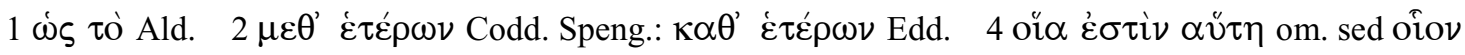

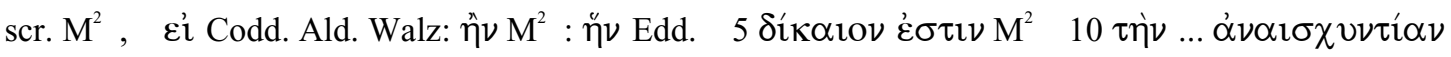

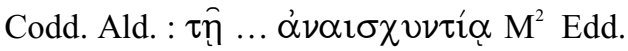


$\beta^{\prime}$. ПЕРI ANA $\triangle \mathrm{I} \Pi \Lambda \Omega \Sigma$ E $\Omega \Sigma$ Н ПА $\Lambda \Lambda \Lambda$ ОГIA $\Sigma$ Н ЕПАNА $\Lambda$ НЧЕ $\Omega \Sigma$.

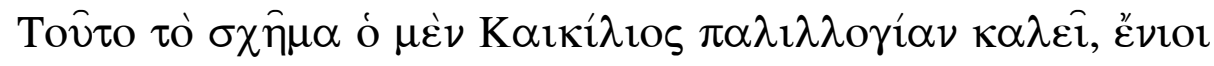

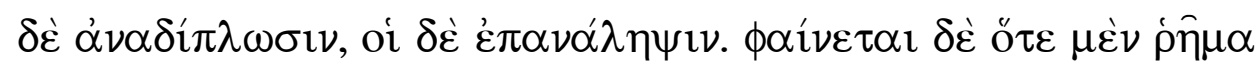

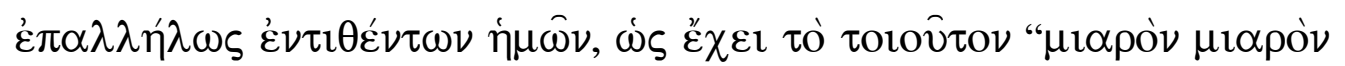

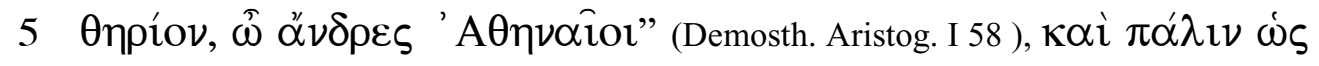

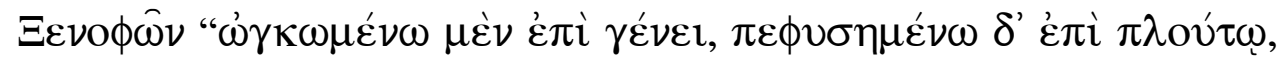

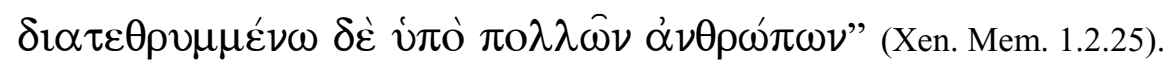

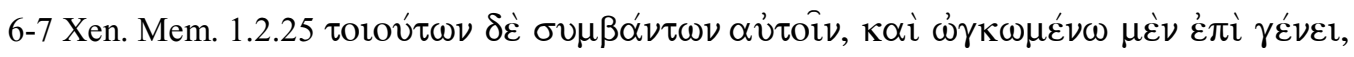

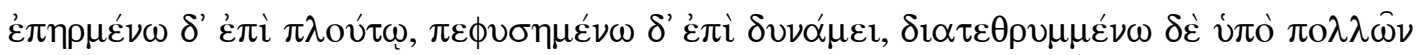
$\dot{\alpha} \nu \theta \rho \omega \dot{\pi} \omega \nu, \ldots$.

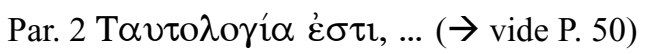

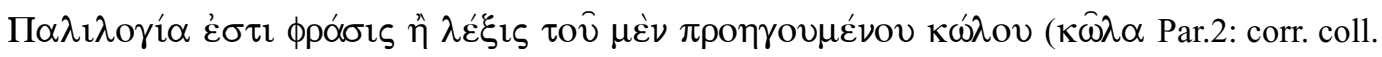

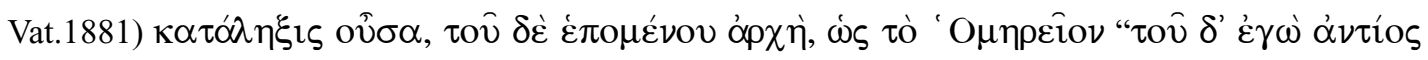

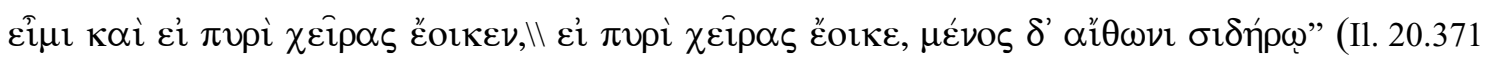

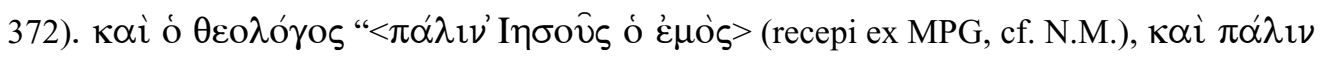

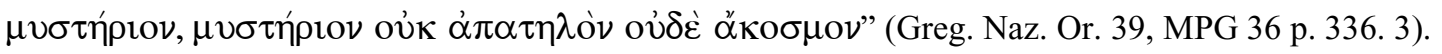

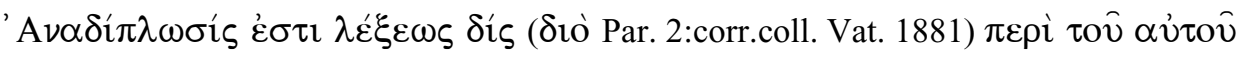

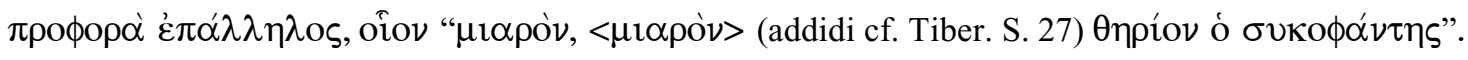

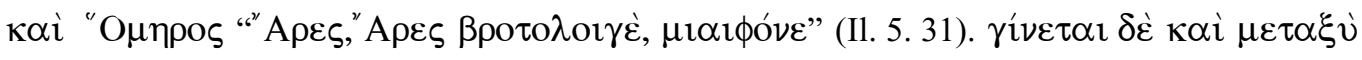

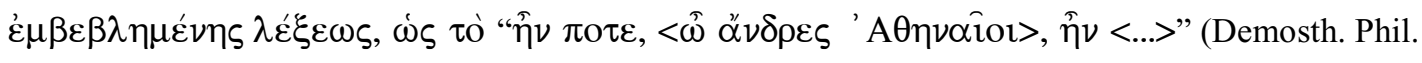

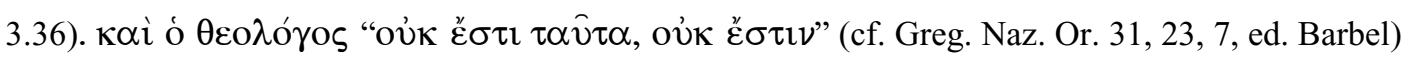

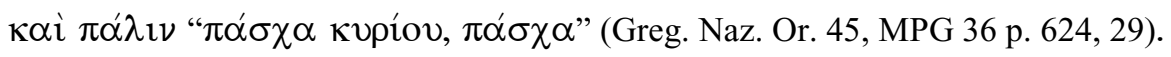

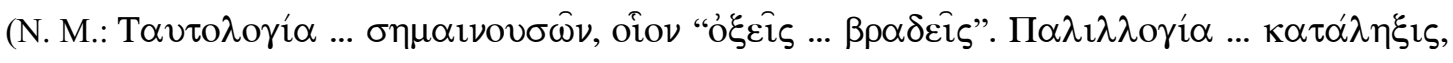

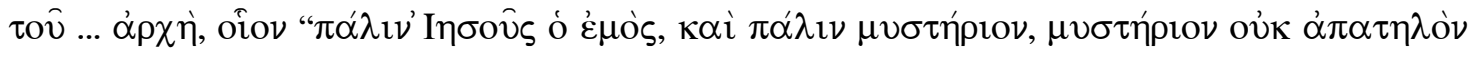

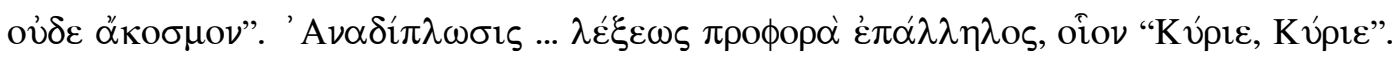

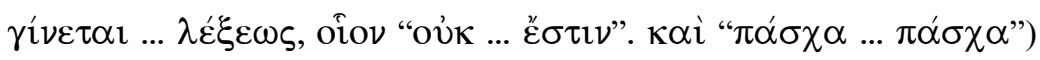

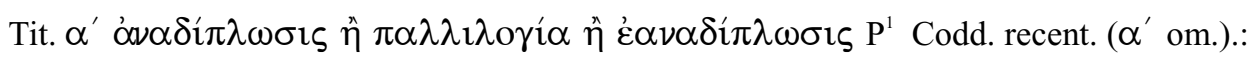

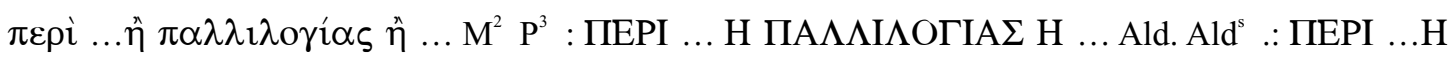
ПА $\Lambda \Lambda \Lambda$ ОГІА $\Sigma$ Н ... $\beta^{\prime}$ Norrm. : $\beta^{\prime}$. ПЕРІ ... Н ПА $\Lambda \Lambda \Lambda$ ОГІА $\Sigma$... Edd.

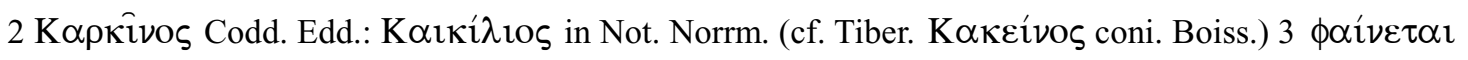

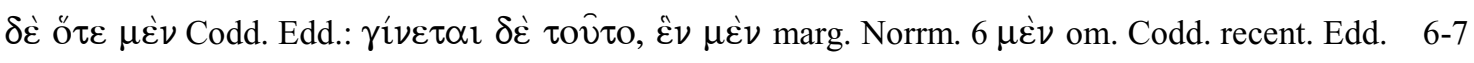

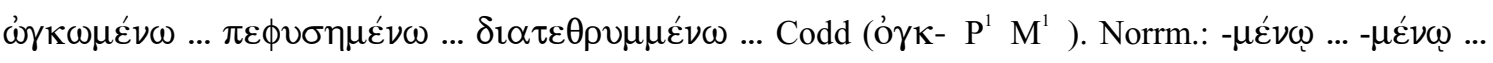

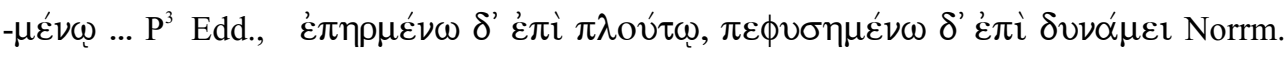




\section{$\gamma^{\prime}$. ПЕРІ ЕПАNАФОРА $\Sigma$.}

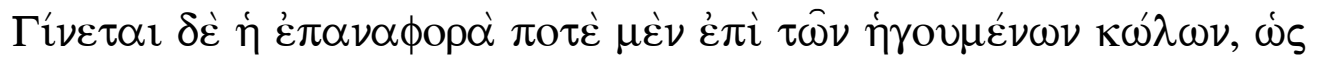

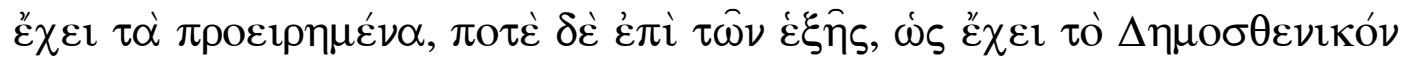

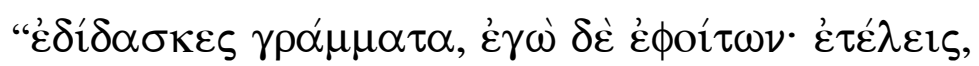

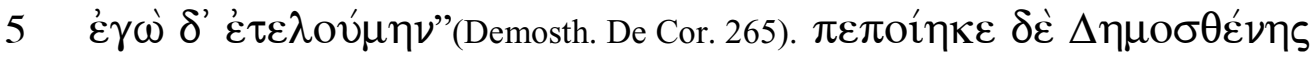

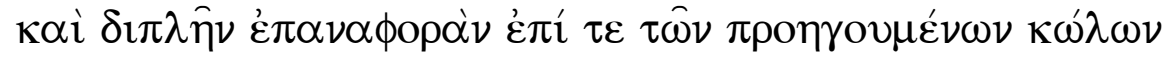

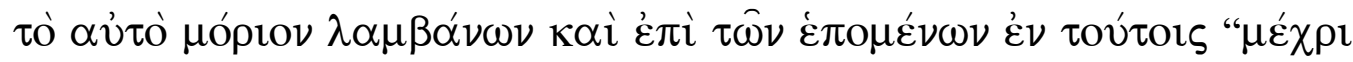

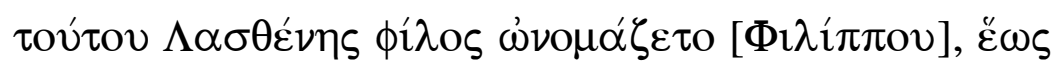

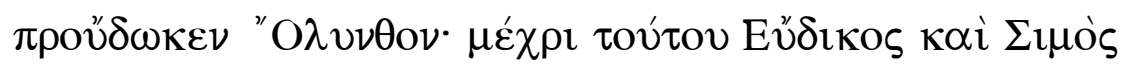

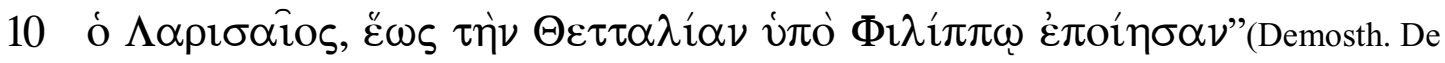

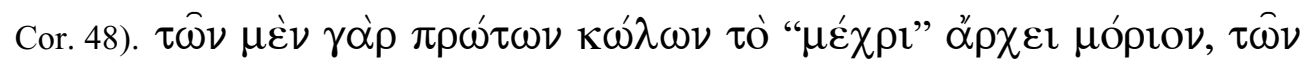

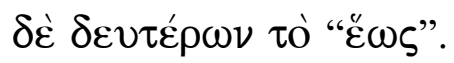

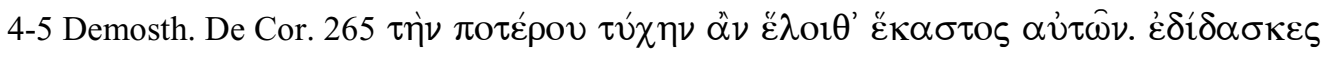

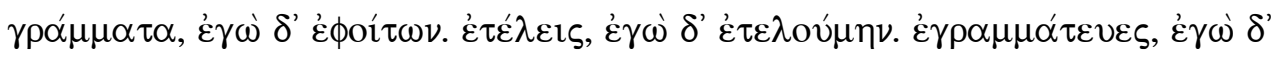

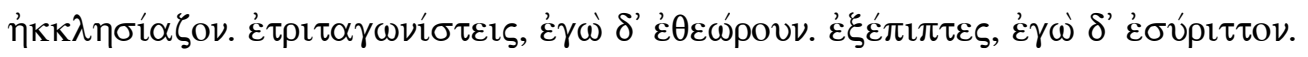

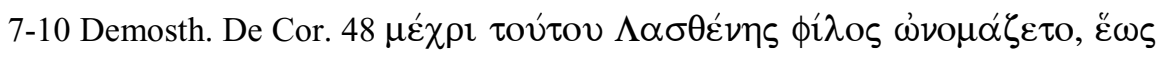

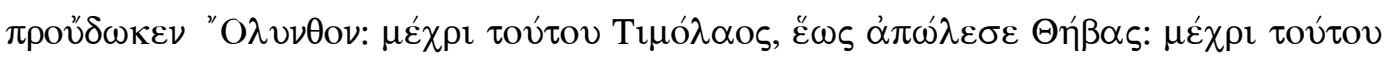

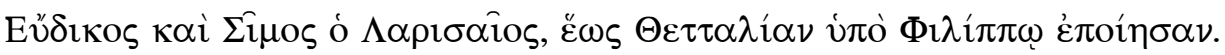

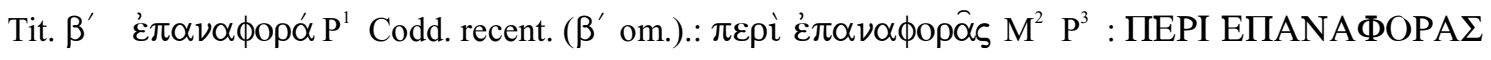
Ald. Ald ${ }^{s}:$ ПЕРI ЕПАNAПОРА $\Sigma \gamma^{\prime}$ Norrm. : $\gamma^{\prime}$. ПЕРI ЕПАNАФOPА $\Sigma$ Edd.

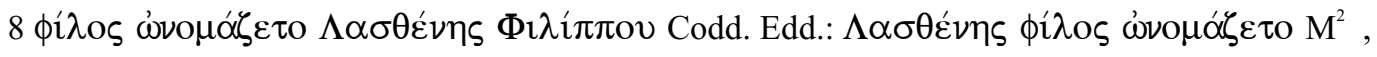

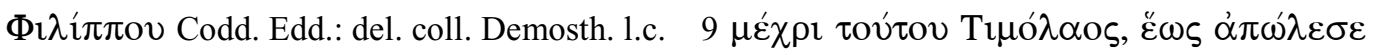

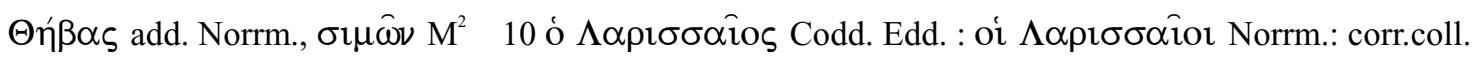

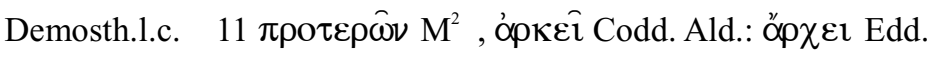




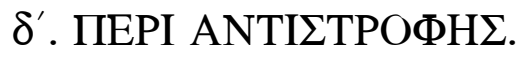

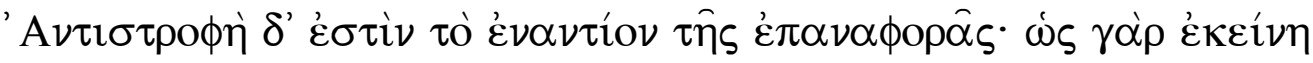

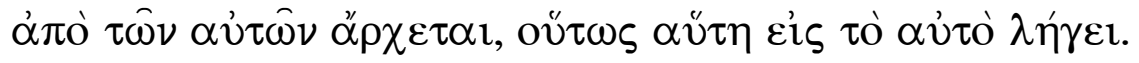

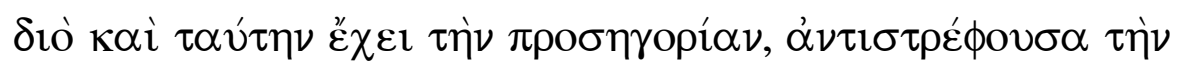

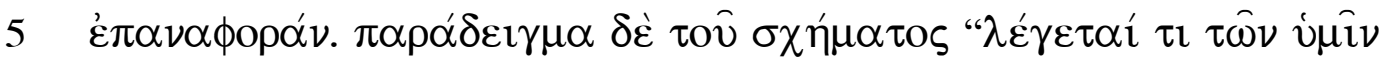

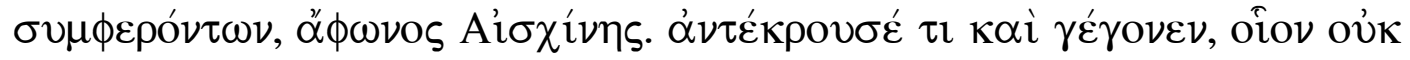

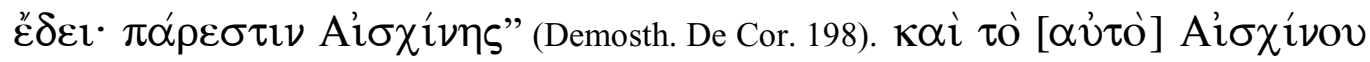

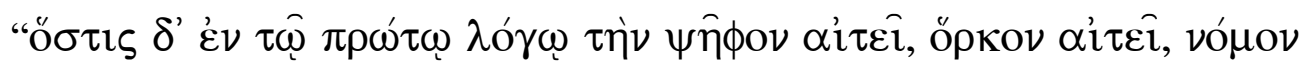
$\alpha i \tau \varepsilon \hat{i}, \delta \eta \mu о \kappa \rho \alpha \tau i \alpha \nu \alpha i \tau \varepsilon \hat{\imath} "$ (Aesch. In Ctes. 198).

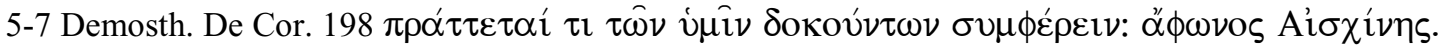

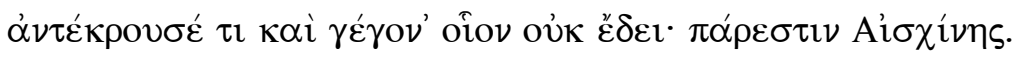

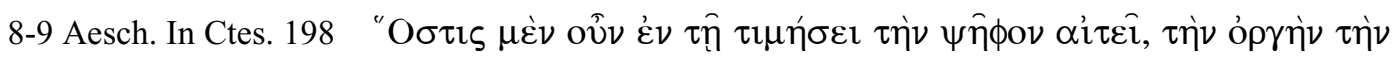

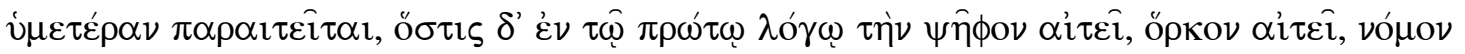

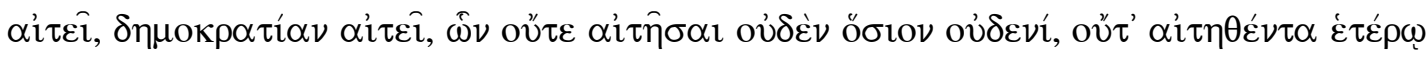

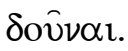

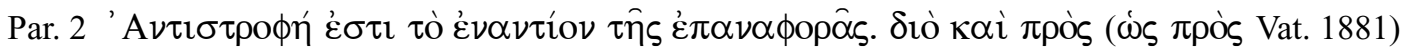

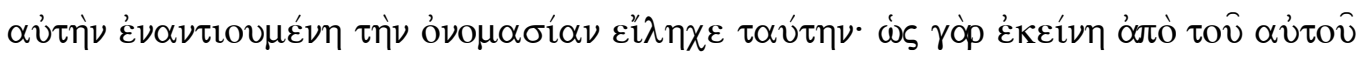

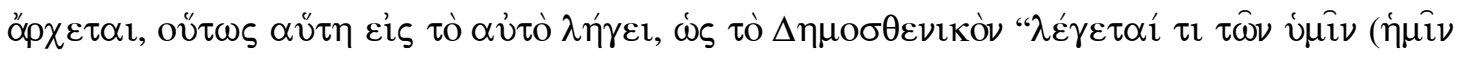

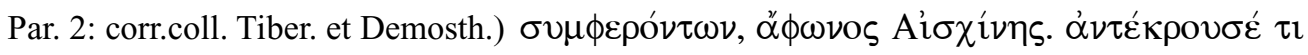

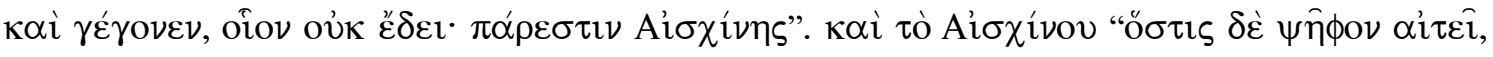

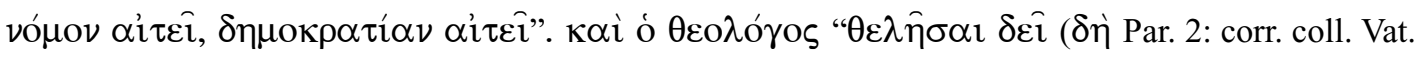

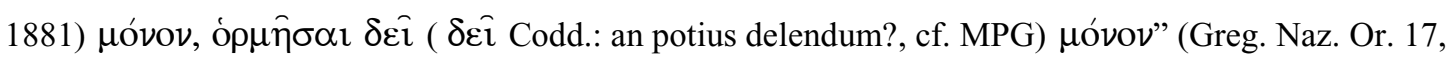

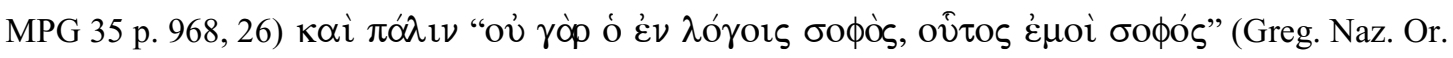

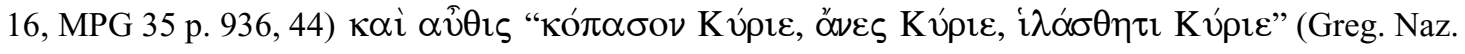
Or. 16, MPG 35 p. 952, 8).

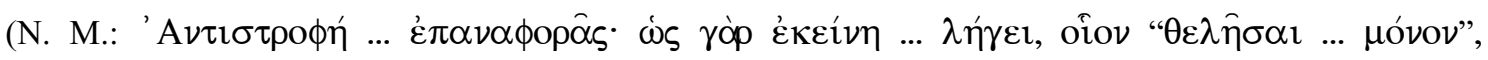

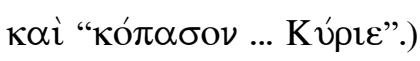

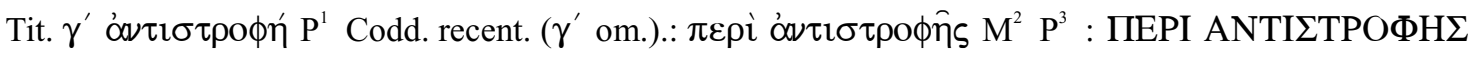
Ald. Ald ${ }^{s} .:$ ПEPI ANTI $\Sigma$ TPO $\Phi H \Sigma \delta^{\prime}$ Norrm. : $\delta^{\prime}$. ПEPI ANTI $\Sigma$ TPO $\Phi H \Sigma$ Edd.

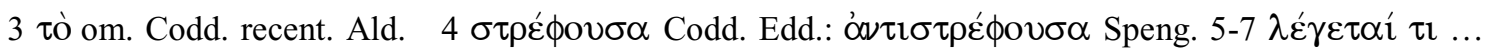

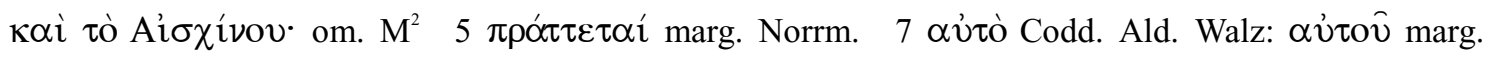

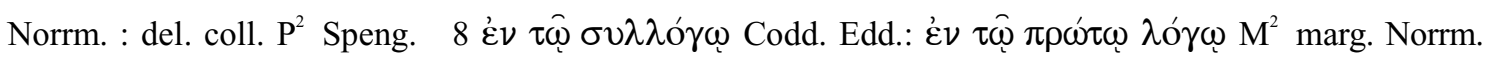
cf. Aisch. $9 \delta \eta \mu о \kappa \rho \alpha \tau i ́ \alpha \nu \mathrm{P}^{1}$ 


\section{$\varepsilon^{\prime}$. ПЕРІ $\Sigma$ YMП $\Lambda \mathrm{OKH} \Sigma \mathrm{H} \Sigma \mathrm{YN} \Theta \mathrm{E} \Sigma \mathrm{E} \Omega \Sigma$.}

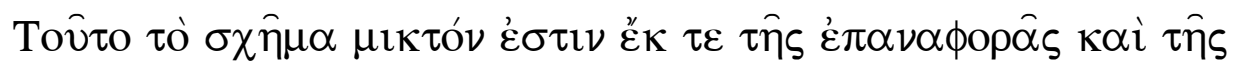

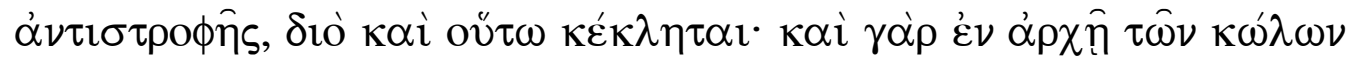

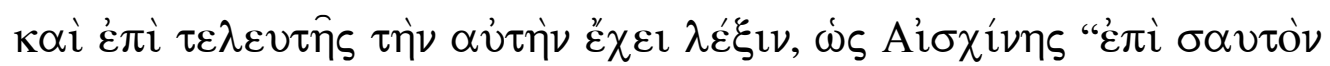

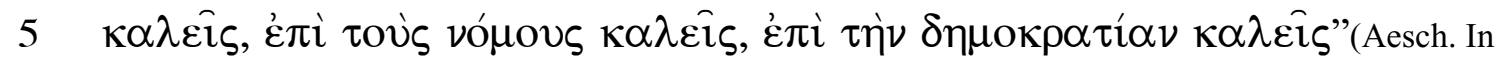
Ctes. 202).

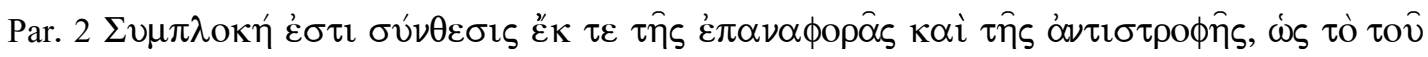

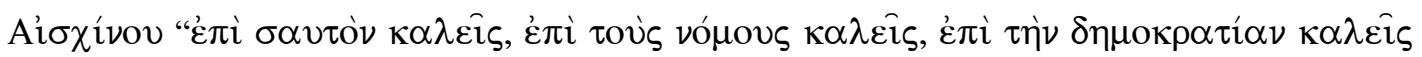

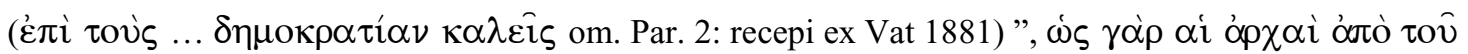
$\alpha$

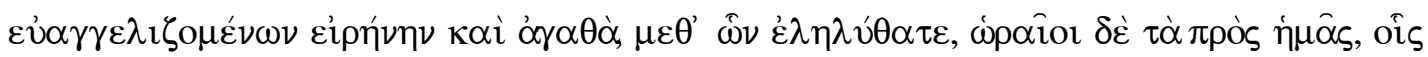

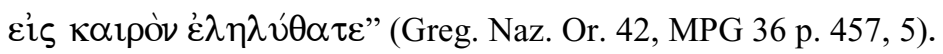

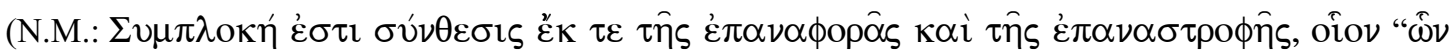

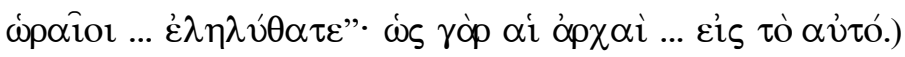

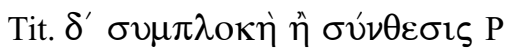




\section{$\varsigma^{\prime}$. ПЕPI $\Sigma$ YN $\Omega$ NYMIA $\Sigma$.}

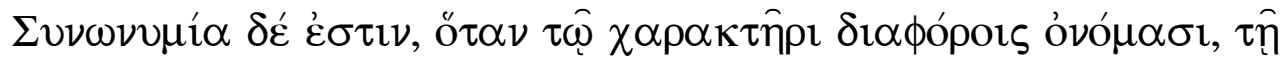

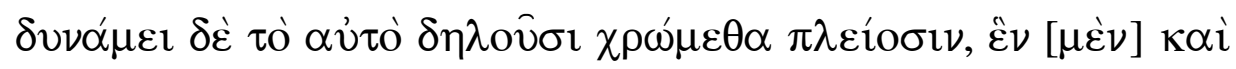

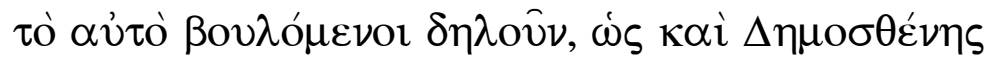

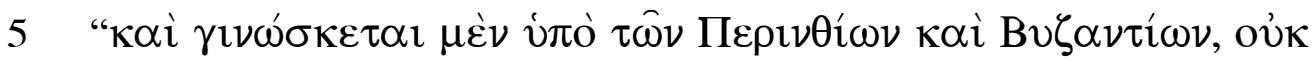

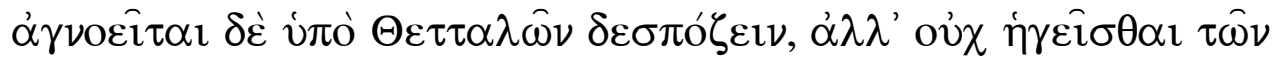

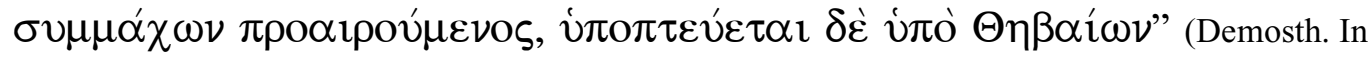
Epist. Philipp. 3-4).

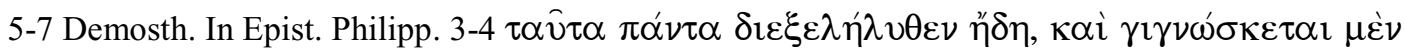

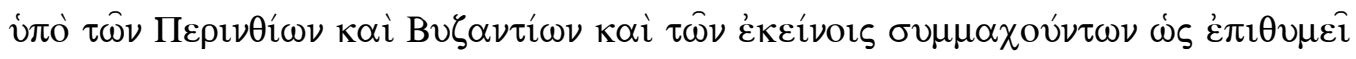

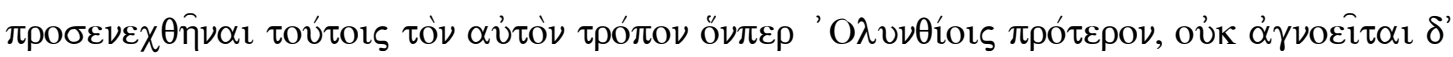

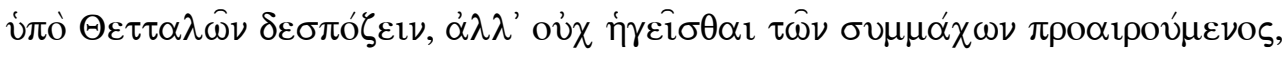

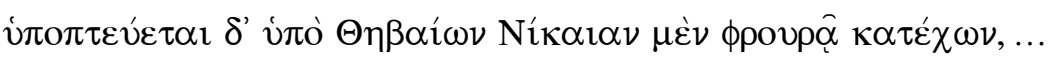

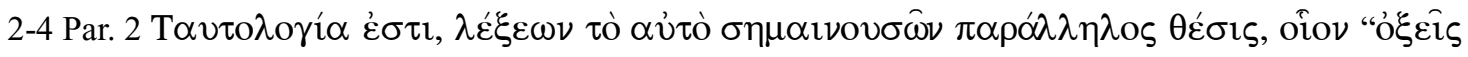

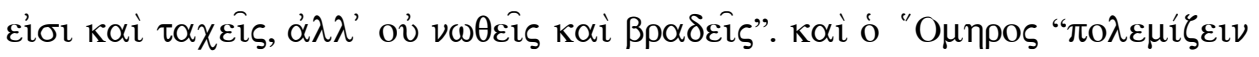

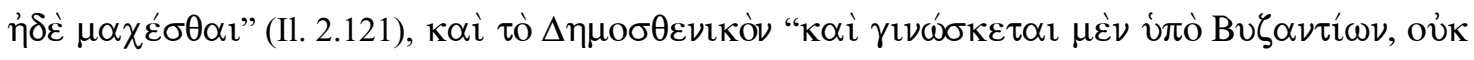

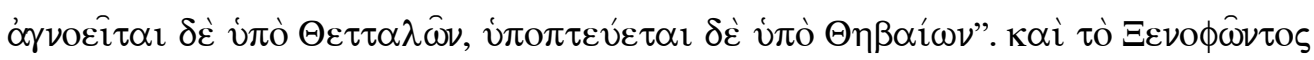

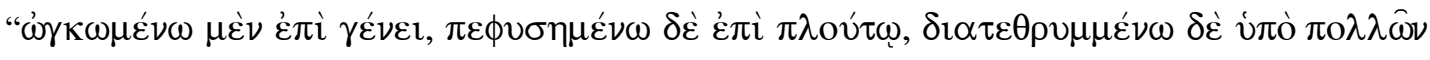

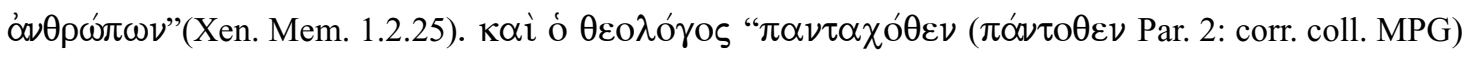

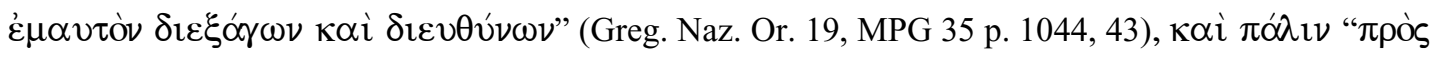

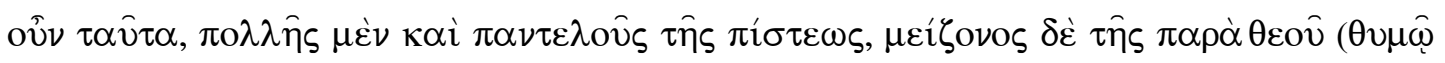

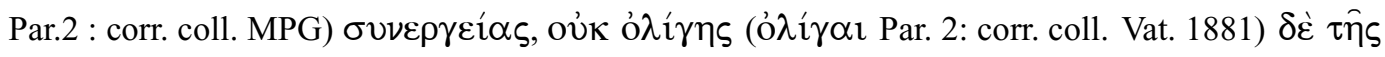

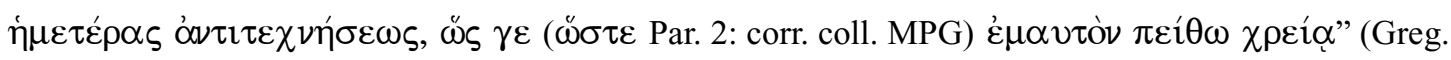

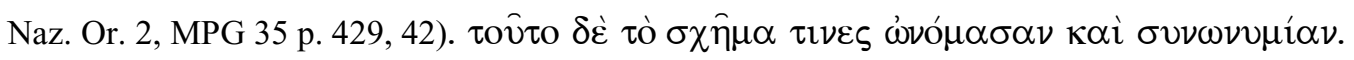

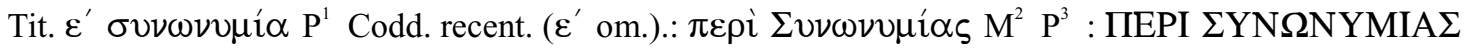
Ald. Ald ${ }^{\varsigma}$. חEPI $\Sigma$ YN $\Omega$ NYMIA $\Sigma \varsigma^{\prime}$ Norrm. $: \varsigma^{\prime}$. ПEPI $\Sigma$ YN $\Omega$ NYMIA $\Sigma$ Edd.

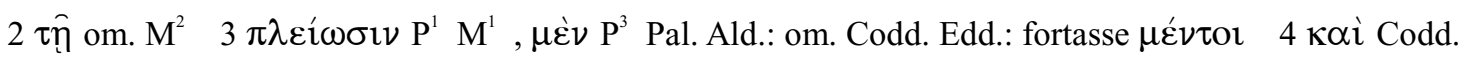

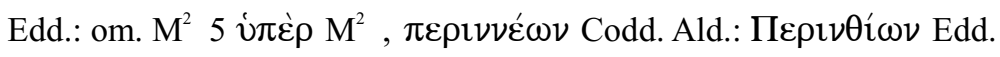




\section{$\zeta^{\prime}$. ПЕPI ЕПАNO $\triangle O Y$.}

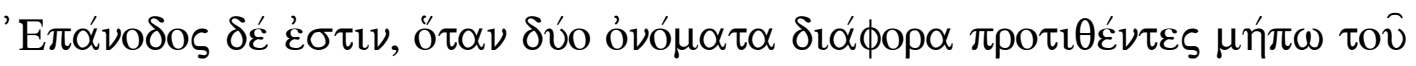

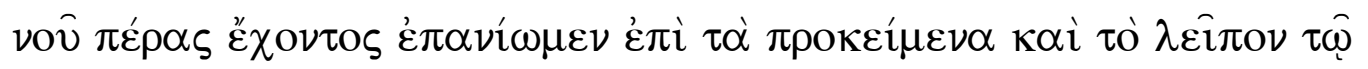

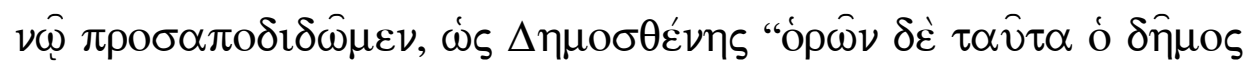

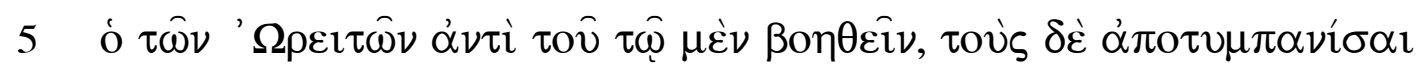

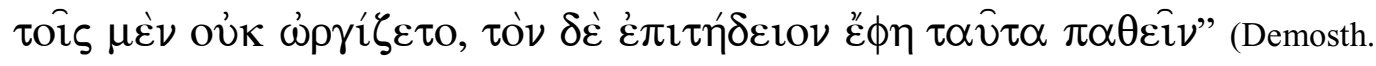

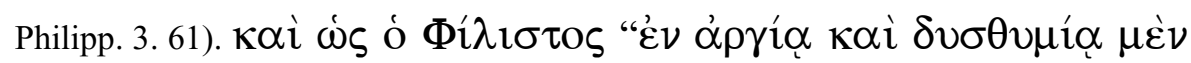

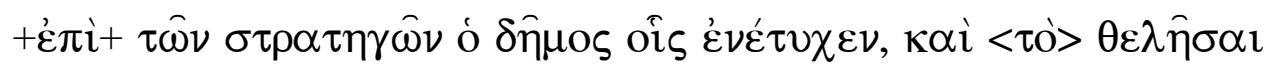

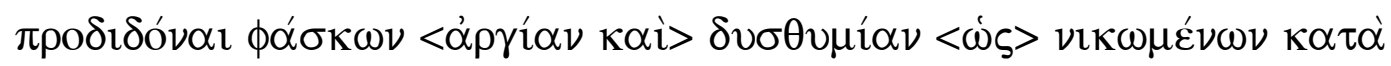

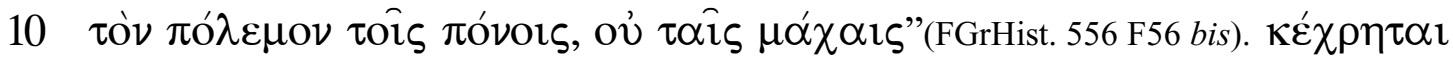

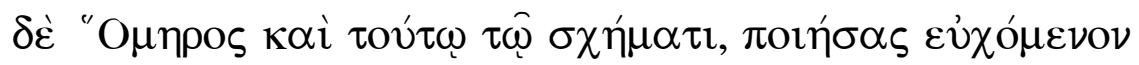

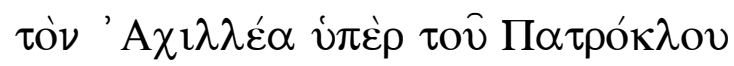

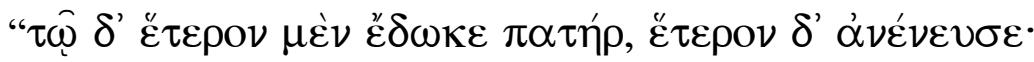

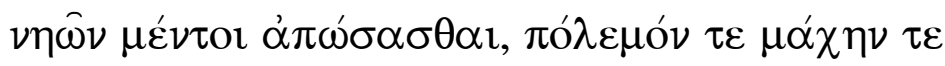

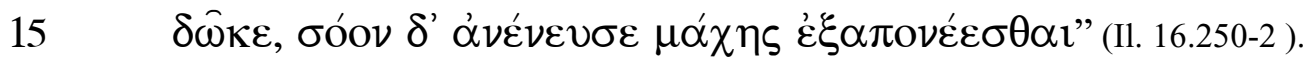

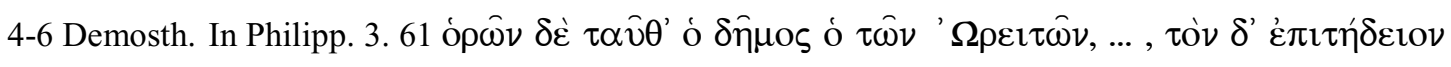

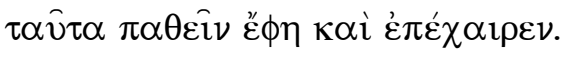

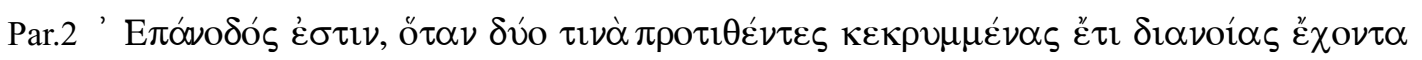

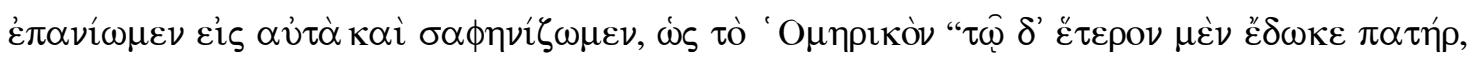

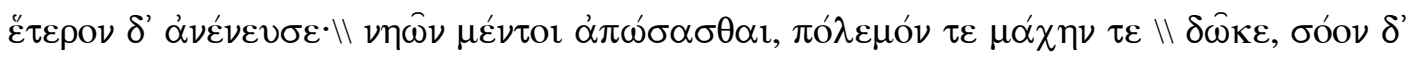

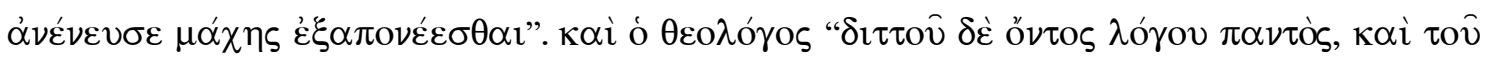

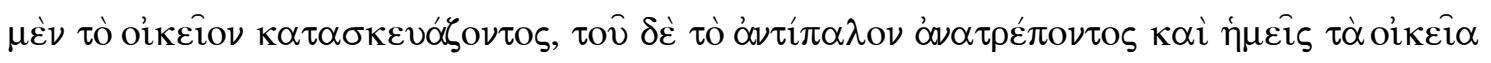

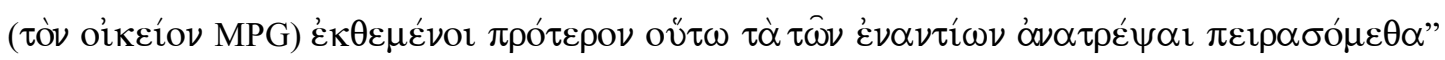

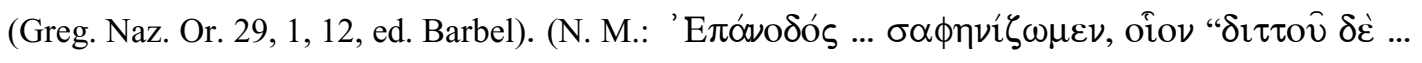
$\pi \varepsilon 1 \rho \alpha \sigma o ́ \mu \varepsilon \theta \alpha "$.

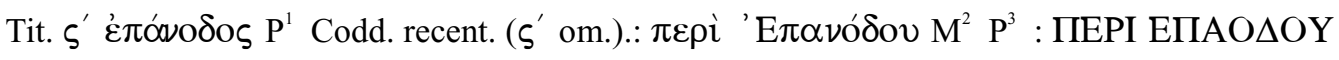
Ald. Ald ${ }^{\mathrm{s}} .:$ ПЕPI ЕПАNO $\Delta \mathrm{OY} \zeta^{\prime}$ Norrm. : $\zeta^{\prime}$. ПЕPI EПANO $\Delta \mathrm{OY}$ Edd.

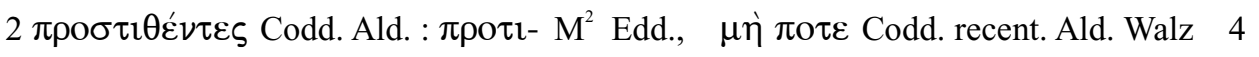

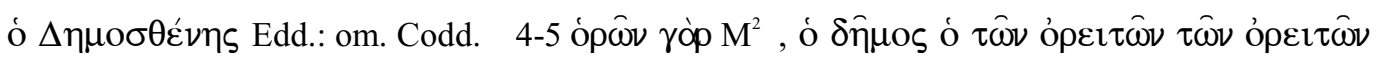

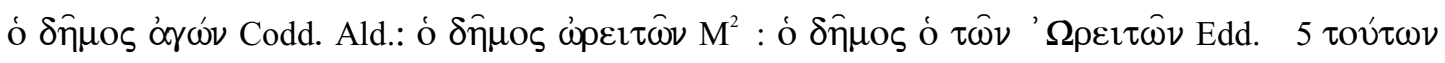

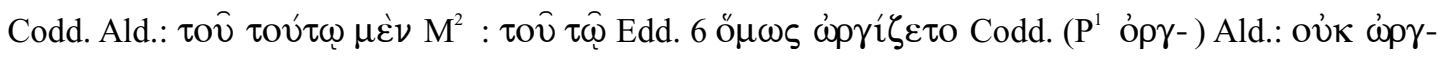

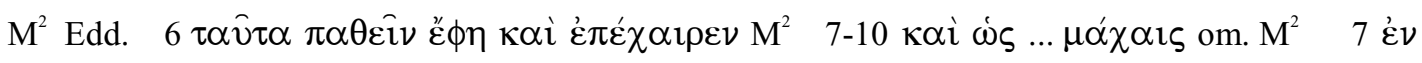

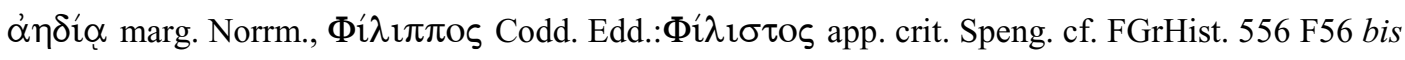

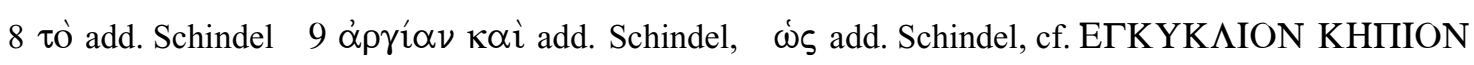

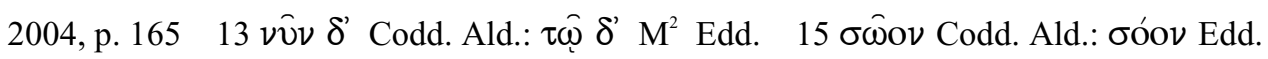


$\eta^{\prime}$. ПЕPI KАIMAKO $\Sigma$.

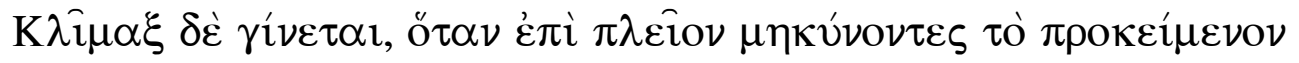

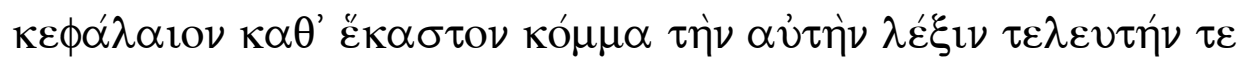

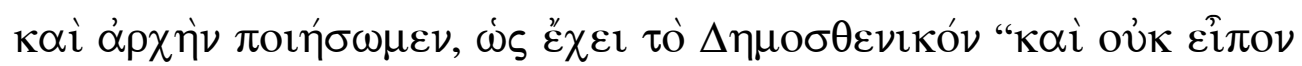

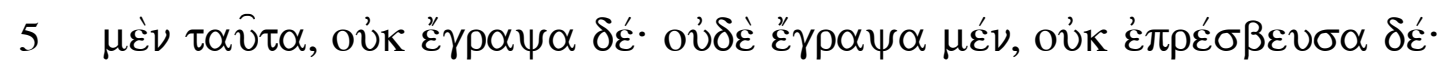

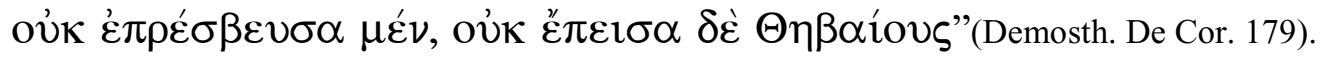

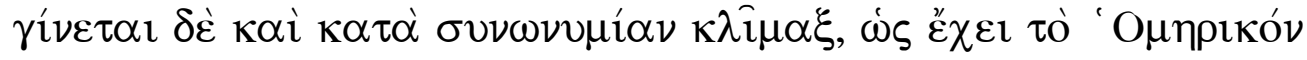

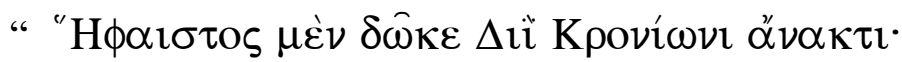

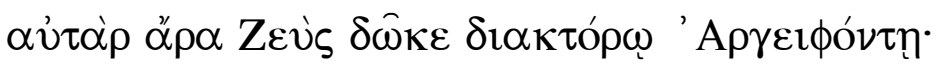

10

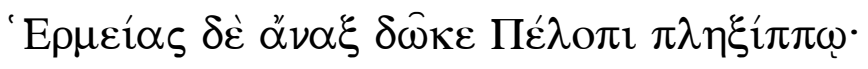

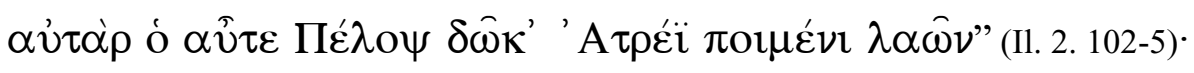

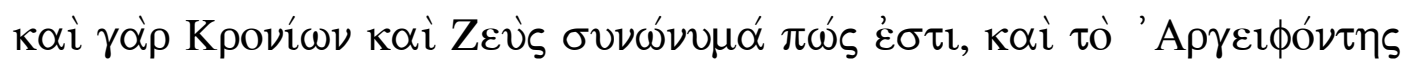

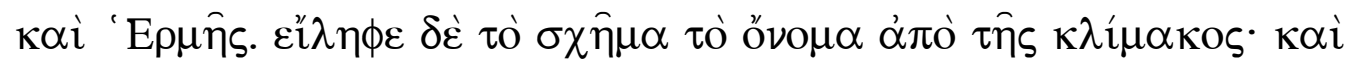

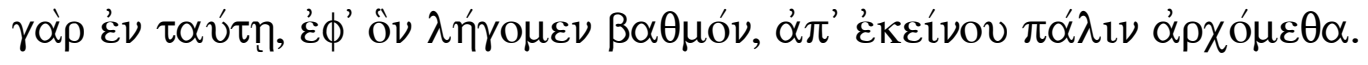

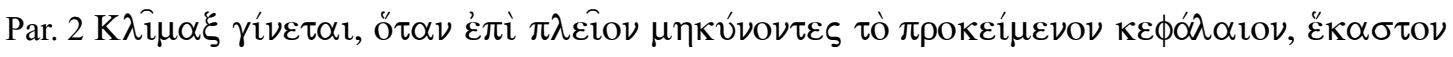

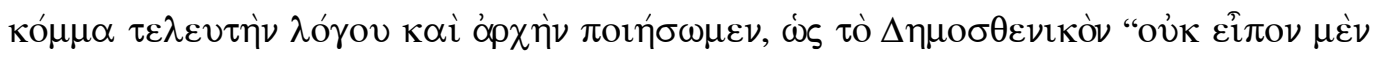

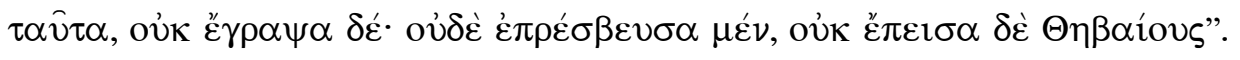

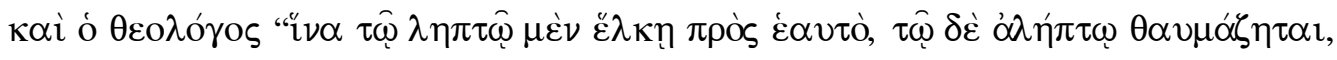

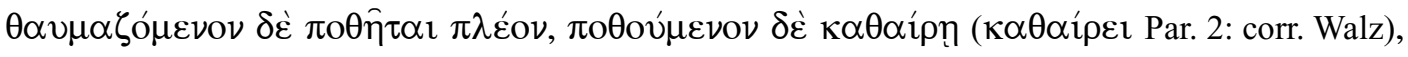

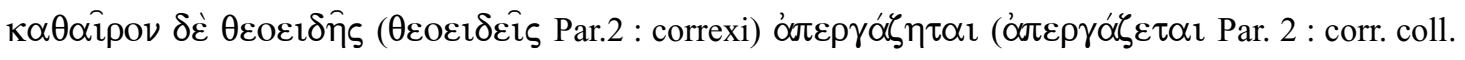

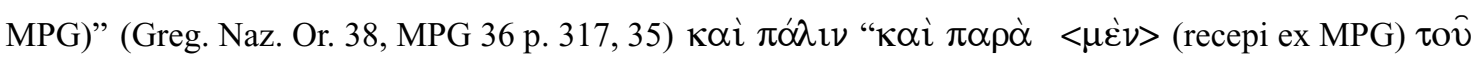

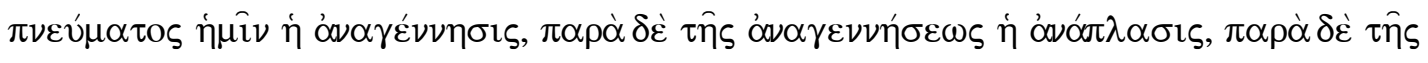

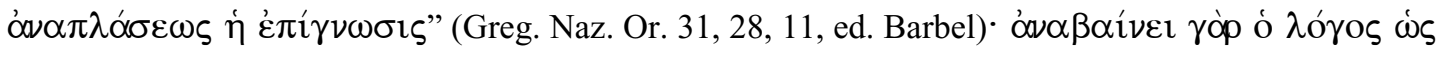
$\delta i \grave{\alpha} \kappa \lambda \dot{\imath} \mu \alpha \kappa o s$.

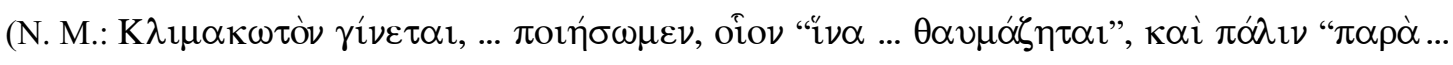

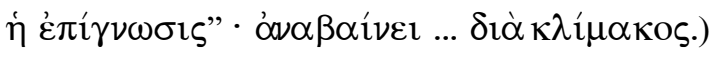

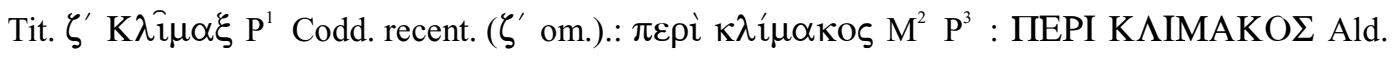

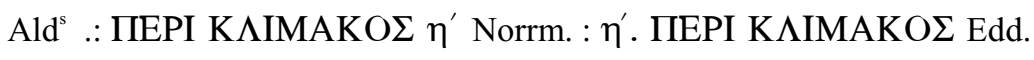

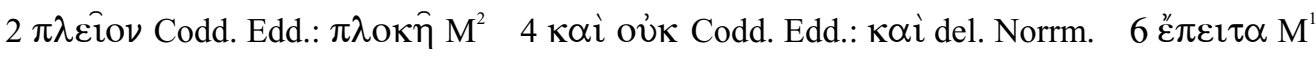

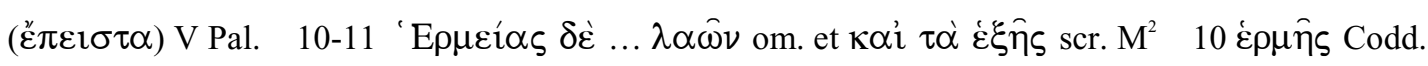

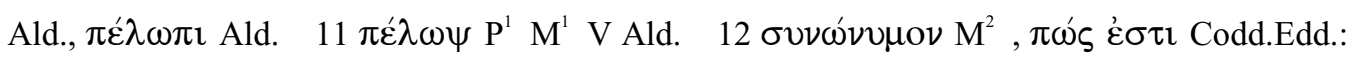

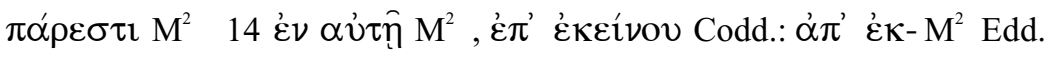




\section{$\theta^{\prime}$. ПЕPI ПРО $\Sigma \Delta \mathrm{IA} \Sigma \mathrm{A} \Phi \mathrm{H} \Sigma \mathrm{E} \Omega \Sigma$.}

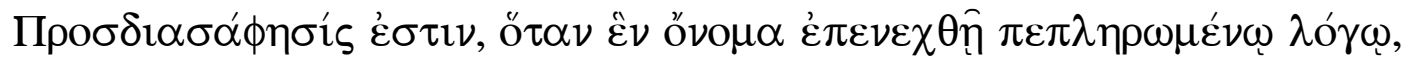

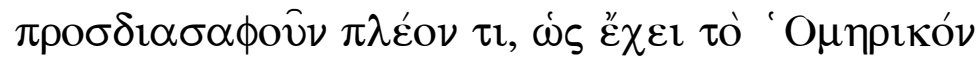

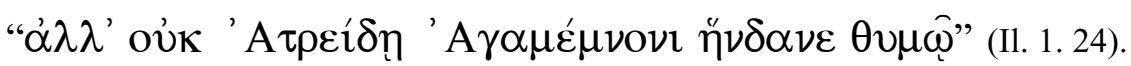

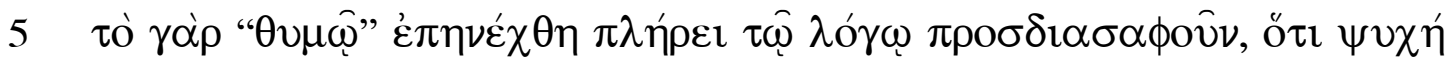

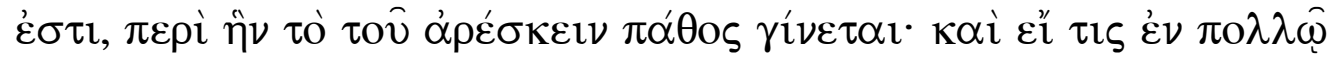

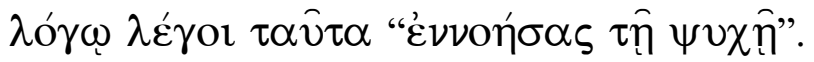

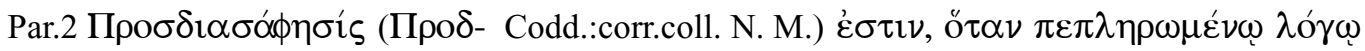

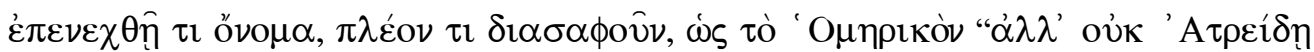

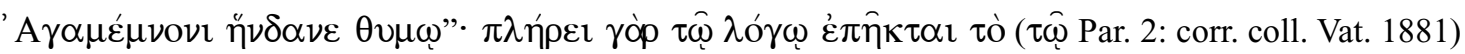

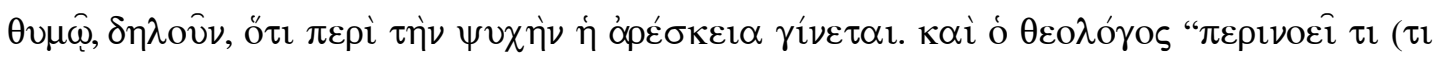

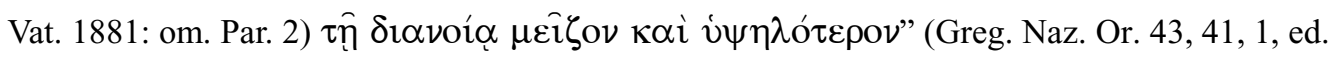
Boulenger).

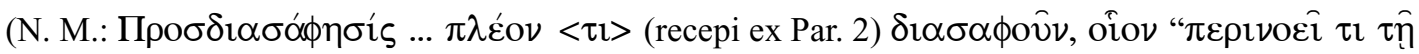

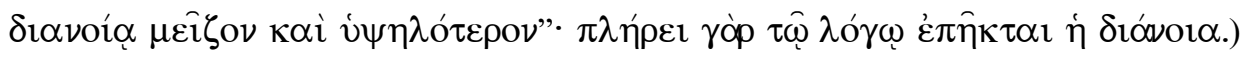

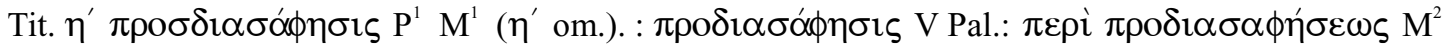
$\mathrm{P}^{3}:$ ПЕРІ ПРО $\Delta$ - Ald.Ald ${ }^{s}:$ ПЕРI ПРО $\Sigma \Delta-\theta^{\prime}$ marg. Norrm. : $\theta^{\prime}$. ПЕРI ПРО $\Sigma \Delta$ - Edd.

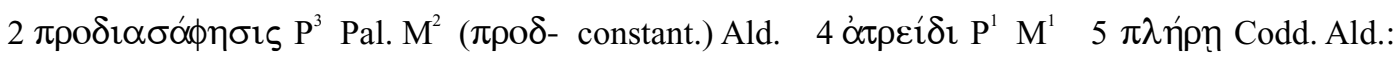
$\pi \lambda \eta \dot{p} \varepsilon \varepsilon \mathrm{M}^{2}$ Edd. 6 عl $\tau \imath \mathrm{M}^{2}$ 


\section{$\imath^{\prime}$. ПЕРI ПЕРІФРА $\Sigma$ Е $\Omega \Sigma$.}

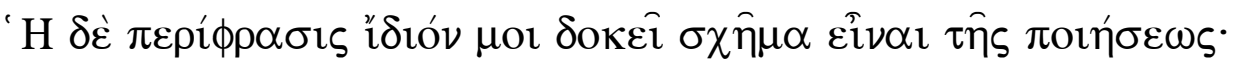

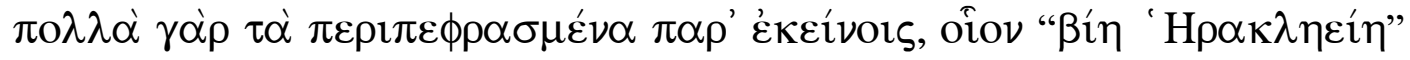

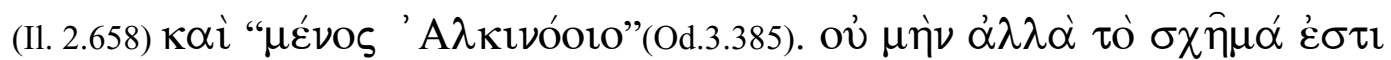

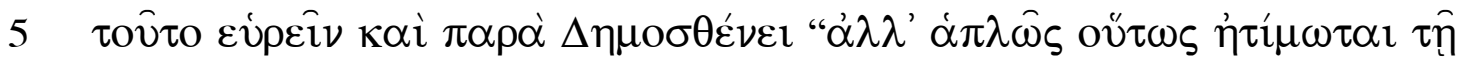

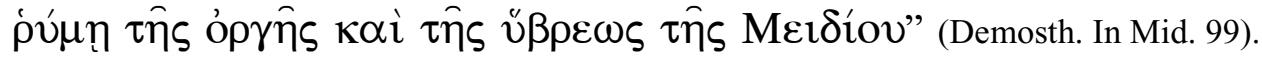

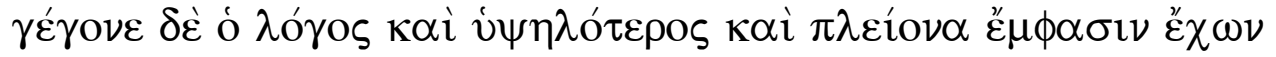

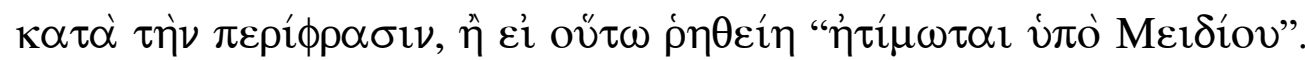

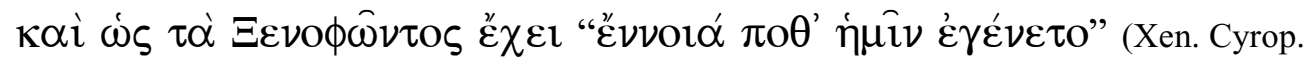

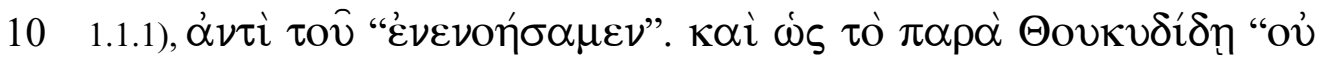

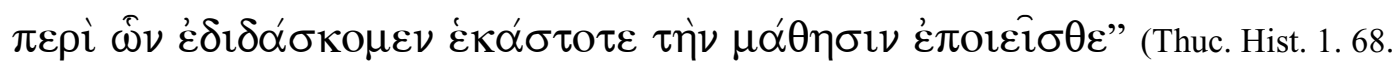

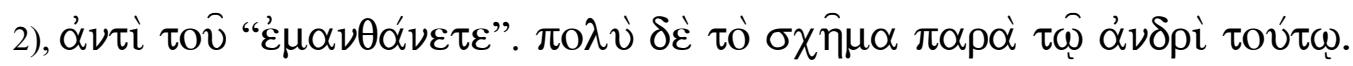

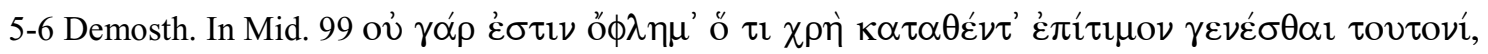

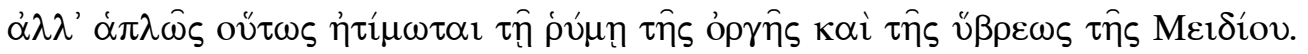

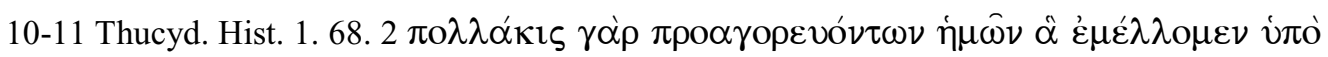

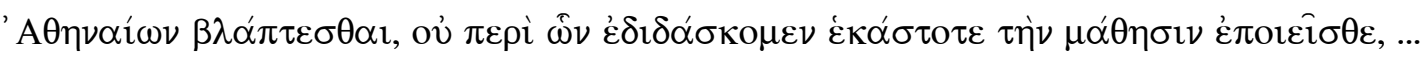

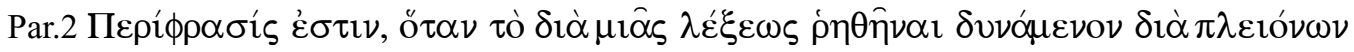

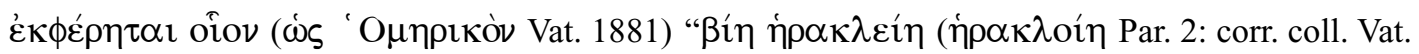

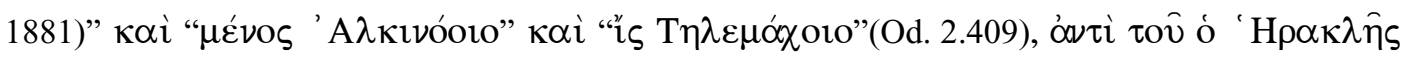

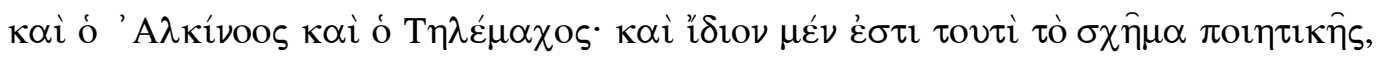

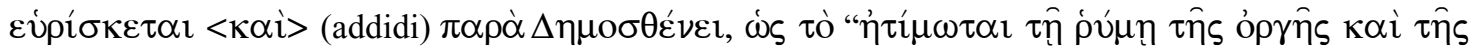

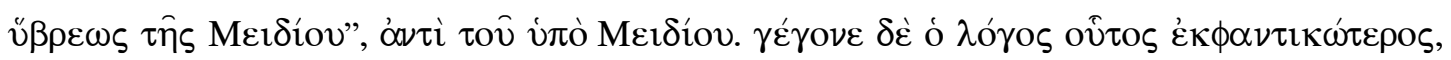

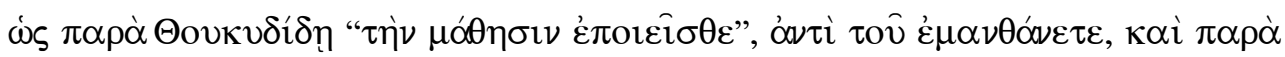

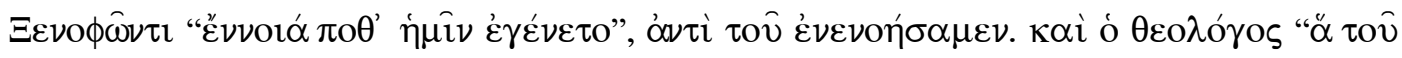

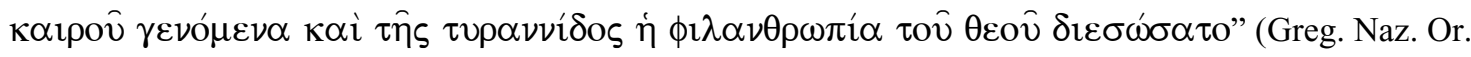

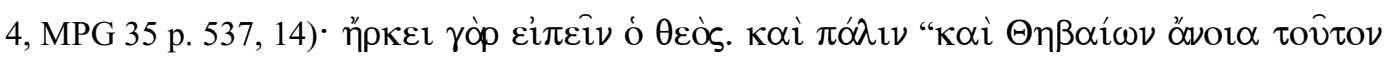

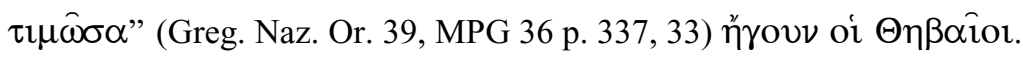

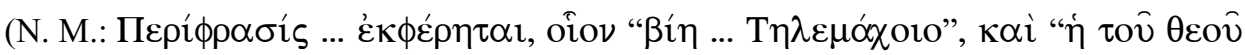

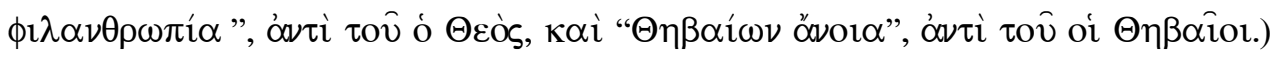

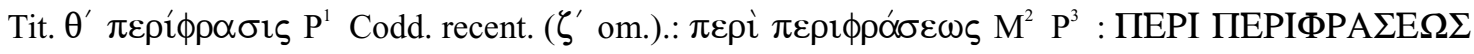
Ald. Ald ${ }^{s} .:$ ПЕРI ПЕРІФРА $\Sigma$ Е $\Omega \Sigma \mathrm{\imath}^{\prime}$ Norrm. : $\imath^{\prime}$. ПЕРI ПЕРІФРА $\Sigma$ E $\Omega \Sigma$ Edd.

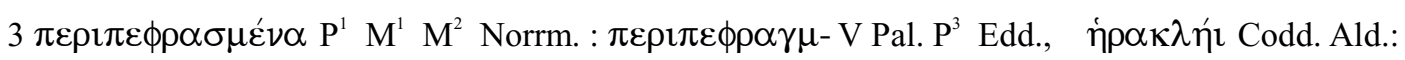

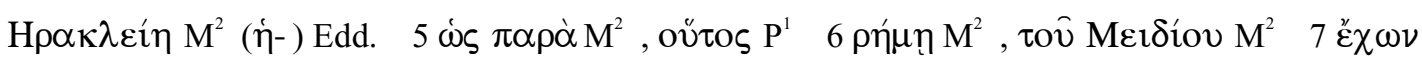
$\mathrm{M}^{2}$ Edd.: om. Codd. Ald. $11 \hat{\omega}$ Codd. recent. Ald. 
$1 \alpha^{\prime}$. ПЕPI П $\Lambda$ EONA $\Sigma$ MOY.

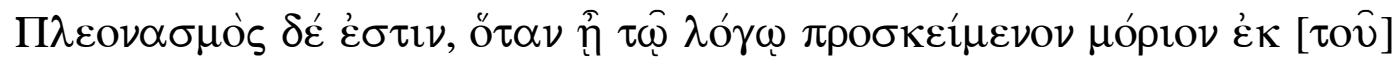

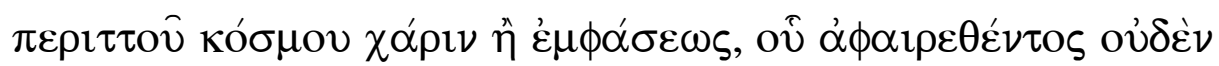

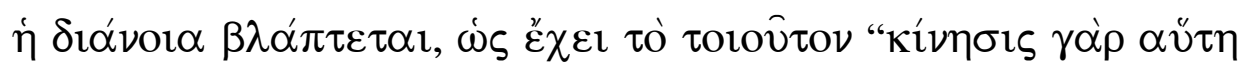

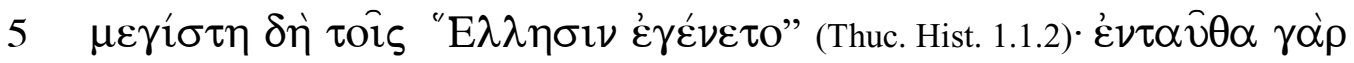

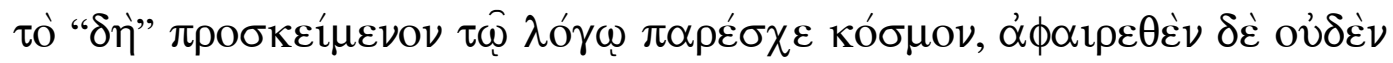

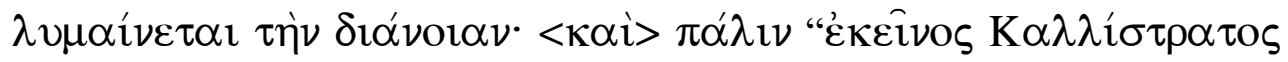

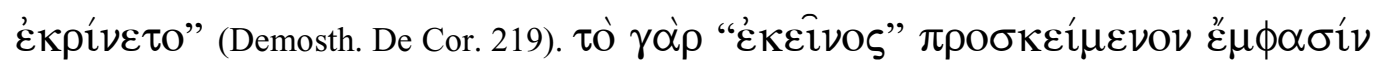

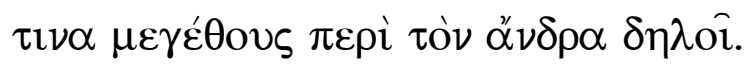

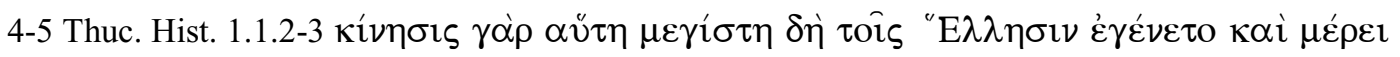

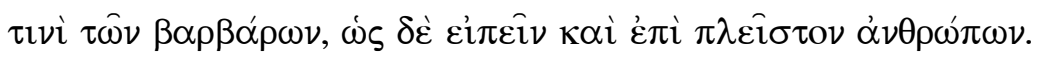

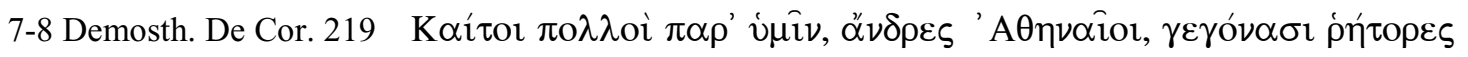

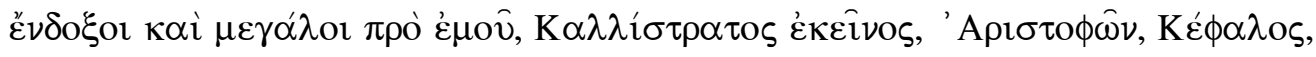

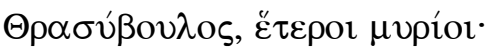

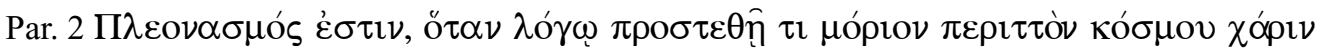

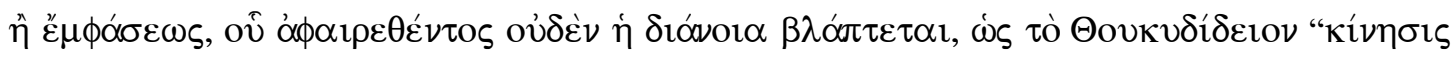

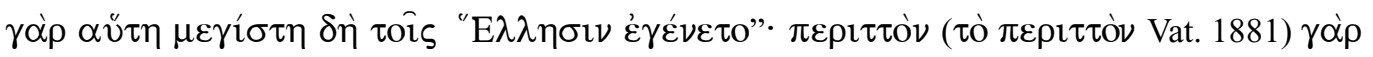

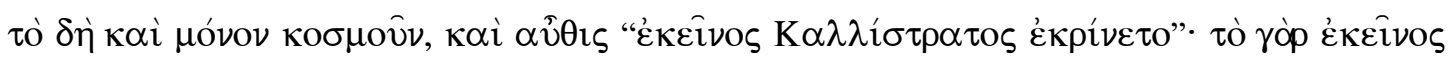

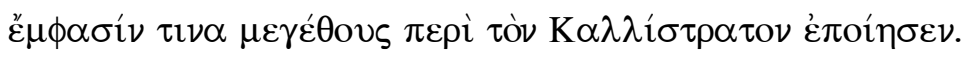

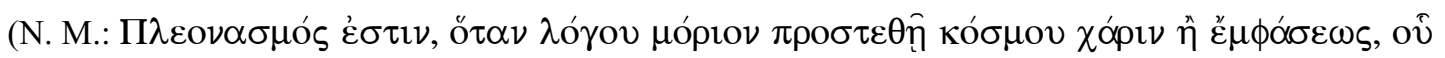

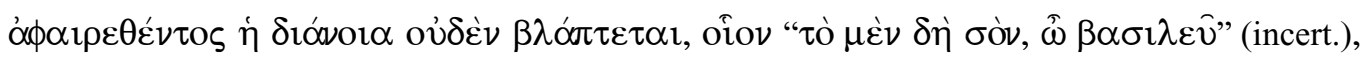

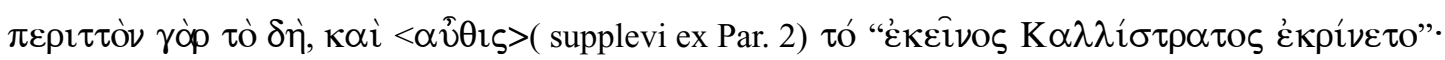

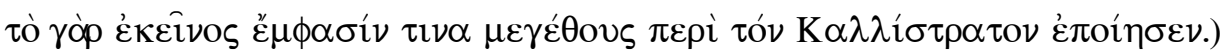

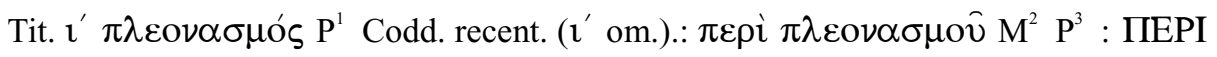

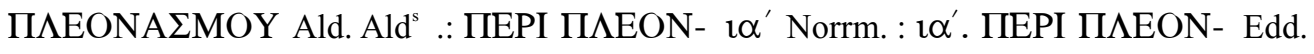

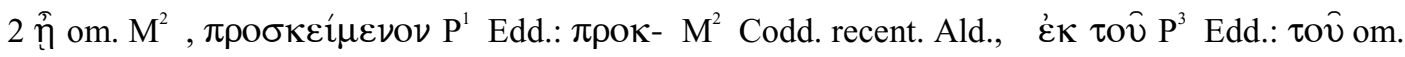
Codd. $3 \pi \alpha \rho \alpha \pi \lambda \eta \rho \omega \sigma \eta ̣$ ante $\kappa o ́ \sigma \mu o v \mathrm{M}^{2}$ : om.Codd. Edd. $6 \pi \rho \circ \sigma \kappa \varepsilon i ́ \mu \varepsilon v o v \mathrm{P}^{1} \mathrm{M}^{2}$ Edd.:

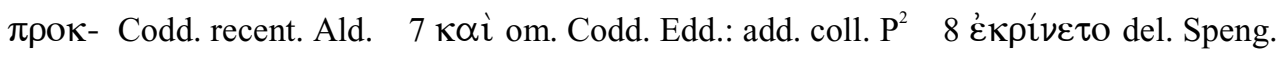
$9 \pi \varepsilon \rho \grave{~ C o d d . ~ E d d .: ~}$ v $\pi \dot{\varepsilon} \rho \mathrm{M}^{2}$ 
$\imath \beta^{\prime}$. ПEPI A $\Sigma$ YN $\Delta$ ETOY H $\Delta \mathrm{IA} \Lambda \mathrm{Y} \Sigma \mathrm{E} \Omega \Sigma$.

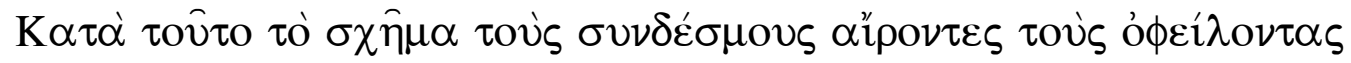

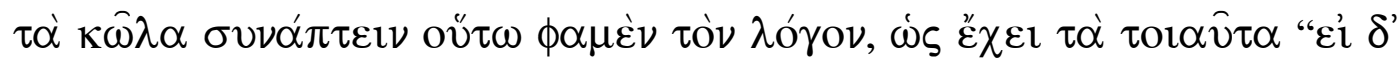

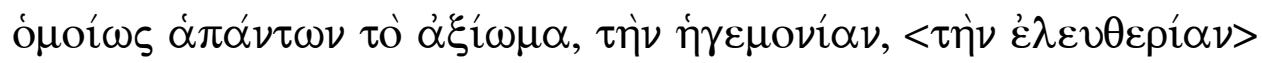

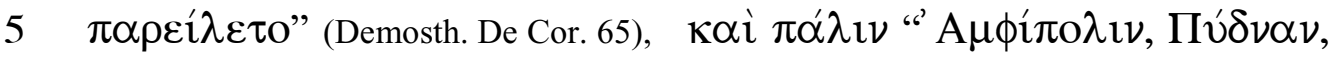

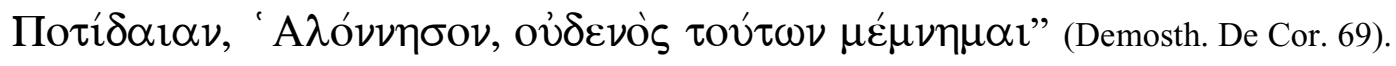

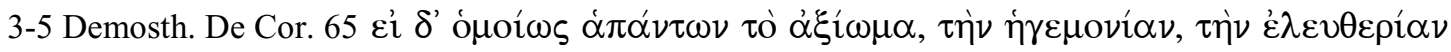
$\pi \varepsilon \rho 1 \varepsilon i ́ \lambda \varepsilon \tau 0, \ldots$

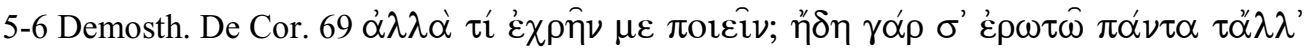

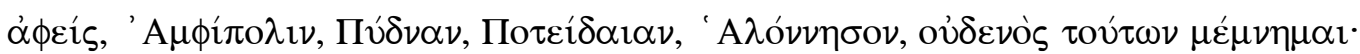

Par. 2 'A

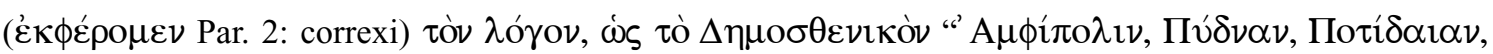

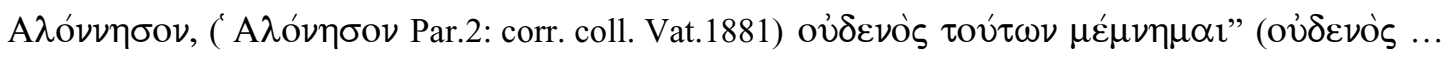

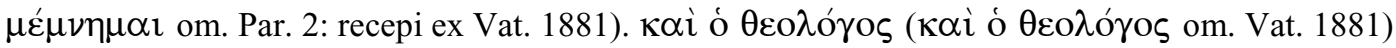

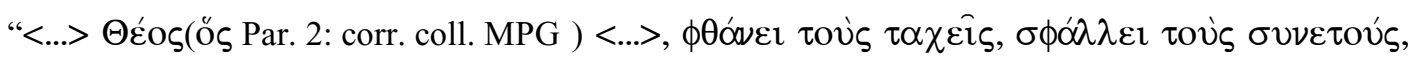

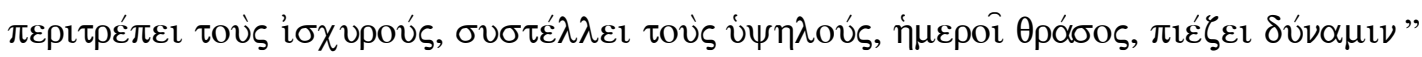
(Greg. Naz. Or. 2, MPG 35 p. 508, 15).

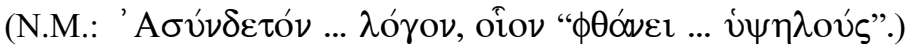

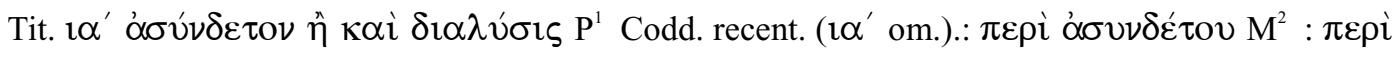

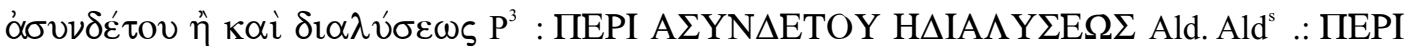
A $\Sigma$ YN $\Delta$ ETOY H $\Delta$ IA $\Lambda$ Y $\Sigma E \Omega \Sigma i \beta^{\prime}$ Norrm. : $\imath \beta^{\prime}$. ПEPI A $\Sigma Y N \Delta E T O Y$ H $\Delta$ IA $\Lambda Y \Sigma E \Omega \Sigma$ Edd.

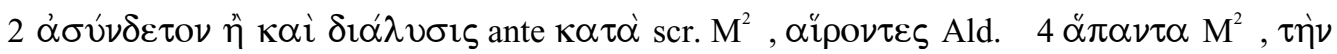

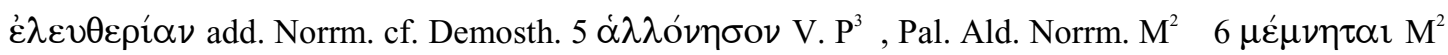




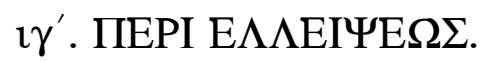

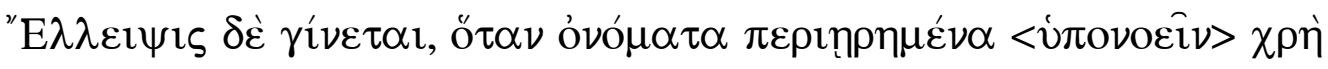

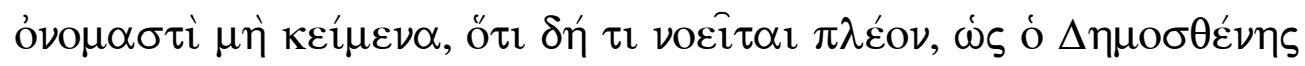

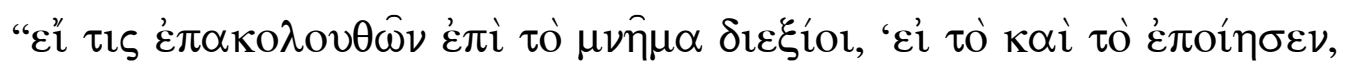

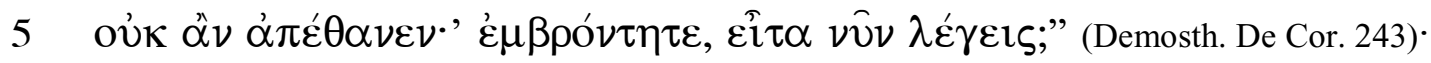

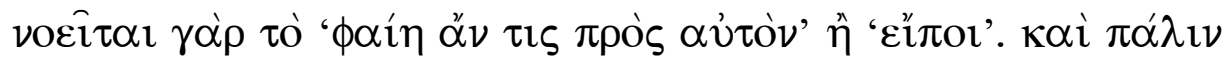

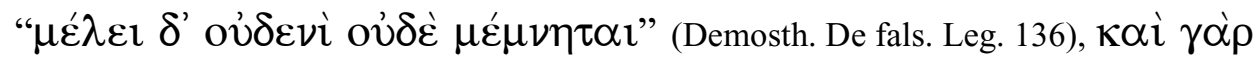

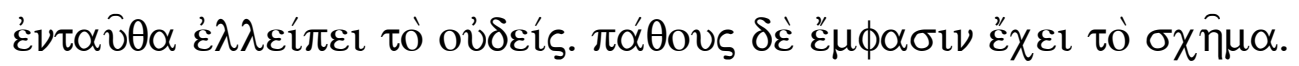

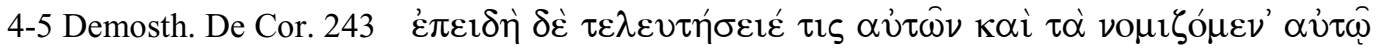

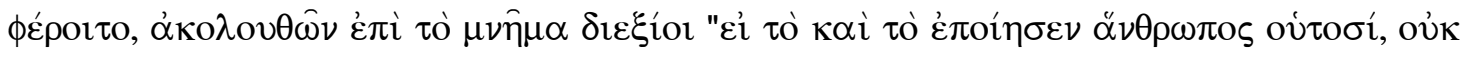

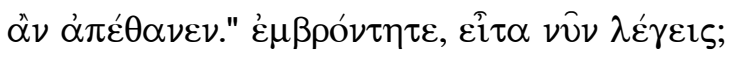

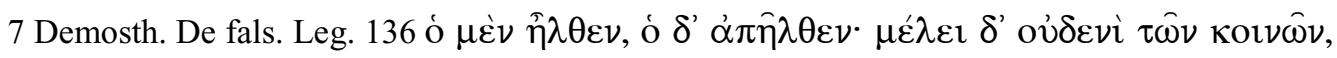

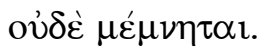

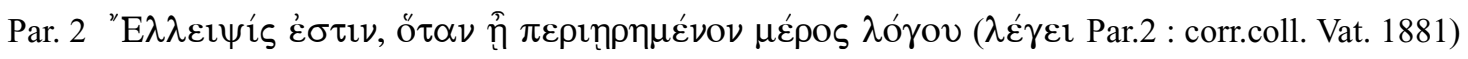

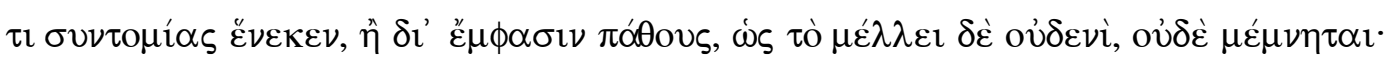

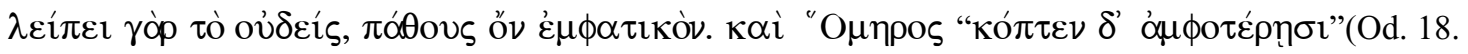

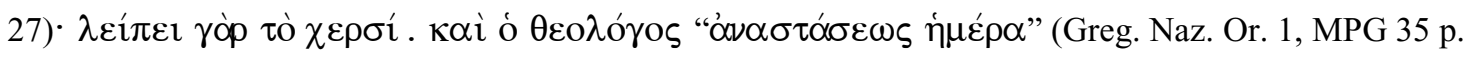

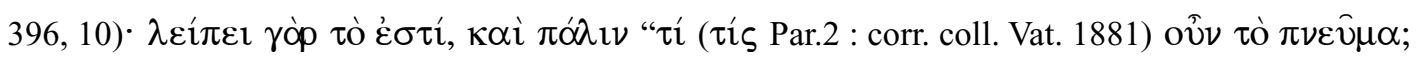

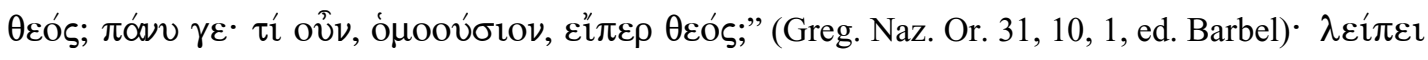

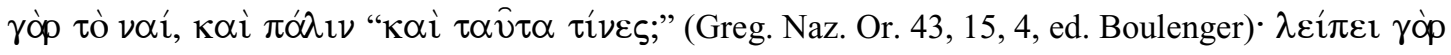

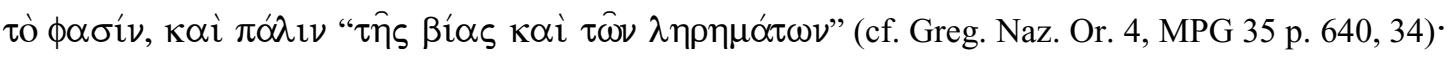

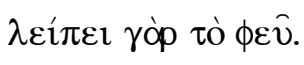

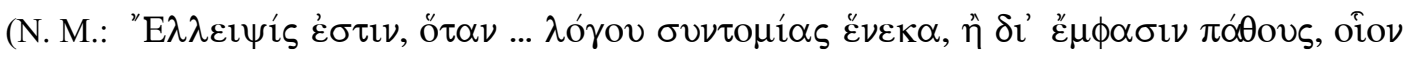

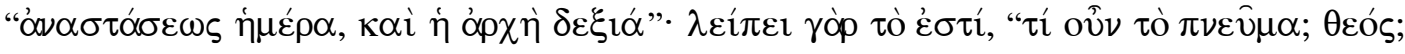

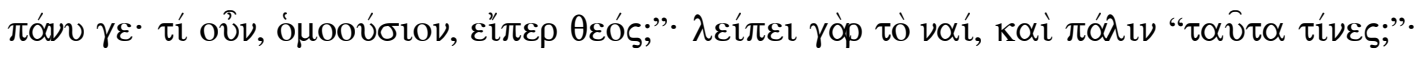

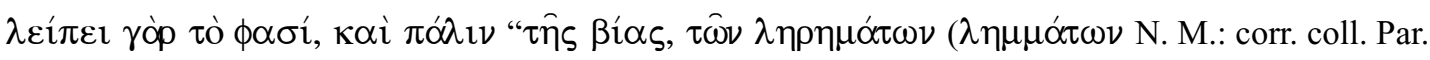

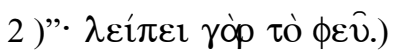

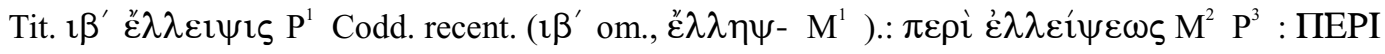

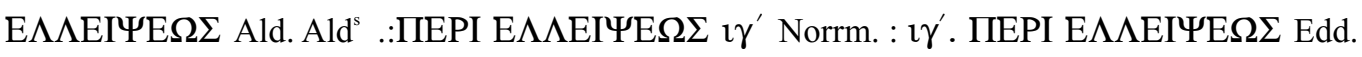

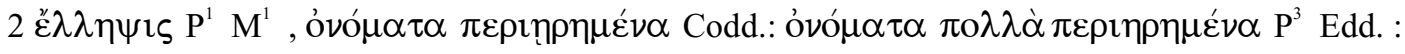

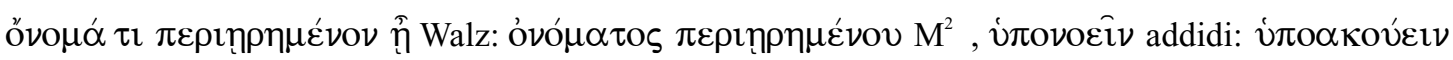

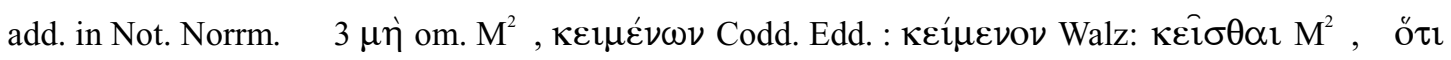

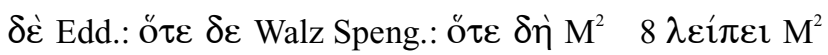


$1 \delta^{\prime}$. ПЕPI A $\Lambda \Lambda \mathrm{OI} \Omega \Sigma \mathrm{E} \Omega \Sigma \mathrm{H} \mathrm{A} \Lambda \Lambda \mathrm{A} \Gamma \mathrm{H} \Sigma$.

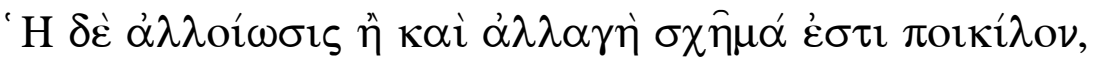

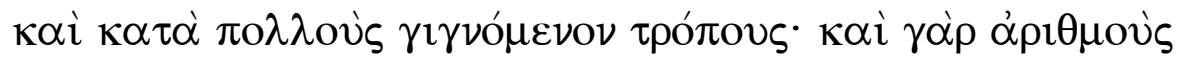

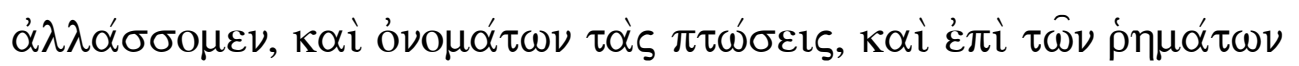

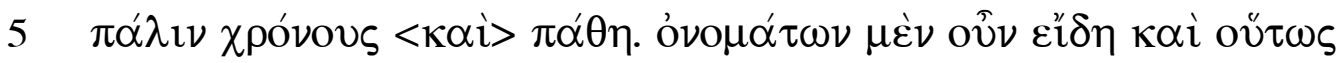

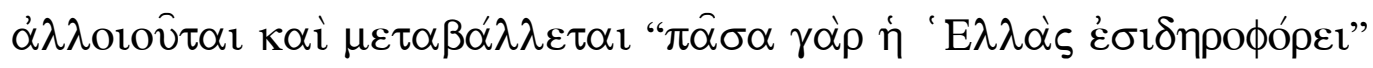

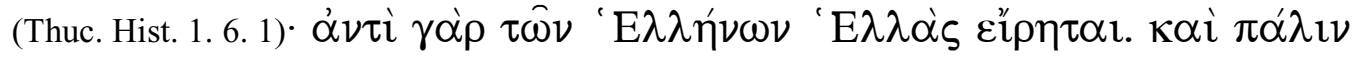

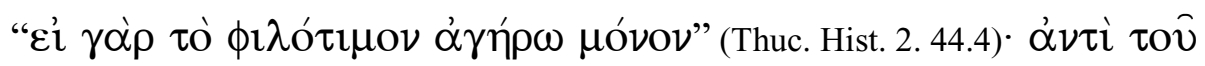

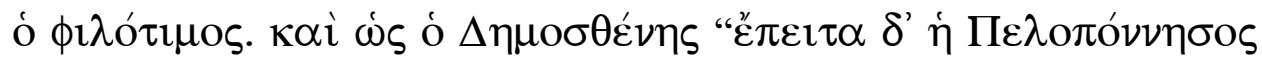

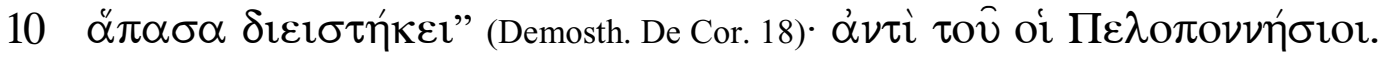

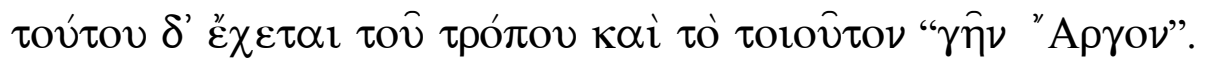

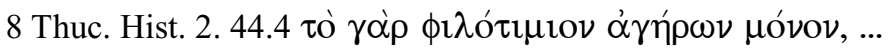

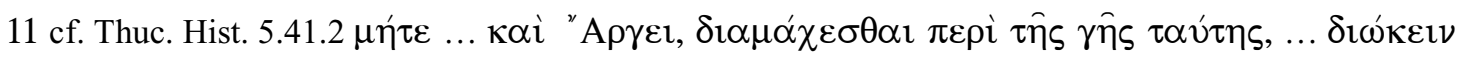

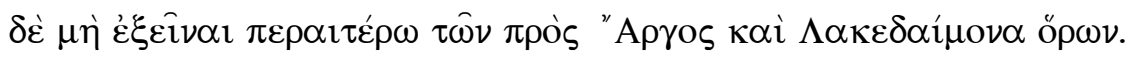

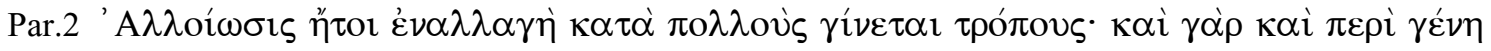

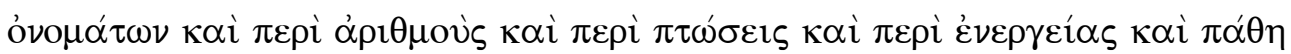

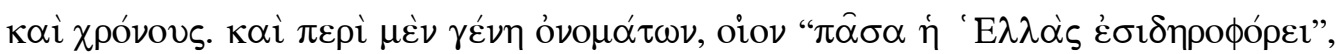

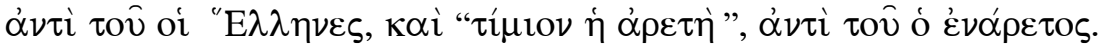

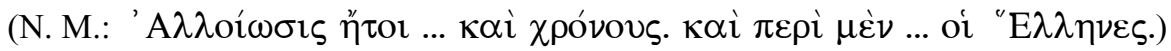

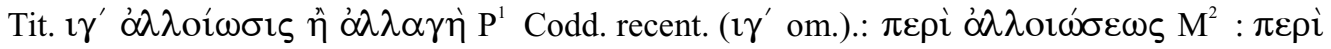

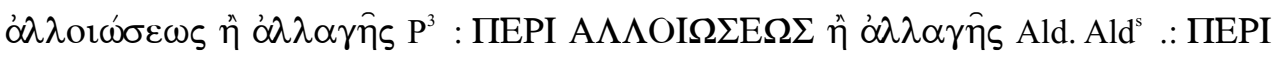
$\mathrm{A} \Lambda \Lambda \mathrm{OI} \Sigma \mathrm{E} \Omega \Sigma \eta_{\eta}^{\prime} \mathrm{A} \Lambda \Lambda \mathrm{A} \Gamma \mathrm{H} \Sigma$ i $\delta^{\prime}$ Norrm. : $1 \delta^{\prime}$. ПEPI A $\Lambda \Lambda \mathrm{OI} \Omega \Sigma \mathrm{E} \Omega \Sigma \mathrm{H} \mathrm{A} \Lambda \Lambda \mathrm{A} \Gamma \mathrm{H} \Sigma$ Edd.

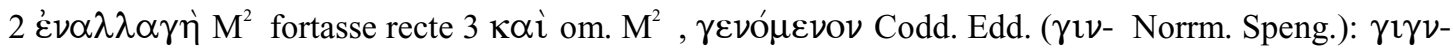

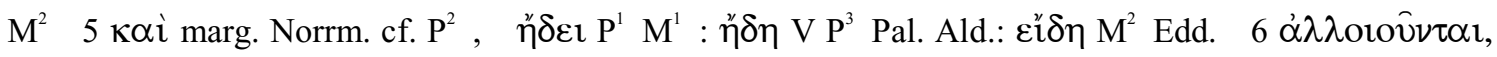

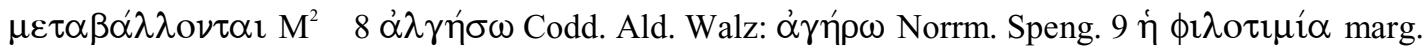

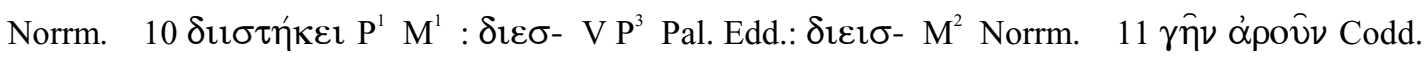
Edd.: $\gamma \tilde{\eta} \nu \alpha ́ \rho \gamma o \nu \mathrm{M}^{2}$ 


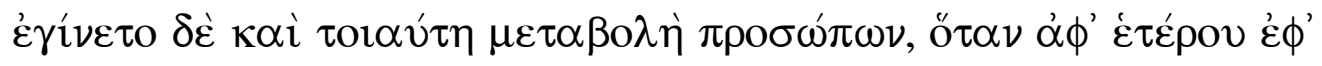

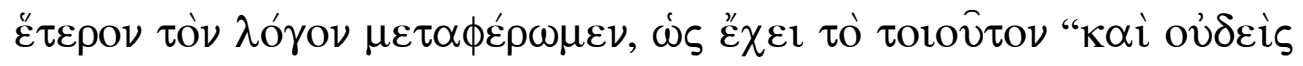

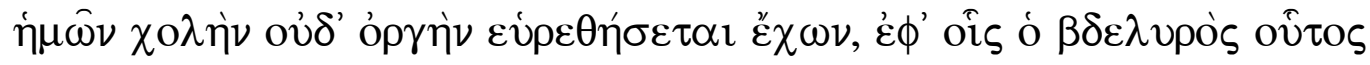

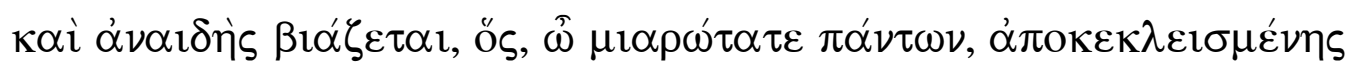

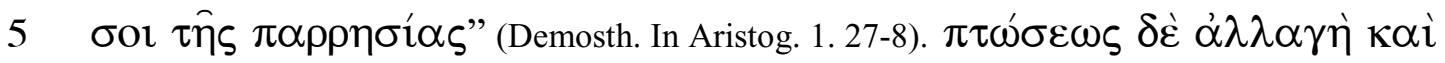

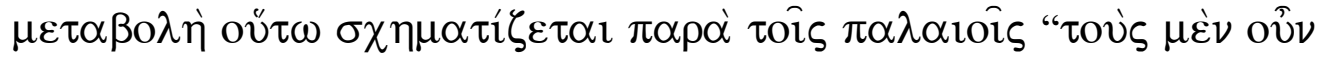

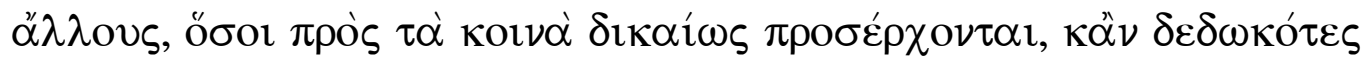

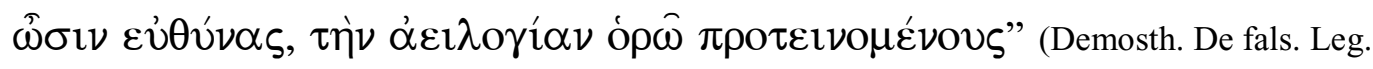

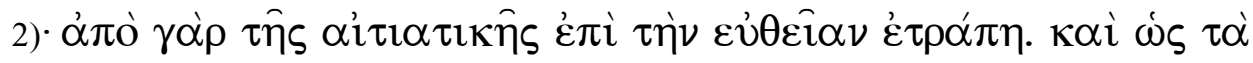

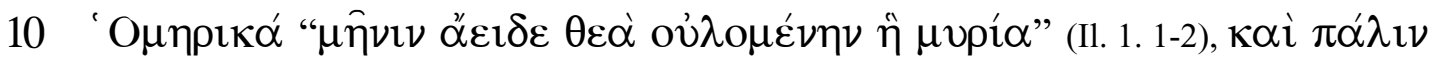

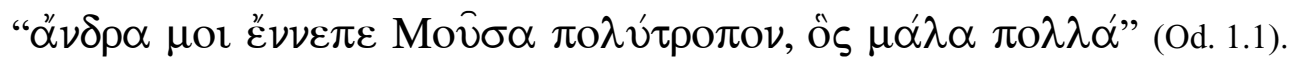

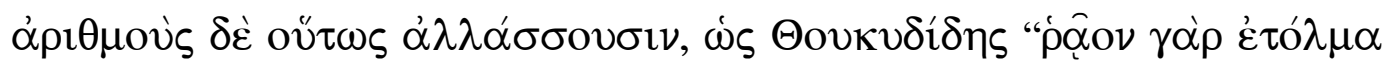

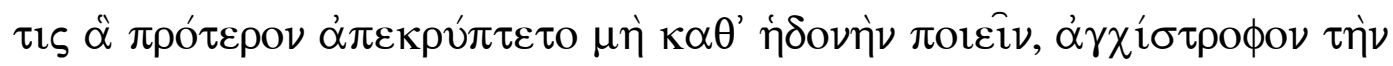

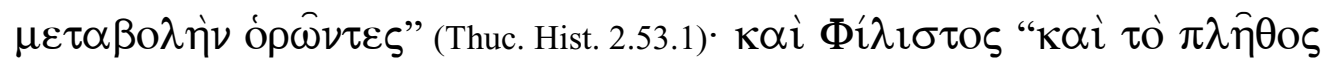

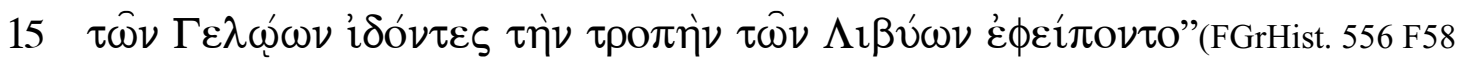
bis).

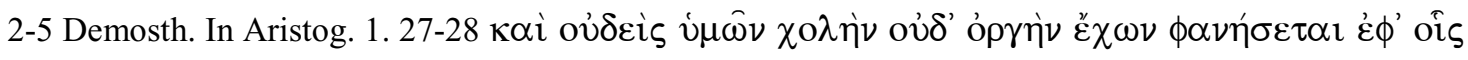

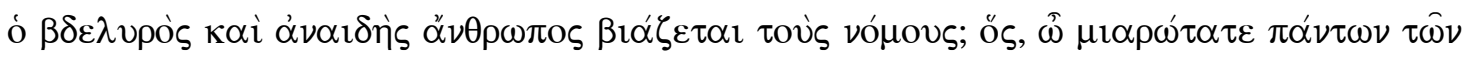

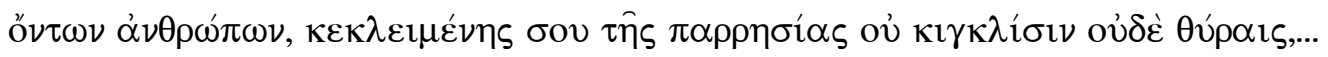

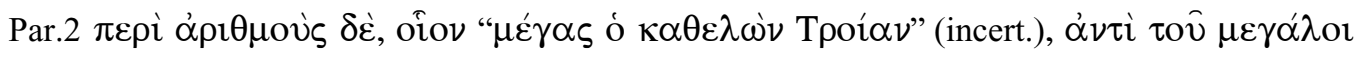

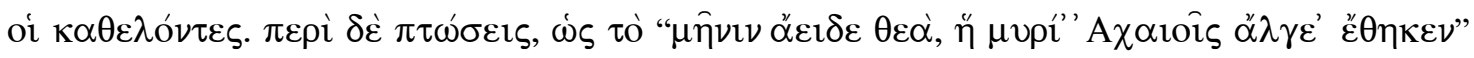

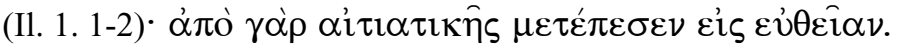

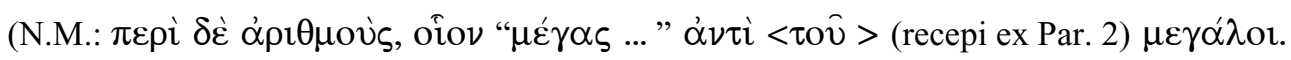

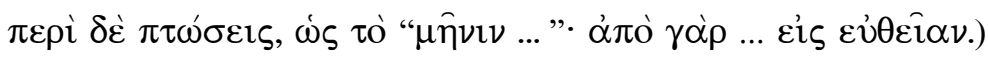

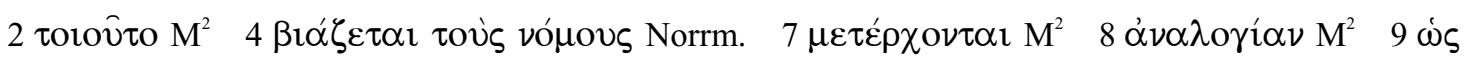

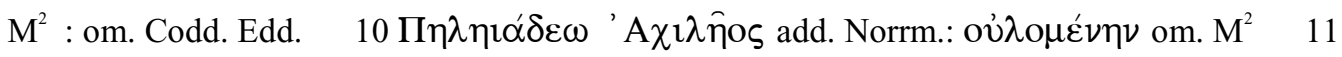

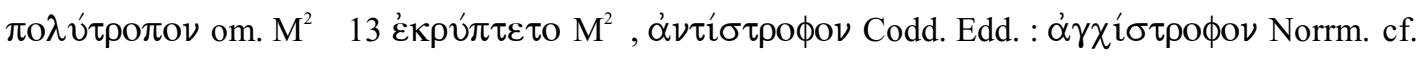
Thuc. 14 Фí $\lambda \imath \pi \pi \circ \varsigma$ Codd. Edd. : $\Phi i ́ \lambda \imath \sigma \tau o \varsigma$ correxi coll. FGrHist. 556 F 58 bis, Schindel, cf.

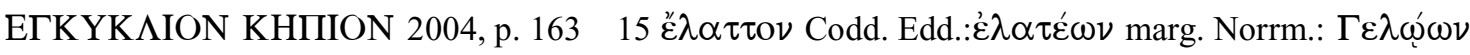
corr. Bloch. FGrHist. 556 F 58 bis (add. ad comm. III b, p. 402), $\tau \hat{\omega} v \lambda$ ı $\beta v ́ \omega \nu$ Codd. Speng.: $\tau \hat{\omega} \nu$ om. Edd. 


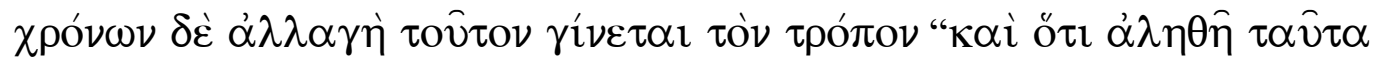

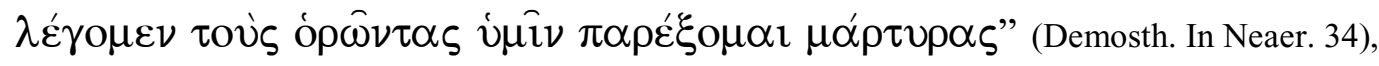

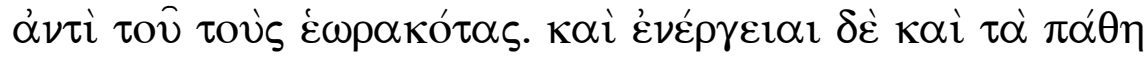

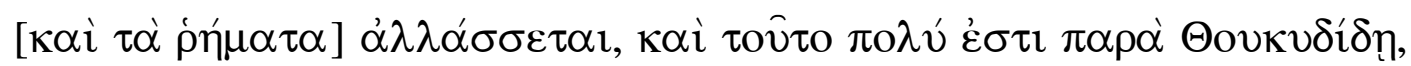

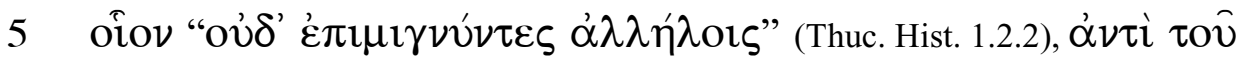

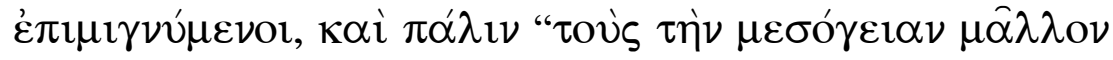

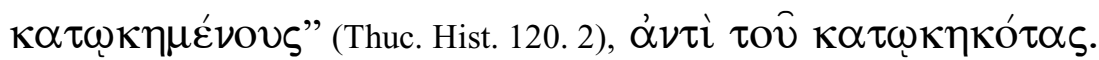

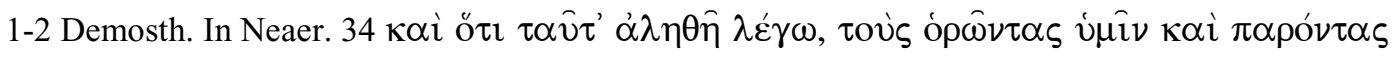

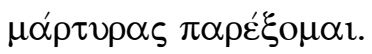

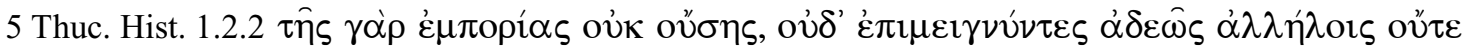

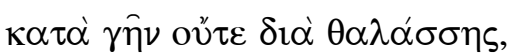

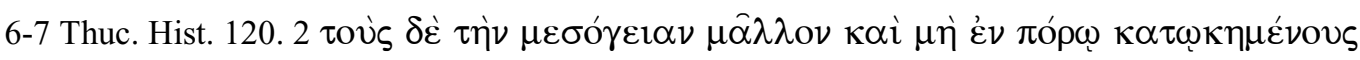

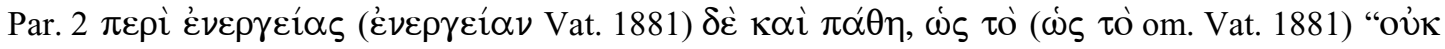

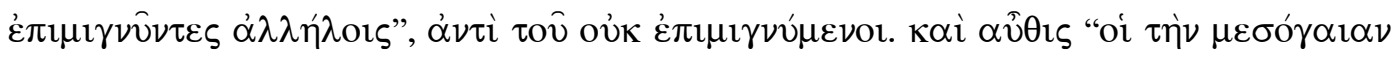

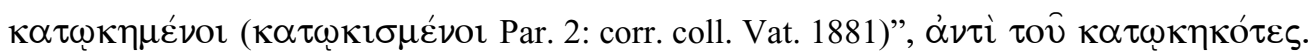

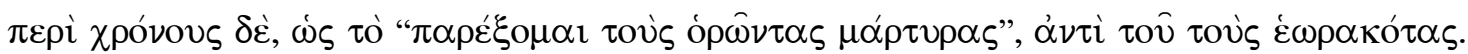

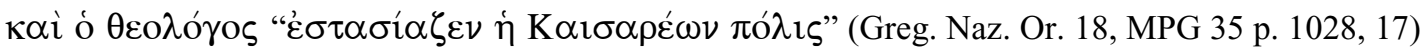

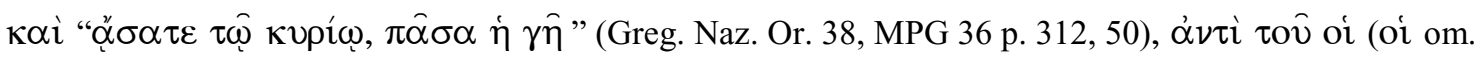

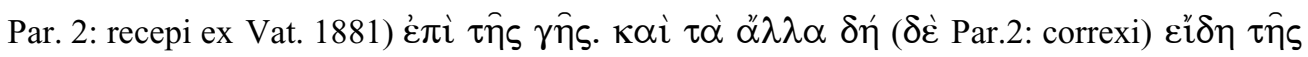

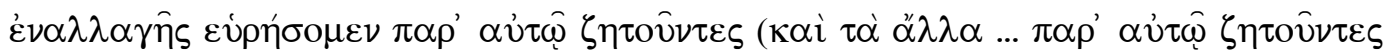
om. Vat. 1881).

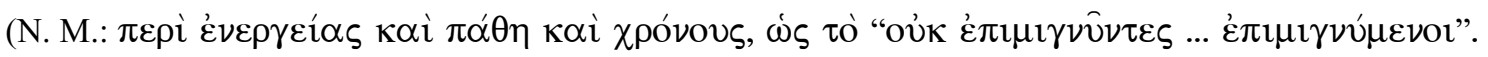

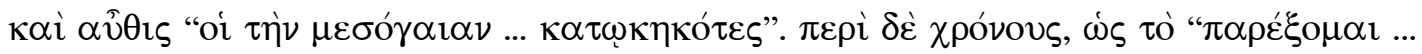

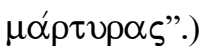

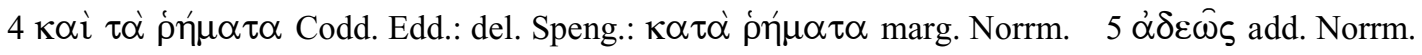

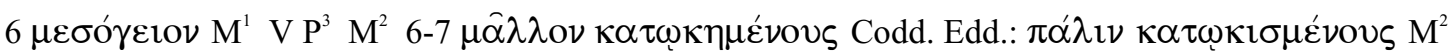


$\imath \varepsilon^{\prime}$. ПЕРІ ПО $\Lambda$ ҮПТТОY.

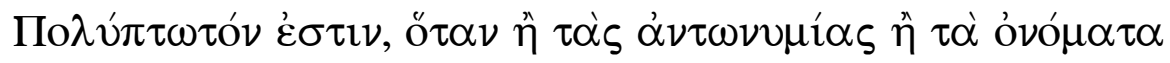

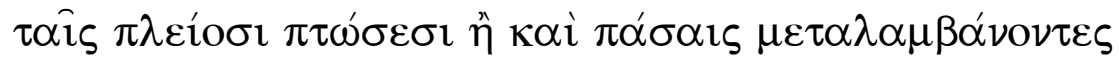

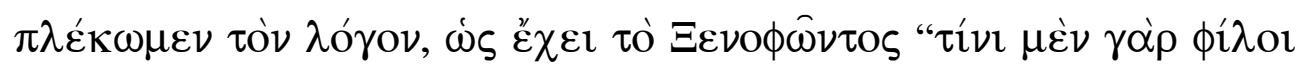

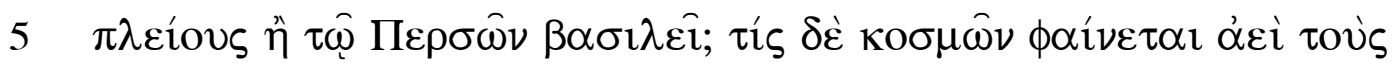

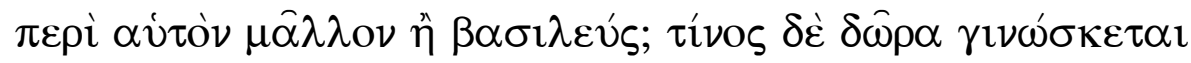

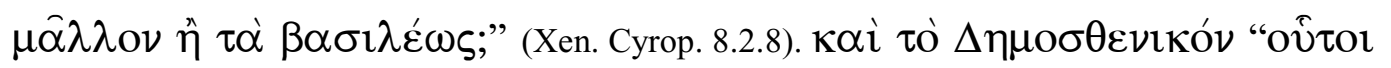

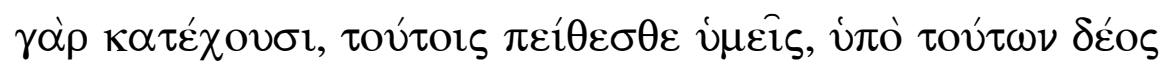

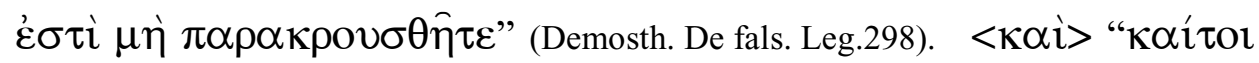

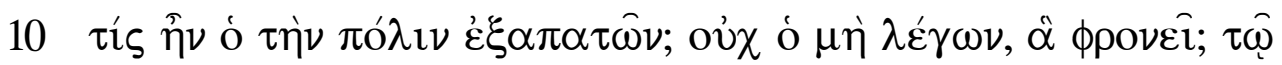

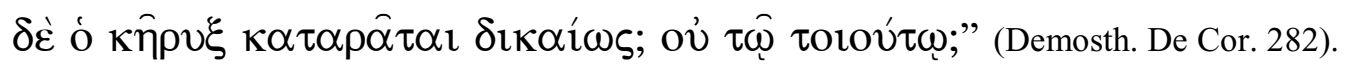

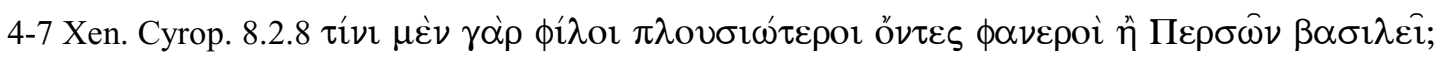

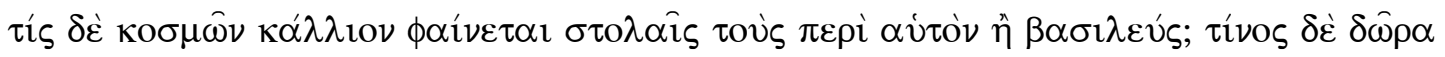

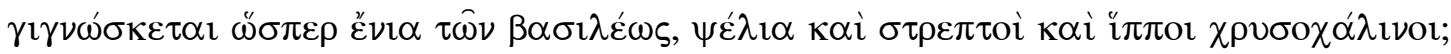
8-9 Demosth. De fals. Leg. 298 oṽ $\dot{\varepsilon} \sigma \tau \grave{\imath} \mu \grave{\eta} \pi \alpha \rho \alpha \kappa \rho \circ \nu \sigma \theta \hat{\eta} \tau \varepsilon$.

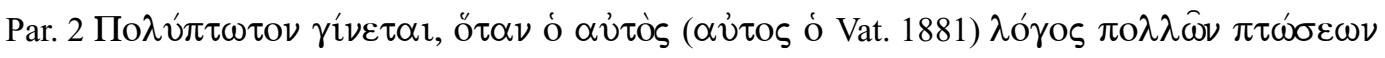

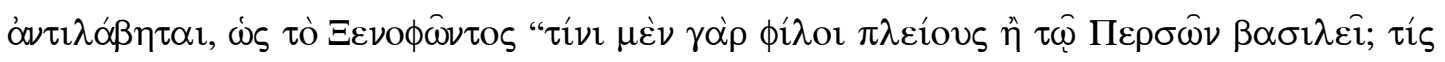

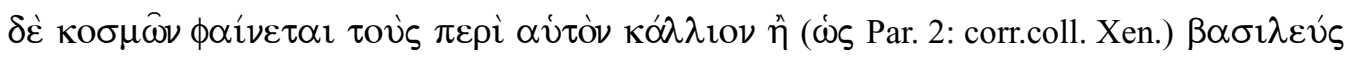

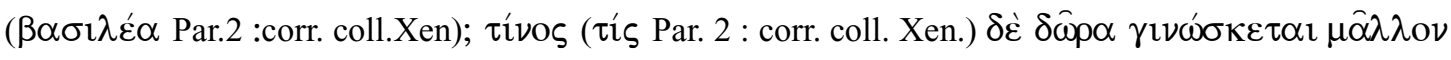

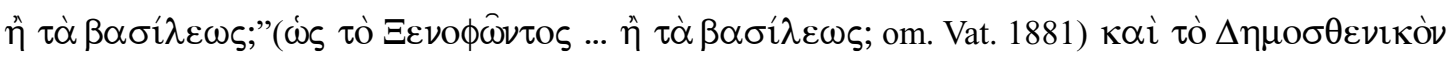

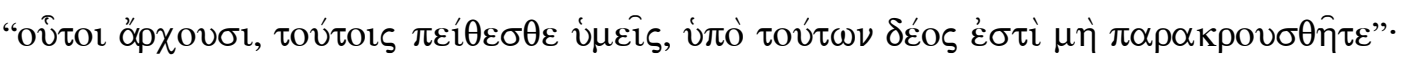

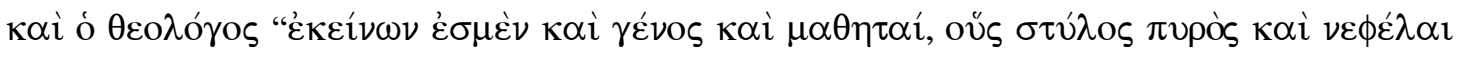

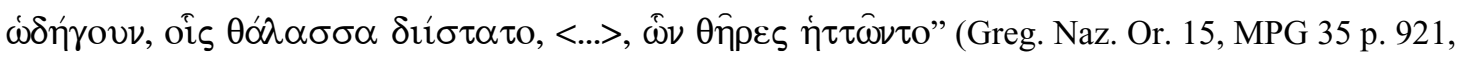

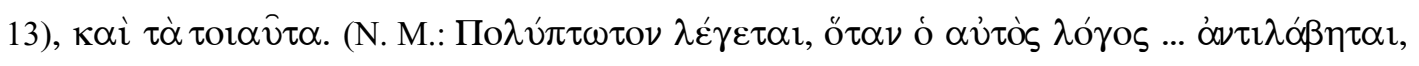

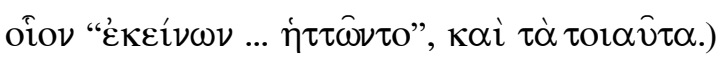

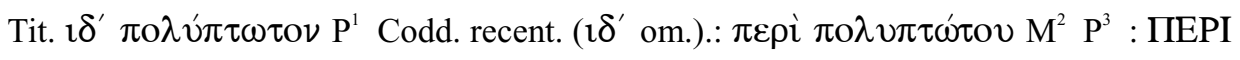
ПО $\Lambda$ ҮПТ $\Omega$ TOY Ald. Ald ${ }^{s}$ : ПЕРI ПО $\Lambda$ ҮПТ- $1 \varepsilon^{\prime}$ Norrm. : $1 \varepsilon^{\prime}$. ПЕРI ПО $\Lambda$ YПТ- Edd.

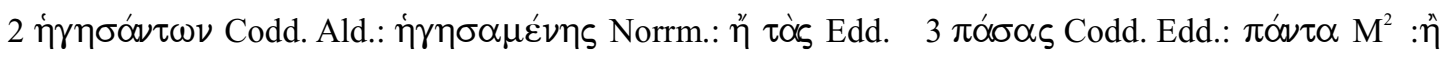

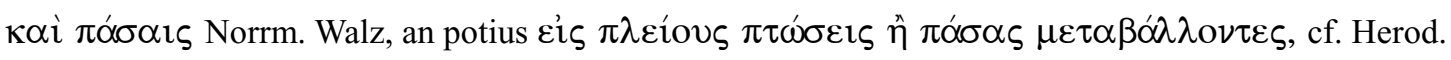

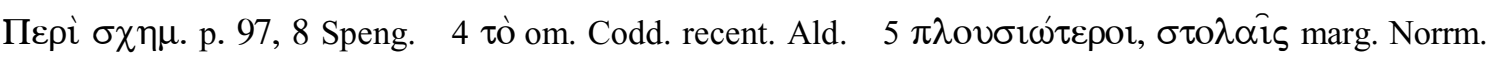

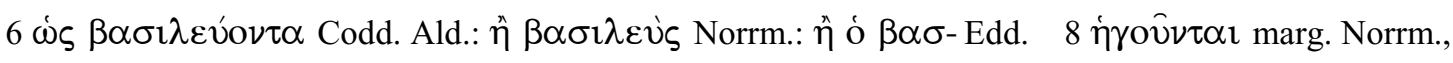

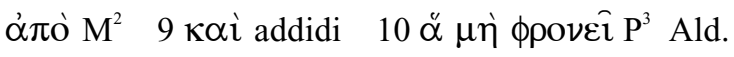


$1 \varsigma^{\prime}$. ПЕPI METABO $\Lambda \mathrm{H} \Sigma$.

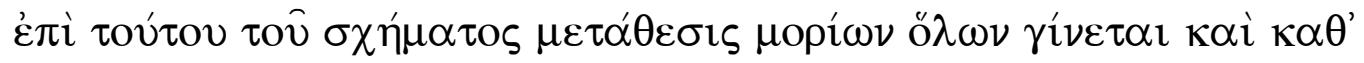

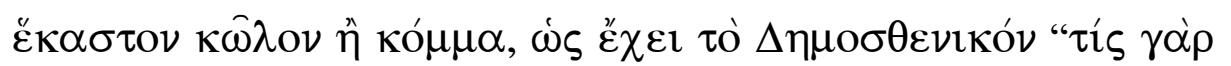

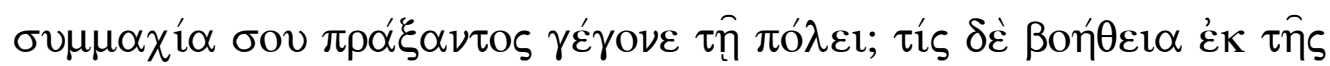

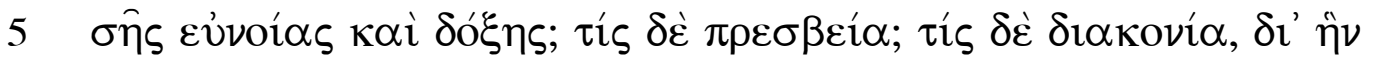

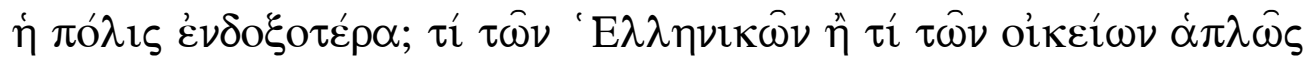

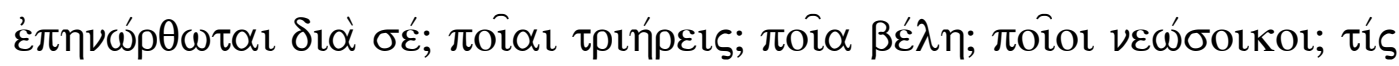

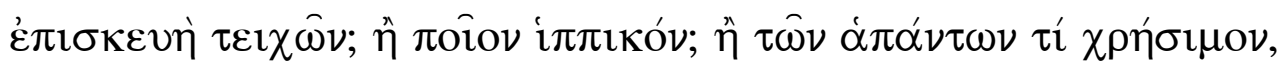

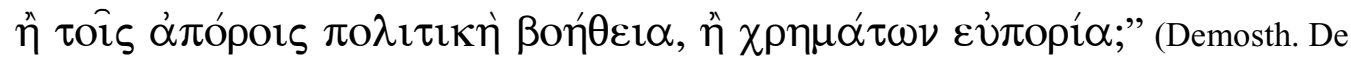

10 Cor. 311-2).

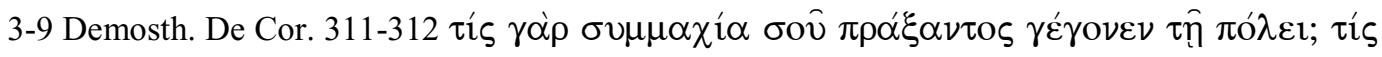

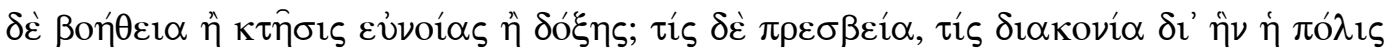

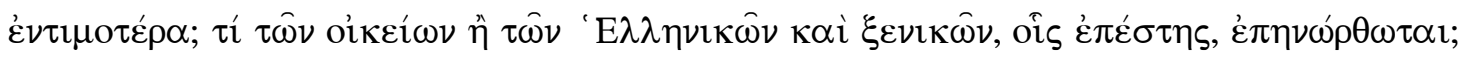

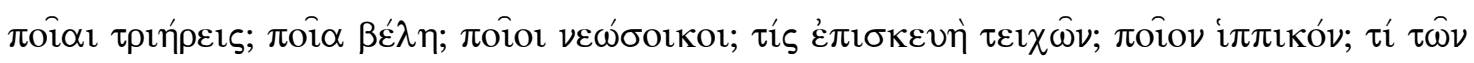

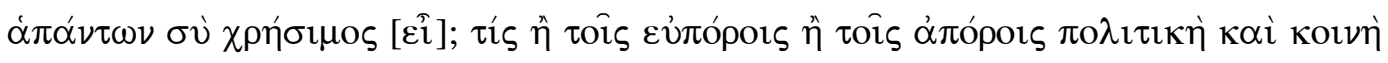

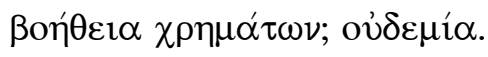

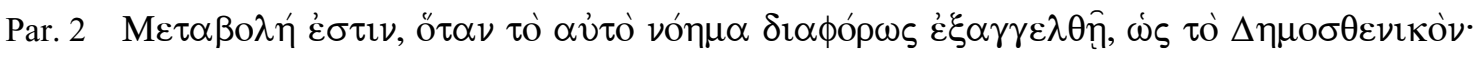

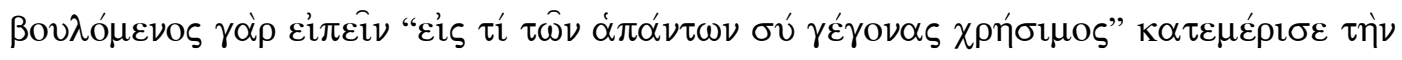

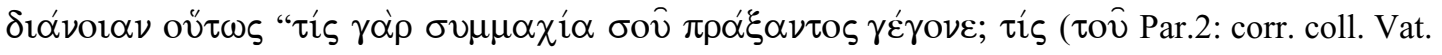

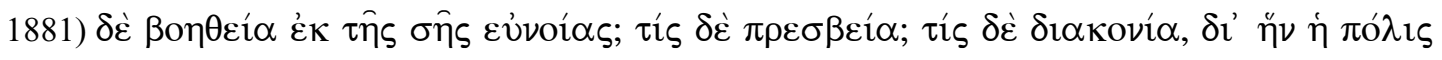

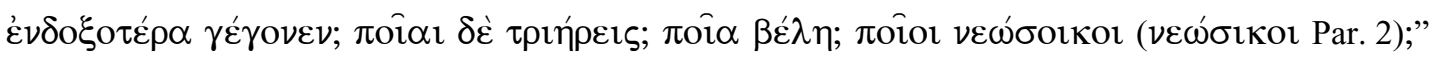

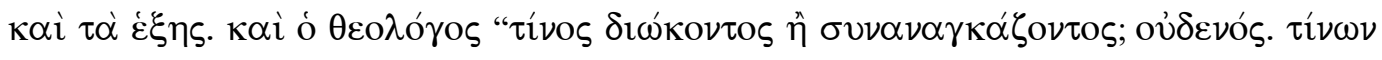

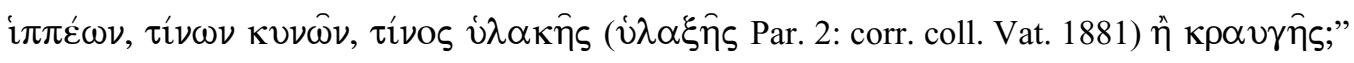
(Greg. Naz. Or. 43, 7, 5, ed. Boulenger).

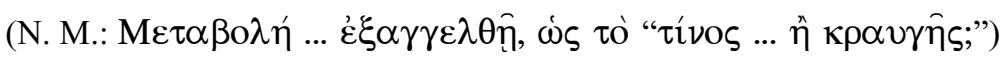

Tit. $1 \varepsilon^{\prime} \mu \varepsilon \tau \alpha \beta o \lambda \eta \mathrm{P}^{1}$ Codd. recent. ( $1 \varepsilon^{\prime}$ om.).: $\pi \varepsilon \rho \grave{~} \mu \varepsilon \tau \alpha \beta 0 \lambda \hat{\eta} \varsigma \mathrm{M}^{2} \mathrm{P}^{3}:$ ПEPI METABO $\Lambda \mathrm{H} \Sigma$ Ald. Ald ${ }^{s} .:$ ПEPI METABO $\Lambda \mathrm{H} \Sigma \iota^{\prime} \varsigma^{\prime}$ Norrm. : $\iota \varsigma^{\prime}$. ПEPI METABO $\Lambda \mathrm{H} \Sigma$ Edd.

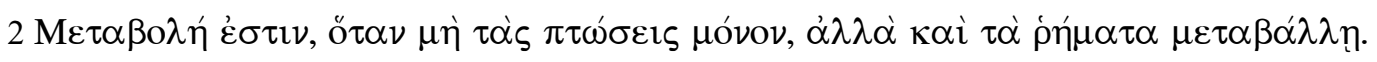

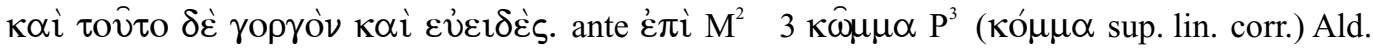

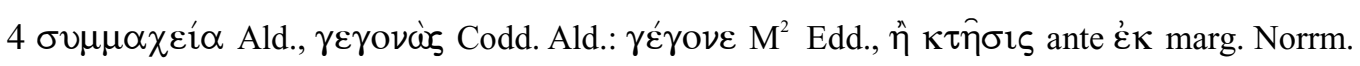

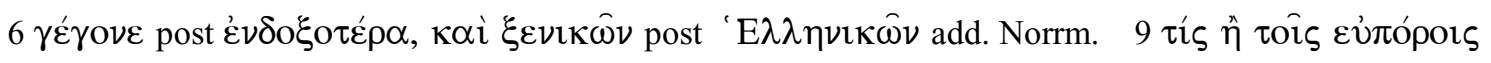

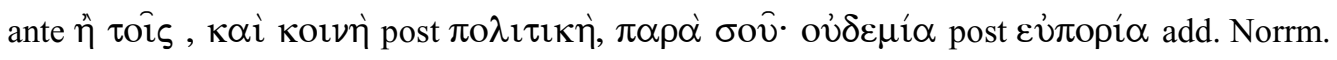


$\imath \zeta^{\prime}$. ПЕPI ZЕYГMATO $\Sigma$.

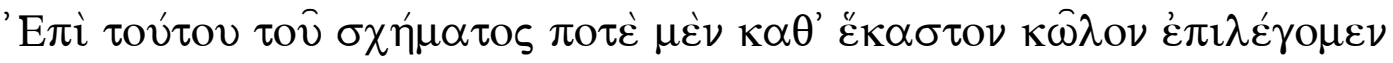

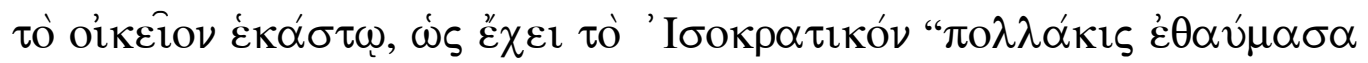

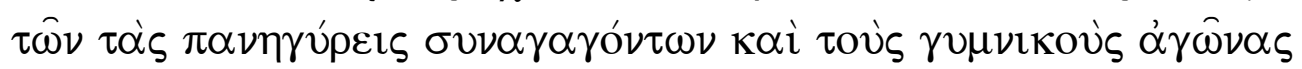

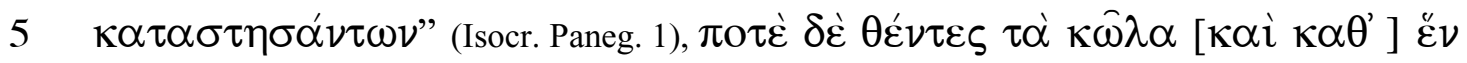

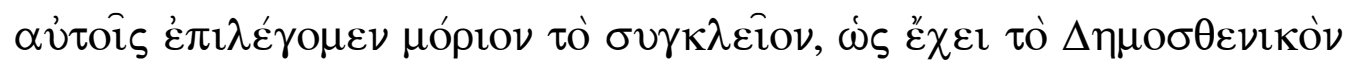

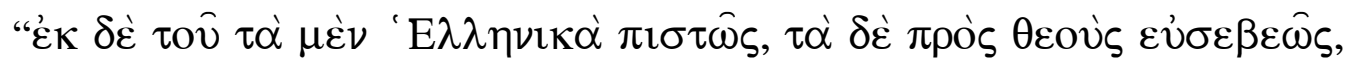

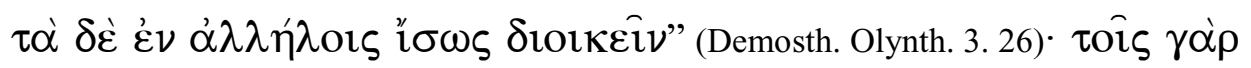

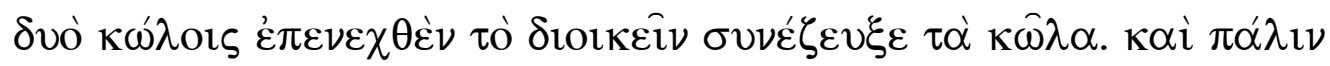

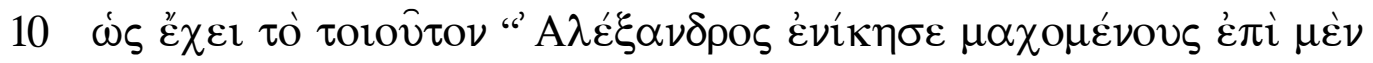

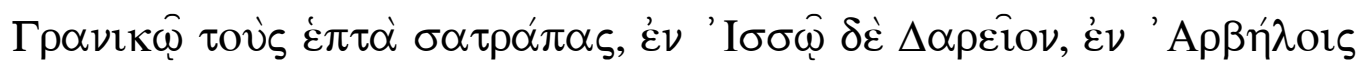

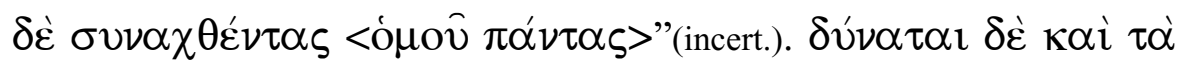

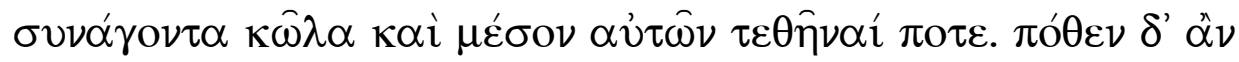

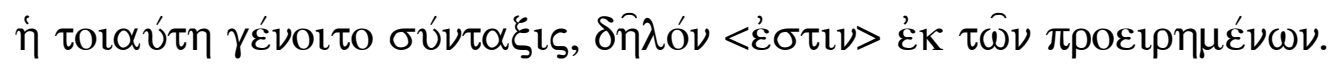

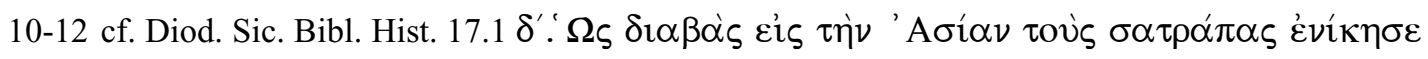

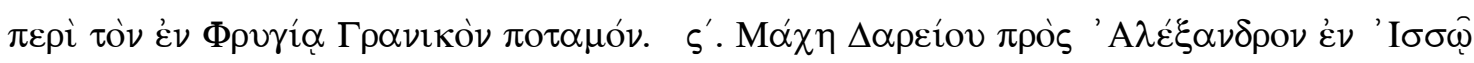

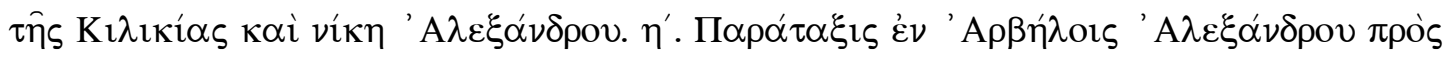

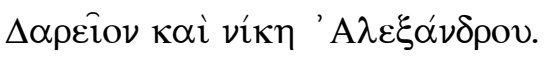

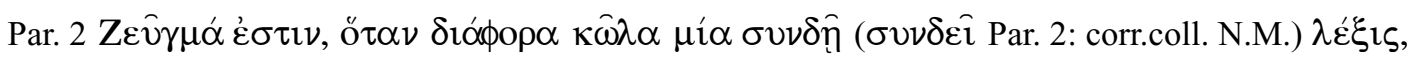

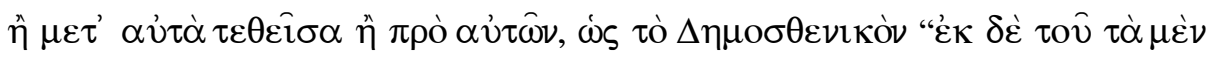

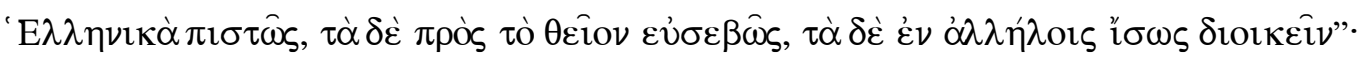

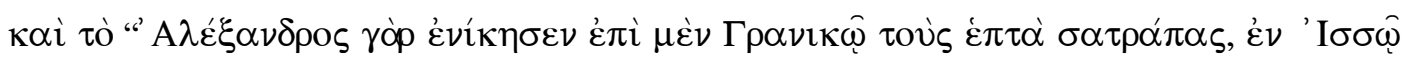

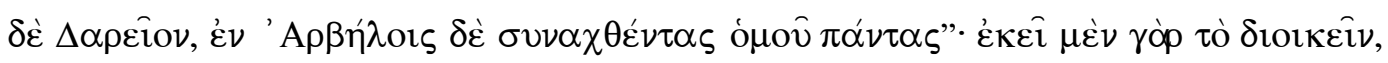

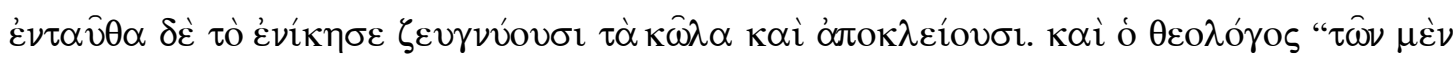

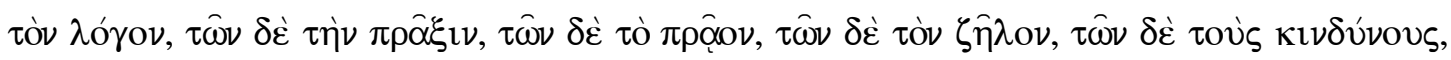

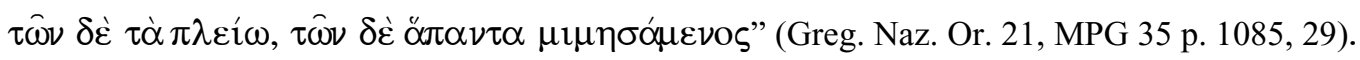

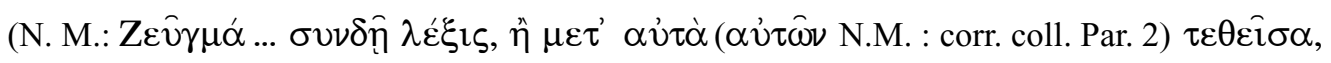

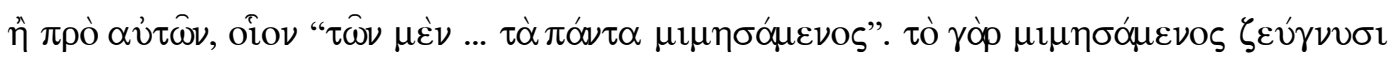
$\tau \grave{\alpha} \kappa \hat{\omega} \lambda \alpha$.)

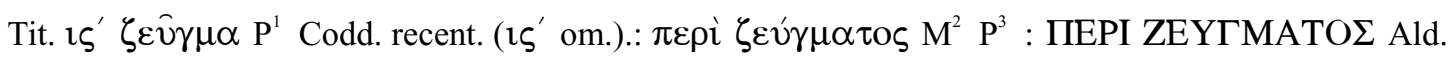
Ald $^{s} .:$ ПЕPI ZEYГMATO $\Sigma$ ¡ $\zeta^{\prime}$ Norrm. : $\iota \zeta^{\prime}$. ПЕPI ZEYГMATO $\Sigma$ Edd.

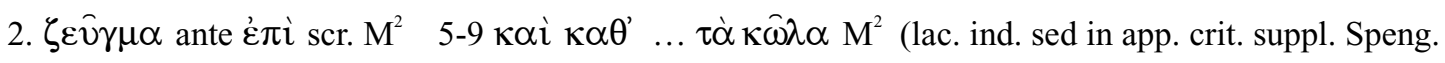

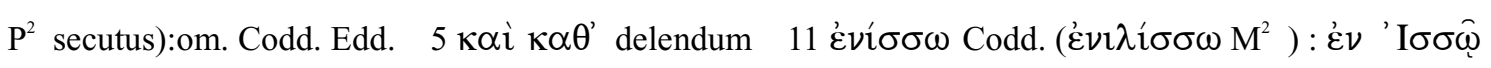

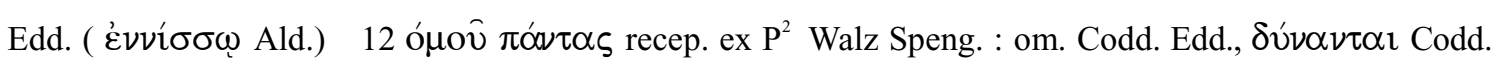

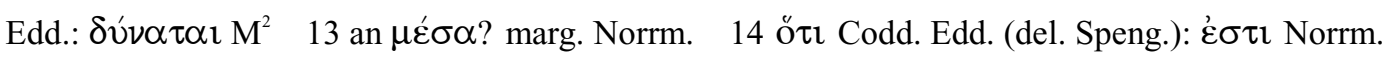


$\imath \eta^{\prime}$. ПЕPI OMOIOTE $\Lambda$ EYTOY.

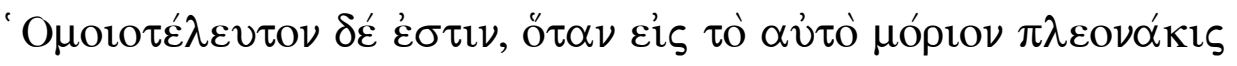

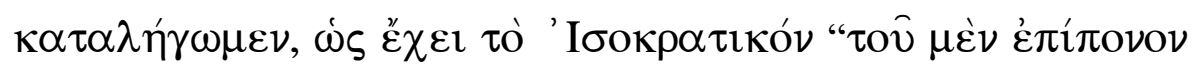

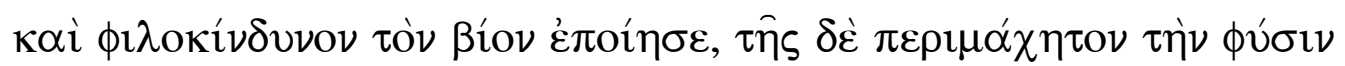

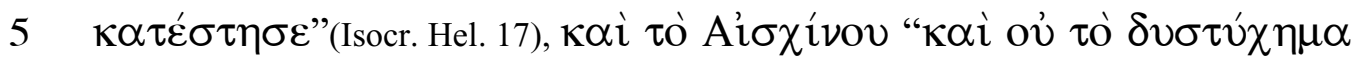

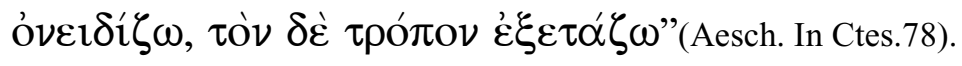

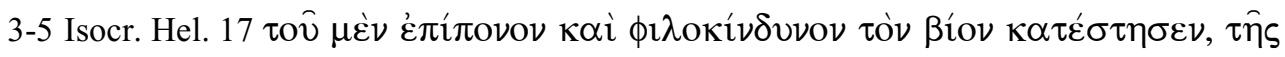

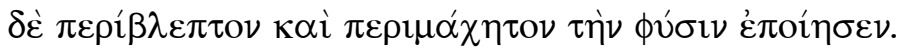

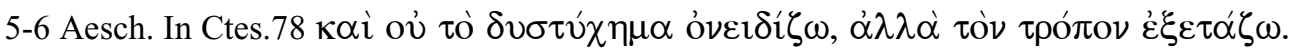

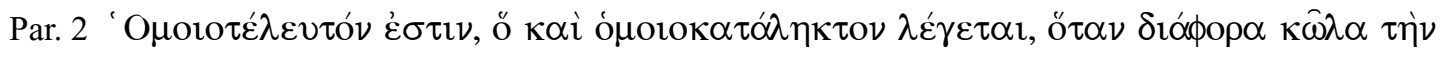

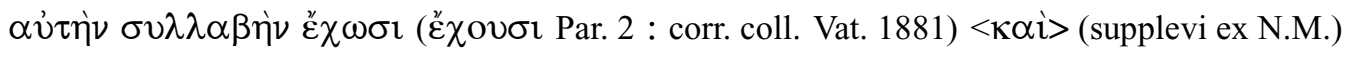

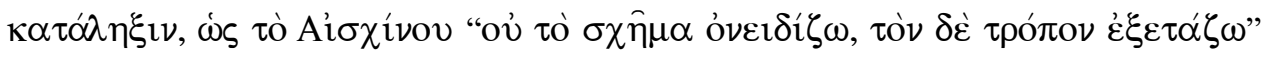

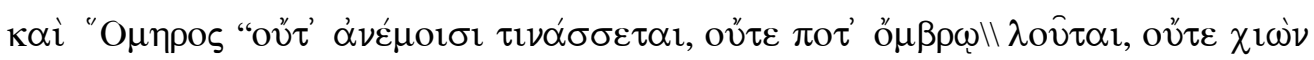

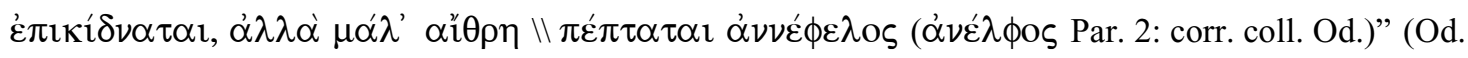

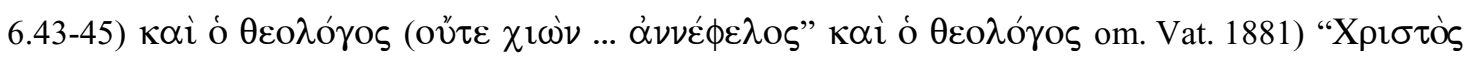

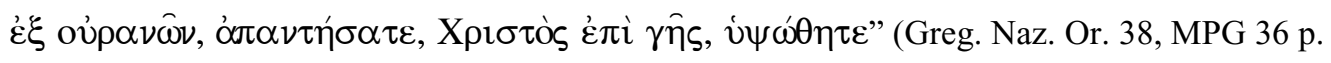
$312,48)$.

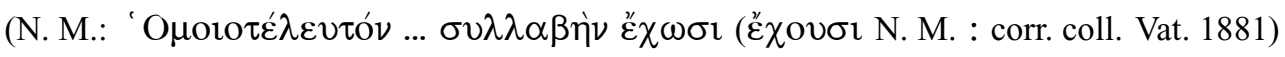

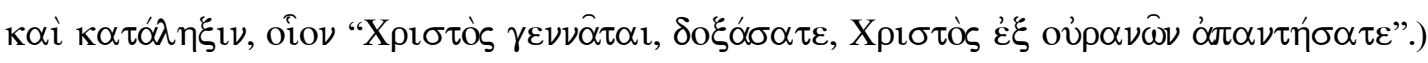

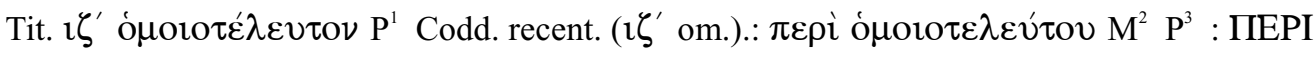

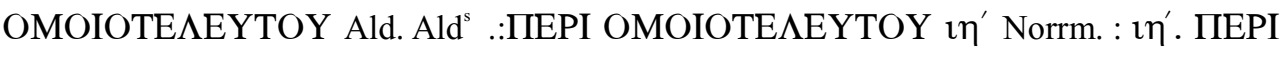
OMOIOTE $\Lambda$ EYTOY Edd.

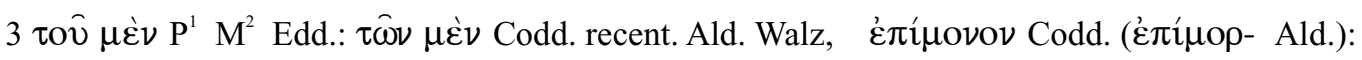

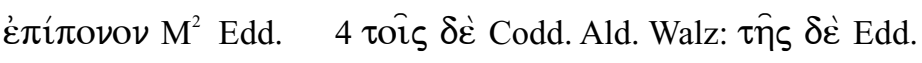


$\imath \theta^{\prime}$. ПЕРI OMOIOПТ $\Omega$ TOY.

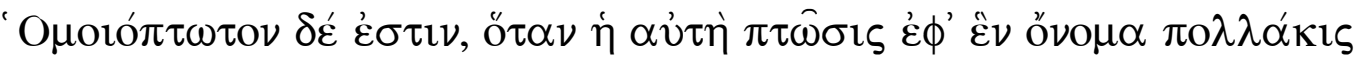

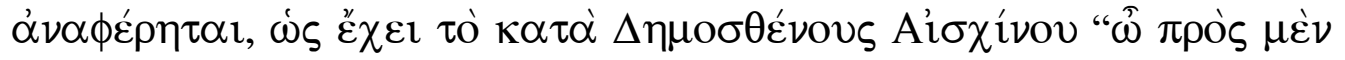

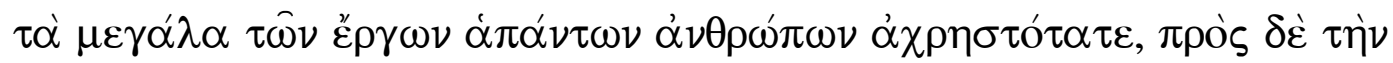

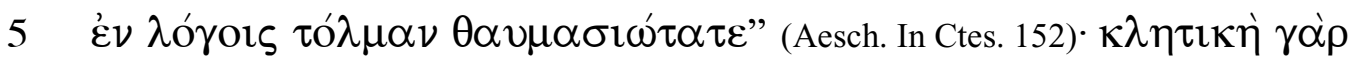

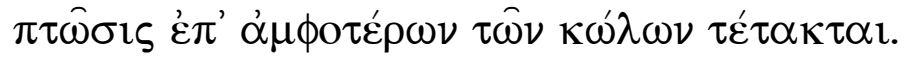

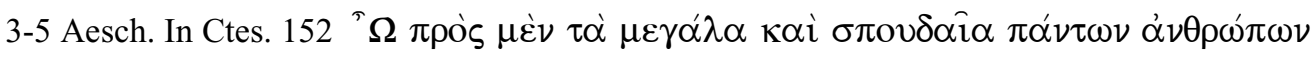

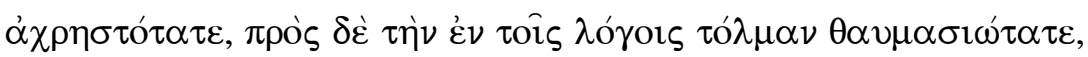

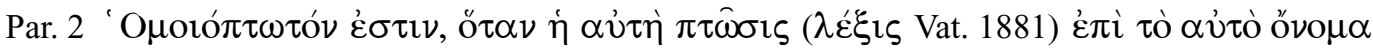

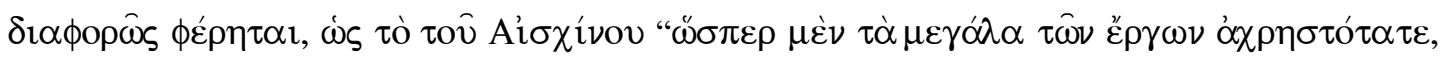

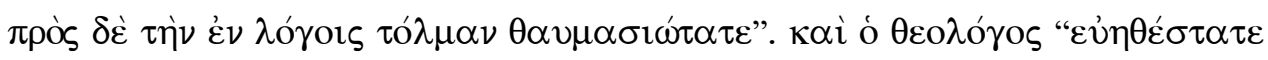

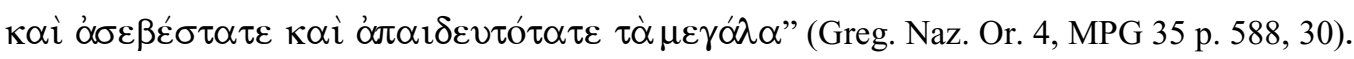

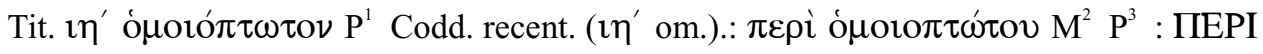

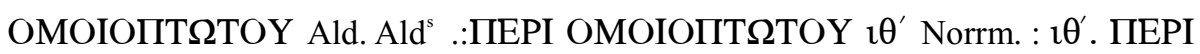
OMOIOПT $\Omega$ TOY Edd.

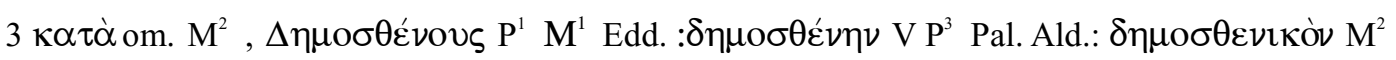

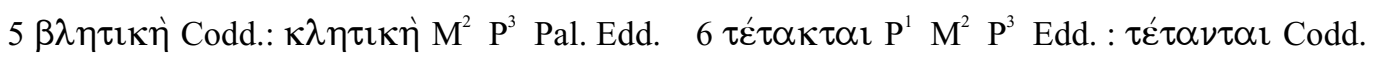
recent. 


\section{$\kappa^{\prime}$. ПЕРI ПАРONOMA $\Sigma$ IA $\Sigma$.}

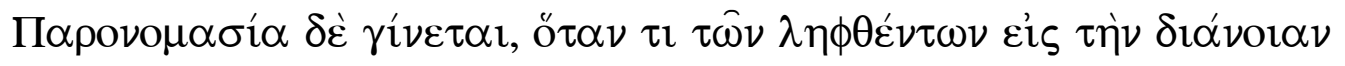

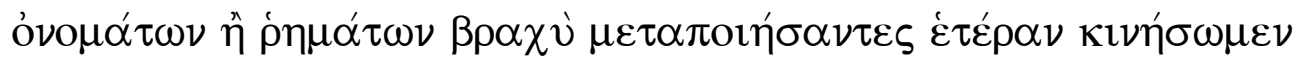

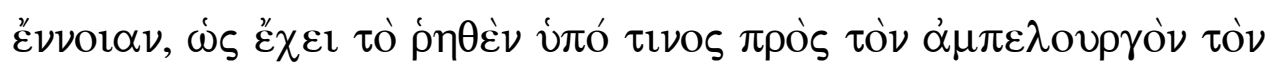

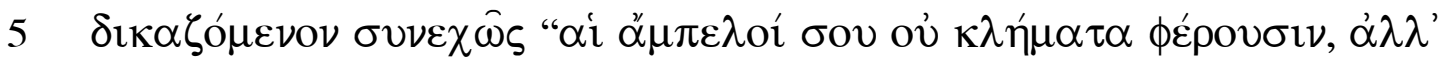

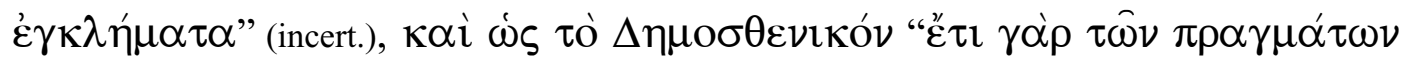

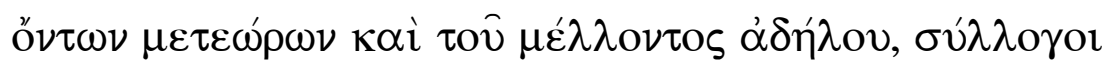

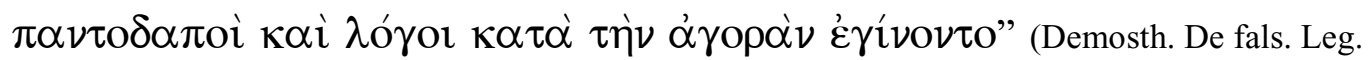

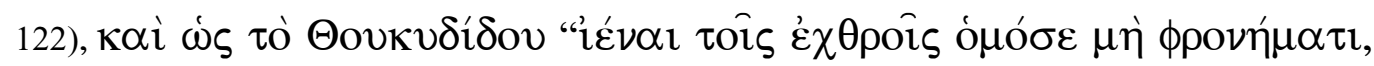

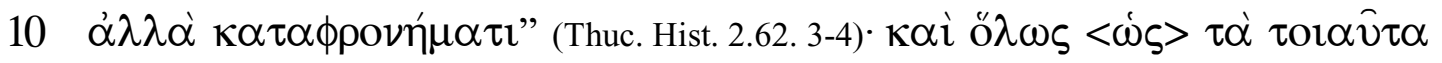

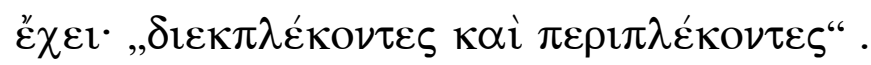

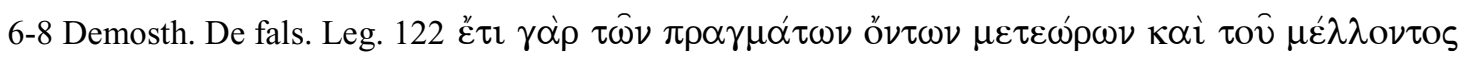

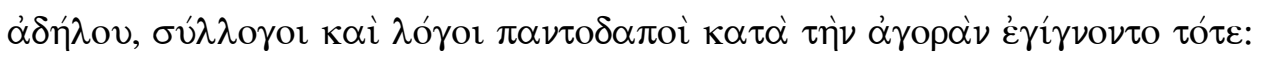

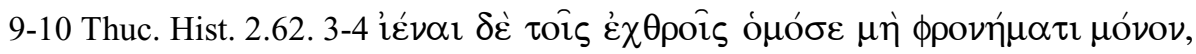
$\alpha \lambda \lambda \alpha \kappa^{\prime} \alpha \grave{i} \kappa \alpha \tau \alpha \phi \rho о \nu \eta \dot{\mu} \mu \tau \tau$

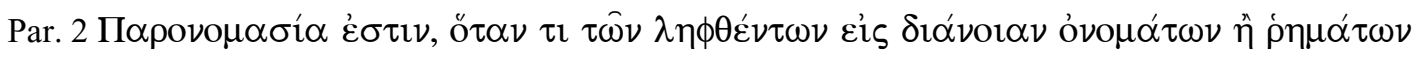

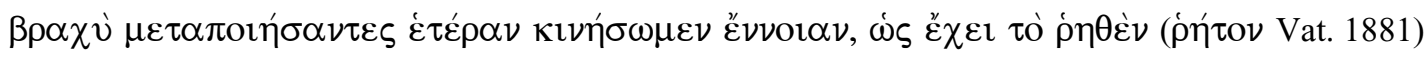

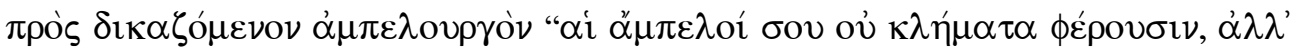

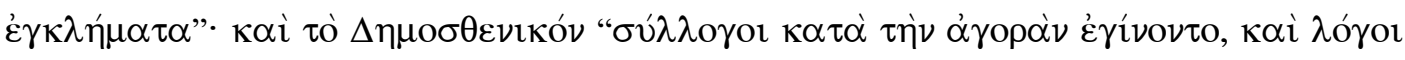

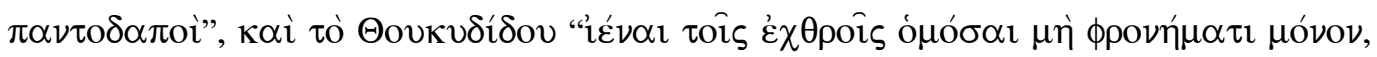

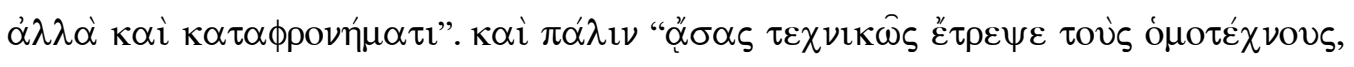

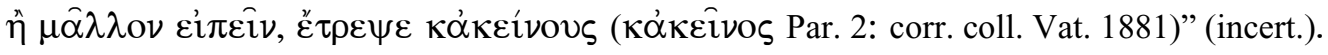

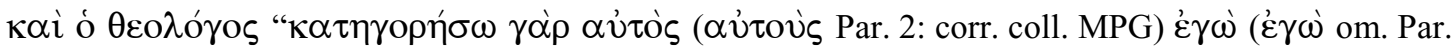

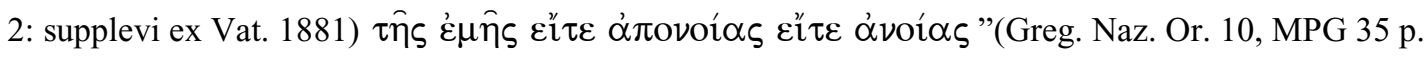

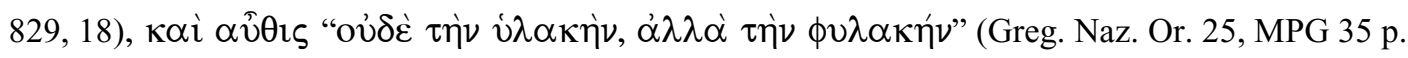

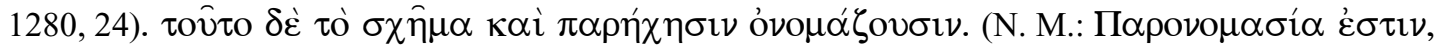

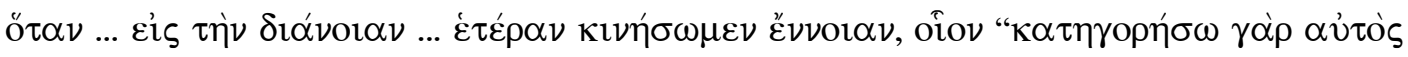

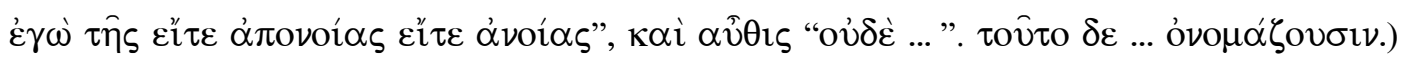

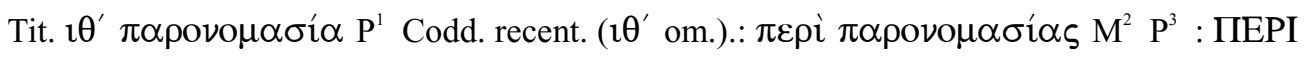
ПАPONOMA $\Sigma$ IA $\Sigma$ Ald. Ald ${ }^{\mathrm{s}}$ : ПЕPI ПАР- $\kappa^{\prime}$ Norrm. : $\kappa^{\prime}$. ПЕPI ПАР- Edd.

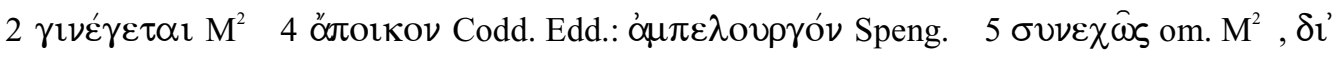

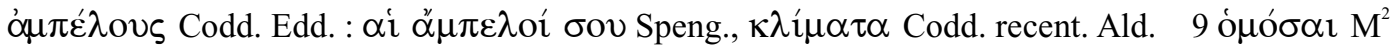

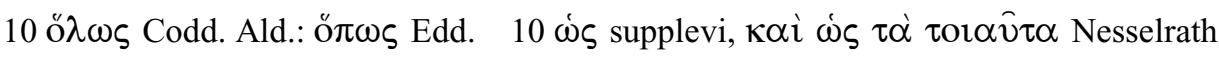

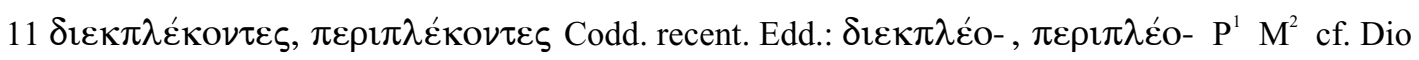

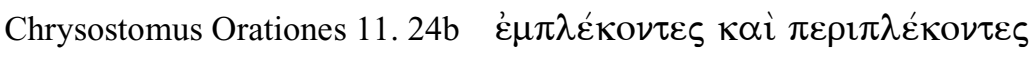




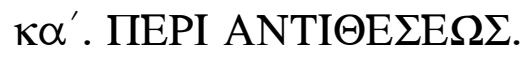

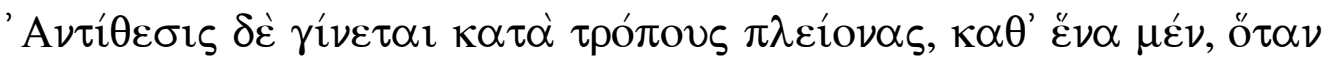

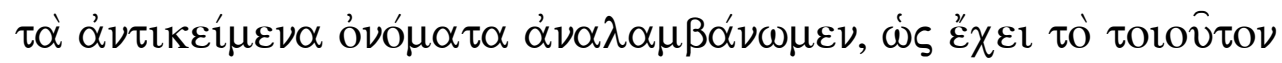

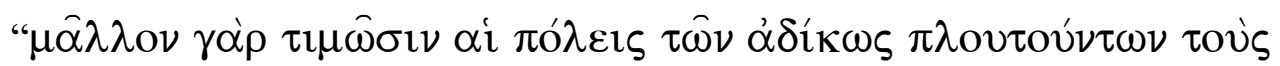

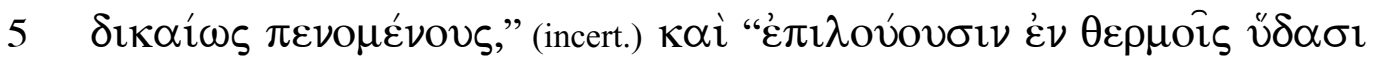

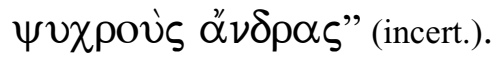

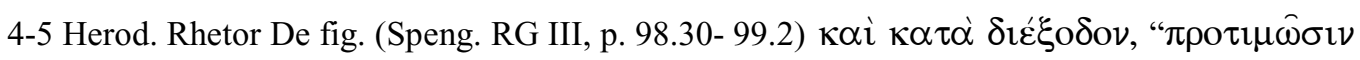

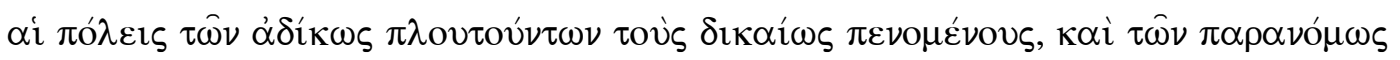

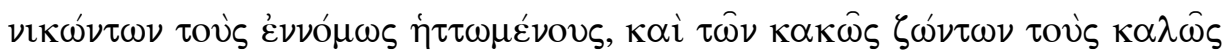

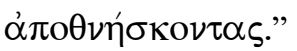

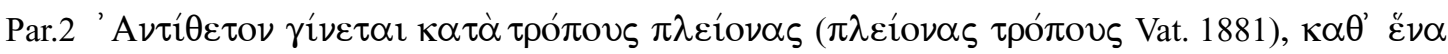

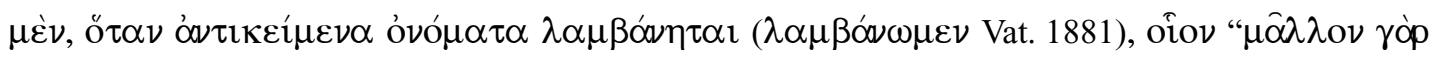

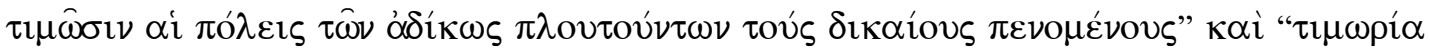

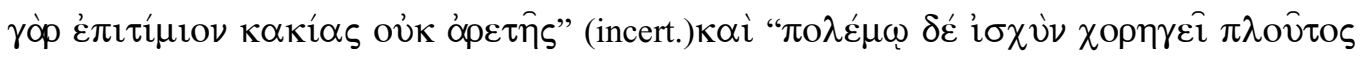

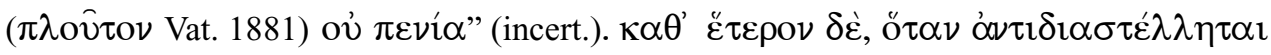

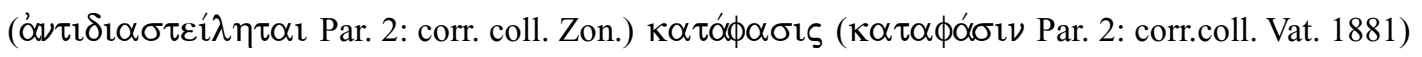

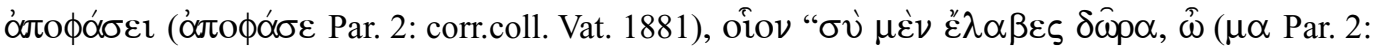

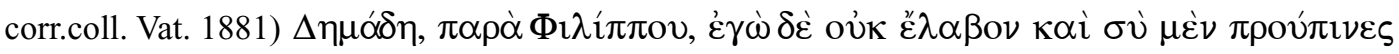

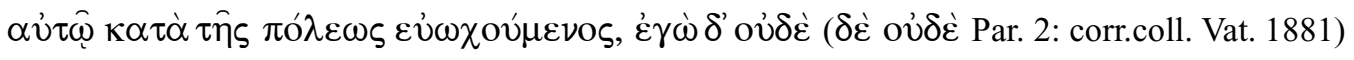

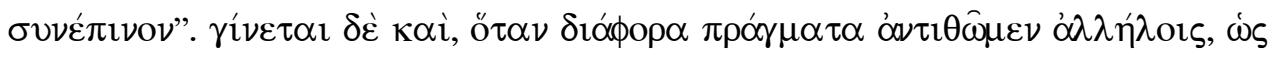

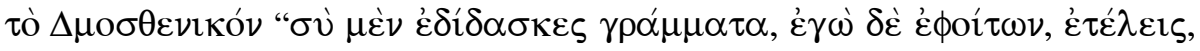

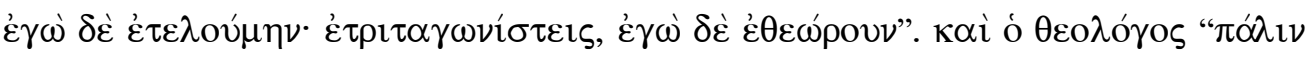

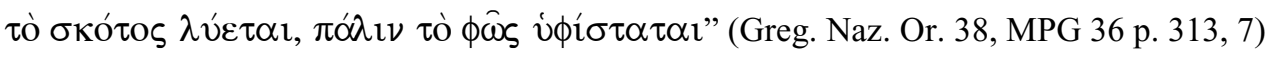

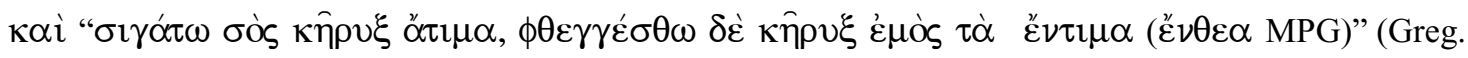

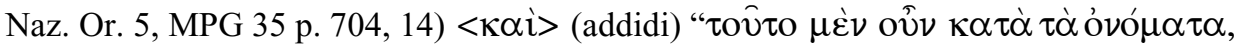

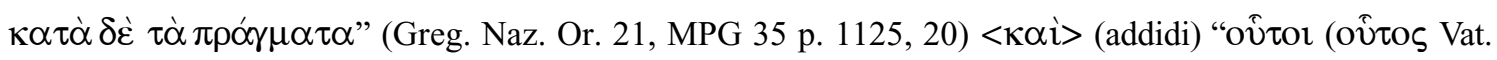

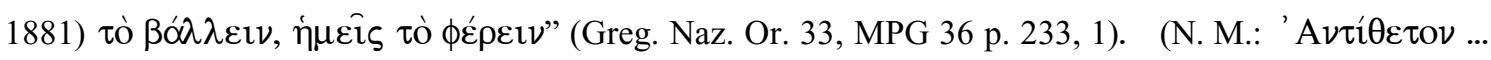

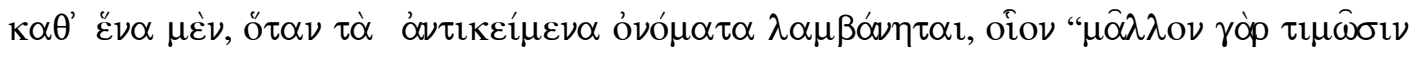

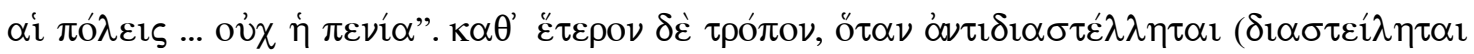

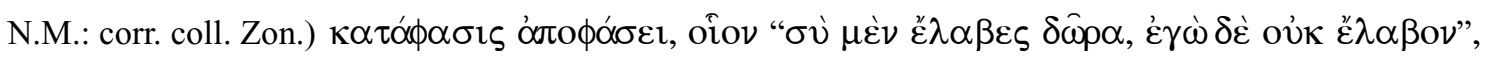

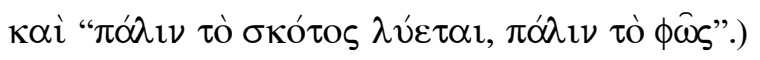

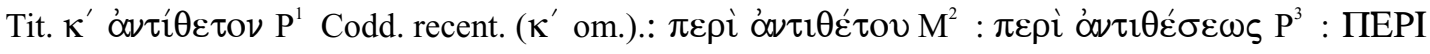

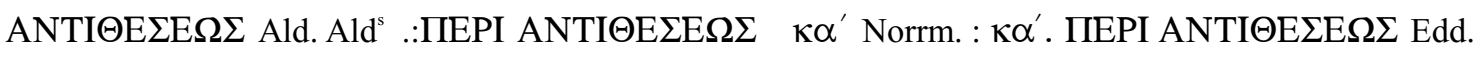

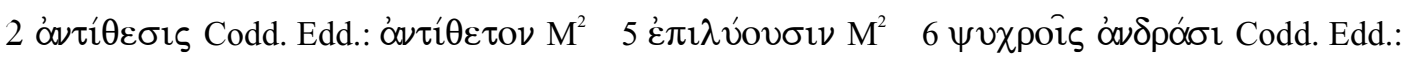

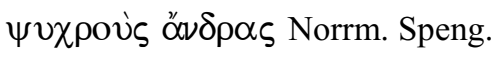




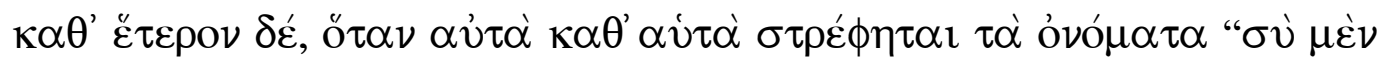

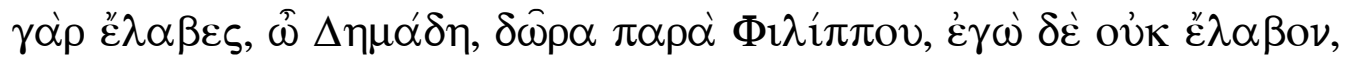

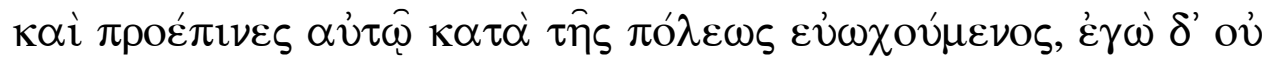

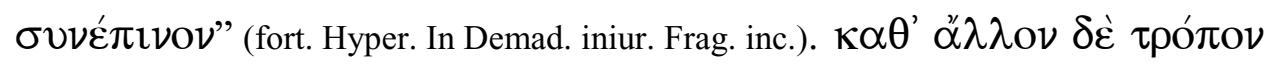

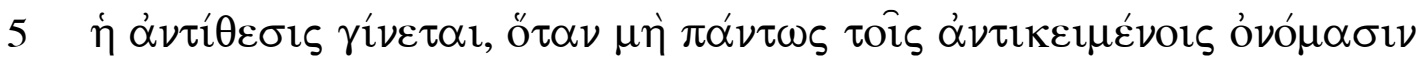

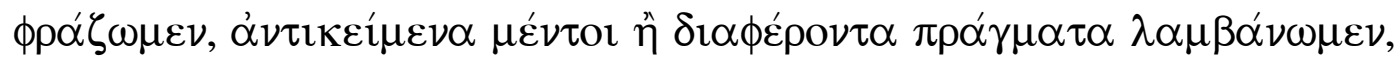

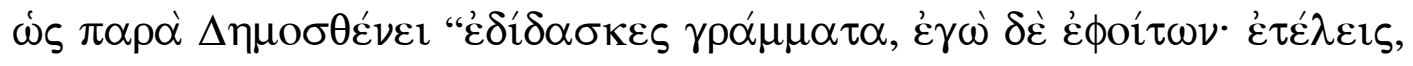

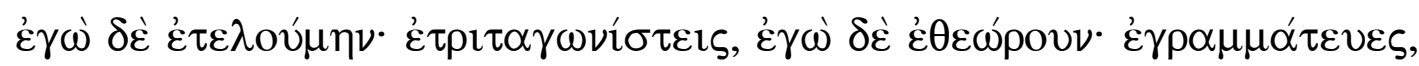

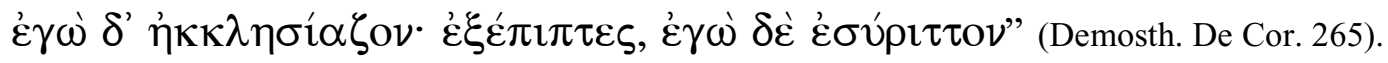

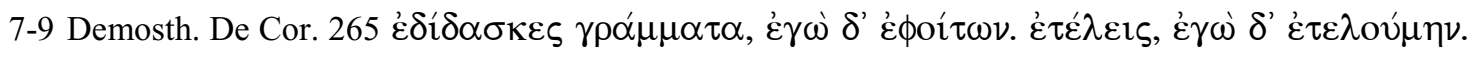

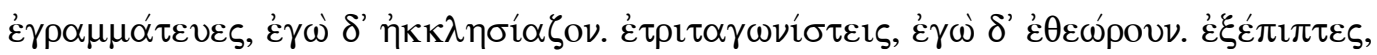

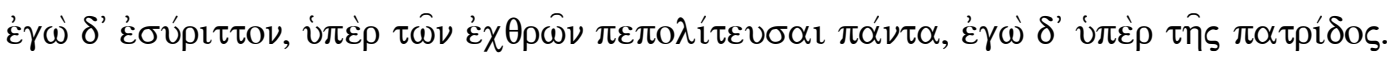

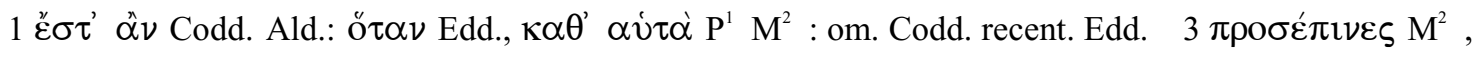

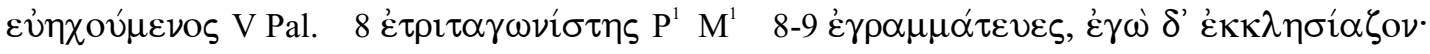

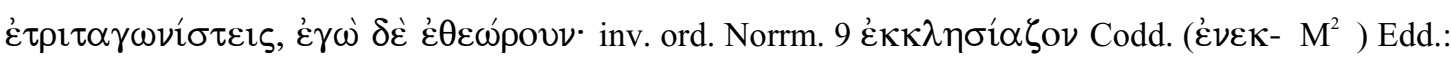

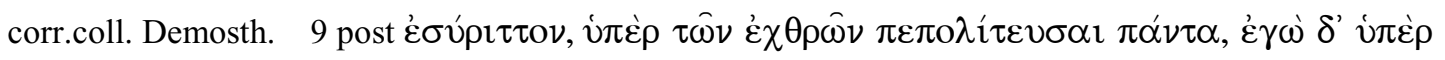

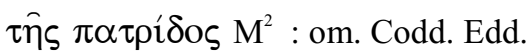




\section{$\kappa \beta^{\prime}$. ПЕPI ANTIMETA $\Theta E \Sigma E \Omega \Sigma$ H $\Sigma$ YГKPI $\Sigma$ E $\Omega \Sigma$ H П $\Lambda$ OKH $\Sigma$.}

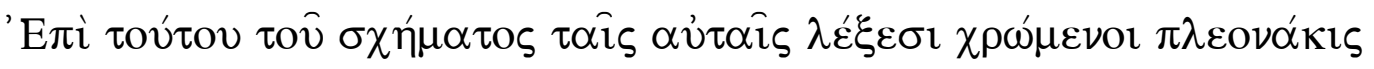

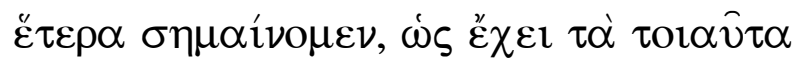

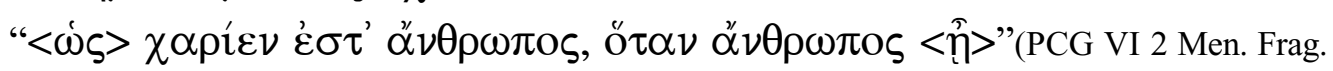
5 inc. 707) $<\kappa \alpha i>$

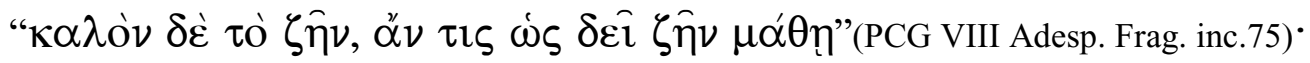

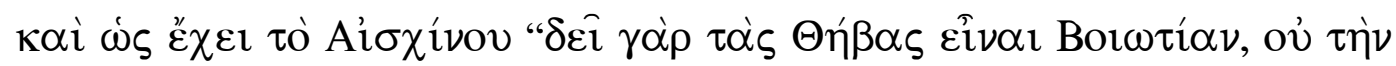

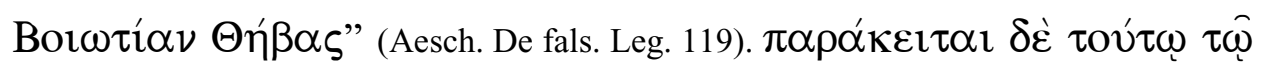

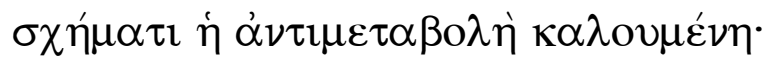

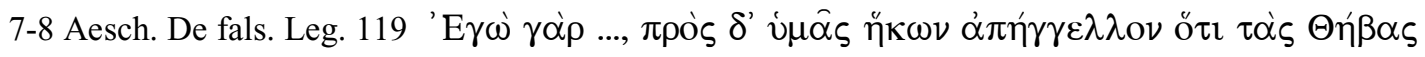

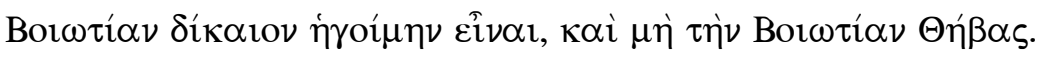

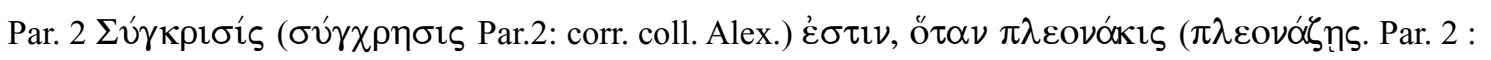

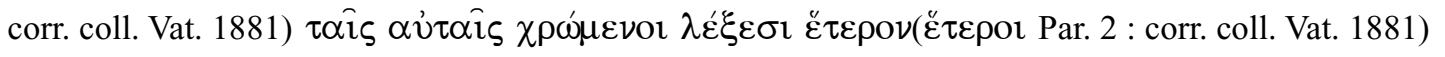

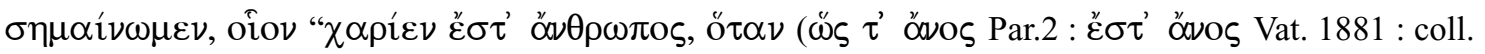

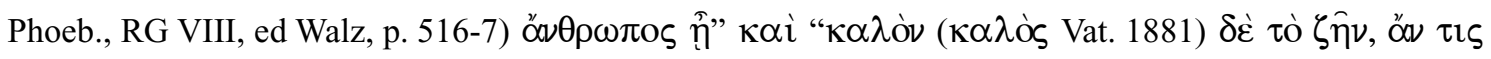

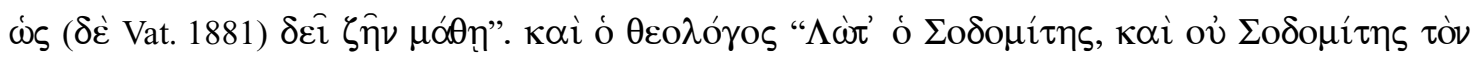

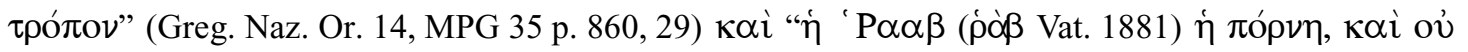

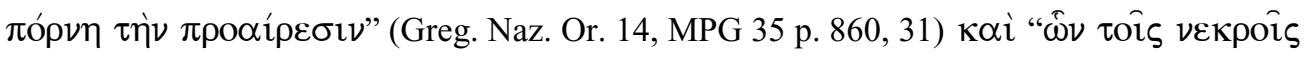

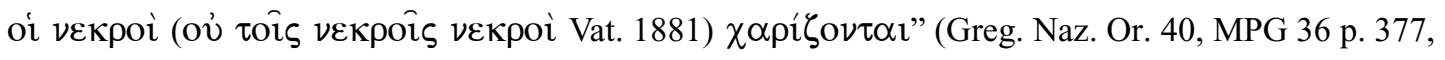

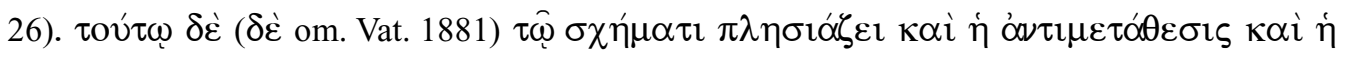

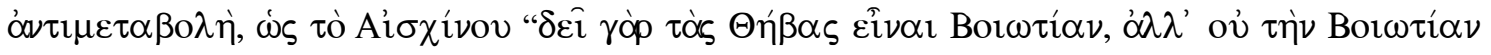

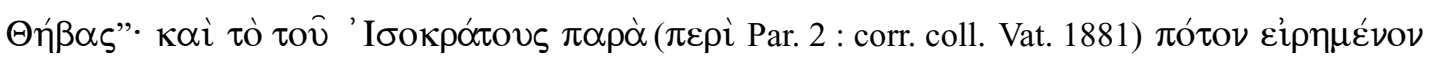

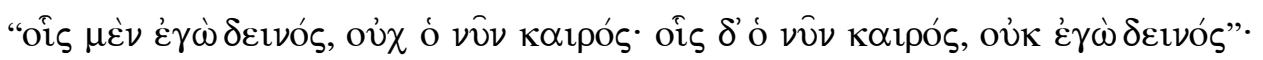

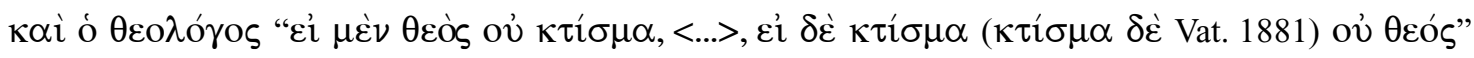

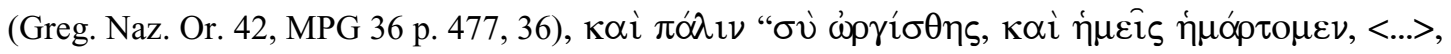

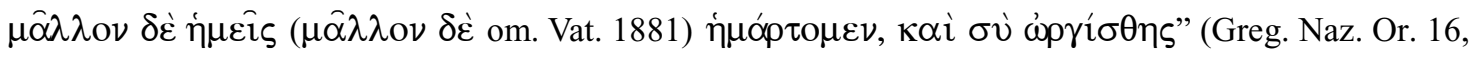

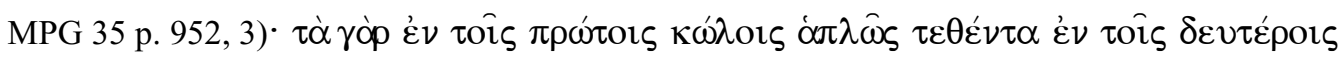

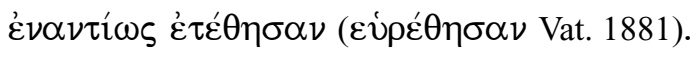

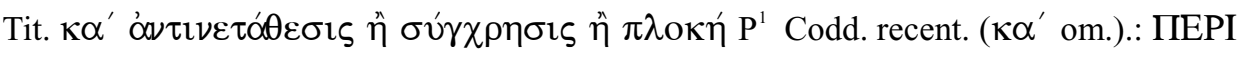

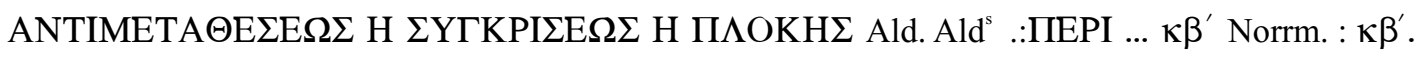

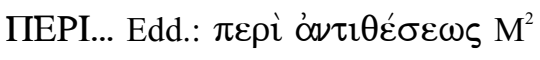

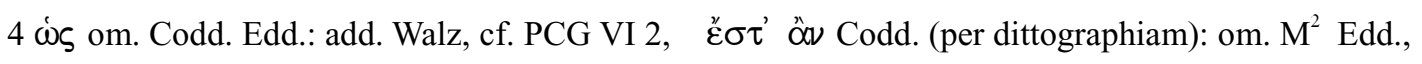

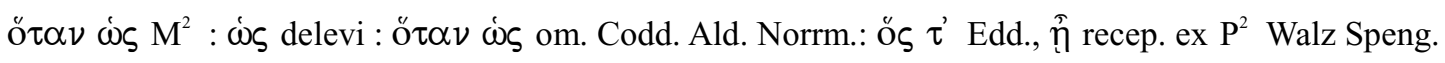

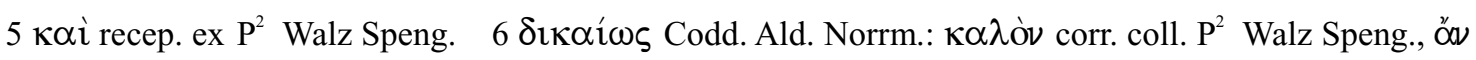

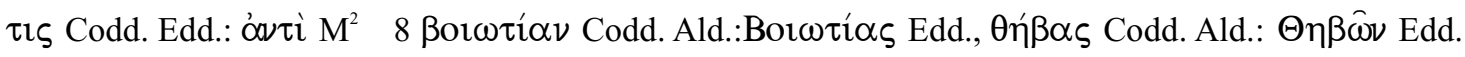




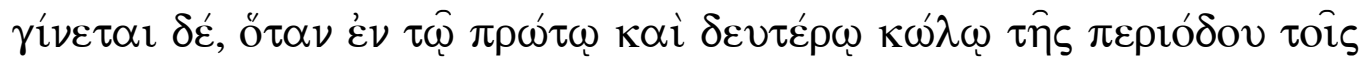

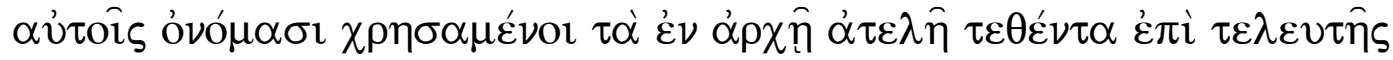

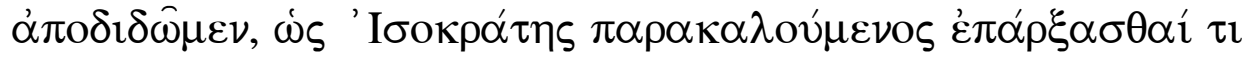

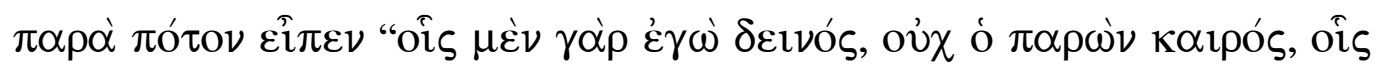

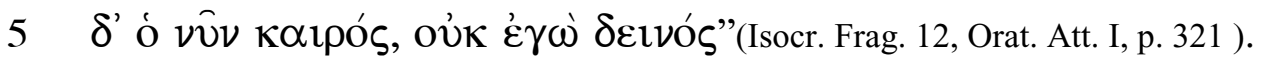

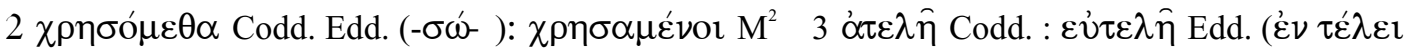
Speng.): an potius $\alpha \dot{\tau} \lambda \hat{\omega} \zeta$ ut $\mathrm{P}^{2}$ ?, $\tau \alpha v \tau \hat{\eta} \varsigma$ Codd. Edd.: $\tau \varepsilon \lambda \varepsilon v \tau \hat{\eta} \varsigma$ app. crit. Speng. cf. $\mathrm{P}^{2}$,

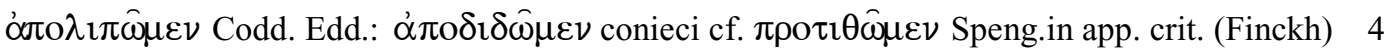

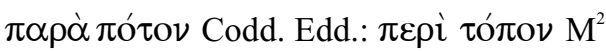


$\kappa \gamma^{\prime}$. ПEPI ANTENANTI $\Omega \Sigma E \Omega \Sigma$.

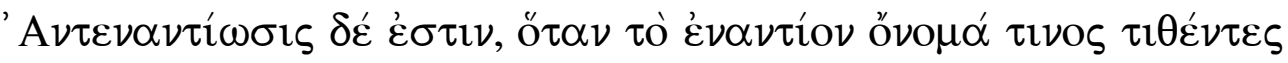

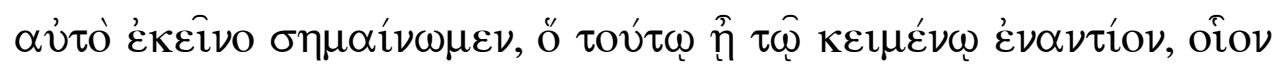

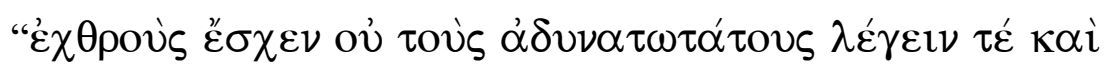

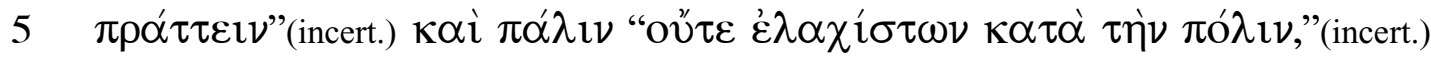

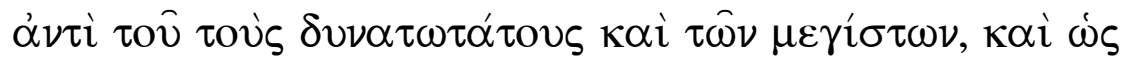

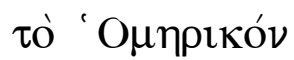

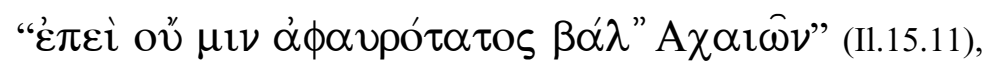

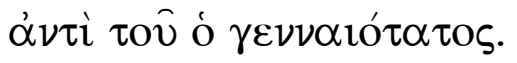

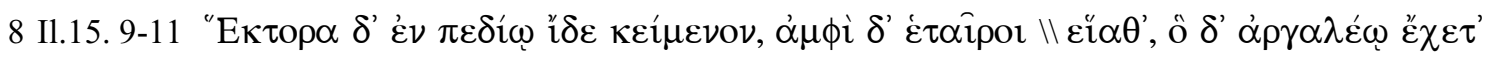

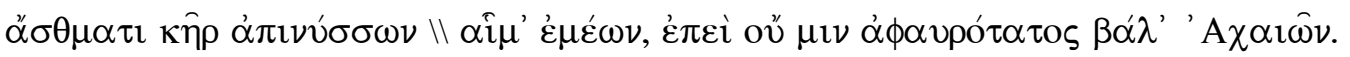

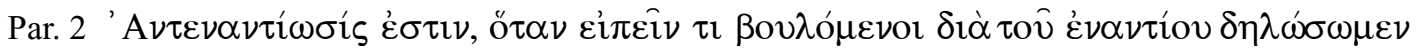

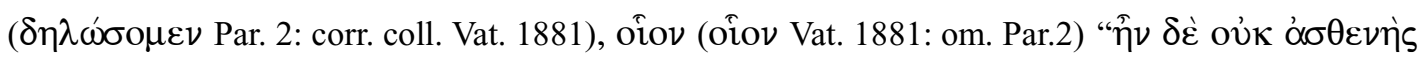

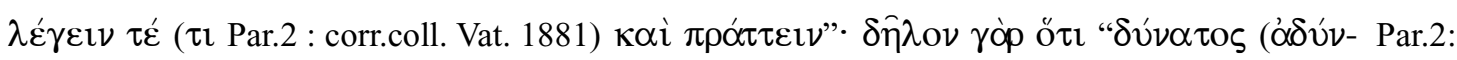

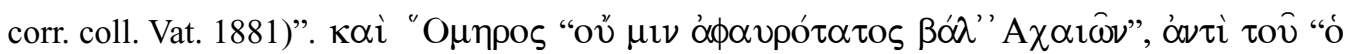

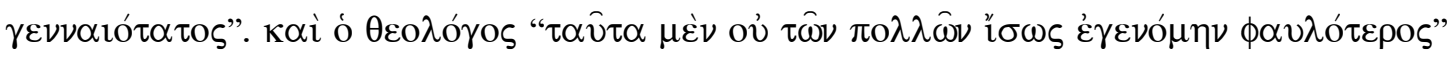
(Greg. Naz. Or. 2, MPG 35 p. 484, 43).

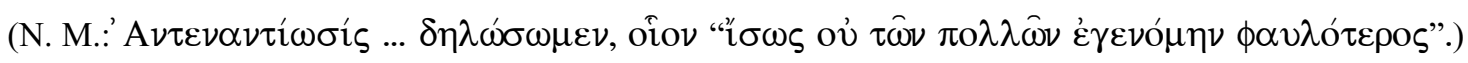

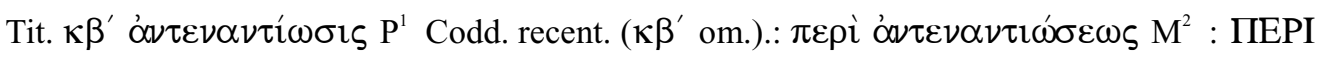
ANTENANTI $\Omega \Sigma$ E $\Omega \Sigma$ Ald. Ald ${ }^{s} .:$ חEPI ANTEN- $\kappa \gamma^{\prime}$ Norrm. $: \kappa \gamma^{\prime}$. ПEPI ANTEN- Edd.

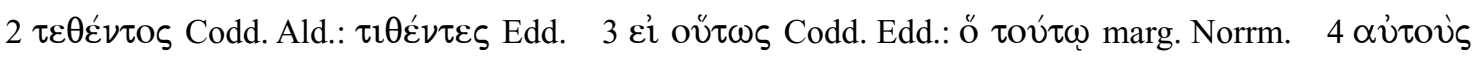

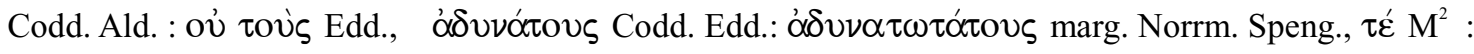

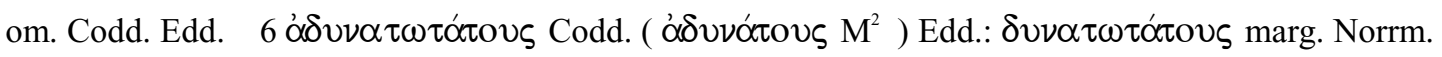
Speng.: corr. coll. Vat. 1881, $\tau \grave{\alpha} \mu \dot{\varepsilon} \gamma 1 \sigma \tau \alpha$ Codd. Edd.: $\tau \hat{\omega} \nu \mu \varepsilon \gamma i ́ \sigma \tau \omega \nu$ marg. Norrm. $8 \mu \eta \nu$ Codd.

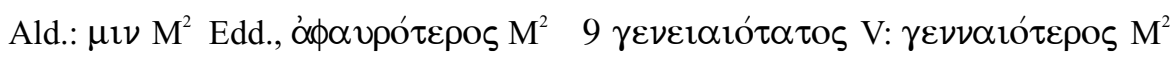


$\kappa \delta^{\prime}$. ПЕРI YПЕРВАТОY.

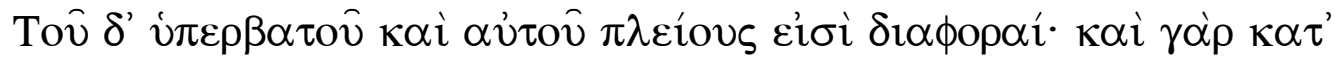

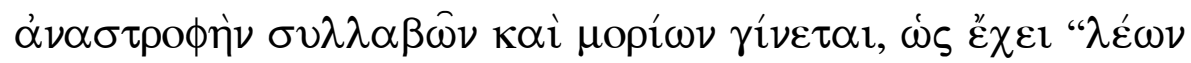

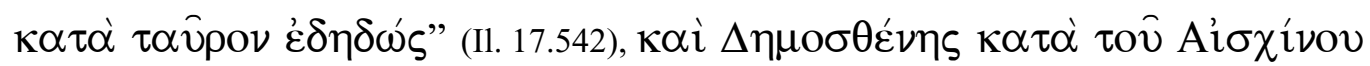

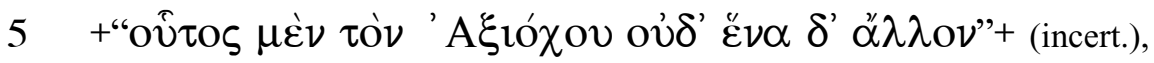

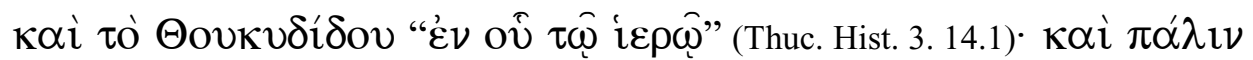

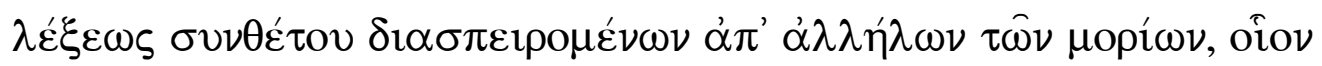

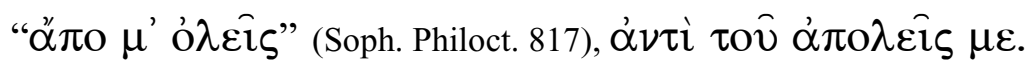

Par.2 'Y

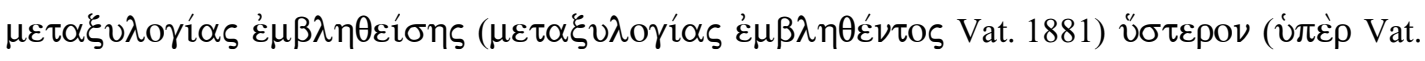

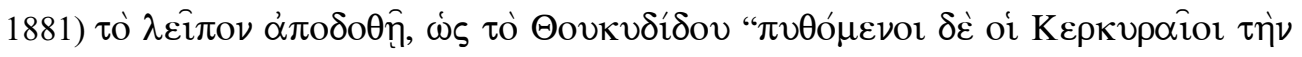

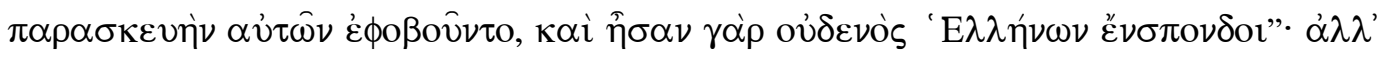

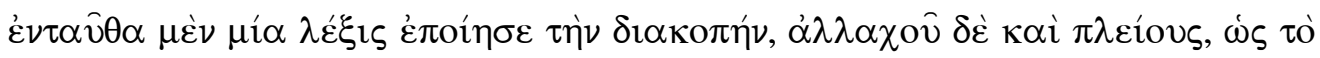

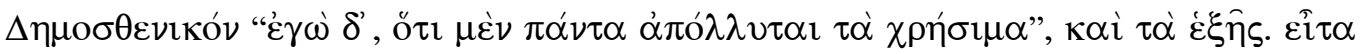

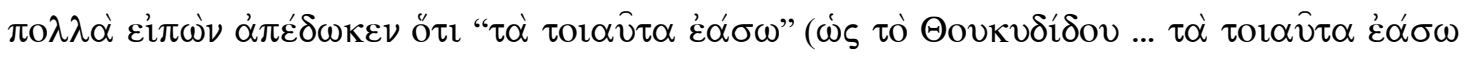

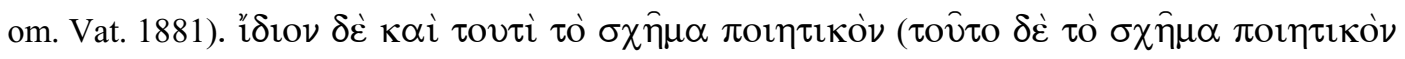

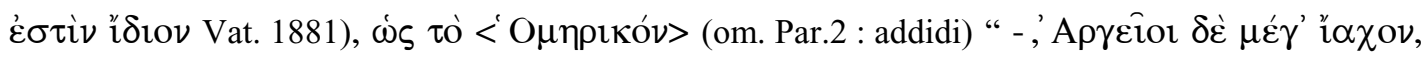

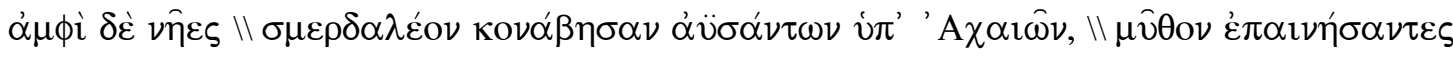

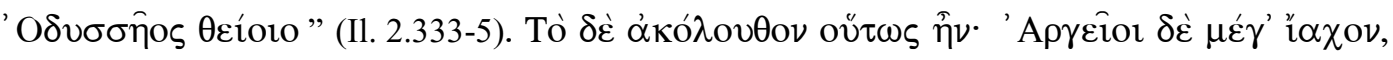

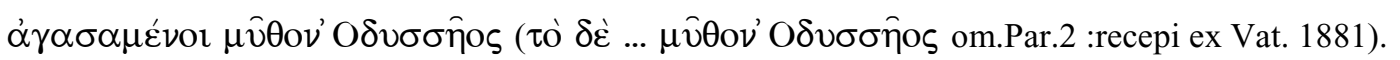

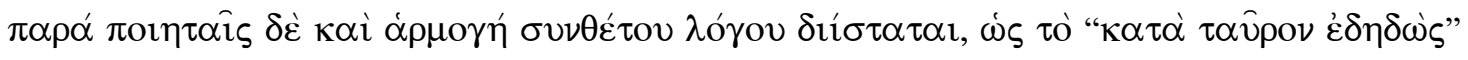

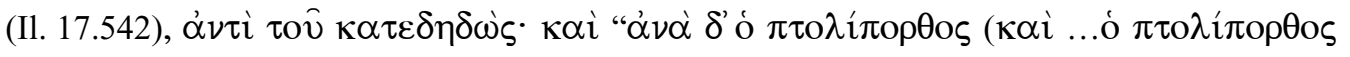

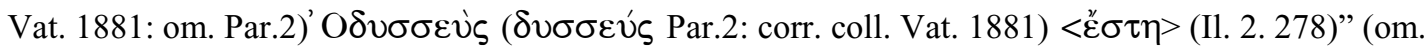

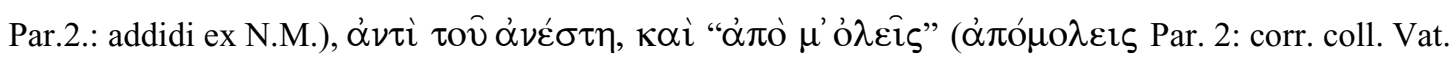

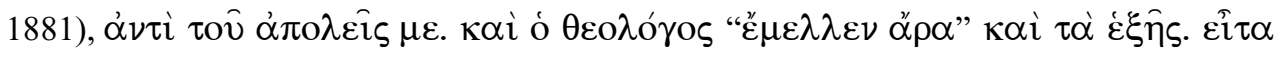

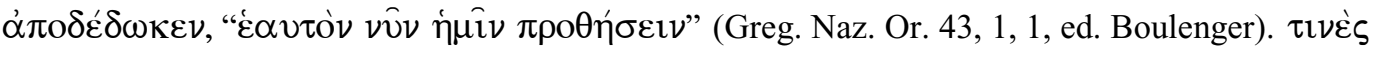

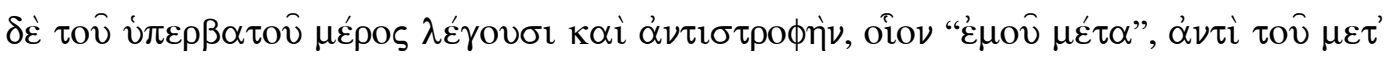

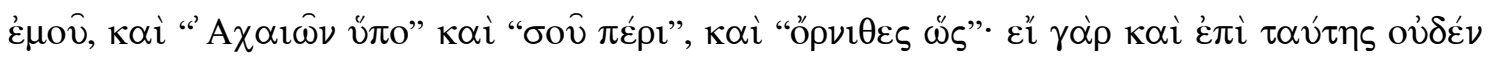

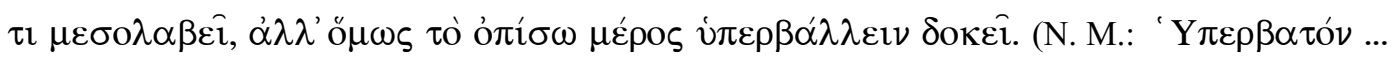

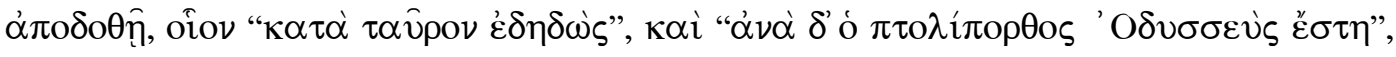

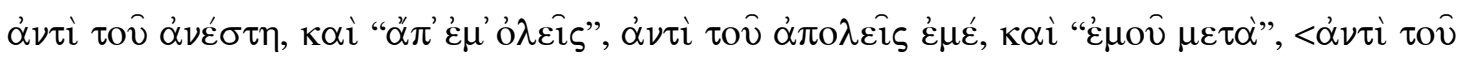

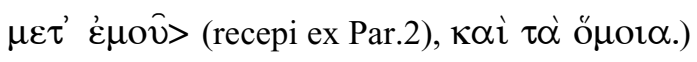

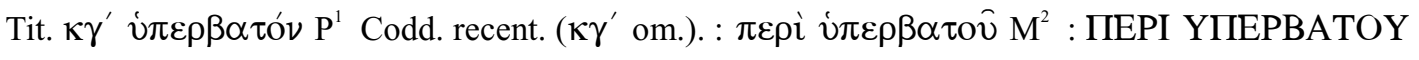
Ald. Ald ${ }^{s} .:$ ПЕРI YПЕРВАТОY $\kappa \delta^{\prime}$ Norrm. : $\kappa \delta^{\prime}$. ПЕРI YПЕРВАTOY Edd.

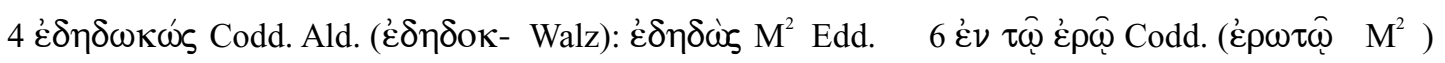
Edd: '̇ंv ỗ $\tau \hat{\omega}$ i $\varepsilon \rho \omega \hat{\omega}$ Norrm. (ỗ om.) Speng. 


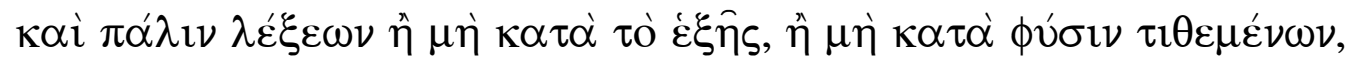

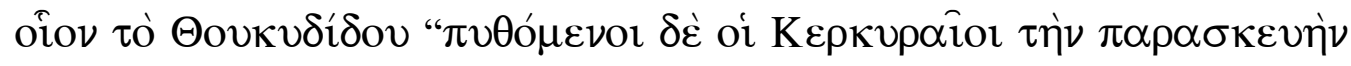

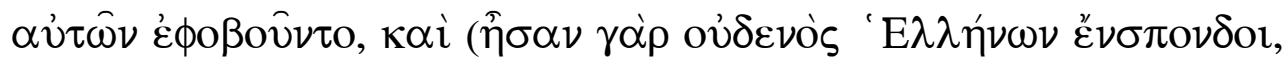

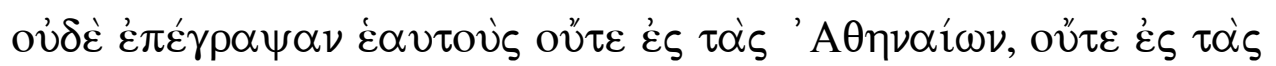

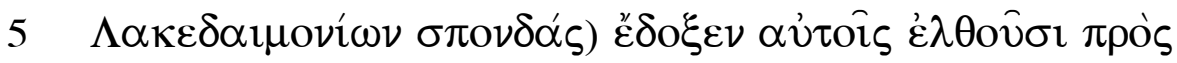

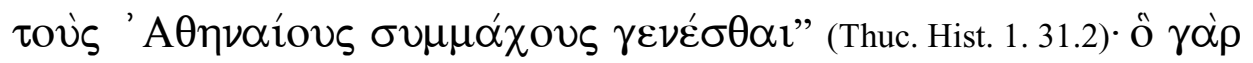

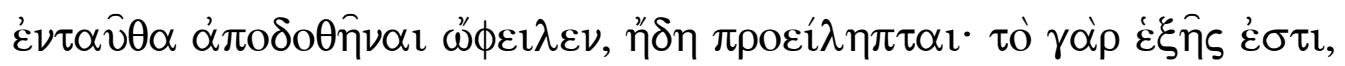

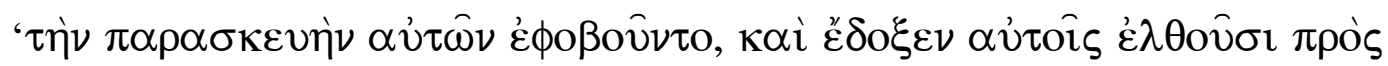

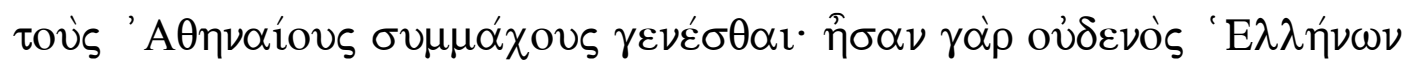

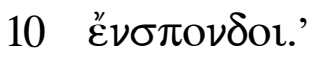

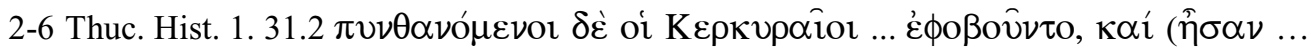

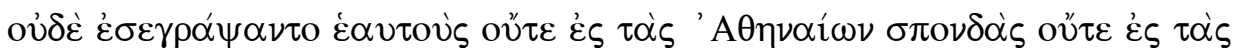

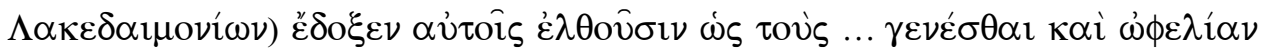

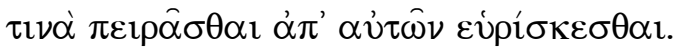

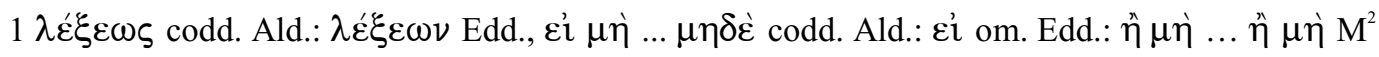

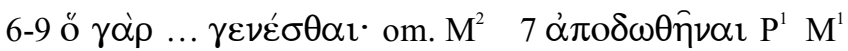




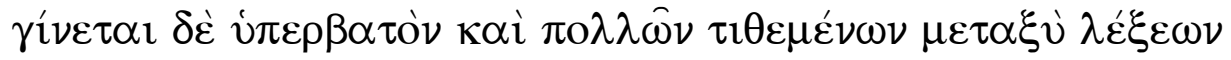

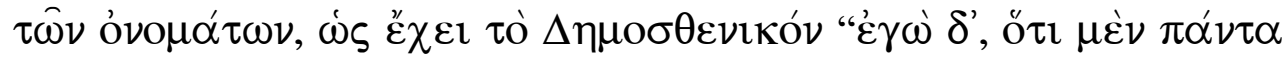

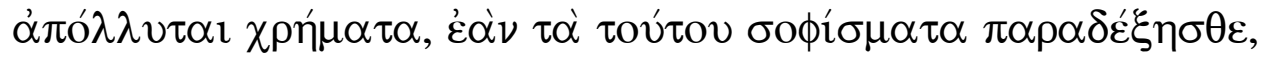

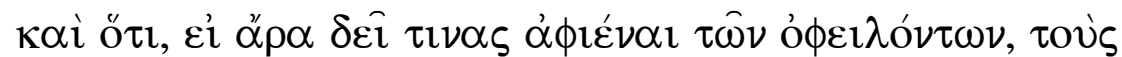

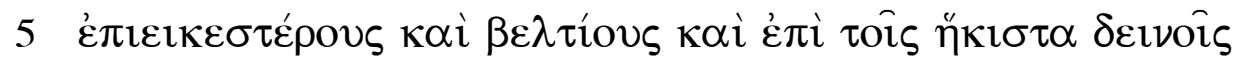

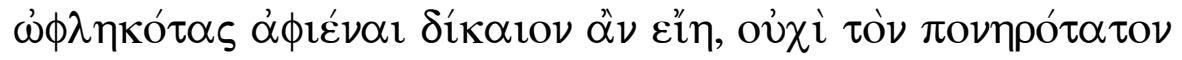

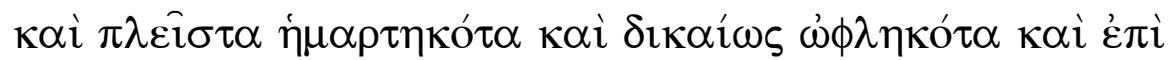

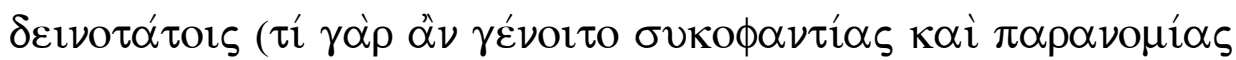

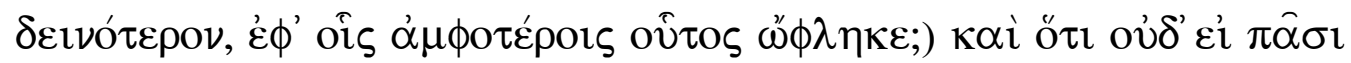

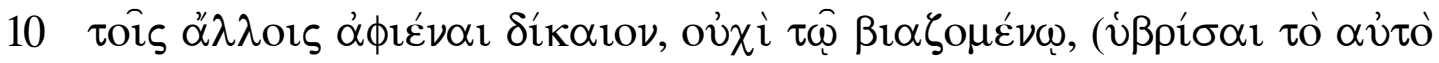

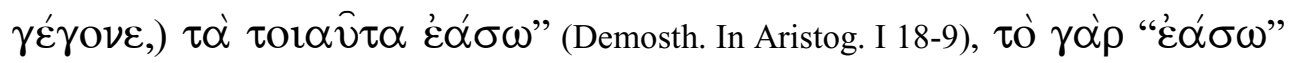

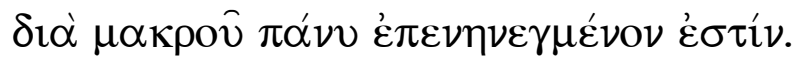

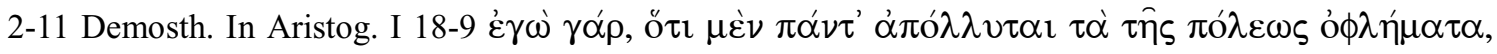

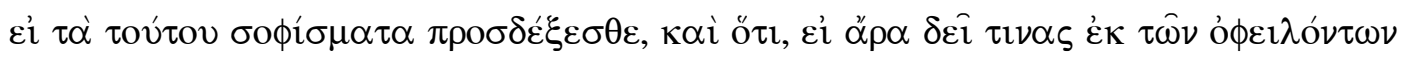

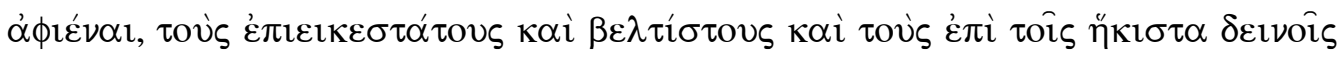

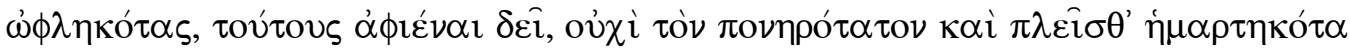

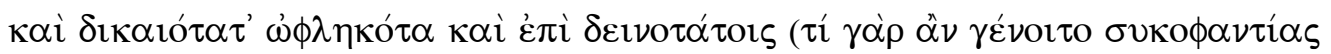

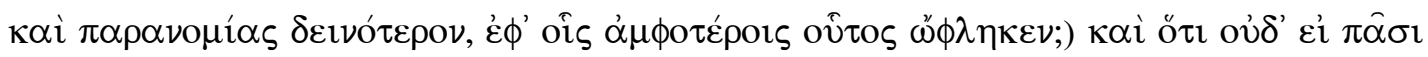

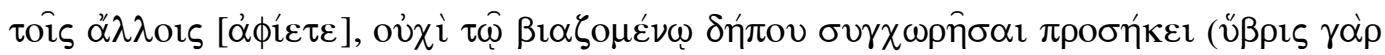

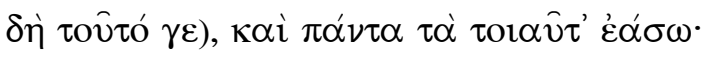

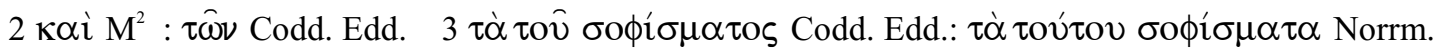

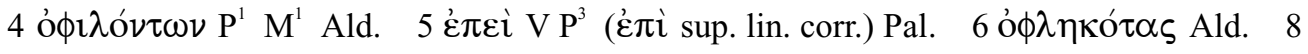

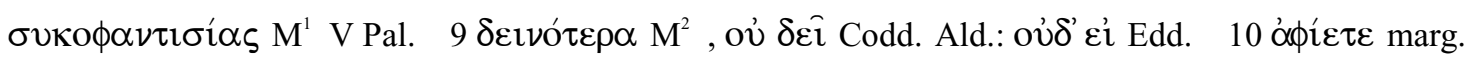

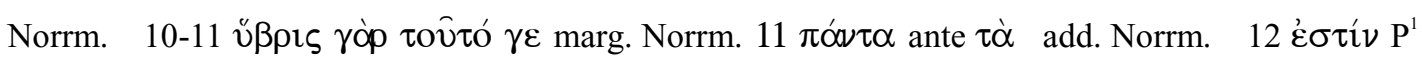
$\mathrm{M}^{2}$ : om. Codd. recent. Edd. 
$\kappa \varepsilon^{\prime}$. ПЕРI ПАРЕМВО $\Lambda \mathrm{H} \Sigma$.

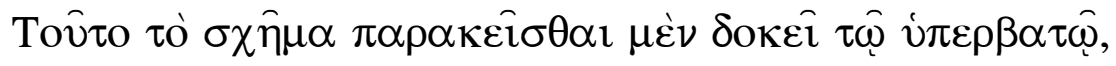

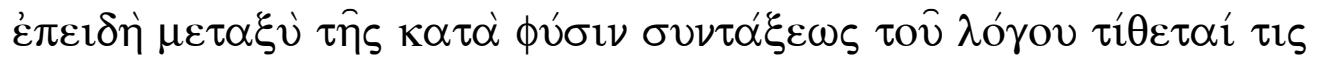

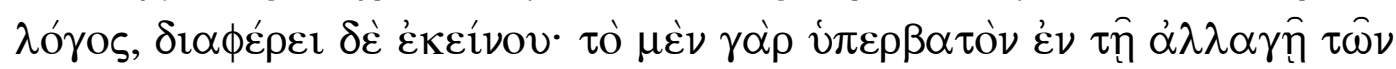

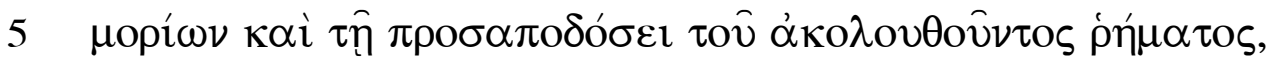

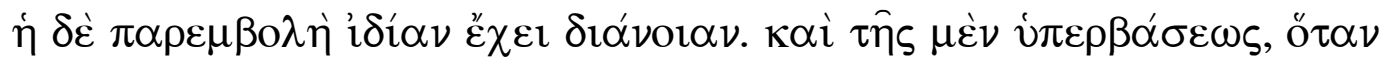

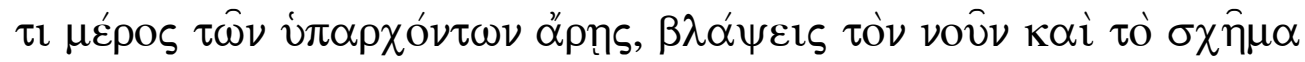

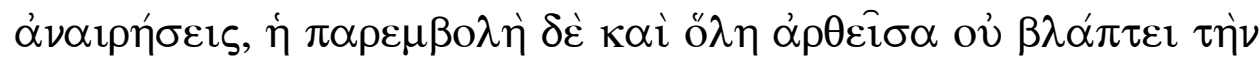

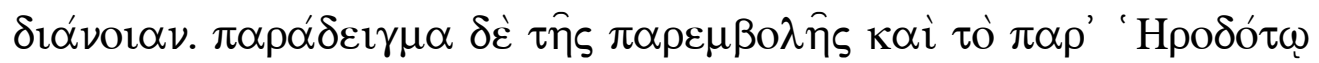

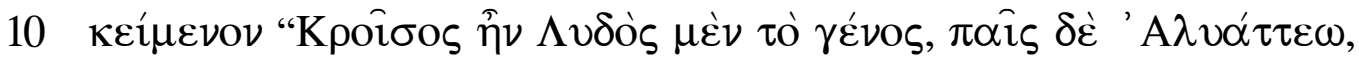

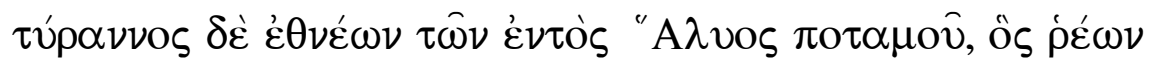

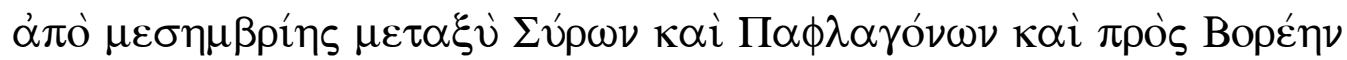

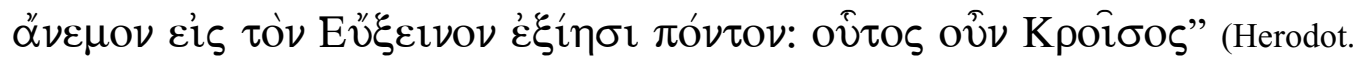

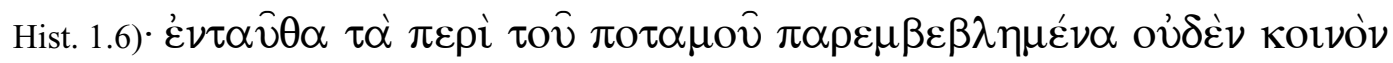

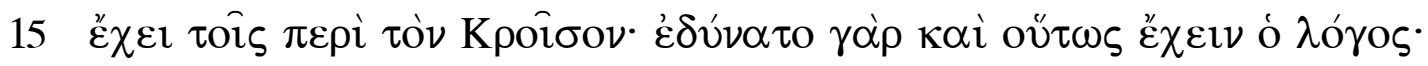

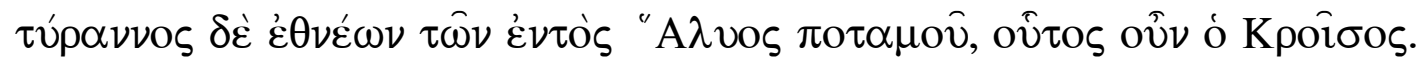

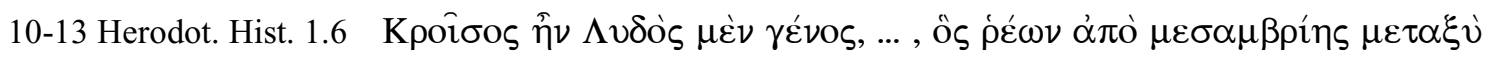

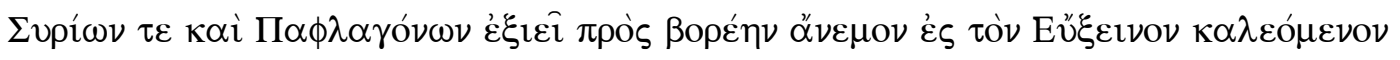

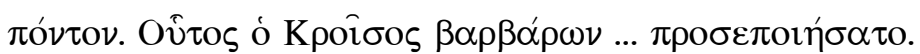

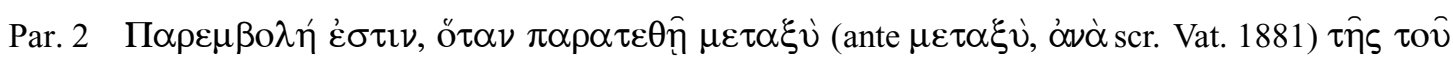

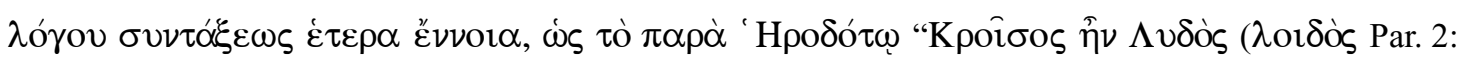

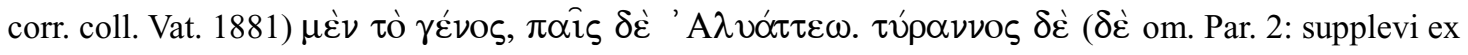

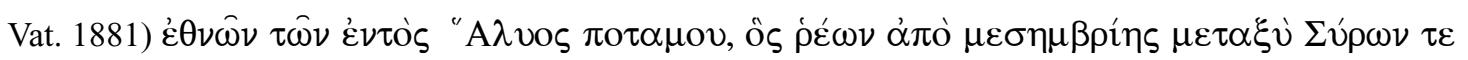

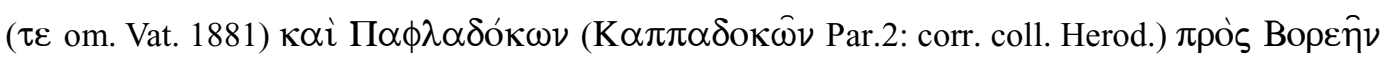

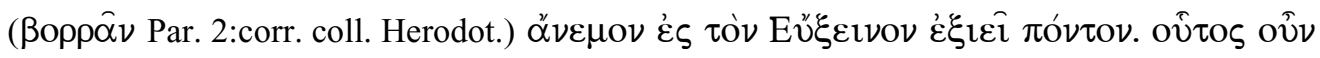

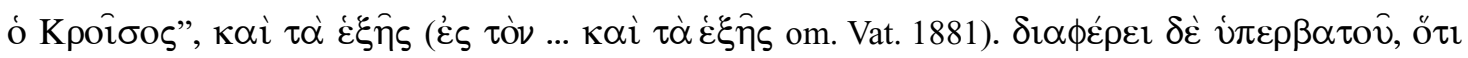

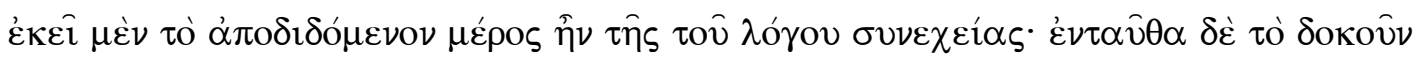

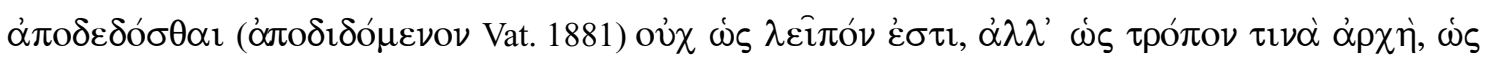

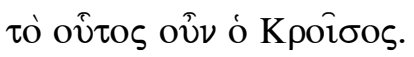

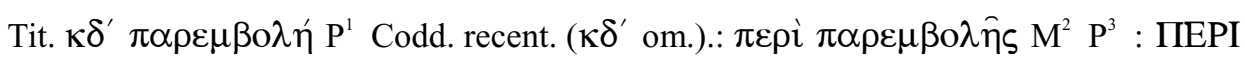
ПАРЕМВО $\Lambda \mathrm{H} \Sigma$ Ald. Ald ${ }^{\mathrm{s}}$ : ПЕРI ПАР- $\kappa \varepsilon^{\prime}$ Norrm. : $\kappa \varepsilon^{\prime}$. ПЕРI ПАР- Edd.

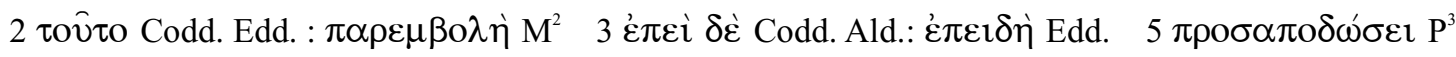

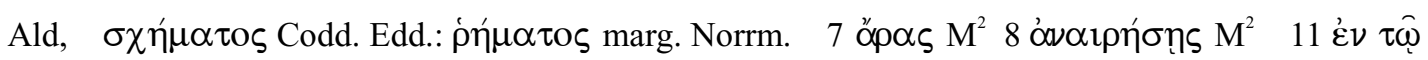

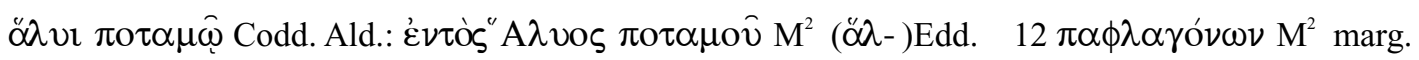

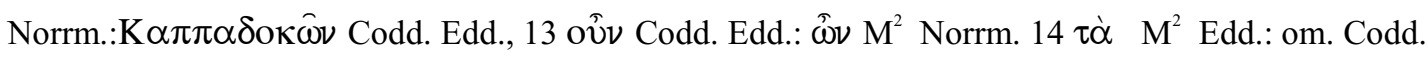
Ald., $\pi \alpha \rho \alpha \beta \varepsilon \beta \lambda \eta \mu \varepsilon ́ v \alpha \mathrm{M}^{2} 16$ $\hat{\omega} \nu$ Codd. Edd.: ov̂v Norrm. Walz cf. $\mathrm{P}^{2}$ 
$\kappa \varsigma^{\prime}$. ПЕРI ПАРI $\Sigma \mathrm{OY}$.

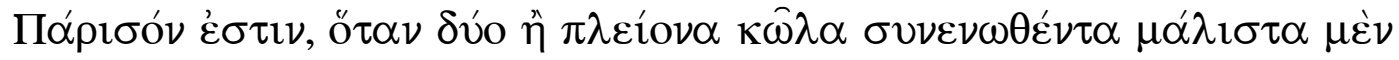

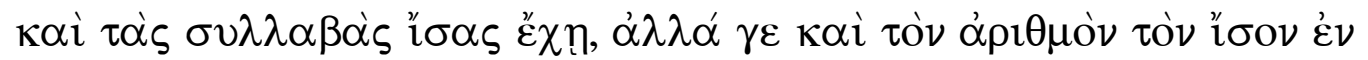

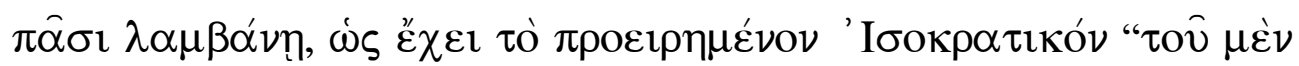

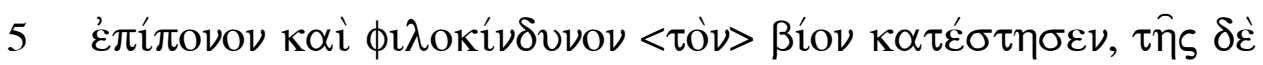

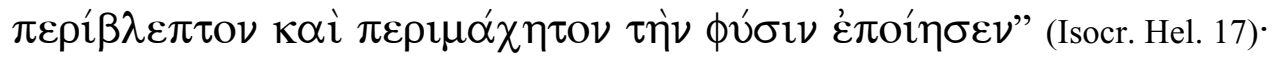

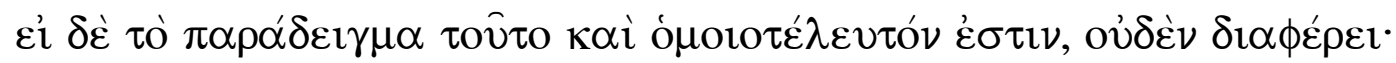

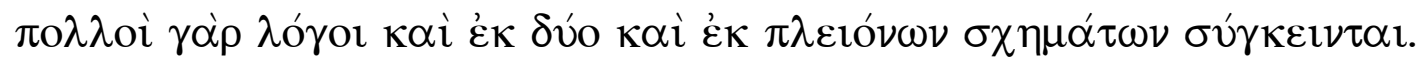

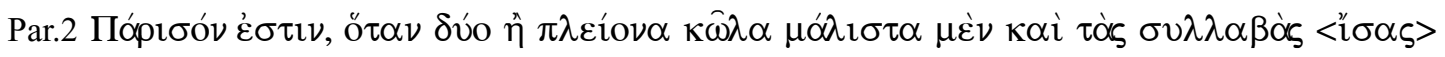

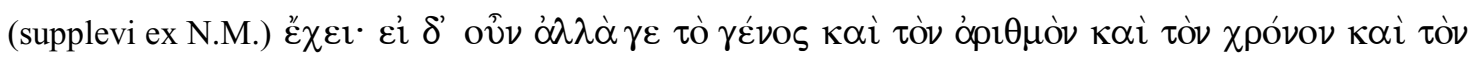

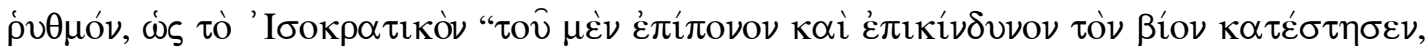

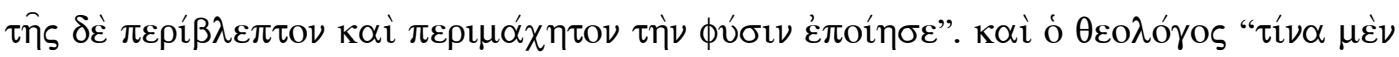

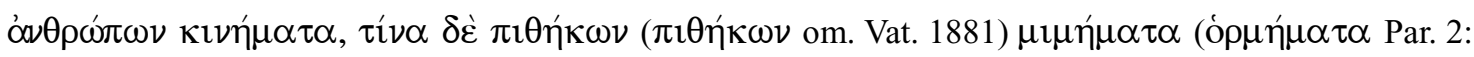

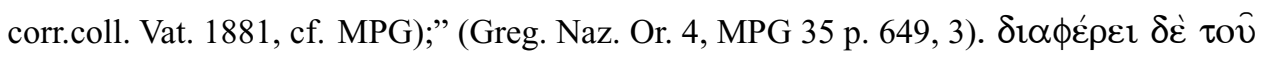

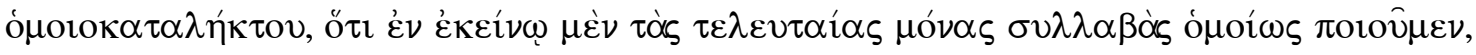

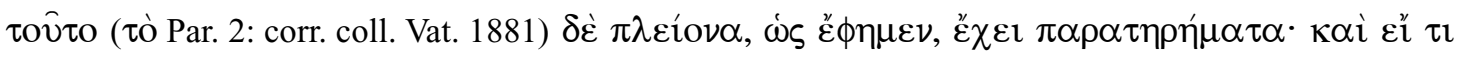

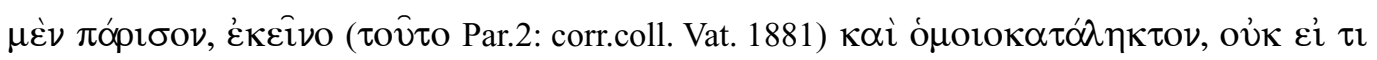

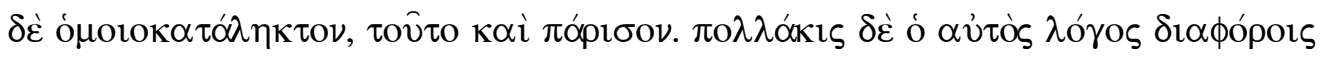

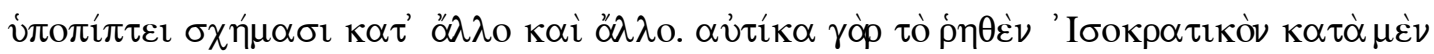

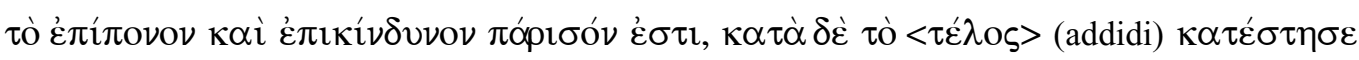

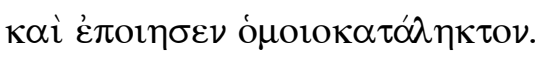

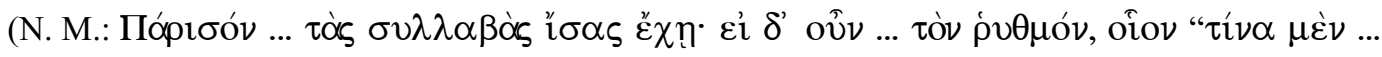

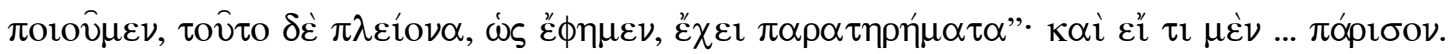

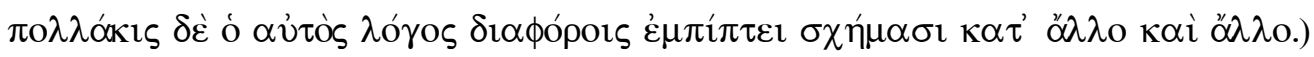

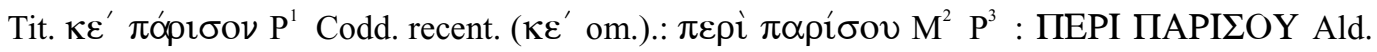
Ald $^{s}$ : П ПЕРI ПАРI $\Sigma O Y ~ \kappa \varsigma^{\prime}$ Norrm. : $\kappa \varsigma^{\prime}$. ПЕPI ПАPI $\Sigma O Y$ Edd.

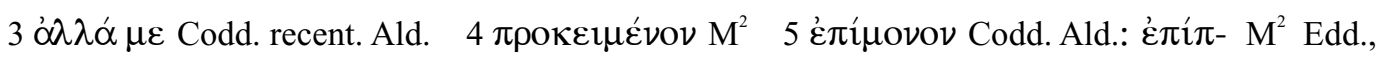

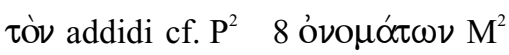


$\kappa \zeta^{\prime}$. ПЕPI ПРО $\Sigma$ YNAПANTH $\Sigma$ E $\Omega \Sigma$.

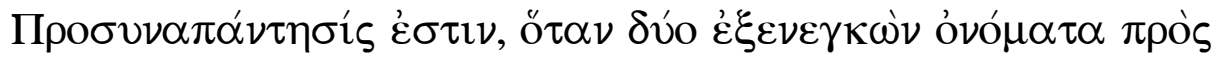

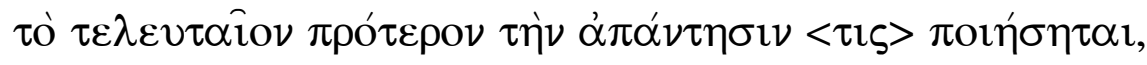

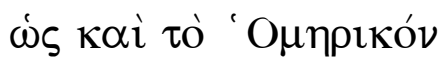

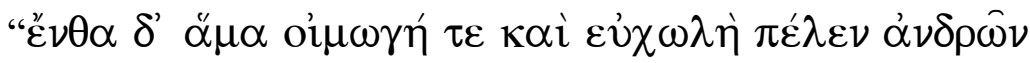

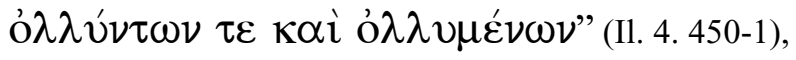

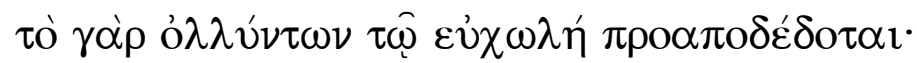

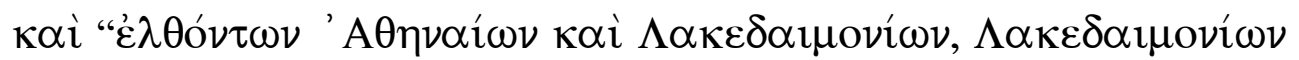

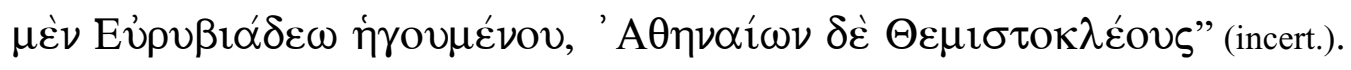

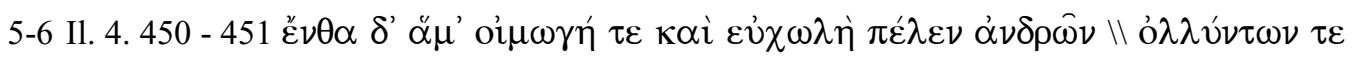

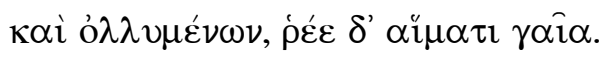

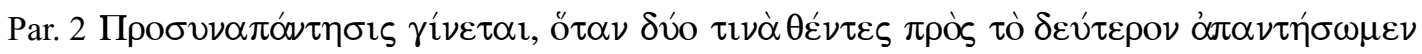

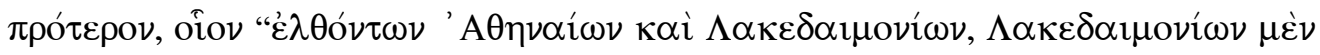

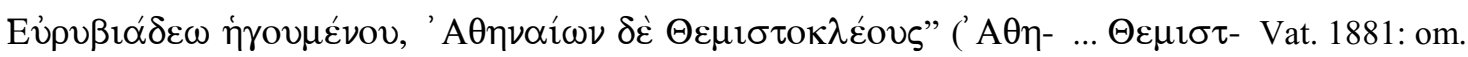

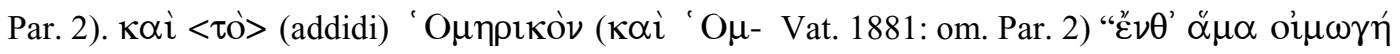

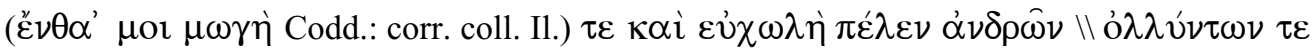

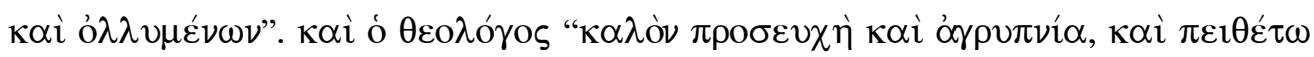

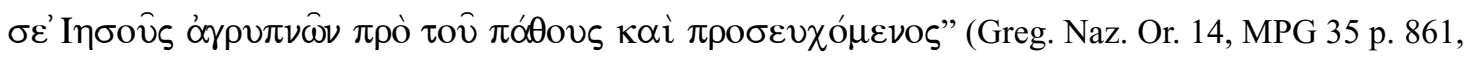

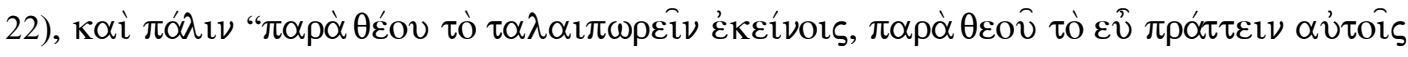

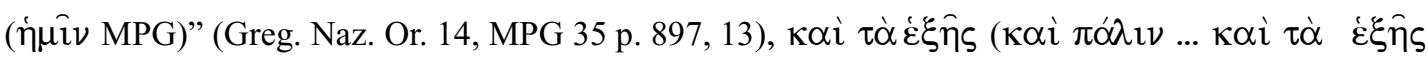
om. Vat. 1881).

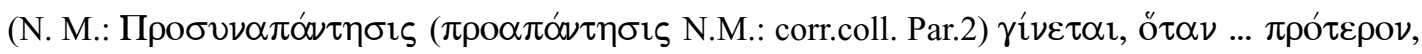

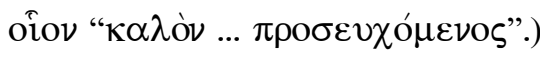

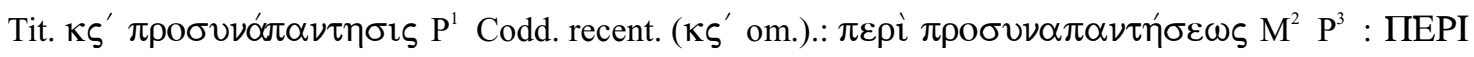
ПРО $\Sigma$ YNAПANTE $\Sigma$ E $\Omega \Sigma$ Ald. Ald ${ }^{s} .:$ ПЕPI ПРO $\Sigma$ YNAПANTH $\Sigma$ E $\Omega \Sigma \kappa \zeta^{\prime}$ Norrm. : $\kappa \zeta^{\prime}$. ПЕРI ПРО $\Sigma$ YNAПANTH $\Sigma$ E $\Omega \Sigma$ Edd.

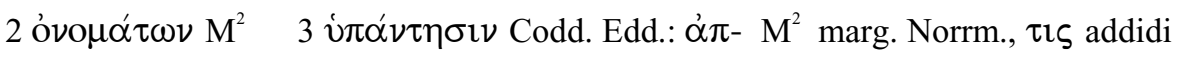

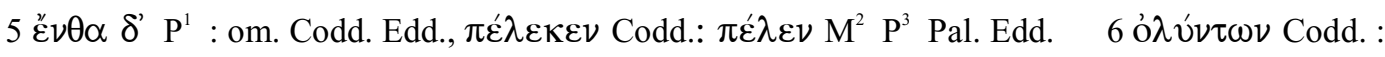

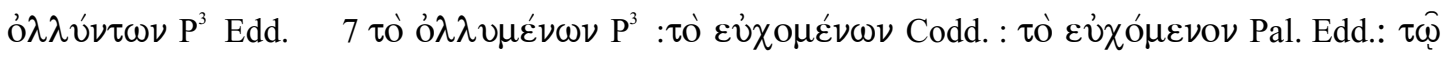

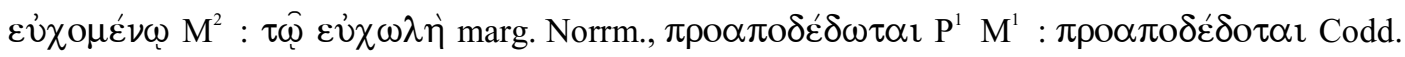

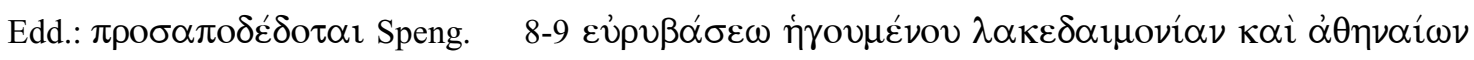

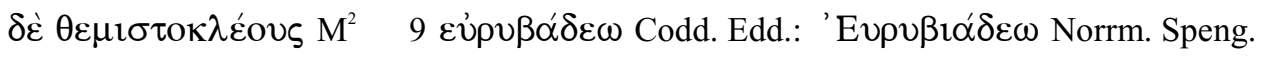




\section{$\kappa \eta^{\prime}$. ПЕРI ЕПITIMH $\Sigma$ E $\Omega \Sigma$}

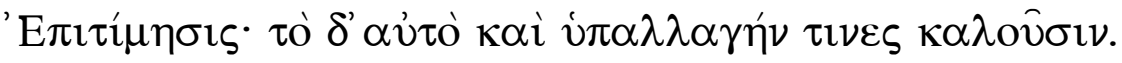

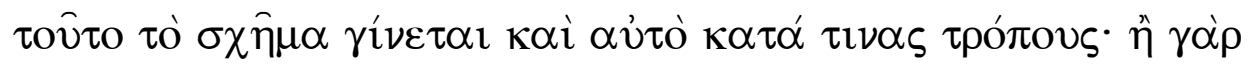

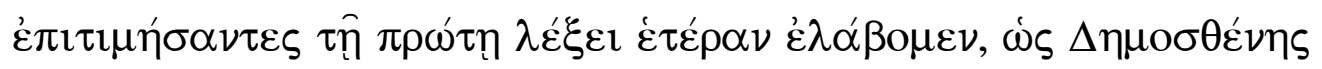

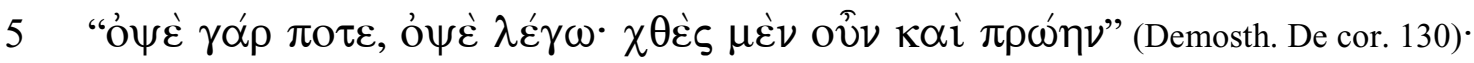

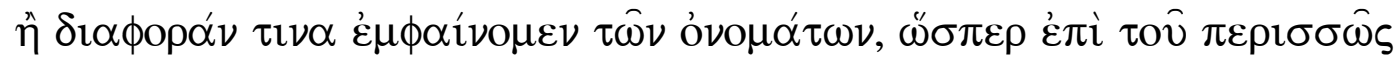

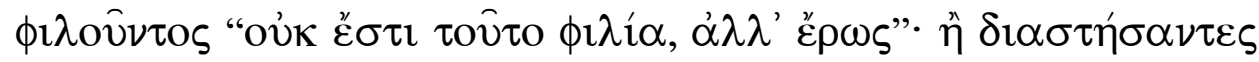

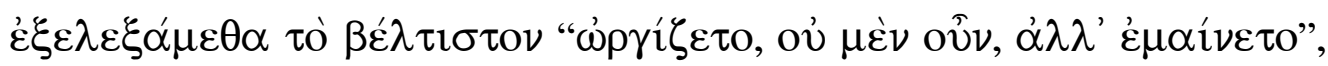

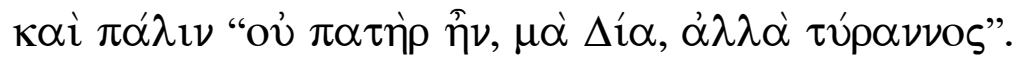

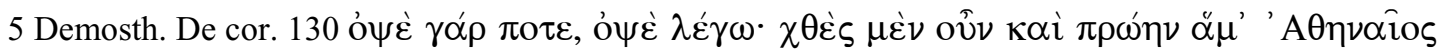

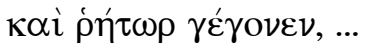

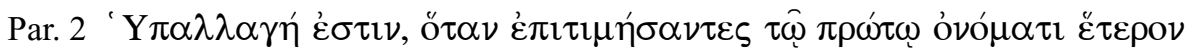

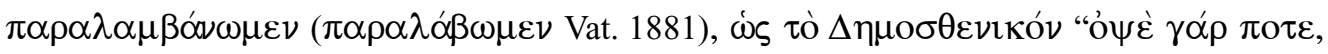

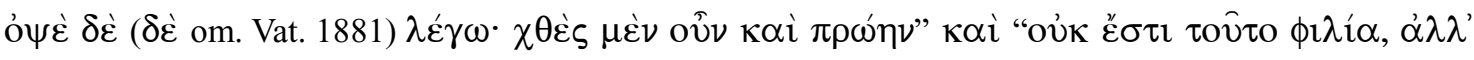

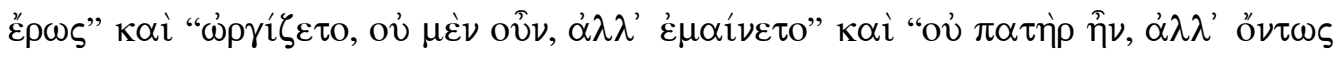

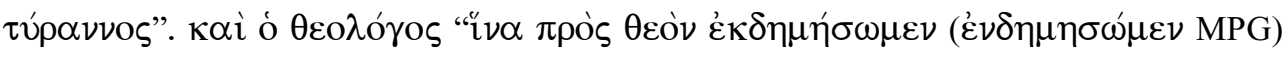

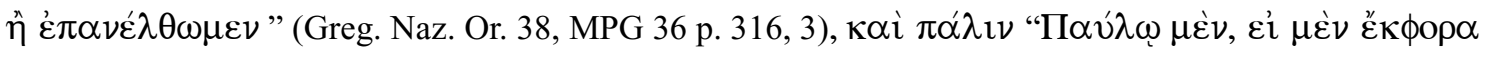

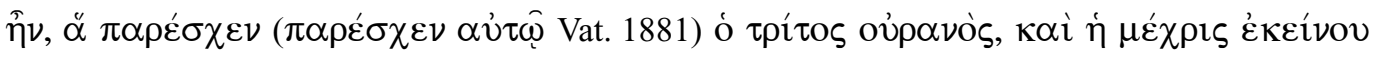

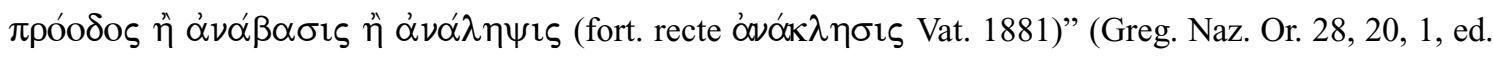
Barbel).

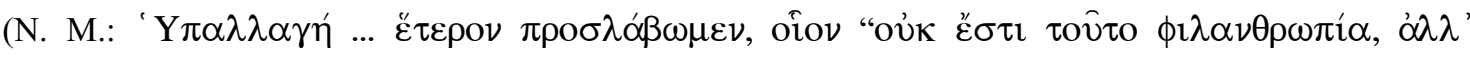

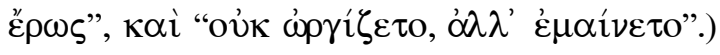

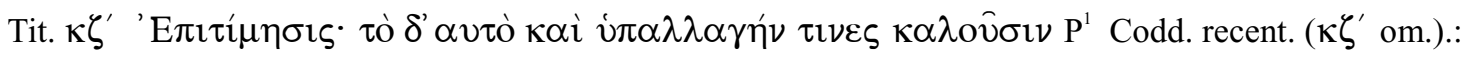

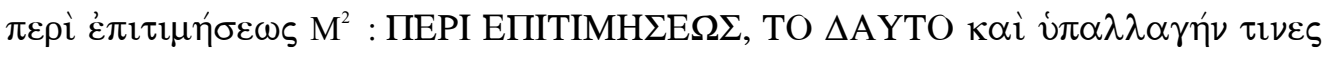

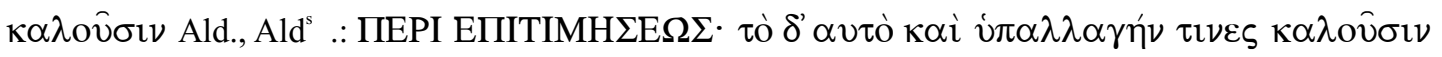
$\kappa \eta^{\prime}$ Norrm. : $\kappa \eta^{\prime}$. ПЕРI ЕПITIMH $\Sigma$ E $\Omega \Sigma$, TO $\Delta^{\prime}$ AYTO ( $\Delta$ AYTO Speng.) KAI YПA $\Lambda \Lambda A \Gamma H N$ TINE $\Sigma$ KA $\Lambda$ OY $\Sigma I N$ Edd.:

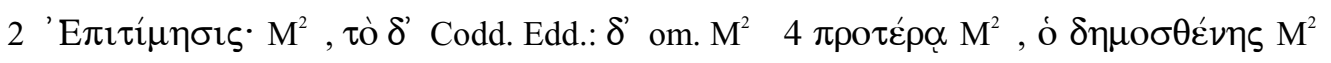

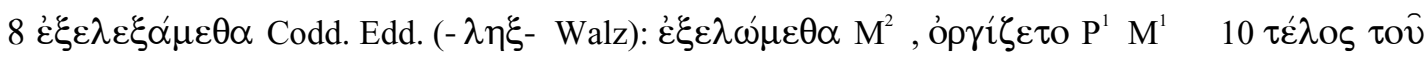
$\pi \varepsilon \rho \grave{i} \sigma \chi \eta \mu \alpha \dot{\alpha} \omega \omega \nu \alpha \dot{\alpha} \varepsilon \varepsilon \xi \alpha \dot{\alpha} \delta \rho \circ v \mathrm{M}^{2}$ :om. Codd. Edd. 


\section{Photokopien}

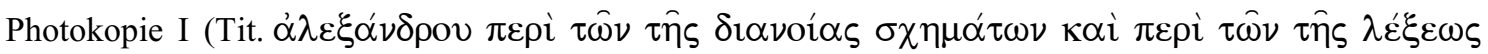
$\sigma \chi \eta \mu \alpha ́ \tau \omega \nu$, Paris. 1741, f. 106)

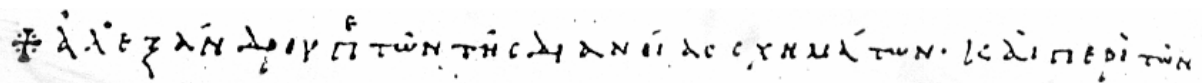

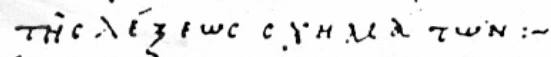

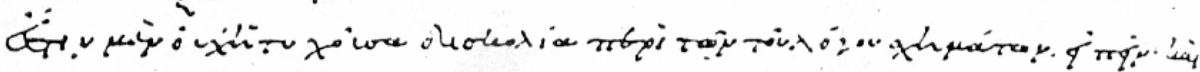

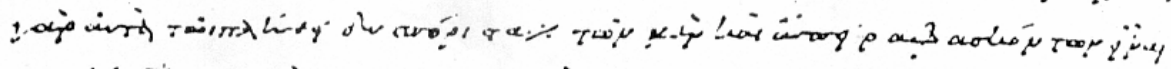

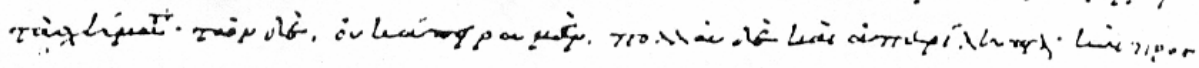

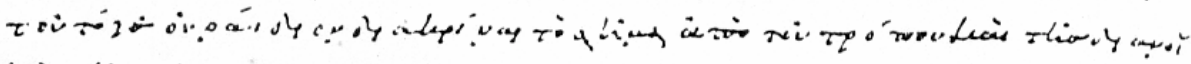

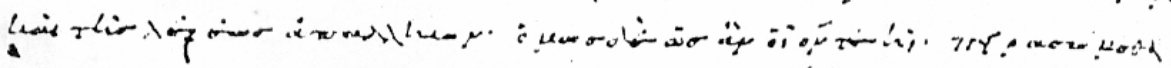

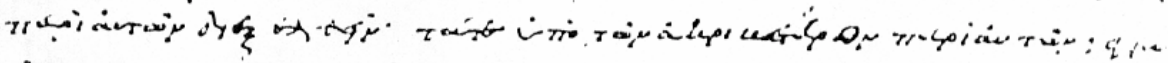

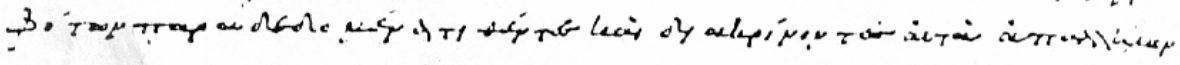

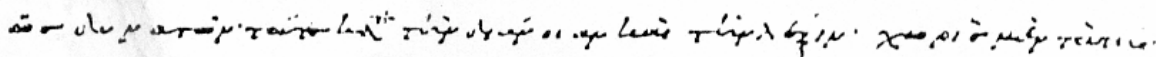

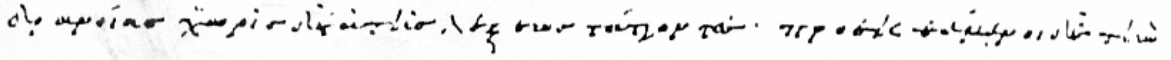

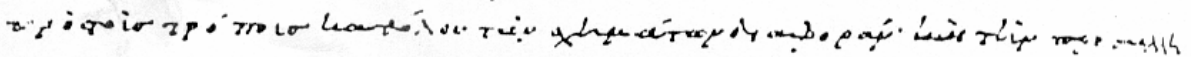

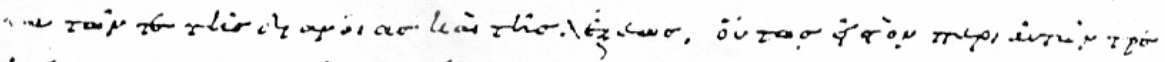

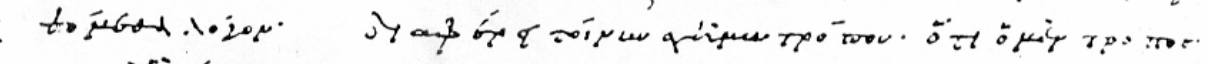

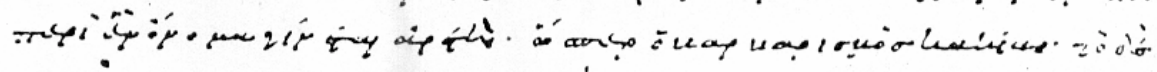
q

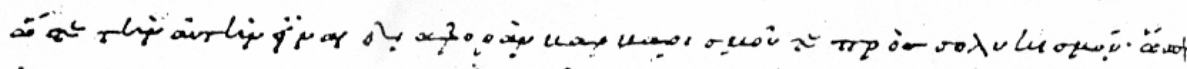
or hatún

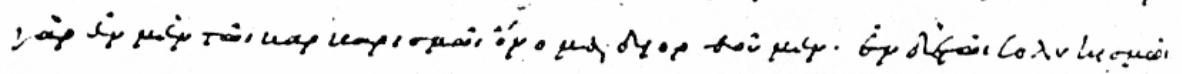

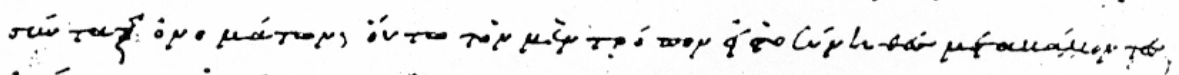

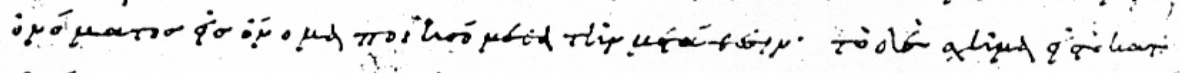

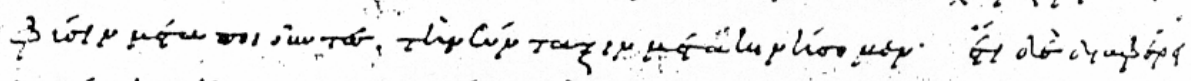

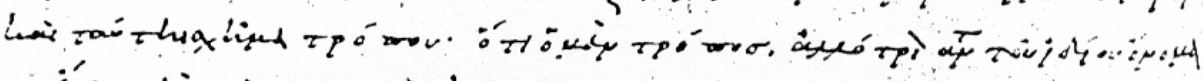

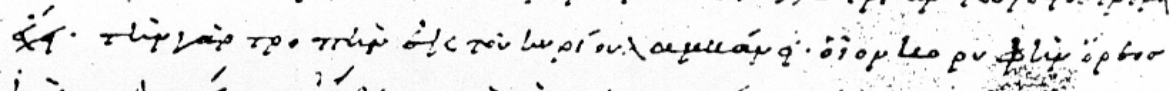

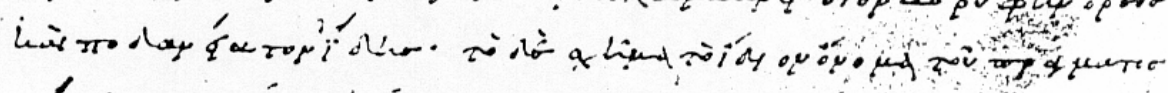

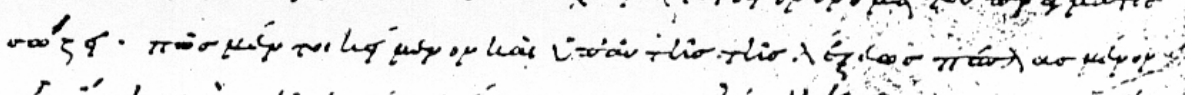

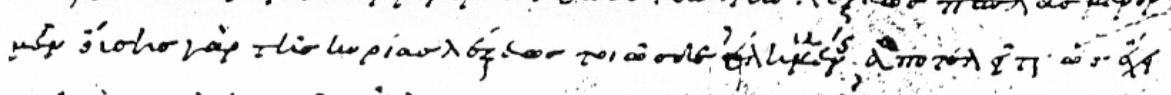
ro

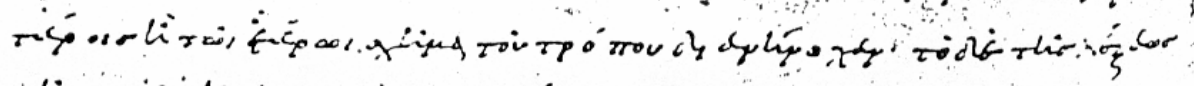

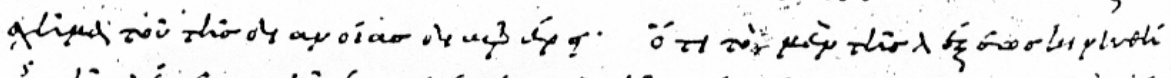

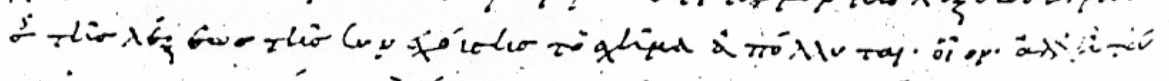

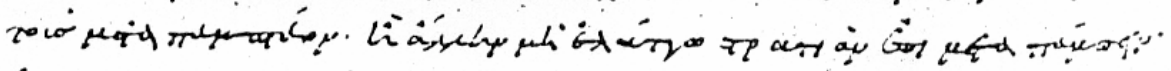

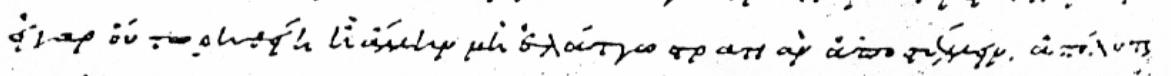

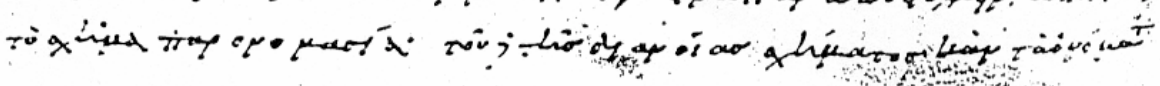




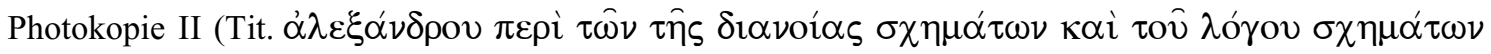
$\mathrm{M}^{2}$, Marc. 512, f. 9)

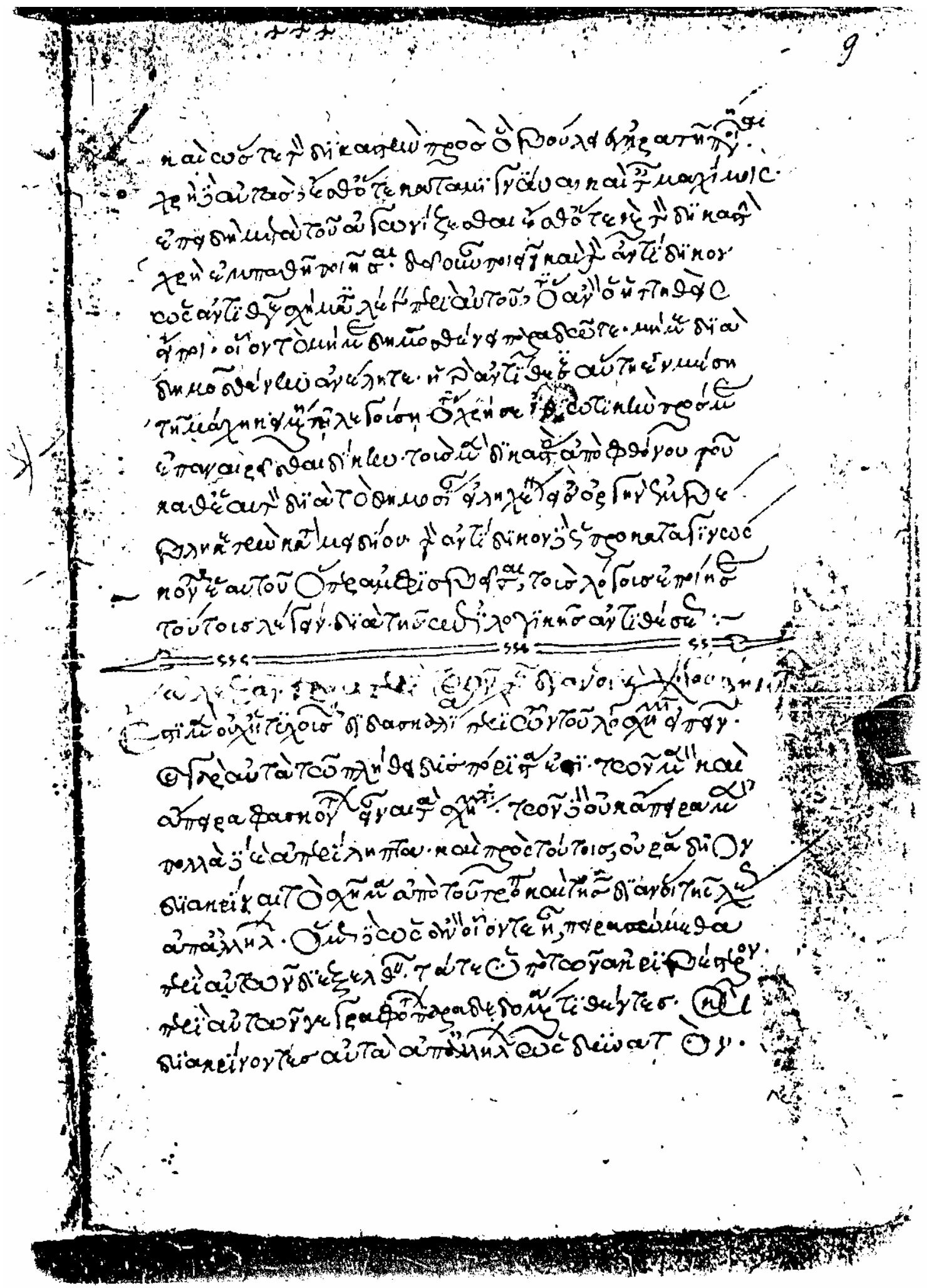




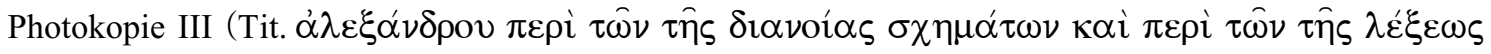
$\sigma \chi \eta \mu \alpha ́ \tau \omega \nu$, Marc. 429, f. $87 \mathrm{v})$

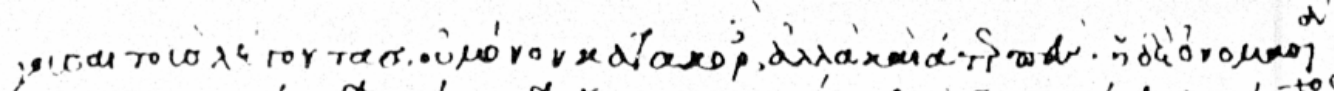

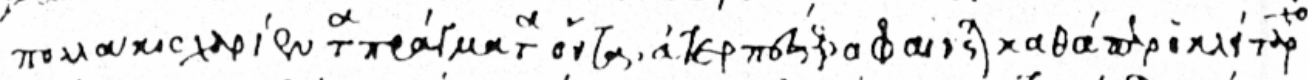

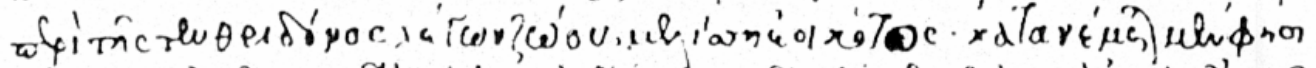

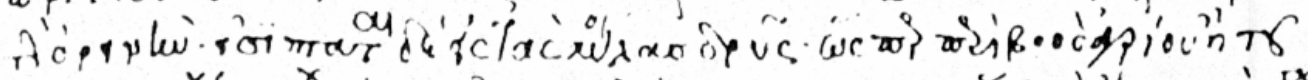

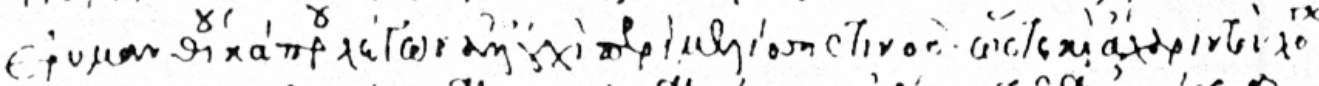

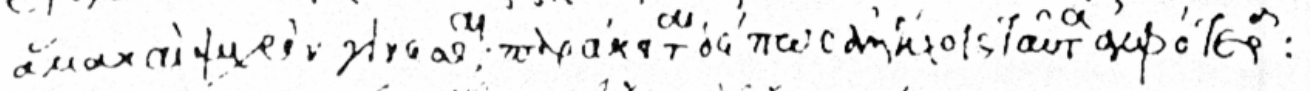

$\rightarrow$

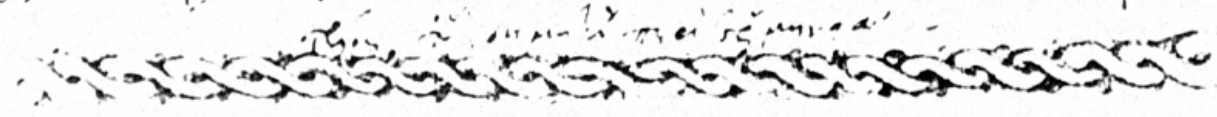

is

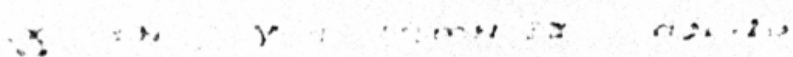

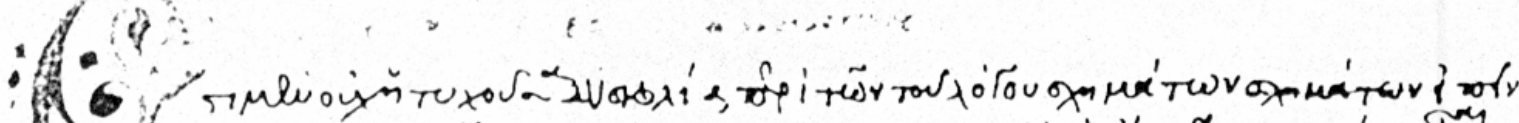

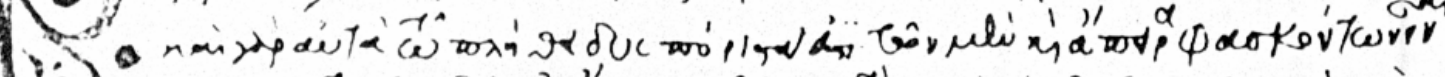

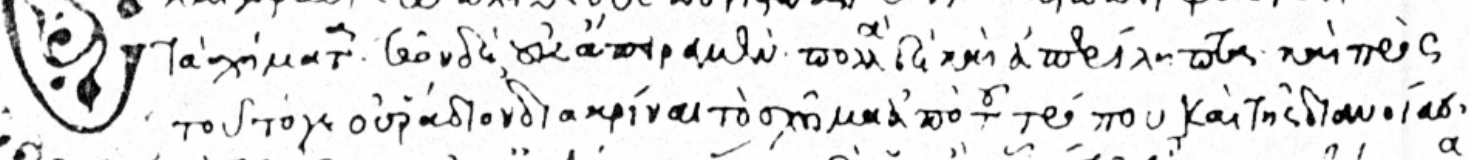

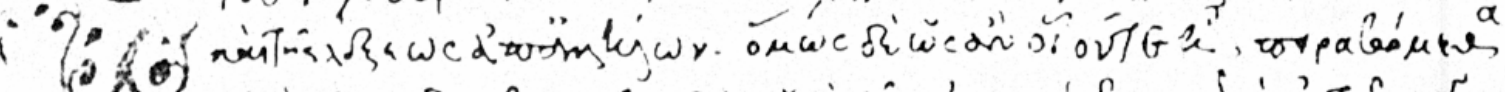

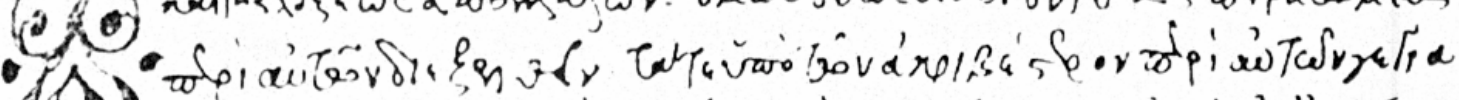

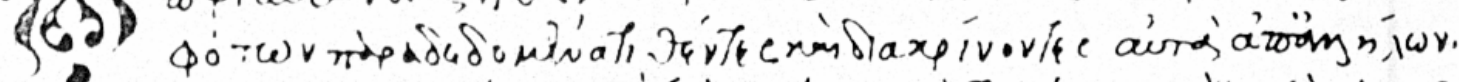

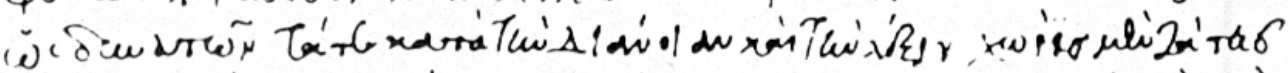

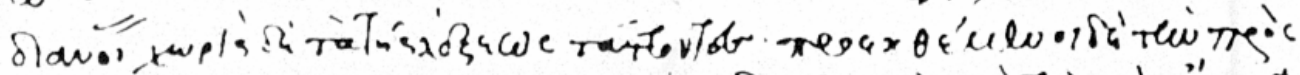

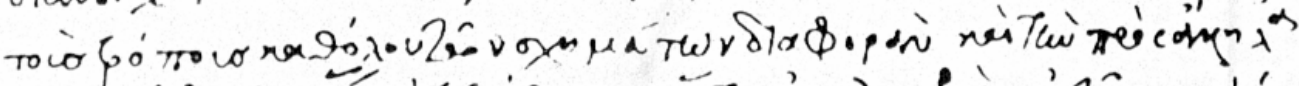

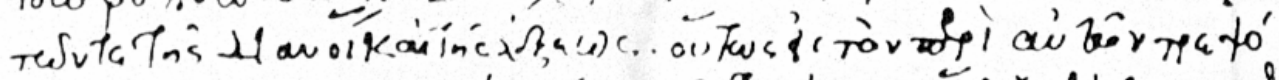

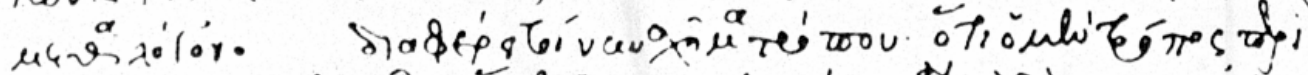

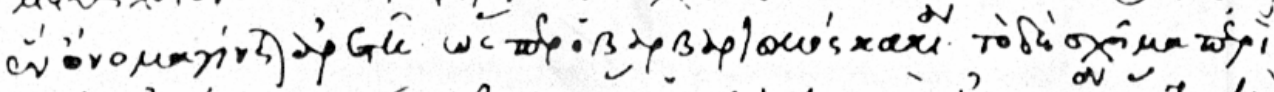

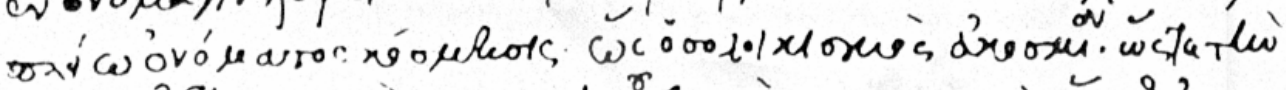

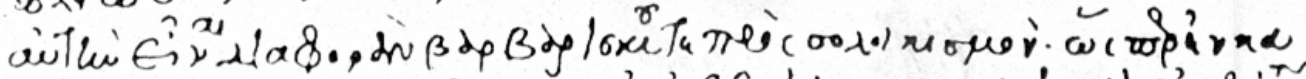

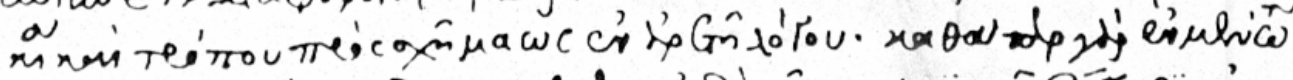

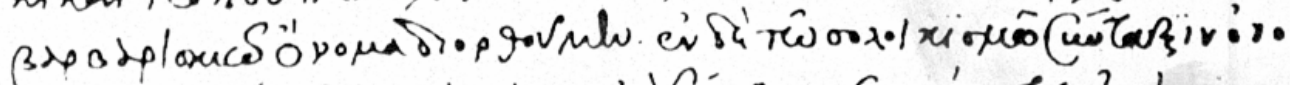

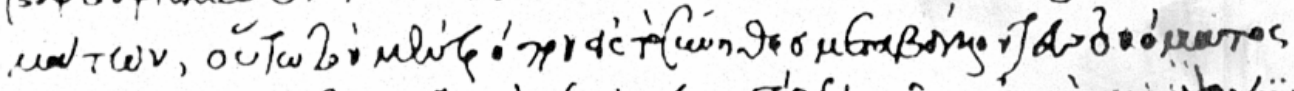

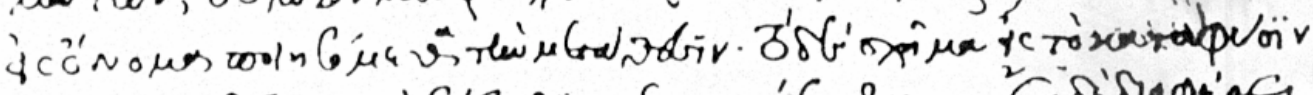

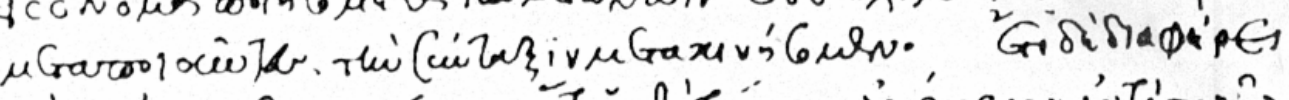

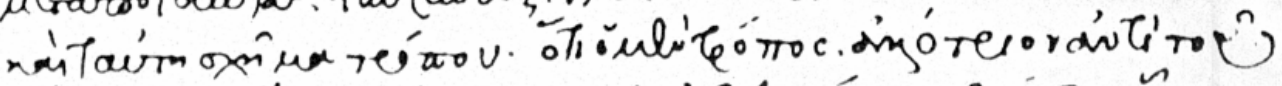

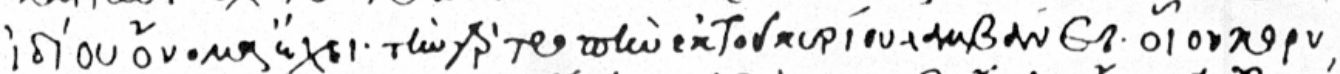

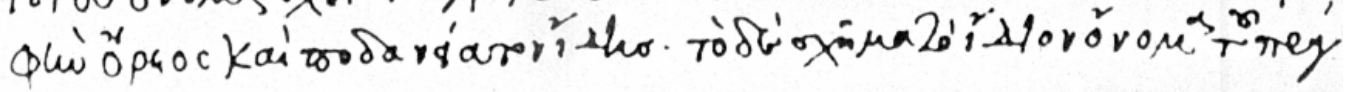




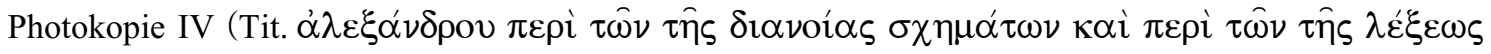
$\sigma \chi \eta \mu \alpha ́ \tau \omega \nu$, Vind. 60, f. 110v)

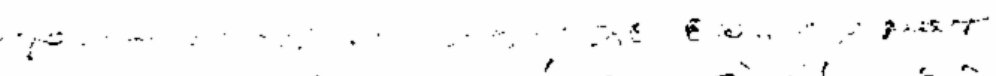

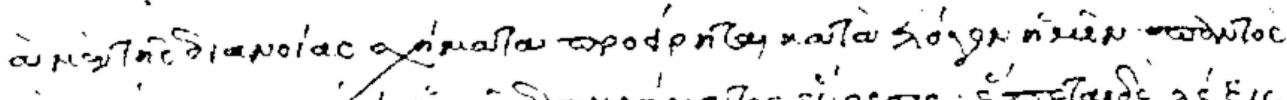

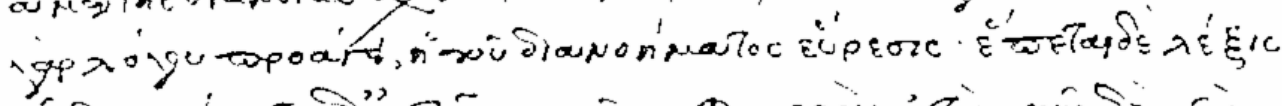

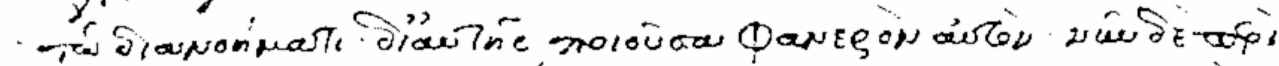

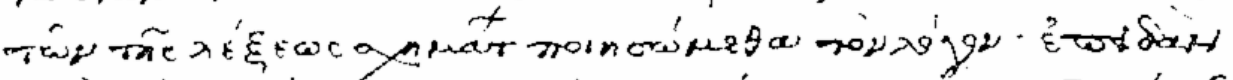

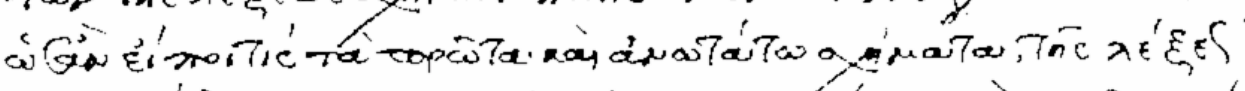

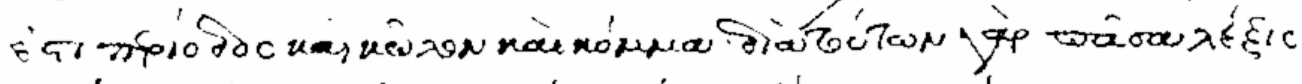

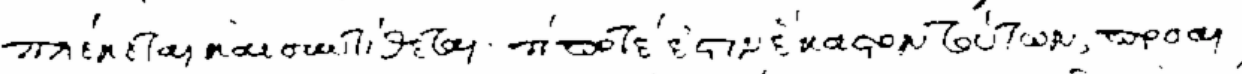

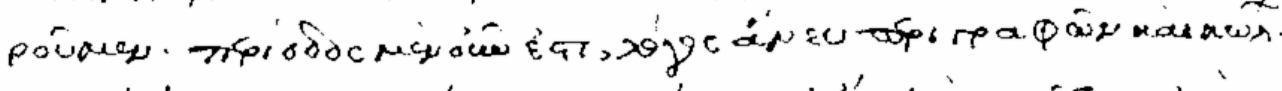

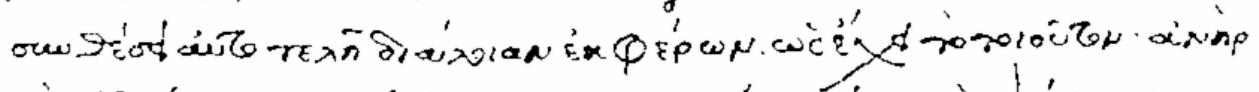

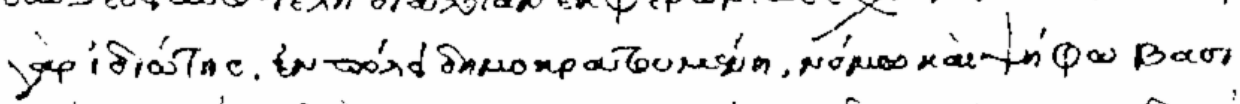

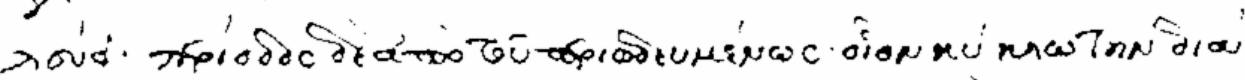

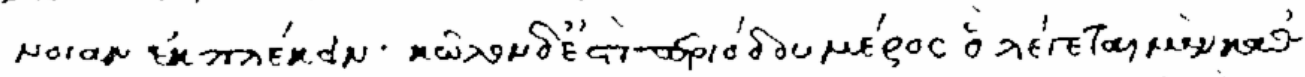

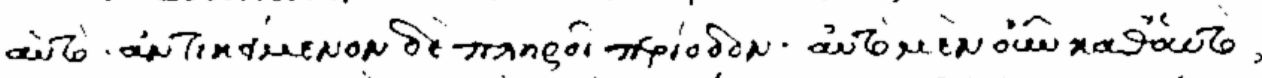

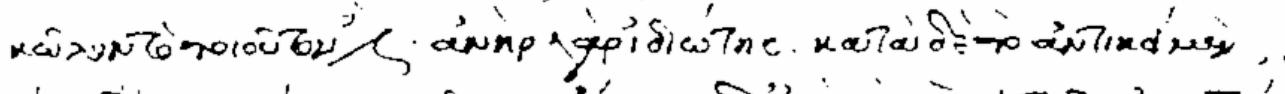

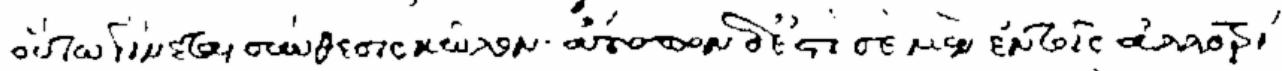

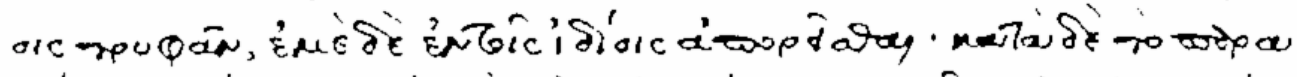

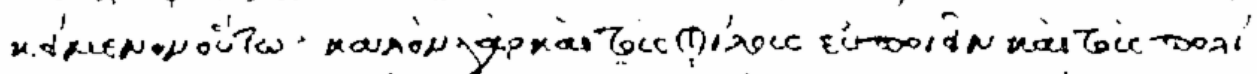

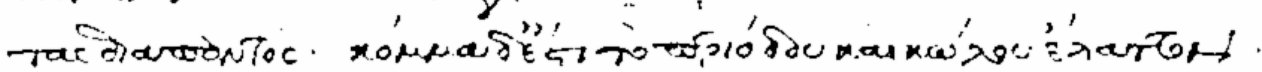

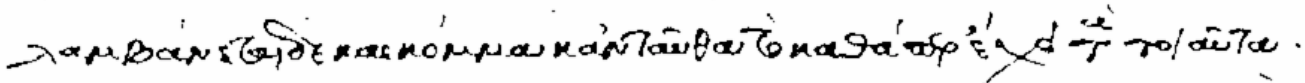

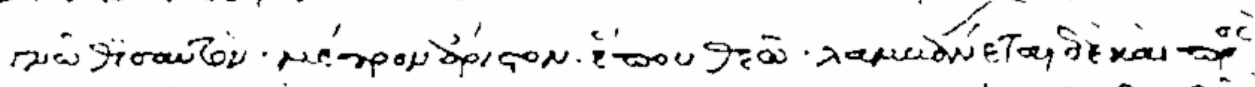

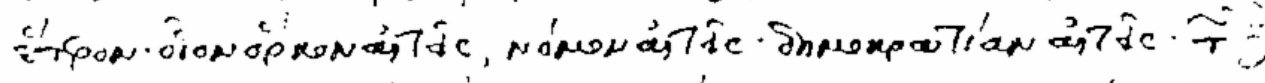

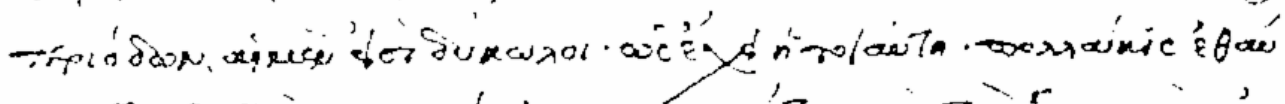

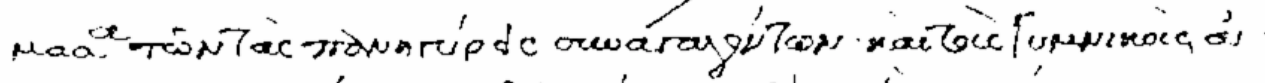

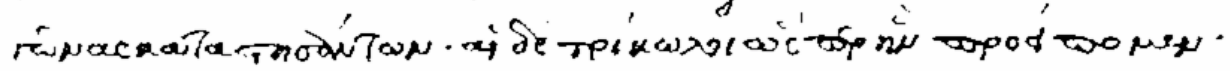

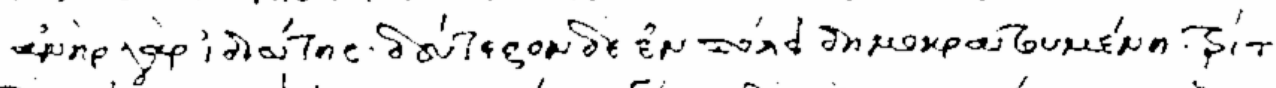

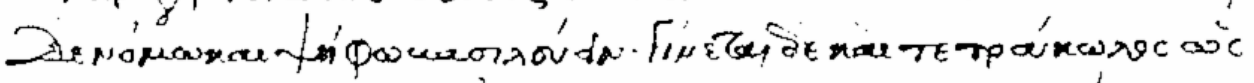

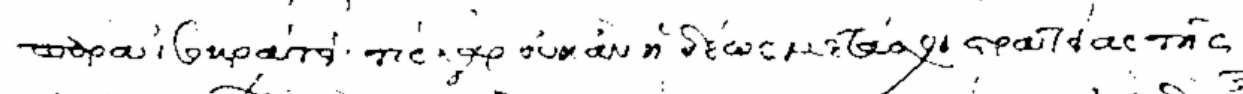

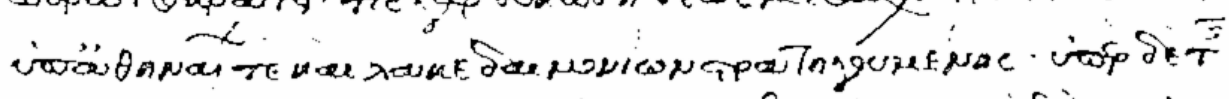

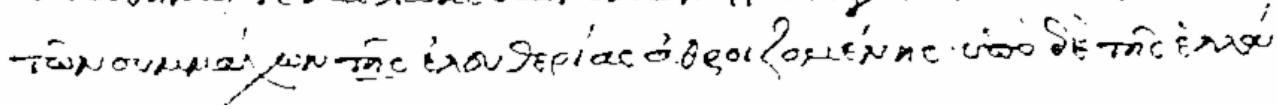




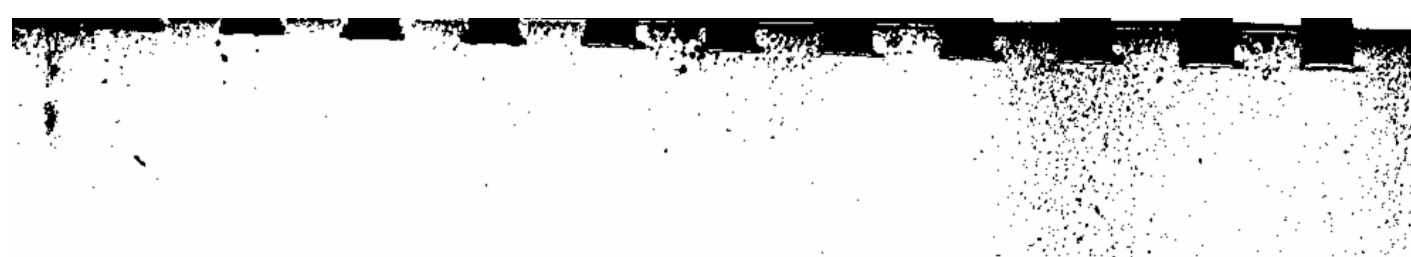

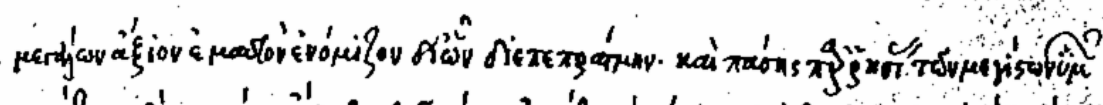

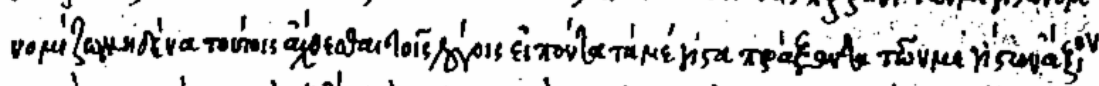

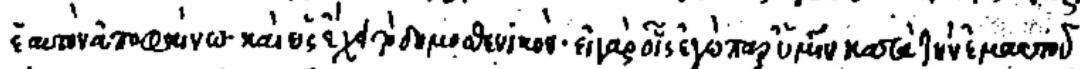

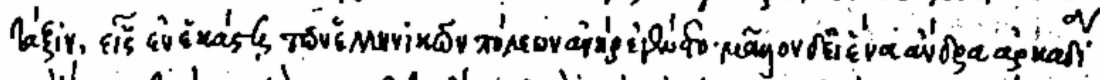

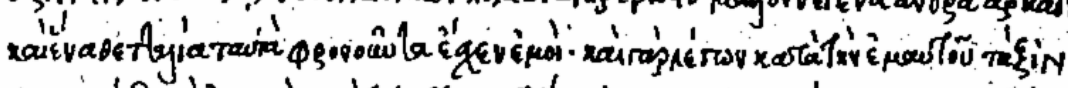

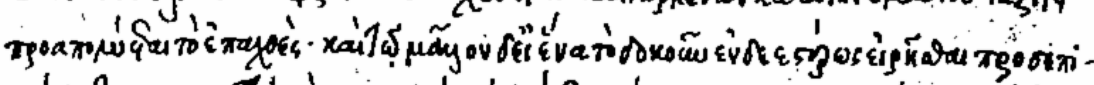

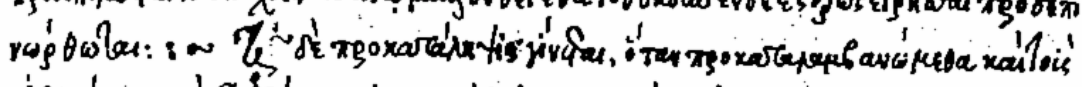

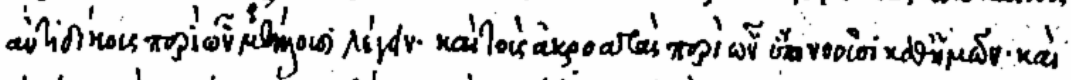

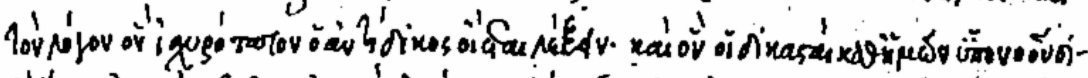

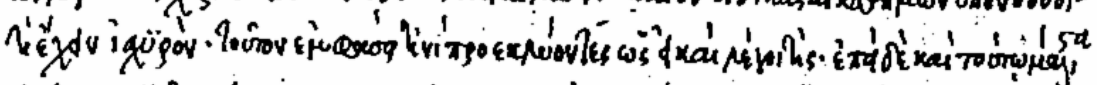

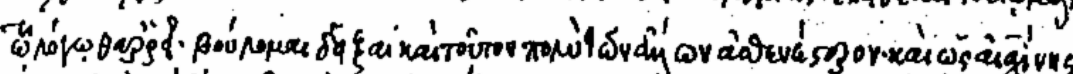

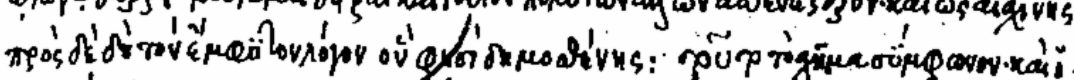

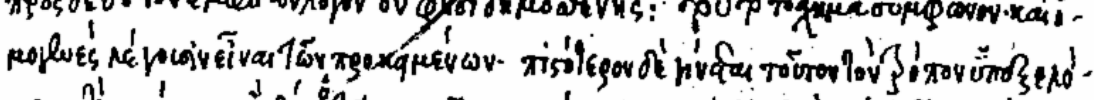

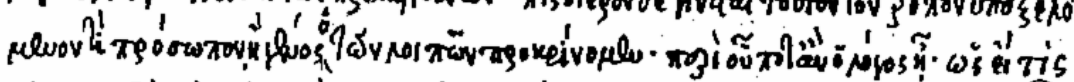

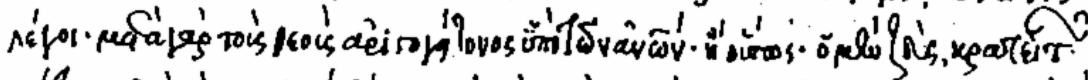
Túf

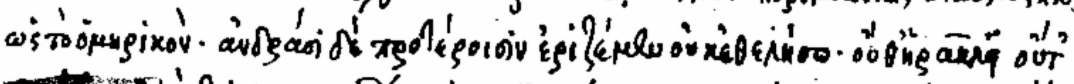

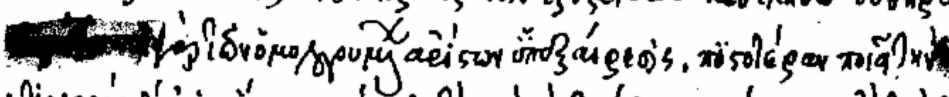

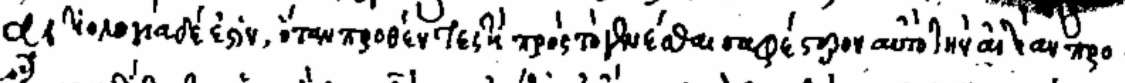

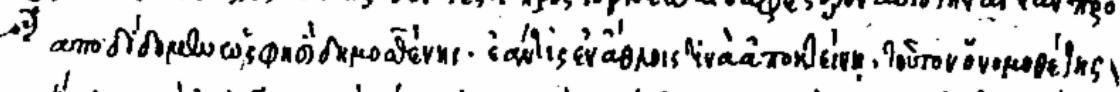

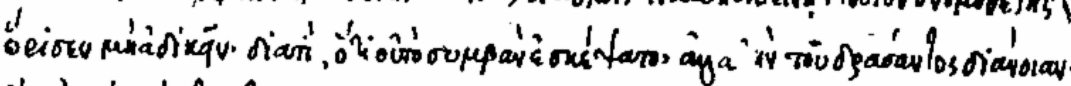

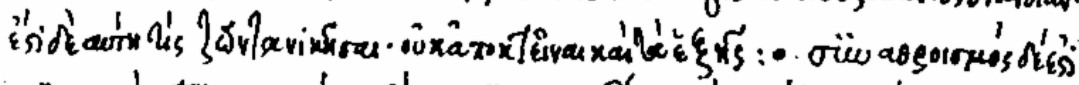

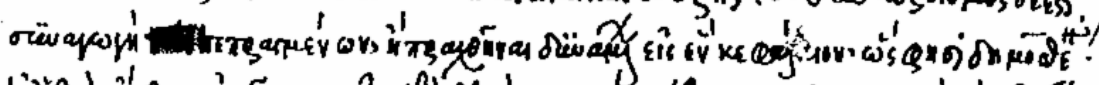

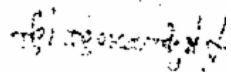

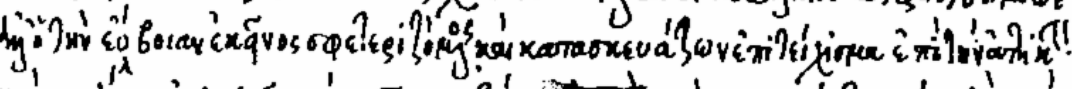

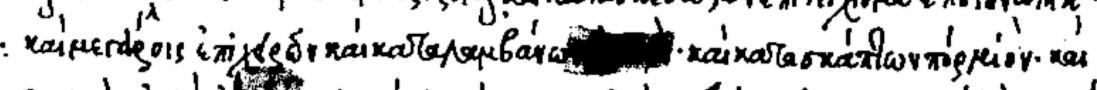

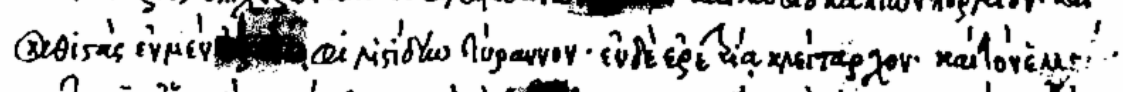

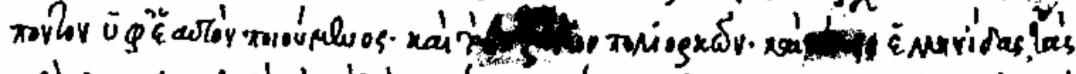

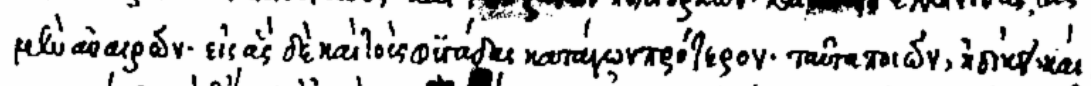

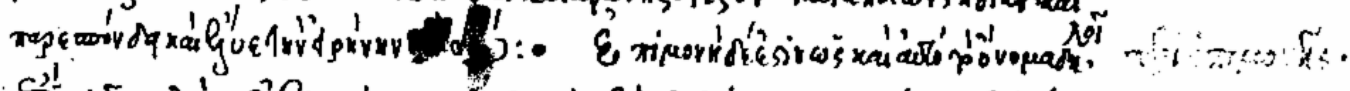

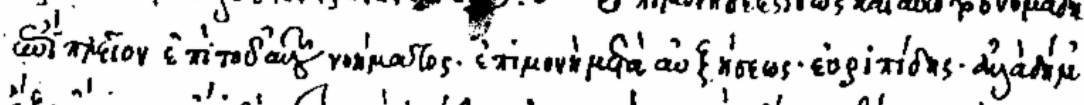

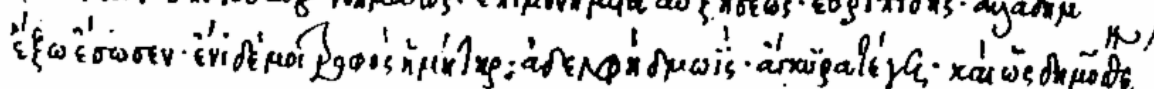




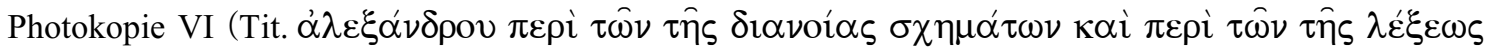
$\sigma \chi \eta \mu \alpha ́ \tau \omega \nu$, Vat. Pal. 66, f. 118r)

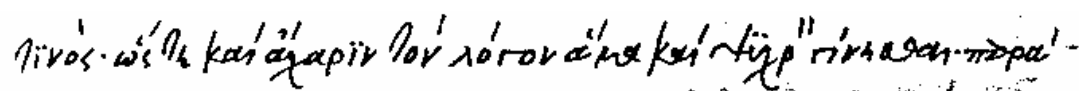

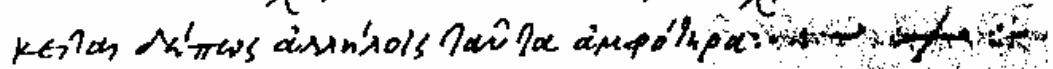

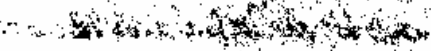

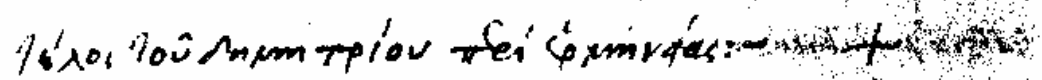

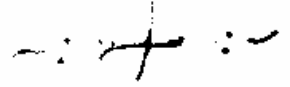

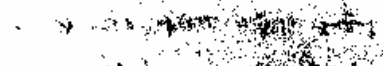

$\therefore \quad+40+4$

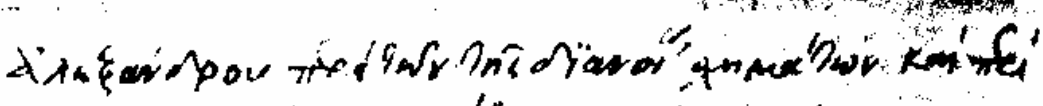

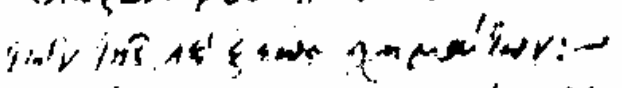

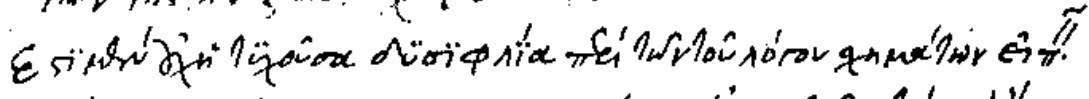

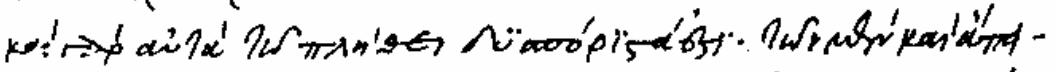

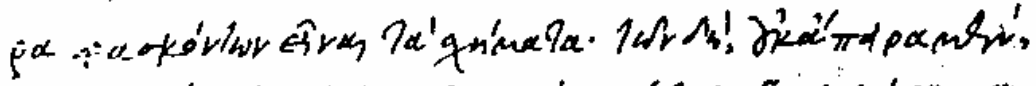

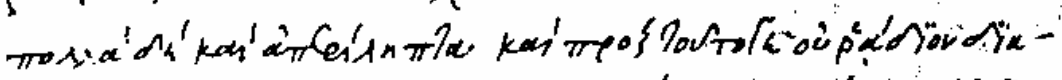

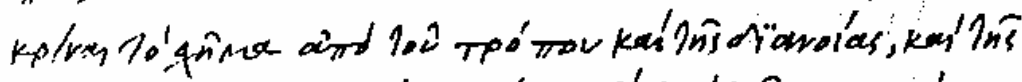

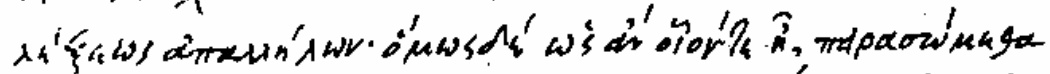

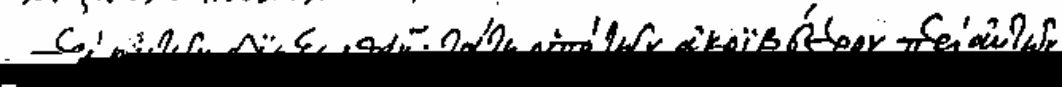


Photokopie VII (Rhetores Graeci, Alexandri De figuris sententiae atque elocutionis, ed. Aldus Manutius, Venedig 1508, p. 582-583)
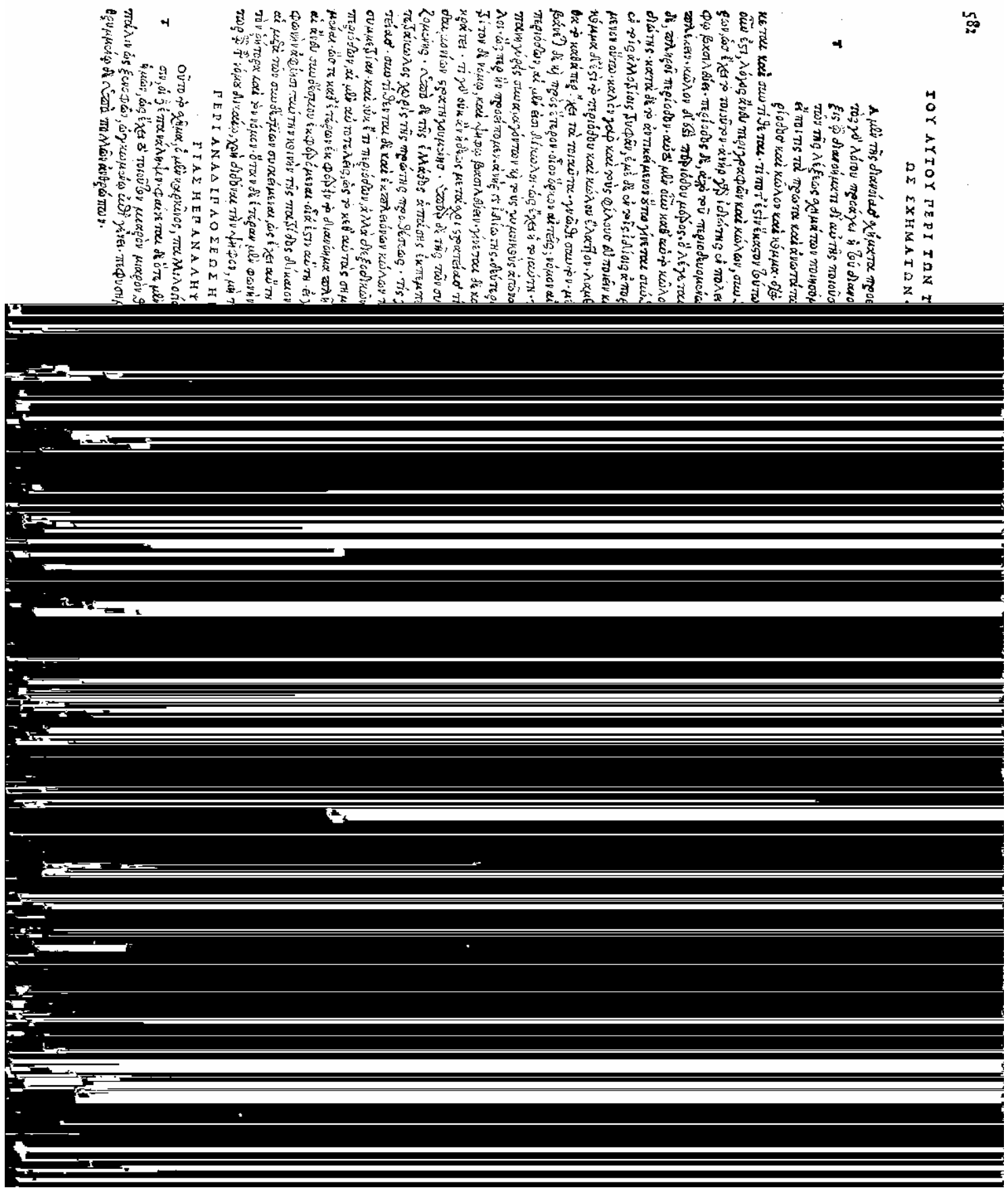


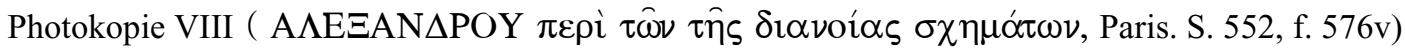

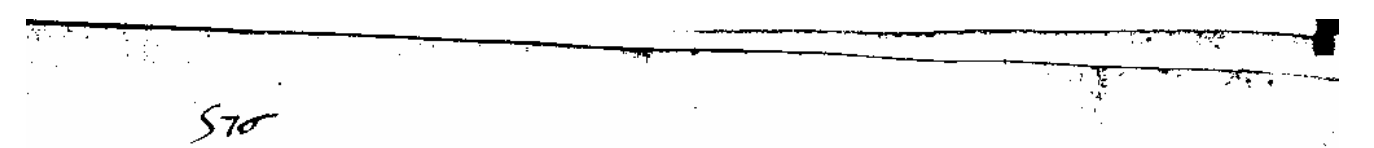

$57 \%$

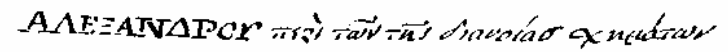

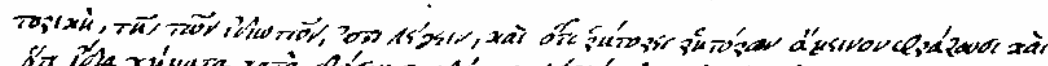

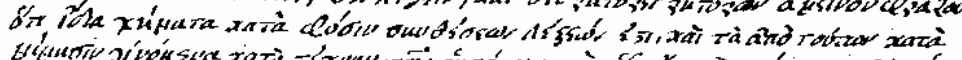

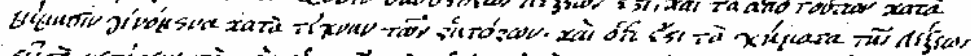

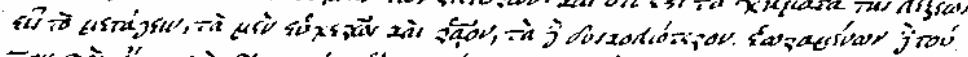

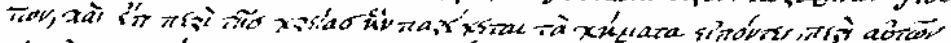

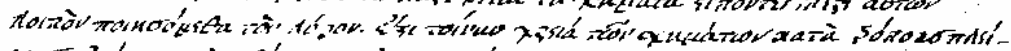

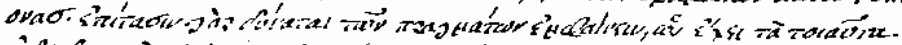

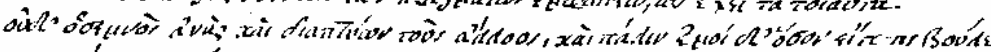

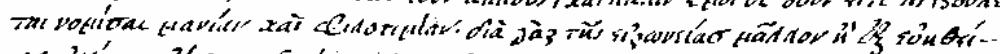

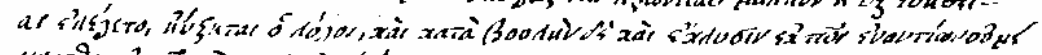

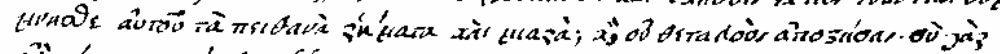

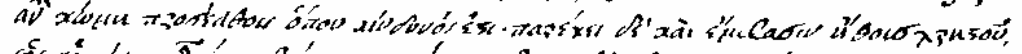

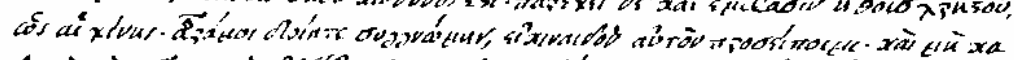
Aa

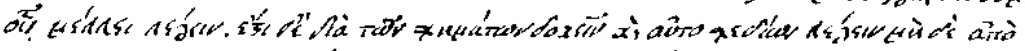
"̈a

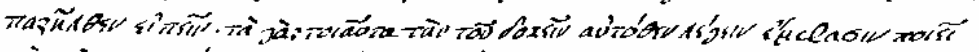

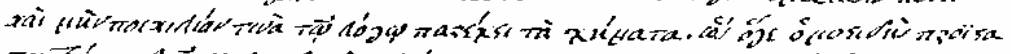

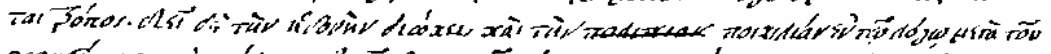

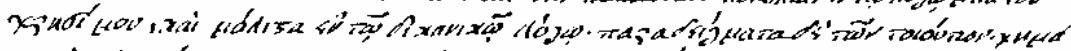

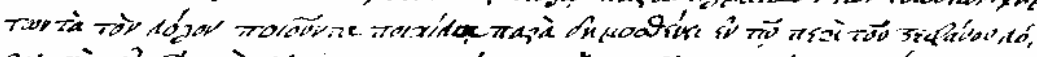

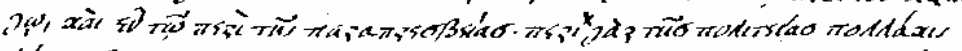

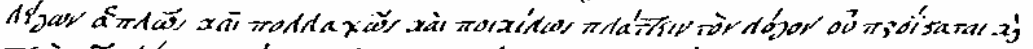

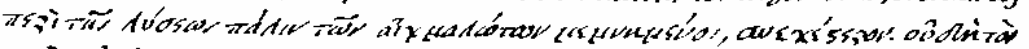

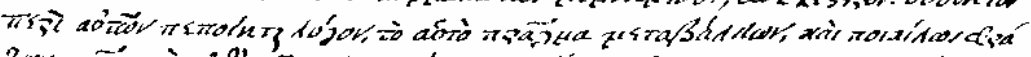

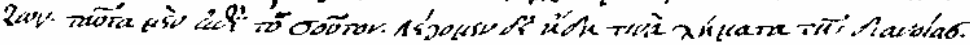

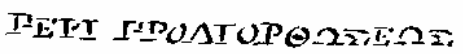

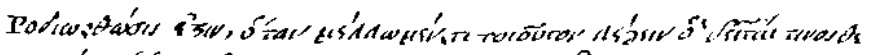

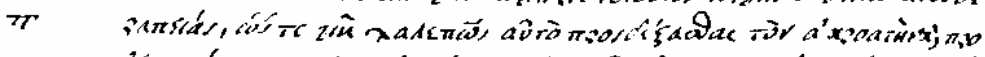

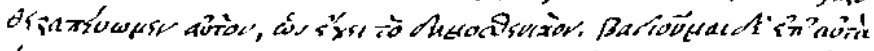

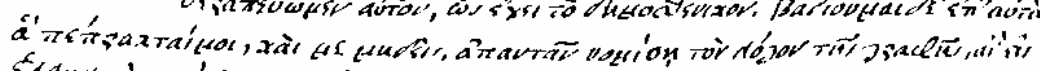

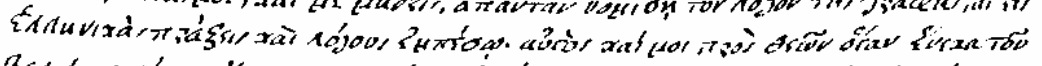

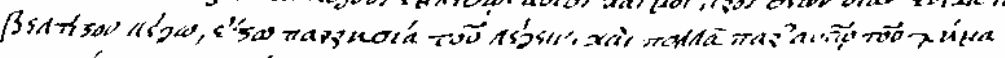

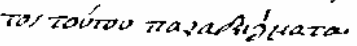

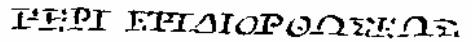

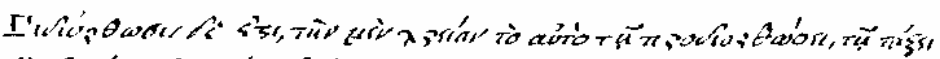

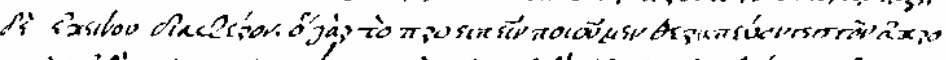

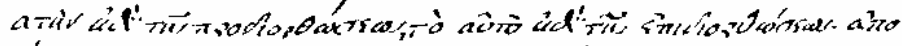

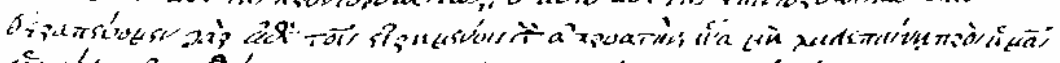

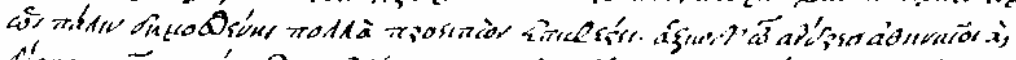

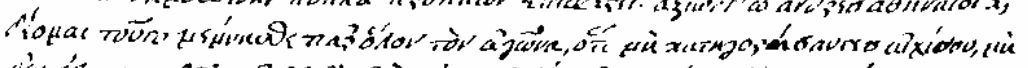

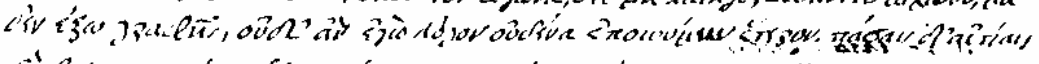

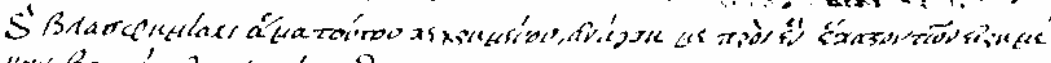

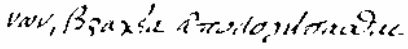


Photokopie IX (Alexandri De figuris sententiae atque elocutionis, ed. L. Norrmannus, Uppsala 1690, p. $1-2)$

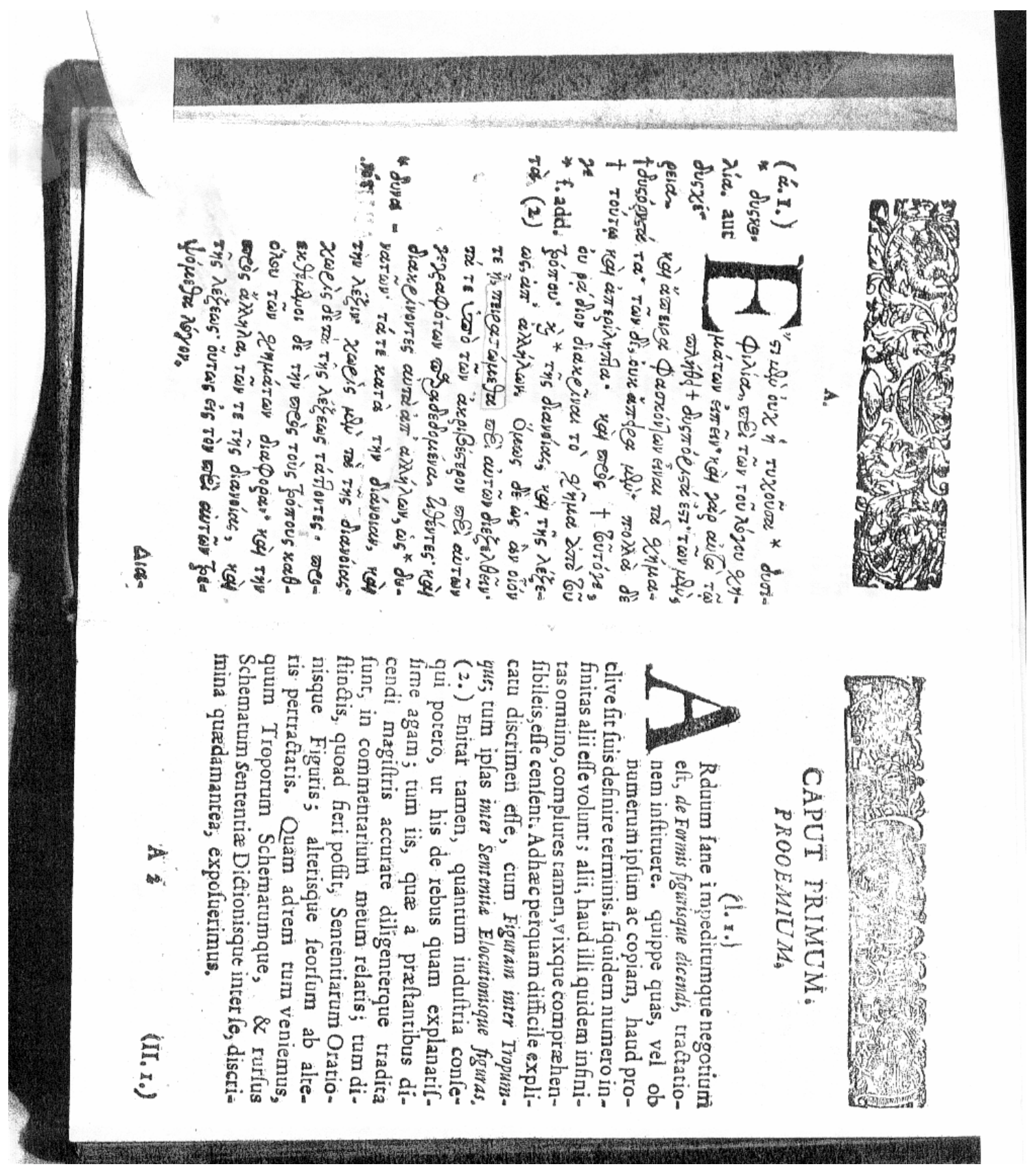




\section{INDICES}

INDEX FIGURARUM

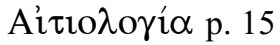

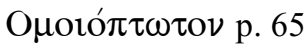

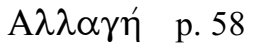

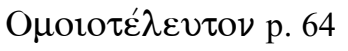

A $\lambda \lambda$ oíwols p. 58

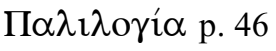

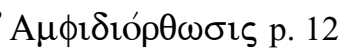

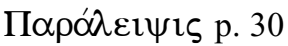

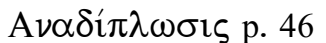

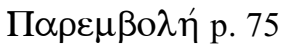

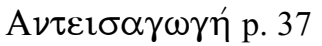

Пópı бov p. 76

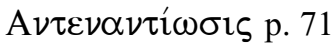

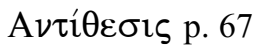

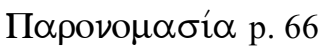

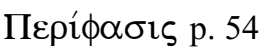

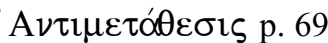

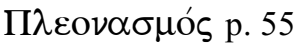

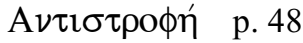

П入оки́ р. 69

A $\pi 0 \sigma \iota \omega ́ \pi \eta \sigma \iota \varsigma$ p. 27

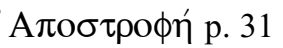

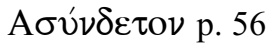

$\Delta$ iódvois p. 56

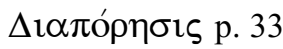

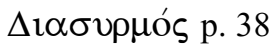

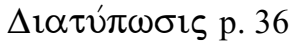

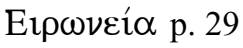

"Е $\lambda \lambda \varepsilon \imath \psi 1 \varsigma$ p. 57

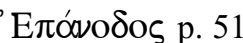

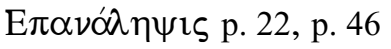

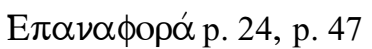

Подט́ $\pi \tau \omega \tau$ ov p. 61

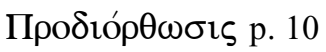

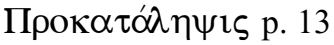

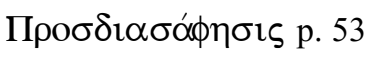

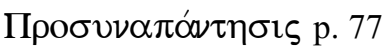

Проб

$\Pi v ́ \sigma \mu \alpha$ р. 35

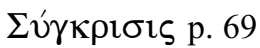

$\Sigma v \mu \pi \lambda$ окиं p. 49

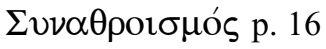

$\Sigma u ́ v \theta \varepsilon \sigma ı \varsigma$ р. 49

$\Sigma v \nu \omega \nu v \mu i ́ \alpha$ p. 50

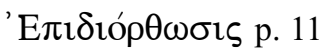

Ү $\pi \varepsilon \xi \alpha ı \rho \varepsilon ́ \sigma 1 \varsigma$ p. 14

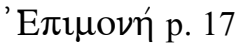

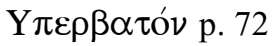

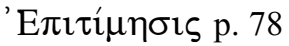

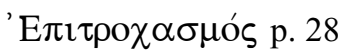

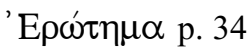

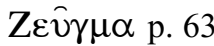

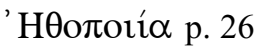

$\mathrm{K} \lambda \hat{\mu} \mu \alpha \xi$ p. 52

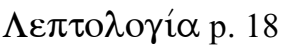

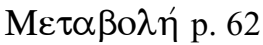

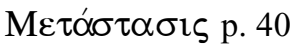




\section{INDEX LOCORUM}

Aeschines

1 In Timarchum 175 p. $26,5-10$

2 De falsa legatione

88 p. $8,15-17 \quad 119$ p. $69,7-8$

3 In Ctesiphontem

16 p. $45,7-10 \quad 17$ p. 13,9

75 p. $23,1-2 \quad 78$ p. $64,5-6$

152 p. $65,3-5 \quad 153$ p. $36,5-8$

166 p. $8,13-14 \quad 166$ p. $39,1-3$

167 p. $8 ., 14-15 \quad 198$ p. $43,10-11$

198 p. $48,8-9 \quad 202$ p. 3,7

202 p. $49,4-5 \quad 211$ p. $26,10-12$

233 p. $42,11-12 \quad 257$ p. $25,9-14$

257-258 p. $21,1-7$

Demosthenes

1 Olynthiaca I 2 p. 20, 4-5

3 Olynthiaca III

15 p. $40 \quad 26$ p. $63,7-8$

4 De pace 15 p. 4,48

8 De Chersoneso

32 p. $10,8-9 \quad 60$ p. $22,11-12$

9 Philippica III

36 p. 22,10 , p. $46 \quad 61$ p. $51,4-6$

11 In epistulam Philippi 3-4 p. 50, 5-7

18 De corona

18 p. $40,3-4$

20 p. $7,2-3$

22 p. $40,8-12$

27 p. $28,4-6$

34 p. $11,7-11$

41 p. $29,7-9$

43 p. $17,6-8$

51 p. $23,6-8$

48 p. $47,7-10$

65 p. $56,3-5$

$58-59$ p. $10,5-7$

71 p. $16,3-10$

103 p. $27,7-8$

69 p. $30,3-6$, p. $56,5-6$

130 p. 78,5
71 p. $34,4-5$

113 p. 25

121 p. $24,7-9$
170 p. $45,4-5 \quad 179$ p. $52,4-6$

198 p. $48,5-7 \quad 208$ p. 22,3

209 p. $35,3-4 \quad 219$ p. $55,7-8$

$232-233$ p. $39,5-7 \quad 243$ p. $57,4-5$

258 p. $8,8 \quad 265$ p. $47,4-5$

265 p. $68,7-9 \quad 282$ p. $61,9-11$

299 p. $25 \quad 304$ p. $12,10-13$

311-312 p. 62, 3-9

19 De falsa legatione

$\begin{array}{ll}2 \text { p. } 59,6-8 & 18 \text { p. } 58,9-10 \\ 65 \text { p. } 36,9-11 & 122 \text { p. } 66,6-8 \\ 136 \text { p. } 57,7 & 169 \text { p. } 9,12-15 \\ 298 \text { p. } 61,7-9 & \\ 21 \text { In Midiam } & \\ 1 \text { p. } 30,10-12 & 15 \text { p. } 30,7-9 \\ 62-63 \text { p. } 32,5-11 & 69 \text { p. } 8,9-10 \\ 74 \text { p. } 17,8-10 & 99 \text { p. } 54,5-6 \\ 110 \text { p. } 9,4 & 147-148 \text { p. } 30 \\ 23 \text { In Aristocratem } & \\ 82 \text { p. } 9,3 & 54 \text { p. } 15,4-7 \\ 156 \text { p. } 33,4-7 & 213 \text { p. } 27,4-6\end{array}$

25 In Aristogitonem I

$18-19$ p. 72 , p. $74,2-11 \quad 21$ p. 14

27-28 p. $59,2-5 \quad 58$ p. $46,4-5$

59 In Neaeram 34 p. $60,1-2$

60 Epitaphius 36 p. 37, 3-6 37 p. 37

Frag. inc. p. 24, 3-4

Frag. inc. p. 13, 7-8

Frag. inc. p. 19, 8-16

Diodorus Siculus

Bibliotheca Historica 17.1 p. 63

Euripides

Medea 509-511 p. 29, 4-6 
Hecuba. 162 -163 p. 6, 25

Orestes $25-27$ p. $27,11-13$

Frag. inc. 866 p. 17, 4-5

Gregorius Nazianzenus

Or. 1, MPG 35 p. 396, 10: p. 57

Or. 2, MPG 35

p. $508,15:$ p. $56, \quad$ p. $429,42:$ p. 50

p. $484,43:$ p. $71, \quad$ p. $489,26:$ p. 17

p. $537,14:$ p. 54

Or. 4 MPG 35

p. $585,5:$ p. $30, \quad$ p. $588,25:$ p. 31

p. $588,30:$ p. 65 , p. $600,28:$ p. 16

p. $609,1:$ p. $36, \quad$ p. $613,28:$ p. 30

cf. p. $640,34:$ p. 57, p. $649,3:$ p. 76

p. $684,7:$ p. $40, \quad$ p. $704,14:$ p. 67

p. $709,29:$ p. 37

Or. 6, MPG 35 p. 744, 47: p. 36

Or. 7, 20, 2 (ed. Boulenger): p. 37

Or. 9, MPG 35

p. 824,20 : p. 11 , p. $824,24:$ p. 11

p. $825,20:$ p. 35

Or. 10, MPG 35 p. 829, 18: p. 66

Or. 14, MPG 35

p. $860,29:$ p. 69 , p. $861,22:$ p. 77

p. $860,31:$ p. $69, \quad$ p. $869,43:$ p. 20

p. $897,13:$ p. 77

Or. 15, MPG 35 p. 921 , 13: p. 61

Or. 16, MPG 35

p. $933,46:$ p. $24, \quad$ p. $936,44:$ p. 48

p. $952,3:$ p. $69, \quad$ p. $952,8:$ p. 48

Or. 17, MPG 35

p. $968,26:$ p. 48 , p. $976,11:$ p. 10

Or. 18, MPG 35

p. $1028,17:$ p. $60, \quad$ p. $1041,28:$ p. 37

Or. 19, MPG 35 p. $1044,43:$ p. $50, \quad$ p. $1061,23: p . ~ 27$

Or. 21, MPG 35

p. $1085,29:$ p. 63 , p. $1125,20:$ p. 67

Or. 24, MPG 35

p. 1173,18 : p. 17 , p. 1177,42 : p. 13

Or. 25, MPG 35

p. $1212,24:$ p. $18, \quad$ p. $1280,24:$ p. 66

Or. 27, 9, 12 (ed. Barbel): p. 14

Or. 28, 20, 1 (ed. Barbel): p. 78

Or. 29 (ed. Barbel)

1, 2: p. 51, 13, 1: p. 13

18, 9: p. 38

Or. 31 (ed. Barbel)

10, 1: p. 57 , cf. $23,7:$ p. 46

28, 11: p. 52

Or. 33, MPG 36

$\begin{array}{ll}\text { p. } 216,37: \text { p. } 24, & \text { p. } 217,29: \text { p. } 31\end{array}$

$\begin{array}{ll}\text { p. } 224,36: \text { p. } 38, & \text { p. } 228,7: \text { p. } 29\end{array}$

p. $233,1:$ p. 67

Or. 38, MPG 36

p. $312,48:$ p. 24 , p. 64

p. $312,50:$ p. 60 , p. $313,7:$ p. 67

p. $313,25:$ p. 25 , p. $316,3:$ p. 78

p. $317,35:$ p. 52 , p. $317,41:$ p. 10

p. $320,44:$ p. $33, \quad$ p. $324,7:$ p. 15

p. $324,39:$ p. $22, \quad$ p. $325,16:$ p. 22

p. $325,19:$ p. 25

Or. 39, MPG 36

p. $337,28:$ p. $27, \quad$ p. $337,33:$ p. 54

p. 356,28 : p. 34

Or. 40, MPG 36

p. $364,27:$ p. 33 , p. $377,26:$ p. 69

Or. 42, MPG 36

p. $457,5:$ p. $49, \quad$ p. $465,35:$ p. 26

p. $469,31:$ p. 17 , p. $472,28:$ p. 10

$\begin{array}{ll}\text { p. } 472,45: \text { p. } 12, & \text { p. } 477,36: \text { p. } 69\end{array}$ 
Or. 43 (ed. Boulenger)

1, 1: p. 72, 7, 5: p. 62

15, 4: p. 57, 19, 3: p. 11

20,3: p. 10, 22, 2: p. 24

41, 1: p. 53, 54, 1: p. 18

58, 4: p. 28

Or. 44, MPG 36 p. 617, 13: p. 37

Or. 45, MPG 36

p. $624,29:$ p. 46 , cf. p. 640,39

Frag. inc.: p. 14, p. 15

(Ps.) Hermogenes

ПEPI METHO $\Delta \mathrm{OY} \Sigma \Delta$ EINOTHTO $\Sigma$

p. 25,24 (Speng. RG III) p. 25

Herodianus

De figuris p. 98, 30- 99, 2 (Speng. RG III) p. 67

Herodotus

Historia 1

6 p. $75,10-13 \quad 66$ p. $23,15-16$
$16.250-252$ p. $51,13-15$

17.542 p. $72,3-4$

20. $371-372$ p. $22,5-6$, p. 46

Odyssea

1.1 p. 59,11

1. $22-23$ p. $23,3-4$

2. 409 p. 54

3.385 p. 54,4

$6.43-45$ p. 64

8. $223-224$ p. $14,9-10$

9. 481 p. 3,3

11.310 p. 14

$16.17-19$ p. $17,12-14$

Horatius

Carmina $3.212-13$ p. 37

Hyperides

In Demosthenem Frag. 2.3 p. 38, 3-7

Frag. inc. 173 p. 38, 8-9

fort. Frag. inc. p. 68, 1-4

Isocrates

Helenae encomium

17 p. $64,3-5$ 17 p. $76,4-6$

Panegyricus

1 p. $44,1-3$, p. $63,3-5 \quad 185$ p. $44,6-9$

Frag. 12 (Orat. Att. I p. 321) p. 70, 4-5

Ps. Longinus

De Sublimitate 12, 38 p. 41

Menander

PCG VI 2 Frag. inc. 420 p. 18, 5-19, 3

PCG VI 2 Frag. inc. 707 p. 69, 4 
PCG VIII Adesp. Frag. 75 p. 69, 6

Philistos

FGrHist. 556 F56 bis p. 51, 7-10

FGrHist. 556 F58 bis p. 59, 14-15

Quintilianus

Institutiones Oratoriae 8, 4. 29, 4.15, 6. 67-76 p.

41

Iulius Rufinianus

De figuris I 38 p. 41

Sophocles

Electra 301 p. $25,5 \quad 1163-1164$ p. 23, 13-14

Philoctetes 817 p. 72,8

Thucydides

Historiae

1.1.2 p. $55,4-5 \quad 1.2 .2$ p. 60,5

1. 6.1 p. $58,6 \quad 120.2$ p. $60,6-7$

1. 31.2 p. $73,6 \quad 1.68 .2$ p. $54,10-11$

2. 44.4 p. $58,8 \quad$ 2. 53.1 p. $59,12-14$

2. $62.3-4$ p. $66,9-10 \quad 3.14 .1$ p. 72,6

5.41 .2 p. $58 \quad 7.15$ p. $3,11-12$

Tryphon

De Tropis $\alpha^{\prime}-\gamma^{\prime}$ p. 41

\section{Xenophon}

Memorabilia 1.2 .25 p. 46, 6-7, p. 50

Cyropaedia 1.1 .1 p. $54,9 \quad 8.2 .8$ p. $61,4-7$

Fragmenta incerta
p. $12,5-10$ p. $25,8-9$
$\begin{array}{lll}\text { p. } 40,5-6 & \text { p. } 43,4-5 & \text { p. } 55\end{array}$
p. 59 p. $63,10-12$ p. $66,5-6$ p 66
p. $67,4-5,5-6 \quad$ p. $71,4-5,5$ p 72, 5
p. $77,8-9$ 
INDEX NOMINUM ET VERBORUM POTIORUM

'A $\gamma \alpha \mu \varepsilon \dot{\varepsilon} \mu \omega \nu$ p. 31, 9; p. 53, 4

'A $\gamma \lambda \alpha i \alpha$ p. 25, 3

'A $\theta \eta \nu \hat{\alpha}$ p. 18, 5

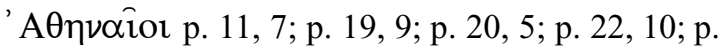
23,1 ; p. 26,11 ; p. 27,7 ;p. 44, 7;p. 46, 5; p. 72, 4, 6,$9 ;$ p. $77,8,9$

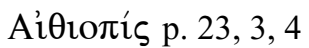

Aíxínns p. 11, 7; p. 13, 9; p. 20, 7; p. 23, 1; p. 25,9 ; p. 26, 4;p. 36, 5; p. 39, 1; p. 48, 6, 7 (bis);

p. 49,4 ; p. 64,5 ; p. 65,3 ; p. 69,7 ; p. 72,4

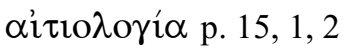

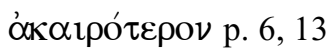

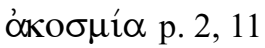

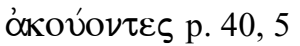

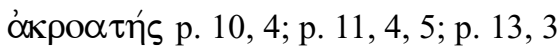

A $\lambda \varepsilon^{\prime} \xi \alpha \nu \delta \rho o \varsigma$ p. 23, 7;p. 63, 10

A $\lambda$ кívous p. 54, 4

ò $\lambda \lambda \alpha \gamma \eta ́$ p. $58,1,2 ;$ p. 59, 5; p. 60, 1; p. 75, 4

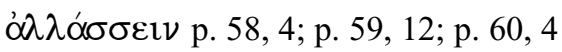

ò $\lambda \lambda \eta \gamma о \rho i \alpha$ p. 7, 17

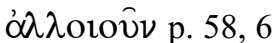

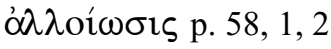

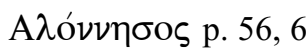

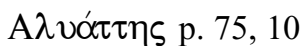

"A $\lambda$ vs p. 75, 11, 16

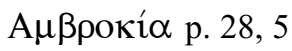

ó $\mu \phi 1 \delta$ เó $\rho \theta \omega \sigma \iota \varsigma$ p. 12, 1

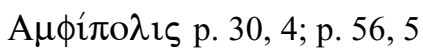

ó $\alpha \delta i ́ \pi \lambda \omega \sigma \imath \varsigma$ p. 23,$10 ;$ p. 46, 1, 3

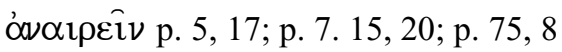

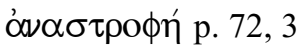

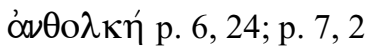

ó $\tau \varepsilon ı 1 \sigma \alpha \gamma \omega \gamma \eta \dot{~ p . ~ 37, ~ 1, ~ 2 ; ~ p . ~ 71, ~ 1, ~} 2$

ó $\tau \varepsilon \nu \alpha \nu \tau i \omega \sigma l \varsigma$ p. 71

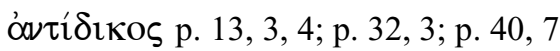

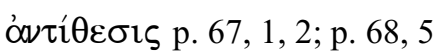

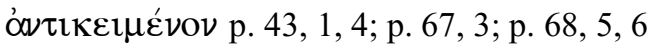

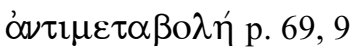

ó $\nu \imath \iota \varepsilon \tau \alpha ́ \theta \varepsilon \sigma ı$ p. 69, 1

ó $\tau \tau \imath \pi \alpha \rho o ́ \theta \varepsilon \sigma ı \varsigma$ р. 19, 18

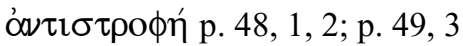

ó

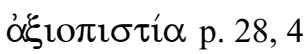

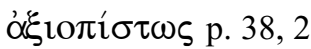

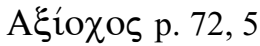

фं $\pi \alpha \nu \tau \hat{\jmath} \sigma \alpha \iota$ p. 35, 2

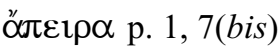

ó $\pi \varepsilon \rho i ́ \lambda \eta \pi \tau \alpha$ p. 1, 7

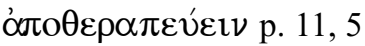

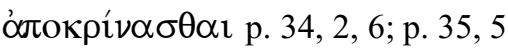

А

о่лорєîv p. 6, 27; p. 7, 4, 5(bis); p. 33, 4; p. 43, 5

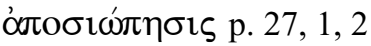

ф́лобтрофи́ p. 31, 1, 2; p. 32, 1

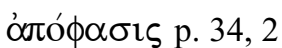

Арßи́коr p. 63, 11

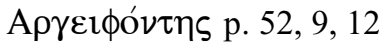

Apros p. 58, 11

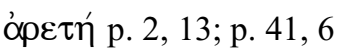

"A $\theta$ $\theta$ ios p. 21, 7; p. 25, 14

öp $\theta \rho o v$ p. 25, 6

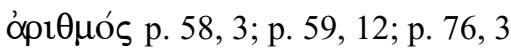

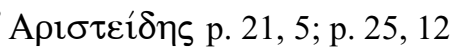

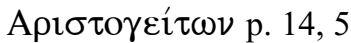

Арк $\alpha \delta i \alpha$ p. 12, 12; p. 23, 15

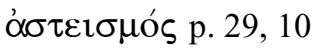

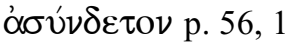

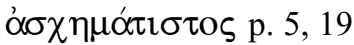

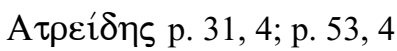

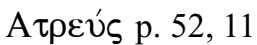




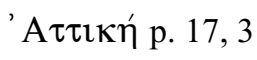

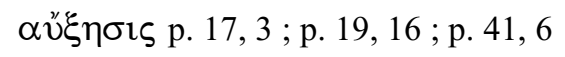

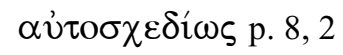

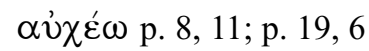

'A $\chi \alpha$ loí p. 31, 4, 6; p. 71, 8

'A $\chi \imath \lambda \lambda \varepsilon v \dot{s}$ p. 51, 12

óффoı

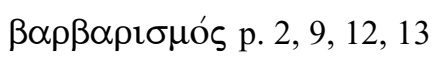

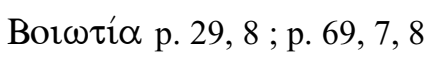

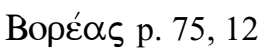

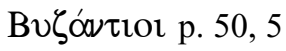

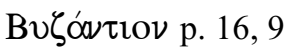

ГЕ

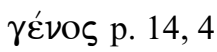

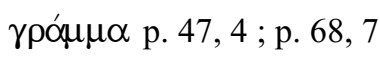

Граиıко́ р. 63, 11

$\Delta \alpha \rho \varepsilon i o s$ p. 63, 11

$\Delta \varepsilon \lambda \phi$ oí p. 36,9

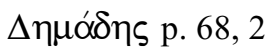

$\Delta \eta \mu о \sigma \theta \varepsilon ́ v \eta$ p. 9,$10 ;$ p. 11, 6; p. 13, 9; p. 15, 3;

p. 16,$3 ;$ p. 17,6 ; p. 23,6 ; p. 24,3 ; p. 26,4 ; p. 27 ,

4; p. 29, 7; p. 30, 3; p. 32, 4; p. 34, 4; p. 36, 9; p.

38,$3 ;$ p. 39,4 ; p. $40,3,8$; p. 48,5 ; p. 50,4 ; p. 51 ,

4; p. 54,5 ; p. 68,7 ; p. 72,4 ; p. 78,4

$\Delta \eta \mu \sigma \theta \varepsilon v i \kappa o ́ v$ p. 10,$4 ;$ p. 12, 10; p. 20, 4; p. 25,

7; p. 28,$4 ;$ p. 48,$3 ;$ p. 52,$4 ;$ p. 58,$9 ;$ p. 61,7 ; p.

62,$3 ;$ p. 63,$6 ;$ p. 65,$3 ;$ p. 66,$6 ;$ p. 74,2

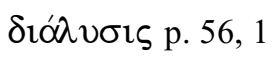

$\delta$ iovón $\mu \alpha$ p. 5, 12; p. 6, 7; p. 42, 3, 4 ; p. 45, 3

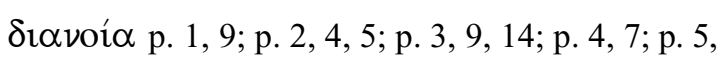
$3,10,11,12,13,15,16,18 ;$ p. 7,$2 ;$ p. 41,$2 ;$ p. 42 ,

2,$10 ;$ p. 45,$2 ;$ p. $55,4,7$; p. 57,3 ; p. 66,$2 ;$ p. 75 , 6,9

$\delta$ ió $\pi \lambda \alpha \sigma i \varsigma$ p. 20, 2

$\delta 1 \alpha \pi \lambda \varepsilon \dot{\kappa} \kappa \iota v$ p. 66, 11

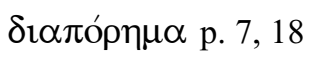

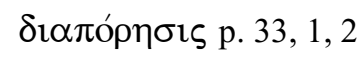

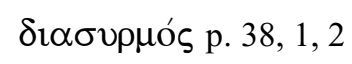

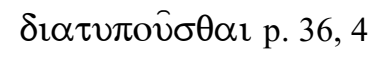

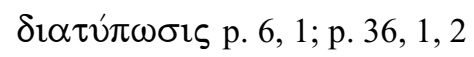

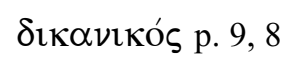

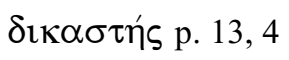

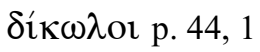

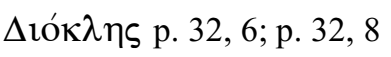

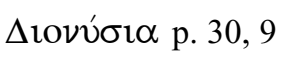

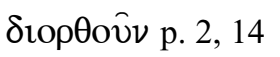

$\Delta$ орі́бко р. 30,5

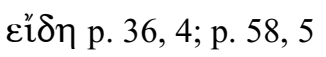

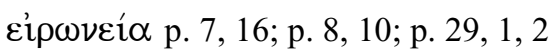

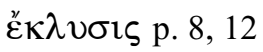

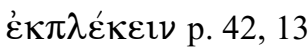

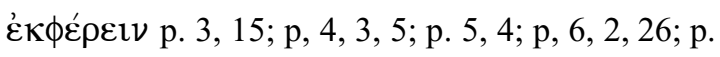

42, 10; p. 45, 2, 3; p. 77, 2

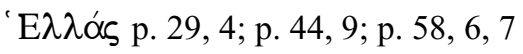

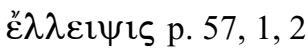

"Е $\lambda \lambda \eta \nu \varepsilon \varsigma$ p. 21, 6; p. 25, 13;p. 39, 5; p. 55, 5; p.

58,$7 ;$ p. $73,3,9$

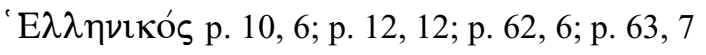

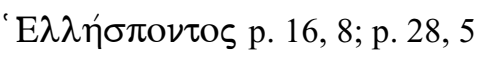

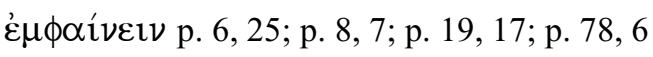

$\varepsilon ้ \mu \phi \alpha \sigma i \varsigma$ p. 8,15 ; p. 9,$5 ;$ p. 13,6 ; p. 41,6 ; p. 55 ,

3,$8 ;$ p. 57,8

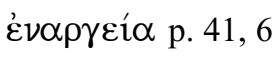

غ่v $\alpha \rho \gamma \eta \dot{\eta} \mu \alpha \alpha$ p. 36, 4

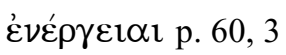

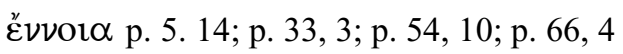

غ̇ $\xi \alpha \alpha \lambda \alpha \xi i \varsigma$ p. 5, 2, 3, 5, 8

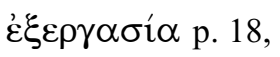

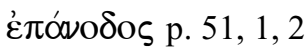

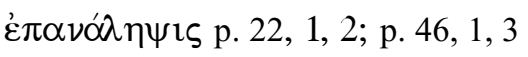

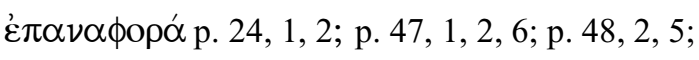

p. 49,2 


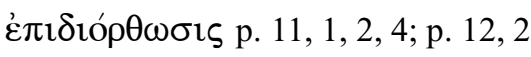

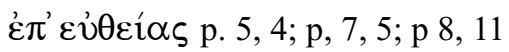

غ̇лцоой́ p. 17, 1, 2, 3; p. 28, 3

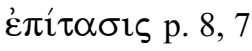

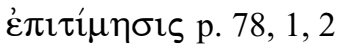

$\dot{\varepsilon} \pi \mathrm{\imath} \tau \rho \chi \chi \alpha \sigma \mu o ́ \varsigma$ p. 28, 1,2

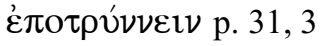

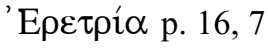

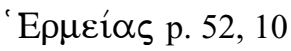

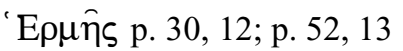

غр

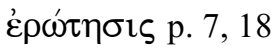

$\dot{\varepsilon} \sigma \chi \eta \mu \alpha \tau i \zeta \xi \iota \nu$ p. $6,15,19,20 ;$ p. 7, 12

Ev้ßor $\alpha$ p. 16, 4

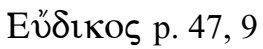

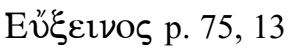

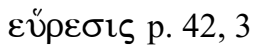

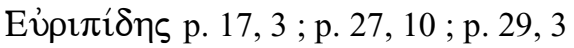

Eủpußióons p. 77, 9

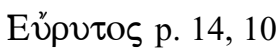

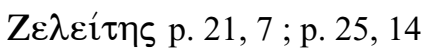

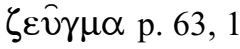

Zzús p. 14, 6; p. 18, 11; p. 52, 8, 9, 12; p. 78, 9

ทंठovท́ p. 9, 7 ; p. 59, 13

$\eta \dot{\eta} \theta$ oлotí $\alpha$ p. 26, 1, 2

${ }^{\top} \mathrm{H} \lambda \mathrm{i \varsigma}$ p. 28,5

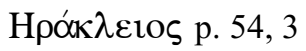

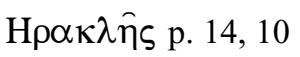

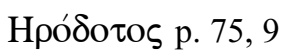

${ }^{\prime} \mathrm{H} \phi \alpha 1 \sigma \tau \mathrm{s}$ p. 52, 8

$\Theta \varepsilon \mu \iota \sigma \tau о \kappa \lambda \bar{\eta} \varsigma$ p. 77, 9

$\theta \varepsilon \rho \alpha \pi \varepsilon i ́ \alpha$ p. 10,3

$\theta \varepsilon \rho \alpha \pi \varepsilon v ́ \varepsilon \iota v$ p. 11,3

$\Theta \varepsilon \tau \tau \alpha \lambda i ́ \alpha$ p. 12,$13 ;$ p. 47.10

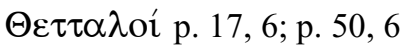

$\Theta \hat{\eta} \beta \alpha \imath$ p. 69, 7, 8
$\Theta \eta \beta \alpha$ iou p. 17, 7; p. 29, 8; p. 50, 7; p. 52, 6

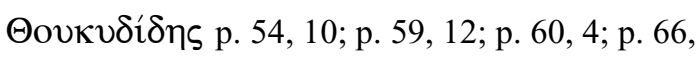
9; p. 72, 6; p. 73, 2

"I $\delta \eta$ p. 3, 3

' Ібокрó́tпs p. 44, 6; p. 70, 3

'Ібократıко́v p. 63, 4; p. 64, 3; p. 76, 4

'I $\sigma \sigma o ́ \varsigma$ p. 63, 11

'Iфıкрó́tпs p. 32, 5; p. 32, 7; p. 32, 9

каıрós p. 6, 13; p. 20, 4, 6; p. 70, 4, 5

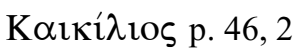

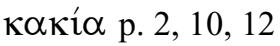

K $\alpha \lambda \lambda i ́ \sigma \tau \rho \alpha \tau o \varsigma$ p. 55, 7

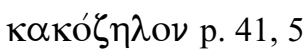

$\kappa \alpha \tau \alpha \beta о \lambda \eta ́$ p. 8,11

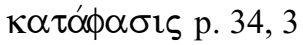

Керкираîoเ p. 73, 2

кєфód $\alpha$ lov p. 16, 3; p. 52, 3

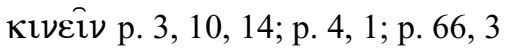

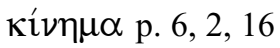

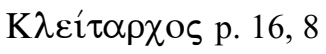

$\kappa \lambda \hat{\imath} \mu \alpha \xi$ p. 52, 1, 2, 7, 13

ко́ $\mu \mu \alpha$ p. 42, 7; p. 43, 7, 8; p. 52, 3; p. 62, 3

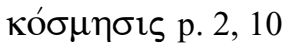

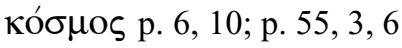

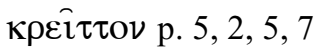

Kpoĩ $\sigma o s$ p. 75, 10, 13, 15

Kpovíwv p. 52, 8, 12

кúpios p. 3, 2, 6

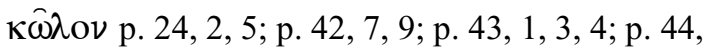
$13 ;$ p. $47,2,6,11 ;$ p. 49,$3 ;$ p. 56,$3 ;$ p. 62,$3 ;$ p. 63 , 5, 9(bis); p. 65,$6 ;$ p. 70,$1 ;$ p. 76, 2

$\Lambda \alpha \kappa \varepsilon \delta \alpha$ uóviol p. 44, 8; p. 73, 5; p. 77, 8(bis)

$\Lambda \alpha \rho i \sigma \alpha i o s$ p. 47,10

$\Lambda \alpha \sigma \theta \dot{v} v \eta$ s p. 47,8

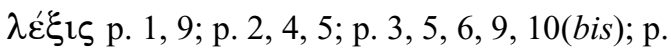

4.2 ; p. $5,2,9,10,14,15 ;$ p. $7,19,20 ;$ p. $8,1,3$; 
p. $42,2,5,6 ;$ p. 52,$3 ;$ p. 69,$2 ;$ p. 73,$1 ;$ p. 74,$1 ;$ p.

77,4

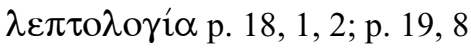

$\lambda \varepsilon \pi \tau o ́ v$ p. 19,5

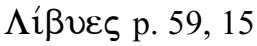

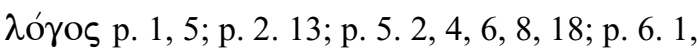
4, 6, 18 (bis), 21; p. 7, 1, 7, 9, 11, 12, 13; p. 8, 6, 11 ; p. 9, 6, 7, 8, 9, 12, 15; p. 12, 4; p. 13, 4, 9; p. 14,4 ; p. 19,5 ; p. 21 , 1; p. 26, 2; p. 27, 2; p. 29, 2 ; p. 32,2 ; p. 36,3 ; p. 38,6 ; p 42, 2, 3, 4, 5, 7; p. 44, 14 ; p. 48,8 ; p. 53, 2; p. 53, 5, 7; p. 55, 3, 6; p. 56, 3 ; p. 61, 4; p.65, 5; p. 66, 8; p. 68, 5; p. 75, 3, 4; p. $75,15,16 ;$ p. 76,8

$\Lambda$ vós p. 75.10

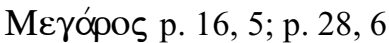

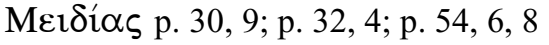

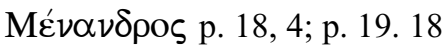

$\mu \varepsilon \tau \alpha \beta \alpha_{\alpha} \lambda \lambda \varepsilon \imath \nu$ p. 2,$15 ;$ p. 9,$15 ;$ p. 58, 6

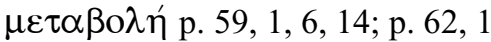

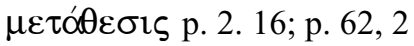

$\mu \varepsilon \tau \alpha \kappa \imath v \varepsilon \hat{\imath} \nu$ p. 2,17

$\mu \varepsilon \tau \alpha \lambda \alpha \mu \beta \alpha^{\prime} \varepsilon \imath \nu$ p. 61,3

$\mu \varepsilon \tau o ́ \sigma \lambda \alpha \sigma i \varsigma$ p. 5,12

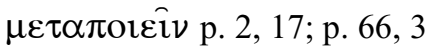

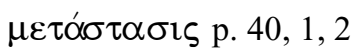

$\mu \varepsilon \tau \alpha \phi \varepsilon ́ \rho \varepsilon \imath \nu$ p. 59, 2

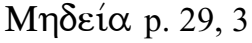

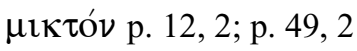

$\mu \iota \mu \hat{\imath} \sigma \theta \alpha \iota$ p. 7,5

$\mu i ́ \mu \eta \mu \alpha$ p. 6,6

$\mu i ́ \mu \eta \sigma$ เs p. 6,23 ; p. 7, 1; p. 8, 1

Mov̂ $\sigma \alpha$ p. 59,11

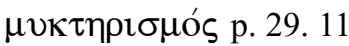

Nipev́s p. 25, 2, 3

vóๆ $\mu \alpha$ p. 19, 4

vôิs p. $51,3,4 ;$ p. 75, 7
$\Xi \varepsilon \nu 0 \phi \hat{\omega} \nu$ p. 46,6 ; p. 54,9 ; p. 61,4

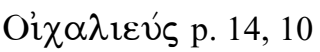

"О $\lambda v \nu \theta 0 \varsigma$ p. 47, 9

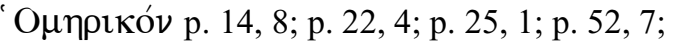

p. $53.3 ;$ p. 50,$10 ;$ p. 71,7 ; p. 77,4

"O $\mu \eta p o s$ p. 51, 11

ómoı́́ $\tau \tau \omega \tau$ ov p. 65, 1, 2

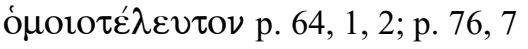

óvo $\mu \alpha$ p. 2, 9, 10, 14, 15(bis); p. 3, 4, 14, 15; p. 4, 7 ; p. 17,2 ; p. 24,2 , 5; p. 50,2 ; p. 51,2 ; p. 52,13 ; p. 53,$2 ;$ p. 57,$2 ;$ p. $58,4,5 ;$ p. 61,$2 ;$ p. 65,$2 ;$ p. 66,$3 ;$ p. 68,$1 ;$ p. 70,$1 ;$ p. 71,$2 ;$ p. 74,$2 ;$ p. 77,2 ; p. 78,6

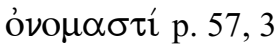

ópos p. 5, 1, 9

$\pi o ́ \theta \eta$ p. 6, 4;p. 58, 5; p. 60, 3

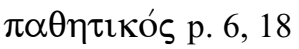

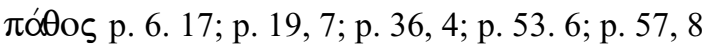

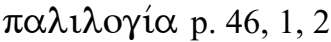

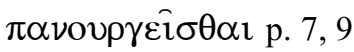

$\pi \alpha \rho \alpha \kappa \varepsilon i ́ \mu \varepsilon v o v$ p. 43, 2, 6

$\pi \alpha \rho o ́ \lambda \varepsilon \imath \psi i \varsigma$ p. 30, 1, 2

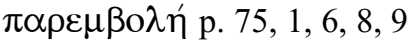

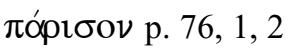

$\pi \alpha \rho \circ \nu^{\prime} \mu \alpha \sigma i \alpha$ p. 3, 14; p. 66, 1, 2

$\pi \alpha \rho p \eta \sigma i ́ \alpha$ p. 10, 8; p. 12, 3; p. 59, 5

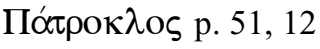

П $\alpha \phi \lambda \alpha \gamma o ́ v \varepsilon \varsigma$ p. 75, 12

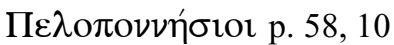

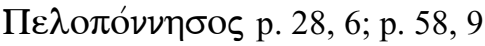

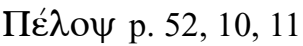

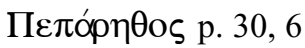

$\pi \varepsilon \rho i ́ \gamma \rho \alpha \phi о \varsigma$ р. 42, 9

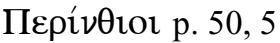

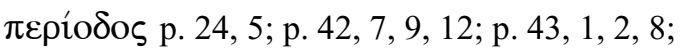

p. $44,1.11,13,14 ;$ p. 45,1 ; p. 70,1 
$\pi \varepsilon \rho \imath \pi \lambda \varepsilon_{\kappa} \kappa \varepsilon \iota \nu$ p. 66,11

$\pi \varepsilon \rho i ́ \phi \alpha \sigma ı \varsigma$ p. 54, 1, 2, 8

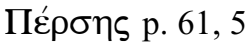

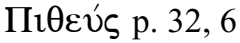

$\pi \lambda \alpha ́ \sigma ı s$ p. 6, 11; p. 7, 21, 22

$\pi \lambda \alpha \tau \tau \varepsilon i \mathrm{i} \nu$ p. 5,$13 ;$ p. 7, 9, 10; p. 9, 12

$\pi \lambda \varepsilon_{\varepsilon} \kappa \varepsilon \imath v$ p. 42,$8 ;$ p. 61,4

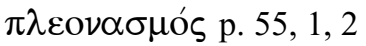

$\pi \lambda \hat{\eta} \theta$ os p. 1, 6; p. 59, 14

$\pi \lambda$ окฑ́ р. 69, 1

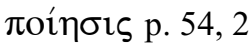

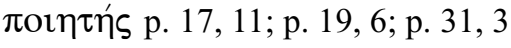

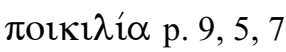

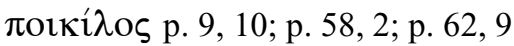

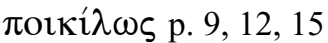

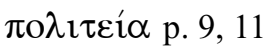

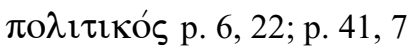

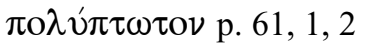

Порөно́s p. 10, 6

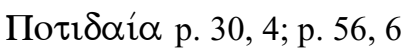

$\pi \rho o ́ \gamma \mu \alpha$ p. 3, 6, 15; p, 8, 7; p. 9, 15; p. 18, 6;

p. 19,12 ; p. 23,2 ; p. 25,7 ; p. 26, 8; p. 33, 2;

p. 36,2 ; p. 39,5 ; p. 40,3 ; p. 66,6 ; p. 68,6

$\pi \rho \alpha v ́ v \varepsilon \imath v$ p. 31,3

$\pi \rho \circ \delta$ เó $\theta \omega \sigma \iota \varsigma$ p. 4, 5; p. 10, 1;

p. $11,2,4 ;$ p. 12,2

$\pi \rho о \theta \varepsilon \rho \alpha \pi \varepsilon v ́ \varepsilon \iota v$ p. 10,4

$\pi \rho о \kappa \alpha \tau \alpha \lambda \alpha \mu \beta \alpha_{\nu \varepsilon \imath \iota}$ р. 13, 2

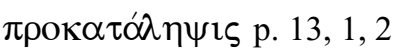

$\pi$ покеí $\mu \varepsilon \nu \alpha$ p. 14, 3

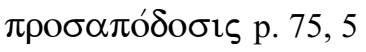

$\pi \rho \circ \sigma \delta 1 \alpha \sigma \alpha ́ \phi \eta \sigma ı \varsigma$ р. 53, 1, 2

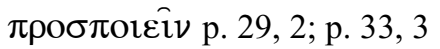

$\pi \rho \circ \sigma \imath \theta \varepsilon \dot{v} \alpha \imath$ p. 4, 1

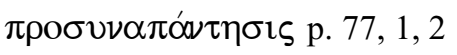

$\pi \rho o ́ \sigma \omega \pi$ ov p. 14, 4; p. 20, 2, 6; p. 26, 2; p. 31,
2 ; p. 36, 2; p. 59, 1

$\pi \rho \circ \sigma \omega \pi$ oлolí $\alpha$ p. 20, 1, 2

$\pi \tau \hat{\omega} \sigma ı \varsigma$ p. 58,$4 ;$ p. 59,$5 ;$ p. 61,$3 ; 65,2,6$

$\Pi v ́ \delta v \alpha$ p. 30,$4 ;$ p. 56,5

$\pi v ́ \sigma \mu \alpha$ p. 7, 18; p. 35, 1, 2

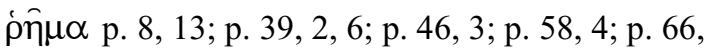

$3 ;$ p. 75,5

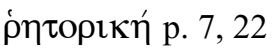

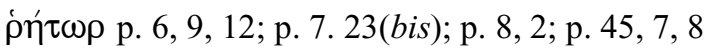

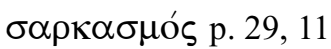

¿غ́pplos p. 30, 5

$\Sigma \imath \kappa \varepsilon \lambda i ́ \alpha$ p. 30, 13

$\Sigma$ ıиós p. 47,9

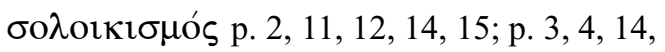

$15 ;$ p. 4,$7 ; 17,2$

$\Sigma o ́ \lambda \omega \nu$ p. 21, 4; p. 25, 11

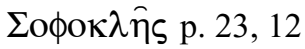

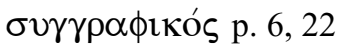

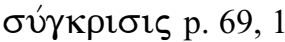

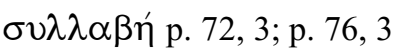

$\Sigma v ́ \mu \eta \theta \varepsilon v$ p. 25,2

$\Sigma v \mu \mu \varepsilon \tau \rho i ́ \alpha$ p. 44, 14

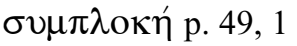

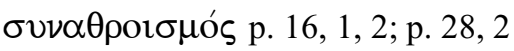

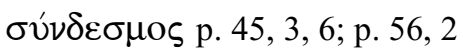

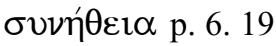

б৩vท́⿴囗十 р. 2, 15

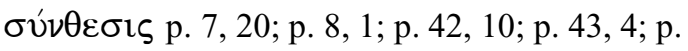

49, 1

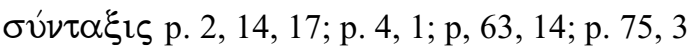

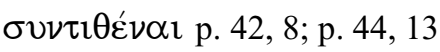

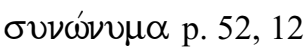

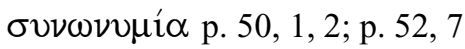

$\Sigma$ úpot p. 75,12

$\sigma \chi \hat{\eta} \mu \alpha$ p. 1, 6, 7, 9; p. 2, 6, 8, 10, 13, 16; p. 3, 1, $4,8,9,10,14 ;$ p. 4, 2, 7; p. 5, 1, 2, 4, 6, 10,11(bis), 


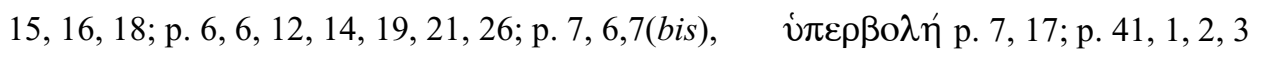

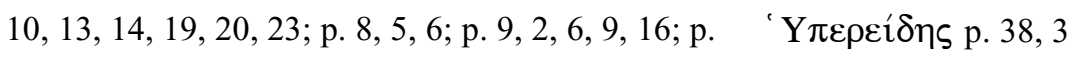

14, 2; p. 19, 17; p. 28, 2; p. 41, 2; p. 42, 1, 2, 5, 6;

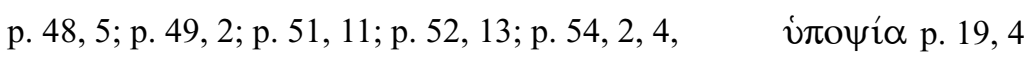

12 ; p. 56,$2 ;$ p. 57,8 ; p. 58,2 ; p. 62,$2 ;$ p. 63,$2 ;$ p. $\quad \Phi i ́ \lambda ı \pi \pi$ os p. 17,7 ; p. 22, 12; p. 26, 10; p. 47,

$69,2,9 ;$ p. 75,$7 ;$ p. 76,$8 ;$ p. $78,3 \quad 10 ;$ p. 68,2

$\sigma \chi \eta \mu \alpha \tau i \zeta \varepsilon ı v$ p. 59,6

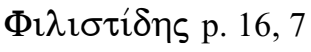

$\sigma \chi \eta \mu \alpha \tau \iota \sigma \mu o ́ s$ p. 6,3

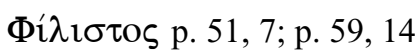

Tó $\alpha \tau \alpha \lambda \circ$ p. 38, 8

$\tau \chi_{\alpha}^{\prime} \xi 1 \varsigma$ p. 11, 2; p. 12, 11; p. 44, 15

Фрúvๆ p. 38, 8

Тع $\varepsilon \varepsilon ́ \alpha$ p. 23, 16

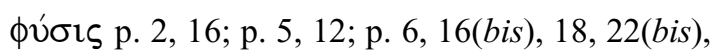

24, 26; p. 7, 8 (bis), 13, 15; p. 8, 1, 3; p. 64, 4;

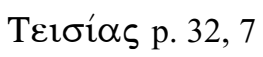

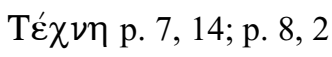

p. 73,$1 ;$ p. 75,$3 ;$ p. 76,6

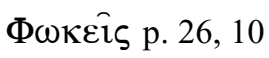

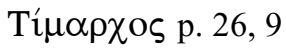

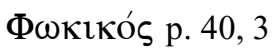

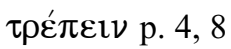

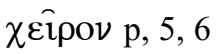

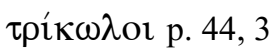

$\chi \lambda \varepsilon v \alpha \sigma \mu$ ós p. 29, 11

троли́ р. 3, 2

$\chi \rho \varepsilon i ́ \alpha$ p. 5, 14; p. 8, 4, 6; p. 11, 2

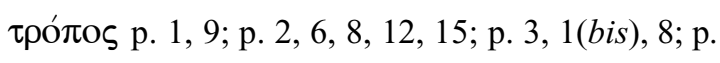

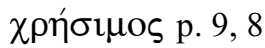

$5,3,8 ;$ p. 7, 10; p. 8,$6 ;$ p. $9,7,16 ;$ p. 14, 3; p. 58,

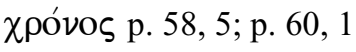

$3 ;$ p. 60,$1 ;$ p. 64,$6 ;$ p. 67,$2 ;$ p. 68,$4 ;$ p. 78,3

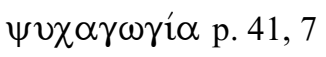

نं $\alpha \lambda \lambda \alpha \gamma \eta \dot{~ p . ~ 78, ~} 2$

భvхท́ p. 6, 1, 3, 5, 6, 15, 16; p. 22, 10; p. 53, 5, 7

vं $\pi \xi \alpha i ́ p \varepsilon \sigma i \varsigma$ p. 14, 1, 11

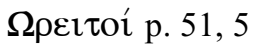

vं $\varepsilon \dot{\rho} \beta \alpha \sigma 1 \varsigma$ p. 75.6

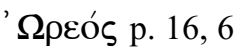

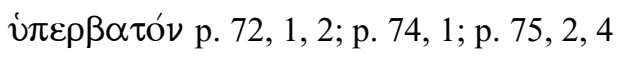

